\title{
Natural Gas Annual 1994
}

\section{November 1995}

\author{
Energy Information Administration \\ Office of Oil and Gas \\ U.S. Department of Energy \\ Washington, DC 20585
}

DISTARUTION OF THIS DOCUMENT IS UMUTED

a

This report was prepared by the Energy Information Administration, the independent statistical and analytical agency within the Department of Energy. The information contained herein should not be construed as advocating or reflecting any policy of the Department of Energy or any other organization. 


\section{Contacts}

The Natural Gas Annual is prepared by the Energy Information Administration, Office of Oil and Gas, Reserves and Natural Gas Division, under the direction of Joan E. Heinkel.

General information for this document may be obtained from Kendrick E. Brown, Jr. (202/586-6077), Chief of the Data Operations Branch. Questions and comments con- cerning the contents of the Natural Gas Annual may be directed to Ann M. Ducca (202/586-6137) and Margo Natof (202/586-6303). Specific inquiries should be referred to the following subject specialists: Supply -Donna Guerrina (202/586-6135), Transmission -- Dolly Tolson (202/586-6664), Demand -- Sylvia Norris (202/586-6106), and Electronic versions -- Sheila Darnell (202/586-6165). 


\section{DISCLAIMER}

Portions of this document may be illegible in electronic image products. Images are produced from the best available original document. 


\section{Preface}

The Natural Gas Annual provides information on the supply and disposition of natural gas to a wide audience including industry, consumers, Federal and State agencies, and educational institutions. The 1994 data are presented in a sequence that follows natural gas (including supplemental supplies) from its production to its end use. This is followed by tables summarizing natural gas supply and disposition from 1990 to 1994 for each Census Division and each State. Annual historical data are shown at the national level.

Much of the analysis in this publication is taken from Natural Gas 1995: Issues and Trends, which was published in November 1995. This analysis report discusses natural gas prices, recent developments in natural gas supply, restructuring of the interstate pipeline industry, deliverability on the transmission network, the role of underground storage, end-use markets, and major influences on gas industry finances.

The data in the Natural Gas Annual 1994 are taken from surveys conducted by the Energy Information Administration (EIA), U.S. Department of Energy (DOE), to fulfill its responsibilities for gathering and reporting energy data. Two EIA surveys provide most of the information presented in this report--the mandatory Form EIA-176, "Annual Report of Natural and Supplemental Gas Supply and Disposition," and the voluntary Form EIA-627, "Annual Quantity and Value of Natural Gas Report." Form EIA-176 was submitted by respondents out of an identified universe of operators of fields, wells, or natural gas processing plants who distribute gas to end users or transport gas to or across a State border; operators of synthetic natural gas plants; natural gas distributors; natural gas pipeline companies; and companies that operate underground natural gas storage facilities. Form EIA-627 was submitted by the appropriate agencies of the 33 natural gas producing States.

Data collected on Form EIA-176 are not proprietary. Selected company-level data are presented in the Demand section.
Other EIA surveys that provided information for this report are Forms EIA-816, "Monthly Natural Gas Liquids Report," and EIA-64A, "Annual Report of the Origin of Natural Gas Liquids Production," for gas processed, plant fuel, and extraction loss data; Form EIA-857, "Monthly Report of Natural Gas Purchases and Deliveries to Consumers," for data on the city gate prices; Forms EIA-759, "Monthly Power Plant Report," and FERC-423, "Monthly Report of Cost and Quality of Fuels for Electric Plants," for data on the quantity and price of natural gas consumed by electric utilities; and Form FPC-14, "Annual Report for Importers and Exporters of Natural Gas," for data on the quantity and price of natural gas imports and exports. The EIA report, U.S. Crude Oil, Natural Gas, and Natural Gas Liquids Reserves, 1994 Annual Report, was the source of the reserves data.

Some of the data are collected under the authority of the Federal Energy Regulatory Commission (FERC), an independent commission within the DOE that has jurisdiction primarily in the regulation of electric utilities and the interstate natural gas industry. The United States Geological Survey (USGS) and the United States Minerals Management Service (MMS) of the Department of the Interior, and the Interstate Oil and Gas Compact Commission (IOGCC) were sources of supplemental information on production, the number of producing gas and gas-condensate wells, and wellhead values. The geographic coverage is the 50 States and the District of Columbia.

All volumes of natural gas in this publication are reported at 14.73 pounds per square inch absolute and 60 degrees Fahrenheit, except where noted. A glossary of terms is provided to assist users in understanding the data presented. A description of the data collection surveys appears in Appendix A. Appendix $B$ describes metric and thermal conversion factors, and Appendix $\mathrm{C}$ lists additional natural gas information sources.

The tables of data that appear in this report are available on personal computer diskettes and the Internet. See the inside cover for further details. 


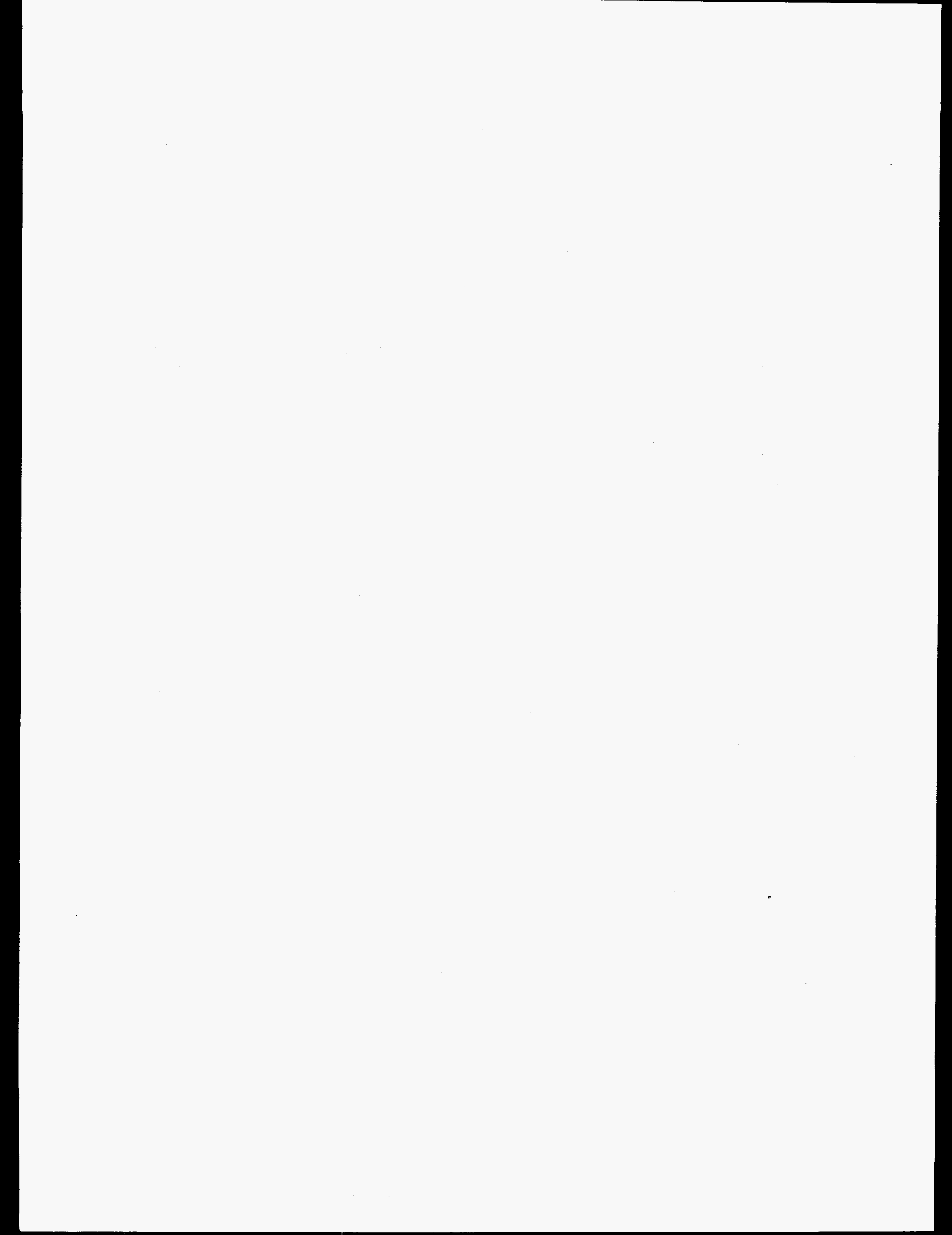




\section{Contents}

Page

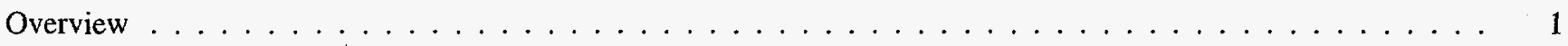

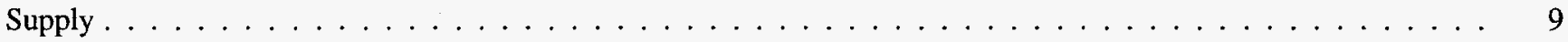

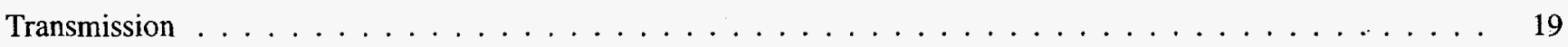

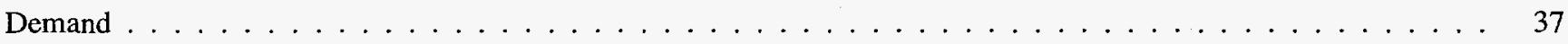

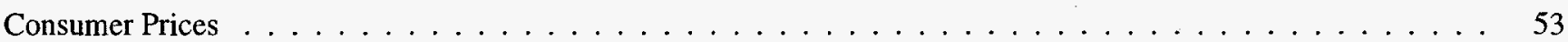

Census Division Summary $\ldots \ldots \ldots \ldots \ldots \ldots$

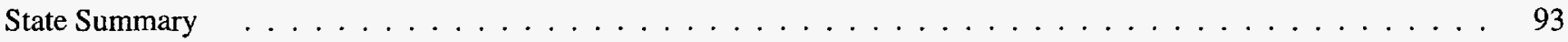

Historical Data . . . . . . . . . . . . . . . . . . . . . . . . . . . . . . 199

Appendices

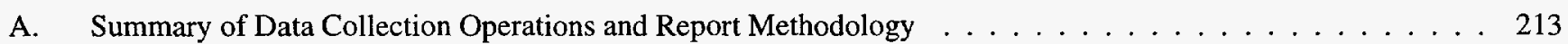

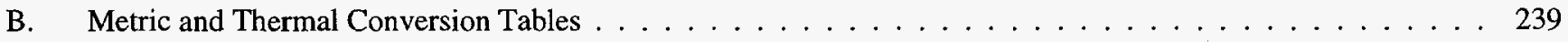

C. Selected Natural Gas and Related Reports . . . . . . . . . . . . . . . . . . . . . . 245

Glossary . . . . . . . . . . . . . . . . . . . . . . . . 247 


\section{Tables}

Page

1. Summary Statistics for Natural Gas in the United States, 1990-1994 . . . . . . . . . . . . . . . . . . . . . . . 4

2. Natural Gas Production, Transmission, and Consumption by State, $1994 \ldots \ldots \ldots \ldots \ldots$

3. Gross Withdrawals and Marketed Production of Natural Gas by State,1990-1994 . . . . . . . . . . . . . . . . 13

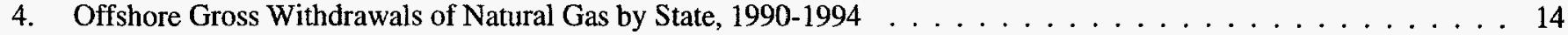

5. Number of Producing Gas and Gas Condensate Wells by State as of December 31, 1990-1994 . . . . . . . . . . . 15

6. Estimated Total Dry Natural Gas Proved Reserves by State, 1990-1994 . . . . . . . . . . . . . . . . . . . . . . . 16

7. Wellhead Value and Marketed Production of Natural Gas by State, 1990-1994 . . . . . . . . . . . . . . . . . . 17

8. Natural Gas Processed, Liquids Extracted, and Estimated Extraction Loss by State, $1994 \ldots \ldots$

9. Interstate Movements and Movements Across U.S. Borders of Natural Gas by State, 1994 . . . . . . . . . . . . . . 24

10. Summary of U.S. Natural Gas Imports and Exports, 1990-1994 . . . . . . . . . . . . . . . . . . . . . . . . 32

11. Additions to and Withdrawals from Gas Storage by State, $1994 \ldots \ldots \ldots$. . . . . . . . . . . . . . 33

12. Underground Natural Gas Storage Capacity by State, December 31, $1994 \ldots \ldots \ldots \ldots$. . . . . . . . . . . 34

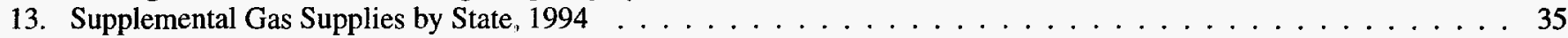

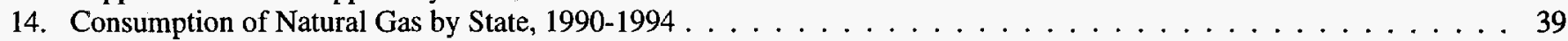

15. Natural Gas Delivered to Consumers by State, 1990-1994 . . . . . . . . . . . . . . . . . . . . . . 40

16. Natural Gas Delivered to Commercial Consumers for the Account of Others by State, 1990-1994 . . . . . . . . . 43

17. Natural Gas Delivered to Industrial Consumers for the Account of Others by State, 1990-1994 . . . . . . . . . . . 45

18. Natural Gas Delivered to Electric Utilities for the Account of Others by State, 1990-1994 . . . . . . . . . . . . . 46

19. Firm Natural Gas Deliveries to Consumers by State, $1994 \ldots \ldots$. . . . . . . . . . . . . . . . . . . . . . . . 47

20. Interruptible Natural Gas Deliveries to Consumers by State, $1994 \ldots \ldots \ldots \ldots \ldots \ldots \ldots$. . . . . . . . . 48

21. Natural Gas Deliveries to Commercial Consumers by State, $1994 \ldots \ldots \ldots \ldots \ldots$

22. Natural Gas Deliveries to Industrial Consumers by State, $1994 \ldots \ldots \ldots \ldots \ldots \ldots \ldots \ldots$

23. Natural Gas Deliveries to Electric Utilities by State, $1994 \ldots \ldots \ldots \ldots \ldots \ldots \ldots \ldots \ldots$

24. Average City Gate Price of Natural Gas in the United States, 1990-1994 . . . . . . . . . . . . . . . . . . 56

25. Average Price of Natural Gas Delivered to Residential Consumers by State, 1990-1994 . . . . . . . . . . . . 57

26. Average Prices of Natural Gas to Consumers by State, $1994 \ldots \ldots \ldots \ldots$. . . . . . . . . . . . . . . 58

27. Price of Natural Gas Deliveries to Commercial Consumers by State, $1994 \ldots \ldots$. . . . . . . . . . . . . . . . . . 61

28. Price of Natural Gas Deliveries to Industrial Consumers by State, $1994 \ldots \ldots \ldots \ldots$. . . . . . . . . . . . 62

29. Prices of Natural Gas Deliveries to Electric Utilities by State, $1994 \ldots \ldots \ldots$. . . . . . . . . . . . . 63

30. Selected Volumes and Prices of Natural Gas Sold to Residential Customers in the United States, 1994 . . . . . . . 64

31. Selected Volumes and Prices of Natural Gas Sold to Commercial Consumers in the United States, 1994 . . . . . . . 65

32. Leading Suppliers of Natural Gas to End Users in the United States, 1994 . . . . . . . . . . . . . . . . . . . . . 66

33. Average Residential Annual Consumption and Cost of Natural Gas per Consumer by State, 1990 - 1994 . . . . . 67

34. Natural Gas Delivered to Consumers by Census Division, $1994 \ldots \ldots \ldots \ldots \ldots \ldots$

35. Average Price of Natural Gas Delivered to Consumers by Census Division, 1993-1994 . . . . . . . . . . . . . . 72

36. Summary Statistics for Natural Gas - New England, 1990-1994 . . . . . . . . . . . . . . . . . . . . . . . . . 72

37. Summary Statistics for Natural Gas - Middle Atlantic, 1990-1994 . . . . . . . . . . . . . . . . . . . . . . . . . . . 74

38. Summary Statistics for Natural Gas - East North Central, 1990-1994 . . . . . . . . . . . . . . . . . . . . . 76

39. Summary Statistics for Natural Gas - West North Central, 1990-1994 . . . . . . . . . . . . . . . . . . . . . 78

40. Summary Statistics for Natural Gas - South Atlantic, 1990-1994 . . . . . . . . . . . . . . . . . . . . . . . . . 80

41. Summary Statistics for Natural Gas - East South Central, 1990-1994 . . . . . . . . . . . . . . . . . . . . . . . 82

42. Summary Statistics for Natural Gas - West South Central, 1990-1994 . . . . . . . . . . . . . . . . . . . . . 84

43. Summary Statistics for Natural Gas - Mountain, 1990-1994 . . . . . . . . . . . . . . . . . . . . . 86

44. Summary Statistics for Natural Gas - Pacific Contiguous, 1990-1994 . . . . . . . . . . . . . . . . . . . 88

45. Summary Statistics for Natural Gas - Pacific Noncontiguous, 1990-1994 . . . . . . . . . . . . . . . . . . . . . . . 90

46. Percent Distribution of Natural Gas Supply and Disposition by State, $1994 \ldots \ldots$. . . . . . . . . . . . . . . . . 94

47. Percent Distribution of Natural Gas Delivered to Consumers by State, $1994 \ldots \ldots$. . . . . . . . . . . . . . . . . . 95

48. Summary Statistics for Natural Gas - Alabama, 1990-1994 . . . . . . . . . . . . . . . . . . . . . . . 96

49. Summary Statistics for Natural Gas - Alaska, 1990-1994 . . . . . . . . . . . . . . . . . . . . . . . . 98

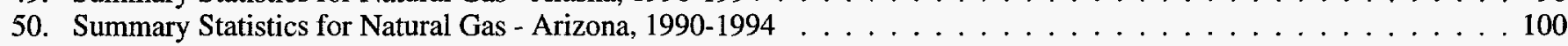

51. Summary Statistics for Natural Gas - Arkansas, 1990-1994 . . . . . . . . . . . . . . . . . . . . . . . . . . 102

52. Summary Statistics for Natural Gas - California, $1990-1994 \ldots \ldots \ldots \ldots$. . . . . . . . . . . . . . . 104

53. Summary Statistics for Natural Gas - Colorado, 1990-1994 . . . . . . . . . . . . . . . . . . . . . 106 
54. Summary Statistics for Natural Gas - Connecticut, 1990-1994 _ . . . . . . . . . . . . . . . . . . . . . 108

55. Summary Statistics for Natural Gas - Delaware, 1990-1994 . . . . . . . . . . . . . . . . . . . . . 110

56. Summary Statistics for Natural Gas - District of Columbia, 1990-1994 _ . . . . . . . . . . . . . . . . . 112

57. Summary Statistics for Natural Gas - Florida, 1990-1994 . . . . . . . . . . . . . . . . . . . . . . . . . . . 114

58. Summary Statistics for Natural Gas - Georgia, 1990-1994 _ . . . . . . . . . . . . . . . . . . . . . . . . 116

59. Summary Statistics for Natural Gas - Hawaii, 1990-1994 . . . . . . . . . . . . . . . . . . . . . . . . 118

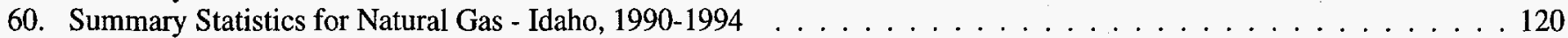

61. Summary Statistics for Natural Gas - Illinois, 1990-1994 . . . . . . . . . . . . . . . . . . . . . . 122

62. Summary Statistics for Natural Gas - Indiana, 1990-1994 . . . . . . . . . . . . . . . . . . . . . . . . . . . . . . 124

63. Summary Statistics for Natural Gas - Iowa, 1990-1994 . . . . . . . . . . . . . . . . . . . . . . . . . . . . 126

64. Summary Statistics for Natural Gas - Kansas, 1990-1994 . . . . . . . . . . . . . . . . . . . . . . . . . . 128

65. Summary Statistics for Natural Gas - Kentucky, 1990-1994 . . . . . . . . . . . . . . . . . . . . . . . . . 130

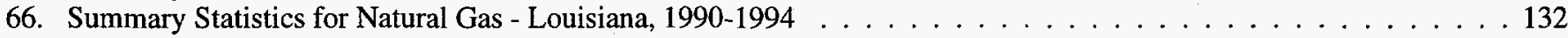

67. Summary Statistics for Natural Gas - Maine, $1990-1994 \ldots \ldots \ldots$. . . . . . . . . . . . . . . . . . . 134

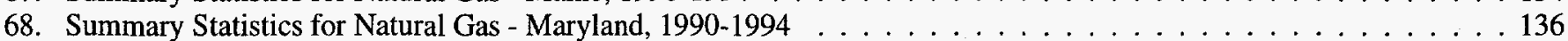

69. Summary Statistics for Natural Gas - Massachusetts, 1990-1994 . . . . . . . . . . . . . . . . . . . . . . . . 138

70. Summary Statistics for Natural Gas - Michigan, 1990-1994 _ . . . . . . . . . . . . . . . . . . . . . . 140

71. Summary Statistics for Natural Gas - Minnesota, 1990-1994 . . . . . . . . . . . . . . . . . . . . . . . . . . . 142

72. Summary Statistics for Natural Gas - Mississippi, 1990-1994 _ . . . . . . . . . . . . . . . . . . . . . . . . . 144

73. Summary Statistics for Natural Gas - Missouri, 1990-1994 . . . . . . . . . . . . . . . . . . . . . . . . . . 146

74. Summary Statistics for Natural Gas - Montana, 1990-1994 . . . . . . . . . . . . . . . . . . . . . . . . . . . 148

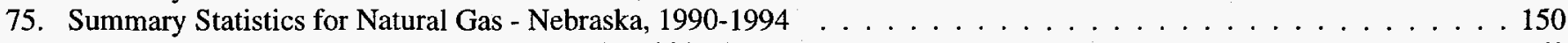

76. Summary Statistics for Natural Gas - Nevada, $1990-1994 \ldots \ldots \ldots$. . . . . . . . . . . . . . . . . . . . . 152

77. Summary Statistics for Natural Gas - New Hampshire, $1990-1994 \ldots \ldots \ldots$

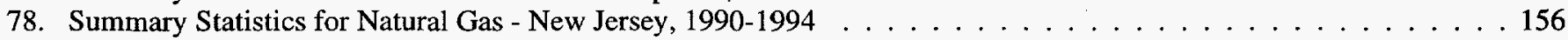

79. Summary Statistics for Natural Gas - New Mexico, 1990-1994 . . . . . . . . . . . . . . . . . . . . . . . . 158

80. Summary Statistics for Natural Gas - New York, 1990-1994 . . . . . . . . . . . . . . . . . . . . . . . 160

81. Summary Statistics for Natural Gas - North Carolina, 1990-1994 _ . . . . . . . . . . . . . . . . . . . . . 162

82. Summary Statistics for Natural Gas - North Dakota, 1990-1994 . . . . . . . . . . . . . . . . . . . . . . . 164

83. Summary Statistics for Natural Gas - Ohio, $1990-1994 \ldots \ldots \ldots \ldots \ldots \ldots$

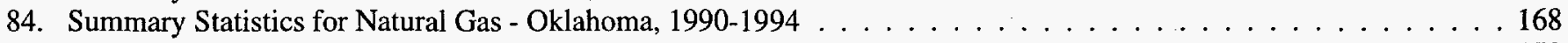

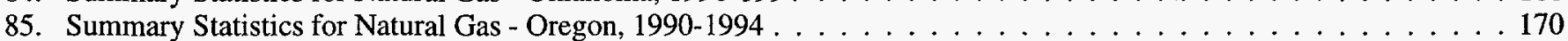

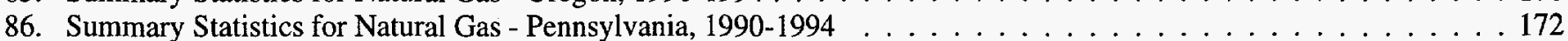

87. Summary Statistics for Natural Gas - Rhode Island, 1990-1994 _ . . . . . . . . . . . . . . . . . . . . . . . . 174

88. Summary Statistics for Natural Gas - South Carolina, 1990-1994 _ . . . . . . . . . . . . . . . . . . . 176

89. Summary Statistics for Natural Gas - South Dakota, $1990-1994 \ldots \ldots \ldots$. . . . . . . . . . . . . . . 178

90. Summary Statistics for Natural Gas - Tennessee, 1990-1994 . . . . . . . . . . . . . . . . . . . . . . . . 180

91. Summary Statistics for Natural Gas - Texas, $1990-1994 \ldots \ldots \ldots \ldots$

92. Summary Statistics for Natural Gas - Utah, $1990-1994 \ldots \ldots \ldots \ldots \ldots$

93. Summary Statistics for Natural Gas - Vermont, 1990-1994 . . . . . . . . . . . . . . . . . . . . . . . . 186

94. Summary Statistics for Natural Gas - Virginia, $1990-1994 \ldots \ldots \ldots \ldots \ldots \ldots$

95. Summary Statistics for Natural Gas - Washington, 1990-1994 . . . . . . . . . . . . . . . . . . . . . . . . . . 190

96. Summary Statistics for Natural Gas - West Virginia, 1990-1994 . . . . . . . . . . . . . . . . . . . . . . . . 192

97. Summary Statistics for Natural Gas - Wisconsin, 1990-1994 . . . . . . . . . . . . . . . . . . . . . . . . . . 194

98. Summary Statistics for Natural Gas - Wyoming, 1990-1994 . . . . . . . . . . . . . . . . . . . . . . . . . 196

99. Quantity and Average Price of Natural Gas Production in the United States, 1930-1994 . . . . . . . . . . . . 201

100. Supply and Disposition of Natural Gas in the United States, 1930-1994 . . . . . . . . . . . . . . . . . . . . . 204

101. Natural Gas Consumption in the United States, 1930-1994 . . . . . . . . . . . . . . . . . . . . . . 207

102. A verage Price of Natural Gas Delivered to U. S. Consumers, 1967-1994 . . . . . . . . . . . . . . . . . 210

A1. Comparison of Electric Utility Natural Gas Consumption Data by State, $1994 \ldots \ldots \ldots$. . . . . . . . . . 231

A2. Natural Gas Unaccounted for by State, 1990-1994 . . . . . . . . . . . . . . . . . . . . . . . . . . . . 232

A3. Natural Gas Processed and Liquids Extracted at Natural Gas Processing Plants by State, 1994 . . . . . . . . . 233

A4. Estimated Composition of Liquids Extracted at Natural Gas Processing Plants and the Resulting Heat Content Extraction Loss by State, 1994

A5. Natural Gas Processed, Liquids Extracted, and Estimated Extraction Loss by State of Origin

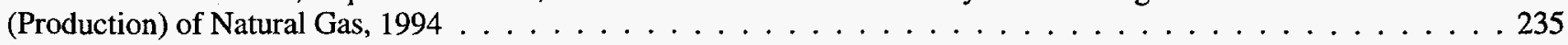

B1. Summary Statistics for Natural Gas in the United States, Metric Equivalents,1990-1994 . . . . . . . . . . . . . 240

B2. Thermal Conversion Factors and Data, 1990-1994 . . . . . . . . . . . . . . . . . . . . . . . . 242 


\section{Illustrations}

1. Natural Gas Flow Diagram, $1994 \ldots \ldots \ldots \ldots$. . . . . . . . . . . . . . . . . . . . . 1

2. Natural Gas Supply and Disposition in the United States, $1994 \ldots \ldots$. . . . . . . . . . . . . . . . . . . . . 6

3. Marketed Production of Natural Gas in the United States, $1994 \ldots \ldots$. . . . . . . . . . . . . . . . . . . . . 9

4. Marketed Production of Natural Gas in Selected States, 1990 - $1994 \ldots \ldots \ldots$

5. Principal Interstate Natural Gas Flow Summary, $1994 \ldots \ldots \ldots \ldots$

6. Locations of Existing Natural Gas Underground Storage Fields in the United States . . . . . . . . . . . . . . . 21

7. Interstate Movements of Natural Gas in the United States, $1994 \ldots \ldots \ldots \ldots$

8. Net Imports as a Percentage of Total Consumption of Natural Gas, 1972 - $1994 \ldots \ldots \ldots$. . . . . . . . . . . . . . . 32

9. Natural Gas Delivered to Consurners in the United States, 1993 - $1994 \ldots \ldots \ldots$. . . . . . . . . . . . . . . 37

10. Natural Gas Delivered to Consunners in the United States, $1994 \ldots \ldots$. . . . . . . . . . . . . . . . . . . . . . . . 42

11. Percent of Natural Gas Deliveries in the United States Representing Deliveries

for the Account of Others, by Consumer Sector, $1990-1994 \ldots \ldots \ldots \ldots \ldots \ldots$. . . . . . . . . . 44

12. Average Price of Natural Gas Delivered to Consumers in the United States, 1993 - $1994 \ldots \ldots$. . . . . . . . . . 53

13. Average City Gate Price of Natural Gas in the United States, $1994 \ldots \ldots \ldots$. . . . . . . . . . . . . . . 55

14. Selected Average Prices of Natural Gas in the United States, $1990-1994 \ldots \ldots \ldots \ldots \ldots$

15. Average Price of Natural Gas Delivered to U.S. Residential Consumers, 1994 . . . . . . . . . . . . . . . . . . . . 59

16. Average Price of Natural Gas Delivered to U.S. Onsystem Commercial Consumers, $1994 \ldots \ldots$

17. Average Price of Natural Gas Delivered to U.S. Onsystem Industrial Consumers, $1994 \ldots \ldots$. . . . . . . . . . . . . 60

18. Average Price of Natural Gas Delivered to U.S. Electric Utilities, $1994 \ldots \ldots$. . . . . . . . . . . . . . . . . . 60

19. Marketed Production of Natural Gas by Census Division, $1994 \ldots \ldots \ldots$

20. Natural Gas Delivered to Consumers by Census Division, $1994 \ldots \ldots \ldots \ldots \ldots \ldots \ldots \ldots$

21. Natural Gas Supply and Disposition in the United States, 1965-1994 . . . . . . . . . . . . . . . . . . . . . . 203

22. Natural Gas Delivered to Consumers in the United States, 1930 - 1994 . . . . . . . . . . . . . . . . . . . . . . 206

23. Average Price of Natural Gas Delivered to U.S. Consumers, 1967 - 1994 . . . . . . . . . . . . . . . . . . . . 209

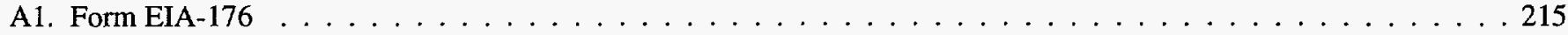

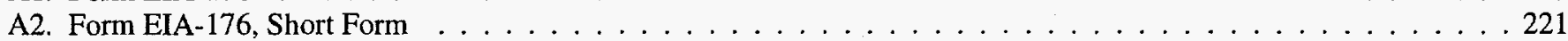

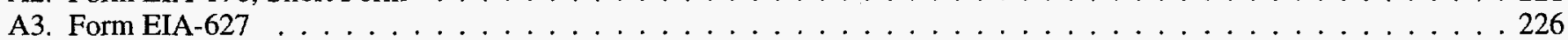




\section{Overview}

The domestic natural gas industry is gradually altering its services and operations to adapt to the continuing competitive pressures between gas suppliers and from other fuel sources. Unlike in the 1970's and 1980's, the competitive aspects of the market are the principal drivers of decisions affecting the supply and demand for natural gas.

Following are some brief discussions on the highlights of 1994; issues facing the natural gas industry and trends in supply, demand, and prices are discussed in the sections that follow.

\section{Highlights}

- End users consumed 20.8 trillion cubic feet of natural gas in 1994, the highest level seen since 1974. End-use consumption in 1994 was 2 percent higher than in 1993 and was largely driven by greater use of natural gas by electric utilities. Increased economic activity in 1994 led to somewhat higher consumption in the commercial and industrial sectors as well, while warmer-than-normal weather overall resulted in a slight decline in residential consumption.

Figure 1. Natural Gas Flow Diagram, 1994 (Trillion Cubic Feet)

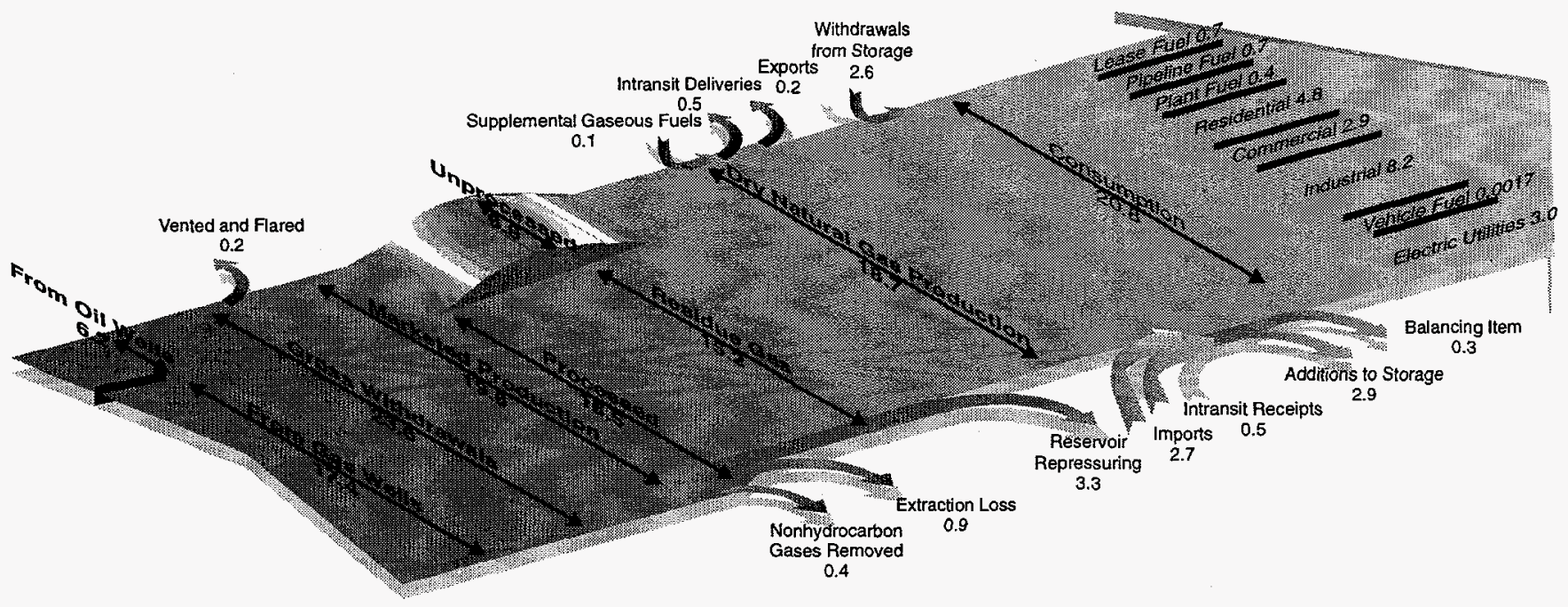

Note: Totals may not add due to independent rounding.

Sources: Energy Information Administration (EIA), Form ElA-176, “Annual Report of Natural and Supplemental Gas Supply and Disposition”; Form ElA-627, “Annual Quantity and Value of Natural Gas Report"; Form EIA-816, "Monthly Natural Gas Liquids Report"; Form ElA-759, "Monthly Power Plant Report"; Form FPC-14, "Annual Report for Importers and Exporters of Natural Gas"; U.S. Crude Oil, Natural Gas, and Natural Gas Liquids Reserves, 1994 Annual Repont, DOE/EIA-0216(94), and the U.S. Minerals Management Service. 
- The average wellhead price of natural gas fell to $\$ 1.88$ in 1994, 8 percent below the 1993 price of $\$ 2.04$. Average wellhead prices generally declined throughout 1994. Prices peaked in February 1994 at $\$ 2.13$ as a result of the January cold wave, gerierally declined to $\$ 1.48$ per thousand cubic feet in October, and then increased by 20 cents in November and another 4 cents in December, the first two months of the next heating season.

- Imports of natural gas from Canada continued its upward trend. Canadian imports in 1994 climbed to a record level of 2.6 billion cubic feet or 12 percent of U.S. consumption. The importance of natural gas imports from Canada is especially significant in the Northeast and Pacific regions which now depend on Canadian sources for over a third of their annual consumption. One of the factors contributing to this increase in imports has been the sharp drop in the price of imported gas from Canada. The average price in 1984 was $\$ 4.08$ per thousand cubic feet, and 10 years later the average price is $\$ 1.87$ per thousand cubic feet, in nominal dollars, a decrease of more than 68 percent. Over the same time period the average wellhead price of gas in the United States in nominal dollars decreased 29 percent.

\section{Other Issues and Events That Impacted the Natural Gas Industry in 1994}

- The first real test of natural gas markets and delivery systems under the Federal Energy Regulatory Commission (FERC) Order 636 occurred during the 1993-1994 heating season when the third week of January 1994 was 40 percent colder than normal for much of the Northeast and the Midwest. Deliveries to residential and commercial consumers in January were up 19 and 30, percent respectively, from the previous year and also were the highest monthly levels recorded since 1973. This resulted in near-record pipeline throughput, storage withdrawals, and gas send-outs by local distribution companies (LDC).

This severe weather challenged the new industry structure and its ability to guarantee the supply of natural gas, now shared by all components in the industry: producers, pipelines, marketers, LDC's, and the end users. The cold weather also reinforced the vital role in underground natural gas storage continues to play in the natural gas industry. Storage, particularly that which is accessed directly by LDC's, gives great flexibility to the distribution system, allowing it to respond quickly to sudden shifts in demand. The severe temperature conditions during January 1994 provide a good example of this flexibility. As average temperatures in much of the Northeast and Midwest plummeted to 20 degrees or more below normal, many companies withdrew record amounts of gas from storage.
The industry currently has the capability to store approximately 8 trillion cubic feet of natural gas in 375 storage sites around the country. Of this, about 46 percent, ( 4 trillion cubic feet) is considered working gas storage that can be withdrawn as necessary to meet demand requirements. On a peak day, the industry has the capability to deliver more than 120 billion cubic feet of natural gas to consumers, and underground storage can supply as much as half of this demand. To service the heavy heating load in the Northeast and Midwest, more than half of the country's working gas capacity is located east of the Mississippi River.

- All parts of the natural gas industry continue to reorganize to improve their competitive positions in a changing market. In 1994, Williams Energy acquired Transco Energy at a cost of $\$ 3$ billion dollars, thus creating the largest gas transmission system in terms of pipeline miles in North America. The combined system consists of more than 35,000 miles of pipelines stretching from the West Coast to the Northeast and the Canadian border to the Gulf of Mexico. Estimates of the total throughput of the system are between 8 and 9 million cubic feet a day. In addition, several other interstate pipeline companies have purchased or set up marketing affiliates, and one large northeastern LDC has formed an exploration, production, and marketing subsidiary.

- The major explosion that took place on the Texas Eastern Gas Transmission pipeline in Edison, New Jersey, in March 1994, prompted an extensive investigation of the event and pipeline safety in general. The National Transportation Safety Board's report confirmed that the probable cause of the New Jersey rupture was damage to the external surface of the pipeline during excavation activity in the area.

- The Department of Transporation's Office of Pipeline Safety (OPS) also investigated the March 1994 explosion and concluded that it was an isolated incident. As part of the OPS study a comprehensive inspection of the six major transmission pipelines with operations in New Jersey was also completed and all were found to be in compliance. As a result of these studies and concern for new approaches to ensuring pipeline safety several pieces of legislation covering pipeline safety have been introduced this year in the House of Representatives.

- The regulatory restructuring of the natural gas industry continued in 1994, but much of the discussion and activity is now shifting to the States. Several State regulatory agencies have instituted the unbundling of services which could eventually include residential and small commercial customers in the future. The focus thus far has been on the industrial and large commercial customer classes. Eventually, like the restructuring of the interstate transmission market to better serve largevolume consumers, the end-user market may be quite different from the one in which consumers obtain their service today. 
- Another development in the restructured interstate transportation market has been the establishment of a secondary market for retrading unneeded firm capacity. The secondary market can be comprised of any capacity transaction other than long-term capacity obtained directly from a pipeline company, including released capacity (under the Order 636 mechanism), interruptible transportation, short-term firm transportation, as well as alternative bundled services. The best known segment of the secondary market is the capacity release market initiated by Order 636 in which releasing and replacement shippers exchange capacity rights through electronic bulletin boards. Capacity release transactions have generated significant revenues for many companies over the last year and a half.
- Electric utility consumption of natural gas increased 10 percent in 1994 to the highest level in several years. This large increase was partly due to more competitive prices and the lack of rain and snowfall in areas, primarily the Northwest, that depend on hydroelectric generation as a power source. Even without these conditions several energy forecasts have natural gas consumption by electric utilities growing at an annual rate of almost 3 percent through the end of the century. (During the first 6 months of 1995, gas consumption by electric utilities was 12 more than the first 6 months of 1994.)

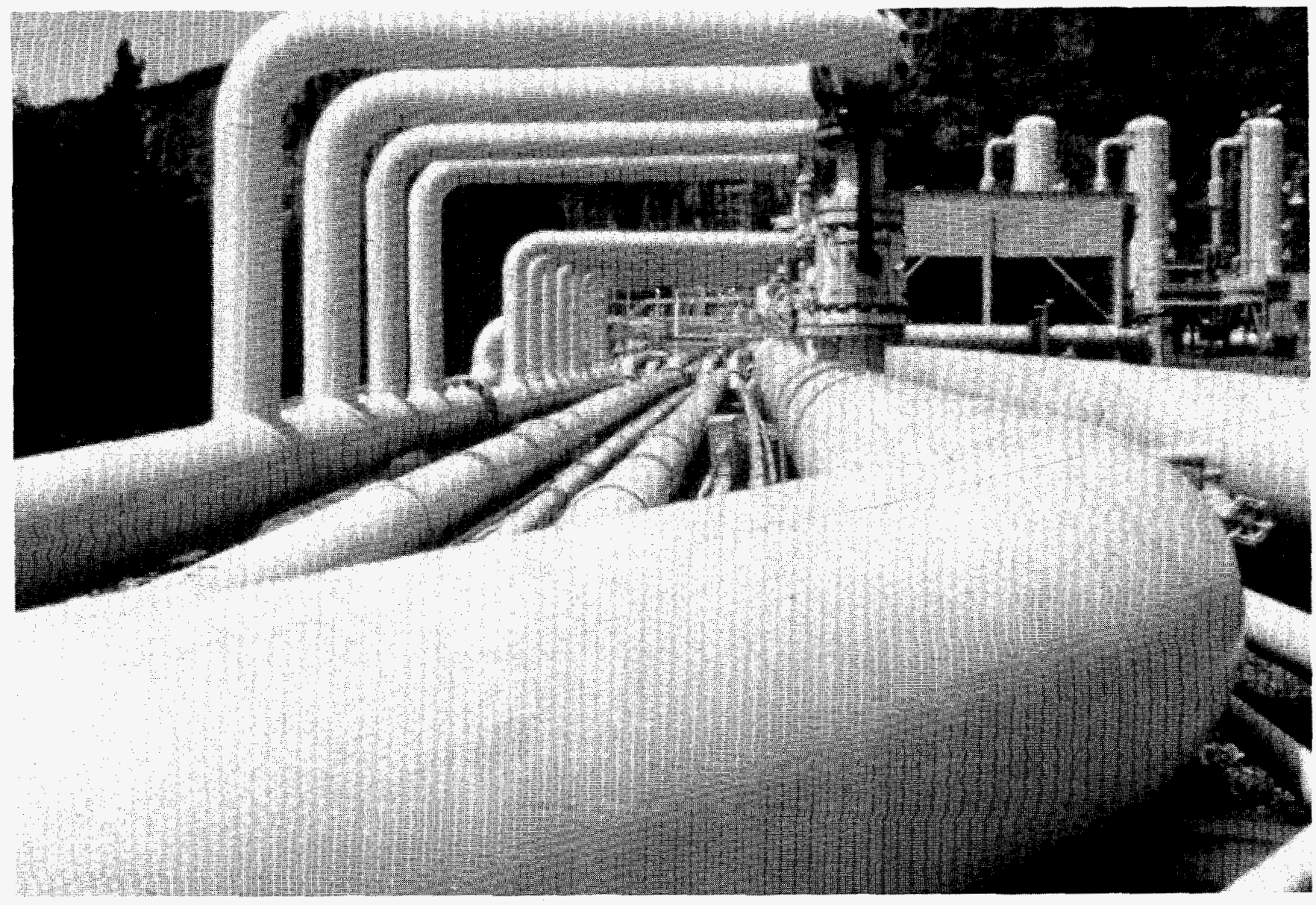

A complex grid of pipelines carry natural gas to customers in the lower-48 States. 
Table 1. Summary Statistics for Natural Gas in the United States, 1990-1994

\begin{tabular}{|c|c|c|c|c|c|}
\hline & 1990 & 1991 & 1992 & 1993 & 1994 \\
\hline 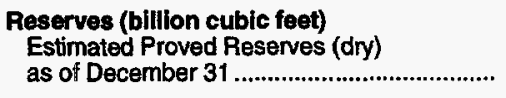 & 169,346 & 167,062 & 165,015 & 162,415 & 163,837 \\
\hline $\begin{array}{l}\text { Number of Cas and Cas Condensate Welis } \\
\text {-Producing at End of Year ................................. }\end{array}$ & $R_{269,100}$ & $R_{276,337}$ & $R_{275,414}$ & $\mathbf{R}_{282,152}$ & 287,206 \\
\hline 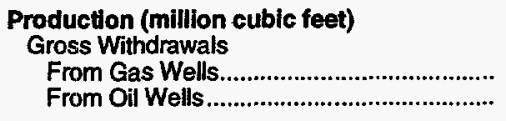 & $\begin{array}{r}16,053,566 \\
5,469,055\end{array}$ & $\begin{array}{r}16,017,626 \\
5,732,482\end{array}$ & $\begin{array}{r}16,164,874 \\
5,967,376\end{array}$ & $\begin{array}{r}R_{16,691,139} \\
R_{6,034,504}\end{array}$ & $\begin{array}{r}17,377,544 \\
6,231,110\end{array}$ \\
\hline Total & $2 \cdot 1,522,622$ & $21,750,108$ & $22,132,249$ & $R_{22,725,642}$ & $23,608,654$ \\
\hline 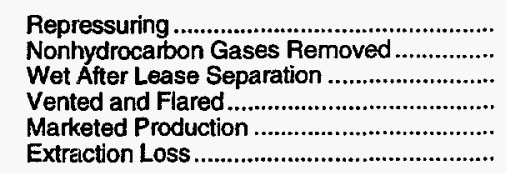 & $\begin{array}{r}-2,489,040 \\
-289,374 \\
18,744,207 \\
-150,415 \\
18,593,792 \\
-784,118\end{array}$ & $\begin{array}{r}-2,771,928 \\
-275,831 \\
18,702,348 \\
-169,909 \\
18,532,439 \\
-834,637\end{array}$ & $\begin{array}{r}-2,972,552 \\
-280,370 \\
18,879,327 \\
-167,519 \\
18,711,808 \\
-871,905\end{array}$ & $\begin{array}{r}R_{-3,103,014} \\
-413,971 \\
R_{19,208,657} \\
R_{-226,743} \\
R_{18,981,915} \\
-886,455\end{array}$ & $\begin{array}{r}-3,332,644 \\
-412,178 \\
19,863,831 \\
-228,336 \\
19,635,495 \\
-888,500\end{array}$ \\
\hline 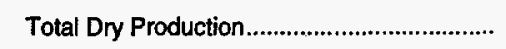 & $17,809,674$ & $17,697,802$ & $17,839,903$ & $R_{18,095,460}$ & $18,746,995$ \\
\hline 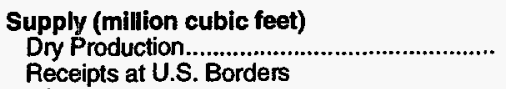 & $17,809,674$ & $17,697,802$ & $17,839,903$ & $R_{18,095,460}$ & $18,746,995$ \\
\hline 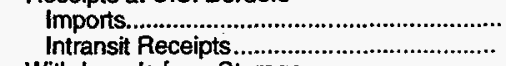 & $\begin{array}{r}1,532,259 \\
356,401\end{array}$ & $\begin{array}{r}1,773,313 \\
362,861\end{array}$ & $\begin{array}{r}2,137,504 \\
486,163\end{array}$ & $\begin{array}{r}2,350,115 \\
324,093\end{array}$ & $\begin{array}{r}2,659,673 \\
487,760\end{array}$ \\
\hline 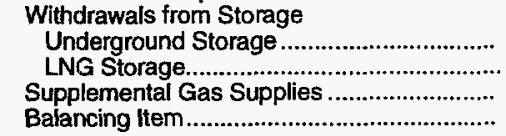 & $\begin{array}{r}1,933,786 \\
52,544 \\
122,806 \\
-151,863\end{array}$ & $\begin{array}{r}2,688,728 \\
63,090 \\
112,606 \\
-499,779\end{array}$ & $\begin{array}{r}2,723,774 \\
48,534 \\
117,919 \\
-507,565\end{array}$ & $\begin{array}{r}2,717,064 \\
82,189 \\
118,999 \\
R_{-109,593}\end{array}$ & $\begin{array}{r}2,491,427 \\
70,689 \\
110,826 \\
-296,577\end{array}$ \\
\hline 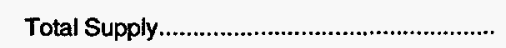 & $21,655,607$ & $22,198,621$ & $22,846,233$ & $\mathbf{R}_{23,578,326}$ & $24,270,793$ \\
\hline 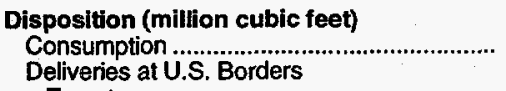 & $18,715,090$ & $19,035,156$ & $19,544,364$ & $R_{20,279,095}$ & $20,755,471$ \\
\hline 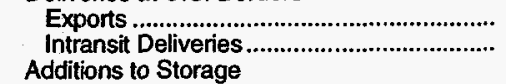 & $\begin{array}{r}85,565 \\
355,688\end{array}$ & $\begin{array}{l}129,244 \\
362,588\end{array}$ & $\begin{array}{l}216,282 \\
486,161\end{array}$ & $\begin{array}{l}140,183 \\
324,093\end{array}$ & $\begin{array}{l}178,066 \\
472,499\end{array}$ \\
\hline 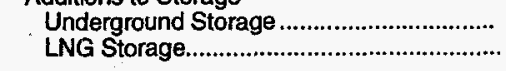 & $\begin{array}{r}2,433,450 \\
65,814\end{array}$ & $\begin{array}{r}2,608,373 \\
63,259\end{array}$ & $\begin{array}{r}2,555,393 \\
44,033\end{array}$ & $\begin{array}{r}2,759,738 \\
75,217\end{array}$ & $\begin{array}{r}2,796,279 \\
68,478\end{array}$ \\
\hline 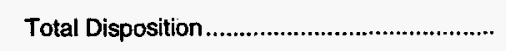 & $21,655,607$ & $22,198,621$ & $22,846,233$ & $\mathbf{R}_{23,578,326}$ & $24,270,793$ \\
\hline 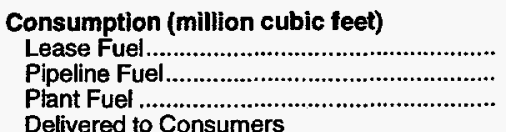 & $\begin{array}{l}807,735 \\
659,816 \\
428,657\end{array}$ & $\begin{array}{l}672,314 \\
601,305 \\
456,954\end{array}$ & $\begin{array}{l}710,250 \\
587,710 \\
460,571\end{array}$ & $\begin{array}{r}R_{723,118} \\
624,308 \\
448,822\end{array}$ & $\begin{array}{l}736,678 \\
685,327 \\
423,878\end{array}$ \\
\hline 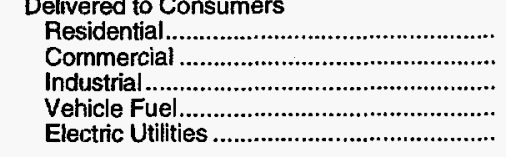 & $\begin{array}{r}4,391,324 \\
2,622,721 \\
7,018,414 \\
270 \\
2,786,153\end{array}$ & $\begin{array}{r}4,555,659 \\
2,728,581 \\
7,230,962 \\
367 \\
2,789,014\end{array}$ & $\begin{array}{r}4,690,065 \\
2,802,751 \\
7,526,898 \\
511 \\
2,765,608\end{array}$ & $\begin{array}{r}\mathbf{R}_{4,956,445} \\
\mathbf{R}_{2,861,569} \\
\mathbf{R}_{7,981,433} \\
960 \\
2,682,440\end{array}$ & $\begin{array}{r}4,847,702 \\
2,895,034 \\
8,177,975 \\
1,730 \\
2,987,146\end{array}$ \\
\hline Total Delivered to Consumers........................ & $15,818,882$ & $17,304,582$ & $17,785,833$ & $R_{18,482,847}$ & $18,909,587$ \\
\hline Total Consumption & $18,715,090$ & $19,035,156$ & $19,544,364$ & $R_{20,279,095}$ & $20,755,471$ \\
\hline 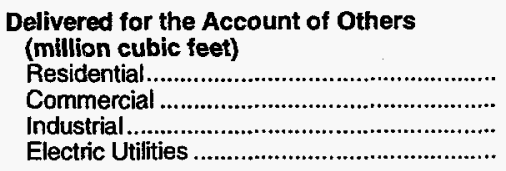 & $\begin{array}{r}31,302 \\
352,521 \\
4,544,535 \\
1,390,340\end{array}$ & $\begin{array}{r}36,440 \\
405,919 \\
4,863,923 \\
1,580,077\end{array}$ & $\begin{array}{r}41,433 \\
471,009 \\
5,248,609 \\
1,697,363\end{array}$ & $\begin{array}{r}44,314 \\
R_{460,097} \\
R_{5,644,894} \\
1,658,438\end{array}$ & $\begin{array}{r}42,338 \\
599,058 \\
6,123,797 \\
2,092,124\end{array}$ \\
\hline
\end{tabular}

See footnotes at end of table. 
Table 1. Summary Statistics for Natural Gas in the United States, 1990-1994 (Continued)

\begin{tabular}{|c|c|c|c|c|c|}
\hline & 1990 & 1991 & 1992 & 1993 & 1994 \\
\hline 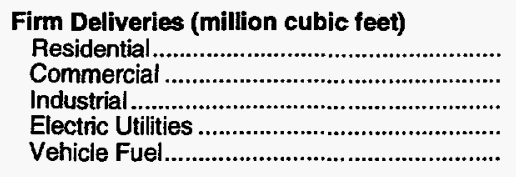 & $\begin{array}{l}\text { NA } \\
\text { NA } \\
\text { NA } \\
\text { NA } \\
\text { NA }\end{array}$ & $\begin{array}{l}\text { NA } \\
\text { NA } \\
\text { NA } \\
\text { NA } \\
\text { NA }\end{array}$ & $\begin{array}{l}\text { NA } \\
\text { NA } \\
\text { NA } \\
\text { NA } \\
\text { NA }\end{array}$ & $\begin{array}{r}4,957,208 \\
R_{2,553,679} \\
R_{4,624,853} \\
R_{1,522,061} \\
792\end{array}$ & $\begin{array}{r}4,843,995 \\
2,559,521 \\
4,754,907 \\
1,580,745 \\
1,325\end{array}$ \\
\hline 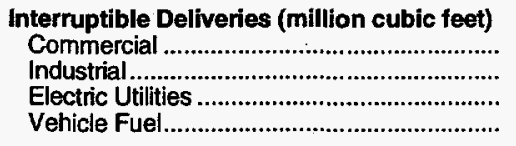 & $\begin{array}{l}\text { NA } \\
\text { NA } \\
\text { NA } \\
\text { NA }\end{array}$ & $\begin{array}{l}\text { NA } \\
\text { NA } \\
\text { NA } \\
\text { NA }\end{array}$ & $\begin{array}{l}\text { NA } \\
\text { NA } \\
\text { NA } \\
\text { NA }\end{array}$ & $\begin{array}{r}R_{307,890} \\
R_{3,356,581} \\
922,962 \\
168\end{array}$ & $\begin{array}{r}335,513 \\
3,423,068 \\
1,210,453 \\
405\end{array}$ \\
\hline 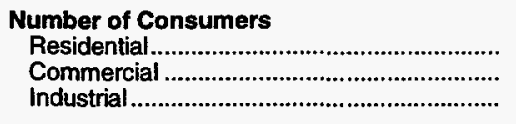 & $\begin{array}{r}50,187,178 \\
4,236,280 \\
218,341\end{array}$ & $\begin{array}{r}51,593,206 \\
4,357,252 \\
216,529\end{array}$ & $\begin{array}{r}52,331,397 \\
4,409,699 \\
209,616\end{array}$ & $\begin{array}{r}52,535,411 \\
\mathrm{R}_{4,464,906} \\
\mathrm{H}_{209,666}\end{array}$ & $\begin{array}{r}53,393,142 \\
4,537,500 \\
202,953\end{array}$ \\
\hline 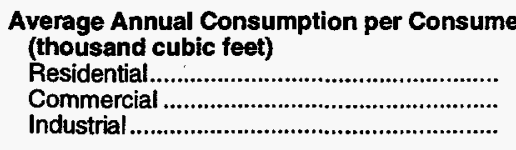 & $\begin{array}{r}87 \\
619 \\
32,144\end{array}$ & $\begin{array}{r}88 \\
626 \\
33,395\end{array}$ & $\begin{array}{r}90 \\
636 \\
35,908\end{array}$ & $\begin{array}{r}94 \\
R_{641} \\
R_{38,067}\end{array}$ & $\begin{array}{r}91 \\
638 \\
40,295\end{array}$ \\
\hline 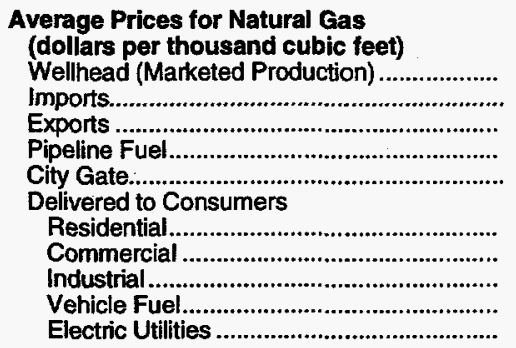 & $\begin{array}{l}1.71 \\
1.94 \\
3.10 \\
1.95 \\
3.03\end{array}$ & $\begin{array}{l}1.64 \\
1.82 \\
2.59 \\
1.87 \\
2.90\end{array}$ & $\begin{array}{l}1.74 \\
1.85 \\
2.25 \\
2.07 \\
3.01\end{array}$ & $\begin{array}{r}\mathbf{R}_{2.04} \\
2.03 \\
2.59 \\
1.97 \\
3.21\end{array}$ & $\begin{array}{l}1.88 \\
1.87 \\
2.50 \\
1.70 \\
3.07\end{array}$ \\
\hline
\end{tabular}

$\mathbf{R}=$ Revised data.

NA $=$ Not Available.

Noles: Beginning in 1987, prices for gas delivered to consumers are calculated using only on-system sales data. No imputations are made for prices of gas delivered for the account of others. In previous years, prices were calculated using reported values and values imputed for gas delivered for the account of others. The United States includes

the 50 states and the District of Columbia. Totals may not equal sum of components due to independent rounding.
Sources: Energy Information Administration (EIA), Form ElA-176, "Annual Report of Natural and Supplemental Gas Supply and Disposition"; Form EIA-627, "Annual Quantity and Value of Natural Gas Report"; Form EIA-857, "Monthly Report of Natural Gas Purchases and Deliveries to Consumers"; Form EIA-816, "Monthly Natural Gas Liquids Report"; Form EIA-759, "Monthly Power Plant Report"; Form FERC-423, "Monthly Report of Cost and Quality of Fuels for Electric Plants"; Form ElA-191, "Underground Gas

Storas

Report, DOE $/ E I A-0216(94)$; and the U.S. Minerals Management Service. 
Figure 2. Natural Gas Supply and Disposition in the United States, 1994

(Trillion Cubic Feet)

Gross Withdrawals From Gas and Oil Wells

23.6

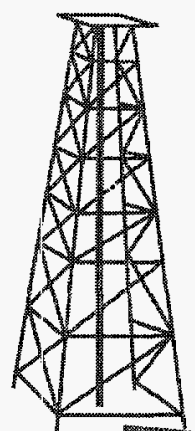

Natural Gas

Storage Facilities

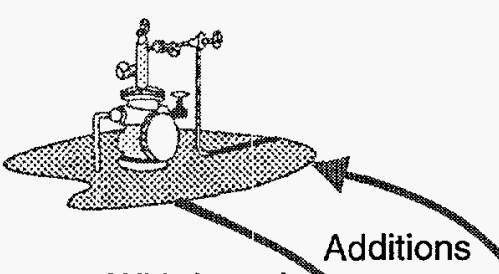

Withdrawals

2.6
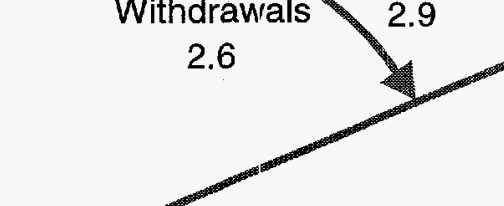

Dry Gas

Production

18.7

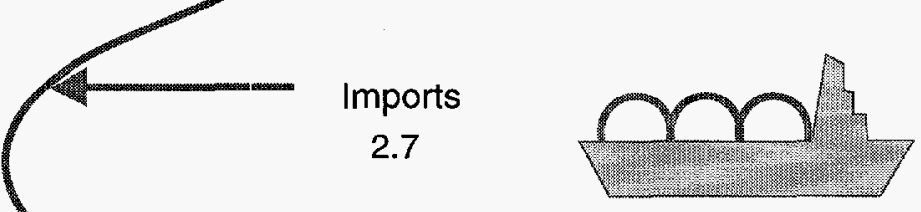

Exports

0.2

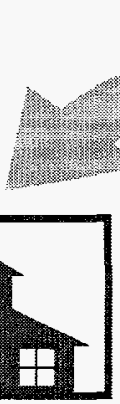

Residential

4.8

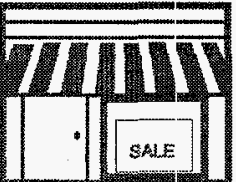

Commercial

2.9

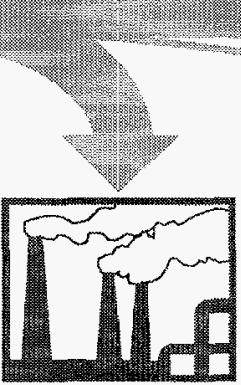

Industrial

8.2

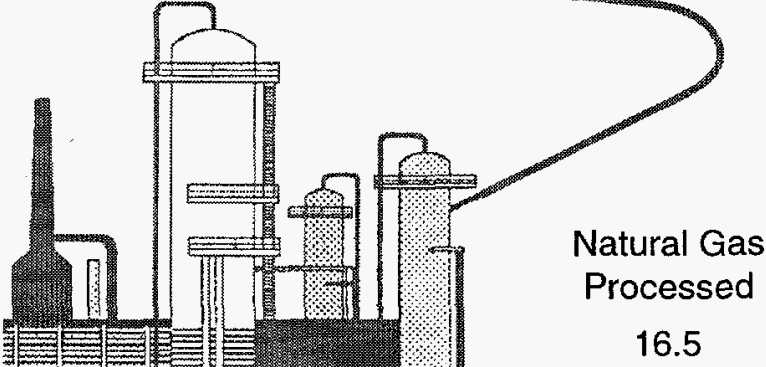

16.5 
Table 2. Natural Gas Production, Transmission, and Consumption by State, 1994 (Million Cubic Feet)

\begin{tabular}{|c|c|c|c|c|c|c|c|c|}
\hline State & $\begin{array}{l}\text { Marketed } \\
\text { Production }\end{array}$ & $\begin{array}{l}\text { Extraction } \\
\text { Loss }\end{array}$ & $\begin{array}{l}\text { Balancing } \\
\text { Item }^{\mathrm{a}}\end{array}$ & $\begin{array}{c}\text { Net } \\
\text { Interstate } \\
\text { Movements }\end{array}$ & $\begin{array}{l}\text { Net Move- } \\
\text { ments Across } \\
\text { U.S. Borders }\end{array}$ & $\begin{array}{l}\text { Net Storage } \\
\text { Changes }\end{array}$ & $\begin{array}{c}\text { Supplemental } \\
\text { Gas } \\
\text { Supplies }\end{array}$ & Consumption \\
\hline $\begin{array}{l}\text { Alabama ................. } \\
\text { Alaska .................. } \\
\text { Arizona ................. } \\
\text { Arkansas ................ } \\
\text { California ............... }\end{array}$ & $\begin{array}{r}515,271 \\
453,425 \\
752 \\
187,673 \\
309,427\end{array}$ & $\begin{array}{r}5,647 \\
30,945 \\
0 \\
553 \\
11,250\end{array}$ & $\begin{array}{r}-46,632 \\
43,398 \\
20,057 \\
-9,054 \\
-57,030\end{array}$ & $\begin{array}{r}-173,112 \\
0 \\
115,147 \\
63,880 \\
1,887,149\end{array}$ & $\begin{array}{r}0 \\
-62,682 \\
-2,459 \\
0 \\
0\end{array}$ & $\begin{array}{r}1,087 \\
0 \\
0 \\
-2,478 \\
4,985\end{array}$ & $\begin{array}{r}69 \\
0 \\
0 \\
0 \\
0\end{array}$ & $\begin{array}{r}288,863 \\
403,197 \\
133,497 \\
244,425 \\
2,123,310\end{array}$ \\
\hline $\begin{array}{l}\text { Colorado............... } \\
\text { Connecticut ............ } \\
\text { D.C........................ } \\
\text { Delaware ............... } \\
\text { Florida .................... }\end{array}$ & $\begin{array}{r}453,207 \\
0 \\
0 \\
0 \\
7,486\end{array}$ & $\begin{array}{r}19,612 \\
0 \\
0 \\
0 \\
1,789\end{array}$ & $\begin{array}{r}48,328 \\
9,463 \\
1,042 \\
-3,781 \\
2,713\end{array}$ & $\begin{array}{r}-213,230 \\
110,478 \\
29,806 \\
49,408 \\
359,143\end{array}$ & $\begin{array}{l}0 \\
0 \\
0 \\
0 \\
0\end{array}$ & $\begin{array}{r}1,100 \\
-61 \\
0 \\
23 \\
0\end{array}$ & $\begin{array}{r}8,168 \\
29 \\
0 \\
3,032 \\
0\end{array}$ & $\begin{array}{r}275,761 \\
120,031 \\
30,849 \\
48,636 \\
367,552\end{array}$ \\
\hline 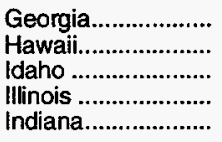 & $\begin{array}{r}0 \\
0 \\
0 \\
333 \\
107\end{array}$ & $\begin{array}{r}0 \\
0 \\
0 \\
80 \\
0\end{array}$ & $\begin{array}{r}9,376 \\
-53 \\
-4,498 \\
-131,627 \\
-80,586\end{array}$ & $\begin{array}{r}331,812 \\
0 \\
-661,486 \\
1,161,327 \\
600,860\end{array}$ & $\begin{array}{r}0 \\
0 \\
723,071 \\
0 \\
0\end{array}$ & $\begin{array}{r}-94 \\
0 \\
182 \\
12,916 \\
3,481\end{array}$ & $\begin{array}{r}123 \\
2,831 \\
0 \\
7,871 \\
2,457\end{array}$ & $\begin{array}{r}341,405 \\
2,778 \\
56,905 \\
1,024,908 \\
519,356\end{array}$ \\
\hline $\begin{array}{l}\text { lowa................... } \\
\text { Kansas ................ } \\
\text { Kentucky............... } \\
\text { Louisiana ................ } \\
\text { Maine..................... }\end{array}$ & $\begin{array}{r}0 \\
712,730 \\
73,081 \\
5,169,705 \\
0\end{array}$ & $\begin{array}{r}0 \\
46,936 \\
2,024 \\
128,583 \\
0\end{array}$ & $\begin{array}{r}-35,943 \\
20,700 \\
56,159 \\
409,022 \\
131\end{array}$ & $\begin{array}{r}287,281 \\
-262,252 \\
85,965 \\
-3,804,900 \\
4,928\end{array}$ & $\begin{array}{r}0 \\
0 \\
0 \\
17,887 \\
0\end{array}$ & $\begin{array}{r}3,109 \\
6,215 \\
4,845 \\
39,364 \\
5\end{array}$ & $\begin{array}{r}123 \\
0 \\
50 \\
0 \\
0\end{array}$ & $\begin{array}{r}248,351 \\
418,027 \\
208,386 \\
1,623,766 \\
5,055\end{array}$ \\
\hline $\begin{array}{l}\text { Maryland................ } \\
\text { Massachusetts ...... } \\
\text { Michigan ................ } \\
\text { Minnesota............. } \\
\text { Mississippi .............. }\end{array}$ & $\begin{array}{r}26 \\
0 \\
222,657 \\
0 \\
63,448\end{array}$ & $\begin{array}{r}0 \\
0 \\
6,371 \\
0 \\
425\end{array}$ & $\begin{array}{r}-45,552 \\
53,615 \\
-197,896 \\
6,163 \\
52,312\end{array}$ & $\begin{array}{r}226,594 \\
243,880 \\
1,495,917 \\
-563,335 \\
167,621\end{array}$ & $\begin{array}{r}0 \\
32,891 \\
-520,977 \\
881,692 \\
0\end{array}$ & $\begin{array}{r}-1,680 \\
-6,820 \\
80,996 \\
994 \\
14,446\end{array}$ & $\begin{array}{r}987 \\
154 \\
13,688 \\
289 \\
0\end{array}$ & $\begin{array}{l}183,734 \\
337,361 \\
926,023 \\
323,815 \\
268,510\end{array}$ \\
\hline $\begin{array}{l}\text { Missouri ................... } \\
\text { Montana ................ } \\
\text { Nebraska ................. } \\
\text { Nevada .............. } \\
\text { New Hampshire..... }\end{array}$ & $\begin{array}{r}8 \\
50,416 \\
2,898 \\
16 \\
0\end{array}$ & $\begin{array}{r}0 \\
631 \\
8 \\
0 \\
0\end{array}$ & $\begin{array}{r}21,145 \\
-7,062 \\
-24,879 \\
-893 \\
4,181\end{array}$ & $\begin{array}{r}245,985 \\
-531,252 \\
150,340 \\
102,628 \\
15,365\end{array}$ & $\begin{array}{r}0 \\
532,768 \\
0 \\
0 \\
0\end{array}$ & $\begin{array}{r}-85 \\
-7,819 \\
2,497 \\
-30 \\
0\end{array}$ & $\begin{array}{r}371 \\
0 \\
890 \\
9 \\
160\end{array}$ & $\begin{array}{r}267,594 \\
52,058 \\
126,744 \\
101,790 \\
19,706\end{array}$ \\
\hline $\begin{array}{l}\text { New Jersey............. } \\
\text { New Mexico........... } \\
\text { New York.............. } \\
\text { North Carolina ....... } \\
\text { North Dakota .......... }\end{array}$ & $\begin{array}{r}0 \\
1,557,689 \\
20,465 \\
0 \\
57,805\end{array}$ & $\begin{array}{r}0 \\
86,607 \\
0 \\
0 \\
5,671\end{array}$ & $\begin{array}{r}48,070 \\
-165,975 \\
26,839 \\
-10,433 \\
-4,439\end{array}$ & $\begin{array}{r}530,317 \\
-1,082,958 \\
376,865 \\
198,606 \\
-63,586\end{array}$ & $\begin{array}{r}0 \\
0 \\
588,983 \\
0 \\
1,617\end{array}$ & $\begin{array}{r}1,270 \\
1,379 \\
2,115 \\
316 \\
0\end{array}$ & $\begin{array}{r}7,737 \\
0 \\
1,460 \\
3 \\
57,127\end{array}$ & $\begin{array}{r}584,853 \\
220,770 \\
1,012,497 \\
187,861 \\
42,853\end{array}$ \\
\hline 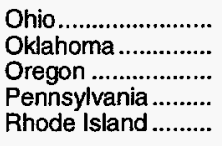 & $\begin{array}{r}132,151 \\
1,934,864 \\
3,221 \\
150,228 \\
0\end{array}$ & $\begin{array}{r}104 \\
101,564 \\
0 \\
513 \\
0\end{array}$ & $\begin{array}{r}-40,803 \\
-303 \\
6,816 \\
-140,774 \\
12,678\end{array}$ & $\begin{array}{r}779,245 \\
-1,242,085 \\
136,833 \\
688,918 \\
58,749\end{array}$ & $\begin{array}{l}0 \\
0 \\
0 \\
0 \\
0\end{array}$ & $\begin{array}{r}28,576 \\
18,838 \\
463 \\
-26 \\
160\end{array}$ & $\begin{array}{r}1,432 \\
0 \\
2 \\
252 \\
0\end{array}$ & $\begin{array}{r}843,345 \\
572,074 \\
146,409 \\
698,138 \\
71,267\end{array}$ \\
\hline $\begin{array}{l}\text { South Carolina....... } \\
\text { South Dakota......... } \\
\text { Tennessee............. } \\
\text { Texas...................... } \\
\text { Utah...................... }\end{array}$ & $\begin{array}{r}0 \\
1,437 \\
1,990 \\
6,353,844 \\
270,858\end{array}$ & $\begin{array}{r}0 \\
0 \\
0 \\
381,020 \\
13,780\end{array}$ & $\begin{array}{r}14,766 \\
-773 \\
24,107 \\
-148,241 \\
-104,650\end{array}$ & $\begin{array}{r}129,772 \\
30,255 \\
220,081 \\
-2,068,270 \\
4,231\end{array}$ & $\begin{array}{r}0 \\
0 \\
0 \\
-37,029 \\
0\end{array}$ & $\begin{array}{r}165 \\
0 \\
106 \\
52,956 \\
19,587\end{array}$ & $\begin{array}{r}154 \\
61 \\
84 \\
1 \\
0\end{array}$ & $\begin{array}{r}144,526 \\
30,980 \\
246,157 \\
3,666,329 \\
137,073\end{array}$ \\
\hline $\begin{array}{l}\text { Vermont................ } \\
\text { Virginia .................. } \\
\text { Washington ........... } \\
\text { West Virginia ......... } \\
\text { Wisconsin ................ } \\
\text { Wyoming ............... }\end{array}$ & $\begin{array}{r}0 \\
50,259 \\
0 \\
182,000 \\
0 \\
696,018\end{array}$ & $\begin{array}{r}0 \\
0 \\
0 \\
10,901 \\
0 \\
33,486\end{array}$ & $\begin{array}{r}-12 \\
38,265 \\
-55,281 \\
94,283 \\
-124,878 \\
121,842\end{array}$ & $\begin{array}{r}-10,789 \\
140,723 \\
-52,739 \\
-105,063 \\
480,425 \\
-675,475\end{array}$ & $\begin{array}{r}18,091 \\
0 \\
323,013 \\
0 \\
0 \\
0\end{array}$ & $\begin{array}{r}0 \\
-121 \\
2,212 \\
14,932 \\
-50 \\
2,584\end{array}$ & $\begin{array}{r}9 \\
1,195 \\
0 \\
0 \\
5 \\
0\end{array}$ & $\begin{array}{r}7,299 \\
230,562 \\
212,782 \\
145,387 \\
355,602 \\
106,316\end{array}$ \\
\hline 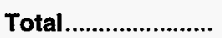 & $19,635,495$ & 888,500 & $-296,577$ & 0 & $2,496,868$ & 302,641 & 110,826 & $20,755,471$ \\
\hline
\end{tabular}

a Balancing Item volumes are equal to Total Disposition (net storage changes plus extraction loss plus consumption) minus Total Supply (marketed production plus net interstate movements plus net movements across U.S. borders plus supplemental gas supplies).

bositive numbers denote net receipts; negative numbers denote net deliveries.

c Negative numbers indicate withdrawals from storage in excess of additions to storage and are, therefore, additions to total supply.

Note: Totals may not equal sum of components due to independent rounding

Sources: Energy Information Administration (EIA), Form ElA-176, "Annual Report of Natural and Supplemental Gas Supply and Disposition"; Form EIA-627, "Annual Quantity and Value of Natural Gas Report"; Form ElA-816, "Monthly Natural Gas Liquids Report"; and the U.S. Minerals Management Service. 


\section{Supply}

\section{Production}

In 1994, natural gas production reached 19.6 trillion cubic feet, the highest level since the early 1980's and 3 percent above production in 1993. Recent production increases reflect the combined benefits of efficiency gains and improved technology. In the deregulated natural gas industry, changing market dynamics have placed continuing pressure on producers to cut costs and improve the efficiency of their operations.

Gross withdrawals of natural gas reached 23.6 trillion cubic feet in 1994 (Table 3). Louisiana, Texas, Oklahoma and New Mexico have continued as the leading producers of natural gas. Together they supplied 15 trillion cubic feet,
76 percent of 1994's marketed natural gas production. The number of producing gas wells and gas-condensate wells reached approximately 287,206 , surpassing (by 2 percent) the all-time high of 282,152 wells reached in 1993 (Table 5).

\section{Wellhead Prices}

Average wellhead prices generally declined throughout 1994, reversing the brief upward trend in 1992 and the beginning of 1993. Several factors contributed to the low prices during the past year, including: increased domestic production, record imports of Canadian gas, and higher

Figure 3. Marketed Production of Natural Gas in the United States, 1994 (Million Cubic Feet)

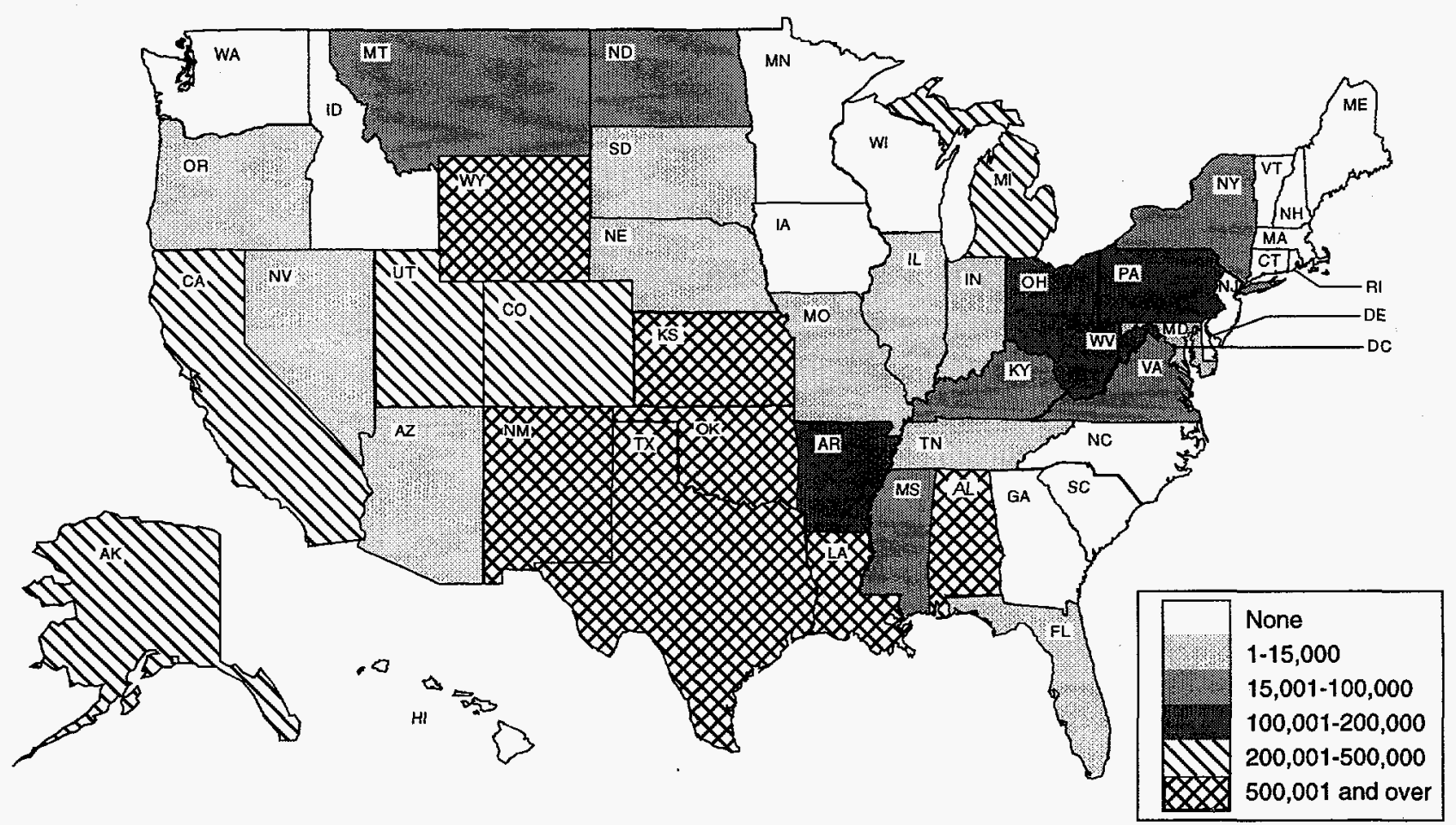

Sources: Illinois, Louisiana, Pennsyivania, South Dakota, and West Virginia, 1994: Energy Information Administration (ElA), U.S. Crude Oil, Natural Gas, and Natural Gas Liquids Reserves, 1994 Annual Report, DOEJEIA-0216(94); and ElA computations. All other data: Form ElA-627, "Annual Quantity and Value of Natural Gas Report" and the United States Minerals Management Service. 
storage levels because of milder than normal weather during most of the period. Wellhead prices dropped almost 8 percent in 1994 to $\$ 1.88$ per thousand cubic feet. Despite being lower than the 1993 price, the 1994 wellhead price is the highest since 1986.

\section{Drilling}

Exploration and development activities incrementally add to existing reserves of natural gas. Indicators that in the past have been good guides to exploration and development activity, and thus to subsequent reserves additions, are the numbers of rotary rigs running and well completions. In 1994, these indicators were generally positive. Rotary rigs drilling for gas rose from 364 to 427 in 1994, exceeding for the first time the number of rigs drilling for oil. Total rigs running also rose from 754 to 775 .

Well completions for gas, both exploratory and development, exceeded those for oil for the second year in a row. Exploratory well completions for gas were up in 1994, rising from 487 to 725 , while development well completions for gas dropped from 9,321 to 8,215 . This led to a drop in total gas well completions from 9,808 to 8,940 . These activities occurred against a background of rising overall exploratory completions, 3,480 to 3,527 , and falling total completions, from 25,270 to 21,019 . This performance resumes a downward trend in total completions which began after the 1990 drilling surge that was caused by the legislative incentive for coalbed methane and tight gas.

\section{Reserves}

U.S. proved reserves of natural gas rose 1 percent in 1994, from 162,415 billion cubic feet in 1993 to 163,837 billion cubic feet, the first gain in 4 years. Proved reserves are those quantities that geological and engineering data demonstrate with reasonable certainty to be recoverable in future years from known reservoirs under existing economic and operating conditions. Large gas discoveries in the Federal offshore, several in deep water, played a major role in this increase.

Total discoveries were up substantially as were revisions and adjustments. These combined to replace 108 percent of gas production, which also increased in 1994. The last increase in gas reserves was in 1990, and the one prior to that was in 1981. While the lower-48 States' gas reserves have been generally declining since the late 1960's, they have declined less than 1 percent per year since natural gas prices peaked in 1983. This is a low reserves decline rate for a period in which rapid changes in price, demand, drilling, and the industry's regulatory environment occurred. These changes left gas prices and drilling at levels much lower in 1994 than in 1993.
The four leading gas producing areas -- Texas, the Gulf of Mexico Federal Offshore, Oklahoma, and Louisiana -- had 1994 proved reserves increases that totaled 3,202 billion cubic feet.

Following several years of growth, coalbed methane reserves declined in 1994 by 472 billion cubic feet, or 5 percent. However, coalbed methane production grew to nearly 5 percent of U.S. dry gas production. Coalbed methane reserves accounted for 6 percent of U.S. natural gas reserves in 1994. No federal tax incentives for new coalbed methane wells have been available for 2 years.

U.S. total discoveries of dry gas reserves were 12,315 billion cubic feet in 1994, an increase of 39 percent over 1993. These discoveries equal two-thirds of 1994 gas production. Total discoveries are those reserves attributable to field extensions, new field discoveries, and new reservoir discoveries in old fields. They result from drilling exploratory wells.

During 1994, the highlights of dry gas reserves discoveries were as follows:

- New field discoveries were 1,894 billion cubic feet, up 111 percent over 1993.

- Field extensions were 6,941 billion cubic feet, up 14 percent over 1993.

- New reservoir discoveries in old fields were 3,480 billion cubic feet, up 86 percent over 1993 and the highest since 1979 .

- Total discoveries per exploratory well were maintained at a high level in 1994. They were more than twice that of the early 1980 's.

- Texas and the Gulf of Mexico Federal Offshore accounted for almost two-thirds of U.S. total discoveries of gas in 1994.

\section{Spot and Futures Market}

\section{Futures Market}

The price of short-term futures contracts fell throughout much of 1994 and remained low in the first half 1995. Although prices rose sharply in response to cold weather in late January and early February 1994, reaching a high of $\$ 2.64$ per million Btu (MMBtu) on February 1, prices fell throughout much of the year. Between 1993 and 1994, domestic dry gas production grew by 3 percent and imports of relatively low-cost Canadian natural gas grew by 9 percent, putting downward pressure on prices. The increased imports were largely due to access to new pipeline capacity and to a weak Canadian dollar that made relatively low-cost 
Canadian gas even more attractive in U.S. markets. Milder than normal weather through the final three quarters of 1994 and the first quarter of $1995 \mathrm{kept}$ residential and commercial consumption down and storage levels up. As a consequence, prices were low compared to those at the same time in 1994.

\section{Spot Market}

The relationship between futures settlement prices and average bid-week prices improved significantly in both 1993 and 1994, compared to 1991 and 1992. A small difference between the two prices means the futures market is more valuable as a "perfect hedging" tool. Overall, the futures price tended to close below the Henry Hub spot price during bid week in 1991 and 1992. However, the spot price was as likely to rise above the futures price as it was to fall below in 1993 and 1994. More importantly, the average difference between these two prices was essentially zero for 1993 and 1994, whereas it was $\$ 0.042$ for 1991 and 1992 . A decreasing spread between the cash and futures prices suggests smaller differences in transactions cost between these two markets. It may also imply gains in informational efficiency in the futures market, probably in response to the influx of more market participants and better information about the market.

\section{Outlook for Supply}

U.S. dry gas production is expected to continue to rise, reaching 18.9 trillion cubic feet in 1995 and 19.5 trillion cubic feet in 1996. Gas production has continued to increase even as drilling has been decreasing in the past 3 years. This is due to the rising level of technological capability in exploration and recovery. This increasing efficiency in gas exploration and development is also expected to result in increased dry gas productive capacity.

Net natural gas imports, especially from Canada, are also expected to grow to about 13 percent of total U.S. demand by 1994 . 
Figure 4. Marketed Production of INatural Gas in Selected States, 1990 - 1994

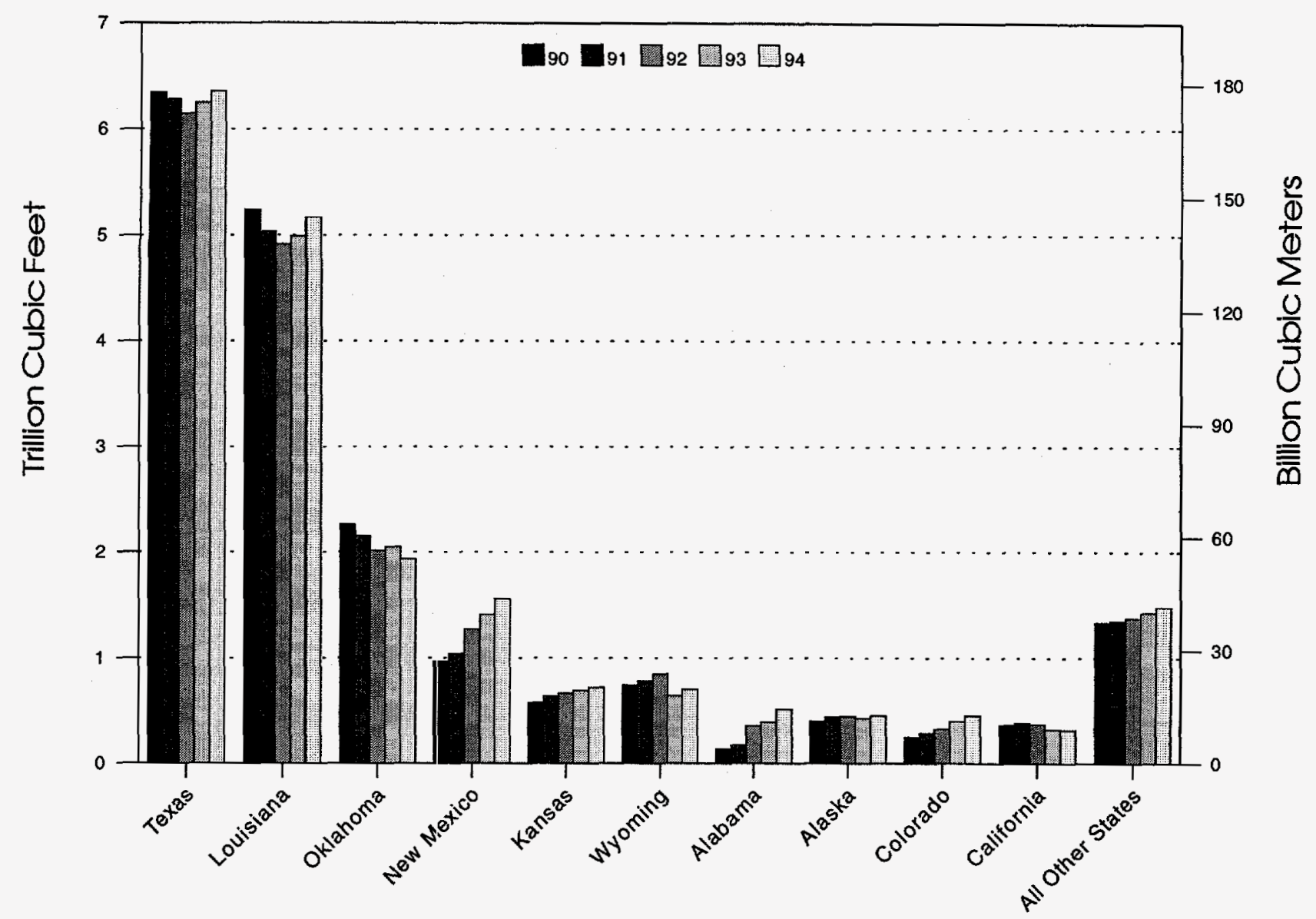

Sources: Illinois, Louisiana, Pennsylvania, South Dakota, and West Virginia, 1994: Energy Information Administration (EIA), U.S. Crude Oil, Natural Gas, and Natura/ Gas Liquids Reserves, 1994 Annual Report, DOE/EIA-0216(\$14); and ElA computations. All other data: Form ElA-627, "Annual Quantity and Value of Natural Gas Report" and the United States Minerals Management Service. 
Table 3. Gross Withdrawals and Marketed Production of Natural Gas by State, 1990-1994 (Million Cubic Feet)

\begin{tabular}{|c|c|c|c|c|c|c|c|}
\hline \multirow{2}{*}{$\begin{array}{l}\text { Year } \\
\text { and } \\
\text { State }\end{array}$} & \multicolumn{3}{|c|}{ Gross Withdrawals } & \multirow{2}{*}{ Repressuring } & \multirow{2}{*}{$\begin{array}{l}\text { Nonhydro- } \\
\text { carbon } \\
\text { Gases } \\
\text { Removed }\end{array}$} & \multirow{2}{*}{$\begin{array}{l}\text { Vented } \\
\text { and } \\
\text { Flared }\end{array}$} & \multirow{2}{*}{$\begin{array}{c}\text { Marketed } \\
\text { Production }\end{array}$} \\
\hline & $\begin{array}{c}\text { From Gas } \\
\text { Welis }\end{array}$ & $\begin{array}{l}\text { From Oil } \\
\text { Wells }\end{array}$ & Total & & & & \\
\hline $\begin{array}{l}1990 \text { Total ............... } \\
1991 \text { Total ............... } \\
1992 \text { Total ............... } \\
1993 \text { Total ................ } \\
1994 \text { Total .............. }\end{array}$ & $\begin{array}{r}16,053,566 \\
16,017,626 \\
16,164,874 \\
R_{16,691,139} \\
17,377,544\end{array}$ & $\begin{array}{r}5,469,055 \\
5,732,482 \\
5,967,376 \\
R_{6,034,504} \\
6,231,110\end{array}$ & $\begin{array}{r}21,522,622 \\
21,750,108 \\
22,132,249 \\
R_{22,725,642} \\
23,608,654\end{array}$ & $\begin{array}{r}2,489,040 \\
2,771,928 \\
2,972,552 \\
R_{3}, 103,014 \\
3,332,644\end{array}$ & $\begin{array}{l}289,374 \\
275,831 \\
280,370 \\
413,971 \\
412,178\end{array}$ & $\begin{array}{r}150,415 \\
169,909 \\
167,519 \\
R_{226,743} \\
228,336\end{array}$ & $\begin{array}{r}18,593,792 \\
18,532,439 \\
18,711,808 \\
R_{18,981,915} \\
19,635,495\end{array}$ \\
\hline $\begin{array}{r}\text { Alabama Total.......... } \\
\text { Onshore............... } \\
\text { State Offshore....... } \\
\text { Federal Offshore... } \\
\text { Alaska Total............. } \\
\text { Onshore............... } \\
\text { State Offshore....... } \\
\text { Federal Offshore... }\end{array}$ & $\begin{array}{r}566,140 \\
219,955 \\
228,878 \\
117,308 \\
180,639 \\
80,839 \\
99,801 \\
0\end{array}$ & $\begin{array}{r}12,722 \\
9,528 \\
0 \\
3,194 \\
2,905,261 \\
2,780,760 \\
124,501 \\
0\end{array}$ & $\begin{array}{r}578,862 \\
229,483 \\
228,878 \\
120,502 \\
3,085,900 \\
2,861,599 \\
224,301 \\
0\end{array}$ & $\begin{array}{r}33,961 \\
33,961 \\
0 \\
0 \\
2,619,236 \\
2,517,259 \\
101,977 \\
0\end{array}$ & $\begin{array}{r}26,980 \\
18,912 \\
8,008 \\
0 \\
0 \\
0 \\
0 \\
0\end{array}$ & $\begin{array}{r}2,650 \\
2,072 \\
577 \\
0 \\
13,240 \\
8,733 \\
4,507 \\
0\end{array}$ & $\begin{array}{r}515,271 \\
174,537 \\
220,233 \\
120,502 \\
453,425 \\
335,608 \\
117,817 \\
0\end{array}$ \\
\hline $\begin{array}{c}\text { Arizona............... } \\
\text { Arkansas............ } \\
\text { California Total........ } \\
\text { Onshore................ } \\
\text { State Offshore...... } \\
\text { Federal Offshore... }\end{array}$ & $\begin{array}{r}711 \\
161,967 \\
113,525 \\
102,461 \\
0 \\
11,064\end{array}$ & $\begin{array}{r}48 \\
33,446 \\
282,227 \\
232,526 \\
7,204 \\
42,497\end{array}$ & $\begin{array}{r}759 \\
195,413 \\
395,752 \\
334,987 \\
7,204 \\
53,561\end{array}$ & $\begin{array}{r}0 \\
7,426 \\
84,369 \\
81,641 \\
0 \\
2,728\end{array}$ & $\begin{array}{r}0 \\
0 \\
1,126 \\
1,126 \\
\text { NA } \\
\text { NA }\end{array}$ & $\begin{array}{r}8 \\
313 \\
830 \\
830 \\
\text { NA } \\
\text { NA }\end{array}$ & $\begin{array}{r}752 \\
187,673 \\
309,427 \\
251,390 \\
7,204 \\
50,833\end{array}$ \\
\hline 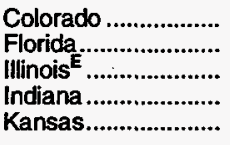 & $\begin{array}{r}365,651 \\
0 \\
323 \\
107 \\
628,900\end{array}$ & $\begin{array}{r}101,379 \\
8,468 \\
10 \\
0 \\
85,759\end{array}$ & $\begin{array}{r}467,031 \\
8,468 \\
333 \\
107 \\
714,659\end{array}$ & $\begin{array}{r}11,347 \\
0 \\
0 \\
\mathrm{NA} \\
1,215\end{array}$ & $\begin{array}{r}0 \\
762 \\
0 \\
\text { NA } \\
\text { NA }\end{array}$ & $\begin{array}{r}2,476 \\
220 \\
0 \\
\text { NA } \\
715\end{array}$ & $\begin{array}{r}453,207 \\
7,486 \\
333 \\
107 \\
712,730\end{array}$ \\
\hline $\begin{array}{l}\text { Kentucky ............... } \\
\text { Louisiana Total ........ } \\
\text { Onshore.................. } \\
\text { State Offshore....... } \\
\text { Federal Offshore... }\end{array}$ & $\begin{array}{r}73,081 \\
4,527,042 \\
1,393,543 \\
137,823 \\
2,995,676\end{array}$ & $\begin{array}{r}0 \\
699,055 \\
159,060 \\
21,690 \\
518,305\end{array}$ & $\begin{array}{r}73,081 \\
5,226,097 \\
1,552,603 \\
159,513 \\
3,513,981\end{array}$ & $\begin{array}{r}\text { * } \\
36,703 \\
1,421 \\
0 \\
35,282\end{array}$ & $\begin{array}{l}\text { * } \\
\text { NA } \\
\text { NA } \\
\text { NA }\end{array}$ & $\begin{array}{r}\star \\
19,689 \\
19,689 \\
\text { NA } \\
\text { NA }\end{array}$ & $\begin{array}{r}73,081 \\
5,169,705 \\
1,531,493 \\
159,513 \\
3,478,699\end{array}$ \\
\hline $\begin{array}{l}\text { Maryland.................. } \\
\text { Michigan ................. } \\
\text { Mississippi ................ } \\
\text { Missouri ................... } \\
\text { Montana.................. }\end{array}$ & $\begin{array}{r}26 \\
136,989 \\
112,205 \\
8 \\
44,350\end{array}$ & $\begin{array}{r}0 \\
91,332 \\
9,597 \\
0 \\
6,722\end{array}$ & $\begin{array}{r}26 \\
228,321 \\
121,802 \\
8 \\
51,072\end{array}$ & $\begin{array}{r}0 \\
2,340 \\
12,083 \\
0 \\
105\end{array}$ & $\begin{array}{r}0 \\
N A \\
44,344 \\
0 \\
N A\end{array}$ & $\begin{array}{r}0 \\
3,324 \\
1,928 \\
0 \\
551\end{array}$ & $\begin{array}{r}26 \\
222,657 \\
63,448 \\
8 \\
50,416\end{array}$ \\
\hline $\begin{array}{l}\text { Nebraska ................ } \\
\text { Nevada ................... } \\
\text { New Mexico ............. } \\
\text { New York................ } \\
\text { North Dakota.......... }\end{array}$ & $\begin{array}{r}2,093 \\
0 \\
1,381,756 \\
19,937 \\
19,592\end{array}$ & $\begin{array}{r}805 \\
16 \\
200,041 \\
539 \\
43,640\end{array}$ & $\begin{array}{r}2,898 \\
16 \\
1,581,797 \\
20,476 \\
63,232\end{array}$ & $\begin{array}{r}* \\
0 \\
18,982 \\
0 \\
2,577\end{array}$ & $\begin{array}{r}* \\
0 \\
3,164 \\
0 \\
358\end{array}$ & $\begin{array}{r}* \\
0 \\
1,963 \\
11 \\
2,492\end{array}$ & $\begin{array}{r}2,898 \\
16 \\
1,557,689 \\
20,465 \\
57,805\end{array}$ \\
\hline $\begin{array}{l}\text { Ohio } \\
\text { Oklahoma .................... } \\
\text { Oregon................... } \\
\text { Pennsylvania E......... } \\
\text { South Dakota.......... }\end{array}$ & $\begin{array}{r}132,151 \\
1,626,858 \\
4,200 \\
148,763 \\
1,000\end{array}$ & $\begin{array}{r}0 \\
308,006 \\
0 \\
1,465 \\
6,264\end{array}$ & $\begin{array}{r}132,151 \\
1,934,864 \\
4,200 \\
150,228 \\
7,264\end{array}$ & $\begin{array}{r}\text { NA } \\
\text { NA } \\
55 \\
\text { NA } \\
22\end{array}$ & $\begin{array}{r}\text { NA } \\
\text { NA } \\
924 \\
\text { NA } \\
0\end{array}$ & $\begin{array}{r}\text { NA } \\
\text { NA } \\
0 \\
\text { NA } \\
5,805\end{array}$ & $\begin{array}{r}132,151 \\
1,934,864 \\
3,221 \\
150,228 \\
1,437\end{array}$ \\
\hline $\begin{array}{c}\text { Tennessee ............... } \\
\text { Texas Total ............. } \\
\text { Onshore ................. } \\
\text { State Offshore....... } \\
\text { Federal Offshore... }\end{array}$ & $\begin{array}{r}0 \\
5,643,577 \\
4,310,086 \\
83,577 \\
1,249,914\end{array}$ & $\begin{array}{r}1,990 \\
1,268,127 \\
1,208,892 \\
996 \\
58,240\end{array}$ & $\begin{array}{r}1,990 \\
6,911,705 \\
5,518,978 \\
84,573 \\
1,308,154\end{array}$ & $\begin{array}{r}\text { NA } \\
319,360 \\
319,360 \\
\text { NA } \\
\text { NA }\end{array}$ & $\begin{array}{r}\text { NA } \\
196,463 \\
196,463 \\
\text { NA } \\
\text { NA }\end{array}$ & $\begin{array}{r}\text { NA } \\
42,037 \\
42,037 \\
\text { NA } \\
\text { NA }\end{array}$ & $\begin{array}{r}1,990 \\
6,353,844 \\
4,961,117 \\
84,573 \\
1,308,154\end{array}$ \\
\hline $\begin{array}{l}\text { Utah } \\
\text { Virginia........................... } \\
\text { West Virginia E.......... } \\
\text { Wyoming................. }\end{array}$ & $\begin{array}{r}304,347 \\
50,259 \\
182,000 \\
949,343\end{array}$ & $\begin{array}{r}42,672 \\
0 \\
0 \\
121,519\end{array}$ & $\begin{array}{r}347,019 \\
50,259 \\
182,000 \\
1,070,862\end{array}$ & $\begin{array}{r}72,798 \\
0 \\
N A \\
110,064\end{array}$ & $\begin{array}{r}\text { NA } \\
0 \\
N A \\
138,056\end{array}$ & $\begin{array}{r}3,363 \\
0 \\
N A \\
126,722\end{array}$ & $\begin{array}{r}270,858 \\
50,259 \\
182,000 \\
696,018\end{array}$ \\
\hline
\end{tabular}

$\mathbf{R}=$ Revised data

$E$ = Estimated Data.

NA = Not Available.

= Volume is less than 500,000 cubic feet.

Nole: Totals may not equal sum of components due to independent rounding

Sources: Illinois, Pennsylvania, and West Virginia, 1994: Energy Information Administration (EIA), U.S. Crude Oil, Natura/ Gas, and Natural Gas Liquids Reserves, 1994 Annual Report, DOE/EIA-0216(94); and EIA computations. All other data: Form EIA-627, "Annual Quantity and Value of Natural Gas Report," and the United States Minerals Management Service. 
Table 4. Offshore Gross Withdrawals of Natural Gas by State, 1990-1994 (Million Cubic Feet)

\begin{tabular}{|c|c|c|c|c|c|c|c|}
\hline \multirow{2}{*}{$\begin{array}{l}\text { Year } \\
\text { and } \\
\text { State }\end{array}$} & \multicolumn{3}{|c|}{ State } & \multicolumn{3}{|c|}{ Federal } & \multirow{2}{*}{$\begin{array}{c}\text { Total } \\
\text { Offshore }\end{array}$} \\
\hline & $\begin{array}{l}\text { From } \\
\text { Gas Wells }\end{array}$ & $\begin{array}{l}\text { From } \\
\text { Oil Welts }\end{array}$ & Total & $\begin{array}{l}\text { From } \\
\text { Gas Wells }\end{array}$ & $\begin{array}{l}\text { From } \\
\text { Oil Wells }\end{array}$ & Total & \\
\hline $\begin{array}{l}1990 \text { Total .................. } \\
\text { Alabama ............... } \\
\text { Alaska.................. } \\
\text { California .............. } \\
\text { Louisiana ............... } \\
\text { Texas.................... }\end{array}$ & $\begin{array}{r}383,544 \\
19,861 \\
94,642 \\
340 \\
161,292 \\
107,409\end{array}$ & $\begin{array}{r}79,1108 \\
0 \\
49,4: 22 \\
7,743 \\
20,948 \\
9 ! 95\end{array}$ & $\begin{array}{r}462,652 \\
19,861 \\
144,064 \\
8,083 \\
182,240 \\
108,404\end{array}$ & $\begin{array}{r}4,562,144 \\
0 \\
0 \\
15,640 \\
3,140,870 \\
1,405,634\end{array}$ & $\begin{array}{r}484,516 \\
0 \\
0 \\
0 \\
34,332 \\
408,654 \\
41,530\end{array}$ & $\begin{array}{r}5,046,660 \\
0 \\
0 \\
49,972 \\
3,549,524 \\
1,447,164\end{array}$ & $\begin{array}{r}5,509,312 \\
19,861 \\
144,064 \\
58,055 \\
3,731,764 \\
1,555,568\end{array}$ \\
\hline $\begin{array}{l}1991 \text { Total ................. } \\
\text { Alabama ............... } \\
\text { Alaska.................. } \\
\text { California ............... } \\
\text { Louisiana ............... } \\
\text { Texas.................... }\end{array}$ & $\begin{array}{r}359,112 \\
32,603 \\
100,733 \\
0 \\
128,891 \\
96,885\end{array}$ & $\begin{array}{r}99,6138 \\
0 \\
70,9332 \\
7,610 \\
19,5 ; 38 \\
1,608\end{array}$ & $\begin{array}{r}458,800 \\
32,603 \\
171,665 \\
7,610 \\
148,429 \\
98,493\end{array}$ & $\begin{array}{r}4,314,407 \\
0 \\
0 \\
16,464 \\
2,946,749 \\
1,351,194\end{array}$ & $\begin{array}{r}535,250 \\
0 \\
0 \\
35,391 \\
455,052 \\
44,807\end{array}$ & $\begin{array}{r}4,849,657 \\
0 \\
0 \\
51,855 \\
3,401,801 \\
1,396,001\end{array}$ & $\begin{array}{r}5,308,457 \\
32,603 \\
171,665 \\
59,465 \\
3,550,230 \\
1,494,494\end{array}$ \\
\hline $\begin{array}{c}1992 \text { Total................. } \\
\text { Alabama ............... } \\
\text { Alaska................... } \\
\text { California .............. } \\
\text { Louisiana ................ } \\
\text { Texas...................... }\end{array}$ & $\begin{array}{r}415,486 \\
112,311 \\
110,067 \\
0 \\
+16,470 \\
76,638\end{array}$ & $\begin{array}{r}136,809 \\
0 \\
106,311 \\
7,2442 \\
21,6: 31 \\
1,6: 5\end{array}$ & $\begin{array}{r}552,294 \\
112,311 \\
216,377 \\
7,242 \\
138,101 \\
78,263\end{array}$ & $\begin{array}{r}4,258,686 \\
79,294 \\
0 \\
13,947 \\
2,867,842 \\
1,297,602\end{array}$ & $\begin{array}{r}513,058 \\
0 \\
0 \\
41,284 \\
436,493 \\
35,281\end{array}$ & $\begin{array}{r}4,771,744 \\
79,294 \\
0 \\
55,231 \\
3,304,336 \\
1,332,883\end{array}$ & $\begin{array}{r}5,324,039 \\
191,605 \\
216,377 \\
62,473 \\
3,442,437 \\
1,411,147\end{array}$ \\
\hline $\begin{array}{l}1993 \text { Total................ } \\
\text { Alabama ................ } \\
\text { Alaska................... } \\
\text { California ............... } \\
\text { Louisiana ............... } \\
\text { Texas.................... }\end{array}$ & $\begin{array}{r}R_{470,487} \\
131,508 \\
127,834 \\
0 \\
R_{133,261} \\
77,883\end{array}$ & $\begin{array}{r}R_{136,9 \cdot 48} \\
0 \\
105,3153 \\
6,4134 \\
R_{23,7: 50} \\
1,3150\end{array}$ & $\begin{array}{r}\mathbf{R}_{607,435} \\
131,508 \\
233,198 \\
6,484 \\
\mathbf{R}_{157,011} \\
79,234\end{array}$ & $\begin{array}{r}4,215,015 \\
86,515 \\
0 \\
0 \\
10,618 \\
2,883,761 \\
1,234,121\end{array}$ & $\begin{array}{r}550,850 \\
0 \\
0 \\
41,532 \\
467,340 \\
41,978\end{array}$ & $\begin{array}{r}4,765,865 \\
86,515 \\
0 \\
0 \\
52,150 \\
3,351,101 \\
1,276,099\end{array}$ & $\begin{array}{r}R_{5,373,300} \\
218,023 \\
233,198 \\
58,635 \\
R_{3,508,112} \\
1,355,333\end{array}$ \\
\hline $\begin{array}{l}1994 \text { Total............... } \\
\text { Alabarna ................. } \\
\text { Alaska.................... } \\
\text { California ................ } \\
\text { Louisiana ............... } \\
\text { Texas...................... }\end{array}$ & $\begin{array}{r}550,079 \\
228,878 \\
99,801 \\
0 \\
137,823 \\
83,577\end{array}$ & $\begin{array}{r}154,390 \\
0 \\
124,501 \\
7,204 \\
21,690 \\
996\end{array}$ & $\begin{array}{r}704,469 \\
228,878 \\
224,301 \\
7,204 \\
159,513 \\
84,573\end{array}$ & $\begin{array}{r}4,373,962 \\
117,308 \\
0 \\
11,064 \\
2,995,676 \\
1,249,914\end{array}$ & $\begin{array}{r}622,235 \\
3,194 \\
0 \\
42,497 \\
518,305 \\
58,240\end{array}$ & $\begin{array}{r}4,996,197 \\
120,502 \\
0 \\
53,561 \\
3,513,981 \\
1,308,154\end{array}$ & $\begin{array}{r}5,700,666 \\
349,380 \\
224,301 \\
60,765 \\
3,673,494 \\
1,392,727\end{array}$ \\
\hline
\end{tabular}

$R=$ Revised data.

Notes: For individual State and U.S. production, see Talble 3. Totals may not equal sum of components due to independent rounding

Source: Appropriate State agencies responses to informlal data requests and the United States Minerals Management Service. 
Table 5. Number of Producing Gas and Gas Condensate Wells by State as of December 31, 1990-1994

\begin{tabular}{|c|c|c|c|c|c|}
\hline State & 1990 & 1991 & 1992 & 1993 & 1994 \\
\hline 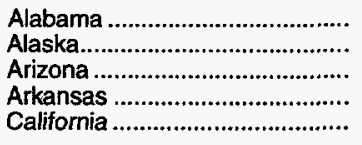 & $\begin{array}{r}2,362 \\
111 \\
5 \\
2,952 \\
1,162\end{array}$ & $\begin{array}{r}3,392 \\
110 \\
6 \\
2,780 \\
1,377\end{array}$ & $\begin{array}{r}3,350 \\
112 \\
6 \\
3,500 \\
1,126\end{array}$ & $\begin{array}{r}3,514 \\
113 \\
6 \\
3,500 \\
R_{1,092}\end{array}$ & $\begin{array}{r}3,565 \\
104 \\
19 \\
3,500 \\
1,261\end{array}$ \\
\hline 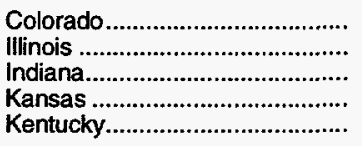 & $\begin{array}{r}5,741 \\
356 \\
1,307 \\
16,980 \\
11,713\end{array}$ & $\begin{array}{r}5,562 \\
373 \\
1,334 \\
17,948 \\
12,169\end{array}$ & $\begin{array}{r}5,912 \\
382 \\
1,333 \\
18,400 \\
12,483\end{array}$ & $\begin{array}{r}6,372 \\
385 \\
1,336 \\
19,472 \\
12,836\end{array}$ & $\begin{array}{r}7,056 \\
390 \\
1,348 \\
19,365 \\
13,036\end{array}$ \\
\hline 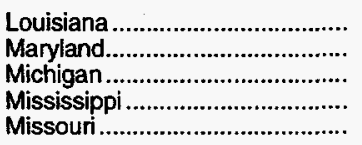 & $\begin{array}{r}16,889 \\
7 \\
1,438 \\
585 \\
8\end{array}$ & $\begin{array}{r}15,271 \\
7 \\
2,620 \\
629 \\
6\end{array}$ & $\begin{array}{r}13,512 \\
9 \\
3,257 \\
507 \\
5\end{array}$ & $\begin{array}{r}R_{15,569} \\
7 \\
5,500 \\
620 \\
8\end{array}$ & $\begin{array}{r}12,958 \\
7 \\
6,000 \\
583 \\
12\end{array}$ \\
\hline 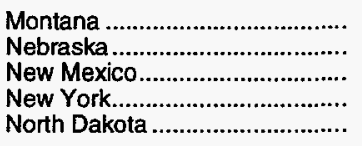 & $\begin{array}{r}2,607 \\
11 \\
17,124 \\
5,525 \\
103\end{array}$ & $\begin{array}{r}2,802 \\
12 \\
20,021 \\
5,737 \\
100\end{array}$ & $\begin{array}{r}2,890 \\
22 \\
18,040 \\
5,906 \\
104\end{array}$ & $\begin{array}{r}3,075 \\
59 \\
20,846 \\
5,757 \\
101\end{array}$ & $\begin{array}{r}2,940 \\
87 \\
23,292 \\
5,884 \\
104\end{array}$ \\
\hline 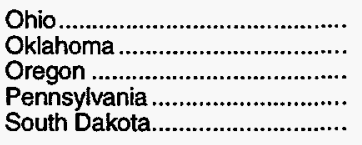 & $\begin{array}{r}34,586 \\
24,547 \\
19 \\
30,300 \\
54\end{array}$ & $\begin{array}{r}34,760 \\
28,216 \\
16 \\
31,000 \\
54\end{array}$ & $\begin{array}{r}34,784 \\
28,902 \\
16 \\
31,000 \\
38\end{array}$ & $\begin{array}{r}34,782 \\
29,118 \\
18 \\
31,100 \\
R_{47}\end{array}$ & $\begin{array}{r}34,731 \\
29,121 \\
19 \\
31,150 \\
55\end{array}$ \\
\hline 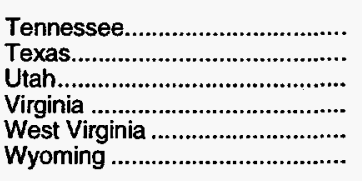 & $\begin{array}{r}R_{0} \\
50,867 \\
822 \\
819 \\
37,500 \\
2,600\end{array}$ & $\begin{array}{r}\mathbf{R}_{0} \\
47,615 \\
913 \\
886 \\
37,800 \\
2,821\end{array}$ & $\begin{array}{r}{ }^{R} 0 \\
46,298 \\
1,006 \\
1,153 \\
38,250 \\
3,111\end{array}$ & $\begin{array}{r}\mathbf{R}_{0} \\
47,101 \\
1,061 \\
1,426 \\
R_{33,716} \\
3,615\end{array}$ & $\begin{array}{r}0 \\
48,654 \\
1,303 \\
1,470 \\
35,250 \\
3,942\end{array}$ \\
\hline Total & $\mathbf{R}_{269,100}$ & $\mathbf{R}_{276,337}$ & $R_{275,414}$ & $R_{282,152}$ & 287,206 \\
\hline
\end{tabular}

$\mathrm{R}=$ Revised data.

Note: Totals may not equal sum of components due to independent rounding.

Sources: Energy Information Administration (EIA), Form EIA-627, "Annual Quantity and Value of Natural Gas Peport," the United States Minerals Management Services (MMS), and Worfd Oil Magazine. 
Table 6. Estimated Total Dry Natural Gas Proved Reserves by State, 1990-1994 (Billion Cubic Feet)

\begin{tabular}{|c|c|c|c|c|c|}
\hline State & 1990 & 1991 . & 1992 & 1993 & 1994 \\
\hline 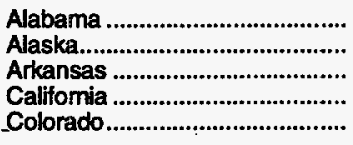 & $\begin{array}{l}4,125 \\
9,300 \\
1,731 \\
3,185 \\
4,555\end{array}$ & $\begin{array}{l}5,414 \\
9,553 \\
1,669 \\
3,004 \\
5,767\end{array}$ & $\begin{array}{l}5,802 \\
9,638 \\
1,750 \\
2,778 \\
6,198\end{array}$ & $\begin{array}{l}5,140 \\
9,907 \\
1,552 \\
2,682 \\
6,722\end{array}$ & $\begin{array}{l}4,830 \\
9,733 \\
1,607 \\
2,402 \\
6,753\end{array}$ \\
\hline 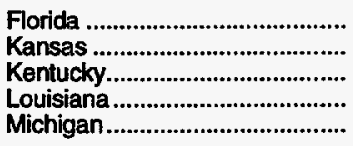 & $\begin{array}{r}45 \\
9,614 \\
1,016 \\
11,728 \\
1,243\end{array}$ & $\begin{array}{r}38 \\
9,358 \\
1,155 \\
10,912 \\
1,334\end{array}$ & $\begin{array}{r}47 \\
9,681 \\
1,084 \\
9,780 \\
1,223\end{array}$ & $\begin{array}{r}50 \\
9,348 \\
1,003 \\
9,174 \\
1,160\end{array}$ & $\begin{array}{r}98 \\
9,156 \\
969 \\
9,748 \\
1,323\end{array}$ \\
\hline 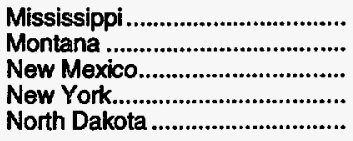 & $\begin{array}{r}1,126 \\
899 \\
17,260 \\
354 \\
586\end{array}$ & $\begin{array}{r}1,057 \\
831 \\
18,539 \\
331 \\
472\end{array}$ & $\begin{array}{r}869 \\
859 \\
18,998 \\
329 \\
496\end{array}$ & $\begin{array}{r}797 \\
673 \\
18,619 \\
264 \\
525\end{array}$ & $\begin{array}{r}650 \\
717 \\
17,228 \\
242 \\
507\end{array}$ \\
\hline 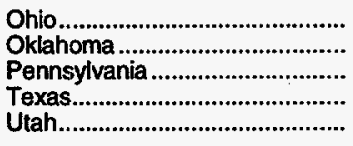 & $\begin{array}{r}1,214 \\
16,151 \\
1,720 \\
38,192 \\
1,510\end{array}$ & $\begin{array}{r}1,181 \\
14,725 \\
1,629 \\
36,174 \\
1,702\end{array}$ & $\begin{array}{r}1,161 \\
13,926 \\
1,528 \\
35,093 \\
1,830\end{array}$ & $\begin{array}{r}1,104 \\
13,289 \\
1,717 \\
34,718 \\
2,040\end{array}$ & $\begin{array}{r}1,094 \\
13,487 \\
1,800 \\
35,974 \\
1,789\end{array}$ \\
\hline $\begin{array}{l}\text { Virginia } \\
\text { West Virginia .............................. } \\
\text { Wyoming .................................. } \\
\text { Federal Offshore ..................... } \\
\text { Miscellaneous }{ }^{\mathrm{a}} \text {............................. }\end{array}$ & $\begin{array}{r}138 \\
2,207 \\
9,944 \\
31,433 \\
70\end{array}$ & $\begin{array}{r}225 \\
2,528 \\
9,941 \\
29,448 \\
75\end{array}$ & $\begin{array}{r}904 \\
2,356 \\
10,826 \\
27,767 \\
92\end{array}$ & $\begin{array}{r}1,322 \\
2,439 \\
10,933 \\
27,143 \\
94\end{array}$ & $\begin{array}{r}1,833 \\
2,565 \\
10,879 \\
28,388 \\
65\end{array}$ \\
\hline 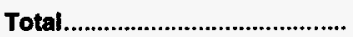 & 169,346 & 167,062 & 165,015 & 162,415 & 163,837 \\
\hline
\end{tabular}

a Includes Arizona, Itinois, Indiana, Maryland, Missouri, Nebraska, Nevada, Oregon, South Dakota, and Tennessee.

Note: Totals may not equal sum of components due to independent rounding.

Sources: Energy Information Administration (EIA), U.S. Crude Oil, Natural Gas, and Natural Gas Liquids Reserves, 1994 Annual Report, DOE/ElA-0216(94). 
Table 7. Wellhead Value and Marketed Production of Natural Gas by State, 1990-1994

\begin{tabular}{|c|c|c|c|c|c|}
\hline \multirow{2}{*}{$\begin{array}{l}\text { Year } \\
\text { and } \\
\text { State }\end{array}$} & \multicolumn{2}{|c|}{ Reported Welihead Value } & \multirow{2}{*}{$\begin{array}{l}\text { Average Wellhead } \\
\text { Price } \\
\text { (dollars per } \\
\text { thousand cubic feet) }\end{array}$} & \multirow{2}{*}{$\begin{array}{l}\text { Marketed } \\
\text { Production } \\
\text { (million } \\
\text { cubic feet) }\end{array}$} & \multirow{2}{*}{$\begin{array}{l}\text { Imputed Wellhead Value } \\
\text { of } \\
\text { Marketed Production } \\
\text { (thousand dollars) }\end{array}$} \\
\hline & $\begin{array}{c}\text { Volumea } \\
\text { (million } \\
\text { cubic feet) }\end{array}$ & $\begin{array}{l}\text { Value } \\
\text { (thousand } \\
\text { dollars) }\end{array}$ & & & \\
\hline 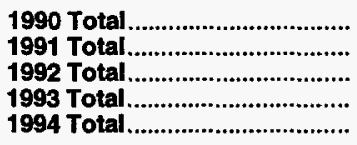 & $\begin{array}{l}16,408,437 \\
15,770,393 \\
15,852,090 \\
15,341,514 \\
15,554,688\end{array}$ & $\begin{array}{l}-- \\
-- \\
-- \\
--\end{array}$ & $\begin{array}{r}1.71 \\
1.64 \\
1.74 \\
\mathrm{R}_{2.04} \\
1.88\end{array}$ & $\begin{array}{r}18,593,792 \\
18,532,439 \\
18,711,808 \\
\mathbf{R}_{18,981,915} \\
19,635,495\end{array}$ & $\begin{array}{r}31,754,803 \\
30,327,779 \\
32,570,827 \\
\mathrm{~A}_{38,810,612} \\
36,849,541\end{array}$ \\
\hline 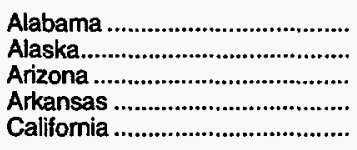 & $\begin{array}{r}70,489 \\
201,891 \\
691 \\
24,326 \\
240,972\end{array}$ & $\begin{array}{r}153,040 \\
257,297 \\
967 \\
64,419 \\
732,115\end{array}$ & $\begin{array}{l}2.17 \\
1.27 \\
1.40 \\
2.65 \\
3.04\end{array}$ & $\begin{array}{r}515,271 \\
453,425 \\
752 \\
187,673 \\
309,427\end{array}$ & $\begin{array}{r}1,118,726 \\
577,858 \\
1,052 \\
496,986 \\
940,092\end{array}$ \\
\hline 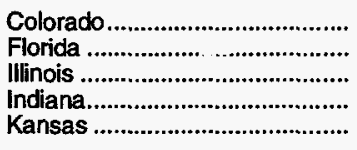 & $\begin{array}{r}441,343 \\
4,940 \\
333 \\
107 \\
714,659\end{array}$ & $\begin{array}{r}612,194 \\
6,324 \\
799 \\
211 \\
1,141,870\end{array}$ & $\begin{array}{l}1.39 \\
1.28 \\
2.40 \\
1.97 \\
1.60\end{array}$ & $\begin{array}{r}453,207 \\
7,486 \\
333 \\
107 \\
712,730\end{array}$ & $\begin{array}{r}628,653 \\
9,583 \\
799 \\
211 \\
1,138,785\end{array}$ \\
\hline 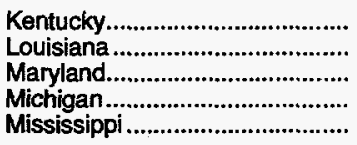 & $\begin{array}{r}73,081 \\
3,562,360 \\
26 \\
216,268 \\
105,646\end{array}$ & $\begin{array}{r}163,938 \\
7,392,495 \\
35 \\
423,474 \\
157,908\end{array}$ & $\begin{array}{l}2.24 \\
2.08 \\
1.37 \\
1.96 \\
1.49\end{array}$ & $\begin{array}{r}73,081 \\
5,169,705 \\
26 \\
222,657 \\
63,448\end{array}$ & $\begin{array}{r}163,938 \\
10,728,016 \\
35 \\
435,984 \\
94,834\end{array}$ \\
\hline 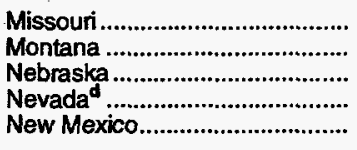 & $\begin{array}{r}8 \\
51,953 \\
2,098 \\
0 \\
1,510,804\end{array}$ & $\begin{array}{r}12 \\
75,597 \\
3,365 \\
0 \\
2,389,173\end{array}$ & $\begin{array}{r}1.49 \\
1.46 \\
1.60 \\
1.58\end{array}$ & $\begin{array}{r}8 \\
50,416 \\
2,898 \\
16 \\
1,557,689\end{array}$ & $\begin{array}{r}12 \\
73,360 \\
4,649 \\
0 \\
2,463,313\end{array}$ \\
\hline 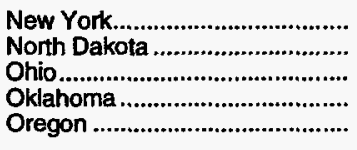 & $\begin{array}{r}19,684 \\
N A \\
130,855 \\
1,879,257 \\
3,221\end{array}$ & $\begin{array}{r}46,257 \\
N A \\
317,729 \\
3,195,421 \\
6,640\end{array}$ & $\begin{array}{r}2.35 \\
\text { NA } \\
2.43 \\
1.70 \\
2.06\end{array}$ & $\begin{array}{r}20,465 \\
57,805 \\
132,151 \\
1,934,864 \\
3,221\end{array}$ & $\begin{array}{r}48,093 \\
N A \\
320,875 \\
3,289,965 \\
6,640\end{array}$ \\
\hline 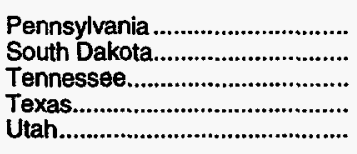 & $\begin{array}{r}187,113 \\
1,000 \\
1,990 \\
5,834,671 \\
154,907\end{array}$ & $\begin{array}{r}515,948 \\
1,735 \\
4,300 \\
11,005,805 \\
238,507\end{array}$ & $\begin{array}{l}2.76 \\
1.73 \\
2.16 \\
1.89 \\
1.54\end{array}$ & $\begin{array}{r}150,228 \\
1,437 \\
1,990 \\
6,353,844 \\
270,858\end{array}$ & $\begin{array}{r}414,242 \\
2,492 \\
4,300 \\
11,985,129 \\
417,034\end{array}$ \\
\hline 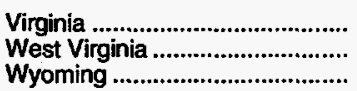 & $\begin{array}{r}50,259 \\
c_{55,453} \\
{ }^{c} 14,283\end{array}$ & $\begin{array}{r}108,058 \\
146,272 \\
29,328\end{array}$ & $\begin{array}{l}2.15 \\
2.64 \\
2.05\end{array}$ & $\begin{array}{r}50,259 \\
182,000 \\
696,018\end{array}$ & $\begin{array}{r}108,058 \\
480,071 \\
1,429,163\end{array}$ \\
\hline
\end{tabular}

a Quantity of production associated with reported wellhead value. A number of States reported values associated with quantities other than marketed production.

b Average wellhead price times marketed production does not equal imputed value of marketed production due to independent rounding.

c Quantity and value data obtained from Energy Information Administration Form EIA-176A, "nnual Report of Natural and Supplemental Gas Supply and Disposition."

d All of Nevada's marketed production was consumed as lease fuel.

R = Revised data.

Note: Totals may not equal sum of components due to independent rounding.

Sources: Illinois and Arkansas, 1994: EIA Computations. All other data: Form ElA-627, "Annual Quantity and Value of Natural Gas Report," and the United States Minerals Management Service. 
Table 8. Natural Gas Processed, Liquids Extracted, and Estimated Extraction Loss by State, 1994

\begin{tabular}{|c|c|c|c|c|}
\hline \multirow[b]{2}{*}{$\begin{array}{c}\text { Plant } \\
\text { Location }\end{array}$} & \multirow{2}{*}{$\begin{array}{c}\text { Natural } \\
\text { Gas } \\
\text { Processed } \\
\text { (million cubic feet) }\end{array}$} & \multirow{2}{*}{$\begin{array}{c}\text { Total } \\
\text { Liquids } \\
\text { Extracted } \\
\text { (thousand barrels) }\end{array}$} & \multicolumn{2}{|c|}{ Extraction Loss } \\
\hline & & & $\begin{array}{c}\text { Volume } \\
\text { (million cubic feet) }\end{array}$ & $\begin{array}{l}\text { Estimated } \\
\text { Heat } \\
\text { Content } \\
\text { (billion Etu) }\end{array}$ \\
\hline 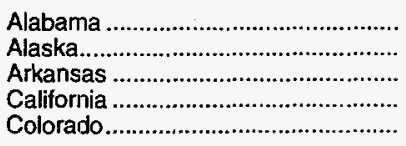 & $\begin{array}{r}136,195 \\
2,667,254 \\
218,710 \\
228,346 \\
353,855\end{array}$ & $\begin{array}{r}4,167 \\
26,071 \\
458 \\
9,425 \\
14,546\end{array}$ & $\begin{array}{r}5,647 \\
30,945 \\
553 \\
11,250 \\
19,612\end{array}$ & $\begin{array}{r}17,557 \\
115,975 \\
1,904 \\
39,921 \\
56,294\end{array}$ \\
\hline 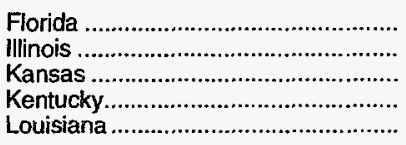 & $\begin{array}{r}10,265 \\
685 \\
965,674 \\
42,877 \\
4,200,126\end{array}$ & $\begin{array}{r}1,768 \\
69 \\
33,762 \\
1,453 \\
91,569\end{array}$ & $\begin{array}{r}1,789 \\
80 \\
46,936 \\
2,024 \\
128,583\end{array}$ & $\begin{array}{r}6,532 \\
299 \\
133,457 \\
5,796 \\
346,671\end{array}$ \\
\hline 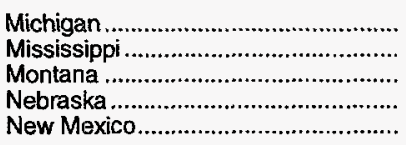 & $\begin{array}{r}196,000 \\
5,052 \\
10,418 \\
586 \\
800,836\end{array}$ & $\begin{array}{r}4,900 \\
337 \\
474 \\
6 \\
60,740\end{array}$ & $\begin{array}{r}6,371 \\
425 \\
631 \\
8 \\
86,607\end{array}$ & $\begin{array}{r}19,487 \\
1,445 \\
1,992 \\
0 \\
223,857\end{array}$ \\
\hline 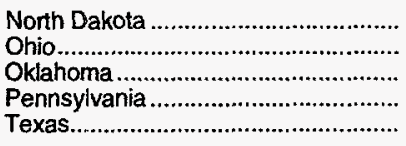 & $\begin{array}{r}55,150 \\
2,930 \\
1,092,734 \\
7,133 \\
4,160,551\end{array}$ & $\begin{array}{r}4,278 \\
78 \\
71,936 \\
394 \\
267,602\end{array}$ & $\begin{array}{r}5,671 \\
104 \\
101,564 \\
513 \\
381,020\end{array}$ & $\begin{array}{r}17,881 \\
328 \\
265,473 \\
1,645 \\
1,012,259\end{array}$ \\
\hline $\begin{array}{l}\text { Utah } \\
\text { West Virginia } \\
\text { Wyoming }\end{array}$ & $\begin{array}{l}368,585 \\
113,365 \\
821,389\end{array}$ & $\begin{array}{r}9,763 \\
7,369 \\
24,818\end{array}$ & $\begin{array}{l}13,780 \\
10,901 \\
33,486\end{array}$ & $\begin{array}{l}38,270 \\
27,572 \\
95,735\end{array}$ \\
\hline 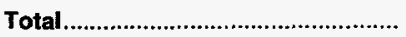 & $16,459,516$ & 635,983 & 888,500 & $2,430,350$ \\
\hline
\end{tabular}

Note: Totals may not equal sum of components due to independent rounding.

Sources: Natural Gas Processed, Total Liquids Extracted, and Extraction Loss Volume: Energy Information Administration (ElA), Form EIA-64A, "Annual Report of the Origin of Natural Gas Liquids Production." Estimated Heat Content of Extraction Loss: Estimated, assuming the makeup to total liquids production as reported on Form ElA-64A for each Stale was proportional to the components and products ultimately separated in the States as reported on the 12 monthly reports on Energy Information Administration, Form EIA-816, "Monthly Natural Gas Liquids Report," and applying the following conversion factors to the individual component and product production estimates: (million Btu extraction loss per barrel of liquid produced): ethane, 3.082; propane, 3.836; normal butane, 4.326; isobutane, 3.974; pentanes plus, 4.620. 


\section{Transmission}

The North American natural gas pipeline network continued to function as an efficient, integrated market system during 1994. In the United States, natural gas flowed predominately northeastward, originating primarily from the major producing centers in Texas and Louisiana. At the U.S./Canadian border, active trading took place, and Canada's role in supplying gas to the United States continued to expand.

In addition, underground storage is becoming more highly integrated into the daily operations of the pipeline system. The maintenance of required deliverability to the customer's destination is often predicated upon the availability of storage inventories. Pipeline deliverability is also being improved with the increasing development of new storage facilities, especially those that provide what is referred to as high-deliverability service. This type of storage facility, which supports rapid transfer of inventories during peak-demand periods, provides transporters and their customers greater assurance that peak-day demand may be met.

\section{Natural Gas Imports and Exports}

Active trading took place from Canada into the United States, and Canada is expected to continue to be a secure source of gas supply for many years to come. In the spring of 1995, the Mexican government took steps to open up their energy industry to foreign investments, perhaps signaling a new era of U.S. participation in the Mexican natural gas market.

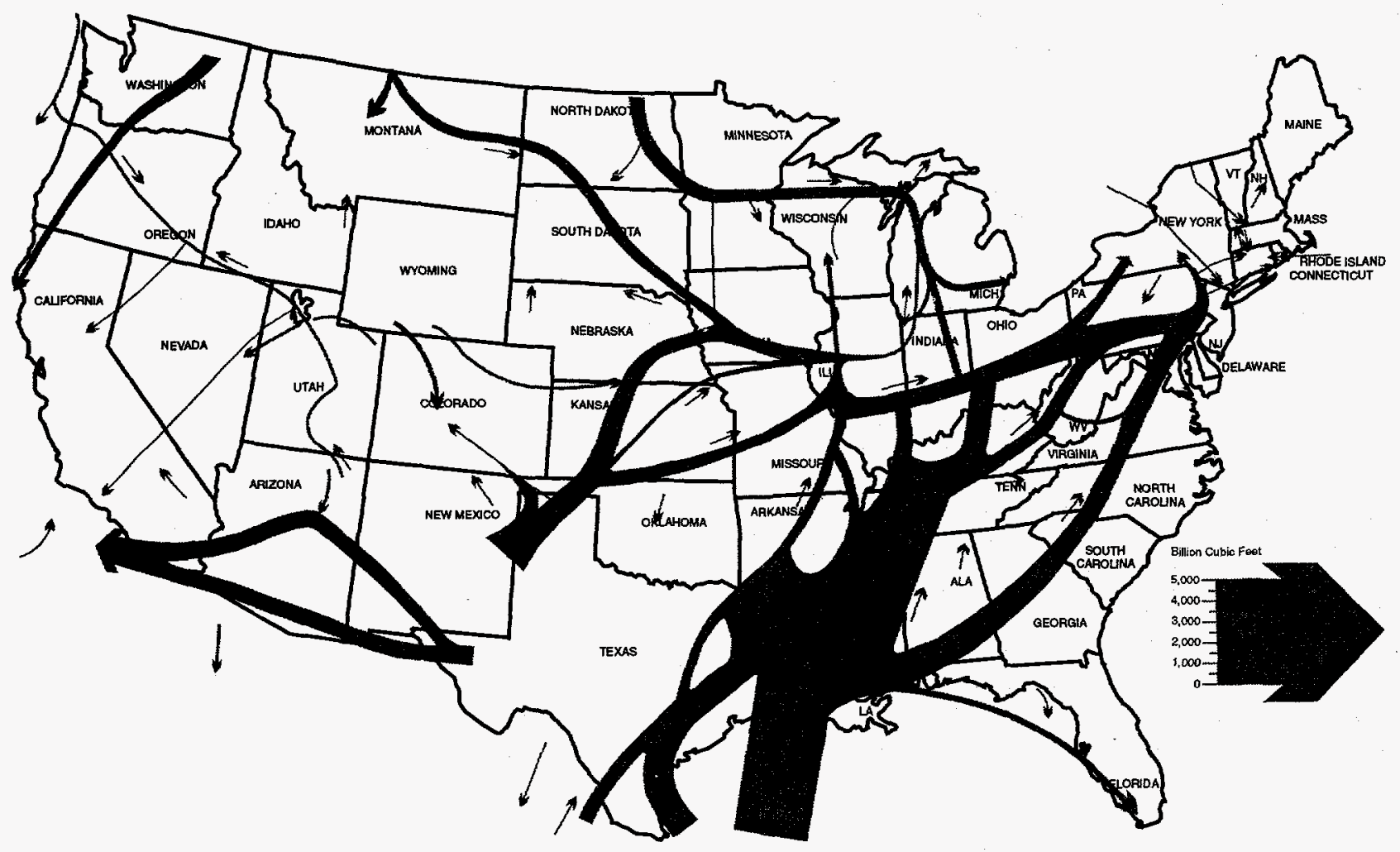


Some of the highlights of 1994 for U.S. natural gas imports and exports are as follows:

- Net imports rose for the eighth consecutive year, reaching 12 percent of U.S. natural gas consumption.

- Pipeline imports from Canada continued to climb to a new record level of 2,566 billion cubic feet.

- From 1993 to 1994, the price of Canadian imports fell to $\$ 1.86$ per thousand cubic feet, an 8-percent decrease.

- Liquefied natural gas (LNG) impoits from Algeria fell 38 percent to 50.8 billion cubic fest.

- In contrast to the decline in Canadian pipeline imports prices, the price of LNG imports from Algeria increased 4 percent.

- Exports to Canada, Mexico, and Japan all rose for an increase in total exports of 15 percent from 1993 to 1994.

\section{Trade with Canada}

Pipeline imports from Canada continued to grow to 2,566 billion cubic feet, a new record level. Competitive prices and additional pipeline capacity, which became available in late 1993 on the Pacific Gas Transmission pipeline, contributed to the increase. Natural gas imports from Canada have almost doubled in the last 6 years, while U.S. gas consumption rose 10 percent during the same time period. Net imports, which are nearly all from Canada, represent a growing share of U.S. natural gas consumption-- 12 percent in 1994 (Figure 8).

Pipeline imports from Canada enter the United States in four regional areas: the Pacific Northwest, the West, the Midwest, and the Northeast. From 1993 to 1994, natural gas imports rose in all regional areas with the largest increase (211 billion cubic feet) occurring in the West. Western imports climbed 41 percent to 723 billion cubic feet, primarily due to the additional capacity on the Pacific Gas Transmission pipeline. They entered the United States through Idaho, and most were destined for California markets.

Gas well completions in Canada have surged since 1992, indicating that Canada will remain a major supplier to the United States. This increase can be attributed to the gas wellhead prices, which in Canadian dollars have improved since 1992, even though the same prices in U.S. dollars have remained low or fallen slightly. Canada's share of the U.S. gas market is expected to continue to grow, and its role as a significant supplier seems secure for years to come.

\section{Trade with Mexico}

In 1994, exports to Mexico were 47 billion cubic feet, 17 percent higher than in 1993 but less than half of the record level reached in 1992. In December 1993 the United States began importing gas from Mexico for the first time since 1984. During 1994, these imports continued through June but represented less than 1 percent of total U.S. natural gas imports. Small amounts of gas have been imported from Mexico during 1995.

In April 1995, the Mexican Congress modified its Constitution to permit foreign investment in natural gas storage, distribution, and transmission. Electric power markets were also opened to foreign investment. Gas exploration and development activities were not opened to outsiders. Pemex will continue to conduct gas exploration and development under government jurisdiction.

Northern Mexico's role as a market for U.S. natural gas exports could expand in the coming years as new power generating capacity is added. Mexico's future plans for power plant construction include fueling by natural gas to solve some of their air-quality problems. It is anticipated that Mexico may meet its future demand for electricity through privately built gas-fired power plants. However, the history of state regulation of the oil and gas industry as well as the electricity industry may cause new development in these areas to be slow.

\section{Liquefied Natural Gas}

Natural gas can be transmitted in a vapor state via pipeline or in a liquid form, liquefied natural gas (LNG). Liquefaction makes overseas transportation possible. A 600-to-1 volume reduction is achieved by lowering the temperature of natural gas to approximately minus 260 degrees Fahrenheit. During 1994, the United States imported LNG from Algeria into Massachusetts and Louisiana and exported LNG from Alaska to Japan. LNG imports represented 2 percent of total imports, while LNG exports were 39 percent of total exports.

During 1994, LNG imports from Algeria fell 38 percent to 51 billion cubic feet. Sonatrach, the state-owned oil and gas company in Algeria, curtailed exports because of a major renovation project on that nation's liquefaction plants starting in August 1994.

LNG exports from Alaska to Japan rose 12 percent from 1993 to 1994 to a record 63 billion cubic feet. The expansion of the facility at Port Nikiski on the Kenai Peninsula 
in southern Alaska made the increase in exports possible. Although the price for these exports fell 5 percent from 1993 to 1994 , it remained strong at $\$ 3.18$ per thousand cubic feet, compared to the price for imports on LNG from Algeria of $\$ 2.28$ per thousand cubic feet.

The Cove Point LNG facility in southern Maryland, mothballed since 1980, plans to offer storage and peaking services for the winter of 1995-1996. In the near term the project calls for the use of domestic gas, but in the long term the facility also expects to be a receiving terminal for LNG tankers. Similar plans have also been announced for the Elba Island LNG facility in Georgia for late 1997.

There are currently four projects proposed to expand United States-International LNG Trade. The Ecoelectrica Project would build a power plant in Puerto Rico to be fueled by LNG, which would be imported from various suppliers. The Trinidad and Tobago Project would develop the natural gas resources off the east coast of Trinidad and construct an LNG facility to export the gas to the Northeast United States and Europe or Puerto Rico. Trinidad is located about 9 miles northeast of Venezuela. These projects could possibly become operational in 2 to 3 years.

The Nigerian LNG Export Project is a plan to construct a new natural gas liquefaction facility off that nation's coast. The facility would export Nigerian gas from the West Coast of Africa mostly to Europe but also to the United States. The
Yukon Pacific LNG Export Project calls for the construction of a new natural gas pipeline parallel to the existing crude oil pipeline in Alaska and construction of a gas liquefaction plant and marine terminal at Valdez. The LNG would be exported from Alaska to Japan and Pacific Rim countries. The service dates for these projects are 2000 or later.

\section{Storage}

Underground storage inventories and operations have become key factors in today's natural gas market. With customers making their own arrangements to ensure supply reliability, they are more conscious of costs involved and are demanding new and more flexible storage services, which has also led to a marked increase in proposals for new storage capacity.

The ability to store natural gas ensures supply reliability during periods of heavy demand by supplementing pipeline capacity and serving as backup supply in case of an interruption in wellhead production. It also enables greater system efficiency by allowing more level production and transmission flows. Currently, the industry has the capability to store approximately 8 trillion cubic feet of natural gas in 397 storage sites around the country. Almost half of this storage capacity is considered working gas storage that can be withdrawn to meet customer demand.

Figure 6. Locations of Existing Natural Gas Underground Storage Fields in the United States

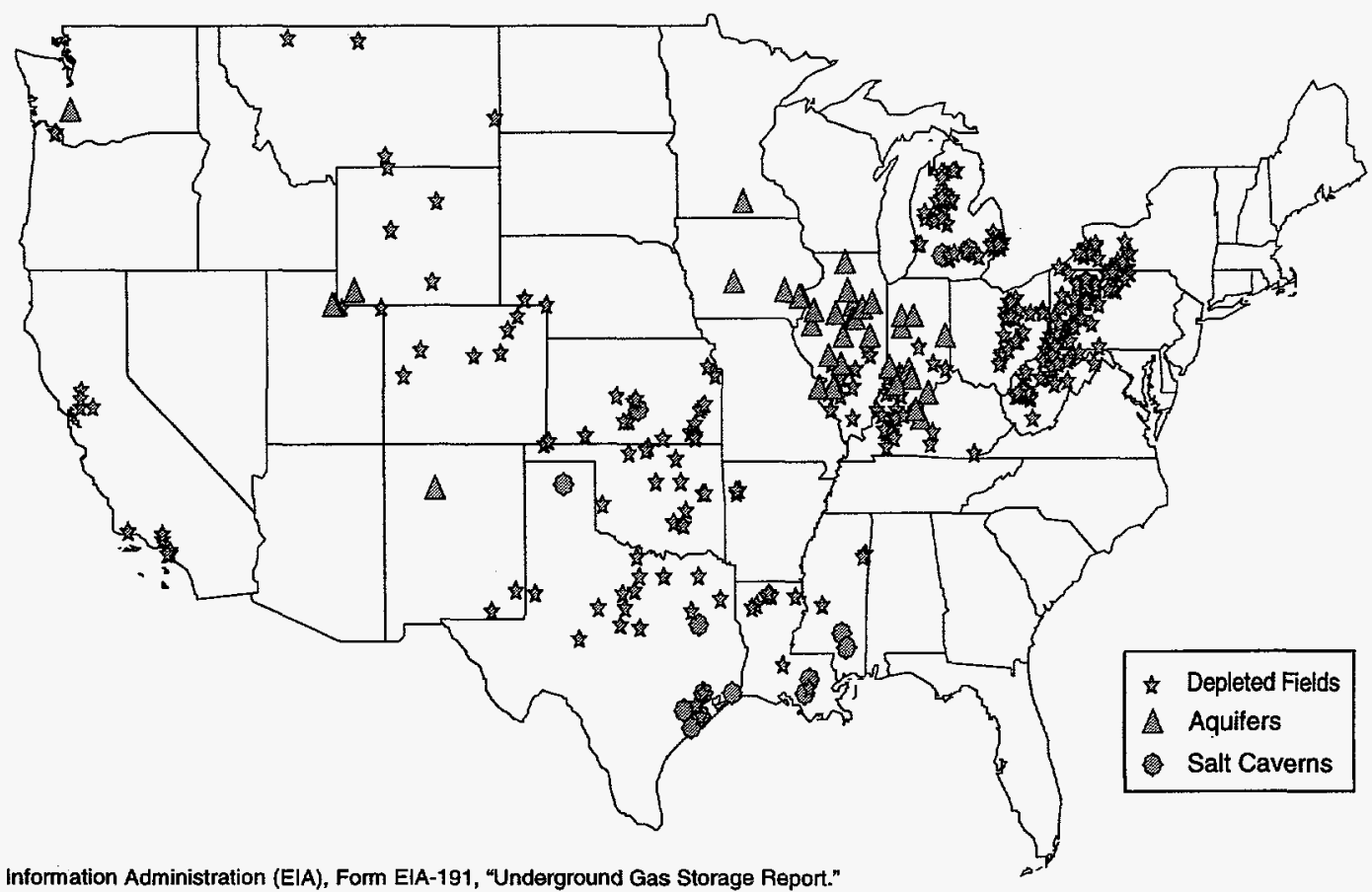

Source: Energy Information Administration (EIA), Form EIA-191, “Underground Gas Storage Report. 
Most of the new storage development is high deliverability storage, particularly salt cavern facilities. Although salt cavern storage facilities usually have much less capacity than traditional storage sites in depleted gas and oil fields, they can be recycled quickly and can convert from injections to withdrawals in only a few hours, providing the flexibility for meeting market requirements. More than one-third of the 21 existing salt cavern storage operations have been brought on line since 1991, adding 29 billion cubic feet of working gas capacity and 3.1 billion cubic feet per day of deliverability. Plans for additional high-deliverability projects account for 71 percent of the possible increase in peak-day deliverability by the end of the decade.

After the extreme cold of the 1993-1994 winter heating season, the level of injections during the warm weather of 1994 increased to adjust for the low level of working gas in storage. The 1994-1995 winter heating season began with 3.1 trillion cubic feet of working gas in storage. This level is 3 percent higher than the level at the beginning of the 1993-1994 heating season.

\section{Pipeline Expansions}

After a period of rapid growth in pipeline capacity, expansion of the system slowed in 1994, with only several small expansion projects completed during the year. About twothirds of the expansions were to enhance deliverability from Canada with most of the rest to enhance service to the Northeast. Overall since 1990, interregional capacity on the interstate natural gas pipeline system has increased by more than 12 percent, or 10 billion cubic feet per day. (Interregional capacity is defined as the capability to deliver gas to regional distribution networks from supply areas as measured at regional boundaries.) The total cost of new pipeline development and expansion implemented during the period is estimated to be about $\$ 6.5$ billion.

The impetus for much of the increase has been the demand potential in the Western and northeastern markets as well as the development of new supplies in western Canada and in Utah, Colorado, and New Mexico. Capacity from Canada into the United States, for instance, increased by 59 percent during the period. Capacity from the Central to the Western Region also increased dramatically, 219 percent, while capacity from the Southwest to the West increased more modestly, 23 percent.

Overall capacity usage rates on the expanded network increased despite some soft regional markets. For instance, annual average use of Canadian import capacity rose by almost 8 percent in 1994 from 1990 levels, increasing on all routes but those into the Western Region. Average capacity usage rates remained unchanged in the Western Region from the 78 percent level in 1990 . The enhanced oil recovery market in California supported high average utilization rates ( 80 percent) on those pipelines extending from the Central Region to the Western Region.

As of May 1995, at least 40 new or expansion pipeline projects of varying sizes were under study, under construction, or before the Federal Energy Regulatory Commission (FERC) for consideration. Concerns about market expansion, surplus of capacity in some areas, and the ability to recover costs have resulted in a slowdown in planned expansions. In 1994, several major proposed projects were either downsized, canceled, postponed, or withdrawn from the FERC approval process. The backlog of 40 expansion projects, 9.7 billion cubic feet per day, if fully implemented, would add 5.3 billion cubic feet per day of pipeline capacity to current interregional capabilities by the end of 1998 . This would represent an increase of 6 percent from the level at the end of 1994.

A growing factor in capacity expansions has been the drive to expand into new market areas and to offer new services. Expansion of trunkline capacity was the larger component of capacity increases during the early part of this decade. Now capacity expansions are focusing on improving services within regions and developing operational flexibility to support new services. One example of this is the Winternet project, which is a joint effort of Texas Eastern Pipeline and CNG Transmission Company. This project will combine capacity expansion into a new market in northern North Carolina with the packaging of storage, peaking support, and other services to support new customers there and in the Northeast. Texas Eastern, in affiliation with its corporate partner Algonquin Transmission Company, has also proposed a similar packet service (The Integrated Transportation Project) to be developed to support customers in the Northeast.

Growth of pipeline capacity into the Midwest Region will remain high during the next several years. The growing Chicago and northern Illinois markets are the target for two major proposed projects, which together represent 1.3 billion cubic feet per day, or 75 percent of the 1.7 billion cubic feet per day of additional capacity slated for development into the region. (From 1991 through 1994, 1.5 billion cubic feet per day was put into service). The two projects are the Northern Border pipeline expansion project $(0.4$ billion cubic feet per day), which has already been filed with the FERC, and the Natural Gas Pipeline of America Company expansion proposal ( 0.9 billion cubic feet per day). The latter project, while not yet before the FERC, has generated enough interest among potential customers that the company may soon provide more specifications on project design. The Northern Border project also targets service expansion into the Indiana market via the Crossroads (oil pipeline conversion) project. 
Proposed capacity expansion into the West is heavily dependent upon construction of the Altamont pipeline. The level of pending capacity additions into the Western Region through 1997 currently stands at 1.2 billion cubic feet per day, compared with the 3.0 billion cubic feet completed between 1991 and 1994. A substantial portion (37 percent) of this proposed capacity expansion is represented by one project -- the Kern River Pipeline expansion, which is predicated upon construction of the Altamont pipeline project, itself several times postponed because of changing market conditions and regulatory requirements. The Southwest Region also reflects a substantial decline in expansion activity during the next several years. Proposed expansions into the Southwest are only 0.3 billion cubic feet per day, compared with 2.8 billion cubic feet per day added from 1991 through 1994.

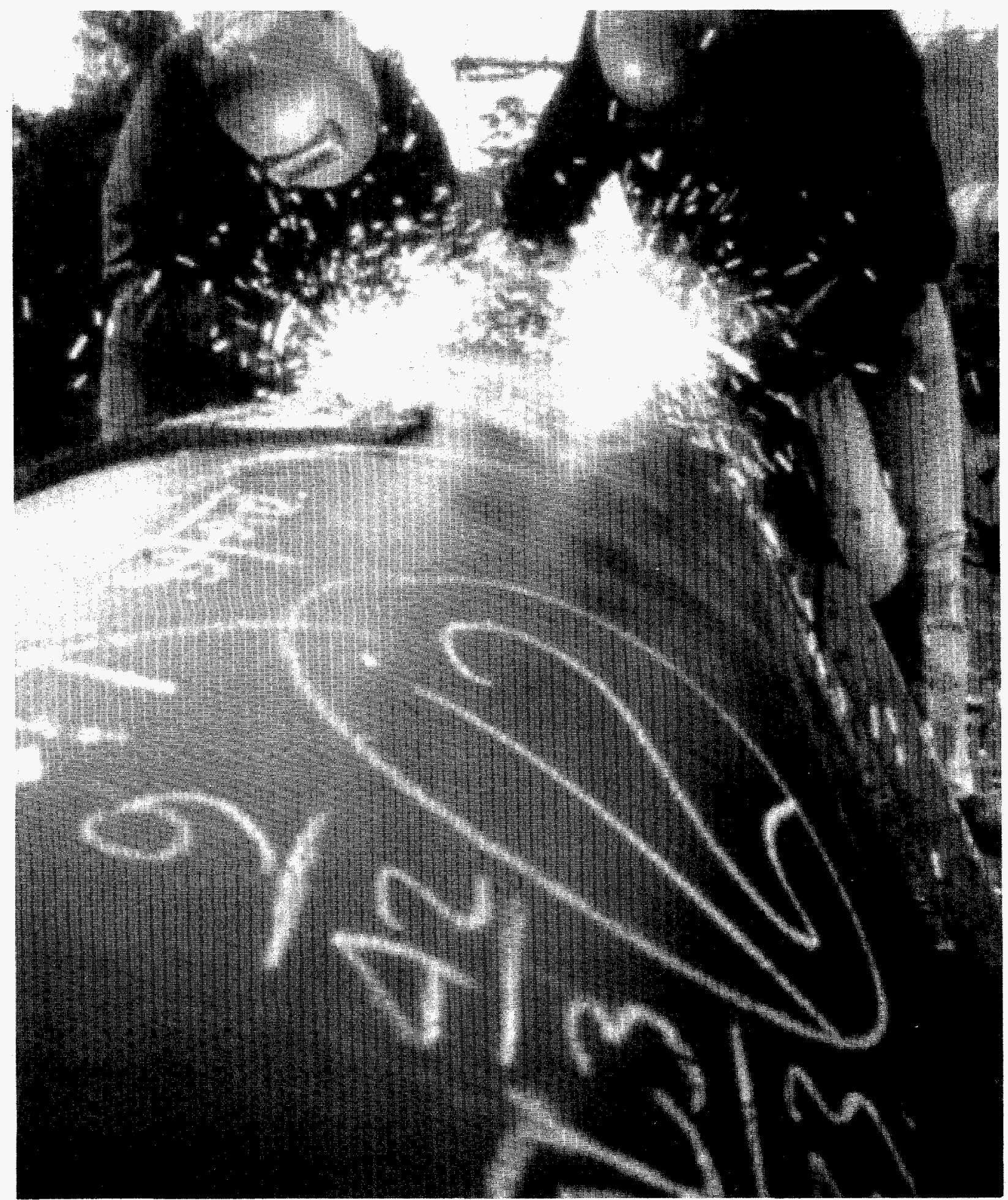

Workmen are welding a 30-inch pipeline section on a natural gas transmission line. 
Table 9. Interstate Movements and Movements Across U.S. Borders of Natural Gas by State, 1994 (Million Cubic Feet)

\begin{tabular}{ll|l|l|}
\hline \multirow{2}{*}{$\begin{array}{l}\text { State or Country } \\
\text { From/To }\end{array}$} & \multicolumn{2}{|c}{ Volume } \\
\cline { 2 - 4 } & $\begin{array}{c}\text { Receipts/ } \\
\text { Imports From }\end{array}$ & $\begin{array}{c}\text { Deliveries/ } \\
\text { Exports To }\end{array}$ & $\begin{array}{c}\text { Net } \\
\text { Sta }\end{array}$
\end{tabular}

Alabama

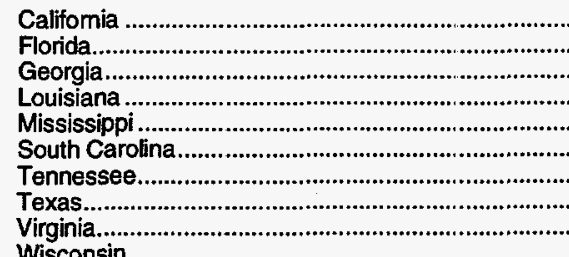

Wisconsin

Total.

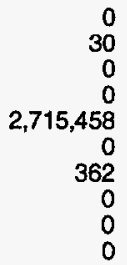

$2,715,849$

Alaska

Japan.

Total.

Arizona

Califomia

Mexico.

Total.

,234,695

Louisiana

Mississippi

Missouri...

Oklahoma .

Texas.

Total.

California

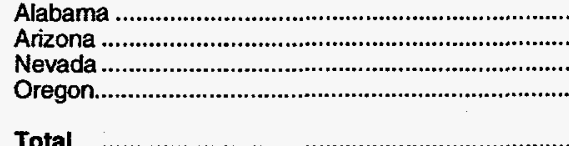

Total.

Kansas

Nebraska.

New Mexico

Oklahoma

Utah...

Wyoming

Total.

Connecticut

Massachusetts

New York.

Rhode Island

Total

$2,041,009$

0
6,208

347,343

471,238

$2,865,799$

${ }_{1}^{b} 6$

$1,079,711$

245,519

561,913

$1,887,149$

0
133,876
0

18,855

106,699

325,777

585,207

1,447

323,129

324,576

0
52,304

52,304

9,225

20,581

29,806

Total.

Maryland.

Virginia.

349,335

Florida

Alabama . $b_{6}$
349,335
$1,371,651$
$b_{2}$
70,961
$b_{34}$
$1,096,867$
$b_{1}$
$b_{83}$
$b_{21}$

$2,888,962$

${ }^{\circ} 62,682$

62,682

$1,079,711$

2,459

0

$1,122,007$

111,745

$2,052,765$

634,208

0
3,200

$2,801,918$

$-349,305$

$-1,371,651$

$2,644,496^{-2}$

$1,096,505$

$-1$

-83
-21

$-173,112$

$-62,682$

$-62,682$

$-1,079,711$

$-2,459$

$-39,837$

$1,234,695$

112,688

$1,929,263$

$-2,052,765$

$-628,000$

347,343

468,038

63,880

$1,079,711$

245,519

561,913

$1,887,149$

67,430

134,823

314,415

70,382

10,616

200,771

798,437

$-67,430$

$-947$

$-314,415$

$-51,527$

96,083

125,006

$-213,230$

1,447

256,482

$-147,451$

110,478

214,098

$-2,896$

52,304

49,408

9,225

20,581

29,806

349,305

See footnotes at end of table. 
Table 9. Interstate Movements and Movements Across U.S. Borders of Natural Gas by State, 1994 (Continued) (Million Cubic Feet)

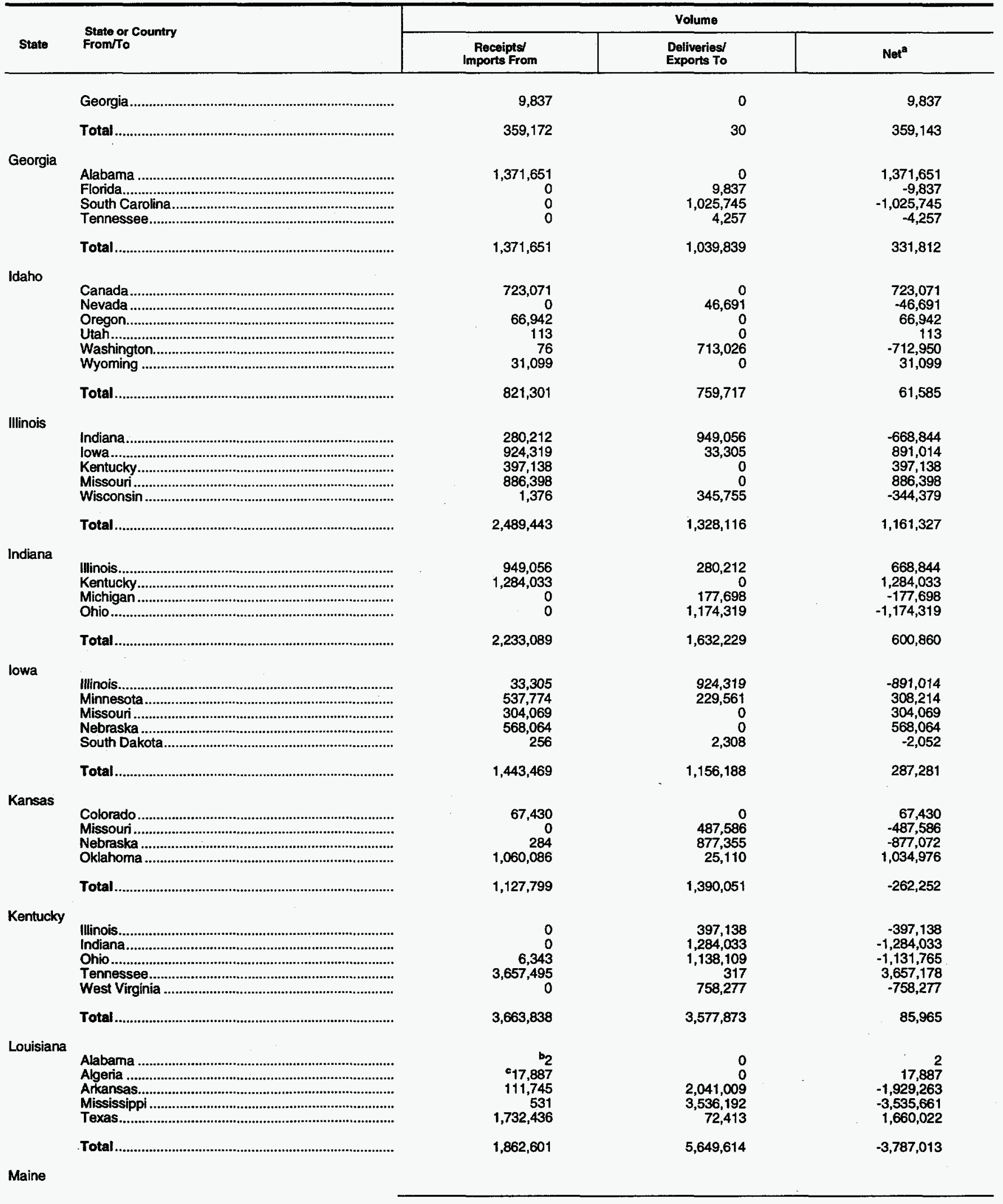

See footnotes at end of table. 
Table 9. Interstate Movements and Movements Across U.S. Borders of Natural Gas by State, 1994 (Continued)

(Million Cubic Feet)

\begin{tabular}{|c|c|c|c|c|}
\hline \multirow[b]{2}{*}{ State } & \multirow{2}{*}{$\begin{array}{l}\text { State or Country } \\
\text { From/To }\end{array}$} & \multicolumn{3}{|c|}{ Volume } \\
\hline & & $\begin{array}{c}\text { Receipts/ } \\
\text { Imports From }\end{array}$ & $\begin{array}{l}\text { Deliveries/ } \\
\text { Exports To }\end{array}$ & Net $^{\mathbf{a}}$ \\
\hline & Massachusetts & $\begin{array}{r}b_{44} \\
4,884\end{array}$ & $\begin{array}{l}0 \\
0\end{array}$ & $\begin{array}{r}44 \\
4,884\end{array}$ \\
\hline & Total & 4,928 & 0 & 4,928 \\
\hline Maryland & 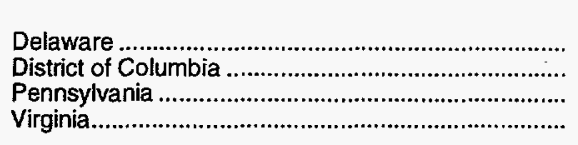 & $\begin{array}{r}2,896 \\
0 \\
23,416 \\
856,866\end{array}$ & $\begin{array}{r}0 \\
9,225 \\
632,250 \\
15,110\end{array}$ & $\begin{array}{r}2,896 \\
-9,225 \\
-608,834 \\
841,756\end{array}$ \\
\hline & Total & 883,179 & 656,585 & 226,594 \\
\hline Massachus & 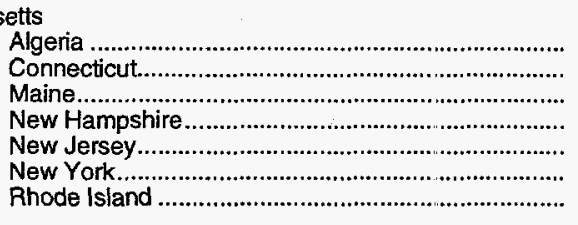 & $\begin{array}{r}32,891 \\
0 \\
0 \\
0 \\
0 \\
166,394 \\
125,766\end{array}$ & $\begin{array}{r}0 \\
1,447 \\
b_{44} \\
9,460 \\
b_{265} \\
0 \\
37,064\end{array}$ & $\begin{array}{r}32,891 \\
-1,447 \\
-44 \\
-9,460 \\
-265 \\
166,394 \\
88,702\end{array}$ \\
\hline & Total & 325,052 & 48,281 & 276,771 \\
\hline Michigan & $\begin{array}{l}\text { Canada } \\
\text { Indiana } \\
\text { Ohio } \\
\text { Wisconsin }\end{array}$ & $\begin{array}{r}17,320 \\
177,698 \\
622,233 \\
695,986\end{array}$ & $\begin{array}{r}538,297 \\
0 \\
0 \\
0\end{array}$ & $\begin{array}{r}-520,977 \\
177,698 \\
622,233 \\
695,986\end{array}$ \\
\hline & Total & $1,513,237$ & 538,297 & 974,940 \\
\hline Minnesota & $\begin{array}{l}\text { Canada } \\
\text { North Dakota } \\
\text { South Dakota } \\
\text { Wisconsin }\end{array}$ & $\begin{array}{r}881,692 \\
229,561 \\
0 \\
583,312 \\
1,224\end{array}$ & $\begin{array}{r}0 \\
537,774 \\
6,422 \\
0 \\
833,235\end{array}$ & $\begin{array}{r}881,692 \\
-308,214 \\
-6,422 \\
583,312 \\
-832,011\end{array}$ \\
\hline & Total & $1,695,788$ & $1,377,432$ & 318,357 \\
\hline Mississippi & 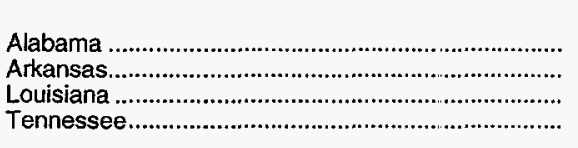 & $\begin{array}{r}70,961 \\
2,052,765 \\
3,536,192 \\
959\end{array}$ & $\begin{array}{r}2,715,458 \\
0 \\
531 \\
2,777,267\end{array}$ & $\begin{array}{r}-2,644,496 \\
2,052,765 \\
3,535,661 \\
-2,776,308\end{array}$ \\
\hline & 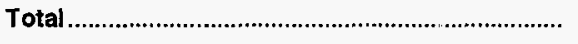 & $5,660,877$ & $5,493,256$ & 167,621 \\
\hline Missouri & 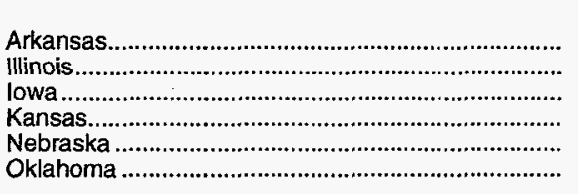 & $\begin{array}{r}634,208 \\
0 \\
0 \\
487,586 \\
306,686 \\
14,181\end{array}$ & $\begin{array}{r}6,208 \\
886,398 \\
304,069 \\
0 \\
0 \\
0\end{array}$ & $\begin{array}{r}628,000 \\
-886,398 \\
-304,069 \\
487,586 \\
306,686 \\
14,181\end{array}$ \\
\hline & Total & $1,442,661$ & $1,196,675$ & 245,985 \\
\hline Montana & $\begin{array}{l}\text { Canada } \\
\text { North Dakota } \\
\text { South Dakota } \\
\text { Wyoming }\end{array}$ & $\begin{array}{r}535,855 \\
12,930 \\
0 \\
7,478\end{array}$ & $\begin{array}{r}3,087 \\
537,121 \\
6,439 \\
8,101\end{array}$ & $\begin{array}{r}532,768 \\
-524,191 \\
-6,439 \\
-622\end{array}$ \\
\hline & Total & 556,263 & 554,747 & 1,516 \\
\hline Nebraska & 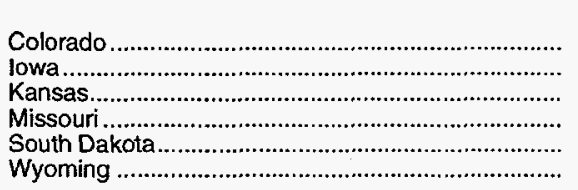 & $\begin{array}{r}134,823 \\
0 \\
877,355 \\
0 \\
0 \\
158,032\end{array}$ & $\begin{array}{r}133,876 \\
568,064 \\
284 \\
306,686 \\
10,962 \\
0\end{array}$ & $\begin{array}{r}947 \\
-568,064 \\
877,072 \\
-306,686 \\
-10,962 \\
158,032\end{array}$ \\
\hline
\end{tabular}

See footnotes at end of table. 
Table 9. Interstate Movements and Movements Across U.S. Borders of Natural Gas by State, 1994 (Continued)

(Million Cubic Feet)

\begin{tabular}{|c|c|c|c|c|}
\hline \multirow[b]{2}{*}{ State } & \multirow{2}{*}{$\begin{array}{l}\text { State or Country } \\
\text { From/To }\end{array}$} & \multicolumn{3}{|c|}{ Volume } \\
\hline & & $\begin{array}{l}\text { Receipts/ } \\
\text { Imports From }\end{array}$ & $\begin{array}{l}\text { Deliveries/ } \\
\text { Exports To }\end{array}$ & Net $^{a}$ \\
\hline \multirow{3}{*}{ Nevada } & Total & $1,170,211$ & $1,019,871$ & 150,340 \\
\hline & $\begin{array}{l}\text { Arizona } \\
\text { California } \\
\text { Idaho } \\
\text { Utah }\end{array}$ & $\begin{array}{r}39,837 \\
0 \\
46,691 \\
261,618\end{array}$ & $\begin{array}{r}0 \\
245,519 \\
0 \\
0\end{array}$ & $\begin{array}{r}39,837 \\
-245,519 \\
46,691 \\
261,618\end{array}$ \\
\hline & Total & 348,147 & 245,519 & 102,628 \\
\hline New Han & 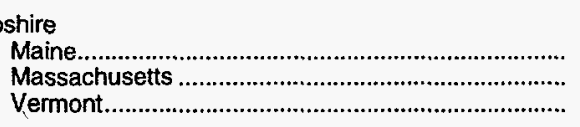 & $\begin{array}{r}0 \\
9,460 \\
10,789\end{array}$ & $\begin{array}{r}4,884 \\
0 \\
0\end{array}$ & $\begin{array}{r}-4,884 \\
9,460 \\
10,789\end{array}$ \\
\hline & Total & 20,249 & 4,884 & 15,365 \\
\hline New Jers & 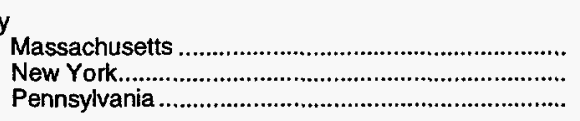 & $\begin{array}{r}b_{265} \\
0 \\
1,119,685\end{array}$ & $\begin{array}{r}0 \\
589,632 \\
0\end{array}$ & $\begin{array}{r}265 \\
-589,632 \\
1,119,685\end{array}$ \\
\hline & 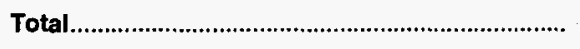 & $1,119,949$ & 589,632 & 530,317 \\
\hline New Mer & 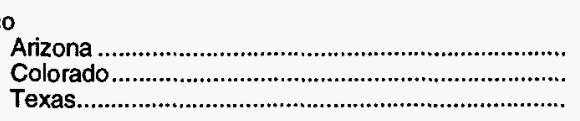 & $\begin{array}{r}0 \\
314,415 \\
20,235\end{array}$ & $\begin{array}{r}1,234,695 \\
0 \\
182,912\end{array}$ & $\begin{array}{r}-1,234,695 \\
314,415 \\
-162,677\end{array}$ \\
\hline & 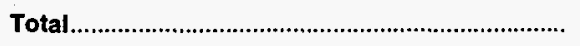 & 334,650 & $1,417,608$ & $-1,082,958$ \\
\hline New Yor & 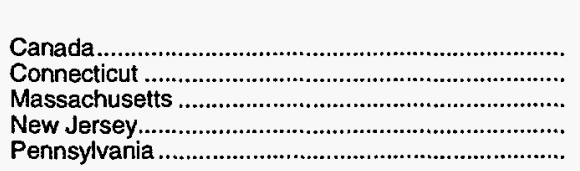 & $\begin{array}{r}588,983 \\
66,647 \\
0 \\
589,632 \\
270,448\end{array}$ & $\begin{array}{r}0 \\
323,129 \\
166,394 \\
0 \\
60,339\end{array}$ & $\begin{array}{r}588,983 \\
-256,482 \\
-166,394 \\
589,632 \\
210,109\end{array}$ \\
\hline & 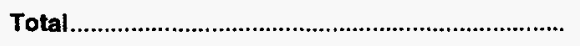 & $1,515,710$ & 549,862 & 965,848 \\
\hline North $\mathrm{Ca}$ & 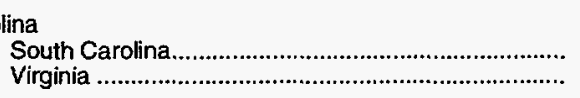 & $\begin{array}{r}896,007 \\
5,115\end{array}$ & 702,517 & $\begin{array}{r}896,007 \\
-697,401\end{array}$ \\
\hline & 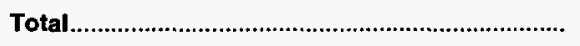 & 901,123 & 702,517 & 198,606 \\
\hline North Da & 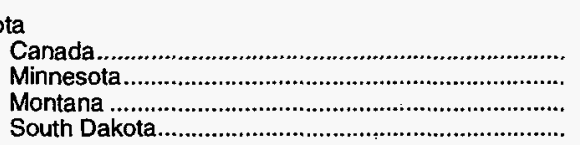 & $\begin{array}{r}1,617 \\
6,422 \\
537,121 \\
0\end{array}$ & $\begin{array}{r}0 \\
0 \\
12,930 \\
594,199\end{array}$ & $\begin{array}{r}1,617 \\
6,422 \\
524,191 \\
-594,199\end{array}$ \\
\hline & Total & 545,160 & 607,129 & $-61,969$ \\
\hline Ohio & 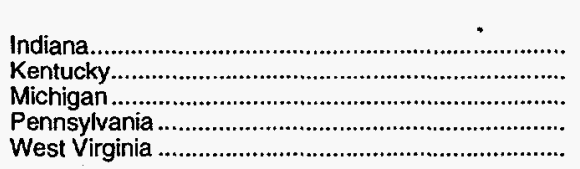 & $\begin{array}{r}1,174,319 \\
1,138,109 \\
0 \\
1,145 \\
304,074\end{array}$ & $\begin{array}{r}0 \\
6,343 \\
622,233 \\
359,479 \\
850,347\end{array}$ & $\begin{array}{r}1,174,319 \\
1,131,765 \\
-622,233 \\
-358,334 \\
-546,272\end{array}$ \\
\hline & 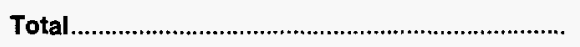 & $2,617,648$ & $1,838,402$ & 779,245 \\
\hline Oklahom & 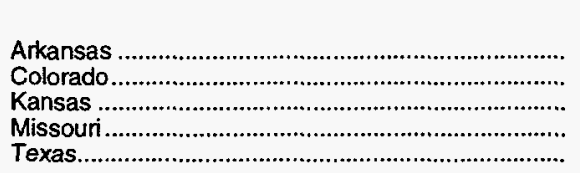 & $\begin{array}{r}0 \\
70,382 \\
25,110 \\
0 \\
448,187\end{array}$ & $\begin{array}{r}347,343 \\
18,855 \\
1,060,086 \\
14,181 \\
345,299\end{array}$ & $\begin{array}{r}-347,343 \\
51,527 \\
-1,034,976 \\
-14,181 \\
102,888\end{array}$ \\
\hline & 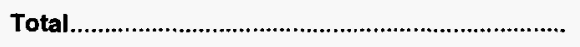 & 543,679 & $1,785,764$ & $-1,242,085$ \\
\hline Oregon & California & $\begin{array}{l}0 \\
0\end{array}$ & $\begin{array}{r}561,913 \\
66,942\end{array}$ & $\begin{array}{r}-561,913 \\
-66,942\end{array}$ \\
\hline
\end{tabular}

See footnotes at end of table. 
Table 9. Interstate Movements and Movements Across U.S. Borders of Natural Gas by State, 1994 (Continued)

(Million Cubic Feet)

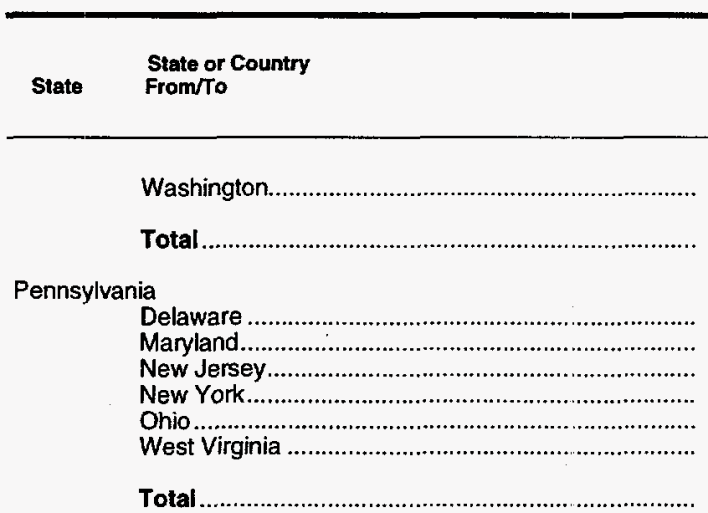

Rhode Island

Connecticut

Massachusetts

Total.

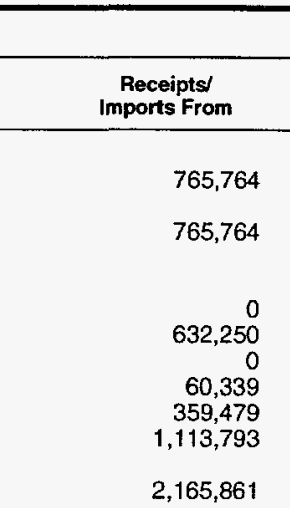

Volume

Deliveries!

Exports To

, 165,81

147,451

37,064

184,516

76

Net $^{a}$

$$
\text { olina }
$$

Alabama

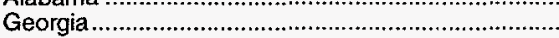

North Carolina.

Total.

$b_{34}$
$1,025,745$
0

$1,025,779$

628,931

765,688

ta

lowa....

Minnesota

Montana.

Nebraska

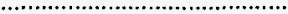

North Dakota .........................................................

Wyoming ...

Total.

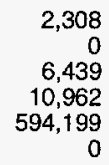

613,908

$$
\begin{array}{r}
52,304 \\
23,416 \\
1,119,685 \\
270,448 \\
1,145 \\
9,945
\end{array}
$$

$1,476,943$

136,833

Alaba

Tennessee

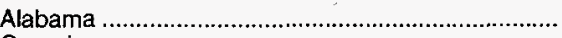

Georgia...

Kentucky.....

Mississippi ..........................................................

Virginia

Total...

$1,096,867$

4,257
317

$2,777,267$

189

$3,878,897$

0
125,766

125,766

$-52,304$

608,834

$-1,119,685$

$-210,109$

358,334

$1,103,848$

688,918

147,451

$-88,702$

58,749

(a)

Texas

Alabama

Arkansas.

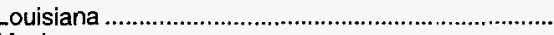

Mexico.

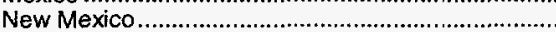

Oklahoma

Total..

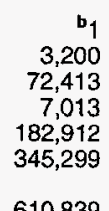

0
0
896,007

896,007

34
$1,025,745$

$-896,007$

129,772

583,312

2,052

$-583,312$
6,439

10,962

594,199

$-85$

583,653

30,255

$1,096,505$

4,257

$-3,657,178$

$2,776,308$

220,081

$3,658,815$

1
$-468,038$

471,238

$1,732,436$

44,041

20,235

448,187

$-1,660,022$

$-37,029$

162,677

$-102,888$

610,839

$2,716,137$

$-2,105,299$

Utah

Colorado.

Idaho...

Nevada....

Wyoming

10,616

106,699

113

261,618

434,030

Total

444,646

440,415

$-96,083$

-113
-618

261,618
362,046

4,231

$18,09 i$
0

18,091

0
10,789

18,091

$-10,789$

10,789

7,302

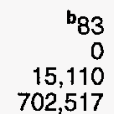

0
20,581

20,581
856,866

83

$-20,581$

$-841,756$

697,401

See footnotes at end of table. 
Table 9. Interstate Movements and Movements Across U.S. Borders of Natural Gas by State, 1994 (Continued)

(Million Cubic Feet)

\begin{tabular}{|c|c|c|c|c|}
\hline \multirow[b]{2}{*}{ State } & \multirow{2}{*}{$\begin{array}{l}\text { State or Country } \\
\text { From/To }\end{array}$} & \multicolumn{3}{|c|}{ Volume } \\
\hline & & $\begin{array}{l}\text { Receipts' } \\
\text { Imports From }\end{array}$ & $\begin{array}{l}\text { Deliveries/ } \\
\text { Exports To }\end{array}$ & $\mathrm{Net}^{\mathrm{a}}$ \\
\hline & 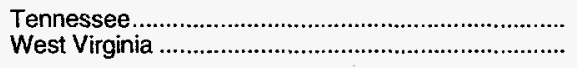 & $\begin{array}{r}0 \\
308,118\end{array}$ & $\begin{array}{r}189 \\
2,352\end{array}$ & $\begin{array}{r}-189 \\
305,765\end{array}$ \\
\hline & 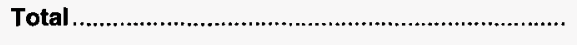 & $1,025,827$ & 885,104 & 140,723 \\
\hline Washing & 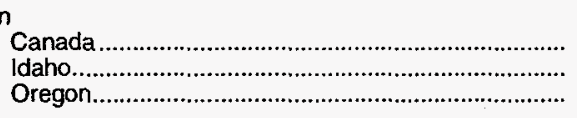 & $\begin{array}{r}323,013 \\
713,026 \\
76\end{array}$ & $\begin{array}{r}0 \\
76 \\
765,764\end{array}$ & $\begin{array}{r}323,013 \\
712,950 \\
-765,688\end{array}$ \\
\hline & Total & $1,036,114$ & 765,840 & 270,274 \\
\hline West Vi & 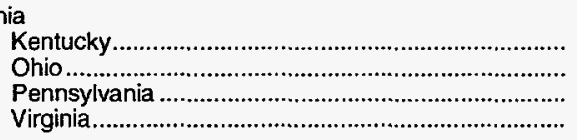 & $\begin{array}{r}758,277 \\
850,347 \\
9,945 \\
2,352\end{array}$ & $\begin{array}{r}0 \\
304,074 \\
1,113,793 \\
308,118\end{array}$ & $\begin{array}{r}758,277 \\
546,272 \\
-1,103,848 \\
-305,765\end{array}$ \\
\hline & Total & $1,620,921$ & $1,725,985$ & $-105,063$ \\
\hline Wiscons & 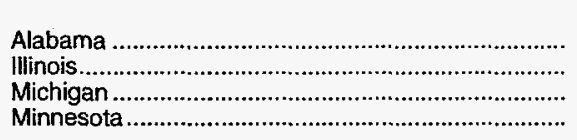 & $\begin{array}{r}b_{21} \\
345,755 \\
0 \\
833,235\end{array}$ & $\begin{array}{r}0 \\
1,376 \\
695,986 \\
1,224\end{array}$ & $\begin{array}{r}21 \\
344,379 \\
-695,986 \\
832,011\end{array}$ \\
\hline & Total & $1,179,011$ & 698,586 & 480,425 \\
\hline Wyomin & 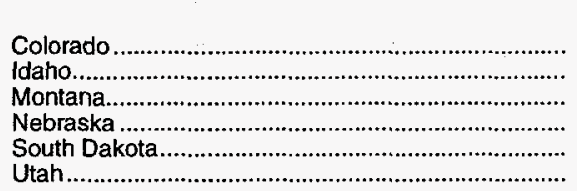 & $\begin{array}{r}200,771 \\
0 \\
8,101 \\
0 \\
85 \\
71,984\end{array}$ & $\begin{array}{r}325,777 \\
31,099 \\
7,478 \\
158,032 \\
0 \\
434,030\end{array}$ & $\begin{array}{r}-125,006 \\
-31,099 \\
622 \\
-158,032 \\
85 \\
-362,046\end{array}$ \\
\hline & Total & 280,941 & 956,416 & $-675,475$ \\
\hline \multicolumn{2}{|c|}{ 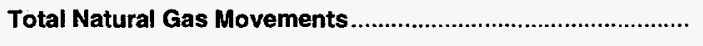 } & $61,117,373$ & $58,620,506$ & $2,496,868$ \\
\hline \multicolumn{2}{|c|}{ 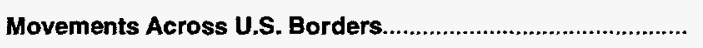 } & $d_{3,147,433}$ & 650,565 & $2,496,868$ \\
\hline U.S. Int & 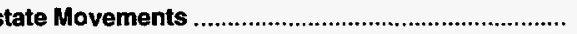 & $57,969,941$ & $57,969,941$ & 0 \\
\hline
\end{tabular}

a Positive numbers denote net receipts; negative numbers denote net deliveries.

b Natural gas transported by truck either as liquefied natural gas (LNG) or compressed natural gas (CNG).

c LNG transported by ship.

d Volumes include 2,659,673 million cubic feet of imports from Mexico, Algeria and Canada, and 487,760 million cubic feet of intransit natural gas receipts from Canada.

e Volumes include 178,066 million cubic feet of exports to Canada, Mexico, and Japan and 472,499 million cubic feet of intransit natural gas deliveries to Canada.

Note: Totals may not equal sum of components due to independent rounding.

Source: Energy Information Administration (EIA), Form EIA-176, "Annual Report of Natural and Supplemental Gas Supply and Disposition." 
Figure 7. Interstate Movements of Natural Gas in the United States, 1994

(Million Cubic Feet)

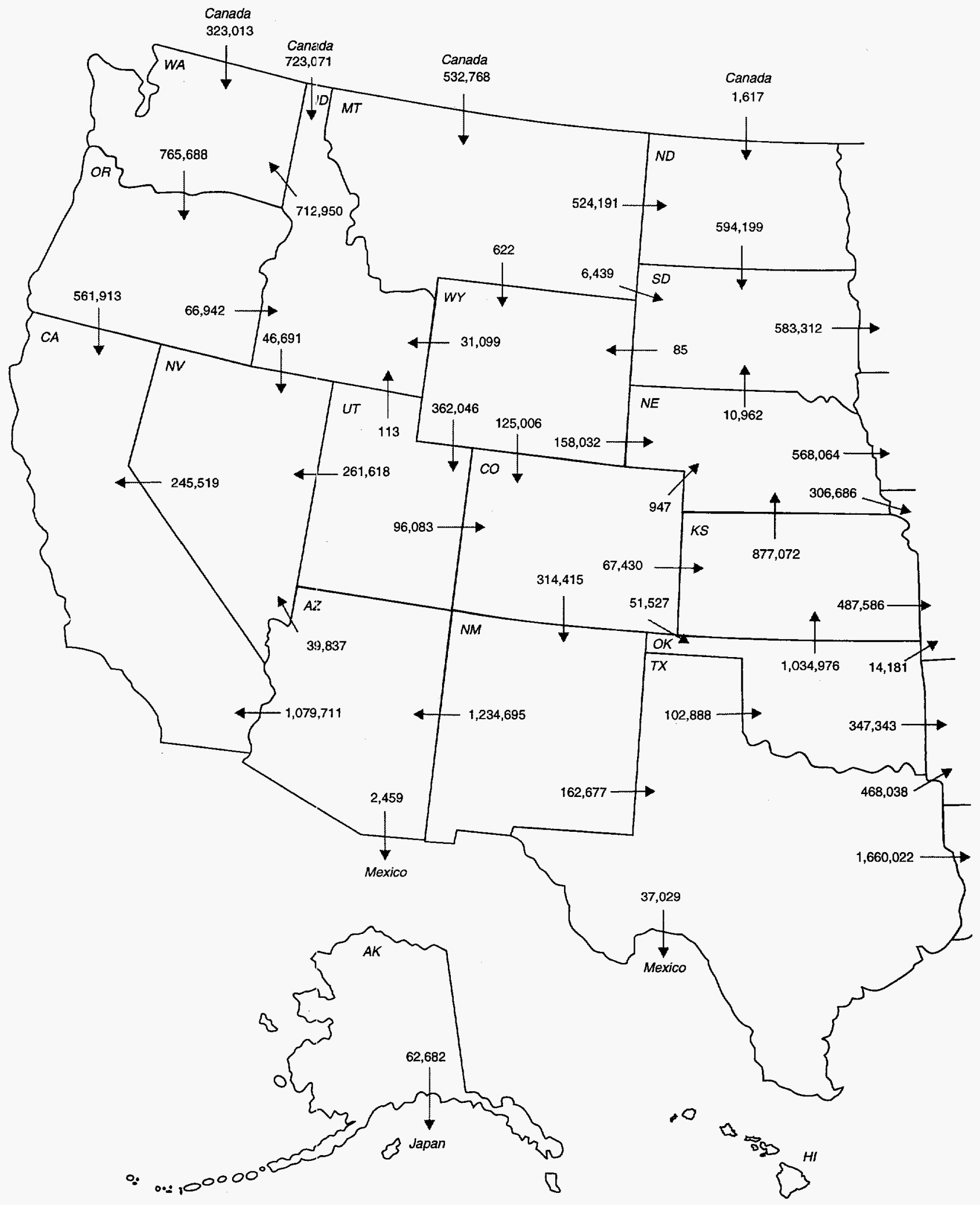




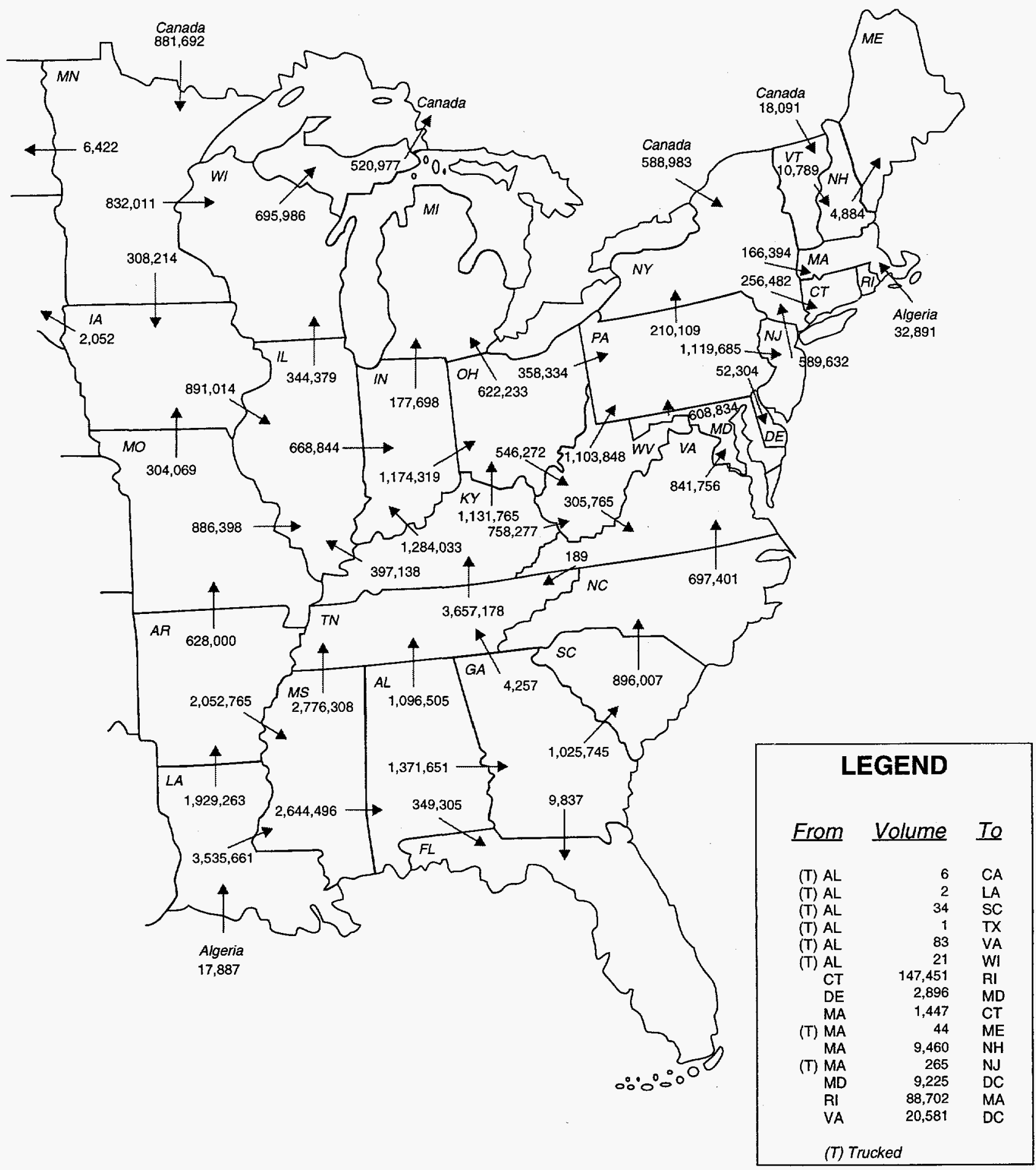

Source: Energy Information Administration (E1A), Form E|A-176, "Annual Report of Natural and Supplemental Gas Supply and Disposition." 
Table 10. Summary of U.S. Natural Gas Imports and Exports, 1990-1994

\begin{tabular}{|c|c|c|c|c|c|}
\hline & 1990 & 1991 & 1992 & 1993 & 1994 \\
\hline \multicolumn{6}{|l|}{ Imports } \\
\hline \multirow{2}{*}{\multicolumn{6}{|c|}{$\begin{array}{l}\text { Volume (million cubic feet) } \\
\text { Pipeline }\end{array}$}} \\
\hline & & & & & \\
\hline 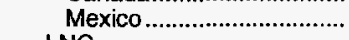 & 0 & 0 & 0 & 1,678 & $\begin{array}{r}2,566,049 \\
7,013\end{array}$ \\
\hline \multicolumn{6}{|l|}{ LNG } \\
\hline 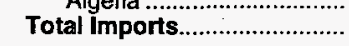 & $\begin{array}{r}04,193 \\
1,532,259\end{array}$ & $1,773,313$ & $2,137,504$ & $\begin{array}{r}81,685 \\
2,350,115\end{array}$ & $\begin{array}{r}50,778 \\
2,623,839\end{array}$ \\
\hline \multicolumn{6}{|l|}{$\begin{array}{l}\text { Average Price (dollars per } \\
\text { thousand cubic feet) }\end{array}$} \\
\hline \multicolumn{6}{|l|}{ Pipeline } \\
\hline Canada & 1.91 & 1.81 & 1.84 & 2.02 & 1.86 \\
\hline 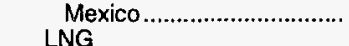 & & & & 1.94 & 1.99 \\
\hline Algeria ................................... & 2.47 & 2.36 & 2.54 & 2.20 & 2.28 \\
\hline 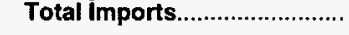 & 1.94 & 1.83 & 1.85 & 2.03 & 1.87 \\
\hline \multirow{2}{*}{\multicolumn{6}{|c|}{$\begin{array}{l}\text { Exports } \\
\text { Volume (million cubic feet) }\end{array}$}} \\
\hline \multirow{2}{*}{\multicolumn{6}{|c|}{ Pipeline }} \\
\hline & & & & & \\
\hline 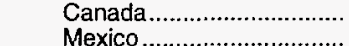 & 17,359 & 14,791 & 67,777 & 44,518 & 52,556 \\
\hline $\begin{array}{l}\text { Mexico } \\
\text { Total Pipeline Exports ....... }\end{array}$ & 15,659 & $\begin{array}{l}60,448 \\
75,239\end{array}$ & $\begin{array}{r}95,973 \\
163750\end{array}$ & 39,676 & 46,500 \\
\hline LNG & 33,018 & 75,239 & 163,750 & 84,195 & 99,057 \\
\hline Japan & 52,546 & 54,005 & 52,532 & 55,989 & 62,682 \\
\hline 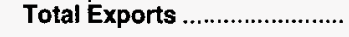 & 85,565 & 129,244 & 216,282 & 140,183 & 161,738 \\
\hline \multirow{2}{*}{\multicolumn{6}{|c|}{$\begin{array}{l}\text { Average Price (dollars per } \\
\text { thousand cubic feet) }\end{array}$}} \\
\hline & & & & & \\
\hline \multicolumn{6}{|l|}{ Pipeline } \\
\hline Canada & 2.70 & 1.91 & 1.83 & 2.14 & 2.42 \\
\hline 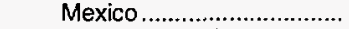 & 1.88 & 1.76 & 1.90 & 2.02 & 1.68 \\
\hline Total Pipeline Exports ....... & 2.31 & 1.79 & 1.88 & 2.08 & 2.08 \\
\hline 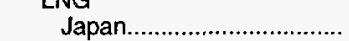 & 3.59 & 3.71 & 3.43 & 3.34 & 3.18 \\
\hline 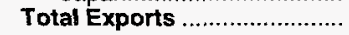 & 3.10 & 2.59 & 2.25 & 2.59 & 2.50 \\
\hline
\end{tabular}

Notes: Totals may not equal sum of components due to independent rounding. Volumes of natural gas for imports and exports in 1994 as shown in Tables 1,2 and 9 may not match due to differences in reporting on the Form EIA-176 and the Form FPC-14. These differences were not resolved at the time of publication.

Source: Energy Information Administration, Form FPC-14, "Annual Report for Importers and Exporters of Natural Gas."

Figure 8. Net Imports as a Percentage of Total Consumption of Natural Gas, 1972 - 1994

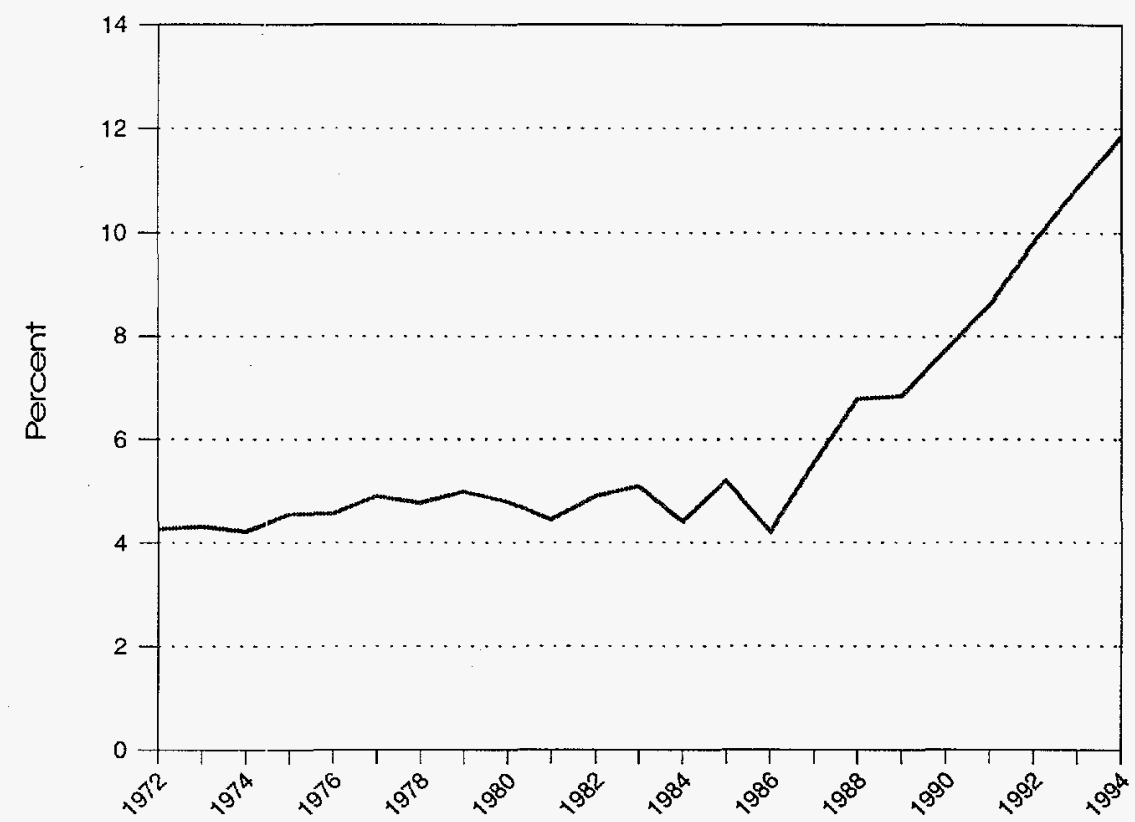

Sources: 1972-1975: Bureau of Mines, Minerals Yearbook, "Natural Gas" chapter. 1976-1978: Energy Information Administration (ElA), Energy Data Reports, Natural Gas Annual. 1979: ElA, Natural Gas Production 1979. 1980-1989: EIA, Form ElA-176, "Annual Report of Natural and Supplemental Gas Supply and Disposition"; Form ElA-759, "Monthly Power Plant Report"; and Form FPC-14, "Annual Report for Importers and Exporters of Natural Gas"; 1990: ElA, Form ElA-176, Form ElA-759, Form FPC-14, and Form EIA-64A, "Annual Report of the Origin of Natural Gas Liquids Production"; 1991-1994: EIA, Form EIA-176, Form EIA-759, Form FPC-14, Form EIA-64A, and Form EIA-627, "Annual Quantity and Value of Natural Gas Report." 
Table 11. Additions to and Withdrawals from Gas Storage by State, 1994 (Million Cubic Feet)

\begin{tabular}{|c|c|c|c|c|c|c|c|}
\hline \multirow{2}{*}{ State } & \multicolumn{3}{|c|}{ Underground Storage } & \multicolumn{3}{|c|}{ LNG Storage } & \multirow{2}{*}{$\begin{array}{c}\text { Net Change } \\
\text { in } \\
\text { Storage }\end{array}$} \\
\hline & Injections & Withdrawals & Net & Additions & Withdrawals & Net & \\
\hline 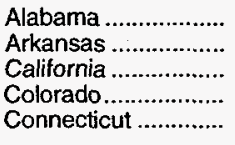 & $\begin{array}{r}694 \\
3,249 \\
155,910 \\
31,185 \\
0\end{array}$ & $\begin{array}{r}55 \\
5,731 \\
150,844 \\
30,085 \\
0\end{array}$ & $\begin{array}{r}639 \\
-2,482 \\
5,066 \\
1,100 \\
0\end{array}$ & $\begin{array}{r}944 \\
73 \\
13 \\
0 \\
1,435\end{array}$ & $\begin{array}{r}497 \\
68 \\
94 \\
0 \\
1,497\end{array}$ & $\begin{array}{r}448 \\
5 \\
-80 \\
0 \\
-61\end{array}$ & $\begin{array}{r}1,087 \\
-2,478 \\
4,985 \\
1,100 \\
-61\end{array}$ \\
\hline 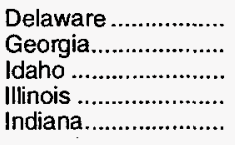 & $\begin{array}{r}0 \\
0 \\
0 \\
235,778 \\
24,656\end{array}$ & $\begin{array}{r}0 \\
0 \\
0 \\
222,871 \\
21,081\end{array}$ & $\begin{array}{r}0 \\
0 \\
0 \\
12,907 \\
3,576\end{array}$ & $\begin{array}{r}225 \\
3,788 \\
554 \\
1,326 \\
2,638\end{array}$ & $\begin{array}{r}202 \\
3,881 \\
373 \\
1,317 \\
2,733\end{array}$ & $\begin{array}{r}23 \\
-94 \\
182 \\
9 \\
-95\end{array}$ & $\begin{array}{r}23 \\
-94 \\
182 \\
12,916 \\
3,481\end{array}$ \\
\hline 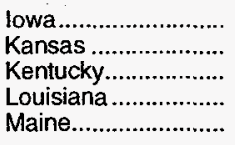 & $\begin{array}{r}70,926 \\
106,069 \\
59,831 \\
224,525 \\
0\end{array}$ & $\begin{array}{r}68,162 \\
99,854 \\
54,986 \\
184,731 \\
0\end{array}$ & $\begin{array}{r}2,764 \\
6,215 \\
4,845 \\
39,794 \\
0\end{array}$ & $\begin{array}{r}3,867 \\
0 \\
0 \\
17,823 \\
27\end{array}$ & $\begin{array}{r}3,521 \\
0 \\
0 \\
18,252 \\
22\end{array}$ & $\begin{array}{r}346 \\
0 \\
0 \\
-429 \\
5\end{array}$ & $\begin{array}{r}3,109 \\
6,215 \\
4,845 \\
39,364 \\
5\end{array}$ \\
\hline $\begin{array}{l}\text { Maryland................. } \\
\text { Massachusetts ........ } \\
\text { Michigan .................... } \\
\text { Minnesota............... } \\
\text { Mississippi ............... }\end{array}$ & $\begin{array}{r}15,316 \\
0 \\
470,220 \\
1,556 \\
64,972\end{array}$ & $\begin{array}{r}17,406 \\
0 \\
389,224 \\
1,192 \\
50,526\end{array}$ & $\begin{array}{r}-2,090 \\
0 \\
80,996 \\
365 \\
14,446\end{array}$ & $\begin{array}{r}939 \\
5,550 \\
0 \\
6,167 \\
0\end{array}$ & $\begin{array}{r}529 \\
12,369 \\
0 \\
5,537 \\
0\end{array}$ & $\begin{array}{r}410 \\
-6,820 \\
0 \\
630 \\
0\end{array}$ & $\begin{array}{r}-1,680 \\
-6,820 \\
80,996 \\
994 \\
14,446\end{array}$ \\
\hline $\begin{array}{l}\text { Missouri .................. } \\
\text { Montana ................. } \\
\text { Nebraska ................. } \\
\text { Nevada .................... } \\
\text { New Jersey.............. }\end{array}$ & $\begin{array}{r}4,997 \\
14,690 \\
12,524 \\
0 \\
0\end{array}$ & $\begin{array}{r}5,082 \\
22,509 \\
10,052 \\
0 \\
0\end{array}$ & $\begin{array}{r}-85 \\
-7,819 \\
2,471 \\
0 \\
0\end{array}$ & $\begin{array}{r}0 \\
0 \\
393 \\
241 \\
4,835\end{array}$ & $\begin{array}{r}0 \\
0 \\
367 \\
271 \\
3,565\end{array}$ & $\begin{array}{r}0 \\
0 \\
26 \\
-30 \\
1,270\end{array}$ & $\begin{array}{r}-85 \\
-7,819 \\
2,497 \\
-30 \\
1,270\end{array}$ \\
\hline $\begin{array}{l}\text { New Mexico............ } \\
\text { New York................ } \\
\text { North Carolina ......... } \\
\text { Ohio .......................... } \\
\text { Oklahoma ................ }\end{array}$ & $\begin{array}{r}18,551 \\
66,627 \\
0 \\
191,660 \\
123,576\end{array}$ & $\begin{array}{r}17,172 \\
64,803 \\
0 \\
163,084 \\
104,738\end{array}$ & $\begin{array}{r}1,379 \\
1,824 \\
0 \\
28,576 \\
18,838\end{array}$ & $\begin{array}{r}0 \\
1,125 \\
2,118 \\
0 \\
0\end{array}$ & $\begin{array}{r}0 \\
835 \\
1,802 \\
0 \\
0\end{array}$ & $\begin{array}{r}0 \\
290 \\
316 \\
0 \\
0\end{array}$ & $\begin{array}{r}1,379 \\
2,115 \\
316 \\
28,576 \\
18,838\end{array}$ \\
\hline $\begin{array}{l}\text { Oregon ................ } \\
\text { Pennsylvania .......... } \\
\text { Rhode Island ............ } \\
\text { South Carolina......... } \\
\text { Tennessee............... }\end{array}$ & $\begin{array}{r}5,956 \\
339,512 \\
0 \\
0 \\
0\end{array}$ & $\begin{array}{r}5,237 \\
340,334 \\
0 \\
0 \\
0\end{array}$ & $\begin{array}{r}720 \\
-823 \\
0 \\
0 \\
0\end{array}$ & $\begin{array}{r}860 \\
5,826 \\
1,156 \\
847 \\
2,985\end{array}$ & $\begin{array}{r}1,117 \\
5,030 \\
996 \\
682 \\
2,879\end{array}$ & $\begin{array}{r}-257 \\
797 \\
160 \\
165 \\
106\end{array}$ & $\begin{array}{l}463 \\
-26 \\
160 \\
165 \\
106\end{array}$ \\
\hline $\begin{array}{l}\text { Texas....................... } \\
\text { Utah........................ } \\
\text { Virginia ................... } \\
\text { Washington ............. } \\
\text { West Virginia ........... }\end{array}$ & $\begin{array}{r}310,273 \\
42,508 \\
0 \\
18,705 \\
171,216\end{array}$ & $\begin{array}{r}257,318 \\
22,921 \\
0 \\
17,133 \\
156,284\end{array}$ & $\begin{array}{r}52,956 \\
19,587 \\
0 \\
1,572 \\
14,932\end{array}$ & $\begin{array}{r}0 \\
0 \\
116 \\
2,340 \\
0\end{array}$ & $\begin{array}{r}0 \\
0 \\
236 \\
1,701 \\
0\end{array}$ & $\begin{array}{r}0 \\
0 \\
-121 \\
640 \\
0\end{array}$ & $\begin{array}{r}52,956 \\
19,587 \\
-121 \\
2,212 \\
14,932\end{array}$ \\
\hline 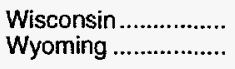 & $\begin{array}{r}0 \\
10,596\end{array}$ & $\begin{array}{r}0 \\
8,012\end{array}$ & $\begin{array}{r}0 \\
2,584\end{array}$ & $\begin{array}{r}266 \\
0\end{array}$ & $\begin{array}{r}316 \\
0\end{array}$ & $\begin{array}{r}-50 \\
0\end{array}$ & $\begin{array}{r}-50 \\
2,584\end{array}$ \\
\hline 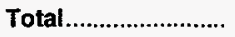 & $2,796,279$ & $2,491,427$ & 304,852 & 68,478 & 70,689 & $-2,211$ & 302,641 \\
\hline
\end{tabular}

Note: Totals may not equal sum of components due to independent rounding.

Source: Energy Information Administration, Form ElA-176, "Annual Report of Natural and Supplemental Gas Supply and Disposition." 
Table 12. Underground Natural Gas Storage Capacity by State, December 31, 1994 (Capacity in Billion Cubic Feet)

\begin{tabular}{|c|c|c|c|c|c|c|c|c|c|}
\hline \multirow{2}{*}{ State } & \multicolumn{2}{|c|}{$\begin{array}{l}\text { Interstate } \\
\text { Companies }\end{array}$} & \multicolumn{2}{|c|}{$\begin{array}{l}\text { Intrastate } \\
\text { Companies }\end{array}$} & \multicolumn{2}{|c|}{$\begin{array}{l}\text { Independent } \\
\text { Companies }\end{array}$} & \multicolumn{3}{|c|}{ Total } \\
\hline & $\begin{array}{l}\text { Number of } \\
\text { Active Fields }\end{array}$ & Capacity & $\begin{array}{l}\text { Number of } \\
\text { Active Fields }\end{array}$ & Capacity & $\begin{array}{l}\text { Number of } \\
\text { Active Fields }\end{array}$ & Capacity & $\begin{array}{l}\text { Number of } \\
\text { Active Fields }\end{array}$ & Capacity & $\begin{array}{l}\text { Percent } \\
\text { of U.S. } \\
\text { Capacity }\end{array}$ \\
\hline $\begin{array}{l}\text { Arkansas ................ } \\
\text { California ................. } \\
\text { Colorado ................. } \\
\text { Illinois ........................ } \\
\text { Indiana ................... }\end{array}$ & $\begin{array}{l}0 \\
0 \\
4 \\
6 \\
5\end{array}$ & $\begin{array}{r}0 \\
0 \\
62 \\
259 \\
12\end{array}$ & $\begin{array}{r}4 \\
10 \\
5 \\
24 \\
22\end{array}$ & $\begin{array}{r}31 \\
472 \\
44 \\
700 \\
96\end{array}$ & $\begin{array}{l}0 \\
0 \\
0 \\
0 \\
0\end{array}$ & $\begin{array}{l}0 \\
0 \\
0 \\
0 \\
0\end{array}$ & $\begin{array}{r}4 \\
10 \\
9 \\
30 \\
27\end{array}$ & $\begin{array}{r}31 \\
472 \\
106 \\
959 \\
109\end{array}$ & $\begin{array}{r}0.39 \\
5.87 \\
1.31 \\
11.92 \\
1.35\end{array}$ \\
\hline $\begin{array}{l}\text { lowa ......................... } \\
\text { Kansas ................... } \\
\text { Kentucky ................ } \\
\text { Louisiana................. } \\
\text { Maryland ................. }\end{array}$ & $\begin{array}{r}4 \\
17 \\
6 \\
8 \\
1\end{array}$ & $\begin{array}{r}270 \\
283 \\
167 \\
530 \\
62\end{array}$ & $\begin{array}{r}0 \\
2 \\
17 \\
3 \\
0\end{array}$ & $\begin{array}{r}0 \\
7 \\
42 \\
21 \\
0\end{array}$ & $\begin{array}{l}0 \\
0 \\
0 \\
0 \\
0\end{array}$ & $\begin{array}{l}0 \\
0 \\
0 \\
0 \\
0\end{array}$ & $\begin{array}{r}4 \\
19 \\
23 \\
11 \\
1\end{array}$ & $\begin{array}{r}270 \\
290 \\
210 \\
552 \\
62\end{array}$ & $\begin{array}{l}3.36 \\
3.61 \\
2.61 \\
6.86 \\
0.77\end{array}$ \\
\hline $\begin{array}{l}\text { Michigan................. } \\
\text { Minnesota ............. } \\
\text { Mississippi ............... } \\
\text { Missouri................... } \\
\text { Montana .................. }\end{array}$ & $\begin{array}{r}30 \\
0 \\
3 \\
0 \\
1\end{array}$ & $\begin{array}{r}780 \\
0 \\
111 \\
0 \\
287\end{array}$ & $\begin{array}{r}17 \\
1 \\
3 \\
1 \\
4\end{array}$ & $\begin{array}{r}267 \\
6 \\
13 \\
31 \\
88\end{array}$ & $\begin{array}{l}0 \\
0 \\
0 \\
0 \\
0\end{array}$ & $\begin{array}{l}0 \\
0 \\
0 \\
0 \\
0\end{array}$ & $\begin{array}{r}47 \\
1 \\
6 \\
1 \\
5\end{array}$ & $\begin{array}{r}1,047 \\
6 \\
124 \\
31 \\
375\end{array}$ & $\begin{array}{r}13.01 \\
0.08 \\
1.54 \\
0.38 \\
4.66\end{array}$ \\
\hline $\begin{array}{l}\text { Nebraska................ } \\
\text { New Mexico ........... } \\
\text { New York................ } \\
\text { Ohio ......................... } \\
\text { Oklahoma............... }\end{array}$ & $\begin{array}{r}2 \\
1 \\
20 \\
15 \\
5\end{array}$ & $\begin{array}{r}93 \\
69 \\
165 \\
437 \\
211\end{array}$ & $\begin{array}{l}0 \\
2 \\
1 \\
8 \\
2\end{array}$ & $\begin{array}{r}0 \\
26 \\
8 \\
158 \\
27\end{array}$ & $\begin{array}{l}0 \\
0 \\
0 \\
0 \\
5\end{array}$ & $\begin{array}{r}0 \\
0 \\
0 \\
0 \\
126\end{array}$ & $\begin{array}{r}2 \\
3 \\
21 \\
23 \\
12\end{array}$ & $\begin{array}{r}93 \\
95 \\
173 \\
595 \\
364\end{array}$ & $\begin{array}{l}1.16 \\
1.18 \\
2.16 \\
7.40 \\
4.52\end{array}$ \\
\hline $\begin{array}{l}\text { Oregon ................... } \\
\text { Pennsylvania.......... } \\
\text { Texas ...................... } \\
\text { Utah ......................... } \\
\text { Washington ............ }\end{array}$ & $\begin{array}{r}0 \\
34 \\
10 \\
3 \\
1\end{array}$ & $\begin{array}{r}0 \\
604 \\
240 \\
122 \\
34\end{array}$ & $\begin{array}{r}2 \\
18 \\
18 \\
0 \\
0\end{array}$ & $\begin{array}{r}12 \\
31 \\
385 \\
0 \\
0\end{array}$ & $\begin{array}{l}0 \\
7 \\
1 \\
1 \\
0\end{array}$ & $\begin{array}{r}0 \\
23 \\
3 \\
1 \\
0\end{array}$ & $\begin{array}{r}2 \\
59 \\
29 \\
4 \\
1\end{array}$ & $\begin{array}{r}12 \\
659 \\
628 \\
122 \\
34\end{array}$ & $\begin{array}{l}0.15 \\
8.19 \\
7.80 \\
1.52 \\
0.42\end{array}$ \\
\hline $\begin{array}{l}\text { West Virginia........... } \\
\text { Wyoming................ }\end{array}$ & $\begin{array}{r}26 \\
3\end{array}$ & $\begin{array}{r}476 \\
76\end{array}$ & $\begin{array}{l}0 \\
4\end{array}$ & $\begin{array}{r}0 \\
30\end{array}$ & $\begin{array}{r}10 \\
0\end{array}$ & $\begin{array}{r}44 \\
0\end{array}$ & $\begin{array}{r}36 \\
7\end{array}$ & $\begin{array}{l}520 \\
106\end{array}$ & $\begin{array}{l}6.47 \\
1.31\end{array}$ \\
\hline Total ....................... & 205 & 5,351 & 168 & 2,495 & 24 & 197 & 397 & 8,043 & 100.00 \\
\hline
\end{tabular}

Note: Totais may not equal sum of components due to independent rounding.

Source: Energy Information Administration (EIA), Form ElA-191, "Underground Gas Storage Report." 
Table 13. Supplemental Gas Supplies by State, 1994 (Million Cubic Feet)

\begin{tabular}{|c|c|c|c|c|c|c|}
\hline State & $\begin{array}{c}\text { Synthetic } \\
\text { Natural } \\
\text { Gas }\end{array}$ & $\begin{array}{l}\text { Propane- } \\
\text { Air }\end{array}$ & $\begin{array}{l}\text { Refinery } \\
\text { Gas }\end{array}$ & $\begin{array}{c}\text { Biomass } \\
\text { Gas }\end{array}$ & Other & Total \\
\hline 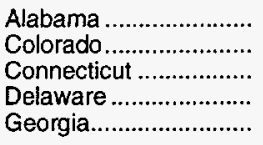 & $\begin{array}{l}0 \\
0 \\
0 \\
0 \\
0\end{array}$ & $\begin{array}{r}5 \\
19 \\
29 \\
2 \\
60\end{array}$ & $\begin{array}{r}0 \\
0 \\
0 \\
3,030 \\
0\end{array}$ & $\begin{array}{r}65 \\
0 \\
0 \\
0 \\
63\end{array}$ & $\begin{array}{r}0 \\
a_{8,149} \\
0 \\
0 \\
0\end{array}$ & $\begin{array}{r}69 \\
8,168 \\
29 \\
3,032 \\
123\end{array}$ \\
\hline 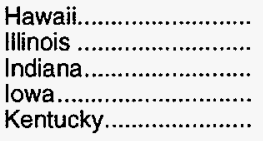 & $\begin{array}{r}2,831 \\
7,734 \\
0 \\
0 \\
0\end{array}$ & $\begin{array}{r}0 \\
137 \\
308 \\
30 \\
50\end{array}$ & $\begin{array}{l}0 \\
0 \\
0 \\
0 \\
0\end{array}$ & $\begin{array}{r}0 \\
0 \\
0 \\
93 \\
0\end{array}$ & $\begin{array}{r}0 \\
0 \\
b_{2,148} \\
0 \\
0\end{array}$ & $\begin{array}{r}2,831 \\
7,871 \\
2,457 \\
123 \\
50\end{array}$ \\
\hline 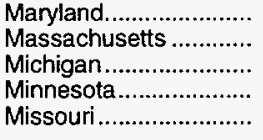 & $\begin{array}{l}0 \\
0 \\
0 \\
0 \\
0\end{array}$ & $\begin{array}{r}987 \\
154 \\
1 \\
289 \\
371\end{array}$ & $\begin{array}{l}0 \\
0 \\
0 \\
0 \\
0\end{array}$ & $\begin{array}{l}0 \\
0 \\
0 \\
0 \\
0\end{array}$ & $\begin{array}{r}0 \\
0 \\
c_{13,687} \\
0 \\
0\end{array}$ & $\begin{array}{r}987 \\
154 \\
13,688 \\
289 \\
371\end{array}$ \\
\hline 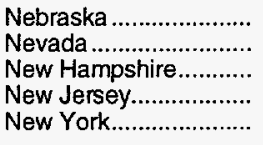 & $\begin{array}{l}0 \\
0 \\
0 \\
0 \\
0\end{array}$ & $\begin{array}{r}18 \\
9 \\
160 \\
44 \\
142\end{array}$ & $\begin{array}{r}0 \\
0 \\
0 \\
5,905 \\
0\end{array}$ & $\begin{array}{r}0 \\
0 \\
0 \\
1,675 \\
1,318\end{array}$ & $\begin{array}{r}a_{872} \\
0 \\
0 \\
d_{114} \\
0\end{array}$ & $\begin{array}{r}890 \\
9 \\
160 \\
7,737 \\
1,460\end{array}$ \\
\hline $\begin{array}{l}\text { North Carolina ............. } \\
\text { North Dakota ............... } \\
\text { Ohio ............................... } \\
\text { Oregon .................... } \\
\text { Pennsylvania ................. }\end{array}$ & $\begin{array}{r}0 \\
57,127 \\
0 \\
0 \\
0\end{array}$ & $\begin{array}{r}3 \\
0 \\
491 \\
2 \\
252\end{array}$ & $\begin{array}{l}0 \\
0 \\
0 \\
0 \\
0\end{array}$ & $\begin{array}{r}0 \\
0 \\
941 \\
0 \\
0\end{array}$ & $\begin{array}{l}0 \\
0 \\
0 \\
0 \\
0\end{array}$ & $\begin{array}{r}3 \\
57,127 \\
1,432 \\
2 \\
252\end{array}$ \\
\hline 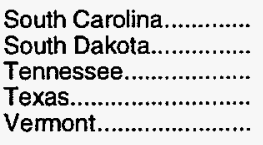 & $\begin{array}{l}0 \\
0 \\
0 \\
0 \\
0\end{array}$ & $\begin{array}{r}154 \\
61 \\
84 \\
1 \\
9\end{array}$ & $\begin{array}{l}0 \\
0 \\
0 \\
0 \\
0\end{array}$ & $\begin{array}{l}0 \\
0 \\
0 \\
0 \\
0\end{array}$ & $\begin{array}{l}0 \\
0 \\
0 \\
0 \\
0\end{array}$ & $\begin{array}{r}154 \\
61 \\
84 \\
1 \\
9\end{array}$ \\
\hline 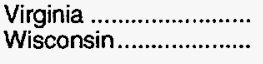 & $\begin{array}{l}0 \\
0\end{array}$ & $\begin{array}{r}1,195 \\
5\end{array}$ & $\begin{array}{l}0 \\
0\end{array}$ & $\begin{array}{l}0 \\
0\end{array}$ & $\begin{array}{l}0 \\
0\end{array}$ & $\begin{array}{r}1,195 \\
5\end{array}$ \\
\hline 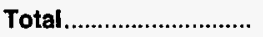 & 67,693 & 5,073 & 8,935 & 4,155 & 24,970 & 110,826 \\
\hline
\end{tabular}

a Air injection for Btu stabilization.

b Coke oven gas.

c Blast furnace gas.

d Manufactured gas.

Note: Totals may not equal sum of components due to independent rounding.

Source: Energy Information Administration (EIA), Form EIA-176, "Annual Report of Nafural and Supplemental Gas Supply and Disposition." 



\section{Demand}

\section{Consumption}

Consumption of natural gas continued to grow, although it showed a slower rate of increase from 1993 to 1994, up 2 percent, compared to the 4 percent increase seen between 1992 and 1993 . The growth did not occur evenly across all consuming sectors. Most of the 476 billion cubic feet increase in consumption was due to increased reliance on natural gas in the electric utility sector. Residential consumption declined and demand by commercial customers was almost flat, while industrial consumption grew by 196 billion cubic feet or 3 percent. Some of the factors that had an impact on 1994 consumption levels by sector are discussed below.

\section{Residential and Commercial}

Despite record low temperatures in the Eastern United States in January, overall the winter of 1993-94 was much more moderate than the exceptionally cold previous heating season. Customers in the residential and commercial sectors, which are driven by weather-related space-heating requirements, responded by decreasing uatural gas consumption. Consumption per residential customer fell from 94 to 91 thousand cubic feet. Although the number of homes using natural gas increased by more than 850 thousand, total deliveries to residential customers declined by 109 billion cubic feet. In the commercial sector, total deliveries increased by 34 billion cubic feet over 1993 . The

Figure 9. Natural Gas Delivered to Consumers in the United States, 1993 - 1994

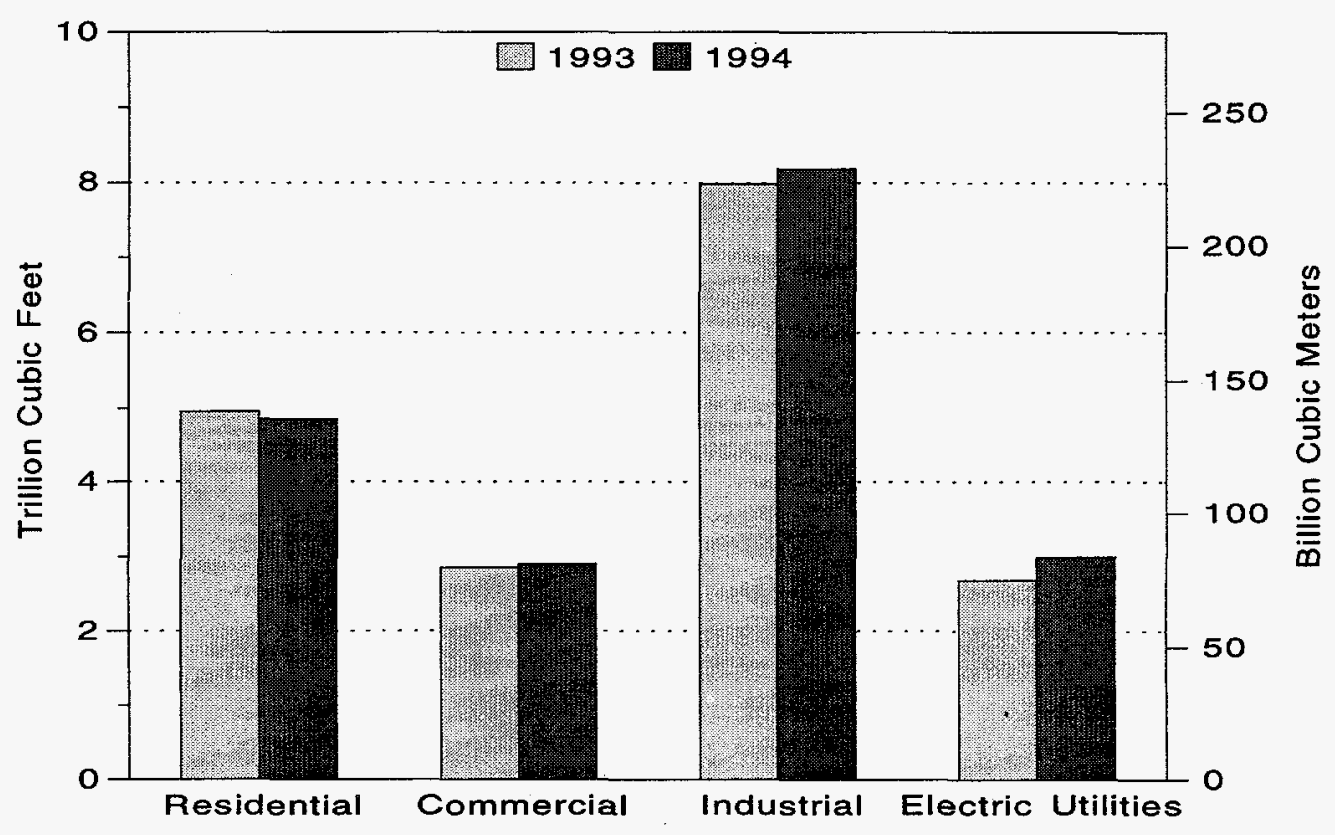

Source: Energy Information Administration (EIA), Form ElA-176, "Annual Report of Natural and Supplemental Gas Supply and Disposition." 
increased number of customers in this sector by 73,000 resulted in the per-customer consumption decreasing from 641 thousand cubic feet to 638 thousand cubic feet.

\section{Industrial}

Natural gas consumption in this sector, while continuing the upward climb of previous years, slowed from a 6 percent increase between 1992 and 1993 to an increase of only 2.5 percent, or 196 billion cubic feet between 1993 and 1994 . Industrial consumption includes deliveries to industrial nonutility power producer consumers and to other nonutility power producer consumers. Much of the increase reported in recent years may be attributed to natural gas consumption by nonutility generators (NUG's). However, the demand for electricity in two of the busiest NUC's growth markets, the Northeast and California, is flat or decreasing and construction of generation facilities by NUG's has decreased dramatically.

\section{Electric Utilities}

Natural gas consumption by electric utilities was notable in 1994, increasing by 305 billion cubic feet or 11 percent to just under 3 trillion cubic feet. Nationally, electric utilities in the United States generated slightly less than 1 percent more power during the year than in 1993. However, the role played by natural gas in that generation changed; 10 percent of electric generation was fueled by natural gas in 1994, compared to 9 percent in 1993. At the same time, the amount of power generated by either hydroelectric facilities or those fueled by petroleum or coal decreased over the year.

Electric utility consumption, shown in this report, is taken from the Form EIA-759, "Monthly Power Plant Report." Electric utilities report receipts of natural gas on this form. The differences between receipts reported on this form and deliveries reported on the Form EIA-176 are shown in Appendix A in Table A1. Further information about natural gas consumption in the electricity sector may be found in the EIA publication, Electric Power Annual, Volume I.

\section{Other Consuming Segments}

The volume of gas consumed in the production, processing, and transmission of natural gas is related to production and consumption volumes. Dry production increased by 652 billion cubic feet, lease fuel increased by 2 percent, while plant fuel declined. Pipeline fuel rose by 10 percent, or 61 billion cubic feet.

Although the amount of natural gas delivered for use as vehicle fuel is very small compared to deliveries to other consuming sectors, in 1994 it was 1.7 billion cubic feet, more than 80 percent greater than in 1993. Natural gas as a vehicle fuel presents one of the strongest areas for new market growth.

\section{Outlook for Demand}

The natural gas market has substantial growth potential and is expected to expand by 2 trillion cubic feet between 1994 and 2000. The majority of this growth is expected in the electric generation and industrial markets as a result of the economic and environmental advantages of natural gas over other generating fuels. (For some companies environmental concerns determine the use of natural gas.) The achievement of this growth will depend on the ability of the industry to develop new services to expand electric power and cogeneration markets to help keep the cost of gas competitive with alternative fuels. In 1996, a record high of 21.9 trillion cubic feet in total gas demand is projected. This growth, which would bring the U.S. gas demand to its highest level since 1973, also depends on a return to normal weather conditions and continued economic growth of over 2 percent.

Due to the weak heating market, residential gas demand in 1994 was less than 1993. Weak demand for gas carried over into the first quarter of 1995. In 1996, mainly due to assumptions of normal weather and also to continued addition of new natural gas customers, demand is expected to be up by 5.6 percent.

Given the relatively low natural gas prices and continued economic growth, particularly in manufactured production, 1995 is likely to show a considerable increase in industrial gas use. In 1996, industrial gas demand is expected to continue to grow but at a somewhat lower rate, along with the economy.

Gas used to generate electricity by electric utilities is expected to continue to grow in 1995, and to remain at about 1995's level in 1996. Moreover, a significant increase in natural gas consumption is driven by its expanding role as a boiler fuel for electric generators.

Market expansion is expected primarily from the demand for electricity (including industrial cogeneration) and the demand for alternative-fuel vehicles. Residential and commercial consumption is expected to remain low, as conservation and efficiency improvements offset the growth in the number of customers. 
Table 14. Consumption of Natural Gas by State, 1990 (Million Cubic Feet)

\begin{tabular}{|c|c|c|c|c|c|}
\hline $\begin{array}{l}\text { Year } \\
\text { and } \\
\text { State }\end{array}$ & Delivered to Consumers & Lease Fuel & Plant Fuel & Pipeline Fuel & Total Consumption \\
\hline 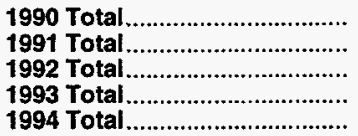 & $\begin{array}{r}16,818,882 \\
17,304,582 \\
17,785,833 \\
R_{18,482,847} \\
18,909,587\end{array}$ & $\begin{array}{r}807,735 \\
672,314 \\
710,250 \\
\mathrm{R} 723,118 \\
736,678\end{array}$ & $\begin{array}{l}428,657 \\
456,954 \\
460,571 \\
448,822 \\
423,878\end{array}$ & $\begin{array}{l}659,816 \\
601,305 \\
587,710 \\
624,308 \\
685,327\end{array}$ & $\begin{array}{r}18,715,090 \\
19,035,156 \\
19,544,364 \\
R_{20,279,095} \\
20,755,471\end{array}$ \\
\hline
\end{tabular}

\begin{tabular}{|c|c|c|c|c|c|}
\hline 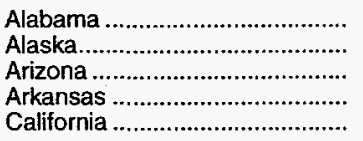 & $\begin{array}{r}260,830 \\
126,045 \\
108,517 \\
227,835 \\
2,041,539\end{array}$ & $\begin{array}{r}9,381 \\
236,781 \\
61 \\
4,283 \\
63,251\end{array}$ & $\begin{array}{r}3,747 \\
37,347 \\
0 \\
467 \\
6,388\end{array}$ & $\begin{array}{r}14,904 \\
3,024 \\
24,919 \\
11,840 \\
12,132\end{array}$ & $\begin{array}{r}288,863 \\
403,197 \\
133,497 \\
244,425 \\
2,123,310\end{array}$ \\
\hline 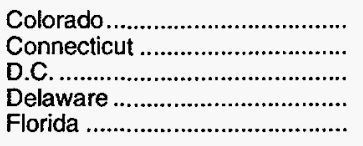 & $\begin{array}{r}241,416 \\
119,334 \\
30,607 \\
48,632 \\
361,428\end{array}$ & $\begin{array}{r}11,865 \\
0 \\
0 \\
0 \\
{ }^{2} 653\end{array}$ & $\begin{array}{r}12,482 \\
0 \\
0 \\
0 \\
167\end{array}$ & $\begin{array}{r}9,998 \\
697 \\
242 \\
4 \\
5,304\end{array}$ & $\begin{array}{r}275,761 \\
120,031 \\
30,849 \\
48,636 \\
367,552\end{array}$ \\
\hline 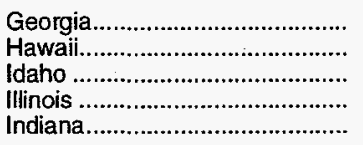 & $\begin{array}{r}334,418 \\
2,778 \\
52,164 \\
1,010,989 \\
512,482\end{array}$ & $\begin{array}{r}0 \\
0 \\
0 \\
10 \\
2\end{array}$ & $\begin{array}{r}0 \\
0 \\
0 \\
101 \\
0\end{array}$ & $\begin{array}{r}6,987 \\
0 \\
4,741 \\
13,808 \\
6,872\end{array}$ & $\begin{array}{r}341,405 \\
2,778 \\
56,905 \\
1,024,908 \\
519,356\end{array}$ \\
\hline 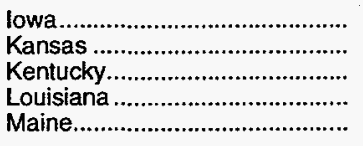 & $\begin{array}{r}237,614 \\
341,677 \\
182,710 \\
1,353,337 \\
5,045\end{array}$ & $\begin{array}{r}0 \\
15,623 \\
2,162 \\
140,571 \\
0\end{array}$ & $\begin{array}{r}0 \\
28,988 \\
611 \\
66,600 \\
0\end{array}$ & $\begin{array}{r}10,737 \\
31,739 \\
22,903 \\
63,257 \\
9\end{array}$ & $\begin{array}{r}248,351 \\
418,027 \\
208,386 \\
1,623,766 \\
5,055\end{array}$ \\
\hline 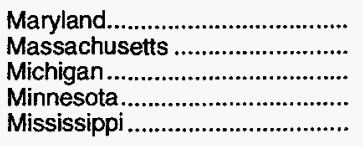 & $\begin{array}{l}181,259 \\
335,544 \\
893,735 \\
306,505 \\
225,730\end{array}$ & $\begin{array}{r}1 \\
0 \\
6,399 \\
0 \\
3,169\end{array}$ & $\begin{array}{r}0 \\
0 \\
3,367 \\
0 \\
463\end{array}$ & $\begin{array}{r}2,475 \\
1,817 \\
22,521 \\
17,310 \\
39,147\end{array}$ & $\begin{array}{l}183,734 \\
337,361 \\
926,023 \\
323,815 \\
268,510\end{array}$ \\
\hline 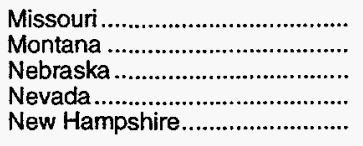 & $\begin{array}{r}264,715 \\
46,274 \\
123,373 \\
101,105 \\
18,732\end{array}$ & $\begin{array}{r}0 \\
2,037 \\
86 \\
16 \\
0\end{array}$ & $\begin{array}{r}0 \\
206 \\
3 \\
0 \\
0\end{array}$ & $\begin{array}{r}2,879 \\
3,542 \\
3,282 \\
669 \\
974\end{array}$ & $\begin{array}{r}267,594 \\
52,058 \\
126,744 \\
101,790 \\
19,706\end{array}$ \\
\hline 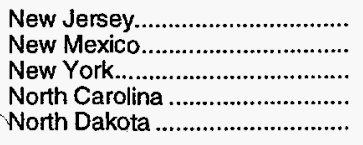 & $\begin{array}{r}582,356 \\
106,849 \\
1,005,676 \\
182,107 \\
27,301\end{array}$ & $\begin{array}{r}0 \\
18,476 \\
781 \\
0 \\
6,247\end{array}$ & $\begin{array}{r}0 \\
36,310 \\
0 \\
0 \\
5,059\end{array}$ & $\begin{array}{r}2,497 \\
59,135 \\
6,039 \\
5,753 \\
4,246\end{array}$ & $\begin{array}{r}584,853 \\
220,770 \\
1,012,497 \\
187,861 \\
42,853\end{array}$ \\
\hline 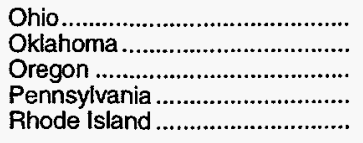 & $\begin{array}{r}824,119 \\
454,889 \\
140,526 \\
656,021 \\
70,901\end{array}$ & $\begin{array}{r}1,296 \\
55,607 \\
95 \\
4,042 \\
0\end{array}$ & $\begin{array}{r}36 \\
35,432 \\
0 \\
116 \\
0\end{array}$ & $\begin{array}{r}17,895 \\
26,146 \\
5,788 \\
37,959 \\
366\end{array}$ & $\begin{array}{r}843,345 \\
572,074 \\
146,409 \\
698,138 \\
71,267\end{array}$ \\
\hline 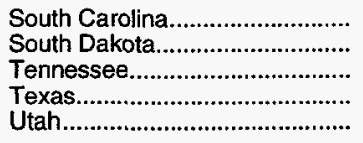 & $\begin{array}{r}141,863 \\
28,002 \\
228,007 \\
3,272,393 \\
120,993\end{array}$ & $\begin{array}{r}0 \\
437 \\
44 \\
139,427 \\
3,322\end{array}$ & $\begin{array}{r}0 \\
0 \\
0 \\
158,826 \\
9,951\end{array}$ & $\begin{array}{r}2,663 \\
2,541 \\
18,106 \\
95,684 \\
2,807\end{array}$ & $\begin{array}{r}144,526 \\
30,980 \\
246,157 \\
3,666,329 \\
137,073\end{array}$ \\
\hline 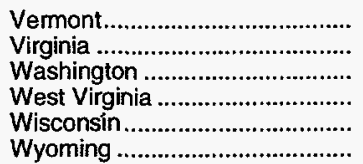 & $\begin{array}{r}7,297 \\
223,122 \\
206,346 \\
107,197 \\
345,748 \\
81,507\end{array}$ & $\begin{array}{r}0 \\
1,102 \\
0 \\
4,774 \\
0 \\
a^{2} 4,676\end{array}$ & $\begin{array}{r}0 \\
0 \\
0 \\
3,272 \\
0 \\
13,939\end{array}$ & $\begin{array}{r}3 \\
6,338 \\
6,437 \\
30,144 \\
9,854 \\
6,193\end{array}$ & $\begin{array}{r}7,299 \\
230,562 \\
212,782 \\
145,387 \\
355,602 \\
106,316\end{array}$ \\
\hline
\end{tabular}

a Lease fuel quantities were estimated by assuming that the proportions of on-system production used as lease fuel by respondents to the Form ElA-176 "Annual Report of Natural and Supplemental Gas Supply and Disposition," were the same as the proportions of gross withdrawals as reported on Form ElA-627, "Annual Quantity and Value of Natural Gas Report," used as lease by all operators. Form ElA-176 respondents reported on-system production totaling 32.8 percent of gross withdrawals in Alaska and 8.3 percent of gross withdrawals in the lower 48 states. The average ratios of volumes "Used in well, lease, and field operations" to "Company-owned natural gas produced on-system computed from Form ElA-176 responses were .02891 for Alaska and .02602 for the lower 48 states. Those ratios were applied to gross withdrawals from all states, where lease fuel was applicable, that did not report lease fuel use on the Form EIA-627.

= Revised data.

Note: Totals may not equal sum of components due to independent rounding.

Sources: Energy Information Administration (EIA), Form EIA-627, "Annual Quantity and Value of Natural Gas Report," estimates based upon Form ElA-176, "Annual Report of Natural and Supplemental Gas Supply and Disposition"; Form EIA-64A, "Annual Report of the Origin of Natural Gas Liquids Production"; and Form EIA-759, "Monthly Power Plant Report." 
Table 15. Natural Gas Delivered to Consumers by State, 1990-1994

\begin{tabular}{|c|c|c|c|c|c|c|}
\hline \multirow{2}{*}{$\begin{array}{l}\text { Year } \\
\text { and } \\
\text { State }\end{array}$} & \multicolumn{2}{|c|}{ Residential } & \multicolumn{2}{|c|}{ Commercial } & \multicolumn{2}{|c|}{ Industrial } \\
\hline & $\begin{array}{l}\text { Volume } \\
\text { (million } \\
\text { cubic feet) }\end{array}$ & Consumers & $\begin{array}{l}\text { Volume } \\
\text { (million } \\
\text { cubic feet) }\end{array}$ & Consumers & $\begin{array}{l}\text { Volume } \\
\text { (million } \\
\text { cubic feet) }\end{array}$ & Consumers \\
\hline $\begin{array}{l}1990 \text { Total.................... } \\
1991 \text { Total................... } \\
1992 \text { Total................... } \\
1993 \text { Total.................. } \\
1994 \text { Total .................. }\end{array}$ & $\begin{array}{r}4,391,324 \\
4,555,659 \\
4,690,065 \\
\mathrm{R}_{4,956,445} \\
4,847,702\end{array}$ & $\begin{array}{l}50,187,178 \\
51,593,206 \\
52,331,397 \\
52,535,411 \\
53,393,142\end{array}$ & $\begin{array}{r}2,622,721 \\
2,728,581 \\
2,802,751 \\
R_{2}, 861,569 \\
2,895,034\end{array}$ & $\begin{array}{r}4,236,280 \\
4,357,252 \\
4,409,699 \\
\mathbf{R}_{4,464,906} \\
4,537,500\end{array}$ & $\begin{array}{r}7,018,414 \\
7,230,962 \\
7,526,898 \\
\mathrm{P} 7,981,433 \\
8,177,975\end{array}$ & $\begin{array}{r}218,341 \\
216,529 \\
209,616 \\
R_{209,666} \\
202,953\end{array}$ \\
\hline 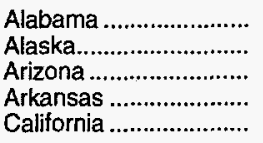 & $\begin{array}{r}49,748 \\
14,895 \\
29,684 \\
41,527 \\
520,959\end{array}$ & $\begin{array}{r}744,294 \\
77,670 \\
635,335 \\
521,176 \\
8,790,733\end{array}$ & $\begin{array}{r}25,526 \\
20,698 \\
29,187 \\
27,410 \\
261,989\end{array}$ & $\begin{array}{r}60,320 \\
12,475 \\
48,568 \\
63,821 \\
411,140\end{array}$ & $\begin{array}{r}181,718 \\
61,404 \\
25,869 \\
133,921 \\
656,751\end{array}$ & $\begin{array}{r}2,477 \\
11 \\
519 \\
1,417 \\
38,750\end{array}$ \\
\hline 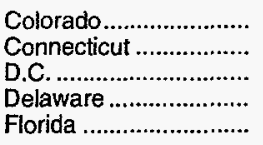 & $\begin{array}{r}99,504 \\
41,600 \\
15,865 \\
8,557 \\
13,855\end{array}$ & $\begin{array}{r}1,073,308 \\
428,157 \\
135,119 \\
100,431 \\
497,777\end{array}$ & $\begin{array}{r}65,870 \\
39,082 \\
14,742 \\
5,459 \\
39,935\end{array}$ & $\begin{array}{r}115,994 \\
45,042 \\
11,132 \\
8,721 \\
47,851\end{array}$ & $\begin{array}{r}71,093 \\
30,647 \\
0 \\
17,216 \\
126,873\end{array}$ & $\begin{array}{r}1,176 \\
3,754 \\
0 \\
252 \\
481\end{array}$ \\
\hline 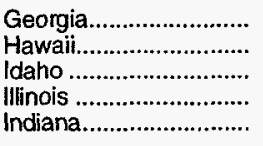 & $\begin{array}{r}105,436 \\
578 \\
12,285 \\
473,788 \\
157,467\end{array}$ & $\begin{array}{r}1,460,141 \\
30,614 \\
162,971 \\
3,418,052 \\
1,438,483\end{array}$ & $\begin{array}{r}54,051 \\
2,200 \\
10,088 \\
197,576 \\
75,819\end{array}$ & $\begin{array}{r}117,980 \\
2,805 \\
22,999 \\
262,308 \\
134,336\end{array}$ & $\begin{array}{r}173,901 \\
0 \\
29,781 \\
305,092 \\
270,128\end{array}$ & $\begin{array}{r}3,277 \\
0 \\
144 \\
25,788 \\
6,314\end{array}$ \\
\hline 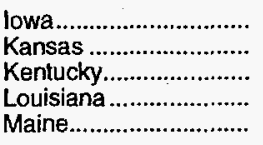 & $\begin{array}{r}78,260 \\
74,156 \\
62,533 \\
52,981 \\
894\end{array}$ & $\begin{array}{r}750,678 \\
773,357 \\
668,774 \\
946,072 \\
14,104\end{array}$ & $\begin{array}{r}47,922 \\
52,253 \\
36,744 \\
24,184 \\
2,381\end{array}$ & $\begin{array}{r}86,918 \\
86,457 \\
73,462 \\
65,879 \\
5,297\end{array}$ & $\begin{array}{r}108,731 \\
187,979 \\
83,081 \\
999,034 \\
1,771\end{array}$ & $\begin{array}{r}1,957 \\
3,560 \\
1,621 \\
1,737 \\
89\end{array}$ \\
\hline $\begin{array}{l}\text { Maryland ..................... } \\
\text { Massachusetts ............ } \\
\text { Michigan ...................... } \\
\text { Minnesota .................. } \\
\text { Mississippi .................... }\end{array}$ & $\begin{array}{r}76,688 \\
119,642 \\
364,588 \\
122,249 \\
27,086\end{array}$ & $\begin{array}{r}824,137 \\
1,179,869 \\
2,717,683 \\
1,049,263 \\
405,312\end{array}$ & $\begin{array}{r}44,136 \\
84,534 \\
183,068 \\
83,933 \\
19,232\end{array}$ & $\begin{array}{r}64,698 \\
92,744 \\
207,629 \\
102,857 \\
43,803\end{array}$ & $\begin{array}{r}47,691 \\
92,798 \\
327,848 \\
94,468 \\
96,863\end{array}$ & $\begin{array}{r}516 \\
8,019 \\
11,308 \\
2,592 \\
1,326\end{array}$ \\
\hline $\begin{array}{l}\text { Missouri ........................ } \\
\text { Montana ........................ } \\
\text { Nebraska ........................ } \\
\text { Nevada ...................... } \\
\text { New Hampshire............. }\end{array}$ & $\begin{array}{r}122,566 \\
18,714 \\
44,397 \\
21,263 \\
6,572\end{array}$ & $\begin{array}{r}1,281,007 \\
194,357 \\
428,201 \\
336,353 \\
72,656\end{array}$ & $\begin{array}{r}66,196 \\
12,981 \\
38,946 \\
18,694 \\
6,412\end{array}$ & $\begin{array}{r}132,378 \\
25,349 \\
62,045 \\
24,521 \\
12,240\end{array}$ & $\begin{array}{r}71,602 \\
13,940 \\
36,960 \\
28,867 \\
4,471\end{array}$ & $\begin{array}{r}3,115 \\
453 \\
766 \\
119 \\
324\end{array}$ \\
\hline $\begin{array}{l}\text { New Jersey................. } \\
\text { New Mexico.................. } \\
\text { New York.................... } \\
\text { North Carolina ............. } \\
\text { North Dakota ................ }\end{array}$ & $\begin{array}{r}216,873 \\
30,868 \\
385,408 \\
47,451 \\
10,661\end{array}$ & $\begin{array}{r}2,089,911 \\
409,095 \\
4,008,868 \\
652,307 \\
93,398\end{array}$ & $\begin{array}{r}132,008 \\
24,964 \\
223,256 \\
38,940 \\
10,783\end{array}$ & $\begin{array}{r}219,061 \\
39,846 \\
301,499 \\
80,739 \\
12,944\end{array}$ & $\begin{array}{r}190,845 \\
18,741 \\
214,438 \\
94,838 \\
5,846\end{array}$ & $\begin{array}{r}10,608 \\
1,097 \\
21,833 \\
3,401 \\
186\end{array}$ \\
\hline $\begin{array}{l}\text { Ohio } \\
\text { Oklahoma ...................... } \\
\text { Oregon ..................... } \\
\text { Pennsylvania ............... } \\
\text { Rhode Island ................. }\end{array}$ & $\begin{array}{r}343,331 \\
69,211 \\
28,848 \\
268,405 \\
17,384\end{array}$ & $\begin{array}{r}2,921,536 \\
845,448 \\
391,845 \\
2,393,053 \\
200,959\end{array}$ & $\begin{array}{r}166,798 \\
36,504 \\
22,960 \\
138,473 \\
12,049\end{array}$ & $\begin{array}{r}245,190 \\
87,494 \\
53,700 \\
206,812 \\
21,208\end{array}$ & $\begin{array}{r}311,123 \\
195,909 \\
62,569 \\
236,417 \\
40,921\end{array}$ & $\begin{array}{r}8,655 \\
2,853 \\
765 \\
6,388 \\
1,064\end{array}$ \\
\hline 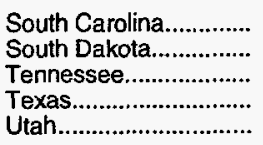 & $\begin{array}{r}23,486 \\
12,056 \\
57,334 \\
213,433 \\
48,922\end{array}$ & $\begin{array}{r}416,773 \\
119,544 \\
768,421 \\
3,350,314 \\
503,583\end{array}$ & $\begin{array}{r}17,870 \\
10,274 \\
50,760 \\
180,232 \\
26,501\end{array}$ & $\begin{array}{r}45,487 \\
15,539 \\
97,943 \\
306,376 \\
39,183\end{array}$ & $\begin{array}{r}97,500 \\
5,508 \\
118,889 \\
1,829,478 \\
36,618\end{array}$ & $\begin{array}{r}1,928 \\
381 \\
2,440 \\
5,222 \\
252\end{array}$ \\
\hline 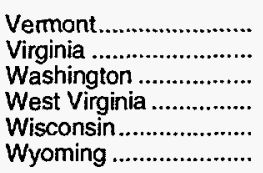 & $\begin{array}{r}2,438 \\
65,176 \\
53,144 \\
35,201 \\
128,175 \\
11,564\end{array}$ & $\begin{array}{r}22,546 \\
721,495 \\
604,315 \\
352,929 \\
1,253,333 \\
117,385\end{array}$ & $\begin{array}{r}2,669 \\
52,944 \\
42,982 \\
24,979 \\
78,609 \\
9,231\end{array}$ & $\begin{array}{r}3,512 \\
72,188 \\
68,118 \\
33,756 \\
119,788 \\
15,348\end{array}$ & $\begin{array}{r}2,023 \\
85,764 \\
107,603 \\
46,774 \\
135,106 \\
60,566\end{array}$ & $\begin{array}{r}24 \\
2,740 \\
3,586 \\
191 \\
7,342 \\
135\end{array}$ \\
\hline
\end{tabular}

See footnotes at end of table. 
Table 15. Natural Gas Delivered to Consumers by State, 1990-1994 (Continued)

\begin{tabular}{|c|c|c|c|c|}
\hline \multirow{2}{*}{$\begin{array}{l}\text { Year } \\
\text { and } \\
\text { State }\end{array}$} & Vehicle Fuel & Electric Utilities & Delivered to Consumers & \multirow{2}{*}{$\begin{array}{l}\text { Heating Value } \\
\text { (Btu per } \\
\text { Cubic Foot) }\end{array}$} \\
\hline & $\begin{array}{l}\text { Volume } \\
\text { (million } \\
\text { cubic teet) }\end{array}$ & $\begin{array}{c}\text { Volume } \\
\text { (million } \\
\text { cubic feet) }\end{array}$ & $\begin{array}{l}\text { Volume } \\
\text { (million } \\
\text { cubic feet) }\end{array}$ & \\
\hline $\begin{array}{l}1990 \text { Total.................. } \\
1991 \text { Total................... } \\
1992 \text { Total.................. } \\
1993 \text { Total................. } \\
1994 \text { Total.................. }\end{array}$ & $\begin{array}{r}270 \\
367 \\
511 \\
960 \\
1,730\end{array}$ & $\begin{array}{r}1007 \\
2,789,014 \\
2,765,608 \\
2,682,440 \\
2,987,146\end{array}$ & $\begin{array}{r}16,818,882 \\
17,304,582 \\
17,785,833 \\
R_{18,482,847} \\
18,909,587\end{array}$ & $\begin{array}{l}1,031 \\
1,030 \\
1,030 \\
1,027 \\
1,028\end{array}$ \\
\hline $\begin{array}{l}\text { Alabama ................... } \\
\text { Alaska ......................... } \\
\text { Arizona ....................... } \\
\text { Arkansas ................... } \\
\text { California .................... }\end{array}$ & $\begin{array}{r}3 \\
0 \\
61 \\
0 \\
550\end{array}$ & $\begin{array}{r}3,834 \\
29,048 \\
23,716 \\
24,977 \\
601,290\end{array}$ & $\begin{array}{r}260,830 \\
126,045 \\
108,517 \\
227,835 \\
2,041,539\end{array}$ & $\begin{array}{l}1,030 \\
1,001 \\
1,027 \\
1,022 \\
1,023\end{array}$ \\
\hline 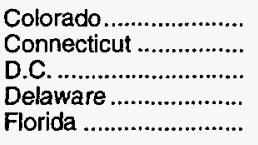 & $\begin{array}{r}68 \\
2 \\
0 \\
1 \\
68\end{array}$ & $\begin{array}{r}4,881 \\
8,002 \\
0 \\
17,399 \\
180,697\end{array}$ & $\begin{array}{r}241,416 \\
119,334 \\
30,607 \\
48,632 \\
361,428\end{array}$ & $\begin{array}{l}1,005 \\
1,030 \\
1,011 \\
1,036 \\
1,068\end{array}$ \\
\hline 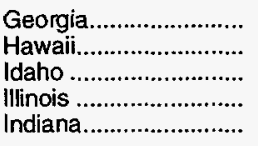 & $\begin{array}{r}3 \\
0 \\
10 \\
28 \\
59\end{array}$ & $\begin{array}{r}1,028 \\
0 \\
0 \\
34,505 \\
9,009\end{array}$ & $\begin{array}{r}334,418 \\
2,778 \\
52,164 \\
1,010,989 \\
512,482\end{array}$ & $\begin{array}{l}1,030 \\
1,051 \\
1,038 \\
1,021 \\
1,013\end{array}$ \\
\hline 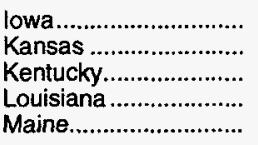 & $\begin{array}{r}5 \\
10 \\
2 \\
22 \\
0\end{array}$ & $\begin{array}{r}2,696 \\
27,279 \\
350 \\
277,116 \\
0\end{array}$ & $\begin{array}{r}237,614 \\
341,677 \\
182,710 \\
1,353,337 \\
5,045\end{array}$ & $\begin{array}{r}1,008 \\
998 \\
1,062 \\
1,040 \\
1,014\end{array}$ \\
\hline $\begin{array}{l}\text { Maryland.................... } \\
\text { Massachusetts ............ } \\
\text { Michigan..................... } \\
\text { Minnesota.................. } \\
\text { Mississippi .................... }\end{array}$ & $\begin{array}{r}25 \\
3 \\
14 \\
29 \\
9\end{array}$ & $\begin{array}{r}12,718 \\
38,567 \\
18,218 \\
5,826 \\
82,541\end{array}$ & $\begin{array}{l}181,259 \\
335,544 \\
893,735 \\
306,505 \\
225,730\end{array}$ & $\begin{array}{l}1,031 \\
1,026 \\
1,021 \\
1,011 \\
1,035\end{array}$ \\
\hline $\begin{array}{l}\text { Missouri ..................... } \\
\text { Montana ...................... } \\
\text { Nebraska ..................... } \\
\text { Nevada .................... } \\
\text { New Hampshire........... }\end{array}$ & $\begin{array}{r}1 \\
6 \\
9 \\
36 \\
\end{array}$ & $\begin{array}{r}4,351 \\
632 \\
3,061 \\
32,246 \\
1,277\end{array}$ & $\begin{array}{r}264,715 \\
46,274 \\
123,373 \\
101,105 \\
18,732\end{array}$ & $\begin{array}{r}1,006 \\
1,024 \\
985 \\
1,035 \\
1,013\end{array}$ \\
\hline $\begin{array}{l}\text { New Jersey................ } \\
\text { New Mexico.................. } \\
\text { New York.................. } \\
\text { North Carolina ............. } \\
\text { North Dakota ............... }\end{array}$ & $\begin{array}{r}4 \\
62 \\
53 \\
8 \\
8\end{array}$ & $\begin{array}{r}42,625 \\
32,214 \\
182,521 \\
871 \\
3\end{array}$ & $\begin{array}{r}582,356 \\
106,849 \\
1,005,676 \\
182,107 \\
27,301\end{array}$ & $\begin{array}{l}1,039 \\
1,003 \\
1,028 \\
1,036 \\
1,058\end{array}$ \\
\hline $\begin{array}{l}\text { Ohio......................... } \\
\text { Oklahoma .................... } \\
\text { Oregon ........................ } \\
\text { Pennsylvania .............. } \\
\text { Rhode Island ................ }\end{array}$ & $\begin{array}{r}48 \\
157 \\
17 \\
10 \\
1\end{array}$ & $\begin{array}{r}2,818 \\
153,109 \\
26,132 \\
12,716 \\
546\end{array}$ & $\begin{array}{r}824,119 \\
454,889 \\
140,526 \\
656,021 \\
70,901\end{array}$ & $\begin{array}{l}1,037 \\
1,028 \\
1,040 \\
1,036 \\
1,029\end{array}$ \\
\hline 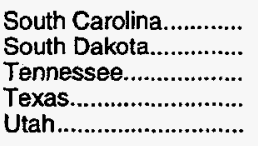 & $\begin{array}{r}2 \\
5 \\
5 \\
45 \\
52\end{array}$ & $\begin{array}{r}3,005 \\
159 \\
1,019 \\
1,049,205 \\
8,900\end{array}$ & $\begin{array}{r}141,863 \\
28,002 \\
228,007 \\
3,272,393 \\
120,993\end{array}$ & $\begin{array}{l}1,031 \\
1,010 \\
1,032 \\
1,037 \\
1,067\end{array}$ \\
\hline 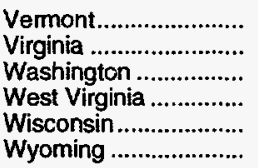 & $\begin{array}{r}0 \\
20 \\
155 \\
0 \\
36 \\
18\end{array}$ & $\begin{array}{r}166 \\
19,219 \\
2,461 \\
243 \\
3,821 \\
129\end{array}$ & $\begin{array}{r}7,297 \\
223,122 \\
206,346 \\
107,197 \\
345,748 \\
81,507\end{array}$ & $\begin{array}{r}996 \\
1,038 \\
1,041 \\
1,064 \\
1,012 \\
1,056\end{array}$ \\
\hline
\end{tabular}

$\mathbf{R}=$ Revised data.

- Less than 500,000 cubic feet.

Note: Totals may not equal sum of components due to independent rounding.

Sources: Energy Information Administration (EIA), Form EIA-176, "Annual Peport of Natural and Supplemental Gas Supply and Disposition" and Form EIA-759, "Monthly Power Plant Report. 
Figure 10. Natural Gas Delivered to Consumers in the United States, 1994

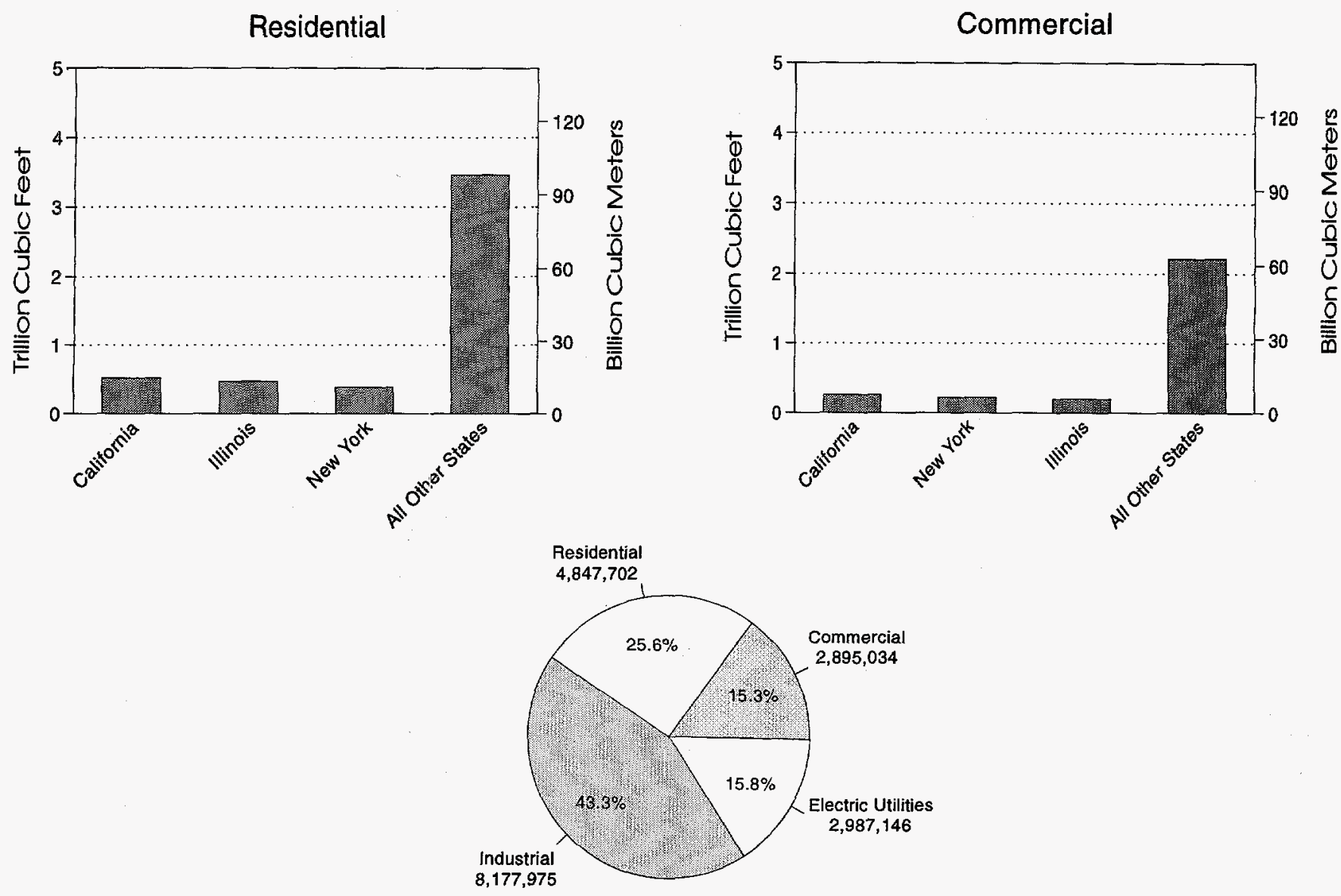

Volumes in Million Cubic Feet

Industrial

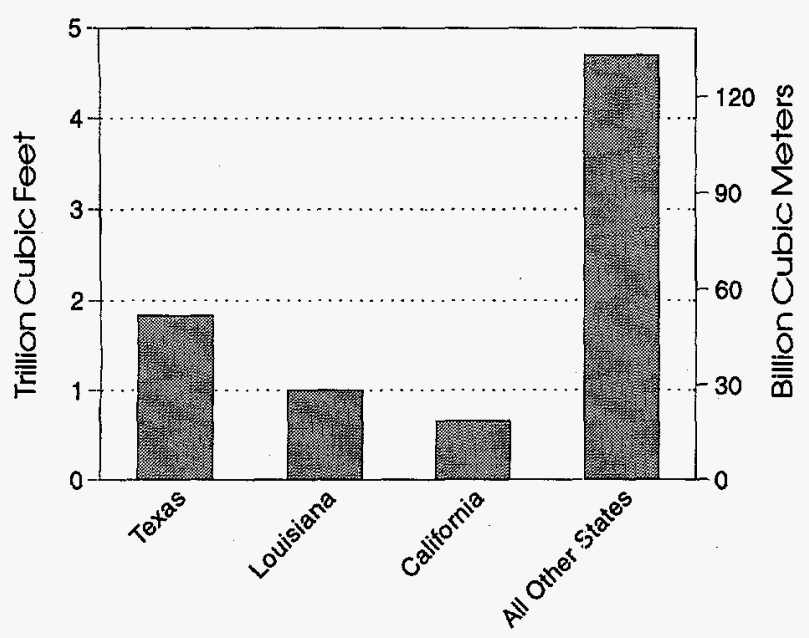

Electric Utilities

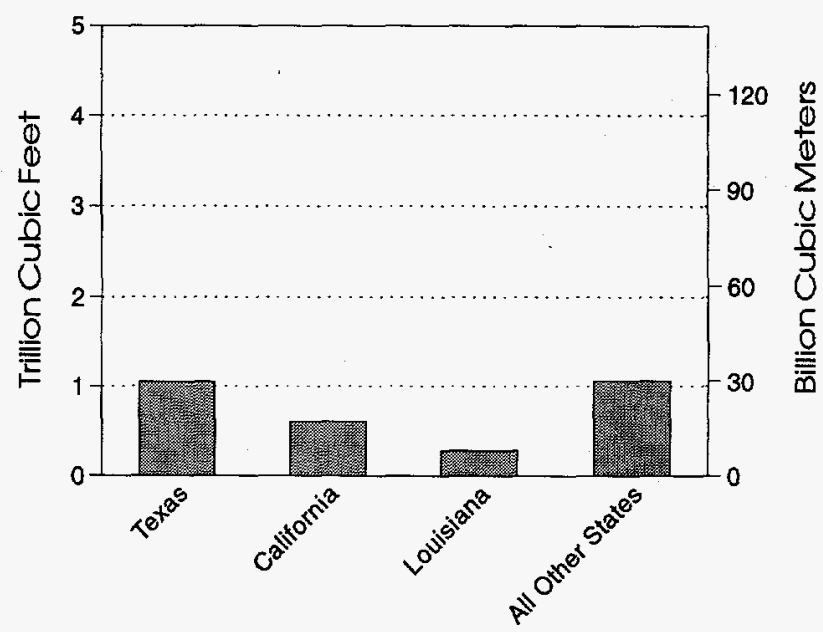

Source: Energy information Administration (EIA), Form ElA-176, "Annual Report of Natural and Supplemental Gas Supply and Disposition." 
Table 16. Natural Gas Delivered to Commercial Consumers for the Account of Others ${ }^{a}$ by State, 1990-1994 (Million Cubic Feet)

\begin{tabular}{|c|c|c|c|c|c|c|c|c|c|c|}
\hline \multirow[b]{2}{*}{ State } & \multicolumn{2}{|c|}{1990} & \multicolumn{2}{|c|}{1991} & \multicolumn{2}{|c|}{1992} & \multicolumn{2}{|c|}{1993} & \multicolumn{2}{|c|}{1994} \\
\hline & $\begin{array}{l}\text { Delivered } \\
\text { for the } \\
\text { Account } \\
\text { of Others }\end{array}$ & $\begin{array}{c}\text { Percent of } \\
\text { Total } \\
\text { Commercial } \\
\text { Deliveries }\end{array}$ & $\begin{array}{l}\text { Delivered } \\
\text { for the } \\
\text { Account } \\
\text { of Others }\end{array}$ & $\begin{array}{c}\text { Percent of } \\
\text { Total } \\
\text { Commercial } \\
\text { Deliveries }\end{array}$ & $\begin{array}{l}\text { Delivered } \\
\text { for the } \\
\text { Account } \\
\text { of Others }\end{array}$ & $\begin{array}{c}\text { Percent of } \\
\text { Total } \\
\text { Commercial } \\
\text { Deliveries }\end{array}$ & $\begin{array}{l}\text { Delivered } \\
\text { for the } \\
\text { Account } \\
\text { of Others }\end{array}$ & $\begin{array}{l}\text { Percent of } \\
\text { Total } \\
\text { Commarcial } \\
\text { Deliveries }\end{array}$ & $\begin{array}{l}\text { Delivered } \\
\text { tor the } \\
\text { Account } \\
\text { of Others }\end{array}$ & $\begin{array}{c}\text { Percent of } \\
\text { Total } \\
\text { Commercial } \\
\text { Deliveries }\end{array}$ \\
\hline $\begin{array}{l}\text { Alabama ............... } \\
\text { Arizona ................ } \\
\text { Arkansas .............. } \\
\text { California .............. } \\
\text { Colorado.............. }\end{array}$ & $\begin{array}{r}4,967 \\
1,219 \\
1,939 \\
38,337 \\
1,800\end{array}$ & $\begin{array}{r}20.5 \\
4.3 \\
7.7 \\
13.4 \\
2.7\end{array}$ & $\begin{array}{r}4,112 \\
1,876 \\
2,198 \\
63,882 \\
2,763\end{array}$ & $\begin{array}{r}17.3 \\
6.8 \\
8.5 \\
22.2 \\
4.0\end{array}$ & $\begin{array}{r}4,868 \\
2,021 \\
2,343 \\
72,782 \\
2,993\end{array}$ & $\begin{array}{r}19.3 \\
7.5 \\
9.3 \\
25.5 \\
4.5\end{array}$ & $\begin{array}{r}4,950 \\
2,336 \\
2,393 \\
57,781 \\
3,241\end{array}$ & $\begin{array}{r}19.2 \\
8.5 \\
8.3 \\
23.1 \\
4.5\end{array}$ & $\begin{array}{r}5,043 \\
2,709 \\
1,351 \\
134,346 \\
3,403\end{array}$ & $\begin{array}{r}19.8 \\
9.3 \\
4.9 \\
51.3 \\
5.2\end{array}$ \\
\hline $\begin{array}{l}\text { Connecticut ......... } \\
\text { D.C..................... } \\
\text { Florida ................. } \\
\text { Georgia................ } \\
\text { Idaho .................... }\end{array}$ & $\begin{array}{r}1,169 \\
0 \\
881 \\
5,721 \\
1,035\end{array}$ & $\begin{array}{r}4.0 \\
-\overline{-} \\
2.4 \\
11.6 \\
12.1\end{array}$ & $\begin{array}{r}1,887 \\
417 \\
1,005 \\
6,395 \\
1,192\end{array}$ & $\begin{array}{r}7.0 \\
2.7 \\
2.6 \\
12.5 \\
12.4\end{array}$ & $\begin{array}{r}1,037 \\
155 \\
964 \\
6,389 \\
1,278\end{array}$ & $\begin{array}{r}3.5 \\
1.0 \\
2.3 \\
11.9 \\
14.3\end{array}$ & $\begin{array}{r}602 \\
332 \\
911 \\
5,487 \\
1,405\end{array}$ & $\begin{array}{r}1.9 \\
2.0 \\
2.2 \\
9.5 \\
13.2\end{array}$ & $\begin{array}{r}7,455 \\
1,343 \\
861 \\
4,304 \\
1,427\end{array}$ & $\begin{array}{r}19.1 \\
9.1 \\
2.2 \\
8.0 \\
14.1\end{array}$ \\
\hline $\begin{array}{l}\text { Illinois .................... } \\
\text { Indiana................... } \\
\text { lowa..................... } \\
\text { Kansas .................. } \\
\text { Kentucky.............. }\end{array}$ & $\begin{array}{r}84,936 \\
2,906 \\
1,068 \\
4,701 \\
1,575\end{array}$ & $\begin{array}{r}42.4 \\
4.3 \\
2.4 \\
8.4 \\
5.0\end{array}$ & $\begin{array}{r}79,512 \\
3,947 \\
1,097 \\
6,321 \\
2,035\end{array}$ & $\begin{array}{r}41.0 \\
5.8 \\
2.4 \\
10.8 \\
6.0\end{array}$ & $\begin{array}{r}83,264 \\
2,319 \\
1,974 \\
8,408 \\
2,451\end{array}$ & $\begin{array}{r}42.3 \\
3.2 \\
4.3 \\
15.6 \\
6.9\end{array}$ & $\begin{array}{r}90,812 \\
3,724 \\
2,648 \\
9,729 \\
2,809\end{array}$ & $\begin{array}{r}44.7 \\
4.8 \\
5.3 \\
17.4 \\
7.4\end{array}$ & $\begin{array}{r}93,206 \\
5,841 \\
4,597 \\
11,295 \\
3,171\end{array}$ & $\begin{array}{r}47.2 \\
7.7 \\
9.6 \\
21.6 \\
8.6\end{array}$ \\
\hline $\begin{array}{l}\text { Louisiana ............. } \\
\text { Maryland............. } \\
\text { Massachusetts .... } \\
\text { Michigan............... } \\
\text { Minnesota............ }\end{array}$ & $\begin{array}{r}0 \\
1,052 \\
32 \\
48,061 \\
3,220\end{array}$ & $\begin{array}{r}-\overline{4} \\
0.1 \\
30.1 \\
4.1\end{array}$ & $\begin{array}{r}233 \\
1,308 \\
96 \\
52,444 \\
4,094\end{array}$ & $\begin{array}{r}0.9 \\
3.4 \\
0.2 \\
31.7 \\
4.8\end{array}$ & $\begin{array}{r}3,552 \\
1,692 \\
140 \\
54,248 \\
3,134\end{array}$ & $\begin{array}{r}12.5 \\
4.0 \\
0.2 \\
31.2 \\
3.8\end{array}$ & $\begin{array}{r}479 \\
1,497 \\
1,625 \\
56,547 \\
2,623\end{array}$ & $\begin{array}{r}1.9 \\
3.4 \\
2.5 \\
31.4 \\
3.0\end{array}$ & $\begin{array}{r}505 \\
1,291 \\
20,132 \\
62,825 \\
3,336\end{array}$ & $\begin{array}{r}2.1 \\
2.9 \\
23.8 \\
34.3 \\
4.0\end{array}$ \\
\hline $\begin{array}{l}\text { Mississippi............ } \\
\text { Missouri.............. } \\
\text { Montana .............. } \\
\text { Nebraska ............. } \\
\text { Nevada ................ }\end{array}$ & $\begin{array}{r}777 \\
8,306 \\
261 \\
2,231 \\
346\end{array}$ & $\begin{array}{r}4.4 \\
14.0 \\
2.1 \\
6.1 \\
2.3\end{array}$ & $\begin{array}{r}731 \\
8,910 \\
327 \\
3,294 \\
1,563\end{array}$ & $\begin{array}{r}4.1 \\
14.1 \\
2.5 \\
8.2 \\
9.2\end{array}$ & $\begin{array}{r}645 \\
8,817 \\
533 \\
4,063 \\
1,889\end{array}$ & $\begin{array}{r}3.6 \\
14.5 \\
4.6 \\
11.8 \\
11.7\end{array}$ & $\begin{array}{r}647 \\
10,710 \\
939 \\
3,142 \\
1,283\end{array}$ & $\begin{array}{r}3.4 \\
15.4 \\
6.8 \\
9.0 \\
7.3\end{array}$ & $\begin{array}{r}647 \\
11,072 \\
1,070 \\
7,726 \\
3,276\end{array}$ & $\begin{array}{r}3.4 \\
16.7 \\
8.2 \\
19.8 \\
17.5\end{array}$ \\
\hline $\begin{array}{l}\text { New Jersey.......... } \\
\text { New Mexico........ } \\
\text { New York............ } \\
\text { North Carolina ..... } \\
\text { North Dakota ....... }\end{array}$ & $\begin{array}{r}5,978 \\
4,008 \\
31,904 \\
1,696 \\
2,585\end{array}$ & $\begin{array}{r}5.2 \\
16.9 \\
16.4 \\
5.4 \\
25.3\end{array}$ & $\begin{array}{r}7,401 \\
5,570 \\
38,556 \\
1,725 \\
3,223\end{array}$ & $\begin{array}{r}6.1 \\
22.3 \\
19.3 \\
5.0 \\
30.0\end{array}$ & $\begin{array}{r}10,012 \\
8,361 \\
48,552 \\
1,497 \\
3,035\end{array}$ & $\begin{array}{r}7.6 \\
30.0 \\
22.4 \\
4.1 \\
31.1\end{array}$ & $\begin{array}{r}10,901 \\
10,459 \\
50,279 \\
561 \\
2,908\end{array}$ & $\begin{array}{r}8.5 \\
37.5 \\
22.8 \\
1.5 \\
27.3\end{array}$ & $\begin{array}{r}11,045 \\
9,395 \\
45,626 \\
1,314 \\
2,199\end{array}$ & $\begin{array}{r}8.4 \\
37.6 \\
20.4 \\
3.4 \\
20.4\end{array}$ \\
\hline $\begin{array}{l}\text { Ohio .................... } \\
\text { Oklahoma ............ } \\
\text { Oregon ............... } \\
\text { Pennsylvania ....... } \\
\text { Rhode Island ....... }\end{array}$ & $\begin{array}{r}18,258 \\
2,944 \\
464 \\
27,144 \\
330\end{array}$ & $\begin{array}{r}12.7 \\
7.9 \\
2.3 \\
21.6 \\
4.1\end{array}$ & $\begin{array}{r}20,033 \\
3,445 \\
477 \\
28,528 \\
0\end{array}$ & $\begin{array}{r}13.3 \\
8.7 \\
2.1 \\
22.7 \\
--\end{array}$ & $\begin{array}{r}23,188 \\
4,052 \\
433 \\
32,481 \\
0\end{array}$ & $\begin{array}{r}14.4 \\
11.5 \\
2.2 \\
24.2 \\
--\end{array}$ & $\begin{array}{r}25,345 \\
4,095 \\
504 \\
29,758 \\
0\end{array}$ & $\begin{array}{r}15.5 \\
10.0 \\
2.1 \\
22.6 \\
--\end{array}$ & $\begin{array}{r}30,807 \\
4,214 \\
430 \\
35,514 \\
0\end{array}$ & $\begin{array}{r}18.5 \\
11.5 \\
1.9 \\
25.6 \\
--\end{array}$ \\
\hline $\begin{array}{l}\text { South Carolina..... } \\
\text { South Dakota....... } \\
\text { Tennessee.......... } \\
\text { Texas................... } \\
\text { Utah................... }\end{array}$ & $\begin{array}{r}341 \\
1,161 \\
1,092 \\
17,645 \\
0\end{array}$ & $\begin{array}{r}2.2 \\
13.6 \\
2.5 \\
10.2 \\
--\end{array}$ & $\begin{array}{r}278 \\
1,723 \\
1,961 \\
19,287 \\
0\end{array}$ & $\begin{array}{r}1.8 \\
18.2 \\
4.3 \\
10.7 \\
-.\end{array}$ & $\begin{array}{r}239 \\
1,603 \\
1,680 \\
37,443 \\
0\end{array}$ & $\begin{array}{r}1.4 \\
17.6 \\
3.6 \\
20.3 \\
.\end{array}$ & $\begin{array}{r}R_{132} \\
1,724 \\
2,129 \\
R_{28,423} \\
0\end{array}$ & $\begin{array}{r}\mathbf{R}_{0.8} \\
16.1 \\
4.2 \\
\mathbf{R}_{16.2} \\
-.\end{array}$ & $\begin{array}{r}265 \\
1,124 \\
2,992 \\
31,742 \\
4,438\end{array}$ & $\begin{array}{r}1.5 \\
10.9 \\
5.9 \\
17.6 \\
16.7\end{array}$ \\
\hline $\begin{array}{l}\text { Virginia ............... } \\
\text { Washington ......... } \\
\text { West Virginia ....... } \\
\text { Wisconsin ............ } \\
\text { Wyoming ............ }\end{array}$ & $\begin{array}{r}2,804 \\
2,462 \\
8,955 \\
6,189 \\
21\end{array}$ & $\begin{array}{r}6.8 \\
6.4 \\
41.9 \\
9.3 \\
0.2\end{array}$ & $\begin{array}{r}2,826 \\
3,247 \\
9,496 \\
6,414 \\
89\end{array}$ & $\begin{array}{r}6.4 \\
7.8 \\
45.1 \\
9.0 \\
1.0\end{array}$ & $\begin{array}{r}4,719 \\
4,831 \\
10,536 \\
6,229 \\
160\end{array}$ & $\begin{array}{r}9.3 \\
12.8 \\
43.1 \\
8.7 \\
2.0\end{array}$ & $\begin{array}{r}5,902 \\
2,671 \\
11,134 \\
4,312 \\
207\end{array}$ & $\begin{array}{r}11.2 \\
6.1 \\
45.7 \\
5.6 \\
2.0\end{array}$ & $\begin{array}{r}7,039 \\
1,993 \\
11,194 \\
5,133 \\
358\end{array}$ & $\begin{array}{r}13.3 \\
4.6 \\
44.8 \\
6.5 \\
3.9\end{array}$ \\
\hline 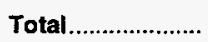 & 352,521 & 13.4 & 405,919 & 14.9 & 471,009 & 16.8 & $\mathbf{R}_{460,097}$ & $\mathbf{R}_{16.1}$ & 599,058 & 20.7 \\
\hline
\end{tabular}

a These deliveries included quantities covered by long-term contracts and gas involved in short-term or spot market sales.

$R=$ Revised data.

Source: Energy information Administration (EIA), Forrn ElA-176, "Annual Report of Natural and Supplemental Gas Supply and Disposition." 
Figure 11. Percent of Natural Gas: Deliveries in the United States Representing Deliveries for the Account of Others, by Consumer Sector, 1990 - 1994

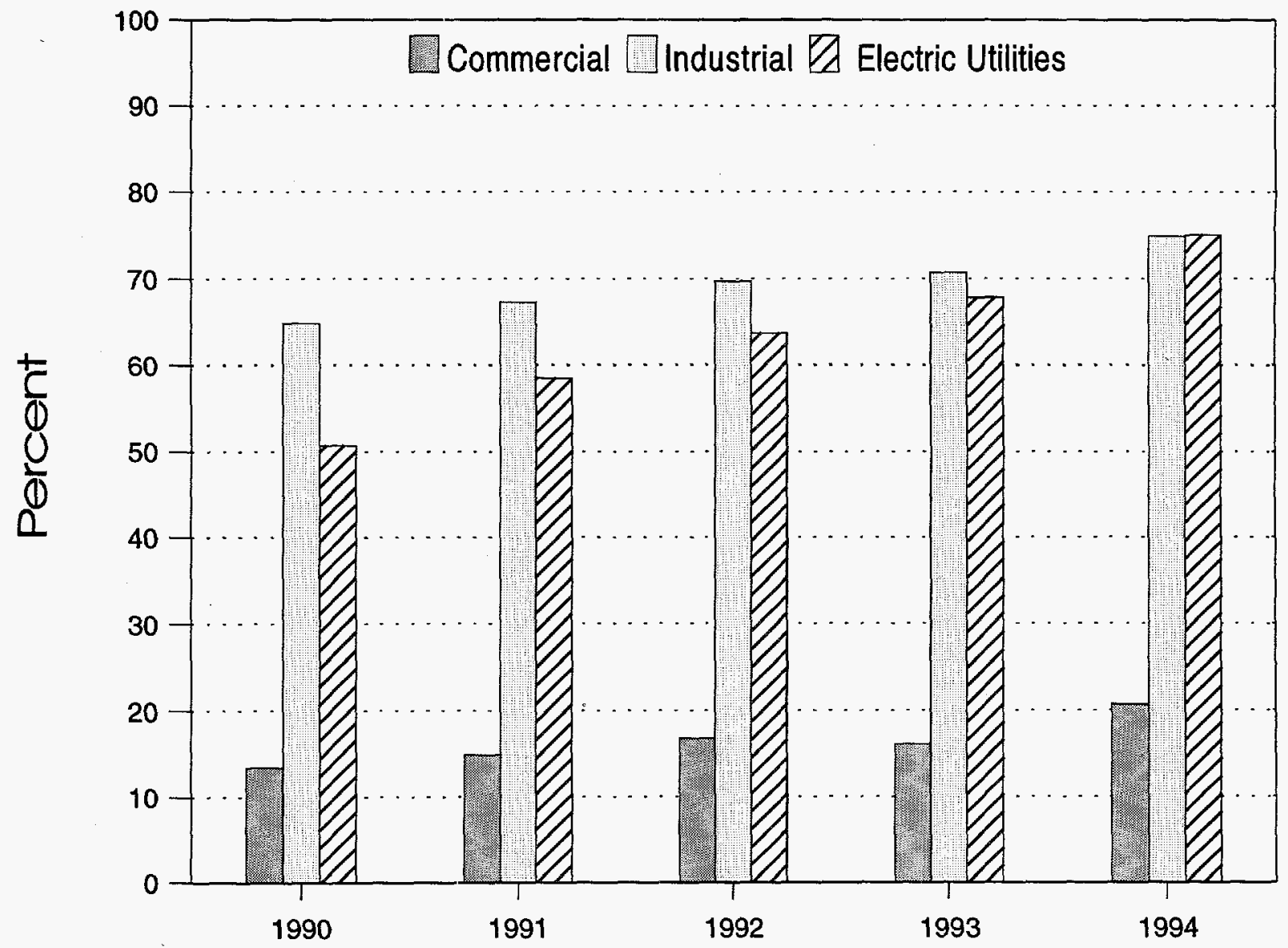

Note: These deliveries included quantities covered by long-term contracts and gas involved in short-term or spot market sales.

Source: Energy Information Administration (EIA), Form EIA-176, "Annual Feport of Natural and Supplemental Gas Supply and Disposition." 
Table 17. Natural Gas Delivered to Industrial Consumers for the Account of Others ${ }^{a}$ by State, 1990-1994 (Million Cubic Feet)

\begin{tabular}{|c|c|c|c|c|c|c|c|c|c|c|}
\hline \multirow[b]{2}{*}{ State } & \multicolumn{2}{|c|}{1990} & \multicolumn{2}{|c|}{1991} & \multicolumn{2}{|c|}{1992} & \multicolumn{2}{|c|}{1993} & \multicolumn{2}{|c|}{1994} \\
\hline & $\begin{array}{l}\text { Delivered } \\
\text { for the } \\
\text { Account } \\
\text { of Others }\end{array}$ & $\begin{array}{l}\text { Percent of } \\
\text { Total } \\
\text { Industrial } \\
\text { Deliveries }\end{array}$ & $\begin{array}{l}\text { Delivered } \\
\text { for the } \\
\text { Account } \\
\text { of Others }\end{array}$ & $\begin{array}{l}\text { Percent of } \\
\text { Total } \\
\text { Industrial } \\
\text { Deliveries }\end{array}$ & $\begin{array}{l}\text { Delivered } \\
\text { for the } \\
\text { Account } \\
\text { of Others }\end{array}$ & $\begin{array}{l}\text { Percent of } \\
\text { Total } \\
\text { Industrial } \\
\text { Deliveries }\end{array}$ & $\begin{array}{l}\text { Delivered } \\
\text { for the } \\
\text { Account } \\
\text { of Others }\end{array}$ & $\begin{array}{l}\text { Percent of } \\
\text { Total } \\
\text { Industrial } \\
\text { Deliveries }\end{array}$ & $\begin{array}{l}\text { Delivered } \\
\text { for the } \\
\text { Account } \\
\text { of Others }\end{array}$ & $\begin{array}{c}\text { Percent of } \\
\text { Total } \\
\text { Industrial } \\
\text { Deliveries }\end{array}$ \\
\hline $\begin{array}{l}\text { Alabama ............... } \\
\text { Alaska................... } \\
\text { Arizona ................ } \\
\text { Arkansas ............. } \\
\text { California ............. }\end{array}$ & $\begin{array}{r}96,889 \\
28,165 \\
10,421 \\
93,332 \\
346,643\end{array}$ & $\begin{array}{l}66.5 \\
36.6 \\
56.7 \\
77.6 \\
61.3\end{array}$ & $\begin{array}{r}106,274 \\
23,363 \\
9,966 \\
84,515 \\
422,738\end{array}$ & $\begin{array}{l}68.6 \\
30.9 \\
51.9 \\
80.6 \\
70.2\end{array}$ & $\begin{array}{r}118,457 \\
24,455 \\
13,925 \\
102,468 \\
430,836\end{array}$ & $\begin{array}{l}70.1 \\
30.2 \\
70.4 \\
86.2 \\
72.5\end{array}$ & $\begin{array}{r}131,817 \\
22,258 \\
15,868 \\
102,902 \\
485,326\end{array}$ & $\begin{array}{l}72.6 \\
29.4 \\
75.0 \\
86.2 \\
73.6\end{array}$ & $\begin{array}{r}132,073 \\
25,523 \\
18,035 \\
115,461 \\
537,503\end{array}$ & $\begin{array}{l}72.7 \\
41.6 \\
69.7 \\
86.2 \\
81.8\end{array}$ \\
\hline $\begin{array}{l}\text { Colorado ............. } \\
\text { Connecticut .......... } \\
\text { Delaware .............. } \\
\text { Florida ................... } \\
\text { Georgia................ }\end{array}$ & $\begin{array}{r}33,799 \\
3,572 \\
4,847 \\
34,996 \\
101,295\end{array}$ & $\begin{array}{l}69.0 \\
14.0 \\
28.4 \\
41.2 \\
62.5\end{array}$ & $\begin{array}{r}36,685 \\
10,370 \\
5,509 \\
49,468 \\
105,563\end{array}$ & $\begin{array}{l}65.3 \\
31.8 \\
34.1 \\
59.3 \\
63.2\end{array}$ & $\begin{array}{r}39,040 \\
12,514 \\
6,316 \\
56,893 \\
111,987\end{array}$ & $\begin{array}{l}67.8 \\
34.4 \\
35.0 \\
67.1 \\
65.0\end{array}$ & $\begin{array}{r}44,465 \\
10,164 \\
4,922 \\
73,610 \\
112,491\end{array}$ & $\begin{array}{r}64.4 \\
27.6 \\
25.3 \\
\mathbf{R} 73.4 \\
67.2\end{array}$ & $\begin{array}{r}51,292 \\
1,435 \\
5,631 \\
106,166 \\
108,859\end{array}$ & $\begin{array}{r}72.1 \\
4.7 \\
32.7 \\
83.7 \\
62.6\end{array}$ \\
\hline $\begin{array}{l}\text { Idaho .................... } \\
\text { lllinois .................... } \\
\text { Indiana.................. } \\
\text { lowa ..................... } \\
\text { Kansas ................. }\end{array}$ & $\begin{array}{r}23,075 \\
221,564 \\
179,733 \\
58,222 \\
95,606\end{array}$ & $\begin{array}{l}98.9 \\
80.4 \\
78.8 \\
64.5 \\
81.8\end{array}$ & $\begin{array}{r}26,565 \\
256,186 \\
178,941 \\
70,066 \\
104,828\end{array}$ & $\begin{array}{l}99.6 \\
84.6 \\
78.6 \\
71.9 \\
84.9\end{array}$ & $\begin{array}{r}26,963 \\
255,365 \\
188,562 \\
83,004 \\
117,419\end{array}$ & $\begin{array}{l}99.7 \\
85.0 \\
76.8 \\
82.4 \\
89.8\end{array}$ & $\begin{array}{r}29,057 \\
265,110 \\
210,303 \\
87,613 \\
120,962\end{array}$ & $\begin{array}{l}99.7 \\
86.9 \\
79.9 \\
85.4 \\
87.0\end{array}$ & $\begin{array}{r}28,932 \\
267,348 \\
233,870 \\
96,016 \\
175,691\end{array}$ & $\begin{array}{l}97.2 \\
87.6 \\
86.6 \\
88.3 \\
93.5\end{array}$ \\
\hline $\begin{array}{l}\text { Kentucky.............. } \\
\text { Louisiana ............. } \\
\text { Maine.................. } \\
\text { Maryland............. } \\
\text { Massachusetts .... }\end{array}$ & $\begin{array}{r}50,673 \\
483,858 \\
70 \\
39,245 \\
4,309\end{array}$ & $\begin{array}{r}74.1 \\
53.2 \\
3.5 \\
63.5 \\
9.7\end{array}$ & $\begin{array}{r}52,422 \\
505,590 \\
91 \\
39,686 \\
15,856\end{array}$ & $\begin{array}{r}73.2 \\
54.7 \\
4.1 \\
84.2 \\
29.0\end{array}$ & $\begin{array}{r}47,776 \\
582,295 \\
0 \\
39,740 \\
38,685\end{array}$ & $\begin{array}{r}64.9 \\
62.4 \\
-- \\
79.9 \\
54.6\end{array}$ & $\begin{array}{r}53,163 \\
670,825 \\
0 \\
38,989 \\
65,739\end{array}$ & $\begin{array}{r}69.9 \\
68.9 \\
-- \\
79.8 \\
69.4\end{array}$ & $\begin{array}{r}57,149 \\
759,747 \\
0 \\
39,697 \\
67,072\end{array}$ & $\begin{array}{r}68.8 \\
76.0 \\
-- \\
83.2 \\
72.3\end{array}$ \\
\hline $\begin{array}{l}\text { Michigan .............. } \\
\text { Minnesota........... } \\
\text { Mississippi ........... } \\
\text { Missouri ................ } \\
\text { Montana .............. }\end{array}$ & $\begin{array}{r}247,139 \\
51,265 \\
51,694 \\
36,711 \\
3,306\end{array}$ & $\begin{array}{l}88.1 \\
58.0 \\
51.3 \\
67.3 \\
35.1\end{array}$ & $\begin{array}{r}242,652 \\
53,727 \\
54,398 \\
42,745 \\
4,493\end{array}$ & $\begin{array}{l}89.2 \\
58.2 \\
52.0 \\
74.7 \\
45.5\end{array}$ & $\begin{array}{r}277,506 \\
53,665 \\
55,973 \\
44,131 \\
10,078\end{array}$ & $\begin{array}{l}90.9 \\
57.7 \\
54.5 \\
75.5 \\
82.5\end{array}$ & $\begin{array}{r}283,775 \\
55,355 \\
60,506 \\
46,608 \\
11,407\end{array}$ & $\begin{array}{l}90.9 \\
56.6 \\
60.4 \\
76.4 \\
89.9\end{array}$ & $\begin{array}{r}292,945 \\
53,928 \\
59,032 \\
56,873 \\
13,395\end{array}$ & $\begin{array}{l}89.4 \\
57.1 \\
60.9 \\
79.4 \\
96.1\end{array}$ \\
\hline $\begin{array}{l}\text { Nebraska .............. } \\
\text { Nevada ................ } \\
\text { New Hampshire... } \\
\text { New Jersey........... } \\
\text { New Mexico........ }\end{array}$ & $\begin{array}{r}14,304 \\
6,457 \\
52 \\
36,790 \\
16,346\end{array}$ & $\begin{array}{r}55.6 \\
86.0 \\
1.6 \\
40.7 \\
84.7\end{array}$ & $\begin{array}{r}13,266 \\
5,687 \\
202 \\
44,419 \\
17,305\end{array}$ & $\begin{array}{r}53.6 \\
85.9 \\
5.9 \\
44.1 \\
89.5\end{array}$ & $\begin{array}{r}15,558 \\
8,569 \\
0 \\
77,276 \\
16,339\end{array}$ & $\begin{array}{r}58.8 \\
92.6 \\
-- \\
44.3 \\
95.7\end{array}$ & $\begin{array}{r}28,068 \\
23,660 \\
0 \\
83,216 \\
16,107\end{array}$ & $\begin{array}{r}72.6 \\
95.7 \\
-- \\
44.1 \\
94.6\end{array}$ & $\begin{array}{r}28,971 \\
28,312 \\
225 \\
81,222 \\
16,884\end{array}$ & $\begin{array}{r}78.4 \\
98.1 \\
5.0 \\
42.6 \\
90.1\end{array}$ \\
\hline $\begin{array}{l}\text { New York............. } \\
\text { North Carolina ..... } \\
\text { North Dakota ....... } \\
\text { Ohio...................... } \\
\text { Oklahoma ............ }\end{array}$ & $\begin{array}{r}60,359 \\
24,962 \\
3,296 \\
239,551 \\
78,309\end{array}$ & $\begin{array}{l}59.6 \\
29.0 \\
75.6 \\
86.0 \\
40.2\end{array}$ & $\begin{array}{r}83,934 \\
23,348 \\
3,901 \\
246,062 \\
104,773\end{array}$ & $\begin{array}{l}70.6 \\
27.5 \\
76.1 \\
88.0 \\
58.0\end{array}$ & $\begin{array}{r}113,074 \\
17,302 \\
4,656 \\
260,609 \\
133,643\end{array}$ & $\begin{array}{l}76.7 \\
19.0 \\
78.4 \\
88.4 \\
76.3\end{array}$ & $\begin{array}{r}125,916 \\
20,068 \\
4,570 \\
270,667 \\
124,925\end{array}$ & $\begin{array}{l}78.5 \\
21.7 \\
75.0 \\
89.8 \\
69.6\end{array}$ & $\begin{array}{r}180,002 \\
38,907 \\
4,402 \\
281,055 \\
148,340\end{array}$ & $\begin{array}{l}83.9 \\
41.0 \\
75.3 \\
90.3 \\
75.7\end{array}$ \\
\hline $\begin{array}{l}\text { Oregon ................ } \\
\text { Pennsylvania ....... } \\
\text { Rhode Island ....... } \\
\text { South Carolina..... } \\
\text { South Dakota....... }\end{array}$ & $\begin{array}{r}38,128 \\
179,492 \\
583 \\
20,817 \\
3,124\end{array}$ & $\begin{array}{l}78.0 \\
76.3 \\
13.3 \\
24.0 \\
53.9\end{array}$ & $\begin{array}{r}44,521 \\
183,097 \\
23,032 \\
25,368 \\
2,090\end{array}$ & $\begin{array}{l}80.9 \\
79.1 \\
85.7 \\
29.6 \\
44.9\end{array}$ & $\begin{array}{r}46,434 \\
182,522 \\
42,457 \\
31,176 \\
2,129\end{array}$ & $\begin{array}{l}79.3 \\
77.1 \\
88.6 \\
33.1 \\
47.4\end{array}$ & $\begin{array}{r}43,558 \\
183,131 \\
41,243 \\
33,507 \\
2,428\end{array}$ & $\begin{array}{l}71.9 \\
75.8 \\
89.6 \\
35.1 \\
48.8\end{array}$ & $\begin{array}{r}42,891 \\
187,980 \\
37,257 \\
22,755 \\
3,449\end{array}$ & $\begin{array}{l}68.6 \\
79.5 \\
91.0 \\
23.3 \\
62.6\end{array}$ \\
\hline $\begin{array}{l}\text { Tennessee........... } \\
\text { Texas................... } \\
\text { Utah................... } \\
\text { Virginia ................ } \\
\text { Washington ......... }\end{array}$ & $\begin{array}{r}43,605 \\
1,231,679 \\
28,108 \\
53,144 \\
36,929\end{array}$ & $\begin{array}{l}39.7 \\
72.0 \\
79.2 \\
71.2 \\
47.1\end{array}$ & $\begin{array}{r}51,462 \\
1,227,979 \\
36,534 \\
42,709 \\
44,526\end{array}$ & $\begin{array}{l}44.4 \\
69.3 \\
84.7 \\
71.2 \\
55.8\end{array}$ & $\begin{array}{r}63,666 \\
1,190,677 \\
36,087 \\
51,223 \\
49,911\end{array}$ & $\begin{array}{l}50.4 \\
68.7 \\
88.3 \\
74.4 \\
62.6\end{array}$ & $\begin{array}{r}66,780 \\
R_{1,258,773} \\
39,387 \\
54,791 \\
54,177\end{array}$ & $\begin{array}{r}53.7 \\
\mathbf{R}_{66.9} \\
93.1 \\
75.1 \\
58.7\end{array}$ & $\begin{array}{r}64,615 \\
1,321,308 \\
32,238 \\
67,341 \\
62,877\end{array}$ & $\begin{array}{l}54.3 \\
72.2 \\
88.0 \\
78.5 \\
58.4\end{array}$ \\
\hline $\begin{array}{l}\text { West Virginia ........ } \\
\text { Wisconsin ........... } \\
\text { Wyorning ............. }\end{array}$ & $\begin{array}{l}36,632 \\
67,869 \\
23,569\end{array}$ & $\begin{array}{l}76.3 \\
55.7 \\
91.6\end{array}$ & $\begin{array}{l}32,142 \\
73,777 \\
31,093\end{array}$ & $\begin{array}{l}78.6 \\
57.3 \\
93.7\end{array}$ & $\begin{array}{l}37,034 \\
76,161 \\
54,053\end{array}$ & $\begin{array}{l}93.8 \\
58.5 \\
96.4\end{array}$ & $\begin{array}{l}40,863 \\
72,890 \\
52,935\end{array}$ & $\begin{array}{r}R_{86.6} \\
54.4 \\
97.6\end{array}$ & $\begin{array}{l}40,684 \\
69,191 \\
59,219\end{array}$ & $\begin{array}{l}87.0 \\
51.2 \\
97.8\end{array}$ \\
\hline 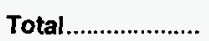 & $4,544,535$ & 64.8 & $4,863,923$ & 67.3 & $5,248,609$ & 69.7 & $\mathbf{R}_{5,644,894}$ & $\mathrm{R}_{70.7}$ & $6,123,797$ & 74.9 \\
\hline
\end{tabular}

a These deliveries included quantities covered by long-term contracts and gas involved in short-term or spot market sales.

H Revised data.

Note: Totals may not equal sum of components due to independent rounding.

Source: Energy Information Administration (EIA), Form EIA-176, "Annual Report of Natural and Supplemental Gas Supply and Disposition." 
Table 18. Natural Gas Delivered to Electric Utilities for the Account of Others ${ }^{\mathrm{a}}$ by State, 1990-1994 (Million Cubic Feet)

\begin{tabular}{|c|c|c|c|c|c|c|c|c|c|c|}
\hline \multirow[b]{2}{*}{ State } & \multicolumn{2}{|c|}{1990} & \multicolumn{2}{|c|}{1991} & \multicolumn{2}{|c|}{1992} & \multicolumn{2}{|c|}{1993} & \multicolumn{2}{|c|}{1994} \\
\hline & $\begin{array}{l}\text { Delivered } \\
\text { for the } \\
\text { Account } \\
\text { of Others }\end{array}$ & $\begin{array}{l}\text { Percent of } \\
\text { Total } \\
\text { Electric } \\
\text { Utility } \\
\text { Deliveries }\end{array}$ & $\begin{array}{l}\text { Delivered } \\
\text { for the } \\
\text { Account } \\
\text { of Others }\end{array}$ & $\begin{array}{c}\text { Percent of } \\
\text { Total } \\
\text { Electric } \\
\text { Utility } \\
\text { Deliveries }\end{array}$ & $\begin{array}{l}\text { Delivered } \\
\text { for the } \\
\text { Account } \\
\text { of Others }\end{array}$ & $\begin{array}{l}\text { Percent of } \\
\text { Total } \\
\text { Electric } \\
\text { Utility } \\
\text { Deliveries }\end{array}$ & $\begin{array}{l}\text { Delivered } \\
\text { for the } \\
\text { Account } \\
\text { of Others }\end{array}$ & $\begin{array}{c}\text { Percent of } \\
\text { Total } \\
\text { Electric } \\
\text { Utility } \\
\text { Deliveries }\end{array}$ & $\begin{array}{l}\text { Delivered } \\
\text { for the } \\
\text { Account } \\
\text { of Others }\end{array}$ & $\begin{array}{c}\text { Percent of } \\
\text { Total } \\
\text { Electric } \\
\text { Utility } \\
\text { Deliveries }\end{array}$ \\
\hline $\begin{array}{l}\text { Alabama ................ } \\
\text { Alaska................. } \\
\text { Arizona ................ } \\
\text { Arkansas ............. } \\
\text { California ............. }\end{array}$ & $\begin{array}{r}2,287 \\
6,495 \\
14,222 \\
29,049 \\
114,142\end{array}$ & $\begin{array}{r}63.7 \\
28.2 \\
63.4 \\
100.0 \\
25.3\end{array}$ & $\begin{array}{r}3,045 \\
0 \\
13,831 \\
25,180 \\
225,773\end{array}$ & $\begin{array}{r}77.5 \\
-. \\
80.4 \\
96.6 \\
49.8\end{array}$ & $\begin{array}{r}2,739 \\
8,925 \\
40,553 \\
25,647 \\
406,235\end{array}$ & $\begin{array}{l}75.2 \\
31.1 \\
99.9 \\
96.7 \\
69.7\end{array}$ & $\begin{array}{r}2,753 \\
7,693 \\
29,866 \\
16,528 \\
342,457\end{array}$ & $\begin{array}{l}61.0 \\
28.9 \\
99.9 \\
93.6 \\
74.1\end{array}$ & $\begin{array}{r}3,271 \\
8,368 \\
33,188 \\
18,625 \\
556,557\end{array}$ & $\begin{array}{l}89.6 \\
29.6 \\
99.9 \\
95.9 \\
93.3\end{array}$ \\
\hline 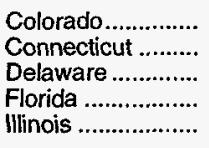 & $\begin{array}{r}4,904 \\
4,796 \\
35 \\
68,783 \\
6,682\end{array}$ & $\begin{array}{r}73.5 \\
100.0 \\
0.3 \\
36.0 \\
87.1\end{array}$ & $\begin{array}{r}6,097 \\
4,518 \\
39 \\
168,412 \\
9,150\end{array}$ & $\begin{array}{r}77.4 \\
100.0 \\
0.3 \\
84.8 \\
89.9\end{array}$ & $\begin{array}{r}3,931 \\
1,881 \\
48 \\
186,524 \\
9,726\end{array}$ & $\begin{array}{r}68.8 \\
100.0 \\
0.5 \\
93.2 \\
91.7\end{array}$ & $\begin{array}{r}3,165 \\
140 \\
58 \\
177,954 \\
14,227\end{array}$ & $\begin{array}{r}66.4 \\
36.5 \\
0.7 \\
97.9 \\
91.4\end{array}$ & $\begin{array}{r}2,685 \\
6,941 \\
16,120 \\
182,379 \\
32,064\end{array}$ & $\begin{array}{l}63.6 \\
88.4 \\
92.3 \\
98.9 \\
95.4\end{array}$ \\
\hline $\begin{array}{l}\text { Indiana.................. } \\
\text { lowa...................... } \\
\text { Kansas ................ } \\
\text { Kentucky............... } \\
\text { Louisiana .............. }\end{array}$ & $\begin{array}{r}5,011 \\
1,137 \\
17,288 \\
0 \\
231,753\end{array}$ & $\begin{array}{r}76.4 \\
38.1 \\
71.1 \\
. .- \\
85.5\end{array}$ & $\begin{array}{r}7,997 \\
1,256 \\
23,059 \\
32 \\
2019,258\end{array}$ & $\begin{array}{l}81.5 \\
33.3 \\
70.7 \\
14.1 \\
82.2\end{array}$ & $\begin{array}{r}6,129 \\
2,163 \\
7,161 \\
0 \\
208,847\end{array}$ & $\begin{array}{r}81.0 \\
65.6 \\
54.6 \\
-- \\
86.8\end{array}$ & $\begin{array}{r}4,204 \\
1,901 \\
8,091 \\
0 \\
182,535\end{array}$ & $\begin{array}{r}85.0 \\
50.6 \\
57.8 \\
-- \\
82.8\end{array}$ & $\begin{array}{r}7,370 \\
1,721 \\
14,617 \\
0 \\
174,324\end{array}$ & $\begin{array}{r}78.3 \\
69.6 \\
71.5 \\
-\overline{83.8}\end{array}$ \\
\hline $\begin{array}{l}\text { Maryland............. } \\
\text { Massachusetts .... } \\
\text { Michigan.............. } \\
\text { Minnesota............ } \\
\text { Mississippi ........... }\end{array}$ & $\begin{array}{r}8,483 \\
18,328 \\
23,015 \\
1,970 \\
62,412\end{array}$ & $\begin{array}{l}48.2 \\
33.0 \\
98.6 \\
57.4 \\
94.9\end{array}$ & $\begin{array}{r}6,430 \\
16,832 \\
20,960 \\
1,657 \\
57,297\end{array}$ & $\begin{array}{l}39.8 \\
44.4 \\
98.1 \\
28.0 \\
94.5\end{array}$ & $\begin{array}{r}3,691 \\
22,672 \\
19,162 \\
1,443 \\
41,304\end{array}$ & $\begin{array}{l}35.4 \\
65.0 \\
98.1 \\
28.1 \\
79.6\end{array}$ & $\begin{array}{r}3,034 \\
9,725 \\
16,324 \\
1,071 \\
26,270\end{array}$ & $\begin{array}{l}43.3 \\
43.0 \\
97.6 \\
46.4 \\
63.4\end{array}$ & $\begin{array}{r}4,186 \\
29,992 \\
17,820 \\
906 \\
31,927\end{array}$ & $\begin{array}{l}32.5 \\
72.5 \\
97.8 \\
22.7 \\
94.9\end{array}$ \\
\hline $\begin{array}{l}\text { Missouri ................ } \\
\text { Montana ............. } \\
\text { Nebraska .............. } \\
\text { Nevada ................ } \\
\text { New Jersey.......... }\end{array}$ & $\begin{array}{r}2,015 \\
118 \\
2,793 \\
23,193 \\
1,432\end{array}$ & $\begin{array}{r}44.4 \\
28.7 \\
79.0 \\
94.7 \\
2.9\end{array}$ & $\begin{array}{r}2,869 \\
178 \\
2,612 \\
21,403 \\
2,056\end{array}$ & $\begin{array}{r}23.2 \\
68.7 \\
77.6 \\
98.5 \\
3.2\end{array}$ & $\begin{array}{r}1,418 \\
99 \\
1,038 \\
28,675 \\
623\end{array}$ & $\begin{array}{r}52.3 \\
44.5 \\
57.9 \\
99.2 \\
1.6\end{array}$ & $\begin{array}{r}2,607 \\
155 \\
484 \\
21,066 \\
3,919\end{array}$ & $\begin{array}{l}51.1 \\
57.4 \\
37.7 \\
99.9 \\
10.0\end{array}$ & $\begin{array}{r}1,246 \\
139 \\
34 \\
32,377 \\
1,637\end{array}$ & $\begin{array}{r}39.5 \\
21.0 \\
5.5 \\
100.0 \\
. \quad 4.6\end{array}$ \\
\hline $\begin{array}{l}\text { New Mexico......... } \\
\text { New York............. } \\
\text { North Carolina ..... } \\
\text { Ohio ...................... } \\
\text { Oklahoma ... ........ }\end{array}$ & $\begin{array}{r}23,532 \\
21,280 \\
2,149 \\
656 \\
160,721\end{array}$ & $\begin{array}{r}95.5 \\
9.6 \\
82.9 \\
57.7 \\
90.2\end{array}$ & $\begin{array}{r}26,421 \\
23,485 \\
2,106 \\
2,695 \\
131,393\end{array}$ & $\begin{array}{l}95.7 \\
11.1 \\
67.5 \\
86.5 \\
81.8\end{array}$ & $\begin{array}{r}17,325 \\
19,893 \\
461 \\
2,147 \\
137,019\end{array}$ & $\begin{array}{r}95.9 \\
9.9 \\
15.4 \\
84.2 \\
86.9\end{array}$ & $\begin{array}{r}20,122 \\
15,455 \\
1,692 \\
2,060 \\
145,216\end{array}$ & $\begin{array}{r}95.9 \\
9.2 \\
61.7 \\
75.7 \\
89.7\end{array}$ & $\begin{array}{r}24,868 \\
14,082 \\
432 \\
1,626 \\
139,435\end{array}$ & $\begin{array}{r}93.6 \\
8.3 \\
50.8 \\
60.5 \\
88.2\end{array}$ \\
\hline $\begin{array}{l}\text { Oregon ............... } \\
\text { Pennsylvania ....... } \\
\text { Rhode Island ....... } \\
\text { South Dakota....... } \\
\text { Tennessee........... }\end{array}$ & $\begin{array}{r}7,458 \\
1,291 \\
1,033 \\
139 \\
563\end{array}$ & $\begin{array}{r}100.0 \\
42.1 \\
99.4 \\
97.3 \\
95.2\end{array}$ & $\begin{array}{r}10,798 \\
1,801 \\
1,771 \\
151 \\
143\end{array}$ & $\begin{array}{r}100.0 \\
73.6 \\
99.9 \\
86.9 \\
64.1\end{array}$ & $\begin{array}{r}12,818 \\
3,252 \\
466 \\
714 \\
219\end{array}$ & $\begin{array}{r}100.0 \\
82.2 \\
100.0 \\
92.6 \\
74.1\end{array}$ & $\begin{array}{r}16,041 \\
3,264 \\
0 \\
142 \\
1,015\end{array}$ & $\begin{array}{r}100.0 \\
36.3 \\
-- \\
91.9 \\
62.5\end{array}$ & $\begin{array}{r}26,310 \\
8,948 \\
0 \\
64 \\
498\end{array}$ & $\begin{array}{r}100.0 \\
63.2 \\
-- \\
100.0 \\
51.3\end{array}$ \\
\hline $\begin{array}{l}\text { Texas................... } \\
\text { Utah..................... } \\
\text { Virginia ................ } \\
\text { Washington ......... } \\
\text { West Virginia ........ } \\
\text { Wisconsin ............ }\end{array}$ & $\begin{array}{r}515,120 \\
445 \\
4,945 \\
48 \\
0 \\
618\end{array}$ & $\begin{array}{r}52.4 \\
87.9 \\
81.4 \\
35.5 \\
-. \\
45.6\end{array}$ & $\begin{array}{r}534,110 \\
4,562 \\
9,441 \\
88 \\
159 \\
1,013\end{array}$ & $\begin{array}{r}56.2 \\
99.6 \\
99.8 \\
63.5 \\
100.0 \\
44.0\end{array}$ & $\begin{array}{r}449,615 \\
5,434 \\
11,669 \\
3,597 \\
204 \\
1,899\end{array}$ & $\begin{array}{r}51.8 \\
99.5 \\
100.0 \\
79.7 \\
100.0 \\
70.4\end{array}$ & $\begin{array}{r}544,255 \\
5,004 \\
25,823 \\
1,195 \\
279 \\
648\end{array}$ & $\begin{array}{r}\mathbf{R}_{62.7} \\
99.9 \\
99.9 \\
100.0 \\
100.0 \\
18.1\end{array}$ & $\begin{array}{r}659,710 \\
7,922 \\
26,706 \\
2,328 \\
244 \\
457\end{array}$ & $\begin{array}{r}66.5 \\
99.6 \\
99.7 \\
100.0 \\
100.0 \\
8.6\end{array}$ \\
\hline 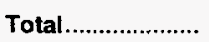 & $1,390,340$ & 50.7 & $1,580,077$ & 58.5 & $1,697,363$ & 63.7 & $1,658,438$ & $\mathbf{R}_{67.8}$ & $2,092,124$ & 75.0 \\
\hline
\end{tabular}

a These deliveries included quantities covered by long-term contracts and gas involved in short-term or spot market sales.

Note: Totals may not equal sum of components due to independent rounding.

Source: Energy Information Administration (EIA), Form EIA-176, "Annual Report of Natural and Supplemental Gas Supply and Disposition. 
Table 19. Firm Natural Gas Deliveries to Consumers by State, 1994 (Million Cubic Feet)

\begin{tabular}{|c|c|c|c|c|c|c|}
\hline State & Residential & Commercial & Industrial & Vehicle Fuel & Electric Utilities & Total \\
\hline 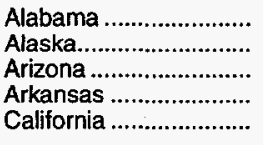 & $\begin{array}{r}49,748 \\
14,895 \\
29,684 \\
41,527 \\
520,959\end{array}$ & $\begin{array}{r}21,008 \\
20,698 \\
28,829 \\
23,654 \\
222,004\end{array}$ & $\begin{array}{r}71,344 \\
57,618 \\
24,898 \\
115,036 \\
399,736\end{array}$ & $\begin{array}{r}3 \\
0 \\
61 \\
0 \\
529\end{array}$ & $\begin{array}{r}83 \\
27,541 \\
32,636 \\
13,517 \\
288,054\end{array}$ & $\begin{array}{r}142,187 \\
120,751 \\
116,109 \\
193,734 \\
1,431,282\end{array}$ \\
\hline 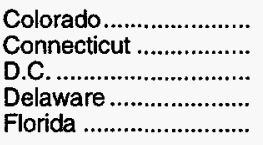 & $\begin{array}{r}99,504 \\
41,265 \\
15,865 \\
8,557 \\
13,855\end{array}$ & $\begin{array}{r}60,755 \\
27,680 \\
6,474 \\
5,459 \\
39,127\end{array}$ & $\begin{array}{r}22,625 \\
14,289 \\
0 \\
10,336 \\
47,194\end{array}$ & $\begin{array}{r}60 \\
1 \\
0 \\
1 \\
67\end{array}$ & $\begin{array}{r}1,532 \\
6,807 \\
0 \\
16,187 \\
169,240\end{array}$ & $\begin{array}{r}184,476 \\
90,041 \\
22,338 \\
40,541 \\
269,482\end{array}$ \\
\hline 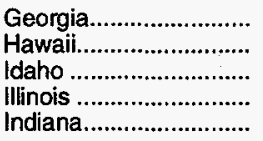 & $\begin{array}{r}105,436 \\
578 \\
12,285 \\
473,788 \\
157,467\end{array}$ & $\begin{array}{r}42,537 \\
1,837 \\
10,088 \\
197,467 \\
70,323\end{array}$ & $\begin{array}{r}46,311 \\
0 \\
26,511 \\
283,176 \\
61,982\end{array}$ & $\begin{array}{r}0 \\
0 \\
10 \\
28 \\
59\end{array}$ & $\begin{array}{r}1,137 \\
0 \\
0 \\
33,596 \\
2,353\end{array}$ & $\begin{array}{r}195,421 \\
2,415 \\
48,894 \\
988,056 \\
292,185\end{array}$ \\
\hline 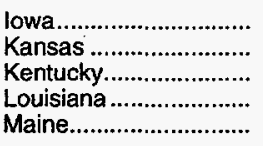 & $\begin{array}{r}78,260 \\
74,156 \\
62,451 \\
52,689 \\
894\end{array}$ & $\begin{array}{r}45,048 \\
43,171 \\
34,466 \\
18,058 \\
2,381\end{array}$ & $\begin{array}{r}92,964 \\
97,904 \\
44,181 \\
592,738 \\
1,166\end{array}$ & $\begin{array}{r}5 \\
10 \\
2 \\
22 \\
0\end{array}$ & $\begin{array}{r}1,826 \\
11,213 \\
267 \\
87,013 \\
0\end{array}$ & $\begin{array}{r}218,103 \\
226,454 \\
141,366 \\
750,520 \\
4,441\end{array}$ \\
\hline $\begin{array}{l}\text { Maryland.................... } \\
\text { Massachusetts ............ } \\
\text { Michigan ........................ } \\
\text { Minnesota ..................... } \\
\text { Mississippi .................... }\end{array}$ & $\begin{array}{r}76,688 \\
119,642 \\
364,588 \\
122,249 \\
27,086\end{array}$ & $\begin{array}{r}36,183 \\
60,879 \\
182,234 \\
62,668 \\
17,748\end{array}$ & $\begin{array}{r}11,836 \\
67,512 \\
294,701 \\
57,965 \\
59,080\end{array}$ & $\begin{array}{r}25 \\
1 \\
14 \\
29 \\
0\end{array}$ & $\begin{array}{r}2,977 \\
21,361 \\
18,019 \\
1,701 \\
1,701\end{array}$ & $\begin{array}{l}127,709 \\
269,395 \\
859,555 \\
244,611 \\
105,615\end{array}$ \\
\hline $\begin{array}{l}\text { Missouri..................... } \\
\text { Montana .................... } \\
\text { Nebraska ...................... } \\
\text { Nevada ...................... } \\
\text { New Hampshire........... }\end{array}$ & $\begin{array}{r}122,566 \\
18,714 \\
44,397 \\
21,263 \\
6,572\end{array}$ & $\begin{array}{r}63,080 \\
12,678 \\
33,550 \\
18,407 \\
6,412\end{array}$ & $\begin{array}{r}57,967 \\
5,580 \\
29,892 \\
28,772 \\
3,634\end{array}$ & $\begin{array}{r}1 \\
6 \\
9 \\
20 \\
*\end{array}$ & $\begin{array}{r}1,243 \\
523 \\
55 \\
31,074 \\
0\end{array}$ & $\begin{array}{r}244,856 \\
37,501 \\
107,903 \\
99,536 \\
16,619\end{array}$ \\
\hline $\begin{array}{l}\text { New Jersey.................. } \\
\text { New Mexico.................. } \\
\text { New York.................... } \\
\text { North Carolina ............. } \\
\text { North Dakota ................ }\end{array}$ & $\begin{array}{r}216,873 \\
30,868 \\
382,713 \\
47,451 \\
10,661\end{array}$ & $\begin{array}{r}103,523 \\
24,849 \\
201,478 \\
36,567 \\
7,937\end{array}$ & $\begin{array}{r}74,939 \\
17,976 \\
135,471 \\
18,816 \\
506\end{array}$ & $\begin{array}{r}4 \\
62 \\
28 \\
8 \\
8\end{array}$ & $\begin{array}{r}29,126 \\
15,213 \\
34,310 \\
149 \\
2\end{array}$ & $\begin{array}{r}424,465 \\
88,968 \\
754,000 \\
102,989 \\
19,114\end{array}$ \\
\hline 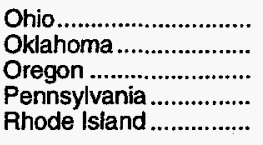 & $\begin{array}{r}343,321 \\
69,211 \\
28,848 \\
268,405 \\
17,384\end{array}$ & $\begin{array}{r}163,205 \\
32,220 \\
22,529 \\
124,236 \\
10,524\end{array}$ & $\begin{array}{r}268,372 \\
40,023 \\
18,733 \\
172,414 \\
39,723\end{array}$ & $\begin{array}{r}48 \\
2 \\
17 \\
7 \\
1\end{array}$ & $\begin{array}{r}985 \\
153,602 \\
26,310 \\
6,195 \\
0\end{array}$ & $\begin{array}{r}775,931 \\
295,057 \\
96,438 \\
571,256 \\
67,633\end{array}$ \\
\hline 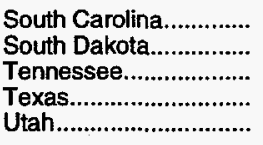 & $\begin{array}{r}23,486 \\
12,056 \\
57,057 \\
213,433 \\
48,922\end{array}$ & $\begin{array}{r}14,435 \\
9,491 \\
46,932 \\
167,798 \\
20,273\end{array}$ & $\begin{array}{r}17,424 \\
2,571 \\
44,500 \\
1,114,766 \\
1,999\end{array}$ & $\begin{array}{r}2 \\
5 \\
5 \\
6 \\
52\end{array}$ & $\begin{array}{r}60 \\
0 \\
0 \\
512,960 \\
6,200\end{array}$ & $\begin{array}{r}55,406 \\
24,124 \\
148,494 \\
2,008,963 \\
77,446\end{array}$ \\
\hline 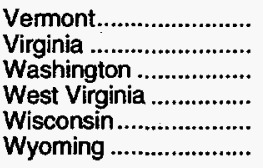 & $\begin{array}{r}2,438 \\
65,176 \\
53,144 \\
35,186 \\
128,175 \\
11,564\end{array}$ & $\begin{array}{r}2,669 \\
39,093 \\
34,631 \\
6,982 \\
70,222 \\
5,025\end{array}$ & $\begin{array}{r}116 \\
51,312 \\
48,545 \\
20,521 \\
28,563 \\
10,670\end{array}$ & $\begin{array}{r}0 \\
18 \\
60 \\
0 \\
10 \\
18\end{array}$ & $\begin{array}{r}0 \\
17,932 \\
2,307 \\
244 \\
3,416 \\
79\end{array}$ & $\begin{array}{r}5,222 \\
173,530 \\
138,687 \\
62,933 \\
230,386 \\
27,355\end{array}$ \\
\hline 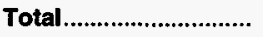 & $4,843,995$ & $2,559,521$ & $4,754,907$ & 1,325 & $1,580,745$ & $13,740,493$ \\
\hline
\end{tabular}

* = Volume is less than 500,000 cubic feet.

Note: Totals may not equal sum of components due to independent rounding.

Source: Energy information Administration (EIA), Form EIA-176, "Annual Report of Natural and Supplemental Gas Supply and Disposition." 
Table 20. Interruptible Natural Gas Deliveries to Consumers by State, 1994 (Million Cubic Feet)

\begin{tabular}{|c|c|c|c|c|c|c|}
\hline State & Residential & Commercial & Industrial & Vehicle Fuel & Electric Utilities & Total \\
\hline 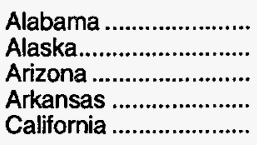 & $\begin{array}{l}0 \\
0 \\
0 \\
0 \\
0\end{array}$ & $\begin{array}{r}4,518 \\
0 \\
357 \\
3,756 \\
39,985\end{array}$ & $\begin{array}{r}110,374 \\
3,786 \\
971 \\
18,884 \\
257,015\end{array}$ & $\begin{array}{r}0 \\
0 \\
0 \\
0 \\
21\end{array}$ & $\begin{array}{r}3,569 \\
706 \\
594 \\
5,903 \\
308,720\end{array}$ & $\begin{array}{r}118,461 \\
4,492 \\
1,922 \\
28,543 \\
605,741\end{array}$ \\
\hline 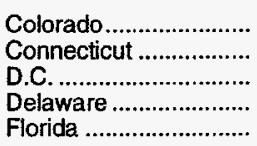 & $\begin{array}{r}0 \\
336 \\
0 \\
0 \\
0\end{array}$ & $\begin{array}{r}5,115 \\
11,402 \\
8,268 \\
0 \\
809\end{array}$ & $\begin{array}{r}48,468 \\
16,358 \\
0 \\
6,880 \\
79,679\end{array}$ & $\begin{array}{l}8 \\
1 \\
0 \\
0 \\
0\end{array}$ & $\begin{array}{r}2,687 \\
1,047 \\
0 \\
1,281 \\
15,105\end{array}$ & $\begin{array}{r}56,278 \\
29,144 \\
8,268 \\
8,161 \\
95,593\end{array}$ \\
\hline 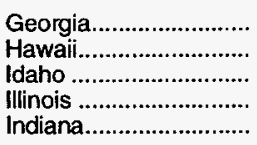 & $\begin{array}{l}0 \\
0 \\
0 \\
0 \\
0\end{array}$ & $\begin{array}{r}11,514 \\
363 \\
0 \\
108 \\
5,496\end{array}$ & $\begin{array}{r}127,590 \\
0 \\
3,270 \\
21,916 \\
208,146\end{array}$ & $\begin{array}{l}2 \\
0 \\
0 \\
0 \\
0\end{array}$ & $\begin{array}{r}0 \\
0 \\
0 \\
2 \\
7,058\end{array}$ & $\begin{array}{r}139,106 \\
363 \\
3,270 \\
22,027 \\
220,699\end{array}$ \\
\hline 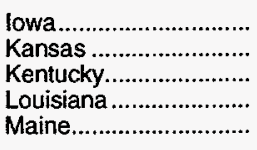 & $\begin{array}{r}0 \\
0 \\
82 \\
292 \\
0\end{array}$ & $\begin{array}{r}2,874 \\
9,082 \\
2,278 \\
6,126 \\
0\end{array}$ & $\begin{array}{r}15,766 \\
90,075 \\
38,900 \\
406,296 \\
604\end{array}$ & $\begin{array}{l}0 \\
0 \\
0 \\
0 \\
0\end{array}$ & $\begin{array}{r}648 \\
9,243 \\
3 \\
121,083 \\
0\end{array}$ & $\begin{array}{r}19,289 \\
108,399 \\
41,262 \\
533,797 \\
604\end{array}$ \\
\hline 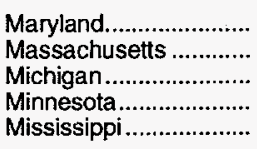 & $\begin{array}{l}0 \\
0 \\
0 \\
0 \\
0\end{array}$ & $\begin{array}{r}7,953 \\
23,656 \\
834 \\
21,265 \\
1,484\end{array}$ & $\begin{array}{l}35,856 \\
25,286 \\
33,148 \\
36,503 \\
37,783\end{array}$ & $\begin{array}{l}0 \\
1 \\
0 \\
0 \\
9\end{array}$ & $\begin{array}{r}9,910 \\
19,983 \\
199 \\
2,296 \\
31,943\end{array}$ & $\begin{array}{l}53,718 \\
68,926 \\
34,180 \\
60,063 \\
71,218\end{array}$ \\
\hline $\begin{array}{l}\text { Missouri ...................... } \\
\text { Montana ....................... } \\
\text { Nebraska .................... } \\
\text { Nevada ...................... } \\
\text { New Hampshire............ }\end{array}$ & $\begin{array}{l}0 \\
0 \\
0 \\
0 \\
0\end{array}$ & $\begin{array}{r}3,116 \\
303 \\
5,396 \\
286 \\
0\end{array}$ & $\begin{array}{r}13,635 \\
8,360 \\
7,069 \\
95 \\
837\end{array}$ & $\begin{array}{r}0 \\
0 \\
0 \\
16 \\
0\end{array}$ & $\begin{array}{r}1,911 \\
139 \\
563 \\
1,303 \\
1,271\end{array}$ & $\begin{array}{r}18,662 \\
8,803 \\
13,027 \\
1,700 \\
2,108\end{array}$ \\
\hline $\begin{array}{l}\text { New Jersey............... } \\
\text { New Mexico................. } \\
\text { New York.................... } \\
\text { North Carolina ............. } \\
\text { North Dakota ................ }\end{array}$ & $\begin{array}{r}0 \\
0 \\
2,695 \\
0 \\
0\end{array}$ & $\begin{array}{r}28,485 \\
115 \\
21,779 \\
2,374 \\
2,846\end{array}$ & $\begin{array}{r}115,906 \\
765 \\
78,967 \\
76,023 \\
5,340\end{array}$ & $\begin{array}{r}0 \\
0 \\
25 \\
0 \\
0\end{array}$ & $\begin{array}{r}6,286 \\
11,360 \\
135,488 \\
703 \\
0\end{array}$ & $\begin{array}{r}150,677 \\
12,240 \\
238,954 \\
79,099 \\
8,187\end{array}$ \\
\hline 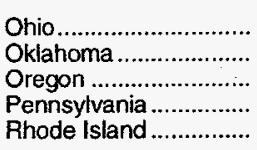 & $\begin{array}{r}11 \\
0 \\
0 \\
0 \\
0\end{array}$ & $\begin{array}{r}3,594 \\
4,284 \\
430 \\
14,237 \\
1,525\end{array}$ & $\begin{array}{r}42,751 \\
155,886 \\
43,835 \\
64,003 \\
1,197\end{array}$ & $\begin{array}{r}0 \\
154 \\
0 \\
3 \\
0\end{array}$ & $\begin{array}{r}1,703 \\
4,457 \\
0 \\
7,958 \\
0\end{array}$ & $\begin{array}{r}48,057 \\
164,782 \\
44,266 \\
86,202 \\
2,723\end{array}$ \\
\hline 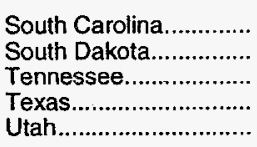 & $\begin{array}{r}0 \\
0 \\
277 \\
0 \\
0\end{array}$ & $\begin{array}{r}3,435 \\
784 \\
3,829 \\
12,433 \\
6,228\end{array}$ & $\begin{array}{r}80,076 \\
2,936 \\
74,389 \\
714,712 \\
34,619\end{array}$ & $\begin{array}{r}0 \\
0 \\
0 \\
40 \\
0\end{array}$ & $\begin{array}{r}2,961 \\
64 \\
971 \\
478,646 \\
1,756\end{array}$ & $\begin{array}{r}86,472 \\
3,784 \\
79,466 \\
1,205,831 \\
42,603\end{array}$ \\
\hline 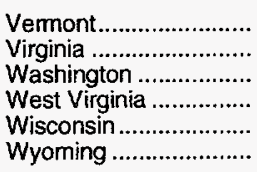 & $\begin{array}{r}0 \\
0 \\
0 \\
15 \\
0 \\
0\end{array}$ & $\begin{array}{r}0 \\
13,851 \\
8,352 \\
17,997 \\
8,386 \\
4,205\end{array}$ & $\begin{array}{r}1,908 \\
34,452 \\
59,059 \\
26,253 \\
106,544 \\
49,896\end{array}$ & $\begin{array}{r}0 \\
2 \\
95 \\
0 \\
26 \\
0\end{array}$ & $\begin{array}{r}165 \\
8,865 \\
20 \\
0 \\
1,885 \\
0\end{array}$ & $\begin{array}{r}2,072 \\
57,170 \\
67,525 \\
44,265 \\
116,841 \\
54,102\end{array}$ \\
\hline 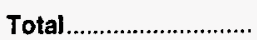 & 3,706 & 335,513 & $3,423,068$ & 405 & $1,210,453$ & $4,973,146$ \\
\hline
\end{tabular}

Note: Totals may not equal sum of components due to independent rounding.

Source: Energy Information Administration (EIA), Form EIA-176, "Annual Report of Natural and Supplemental Gas Supply and Disposition." 
Table 21. Natural Gas Deliveries to Commercial Consumers by State, 1994 (Million Cubic Feet)

\begin{tabular}{|c|c|c|c|c|c|c|c|}
\hline \multirow{2}{*}{ State } & \multicolumn{3}{|c|}{ Firm } & \multicolumn{3}{|c|}{ Interruptible } & \multirow{2}{*}{ Total Volume } \\
\hline & $\begin{array}{l}\text { Sales } \\
\text { Volume }\end{array}$ & $\begin{array}{l}\text { Transported } \\
\text { Volume }\end{array}$ & $\begin{array}{l}\text { Total } \\
\text { Volume }\end{array}$ & $\begin{array}{c}\text { Sales } \\
\text { Volume }\end{array}$ & $\begin{array}{l}\text { Transported } \\
\text { Volume }\end{array}$ & $\begin{array}{l}\text { Total } \\
\text { Volume }\end{array}$ & \\
\hline 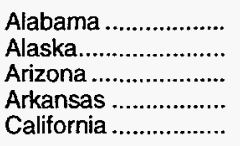 & $\begin{array}{r}19,944 \\
20,698 \\
26,205 \\
22,310 \\
119,882\end{array}$ & $\begin{array}{r}1,064 \\
0 \\
2,624 \\
1,344 \\
102,122\end{array}$ & $\begin{array}{r}21,008 \\
20,698 \\
28,829 \\
23,654 \\
222,004\end{array}$ & $\begin{array}{r}538 \\
0 \\
273 \\
3,749 \\
7,761\end{array}$ & $\begin{array}{r}3,979 \\
0 \\
84 \\
7 \\
32,224\end{array}$ & $\begin{array}{r}4,518 \\
0 \\
357 \\
3,756 \\
39,985\end{array}$ & $\begin{array}{r}25,526 \\
20,698 \\
29,187 \\
27,410 \\
261,989\end{array}$ \\
\hline $\begin{array}{l}\text { Colorado................. } \\
\text { Connecticut .............. } \\
\text { D.C........................... } \\
\text { Delaware .................. } \\
\text { Florida ..................... }\end{array}$ & $\begin{array}{r}59,660 \\
23,486 \\
6,474 \\
5,459 \\
38,267\end{array}$ & $\begin{array}{r}1,095 \\
4,194 \\
0 \\
0 \\
860\end{array}$ & $\begin{array}{r}60,755 \\
27,680 \\
6,474 \\
5,459 \\
39,127\end{array}$ & $\begin{array}{r}2,807 \\
8,141 \\
6,926 \\
0 \\
807\end{array}$ & $\begin{array}{r}2,308 \\
3,261 \\
1,343 \\
0 \\
1\end{array}$ & $\begin{array}{r}5,115 \\
11,402 \\
8,268 \\
0 \\
809\end{array}$ & $\begin{array}{r}65,870 \\
39,082 \\
14,742 \\
5,459 \\
39,935\end{array}$ \\
\hline 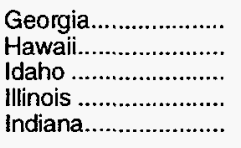 & $\begin{array}{r}42,537 \\
1,837 \\
8,662 \\
104,326 \\
66,621\end{array}$ & $\begin{array}{r}0 \\
0 \\
1,427 \\
93,141 \\
3,702\end{array}$ & $\begin{array}{r}42,537 \\
1,837 \\
10,088 \\
197,467 \\
70,323\end{array}$ & $\begin{array}{r}7,210 \\
363 \\
0 \\
43 \\
3,357\end{array}$ & $\begin{array}{r}4,304 \\
0 \\
0 \\
65 \\
2,139\end{array}$ & $\begin{array}{r}11,514 \\
363 \\
0 \\
108 \\
5,496\end{array}$ & $\begin{array}{r}54,051 \\
2,200 \\
10,088 \\
197,576 \\
75,819\end{array}$ \\
\hline 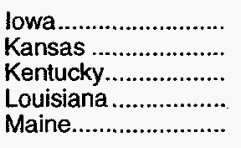 & $\begin{array}{r}40,529 \\
39,657 \\
31,904 \\
17,556 \\
2,381\end{array}$ & $\begin{array}{r}4,519 \\
3,514 \\
2,562 \\
501 \\
0\end{array}$ & $\begin{array}{c}45,048 \\
43,171 \\
34,466 \\
18,058 \\
2,381\end{array}$ & $\begin{array}{r}2,796 \\
1,301 \\
1,669 \\
6,123 \\
0\end{array}$ & $\begin{array}{r}78 \\
7,781 \\
609 \\
3 \\
0\end{array}$ & $\begin{array}{r}2,874 \\
9,082 \\
2,278 \\
6,126 \\
0\end{array}$ & $\begin{array}{r}47,922 \\
52,253 \\
36,744 \\
24,184 \\
2,381\end{array}$ \\
\hline $\begin{array}{l}\text { Maryland.................. } \\
\text { Massachusetts ........ } \\
\text { Michigan.................. } \\
\text { Minnesota ................. } \\
\text { Mississippi ................ }\end{array}$ & $\begin{array}{r}35,228 \\
58,313 \\
120,166 \\
60,907 \\
17,242\end{array}$ & $\begin{array}{r}955 \\
2,566 \\
62,069 \\
1,761 \\
506\end{array}$ & $\begin{array}{r}36,183 \\
60,879 \\
182,234 \\
62,668 \\
17,748\end{array}$ & $\begin{array}{r}7,617 \\
6,089 \\
77 \\
19,689 \\
1,342\end{array}$ & $\begin{array}{r}335 \\
17,566 \\
756 \\
1,575 \\
141\end{array}$ & $\begin{array}{r}7,953 \\
23,656 \\
834 \\
21,265 \\
1,484\end{array}$ & $\begin{array}{r}44,136 \\
84,534 \\
183,068 \\
83,933 \\
19,232\end{array}$ \\
\hline $\begin{array}{l}\text { Missouri................... } \\
\text { Montana ................... } \\
\text { Nebraska ................ } \\
\text { Nevada .................... } \\
\text { New Hampshire....... }\end{array}$ & $\begin{array}{r}53,748 \\
11,900 \\
25,824 \\
15,417 \\
6,412\end{array}$ & $\begin{array}{r}9,332 \\
778 \\
7,726 \\
2,990 \\
0\end{array}$ & $\begin{array}{r}63,080 \\
12,678 \\
33,550 \\
18,407 \\
6,412\end{array}$ & $\begin{array}{r}1,375 \\
11 \\
5,396 \\
0 \\
0\end{array}$ & $\begin{array}{r}1,741 \\
292 \\
0 \\
286 \\
0\end{array}$ & $\begin{array}{r}3,116 \\
303 \\
5,396 \\
286 \\
0\end{array}$ & $\begin{array}{r}66,196 \\
12,981 \\
38,946 \\
18,694 \\
6,412\end{array}$ \\
\hline $\begin{array}{l}\text { New Jersey.............. } \\
\text { New Mexico............. } \\
\text { New York............... } \\
\text { North Carolina .......... } \\
\text { North Dakota ............ }\end{array}$ & $\begin{array}{r}97,758 \\
15,453 \\
161,176 \\
35,376 \\
7,937\end{array}$ & $\begin{array}{r}5,765 \\
9,395 \\
40,302 \\
1,191 \\
0\end{array}$ & $\begin{array}{r}103,523 \\
24,849 \\
201,478 \\
36,567 \\
7,937\end{array}$ & $\begin{array}{r}23,205 \\
115 \\
16,454 \\
2,251 \\
647\end{array}$ & $\begin{array}{r}5,280 \\
0 \\
5,325 \\
123 \\
2,199\end{array}$ & $\begin{array}{r}28,485 \\
115 \\
21,779 \\
2,374 \\
2,846\end{array}$ & $\begin{array}{r}132,008 \\
24,964 \\
223,256 \\
38,940 \\
10,783\end{array}$ \\
\hline 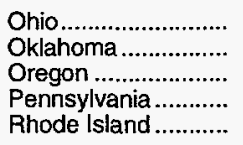 & $\begin{array}{r}134,654 \\
30,442 \\
22,529 \\
93,491 \\
10,524\end{array}$ & $\begin{array}{r}28,551 \\
1,777 \\
0 \\
30,745 \\
0\end{array}$ & $\begin{array}{r}163,205 \\
32,220 \\
22,529 \\
124,236 \\
10,524\end{array}$ & $\begin{array}{r}1,337 \\
1,847 \\
0 \\
9,468 \\
1,525\end{array}$ & $\begin{array}{r}2,256 \\
2,437 \\
430 \\
4,769 \\
0\end{array}$ & $\begin{array}{r}3,594 \\
4,284 \\
430 \\
14,237 \\
1,525\end{array}$ & $\begin{array}{r}166,798 \\
36,504 \\
22,960 \\
138,473 \\
12,049\end{array}$ \\
\hline $\begin{array}{l}\text { South Carolina.......... } \\
\text { South Dakota........... } \\
\text { Tennessee............... } \\
\text { Texas......................... } \\
\text { Utah.......................... }\end{array}$ & $\begin{array}{r}14,304 \\
8,705 \\
44,503 \\
143,302 \\
20,273\end{array}$ & $\begin{array}{r}131 \\
786 \\
2,428 \\
24,496 \\
0\end{array}$ & $\begin{array}{r}14,435 \\
9,491 \\
46,932 \\
167,798 \\
20,273\end{array}$ & $\begin{array}{r}3,301 \\
445 \\
3,265 \\
5,187 \\
1,789\end{array}$ & $\begin{array}{r}134 \\
338 \\
564 \\
7,246 \\
4,438\end{array}$ & $\begin{array}{r}3,435 \\
784 \\
3,829 \\
12,433 \\
6,228\end{array}$ & $\begin{array}{r}17,870 \\
10,274 \\
50,760 \\
180,232 \\
26,501\end{array}$ \\
\hline $\begin{array}{l}\text { Vermont ................. } \\
\text { Virginia .................... } \\
\text { Washington ............. } \\
\text { West Virginia ............ } \\
\text { Wisconsin ................. } \\
\text { Wyoming ................. }\end{array}$ & $\begin{array}{r}2,669 \\
36,397 \\
33,819 \\
6,078 \\
67,691 \\
4,875\end{array}$ & $\begin{array}{r}0 \\
2,696 \\
811 \\
904 \\
2,531 \\
150\end{array}$ & $\begin{array}{r}2,669 \\
39,093 \\
34,631 \\
6,982 \\
70,222 \\
5,025\end{array}$ & $\begin{array}{r}0 \\
9,507 \\
7,170 \\
7,707 \\
5,784 \\
3,998\end{array}$ & $\begin{array}{r}0 \\
4,343 \\
1,182 \\
10,290 \\
2,602 \\
208\end{array}$ & $\begin{array}{r}0 \\
13,851 \\
8,352 \\
17,997 \\
8,386 \\
4,205\end{array}$ & $\begin{array}{r}2,669 \\
52,944 \\
42,982 \\
24,979 \\
78,609 \\
9,231\end{array}$ \\
\hline Total ............................. & $2,095,510$ & 464,011 & $2,559,521$ & 200,466 & 135,046 & 335,513 & $2,895,034$ \\
\hline
\end{tabular}

Note: Totals may not equal sum of components due to independent rounding.

Source: Energy Information Administration (EIA), Form EIA-176, "Annual Report of Natural and Supplemental Gas Supply and Disposition. 
Table 22. Natural Gas Deliveries to Industrial Consumers by State, 1994 (Million Cubic Feet)

\begin{tabular}{|c|c|c|c|c|c|c|c|}
\hline \multirow{2}{*}{ State } & \multicolumn{3}{|c|}{ Firm } & \multicolumn{3}{|c|}{ Interruptible } & \multirow{2}{*}{ Total Volume } \\
\hline & $\begin{array}{c}\text { Sales } \\
\text { Volume }\end{array}$ & $\begin{array}{l}\text { Transported } \\
\text { Volume }\end{array}$ & $\begin{array}{l}\text { Total } \\
\text { Volume }\end{array}$ & $\begin{array}{c}\text { Sales } \\
\text { Volume }\end{array}$ & $\begin{array}{c}\text { Transported } \\
\text { Volume }\end{array}$ & $\begin{array}{l}\text { Total } \\
\text { Volume }\end{array}$ & \\
\hline $\begin{array}{l}\text { Alabama ................... } \\
\text { Alaska...................... } \\
\text { Arizona .................... } \\
\text { Arkansas .................... } \\
\text { California ................ }\end{array}$ & $\begin{array}{r}12,276 \\
35,882 \\
7,539 \\
7,852 \\
96,508\end{array}$ & $\begin{array}{r}59,067 \\
21,736 \\
17,360 \\
107,184 \\
303,228\end{array}$ & $\begin{array}{r}71,344 \\
57,618 \\
24,898 \\
115,036 \\
399,736\end{array}$ & $\begin{array}{r}37,368 \\
0 \\
296 \\
10,608 \\
22,740\end{array}$ & $\begin{array}{r}73,006 \\
3,786 \\
675 \\
8,277 \\
234,275\end{array}$ & $\begin{array}{r}110,374 \\
3,786 \\
971 \\
18,884 \\
257,015\end{array}$ & $\begin{array}{r}181,718 \\
61,404 \\
25,869 \\
133,921 \\
656,751\end{array}$ \\
\hline $\begin{array}{l}\text { Colorado................... } \\
\text { Connecticut ............. } \\
\text { Delaware ................. } \\
\text { Florida ....................... } \\
\text { Georgia.................... }\end{array}$ & $\begin{array}{r}6,654 \\
14,095 \\
4,705 \\
8,347 \\
13,725\end{array}$ & $\begin{array}{r}15,970 \\
194 \\
5,631 \\
38,847 \\
32,585\end{array}$ & $\begin{array}{l}22,625 \\
14,289 \\
10,336 \\
47,194 \\
46,311\end{array}$ & $\begin{array}{r}13,146 \\
15,118 \\
6,880 \\
12,360 \\
51,316\end{array}$ & $\begin{array}{r}35,322 \\
1,241 \\
0 \\
67,319 \\
76,274\end{array}$ & $\begin{array}{r}48,468 \\
16,358 \\
6,880 \\
79,679 \\
127,590\end{array}$ & $\begin{array}{r}71,093 \\
30,647 \\
17,216 \\
126,873 \\
173,901\end{array}$ \\
\hline 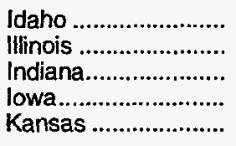 & $\begin{array}{r}467 \\
32,642 \\
28,181 \\
8,073 \\
3,393\end{array}$ & $\begin{array}{r}26,044 \\
250,534 \\
33,801 \\
84,891 \\
94,511\end{array}$ & $\begin{array}{r}26,511 \\
283,176 \\
61,982 \\
92,964 \\
97,904\end{array}$ & $\begin{array}{r}382 \\
5,102 \\
8,076 \\
4,641 \\
8,895\end{array}$ & $\begin{array}{r}2,888 \\
16,814 \\
200,070 \\
11,125 \\
81,180\end{array}$ & $\begin{array}{r}3,270 \\
21,916 \\
208,146 \\
15,766 \\
90,075\end{array}$ & $\begin{array}{r}29,781 \\
305,092 \\
270,128 \\
108,731 \\
187,979\end{array}$ \\
\hline $\begin{array}{l}\text { Kentucky ................. } \\
\text { Louisiana ................... } \\
\text { Maine...................... } \\
\text { Maryland................. } \\
\text { Massachusetts ......... }\end{array}$ & $\begin{array}{r}13,607 \\
168,431 \\
1,166 \\
362 \\
16,112\end{array}$ & $\begin{array}{r}30,574 \\
424,307 \\
0 \\
11,473 \\
51,400\end{array}$ & $\begin{array}{r}44,181 \\
592,738 \\
1,166 \\
11,836 \\
67,512\end{array}$ & $\begin{array}{r}12,325 \\
70,856 \\
604 \\
7,633 \\
9,615\end{array}$ & $\begin{array}{r}26,575 \\
335,440 \\
0 \\
28,223 \\
15,672\end{array}$ & $\begin{array}{r}38,900 \\
406,296 \\
604 \\
35,856 \\
25,286\end{array}$ & $\begin{array}{r}83,081 \\
999,034 \\
1,771 \\
47,691 \\
92,798\end{array}$ \\
\hline $\begin{array}{l}\text { Michigan ................... } \\
\text { Minnesota............... } \\
\text { Mississippi ............... } \\
\text { Missouri .................... } \\
\text { Montana .................. }\end{array}$ & $\begin{array}{r}33,961 \\
9,146 \\
15,067 \\
10,009 \\
545\end{array}$ & $\begin{array}{r}260,739 \\
48,1319 \\
44,013 \\
47,958 \\
5,036\end{array}$ & $\begin{array}{r}294,701 \\
57,965 \\
59,080 \\
57,967 \\
5,580\end{array}$ & $\begin{array}{r}942 \\
31,394 \\
22,763 \\
4,720 \\
1\end{array}$ & $\begin{array}{r}32,205 \\
5,109 \\
15,019 \\
8,915 \\
8,359\end{array}$ & $\begin{array}{r}33,148 \\
36,503 \\
37,783 \\
13,635 \\
8,360\end{array}$ & $\begin{array}{r}327,848 \\
94,468 \\
96,863 \\
71,602 \\
13,940\end{array}$ \\
\hline $\begin{array}{l}\text { Nebraska .................. } \\
\text { Nevada .................. } \\
\text { New Hampshire........ } \\
\text { New Jersey............... } \\
\text { New Mexico.............. }\end{array}$ & $\begin{array}{r}2,957 \\
554 \\
3,409 \\
28,883 \\
1,839\end{array}$ & $\begin{array}{r}26,935 \\
28,218 \\
2.25 \\
46,056 \\
16,137\end{array}$ & $\begin{array}{r}29,892 \\
28,772 \\
3,634 \\
74,939 \\
17,976\end{array}$ & $\begin{array}{r}5,032 \\
0 \\
837 \\
80,741 \\
18\end{array}$ & $\begin{array}{r}2,037 \\
95 \\
0 \\
35,165 \\
747\end{array}$ & $\begin{array}{r}7,069 \\
95 \\
837 \\
115,906 \\
765\end{array}$ & $\begin{array}{r}36,960 \\
28,867 \\
4,471 \\
190,845 \\
18,741\end{array}$ \\
\hline $\begin{array}{l}\text { New York................. } \\
\text { North Carolina .......... } \\
\text { North Dakota ............ } \\
\text { Ohio........................... } \\
\text { Oklahoma ................. }\end{array}$ & $\begin{array}{r}24,754 \\
12,219 \\
173 \\
27,551 \\
19,196\end{array}$ & $\begin{array}{r}110,717 \\
6,596 \\
333 \\
240,821 \\
20,826\end{array}$ & $\begin{array}{r}135,471 \\
18,816 \\
506 \\
268,372 \\
40,023\end{array}$ & $\begin{array}{r}9,683 \\
43,711 \\
1,271 \\
2,517 \\
28,373\end{array}$ & $\begin{array}{r}69,284 \\
32,311 \\
4,069 \\
40,234 \\
127,514\end{array}$ & $\begin{array}{r}78,967 \\
76,023 \\
5,340 \\
42,751 \\
155,886\end{array}$ & $\begin{array}{r}214,438 \\
94,838 \\
5,846 \\
311,123 \\
195,909\end{array}$ \\
\hline $\begin{array}{l}\text { Oregon ................... } \\
\text { Pennsylvania .......... } \\
\text { Rhode Island ........... } \\
\text { South Carolina.......... } \\
\text { South Dakota........... }\end{array}$ & $\begin{array}{r}11,403 \\
31,793 \\
2,470 \\
12,794 \\
1,323\end{array}$ & $\begin{array}{r}7,330 \\
140,621 \\
37,253 \\
4,629 \\
1,248\end{array}$ & $\begin{array}{r}18,733 \\
172,414 \\
39,723 \\
17,424 \\
2,571\end{array}$ & $\begin{array}{r}8,274 \\
16,645 \\
1,193 \\
61,951 \\
736\end{array}$ & $\begin{array}{r}35,561 \\
47,359 \\
4 \\
18,125 \\
2,201\end{array}$ & $\begin{array}{r}43,835 \\
64,003 \\
1,197 \\
80,076 \\
2,936\end{array}$ & $\begin{array}{r}62,569 \\
236,417 \\
40,921 \\
97,500 \\
5,508\end{array}$ \\
\hline 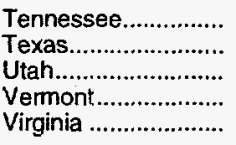 & $\begin{array}{r}27,719 \\
453,167 \\
1,999 \\
116 \\
7,641\end{array}$ & $\begin{array}{r}16,781 \\
661,600 \\
0 \\
0 \\
43,670\end{array}$ & $\begin{array}{r}44,500 \\
1,114,766 \\
1,999 \\
116 \\
51,312\end{array}$ & $\begin{array}{r}26,555 \\
55,004 \\
2,381 \\
1,908 \\
10,781\end{array}$ & $\begin{array}{r}47,834 \\
659,708 \\
32,238 \\
0 \\
23,671\end{array}$ & $\begin{array}{r}74,389 \\
714,712 \\
34,619 \\
1,908 \\
34,452\end{array}$ & $\begin{array}{r}118,889 \\
1,829,478 \\
36,618 \\
2,023 \\
85,764\end{array}$ \\
\hline $\begin{array}{l}\text { Washington ............. } \\
\text { West Virginia ............ } \\
\text { Wisconsin ................ } \\
\text { Wyoming ............... }\end{array}$ & $\begin{array}{r}35,492 \\
5,651 \\
24,215 \\
660\end{array}$ & $\begin{array}{r}13,052 \\
14,870 \\
4,347 \\
10,010\end{array}$ & $\begin{array}{l}48,545 \\
20,521 \\
28,563 \\
10,670\end{array}$ & $\begin{array}{r}9,234 \\
439 \\
41,700 \\
687\end{array}$ & $\begin{array}{l}49,825 \\
25,814 \\
64,843 \\
49,209\end{array}$ & $\begin{array}{r}59,059 \\
26,253 \\
106,544 \\
49,896\end{array}$ & $\begin{array}{r}107,603 \\
46,774 \\
135,106 \\
60,566\end{array}$ \\
\hline 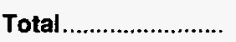 & $1,286,724$ & $3,468,1132$ & $4,754,907$ & 767,453 & $2,655,615$ & $3,423,068$ & $8,177,975$ \\
\hline
\end{tabular}

Note: Totals may not equal sum of components due to independent rounding.

Source: Energy Information Administration (EIA), Form EIA-176, "Annual Report of Natural and Supplemental Gas Supply and Disposition." 
Table 23. Natural Gas Deliveries to Electric Utilities by State, 1994 (Million Cubic Feet)

\begin{tabular}{|c|c|c|c|c|c|c|c|}
\hline \multirow[b]{2}{*}{ State } & \multicolumn{3}{|c|}{ Firm } & \multicolumn{3}{|c|}{ Interruptible } & \multirow[b]{2}{*}{ Total Volume } \\
\hline & $\begin{array}{c}\text { Sales } \\
\text { Volume }\end{array}$ & $\begin{array}{l}\text { Transported } \\
\text { Volume }\end{array}$ & $\begin{array}{c}\text { Total } \\
\text { Volume }\end{array}$ & $\begin{array}{c}\text { Sales } \\
\text { Volume }\end{array}$ & $\begin{array}{c}\text { Transported } \\
\text { Volume }\end{array}$ & $\begin{array}{l}\text { Total } \\
\text { Volume }\end{array}$ & \\
\hline 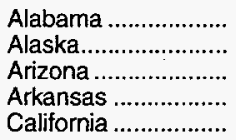 & $\begin{array}{r}0 \\
19,172 \\
42 \\
794 \\
46\end{array}$ & $\begin{array}{r}83 \\
8,368 \\
32,594 \\
12,723 \\
288,008\end{array}$ & $\begin{array}{r}83 \\
27,541 \\
32,636 \\
13,517 \\
288,054\end{array}$ & $\begin{array}{r}382 \\
706 \\
0 \\
0 \\
40,171\end{array}$ & $\begin{array}{r}3,187 \\
0 \\
594 \\
5,903 \\
268,549\end{array}$ & $\begin{array}{r}3,569 \\
706 \\
594 \\
5,903 \\
308,720\end{array}$ & $\begin{array}{r}3,652 \\
28,246 \\
33,230 \\
19,419 \\
596,774\end{array}$ \\
\hline 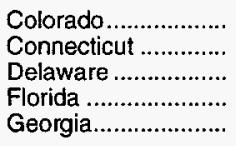 & $\begin{array}{r}1,532 \\
0 \\
68 \\
1,888 \\
1,137\end{array}$ & $\begin{array}{r}0 \\
6,807 \\
16,120 \\
167,352 \\
0\end{array}$ & $\begin{array}{r}1,532 \\
6,807 \\
16,187 \\
169,240 \\
1,137\end{array}$ & $\begin{array}{r}2 \\
913 \\
1,281 \\
78 \\
0\end{array}$ & $\begin{array}{r}2,685 \\
134 \\
0 \\
15,028 \\
0\end{array}$ & $\begin{array}{r}2,687 \\
1,047 \\
1,281 \\
15,105 \\
0\end{array}$ & $\begin{array}{r}4,219 \\
7,854 \\
17,468 \\
184,345 \\
1,137\end{array}$ \\
\hline 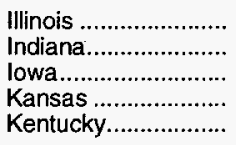 & $\begin{array}{r}1,532 \\
2,041 \\
644 \\
1,169 \\
267\end{array}$ & $\begin{array}{r}32,064 \\
312 \\
1,181 \\
10,044 \\
0\end{array}$ & $\begin{array}{r}33,596 \\
2,353 \\
1,826 \\
11,213 \\
267\end{array}$ & $\begin{array}{r}2 \\
0 \\
108 \\
4,670 \\
3\end{array}$ & $\begin{array}{r}0 \\
7,058 \\
540 \\
4,573 \\
0\end{array}$ & $\begin{array}{r}2 \\
7,058 \\
648 \\
9,243 \\
3\end{array}$ & $\begin{array}{r}33,599 \\
9,411 \\
2,474 \\
20,456 \\
269\end{array}$ \\
\hline $\begin{array}{l}\text { Louisiana ................. } \\
\text { Maryland................. } \\
\text { Massachusetts ........ } \\
\text { Michigan .................. } \\
\text { Minnesota............... }\end{array}$ & $\begin{array}{r}18,503 \\
0 \\
9,706 \\
387 \\
1,701\end{array}$ & $\begin{array}{r}68,510 \\
2,977 \\
11,655 \\
17,632 \\
0\end{array}$ & $\begin{array}{r}87,013 \\
2,977 \\
21,361 \\
18,019 \\
1,701\end{array}$ & $\begin{array}{r}15,269 \\
8,701 \\
1,646 \\
11 \\
1,390\end{array}$ & $\begin{array}{r}105,814 \\
1,209 \\
18,336 \\
188 \\
906\end{array}$ & $\begin{array}{r}121,083 \\
9,910 \\
19,983 \\
199 \\
2,296\end{array}$ & $\begin{array}{r}208,096 \\
12,887 \\
41,343 \\
18,218 \\
3,996\end{array}$ \\
\hline $\begin{array}{l}\text { Mississippi ................ } \\
\text { Missouri.................. } \\
\text { Montana ................. } \\
\text { Nebraska ................. } \\
\text { Nevada ................... }\end{array}$ & $\begin{array}{r}1,367 \\
29 \\
523 \\
55 \\
0\end{array}$ & $\begin{array}{r}334 \\
1,214 \\
0 \\
0 \\
31,074\end{array}$ & $\begin{array}{r}1,701 \\
1,243 \\
523 \\
55 \\
31,074\end{array}$ & $\begin{array}{r}349 \\
1,879 \\
0 \\
528 \\
0\end{array}$ & $\begin{array}{r}31,594 \\
33 \\
139 \\
34 \\
1,303\end{array}$ & $\begin{array}{r}31,943 \\
1,911 \\
139 \\
563 \\
1,303\end{array}$ & $\begin{array}{r}33,643 \\
3,154 \\
663 \\
618 \\
32,377\end{array}$ \\
\hline $\begin{array}{l}\text { New Hampshire....... } \\
\text { New Jersey............. } \\
\text { New Mexico............ } \\
\text { New York................ } \\
\text { North Carolina ......... }\end{array}$ & $\begin{array}{r}0 \\
27,496 \\
1,566 \\
24,904 \\
104\end{array}$ & $\begin{array}{r}0 \\
1,629 \\
13,648 \\
9,406 \\
44\end{array}$ & $\begin{array}{r}0 \\
29,126 \\
15,213 \\
34,310 \\
149\end{array}$ & $\begin{array}{r}1,271 \\
6,278 \\
140 \\
130,812 \\
315\end{array}$ & $\begin{array}{r}0 \\
8 \\
11,220 \\
4,675 \\
388\end{array}$ & $\begin{array}{r}1,271 \\
6,286 \\
11,360 \\
135,488 \\
703\end{array}$ & $\begin{array}{r}1,271 \\
35,412 \\
26,573 \\
169,798 \\
852\end{array}$ \\
\hline 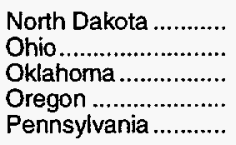 & $\begin{array}{r}2 \\
896 \\
17,323 \\
0 \\
4,439\end{array}$ & $\begin{array}{r}0 \\
89 \\
136,279 \\
26,310 \\
1,755\end{array}$ & $\begin{array}{r}2 \\
985 \\
153,602 \\
26,310 \\
6,195\end{array}$ & $\begin{array}{r}0 \\
166 \\
1,302 \\
0 \\
766\end{array}$ & $\begin{array}{r}0 \\
1,536 \\
3,155 \\
0 \\
7,192\end{array}$ & $\begin{array}{r}0 \\
1,703 \\
4,457 \\
0 \\
7,958\end{array}$ & $\begin{array}{r}2 \\
2,688 \\
158,059 \\
26,310 \\
14,153\end{array}$ \\
\hline $\begin{array}{l}\text { South Carolina......... } \\
\text { South Dakota........... } \\
\text { Tennessee............... } \\
\text { Texas........................ } \\
\text { Utah........................ }\end{array}$ & $\begin{array}{r}60 \\
0 \\
0 \\
311,650 \\
0\end{array}$ & $\begin{array}{r}0 \\
0 \\
0 \\
201,310 \\
6,200\end{array}$ & $\begin{array}{r}60 \\
0 \\
0 \\
512,960 \\
6,200\end{array}$ & $\begin{array}{r}2,961 \\
0 \\
473 \\
20,246 \\
34\end{array}$ & $\begin{array}{r}0 \\
64 \\
498 \\
458,400 \\
1,723\end{array}$ & $\begin{array}{r}2,961 \\
64 \\
971 \\
478,646 \\
1,756\end{array}$ & $\begin{array}{r}3,021 \\
64 \\
971 \\
991,606 \\
7,956\end{array}$ \\
\hline $\begin{array}{l}\text { Vermont.................. } \\
\text { Virginia ................... } \\
\text { Washington ............. } \\
\text { West Virginia .......... } \\
\text { Wisconsin ................. } \\
\text { Wyoming ................. }\end{array}$ & $\begin{array}{r}0 \\
0 \\
0 \\
0 \\
3,416 \\
79\end{array}$ & $\begin{array}{r}0 \\
17,932 \\
2,307 \\
244 \\
0 \\
0\end{array}$ & $\begin{array}{r}0 \\
17,932 \\
2,307 \\
244 \\
3,416 \\
79\end{array}$ & $\begin{array}{r}165 \\
91 \\
0 \\
0 \\
1,428 \\
0\end{array}$ & $\begin{array}{r}0 \\
8,774 \\
20 \\
0 \\
457 \\
0\end{array}$ & $\begin{array}{r}165 \\
8,865 \\
20 \\
0 \\
1,885 \\
0\end{array}$ & $\begin{array}{r}165 \\
26,797 \\
2,328 \\
244 \\
5,301 \\
79\end{array}$ \\
\hline Total.......................... & 454,539 & $1,126,206$ & $1,580,745$ & 244,536 & 965,918 & $1,210,453$ & $2,791,198$ \\
\hline
\end{tabular}

Notes: Deliveries to electric utilities are reported on Form EIA-759, "Annual Report of Natural and Supplemental Gas Supply and Disposition." See the discussion on electric utility data and Table A1 in Appendix A for a comparison of reporting to these two forms. Totals may not equal sum of the components due to independent rounding.

Source: Energy Information Administration (EIA), Form EIA-176, "Annual Report of Natural and Supplemental Gas Supply and Disposition." 



\section{Consumer Prices}

The upward trend in natural gas prices (as discussed for wellhead prices in the Supply section) was also reflected in consumer markets. Residential and commercial prices rose approximately 4 percent between 1993 and 1994, while the price to onsystem industrial customers fell slightly. The price of natural gas to electric utilities was reduced by 13 percent, from $\$ 2.61$ to $\$ 2.28$ per thousand cubic feet.

Prices for deliveries of natural gas to consumers, except electric utilities, are calculated from reports to Form EIA176, "Annual Report of Natural and Supplemental Gas Supply and Disposition." City gate prices are calculated from reports to the Form EIA-857, "Monthly Report of Natural Gas Purchases and Deliveries to Consumers." However these forms survey companies who deliver gas, neither consumers of natural gas nor marketing companies that sell but do not deliver gas are respondents.
Prices for natural gas are also reported to the EIA on the Form FERC-423, "Monthly Report of Cost and Quality of Fuels for Electric Plants," a consumer survey form. The respondents are all large regulated electric utilities that report consumption and prices of fuels and represent most of the volumes delivered to electric utilities. These prices are also published in the EIA report, Cost and Quality of Fuels for Electric Utility Plants.

With the unbundling of services in the natural gas industry under FERC Order 636, pipelines now provide transportation service for their end-user customers. In this report, those volumes are described as deliveries of gas for the account of others. Local distribution companies (LDC's) also provide transportation-only services. When companies that deliver gas are able to report costs, the volumes associated with these costs are described as onsystem sales.

Figure 12. Average Price of Natural Gas Delivered to Consumers in the United States, 1993 - 1994

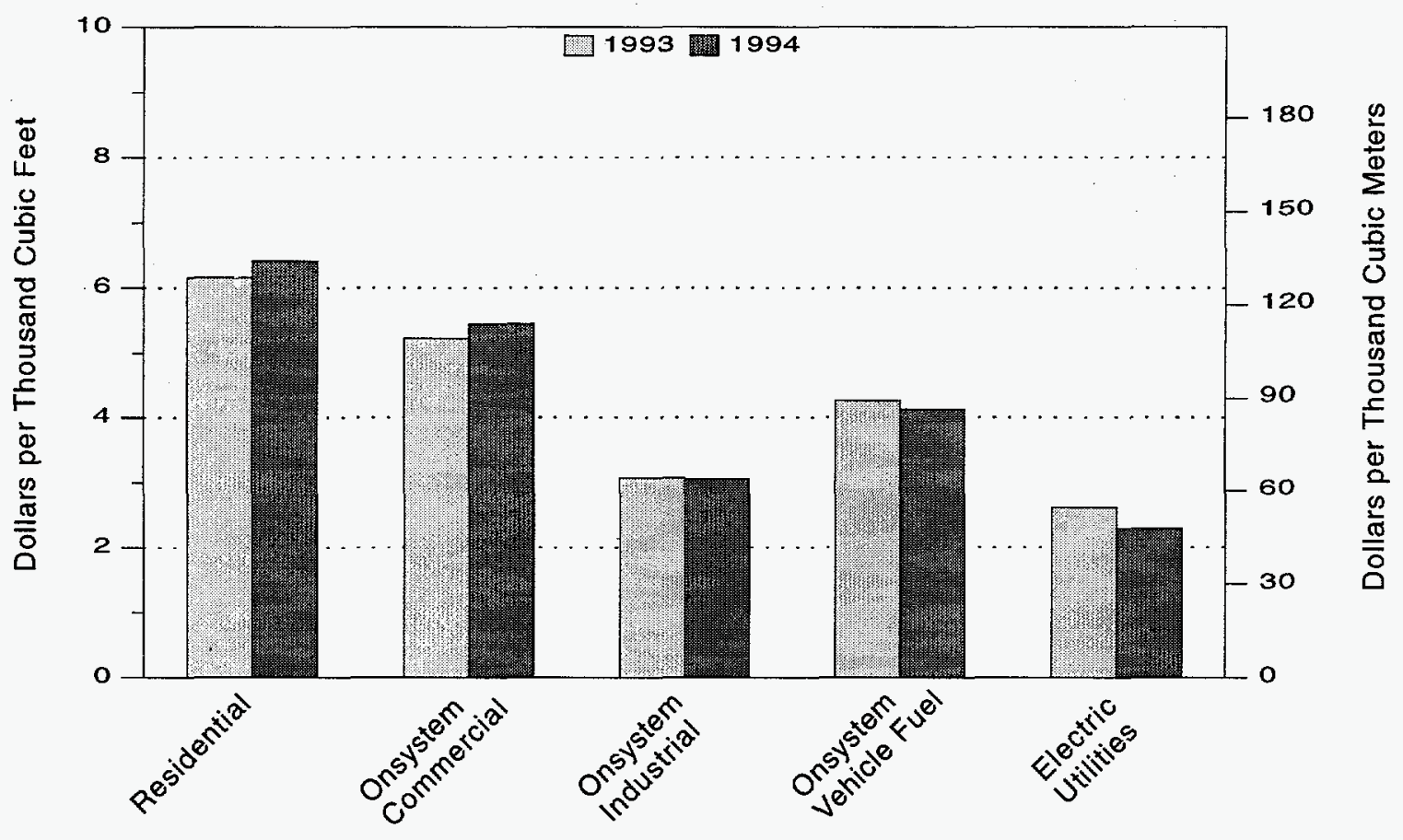

Note: Onsystem sales deliveries represent 79.2 percent of commercial deliveries, 25.3 percent of industrial deliveris, and 84.2 percent of vehicle fuel deliveries. Sources: Energy Information Administration (EIA), Form EIA-176, "Annual Report of Natural and Supplemental Gas Supply and Disposition" and Federal Energy Regulatory Commission (FERC), Form FERC-423, "Monthly Report of Cost and Quality of Fuels for Electric Plants." 
If transportation-only services are provided, companies do not know the sales price of the gas and do not report a revenue amount on the Form EIA-176. Thus, prices in this publication, especially those for the industrial and commercial sectors, are often associated with relatively small volumes of the total gas delivered because they are reported by those that deliver gas and not by consumers. Prices to electric utilities, because they are derived from a survey of the utilities themselves, represent most of the volumes. The coverage that prices represent varies by consumer sector as discussed in the following sections and as shown in the tables that follow. All average prices are computed by dividing the reported revenue by its associated sales volume.

\section{Residential}

In the residential sector, most of the natural gas is delivered and sold by LDC's. Because their individual use is so low, residential consumers are usually not in a position to enter contracts on an individual basis. Therefore, the residential prices in this report represent virtually all of the volumes delivered to this end-user sector.

\section{Commercial}

The percentage of deliveries for the account of others to the commercial sector has grown dramatically in 1994 , increasing from 16 to 21 percent of commercial volumes. Still, the price reflects approximately 80 percent of commercial volumes. For most States, prices for commercial deliveries are associated with a large percentage of the volume of deliveries in the State.

\section{Industrial}

Since 1987, deliveries to the industrial sector for the account of others have represented more than half of all deliveries to that sector. As the industry has evolved and large customers have taken advantage of purchasing their gas needs from a variety of sources, the percentage of these deliveries has continued to rise, reaching 75 percent in 1994 . The prices in this report for industrial deliveries apply to a relatively small proportion of deliveries in most States.

\section{Vehicle Fuel}

Much of the natural gas delivered for vehicle fuel represents deliveries to fueling stations that are used primarily orexclusively by respondents' fleet vehicles. Thus, the prices are often those associated with the operation of fleet vehicles.

\section{Electric Utilities}

Prices for natural gas consumed by electric utilities are reported on the Form FERC-423 and shown in Table 26. These prices are associated with consumption by all large electric utilities.

Facilities that generate electricity but are not regulated are known as nonutility power producers. A nonutility power producer may be a commercial facility, an industrial facility, or a facility that produces electricity for sale. Deliveries of natural gas to nonutility power producers are reported on the Form EIA-176. The volumes and associated revenues for deliveries to nonutility producers who are primarily commercial establishments are included in the calculation of commercial prices. Those for deliveries to both nonutility producers who are primarily industrial establishments and establishments that produce electricity for sale are included in the calculation of industrial prices.

\section{City Gate}

Reflecting the general decrease in prices at the wellhead, prices paid at the city gate declined by 4 percent in 1994 from $\$ 3.21$ to $\$ 3.07$ per thousand cubic feet. City gate prices represent the total cost paid by gas distribution companies for gas received at a point where the gas is transferred from a pipeline company or transmission system. The price is intended to reflect all charges for the acquisition, storage, and transportation as well as other charges associated with the LDC's obtaining the gas for sale to consumers. City gate revenues and volumes are reported monthly on Form EIA857 by companies that deliver gas to consumers. The monthly revenues reported for 1994 were summed and divided by the sum of the monthly volumes reported to calculate annual prices. 
Figure 13. Average City Gate Price of Natural Gas in the United States, 1994 (Dollars per Thousand Cubic Feet)

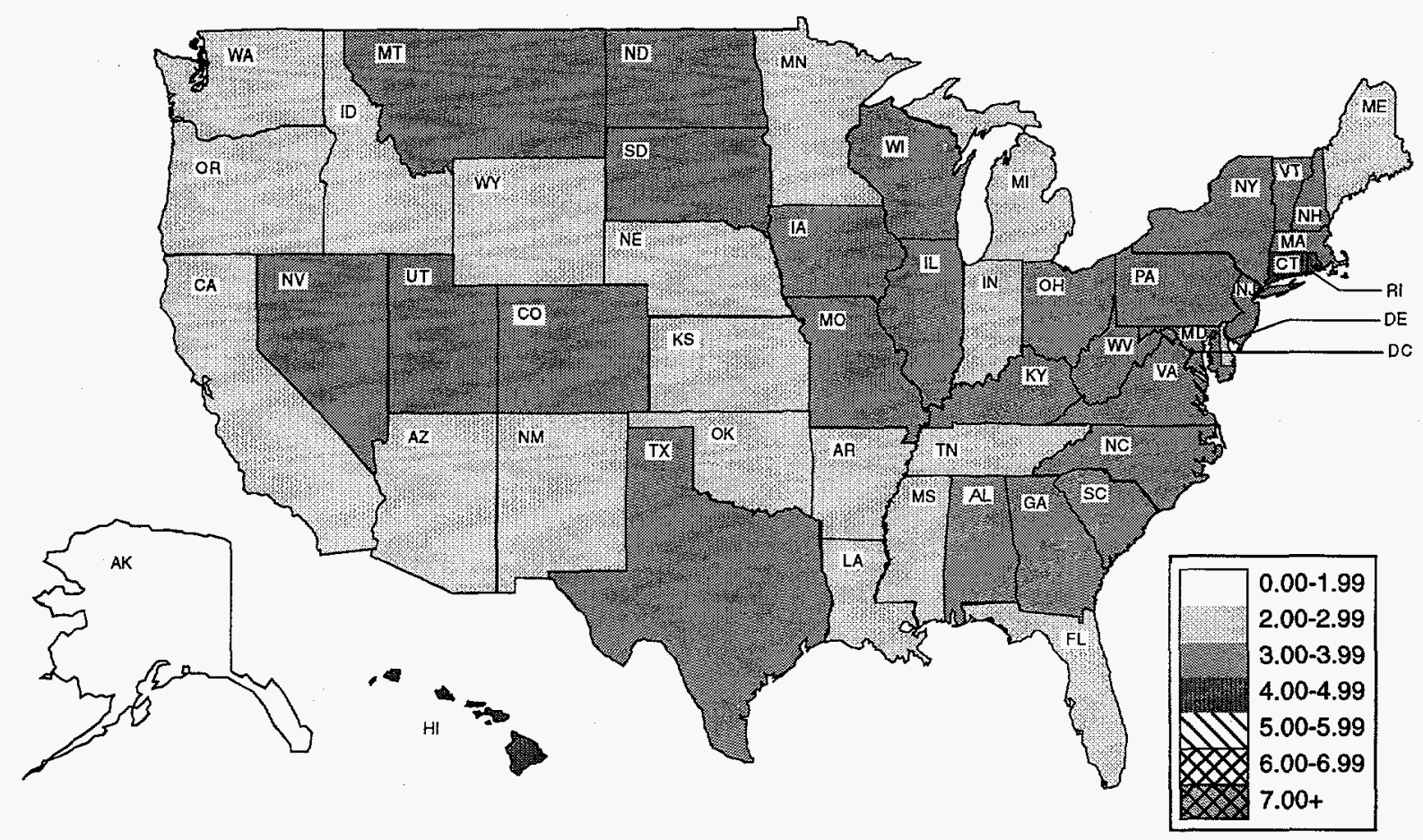

Source: Energy Information Administration (EIA), Form EIA-857, "Monthly Report of Natural Gas Purchases and Deliveries to Consumers."

Figure 14. Selected Average Prices of Natural Gas in the United States, 1990 - 1994

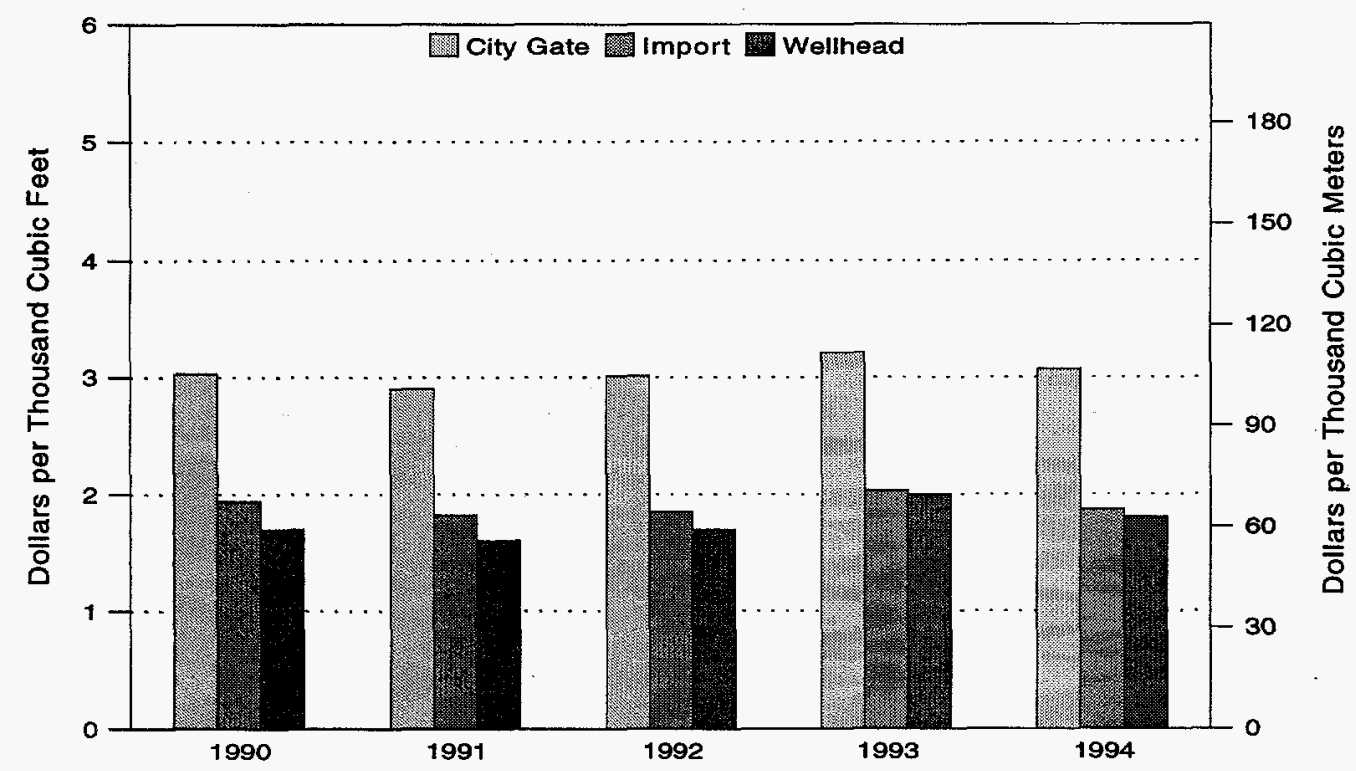

Sources: Energy Information Administration (ElA), Form ElA-857, "Monthly Report of Natural Gas Purchases and Deliveries to Consumers"; Form FPC-14, "Annual Report for Importers and Exporters of Natural Gas" and Form EIA-627, "Annual Quantity and Value of Natural Gas Report." 
Table 24. Average City Gate Price of Natural Gas in the United States, 1990-1994 (Dollars per Thousand Cubic Feet)

\begin{tabular}{|c|c|c|c|c|c|}
\hline \multirow{2}{*}{ State } & \multicolumn{5}{|c|}{ Average Price } \\
\hline & 1990 & 1991 & 1992 & 1993 & 1994 \\
\hline 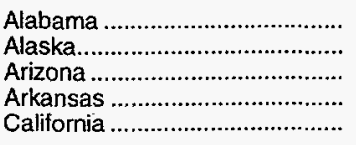 & $\begin{array}{l}3.13 \\
0.34 \\
2.73 \\
2.41 \\
2.90\end{array}$ & $\begin{array}{l}3.11 \\
0.32 \\
2.45 \\
2.45 \\
2.80\end{array}$ & $\begin{array}{l}3.21 \\
0.34 \\
2.33 \\
2.60 \\
2.72\end{array}$ & $\begin{array}{l}3.51 \\
0.33 \\
2.62 \\
2.66 \\
2.85\end{array}$ & $\begin{array}{l}3.44 \\
1.62 \\
2.53 \\
2.54 \\
2.57\end{array}$ \\
\hline 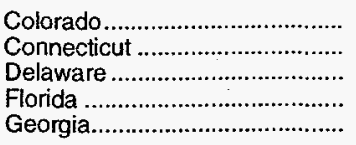 & $\begin{array}{l}2.94 \\
3.66 \\
2.76 \\
2.71 \\
3.43\end{array}$ & $\begin{array}{l}2.85 \\
3.50 \\
2.54 \\
2.51 \\
3.38\end{array}$ & $\begin{array}{l}2.85 \\
3.73 \\
2.83 \\
2.61 \\
3.28\end{array}$ & $\begin{array}{l}2.95 \\
3.87 \\
3.24 \\
2.76 \\
3.77\end{array}$ & $\begin{array}{l}3.31 \\
4.17 \\
2.95 \\
2.78 \\
3.54\end{array}$ \\
\hline 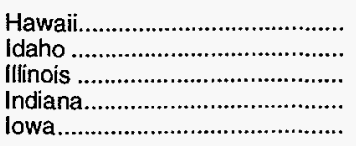 & $\begin{array}{l}7.67 \\
2.08 \\
3.09 \\
3.15 \\
2.86\end{array}$ & $\begin{array}{l}8.74 \\
2.14 \\
2.91 \\
3.05 \\
2.73\end{array}$ & $\begin{array}{l}7.72 \\
2.18 \\
3.20 \\
3.08 \\
3.20\end{array}$ & $\begin{array}{l}5.61 \\
2.26 \\
3.30 \\
3.18 \\
3.24\end{array}$ & $\begin{array}{l}4.94 \\
2.46 \\
3.02 \\
2.98 \\
3.15\end{array}$ \\
\hline 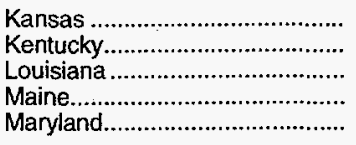 & $\begin{array}{l}2.76 \\
3.07 \\
2.97 \\
3.06 \\
3.16\end{array}$ & $\begin{array}{l}2.62 \\
2.83 \\
2.56 \\
3.00 \\
3.05\end{array}$ & $\begin{array}{l}2.50 \\
3.02 \\
2.48 \\
3.17 \\
3.20\end{array}$ & $\begin{array}{l}2.80 \\
3.21 \\
2.72 \\
3.69 \\
3.53\end{array}$ & $\begin{array}{l}2.86 \\
3.13 \\
2.54 \\
2.98 \\
3.38\end{array}$ \\
\hline 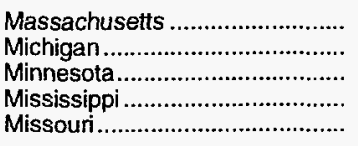 & $\begin{array}{l}3.34 \\
3.12 \\
2.83 \\
2.89 \\
3.14\end{array}$ & $\begin{array}{l}3.37 \\
3.08 \\
2.63 \\
2.55 \\
2.92\end{array}$ & $\begin{array}{l}3.52 \\
3.04 \\
2.92 \\
2.62 \\
2.86\end{array}$ & $\begin{array}{l}3.98 \\
2.89 \\
3.11 \\
2.90 \\
3.20\end{array}$ & $\begin{array}{l}3.98 \\
2.70 \\
2.85 \\
2.83 \\
3.05\end{array}$ \\
\hline 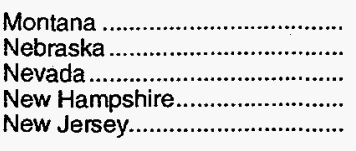 & $\begin{array}{l}3.30 \\
2.95 \\
2.75 \\
3.51 \\
3.23\end{array}$ & $\begin{array}{l}3.69 \\
2.75 \\
2.33 \\
3.40 \\
3.14\end{array}$ & $\begin{array}{l}3.45 \\
2.91 \\
2.37 \\
3.58 \\
3.29\end{array}$ & $\begin{array}{l}3.29 \\
3.46 \\
3.03 \\
3.76 \\
3.54\end{array}$ & $\begin{array}{l}3.49 \\
2.98 \\
3.18 \\
3.49 \\
3.33\end{array}$ \\
\hline 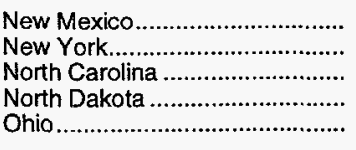 & $\begin{array}{l}2.63 \\
3.05 \\
2.88 \\
3.07 \\
3.09\end{array}$ & $\begin{array}{l}2.49 \\
2.92 \\
2.69 \\
3.49 \\
3.05\end{array}$ & $\begin{array}{l}2.25 \\
3.01 \\
2.88 \\
3.28 \\
3.26\end{array}$ & $\begin{array}{l}2.39 \\
3.32 \\
3.15 \\
3.29 \\
3.52\end{array}$ & $\begin{array}{l}2.02 \\
3.02 \\
3.27 \\
3.15 \\
3.48\end{array}$ \\
\hline 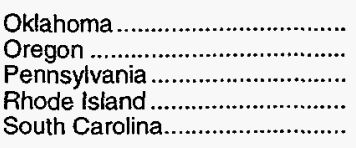 & $\begin{array}{l}2.03 \\
2.47 \\
3.47 \\
3.71 \\
3.14\end{array}$ & $\begin{array}{l}2.04 \\
2.39 \\
3.27 \\
3.68 \\
2.95\end{array}$ & $\begin{array}{l}2.22 \\
2.34 \\
3.29 \\
3.82 \\
3.23\end{array}$ & $\begin{array}{l}2.45 \\
2.48 \\
3.41 \\
4.41 \\
3.54\end{array}$ & $\begin{array}{l}2.46 \\
2.73 \\
3.46 \\
4.17 \\
3.67\end{array}$ \\
\hline 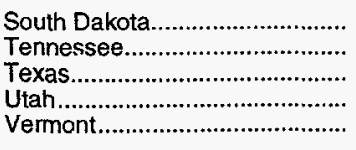 & $\begin{array}{l}3.12 \\
2.88 \\
3.14 \\
3.91 \\
2.88\end{array}$ & $\begin{array}{l}3.11 \\
2.73 \\
2.88 \\
3.89 \\
2.87\end{array}$ & $\begin{array}{l}3.10 \\
2.90 \\
3.06 \\
4.09 \\
2.93\end{array}$ & $\begin{array}{l}3.35 \\
2.93 \\
3.32 \\
2.63 \\
2.96\end{array}$ & $\begin{array}{l}3.35 \\
2.71 \\
3.00 \\
3.31 \\
3.11\end{array}$ \\
\hline 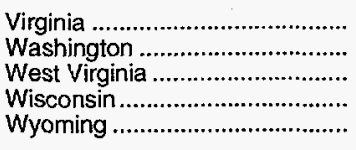 & $\begin{array}{l}3.09 \\
1.95 \\
3.54 \\
3.34 \\
3.00\end{array}$ & $\begin{array}{l}2.76 \\
1.91 \\
3.58 \\
3.17 \\
3.04\end{array}$ & $\begin{array}{l}2.91 \\
1.90 \\
3.23 \\
3.36 \\
2.90\end{array}$ & $\begin{array}{l}3.33 \\
2.39 \\
3.39 \\
3.70 \\
2.80\end{array}$ & $\begin{array}{l}3.44 \\
2.54 \\
3.26 \\
3.42 \\
2.91\end{array}$ \\
\hline 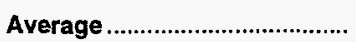 & 3.03 & 2.90 & 3.01 & 3.21 & 3.07 \\
\hline
\end{tabular}

Source: Energy Information Administration (EIA), Forn EIA-857, "Monthly Report of Natural Gas Purchases and Deliveries to Consumers." 
Table 25. Average Price of Natural Gas Delivered to Residential Consumers by State, 1990-1994 (Dollars per Thousand Cubic Feet)

\begin{tabular}{|c|c|c|c|c|c|}
\hline State & 1990 & 1991 & 1992 & 1993 & 1994 \\
\hline 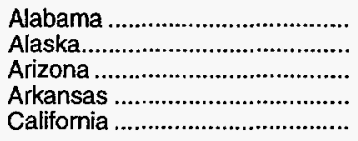 & $\begin{array}{l}6.57 \\
3.79 \\
6.85 \\
5.10 \\
5.78\end{array}$ & $\begin{array}{l}7.05 \\
4.18 \\
6.99 \\
4.98 \\
6.27\end{array}$ & $\begin{array}{l}6.74 \\
3.79 \\
7.24 \\
5.10 \\
5.97\end{array}$ & $\begin{array}{l}7.10 \\
3.96 \\
7.20 \\
5.38 \\
6.23\end{array}$ & $\begin{array}{l}7.41 \\
3.60 \\
7.54 \\
5.71 \\
6.39\end{array}$ \\
\hline 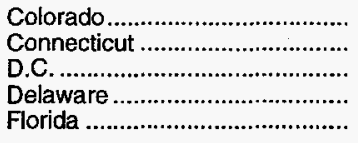 & $\begin{array}{l}4.57 \\
8.58 \\
7.18 \\
6.13 \\
8.47\end{array}$ & $\begin{array}{l}4.59 \\
8.74 \\
7.07 \\
5.86 \\
8.98\end{array}$ & $\begin{array}{l}4.56 \\
8.96 \\
7.61 \\
6.13 \\
9.08\end{array}$ & $\begin{array}{r}4.52 \\
9.43 \\
8.34 \\
6.70 \\
\mathbf{R}_{1} 0.02\end{array}$ & $\begin{array}{r}4.92 \\
10.14 \\
8.29 \\
7.43 \\
9.98\end{array}$ \\
\hline 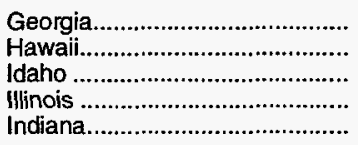 & $\begin{array}{r}6.82 \\
16.45 \\
5.05 \\
5.06 \\
5.38\end{array}$ & $\begin{array}{r}6.70 \\
22.93 \\
5.19 \\
4.95 \\
5.46\end{array}$ & $\begin{array}{r}6.44 \\
18.03 \\
5.23 \\
5.09 \\
5.43\end{array}$ & $\begin{array}{r}6.80 \\
17.51 \\
5.38 \\
5.52 \\
5.76\end{array}$ & $\begin{array}{r}7.32 \\
16.83 \\
5.29 \\
5.50 \\
6.24\end{array}$ \\
\hline 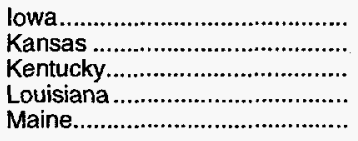 & $\begin{array}{l}4.99 \\
4.48 \\
4.93 \\
6.09 \\
7.61\end{array}$ & $\begin{array}{l}4.81 \\
4.38 \\
4.87 \\
5.77 \\
6.86\end{array}$ & $\begin{array}{l}5.23 \\
4.70 \\
5.01 \\
5.60 \\
6.95\end{array}$ & $\begin{array}{l}5.48 \\
4.91 \\
5.25 \\
6.09 \\
7.47\end{array}$ & $\begin{array}{l}5.40 \\
5.11 \\
5.46 \\
6.24 \\
7.83\end{array}$ \\
\hline 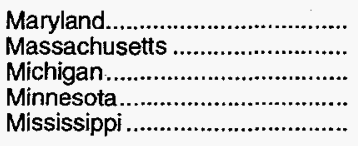 & $\begin{array}{l}6.45 \\
7.82 \\
5.02 \\
4.63 \\
5.33\end{array}$ & $\begin{array}{l}6.16 \\
8.11 \\
5.07 \\
4.52 \\
5.21\end{array}$ & $\begin{array}{l}6.43 \\
7.92 \\
5.06 \\
4.86 \\
4.95\end{array}$ & $\begin{array}{l}7.08 \\
8.33 \\
5.04 \\
5.31 \\
5.23\end{array}$ & $\begin{array}{l}6.95 \\
8.94 \\
4.98 \\
5.18 \\
5.46\end{array}$ \\
\hline 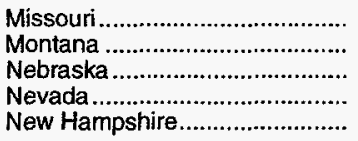 & $\begin{array}{l}5.21 \\
4.59 \\
4.60 \\
5.66 \\
7.41\end{array}$ & $\begin{array}{l}5.14 \\
4.52 \\
4.64 \\
5.61 \\
7.14\end{array}$ & $\begin{array}{l}5.11 \\
4.80 \\
4.82 \\
5.59 \\
7.55\end{array}$ & $\begin{array}{l}5.37 \\
4.92 \\
4.96 \\
5.69 \\
7.66\end{array}$ & $\begin{array}{l}5.43 \\
5.23 \\
5.01 \\
6.66 \\
7.96\end{array}$ \\
\hline 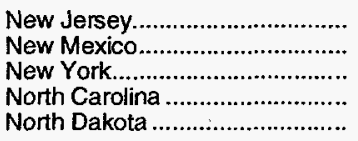 & $\begin{array}{l}6.60 \\
5.67 \\
7.40 \\
6.17 \\
4.70\end{array}$ & $\begin{array}{l}6.73 \\
5.40 \\
7.35 \\
6.24 \\
4.82\end{array}$ & $\begin{array}{l}6.94 \\
4.75 \\
7.58 \\
6.60 \\
5.00\end{array}$ & $\begin{array}{l}6.99 \\
5.46 \\
8.15 \\
6.99 \\
5.23\end{array}$ & $\begin{array}{l}7.11 \\
5.61 \\
8.75 \\
7.30 \\
5.19\end{array}$ \\
\hline 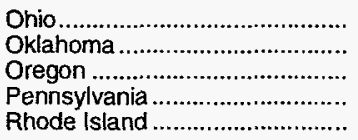 & $\begin{array}{l}5.29 \\
4.80 \\
6.27 \\
6.61 \\
7.22\end{array}$ & $\begin{array}{l}5.28 \\
4.72 \\
6.13 \\
6.76 \\
7.63\end{array}$ & $\begin{array}{l}5.20 \\
4.96 \\
6.17 \\
6.60 \\
7.68\end{array}$ & $\begin{array}{l}5.71 \\
4.94 \\
6.42 \\
6.84 \\
8.17\end{array}$ & $\begin{array}{l}5.88 \\
5.50 \\
6.99 \\
7.44 \\
9.12\end{array}$ \\
\hline 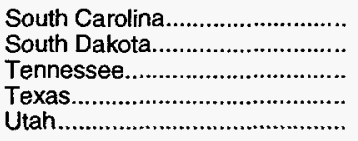 & $\begin{array}{l}7.17 \\
5.14 \\
5.11 \\
5.78 \\
5.28\end{array}$ & $\begin{array}{l}6.98 \\
4.94 \\
5.19 \\
5.71 \\
5.44\end{array}$ & $\begin{array}{l}7.03 \\
5.15 \\
5.50 \\
5.78 \\
5.44\end{array}$ & $\begin{array}{l}7.14 \\
5.30 \\
5.69 \\
5.91 \\
5.13\end{array}$ & $\begin{array}{l}7.65 \\
5.27 \\
6.13 \\
5.99 \\
4.96\end{array}$ \\
\hline 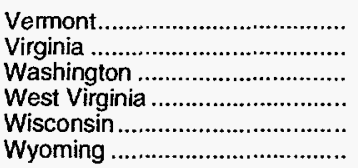 & $\begin{array}{l}5.79 \\
6.75 \\
5.02 \\
6.46 \\
5.74 \\
4.84\end{array}$ & $\begin{array}{l}6.23 \\
6.80 \\
4.68 \\
6.50 \\
5.61 \\
4.74\end{array}$ & $\begin{array}{l}6.70 \\
6.69 \\
5.00 \\
6.31 \\
5.87 \\
4.72\end{array}$ & $\begin{array}{l}6.19 \\
7.51 \\
5.23 \\
6.45 \\
6.34 \\
4.77\end{array}$ & $\begin{array}{l}6.94 \\
7.63 \\
5.70 \\
6.66 \\
6.28 \\
5.10\end{array}$ \\
\hline 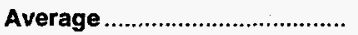 & 5.80 & 5.82 & 5.89 & 6.16 & 6.41 \\
\hline
\end{tabular}

R = Revised data.

Source: Energy Information Administration (EIA), Form-176, "Annual Report of Natural and Supplemental Gas Supply and Disposition." 
Table 26. Average Prices of Natural Gas to Consumers by State, 1994 (Dollars per Thousand Cubic Feet)

\begin{tabular}{|c|c|c|c|c|c|c|c|}
\hline \multirow[b]{2}{*}{ State } & \multicolumn{2}{|c|}{ Commercial } & \multicolumn{2}{|c|}{ Industrial } & \multicolumn{2}{|c|}{ Vehicle Fuel } & \multirow{2}{*}{$\begin{array}{l}\text { Electric Utilities } \\
\text { Average Price }\end{array}$} \\
\hline & Average Price & $\begin{array}{c}\text { Percentage of } \\
\text { Total } \\
\text { Volume tlelivered }\end{array}$ & Average Price & $\begin{array}{c}\text { Percentage of } \\
\text { Total } \\
\text { Volume Delivered }\end{array}$ & Average Price & $\begin{array}{c}\text { Percentage of } \\
\text { Total } \\
\text { Volume Delivered }\end{array}$ & \\
\hline 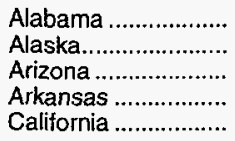 & $\begin{array}{l}6.38 \\
2.48 \\
5.27 \\
4.58 \\
7.12\end{array}$ & $\begin{array}{r}30.2 \\
100.0 \\
90.7 \\
95.1 \\
48.7\end{array}$ & $\begin{array}{l}3.26 \\
1.42 \\
3.57 \\
3.28 \\
3.25\end{array}$ & $\begin{array}{l}27.3 \\
58.4 \\
30.3 \\
13.8 \\
18.2\end{array}$ & $\begin{array}{r}4.24 \\
-- \\
3.93 \\
-\overline{-} \\
5.09\end{array}$ & $\begin{array}{r}100.0 \\
-- \\
100.0 \\
-- \\
65 . \overline{7}\end{array}$ & $\begin{array}{l}2.37 \\
0.72 \\
2.23 \\
1.87 \\
2.56\end{array}$ \\
\hline 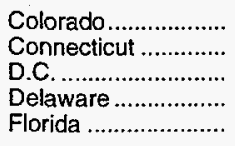 & $\begin{array}{l}4.37 \\
7.39 \\
6.16 \\
6.17 \\
5.54\end{array}$ & $\begin{array}{r}94.8 \\
30.9 \\
90.9 \\
100.0 \\
97.8\end{array}$ & $\begin{array}{r}2.38 \\
4.49 \\
\quad \ldots \\
3.43 \\
3.51\end{array}$ & $\begin{array}{r}27.9 \\
95.3 \\
-0 \\
67.3 \\
16.3\end{array}$ & $\begin{array}{r}3.55 \\
7.74 \\
-- \\
-- \\
4.36\end{array}$ & $\begin{array}{r}88.0 \\
100.0 \\
-- \\
-- \\
100.0\end{array}$ & $\begin{array}{r}2.21 \\
1.99 \\
-- \\
2.43 \\
2.18\end{array}$ \\
\hline 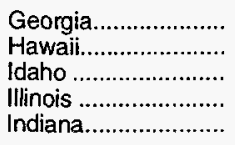 & $\begin{array}{r}6.18 \\
12.40 \\
5.01 \\
5.12 \\
5.33\end{array}$ & $\begin{array}{r}92.0 \\
100.0 \\
85.9 \\
52.8 \\
92.3\end{array}$ & $\begin{array}{r}3.90 \\
3 .- \\
4.35 \\
4.60\end{array}$ & $\begin{array}{r}37.4 \\
-- \\
2.9 \\
12.4 \\
13.4\end{array}$ & $\begin{array}{r}4.07 \\
-- \\
-- \\
3.26 \\
5.11\end{array}$ & $\begin{array}{r}100.0 \\
-- \\
-- \\
93.6 \\
100.0\end{array}$ & $\begin{array}{r}3.29 \\
-- \\
2.04 \\
2.72\end{array}$ \\
\hline 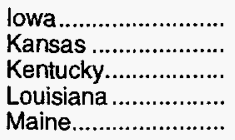 & $\begin{array}{l}4.51 \\
4.12 \\
4.98 \\
5.42 \\
6.97\end{array}$ & $\begin{array}{r}90.4 \\
78.4 \\
91.4 \\
97.9 \\
100.0\end{array}$ & $\begin{array}{l}3.99 \\
2.75 \\
3.64 \\
2.17 \\
4.79\end{array}$ & $\begin{array}{r}11.7 \\
6.5 \\
31.2 \\
24.0 \\
100.0\end{array}$ & $\begin{array}{r}3.51 \\
3.18 \\
4.62 \\
2.36 \\
--\end{array}$ & $\begin{array}{r}100.0 \\
100.0 \\
100.0 \\
100.0 \\
--\end{array}$ & $\begin{array}{r}3.18 \\
1.89 \\
2.93 \\
2.17 \\
--\end{array}$ \\
\hline $\begin{array}{l}\text { Maryland.................. } \\
\text { Massachusetts ........ } \\
\text { Michigan ................. } \\
\text { Minnesota ................ } \\
\text { Mississippi ............... }\end{array}$ & $\begin{array}{l}5.46 \\
6.82 \\
4.68 \\
4.36 \\
4.56\end{array}$ & $\begin{array}{l}97.1 \\
76.2 \\
65.7 \\
96.0 \\
96.6\end{array}$ & $\begin{array}{l}4.04 \\
5.25 \\
3.93 \\
2.87 \\
2.98\end{array}$ & $\begin{array}{l}16.8 \\
27.7 \\
10.6 \\
42.9 \\
39.1\end{array}$ & $\begin{array}{l}3.76 \\
5.66 \\
2.84 \\
1.90 \\
2.82\end{array}$ & $\begin{array}{r}100.0 \\
100.0 \\
97.1 \\
100.0 \\
100.0\end{array}$ & $\begin{array}{l}2.57 \\
2.32 \\
0.97 \\
2.14 \\
1.98\end{array}$ \\
\hline $\begin{array}{l}\text { Missouri................... } \\
\text { Montana ................... } \\
\text { Nebraska ................. } \\
\text { Nevada ..................... } \\
\text { New Hampshire....... }\end{array}$ & $\begin{array}{l}4.85 \\
4.91 \\
4.24 \\
5.36 \\
7.17\end{array}$ & $\begin{array}{r}33.3 \\
91.8 \\
80.2 \\
82.5 \\
100.0\end{array}$ & $\begin{array}{l}4.18 \\
4.91 \\
3.12 \\
5.67 \\
4.44\end{array}$ & $\begin{array}{r}20.6 \\
3.9 \\
21.6 \\
1.9 \\
95.0\end{array}$ & $\begin{array}{l}4.37 \\
4.34 \\
4.67 \\
3.81 \\
6.21\end{array}$ & $\begin{array}{r}100.0 \\
100.0 \\
100.0 \\
98.7 \\
100.0\end{array}$ & $\begin{array}{l}1.90 \\
1.21 \\
2.02 \\
1.99 \\
2.13\end{array}$ \\
\hline $\begin{array}{l}\text { New Jersey............. } \\
\text { New Mexico............... } \\
\text { New York................ } \\
\text { North Carolina .......... } \\
\text { North Dakota ............ }\end{array}$ & $\begin{array}{l}6.03 \\
4.41 \\
6.51 \\
5.56 \\
4.48\end{array}$ & $\begin{array}{l}91.6 \\
62.4 \\
79.6 \\
96.6 \\
79.6\end{array}$ & $\begin{array}{l}3.64 \\
3.39 \\
5.22 \\
3.68 \\
3.31\end{array}$ & $\begin{array}{r}57.4 \\
9.9 \\
16.1 \\
59.0 \\
24.7\end{array}$ & $\begin{array}{l}4.26 \\
5.11 \\
3.64 \\
4.44 \\
4.19\end{array}$ & $\begin{array}{r}100.0 \\
72.4 \\
100.0 \\
100.0 \\
100.0\end{array}$ & $\begin{array}{l}2.17 \\
1.99 \\
2.30 \\
3.38 \\
4.11\end{array}$ \\
\hline $\begin{array}{l}\text { Ohio ...................... } \\
\text { Oklahoma ................. } \\
\text { Oregon .................. } \\
\text { Pennsylvania ........... } \\
\text { Rhode Island ............ }\end{array}$ & $\begin{array}{l}5.38 \\
4.73 \\
5.52 \\
6.50 \\
7.57\end{array}$ & $\begin{array}{r}81.5 \\
88.5 \\
98.1 \\
74.4 \\
100.0\end{array}$ & $\begin{array}{l}4.45 \\
2.14 \\
3.61 \\
4.01 \\
4.43\end{array}$ & $\begin{array}{r}9.7 \\
24.3 \\
31.5 \\
20.5 \\
9.0\end{array}$ & $\begin{array}{l}4.87 \\
2.36 \\
4.91 \\
6.46 \\
7.09\end{array}$ & $\begin{array}{l}100.0 \\
100.0 \\
100.0 \\
100.0 \\
100.0\end{array}$ & $\begin{array}{l}3.85 \\
2.76 \\
1.85 \\
2.36 \\
2.29\end{array}$ \\
\hline $\begin{array}{l}\text { South Carolina.......... } \\
\text { South Dakota........... } \\
\text { Tennessee............... } \\
\text { Texas....................... } \\
\text { Utah....................... }\end{array}$ & $\begin{array}{l}6.11 \\
4.35 \\
5.56 \\
4.33 \\
3.84\end{array}$ & $\begin{array}{l}98.5 \\
89.1 \\
94.1 \\
82.4 \\
83.3\end{array}$ & $\begin{array}{l}3.32 \\
3.72 \\
3.84 \\
2.20 \\
2.74\end{array}$ & $\begin{array}{l}76.7 \\
37.4 \\
45.7 \\
27.8 \\
12.0\end{array}$ & $\begin{array}{l}3.40 \\
3.17 \\
5.69 \\
3.41 \\
4.90\end{array}$ & $\begin{array}{l}100.0 \\
100.0 \\
100.0 \\
100.0 \\
100.0\end{array}$ & $\begin{array}{l}1.71 \\
2.65 \\
2.58 \\
2.20 \\
2.42\end{array}$ \\
\hline $\begin{array}{l}\text { Vermont................. } \\
\text { Virginia .................... } \\
\text { Washington ............. } \\
\text { West Virginia ............ } \\
\text { Wisconsin .................. } \\
\text { Wyoming .................. }\end{array}$ & $\begin{array}{l}5.60 \\
5.67 \\
4.90 \\
5.91 \\
4.91 \\
4.45\end{array}$ & $\begin{array}{r}100.0 \\
86.7 \\
95.4 \\
55.2 \\
93.5 \\
96.1\end{array}$ & $\begin{array}{l}3.47 \\
3.15 \\
2.95 \\
2.93 \\
3.36 \\
3.51\end{array}$ & $\begin{array}{r}100.0 \\
21.5 \\
41.6 \\
13.0 \\
48.8 \\
2.2\end{array}$ & $\begin{array}{l}-- \\
2.57 \\
4.29 \\
3.82 \\
3.41 \\
4.62\end{array}$ & $\begin{array}{l}100.0 \\
100.0 \\
100.0 \\
100.0 \\
100.0\end{array}$ & $\begin{array}{l}2.31 \\
2.66 \\
4.95 \\
4.00 \\
2.66 \\
5.80\end{array}$ \\
\hline Average .................... & 5.44 & 79.3 & 3.05 & 25.1 & 4.13 & 86.9 & 2.28 \\
\hline
\end{tabular}

Note: Totals may not equal sum of components due to independent rounding.

Sources: Energy Information Administration (EIA), Form ElA-176, "Annual Report of Natural and Supplemental Gas Supply and Disposition" and Federal Energy Regulatory Commission (FERC), Form FERC-423, "Monthly Report of Cost and Quality of Fuels for Electric Plants." 
Figure 15. Average Price of Natural Gas Delivered to U.S. Residential Consumers, 1994 (Dollars per Thousand Cubic Feet)

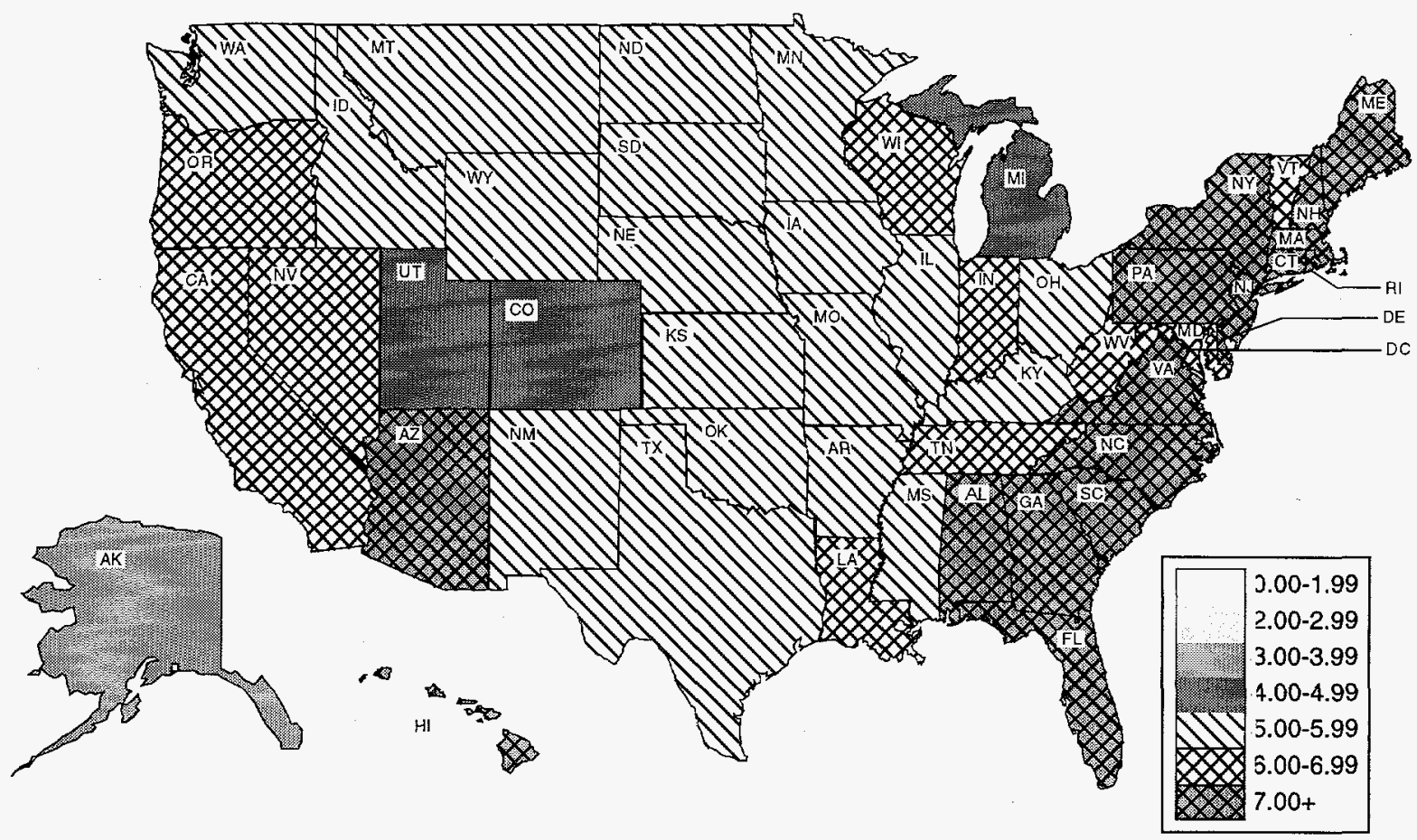

Source: Energy Information Administration, Form ElA-176, “Annual Report of Natural and Supplemental Gas Supply and Disposition.”

Figure 16. Average Price of Natural Gas Delivered to U.S. Onsystem Commercial Consumers, 1994 (Dollars per Thousand Cubic Feet)

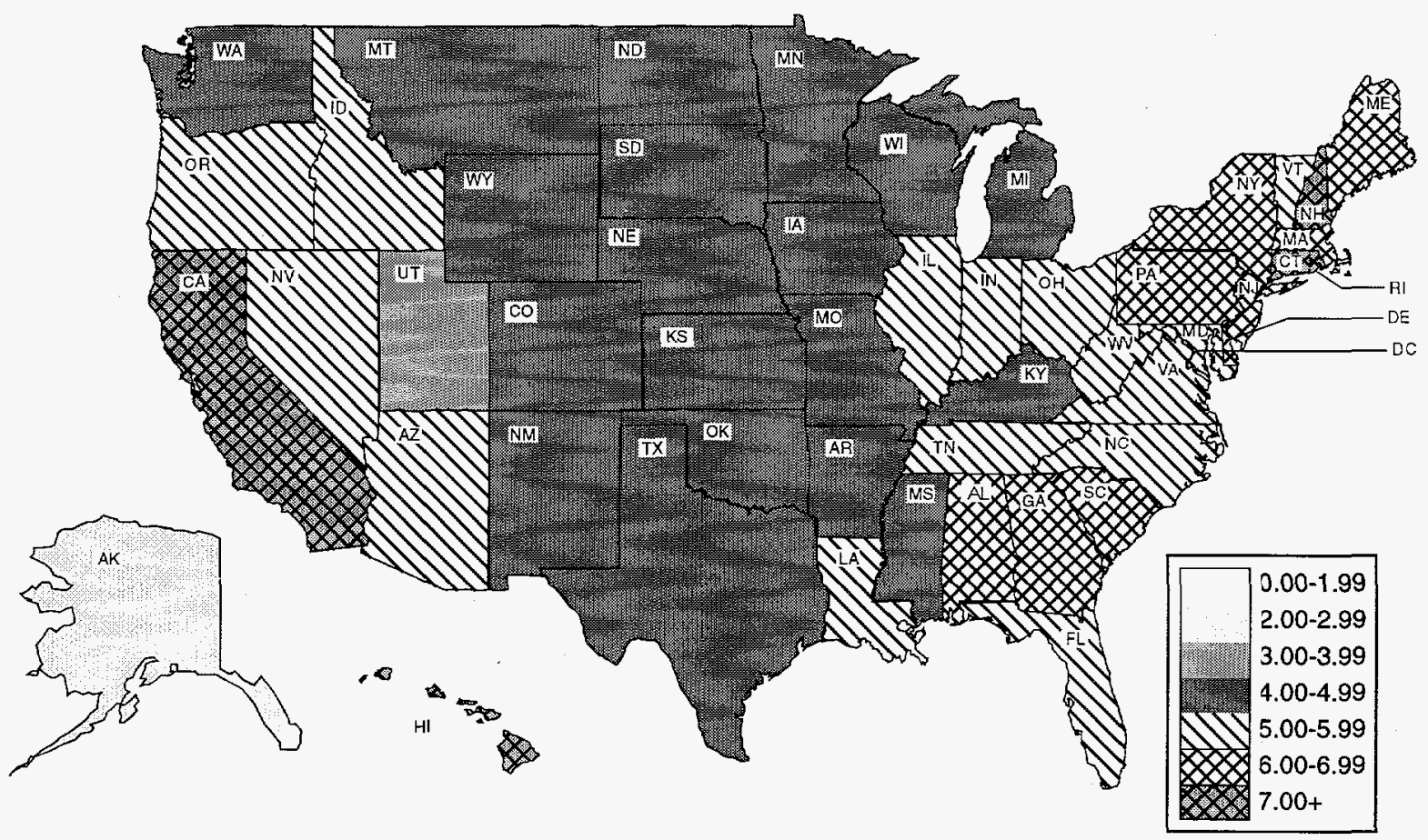

Note: Commercial prices include natural gas delivered for use as vehicle fuel.

Source: Energy Information Administration, Form ElA-176, "Annual Report of Natural and Supplemental Gas Supply and Disposition." 
Figure 17. Average Price of Natural Gas Delivered to U.S. Onsystem Industrial Consumers, 1994 (Dollars per Thousand Cubic Feet)

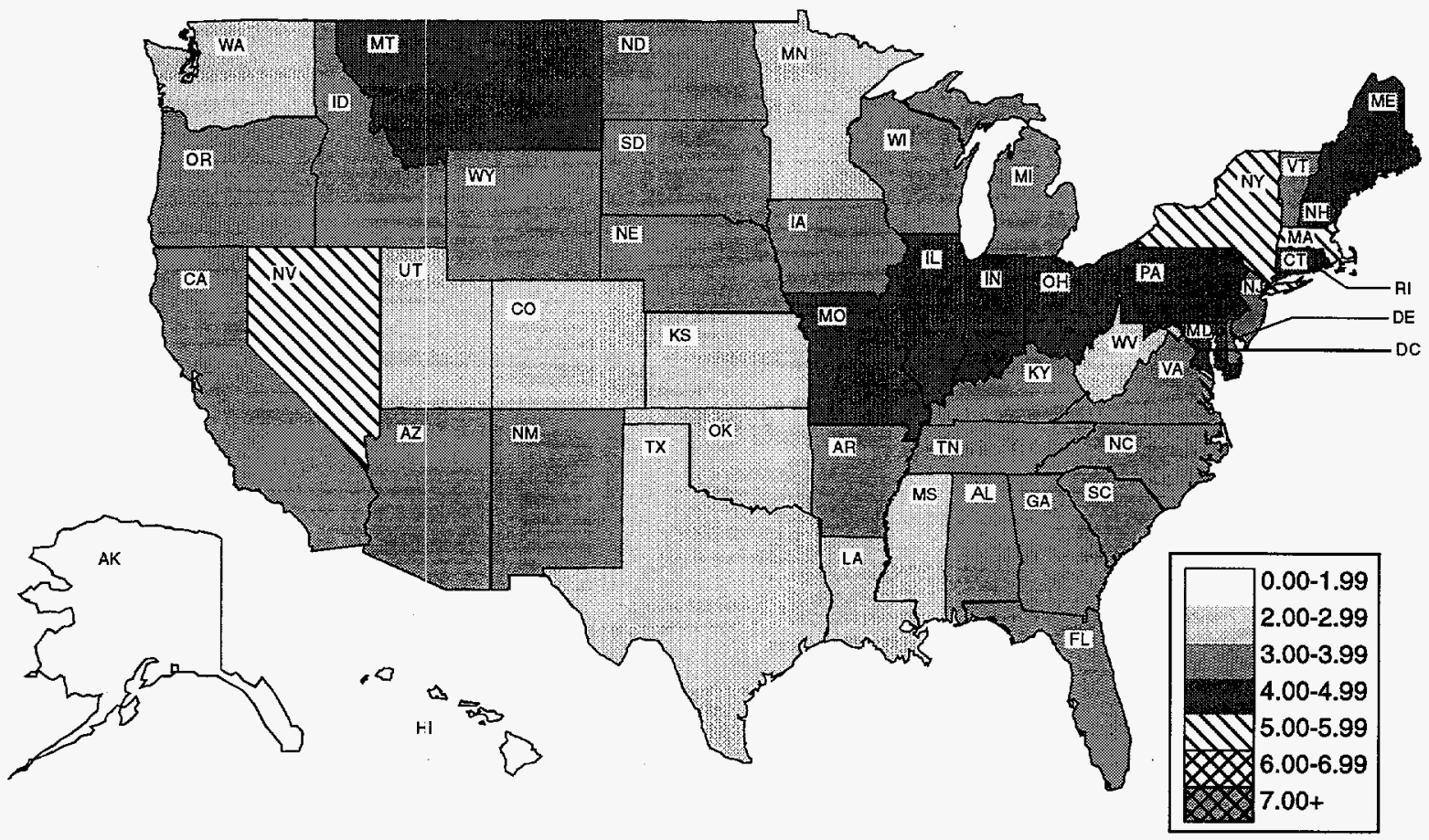

Source: Energy Information Administration, Form EIA-176, “Annual Report of Natural and Supplemental Gas Supply and Disposition.”

Figure 18. Average Price of Natural Gas Delivered to U.S. Electric Utilities, 1994 (Dollars per Thousand Cubic Feet)

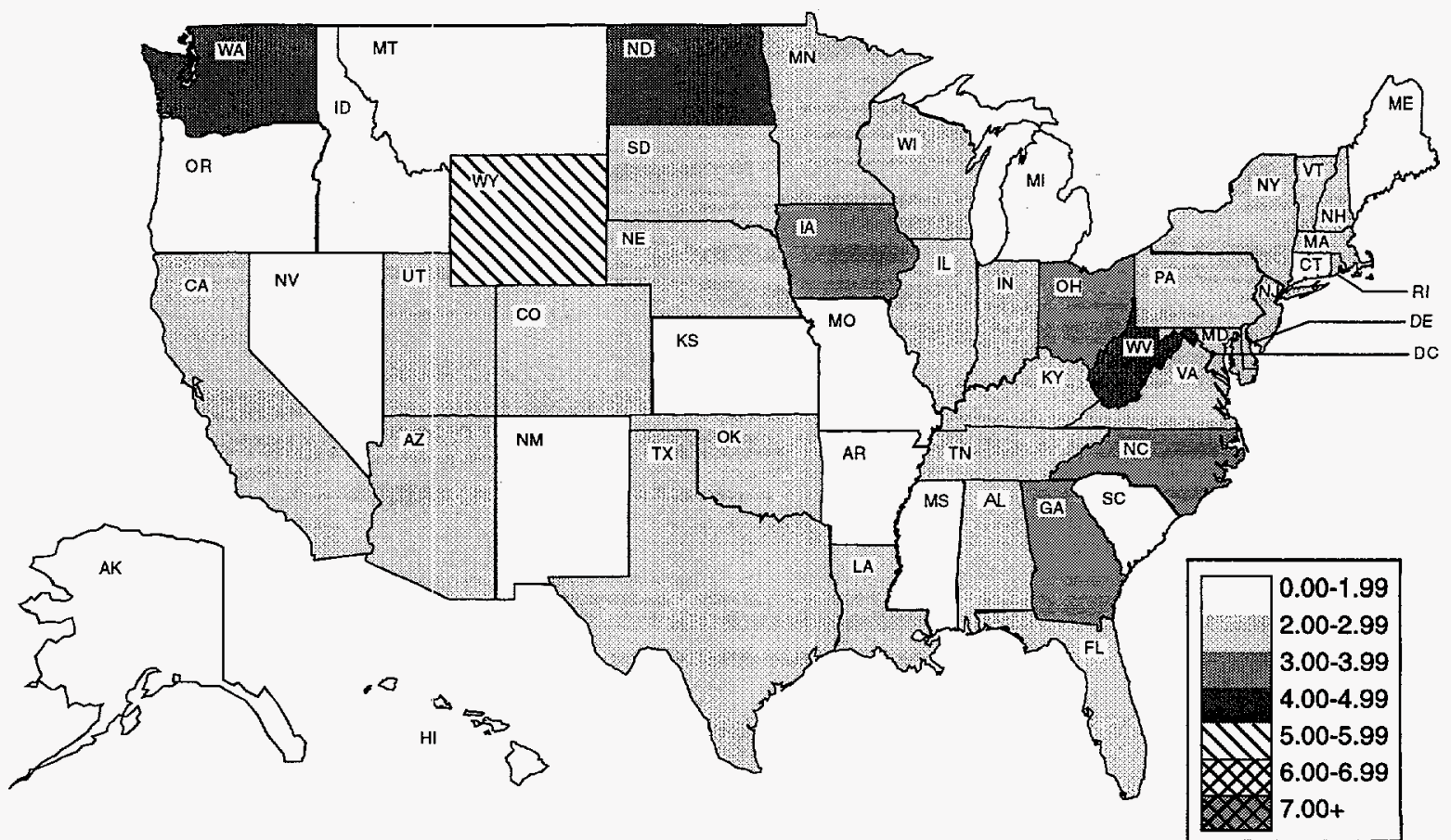

Sources: Federal Energy Regulatory Commission (FERC), Form FERC-423, "Monthly Report of Cost and Quality of Fuels for Electric Plants" and Form EIA-176, "Annual Report of Natural and Supplemental Gas Supply and Disposition." 
Table 27. Price of Natural Gas Deliveries to Commercial Consumers by State, 1994 (Dollars per Thousand Cubic Feet)

\begin{tabular}{|c|c|c|c|c|c|c|}
\hline \multirow{2}{*}{ State } & \multicolumn{2}{|c|}{ Firm } & \multicolumn{2}{|c|}{ Interruplible } & \multicolumn{2}{|c|}{ Total } \\
\hline & Average Price & $\begin{array}{l}\text { Percentage of Total } \\
\text { Volume Delivered }\end{array}$ & Average Price & $\begin{array}{l}\text { Percentage of Total } \\
\text { Volume Delivered }\end{array}$ & Total Average Price & $\begin{array}{l}\text { Percentage of Total } \\
\text { Volume Delivered }\end{array}$ \\
\hline 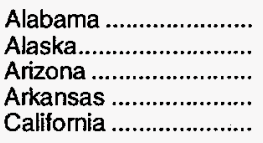 & $\begin{array}{l}6.43 \\
2.48 \\
5.28 \\
4.77 \\
7.35\end{array}$ & $\begin{array}{r}94.9 \\
100.0 \\
90.9 \\
94.3 \\
54.0\end{array}$ & $\begin{array}{r}4.44 \\
-- \\
4.60 \\
3.45 \\
3.63\end{array}$ & $\begin{array}{r}11.9 \\
-- \\
76.4 \\
99.8 \\
19.4\end{array}$ & $\begin{array}{l}6.38 \\
2.48 \\
5.27 \\
4.58 \\
7.12\end{array}$ & $\begin{array}{r}80.2 \\
100.0 \\
90.7 \\
95.1 \\
48.7\end{array}$ \\
\hline 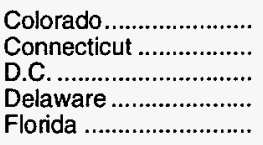 & $\begin{array}{l}4.31 \\
8.60 \\
7.98 \\
6.17 \\
5.56\end{array}$ & $\begin{array}{r}98.2 \\
84.8 \\
100.0 \\
100.0 \\
97.8\end{array}$ & $\begin{array}{r}5.75 \\
3.91 \\
4.46 \\
--- \\
4.56\end{array}$ & $\begin{array}{r}54.9 \\
71.4 \\
83.8 \\
-- \\
99.8\end{array}$ & $\begin{array}{l}4.37 \\
7.39 \\
6.16 \\
6.17 \\
5.54\end{array}$ & $\begin{array}{r}94.8 \\
80.9 \\
90.9 \\
100.0 \\
97.8\end{array}$ \\
\hline 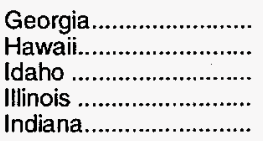 & $\begin{array}{r}6.57 \\
13.50 \\
5.01 \\
5.12 \\
5.39\end{array}$ & $\begin{array}{r}100.0 \\
100.0 \\
85.9 \\
52.8 \\
94.7\end{array}$ & $\begin{array}{r}3.91 \\
6.87 \\
-- \\
3.89 \\
4.00\end{array}$ & $\begin{array}{r}62.6 \\
100.0 \\
-\overline{4} \\
40.0 \\
61.1\end{array}$ & $\begin{array}{r}6.18 \\
12.40 \\
5.01 \\
5.12 \\
5.33\end{array}$ & $\begin{array}{r}92.0 \\
100.0 \\
85.9 \\
52.8 \\
92.3\end{array}$ \\
\hline 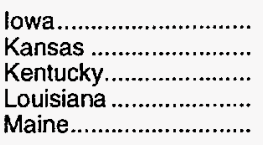 & $\begin{array}{l}4.60 \\
4.16 \\
5.04 \\
5.60 \\
6.97\end{array}$ & $\begin{array}{r}90.0 \\
91.9 \\
92.6 \\
97.2 \\
100.0\end{array}$ & $\begin{array}{r}3.20 \\
3.00 \\
3.86 \\
4.91 \\
--\end{array}$ & $\begin{array}{r}97.3 \\
14.3 \\
73.3 \\
99.9 \\
--\end{array}$ & $\begin{array}{l}4.51 \\
4.12 \\
4.98 \\
5.42 \\
6.97\end{array}$ & $\begin{array}{r}90.4 \\
78.4 \\
91.4 \\
97.9 \\
100.0\end{array}$ \\
\hline $\begin{array}{l}\text { Maryland..................... } \\
\text { Massachusetts ............ } \\
\text { Michigan ...................... } \\
\text { Minnesota.................... } \\
\text { Mississippi ................... }\end{array}$ & $\begin{array}{l}5.73 \\
7.23 \\
4.68 \\
4.88 \\
4.68\end{array}$ & $\begin{array}{l}97.4 \\
95.8 \\
65.9 \\
97.2 \\
97.1\end{array}$ & $\begin{array}{l}4.19 \\
2.98 \\
4.38 \\
2.77 \\
2.99\end{array}$ & $\begin{array}{r}95.8 \\
25.7 \\
9.3 \\
92.6 \\
90.5\end{array}$ & $\begin{array}{l}5.46 \\
6.82 \\
4.68 \\
4.36 \\
4.56\end{array}$ & $\begin{array}{l}97.1 \\
76.2 \\
65.7 \\
96.0 \\
96.6\end{array}$ \\
\hline 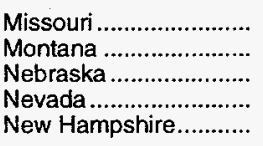 & $\begin{array}{l}4.90 \\
4.91 \\
4.53 \\
5.36 \\
7.17\end{array}$ & $\begin{array}{r}85.2 \\
93.9 \\
77.0 \\
83.8 \\
100.0\end{array}$ & $\begin{array}{r}3.13 \\
4.89 \\
2.83 \\
-- \\
--\end{array}$ & $\begin{array}{r}44.1 \\
3.7 \\
100.0 \\
- \\
--\end{array}$ & $\begin{array}{l}4.85 \\
4.91 \\
4.24 \\
5.36 \\
7.17\end{array}$ & $\begin{array}{r}83.3 \\
91.8 \\
80.2 \\
82.5 \\
100.0\end{array}$ \\
\hline $\begin{array}{l}\text { New Jersey................. } \\
\text { New Mexico................ } \\
\text { New York................... } \\
\text { North Carolina ............. } \\
\text { North Dakota .............. }\end{array}$ & $\begin{array}{l}6.84 \\
4.41 \\
6.74 \\
5.70 \\
4.62\end{array}$ & $\begin{array}{r}94.4 \\
62.2 \\
80.0 \\
96.7 \\
100.0\end{array}$ & $\begin{array}{l}2.62 \\
4.45 \\
4.26 \\
3.35 \\
2.71\end{array}$ & $\begin{array}{r}81.5 \\
100.0 \\
75.6 \\
94.8 \\
22.7\end{array}$ & $\begin{array}{l}6.03 \\
4.41 \\
6.51 \\
5.56 \\
4.48\end{array}$ & $\begin{array}{l}91.6 \\
62.4 \\
79.6 \\
96.6 \\
79.6\end{array}$ \\
\hline $\begin{array}{l}\text { Ohio } \\
\text { Oklahoma ...................... } \\
\text { Oregon ...................... } \\
\text { Pennsylvania ............... } \\
\text { Rhode Island ............... }\end{array}$ & $\begin{array}{l}5.40 \\
4.83 \\
5.52 \\
6.80 \\
8.19\end{array}$ & $\begin{array}{r}82.5 \\
94.5 \\
100.0 \\
75.3 \\
100.0\end{array}$ & $\begin{array}{r}3.49 \\
3.00 \\
-- \\
3.60 \\
3.31\end{array}$ & $\begin{array}{r}37.2 \\
43.1 \\
- \\
66.5 \\
100.0\end{array}$ & $\begin{array}{l}5.38 \\
4.73 \\
5.52 \\
6.50 \\
7.57\end{array}$ & $\begin{array}{r}81.5 \\
88.5 \\
98.1 \\
74.4 \\
100.0\end{array}$ \\
\hline 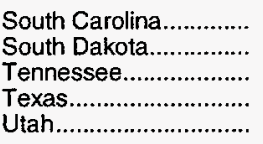 & $\begin{array}{l}6.73 \\
4.43 \\
5.71 \\
4.40 \\
3.96\end{array}$ & $\begin{array}{r}99.1 \\
91.7 \\
94.8 \\
85.4 \\
100.0\end{array}$ & $\begin{array}{l}3.42 \\
2.83 \\
3.43 \\
2.32 \\
2.49\end{array}$ & $\begin{array}{l}96.1 \\
56.9 \\
85.3 \\
41.7 \\
28.7\end{array}$ & $\begin{array}{l}6.11 \\
4.35 \\
5.56 \\
4.33 \\
3.84\end{array}$ & $\begin{array}{l}98.5 \\
89.1 \\
94.1 \\
82.4 \\
83.3\end{array}$ \\
\hline $\begin{array}{l}\text { Vermont................. } \\
\text { Virginia ...................... } \\
\text { Washington ................ } \\
\text { West Virginia ............... } \\
\text { Wisconsin ...................... } \\
\text { Wyoming .................... }\end{array}$ & $\begin{array}{l}5.60 \\
5.98 \\
5.13 \\
6.38 \\
5.06 \\
4.28\end{array}$ & $\begin{array}{r}100.0 \\
93.1 \\
97.7 \\
87.1 \\
96.4 \\
97.0\end{array}$ & $\begin{array}{l}-- \\
4.47 \\
3.80 \\
5.54 \\
3.04 \\
4.66\end{array}$ & $\begin{array}{l}68.6 \\
85.8 \\
42.8 \\
69.0 \\
95.1\end{array}$ & $\begin{array}{l}5.60 \\
5.67 \\
4.90 \\
5.91 \\
4.91 \\
4.45\end{array}$ & $\begin{array}{r}100.0 \\
86.7 \\
95.4 \\
55.2 \\
93.5 \\
96.1\end{array}$ \\
\hline Average ........................ & 5.61 & 81.9 & 3.63 & 59.7 & 5.44 & 79.3 \\
\hline
\end{tabular}

Note: Totals may not equal sum of components due to independent rounding.

Source: Energy Information Administration (EIA), Form E|A-176, "Annual Report of Natural and Supplemental Gas Supply and Disposition." 
Table 28. Price of Natural Gas Deliveries to Industrial Consumers by State, 1994 (Dollars per Thousand Cubic Feet)

\begin{tabular}{|c|c|c|c|c|c|c|}
\hline \multirow{2}{*}{ State } & \multicolumn{2}{|c|}{ Firm } & \multicolumn{2}{|c|}{ Interruptible } & \multicolumn{2}{|c|}{ Total } \\
\hline & Average Price & $\begin{array}{l}\text { Percentage of Total } \\
\text { Volume Delivered }\end{array}$ & Average Price & $\begin{array}{l}\text { Percentage of Total } \\
\text { Volume Delivered }\end{array}$ & Total Average Price & $\begin{array}{l}\text { Percentage of Total } \\
\text { Volume Delivered }\end{array}$ \\
\hline 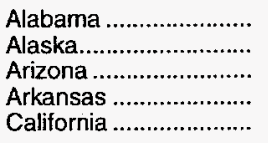 & $\begin{array}{l}3.97 \\
1.42 \\
3.53 \\
3.46 \\
3.20\end{array}$ & $\begin{array}{r}17.2 \\
62.3 \\
30.3 \\
6.8 \\
24.1\end{array}$ & $\begin{array}{r}3.02 \\
-- \\
4.66 \\
3.15 \\
3.45\end{array}$ & $\begin{array}{r}33.9 \\
-- \\
30.4 \\
56.2 \\
8.8\end{array}$ & $\begin{array}{l}3.26 \\
1.42 \\
3.57 \\
3.28 \\
3.25\end{array}$ & $\begin{array}{l}27.3 \\
58.4 \\
30.3 \\
13.8 \\
18.2\end{array}$ \\
\hline 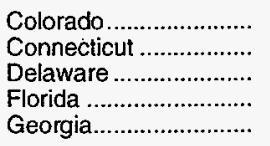 & $\begin{array}{l}2.63 \\
5.92 \\
4.52 \\
3.60 \\
5.21\end{array}$ & $\begin{array}{l}29.4 \\
98.6 \\
45.5 \\
17.7 \\
29.6\end{array}$ & $\begin{array}{l}2.25 \\
3.16 \\
2.69 \\
3.45 \\
3.55\end{array}$ & $\begin{array}{r}27.1 \\
92.4 \\
100.0 \\
15.5 \\
40.2\end{array}$ & $\begin{array}{l}2.38 \\
4.49 \\
3.43 \\
3.51 \\
3.90\end{array}$ & $\begin{array}{l}27.9 \\
95.3 \\
67.3 \\
16.3 \\
37.4\end{array}$ \\
\hline 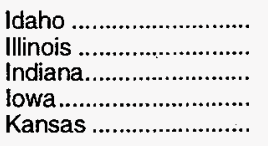 & $\begin{array}{l}3.73 \\
4.61 \\
4.75 \\
4.62 \\
3.25\end{array}$ & $\begin{array}{r}1.8 \\
11.5 \\
45.5 \\
8.7 \\
3.5\end{array}$ & $\begin{array}{l}3.99 \\
2.97 \\
4.05 \\
2.88 \\
2.56\end{array}$ & $\begin{array}{r}11.7 \\
23.3 \\
3.9 \\
29.4 \\
9.9\end{array}$ & $\begin{array}{l}3.85 \\
4.39 \\
4.60 \\
3.99 \\
2.75\end{array}$ & $\begin{array}{r}2.9 \\
12.4 \\
13.4 \\
11.7 \\
6.5\end{array}$ \\
\hline $\begin{array}{l}\text { Kentucky................... } \\
\text { Louisiana ...................... } \\
\text { Maine............................ } \\
\text { Maryland.................... } \\
\text { Massachusetts ............. }\end{array}$ & $\begin{array}{l}4.02 \\
2.14 \\
5.77 \\
6.15 \\
6.69\end{array}$ & $\begin{array}{r}30.8 \\
28.4 \\
100.0 \\
3.1 \\
23.9\end{array}$ & $\begin{array}{l}3.23 \\
2.22 \\
2.91 \\
3.94 \\
2.82\end{array}$ & $\begin{array}{r}31.7 \\
17.4 \\
100.0 \\
21.3 \\
38.0\end{array}$ & $\begin{array}{l}3.64 \\
2.17 \\
4.79 \\
4.04 \\
5.25\end{array}$ & $\begin{array}{r}31.2 \\
24.0 \\
100.0 \\
16.8 \\
27.7\end{array}$ \\
\hline 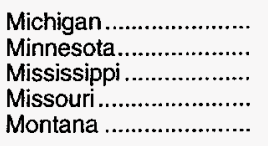 & $\begin{array}{l}3.94 \\
4.36 \\
2.98 \\
4.62 \\
4.91\end{array}$ & $\begin{array}{r}11.5 \\
15.8 \\
25.5 \\
17.3 \\
9.8\end{array}$ & $\begin{array}{l}3.53 \\
2.43 \\
2.98 \\
3.24 \\
4.61\end{array}$ & $\begin{array}{r}2.8 \\
86.0 \\
60.2 \\
34.6 \\
\ldots\end{array}$ & $\begin{array}{l}3.93 \\
2.87 \\
2.98 \\
4.18 \\
4.91\end{array}$ & $\begin{array}{r}10.6 \\
42.9 \\
39.1 \\
20.6 \\
3.9\end{array}$ \\
\hline $\begin{array}{l}\text { Nebraska ..................... } \\
\text { Nevada ...................... } \\
\text { New Hampshire........... } \\
\text { New Jersey................... } \\
\text { New Mexico................. }\end{array}$ & $\begin{array}{l}3.90 \\
5.67 \\
4.81 \\
4.37 \\
3.39\end{array}$ & $\begin{array}{r}9.9 \\
1.9 \\
93.8 \\
38.5 \\
10.2\end{array}$ & $\begin{array}{r}2.66 \\
-- \\
2.90 \\
3.38 \\
4.05\end{array}$ & $\begin{array}{r}71.2 \\
-- \\
100.0 \\
69.7 \\
2.4\end{array}$ & $\begin{array}{l}3.12 \\
5.67 \\
4.44 \\
3.64 \\
3.39\end{array}$ & $\begin{array}{r}21.6 \\
1.9 \\
95.0 \\
57.4 \\
9.9\end{array}$ \\
\hline $\begin{array}{l}\text { New York } \\
\text { North Carolina .............. } \\
\text { North Dakota .............. } \\
\text { Ohio ............................ } \\
\text { Oklahoma .................. }\end{array}$ & $\begin{array}{l}5.84 \\
3.84 \\
4.44 \\
4.53 \\
2.48\end{array}$ & $\begin{array}{l}18.3 \\
64.9 \\
34.1 \\
10.3 \\
48.0\end{array}$ & $\begin{array}{l}3.62 \\
3.64 \\
3.16 \\
3.57 \\
1.91\end{array}$ & $\begin{array}{r}12.3 \\
57.5 \\
23.8 \\
5.9 \\
18.2\end{array}$ & $\begin{array}{l}5.22 \\
3.68 \\
3.31 \\
4.45 \\
2.14\end{array}$ & $\begin{array}{r}16.1 \\
59.0 \\
24.7 \\
9.7 \\
24.3\end{array}$ \\
\hline $\begin{array}{l}\text { Oregón } \\
\text { Pennsylvania .................. } \\
\text { Rhode Island .............. } \\
\text { South Carolina.............. } \\
\text { South Dakota............... }\end{array}$ & $\begin{array}{l}3.90 \\
4.21 \\
5.09 \\
4.44 \\
4.15\end{array}$ & $\begin{array}{r}60.9 \\
18.4 \\
6.2 \\
73.4 \\
51.5\end{array}$ & $\begin{array}{l}3.20 \\
3.65 \\
3.08 \\
3.09 \\
2.94\end{array}$ & $\begin{array}{l}18.9 \\
26.0 \\
99.7 \\
77.4 \\
25.1\end{array}$ & $\begin{array}{l}3.61 \\
4.01 \\
4.43 \\
3.32 \\
3.72\end{array}$ & $\begin{array}{r}31.5 \\
20.5 \\
9.0 \\
76.7 \\
37.4\end{array}$ \\
\hline 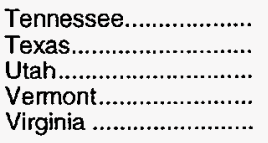 & $\begin{array}{l}4.26 \\
2.16 \\
2.91 \\
4.95 \\
4.06\end{array}$ & $\begin{array}{r}62.3 \\
40.7 \\
100.0 \\
100.0 \\
14.9\end{array}$ & $\begin{array}{l}3.40 \\
2.60 \\
2.61 \\
3.38 \\
2.50\end{array}$ & $\begin{array}{r}35.7 \\
7.7 \\
6.9 \\
100.0 \\
31.3\end{array}$ & $\begin{array}{l}3.84 \\
2.20 \\
2.74 \\
3.47 \\
3.15\end{array}$ & $\begin{array}{r}45.7 \\
27.8 \\
12.0 \\
100.0 \\
21.5\end{array}$ \\
\hline $\begin{array}{l}\text { Washington ................ } \\
\text { West Virginia ............... } \\
\text { Wisconsin .................... } \\
\text { Wyoming ................... }\end{array}$ & $\begin{array}{l}2.88 \\
2.91 \\
4.34 \\
3.37\end{array}$ & $\begin{array}{r}73.1 \\
27.5 \\
84.8 \\
6.2\end{array}$ & $\begin{array}{l}3.23 \\
3.20 \\
2.80 \\
3.64\end{array}$ & $\begin{array}{r}15.6 \\
1.7 \\
39.1 \\
1.4\end{array}$ & $\begin{array}{l}2.95 \\
2.93 \\
3.36 \\
3.51\end{array}$ & $\begin{array}{r}41.6 \\
13.0 \\
48.8 \\
2.2\end{array}$ \\
\hline 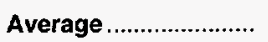 & 3.08 & 27.1 & 3.01 & 22.4 & 3.05 & 25.1 \\
\hline
\end{tabular}

Note: Totals may not equal sum of components due to independent rounding.

Source: Energy Information Administration (EIA), Form ElA-176, "Annual Report of Natural and Supplemental Gas Supply and Disposition." 
Table 29. Prices of Natural Gas Deliveries to Electric Utilities by State, 1994 (Dollars per Thousand Cubic Feet)

\begin{tabular}{|c|c|c|c|c|}
\hline \multirow{2}{*}{ State } & \multicolumn{2}{|c|}{ Firm } & \multicolumn{2}{|c|}{ Interruptible } \\
\hline & Average Price & $\begin{array}{l}\text { Percentage of Total Volume } \\
\text { Delivered }\end{array}$ & Average Price & $\begin{array}{c}\text { Percentage of Total Volume } \\
\text { Delivered }\end{array}$ \\
\hline 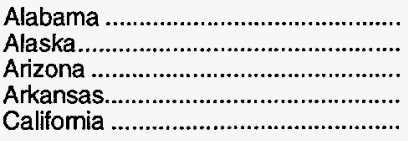 & $\begin{array}{l}1.05 \\
3.13 \\
2.19 \\
2.80\end{array}$ & $\begin{array}{r}-- \\
69.6 \\
0.1 \\
5.9 \\
--\end{array}$ & $\begin{array}{r}2.29 \\
0.32 \\
-- \\
2.97\end{array}$ & $\begin{array}{r}10.7 \\
100.0 \\
\cdots \\
13.0\end{array}$ \\
\hline 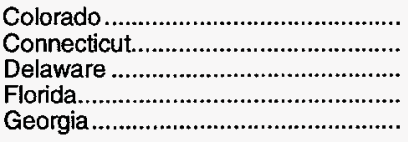 & $\begin{array}{r}2.33 \\
-- \\
4.03 \\
2.85 \\
2.56\end{array}$ & $\begin{array}{r}100.0 \\
-- \\
0.4 \\
1.1 \\
100.0\end{array}$ & $\begin{array}{r}3.24 \\
2.28 \\
2.70 \\
3.17 \\
--\end{array}$ & $\begin{array}{r}0.1 \\
87.2 \\
100.0 \\
0.5 \\
--\end{array}$ \\
\hline 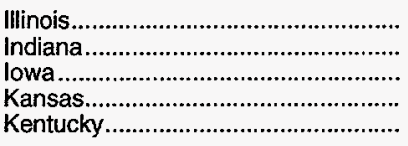 & $\begin{array}{l}5.65 \\
2.26 \\
3.86 \\
2.20 \\
2.74\end{array}$ & $\begin{array}{r}4.6 \\
86.7 \\
35.3 \\
10.4 \\
100.0\end{array}$ & $\begin{array}{r}4.73 \\
2 . \\
2.50 \\
2.09 \\
5.10\end{array}$ & $\begin{array}{r}100.0 \\
-.- \\
16.7 \\
50.5 \\
100.0\end{array}$ \\
\hline 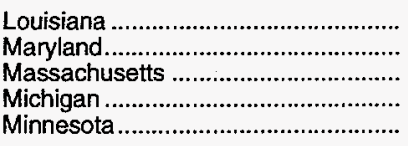 & $\begin{array}{l}2.22 \\
-- \\
2.15 \\
4.00 \\
1.26\end{array}$ & $\begin{array}{r}21.3 \\
-- \\
45.4 \\
2.1 \\
100.0\end{array}$ & $\begin{array}{l}2.34 \\
2.61 \\
2.07 \\
4.25 \\
2.08\end{array}$ & $\begin{array}{r}12.6 \\
87.8 \\
8.2 \\
5.5 \\
60.5\end{array}$ \\
\hline 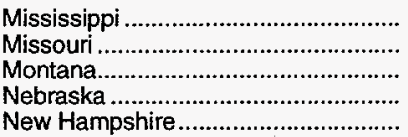 & $\begin{array}{r}2.08 \\
4.49 \\
2.68 \\
2.39 \\
--\end{array}$ & $\begin{array}{r}80.4 \\
2.4 \\
100.0 \\
100.0 \\
--\end{array}$ & $\begin{array}{r}2.41 \\
1.63 \\
2.20 \\
2.14\end{array}$ & $\begin{array}{r}1.1 \\
98.3 \\
-. \\
93.9 \\
100.0\end{array}$ \\
\hline 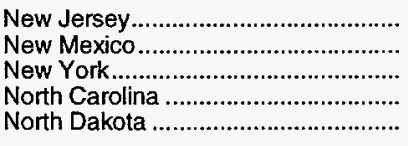 & $\begin{array}{l}2.15 \\
3.15 \\
2.44 \\
3.49 \\
4.47\end{array}$ & $\begin{array}{r}94.4 \\
10.3 \\
72.6 \\
70.2 \\
100.0\end{array}$ & $\begin{array}{r}2.58 \\
2.84 \\
2.50 \\
3.89 \\
--\end{array}$ & $\begin{array}{r}99.9 \\
1.2 \\
96.5 \\
44.8 \\
--\end{array}$ \\
\hline 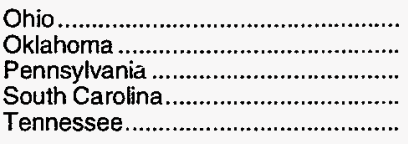 & $\begin{array}{r}4.47 \\
1.81 \\
2.73 \\
4.26 \\
--\end{array}$ & $\begin{array}{r}90.9 \\
11.3 \\
71.7 \\
100.0 \\
--\end{array}$ & $\begin{array}{l}4.07 \\
1.96 \\
2.98 \\
1.94 \\
2.58\end{array}$ & $\begin{array}{r}9.8 \\
29.2 \\
9.6 \\
100.0 \\
48.7\end{array}$ \\
\hline 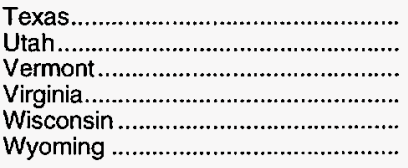 & $\begin{array}{r}2.37 \\
-- \\
-- \\
- \\
2.46 \\
3.47\end{array}$ & $\begin{array}{r}60.8 \\
- \\
- \\
- \\
100.0 \\
100.0\end{array}$ & $\begin{array}{r}3.41 \\
3.89 \\
2.08 \\
1.43 \\
2.37 \\
--\end{array}$ & $\begin{array}{r}4.2 \\
1.9 \\
100.0 \\
1.0 \\
75.7 \\
--\end{array}$ \\
\hline Average & 2.29 & 28.8 & 2.61 & 20.2 \\
\hline
\end{tabular}

Notes: States without direct sales are not included. Totals may not equal sum of components due to independent rounding.

Source: Energy Information Administration (EIA), Form EIA-176, "Annual Report of Natural and Supplemental Gas Supply and Disposition." 
Table 30. Selected Volumes and Prices of Natural Gas Sold to Residential Customers in the United States, 1994 (Volumes in Thousand Cubic Feet, Prices in Dollars per Thousand Cubic Feet)

\begin{tabular}{|c|c|c|c|}
\hline Company & State & Volume & Prices \\
\hline 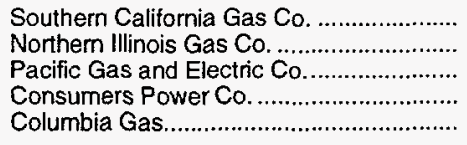 & $\begin{array}{l}\text { CA } \\
\text { IL } \\
\text { CA } \\
\text { MI } \\
\text { OH,PA }\end{array}$ & $\begin{array}{l}253,942,996 \\
223,666,564 \\
215,199,142 \\
170,438,536 \\
164,442,595\end{array}$ & $\begin{array}{l}6.71 \\
4.76 \\
5.92 \\
4.86 \\
6.43\end{array}$ \\
\hline 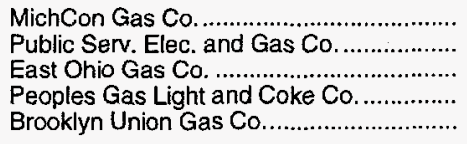 & $\begin{array}{l}\mathrm{MI} \\
\mathrm{NJ} \\
\mathrm{OH} \\
\mathrm{IL} \\
\mathrm{NY}\end{array}$ & $\begin{array}{r}149,272,559 \\
138,930,616 \\
138,679,151 \\
112,546,778 \\
96,484,205\end{array}$ & $\begin{array}{r}5.11 \\
6.53 \\
5.54 \\
6.82 \\
10.06\end{array}$ \\
\hline $\begin{array}{l}\text { Kansas Power and Light Co..................... } \\
\text { Lone Star Gas Co.................................. } \\
\text { National Fuel Gas Distribution.................. } \\
\text { Atlanta Gas Light Co............................ } \\
\text { Washington Gas Light Co. .................... }\end{array}$ & $\begin{array}{l}\text { KS,MO } \\
\text { TX } \\
\text { NY,FA } \\
\text { GA } \\
\text { MD, YA,DC }\end{array}$ & $\begin{array}{l}94,328,824 \\
91,267,209 \\
87,857,739 \\
87,712,759 \\
75,812,140\end{array}$ & $\begin{array}{l}4.91 \\
5.96 \\
7.48 \\
7.44 \\
7.52\end{array}$ \\
\hline 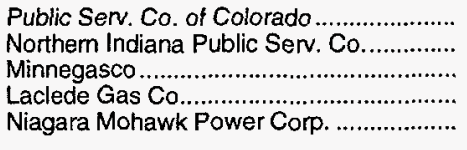 & $\begin{array}{l}\text { CO } \\
\text { IN } \\
\text { MN,SD } \\
\text { MO } \\
\text { NY }\end{array}$ & $\begin{array}{l}75,000,910 \\
65,885,605 \\
65,420,459 \\
56,036,170 \\
54,551,040\end{array}$ & $\begin{array}{l}4.88 \\
6.28 \\
5.21 \\
6.01 \\
7.26\end{array}$ \\
\hline 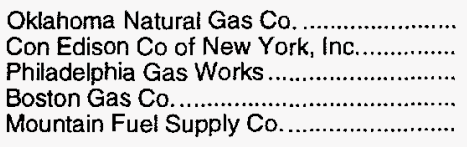 & $\begin{array}{l}\text { OK } \\
\text { NY } \\
\text { PA } \\
\text { MA } \\
\text { UT }\end{array}$ & $\begin{array}{l}54,420,719 \\
52,944,060 \\
50,814,576 \\
50,276,879 \\
48,529,451\end{array}$ & $\begin{array}{r}5.44 \\
10.13 \\
7.94 \\
8.73 \\
4.95\end{array}$ \\
\hline 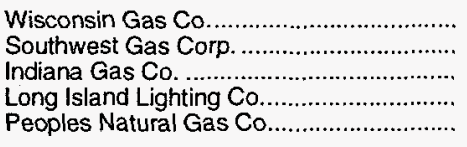 & $\begin{array}{l}\text { WI } \\
\text { AZ,CA,NV } \\
\text { IN } \\
\text { NY } \\
\text { PA }\end{array}$ & $\begin{array}{l}46,368,613 \\
44,803,552 \\
43,524,355 \\
39,648,794 \\
39,458,804\end{array}$ & $\begin{array}{l}6.59 \\
7.54 \\
6.70 \\
9.72 \\
7.03\end{array}$ \\
\hline 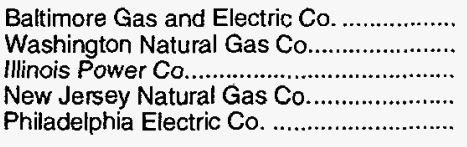 & $\begin{array}{l}\text { MD } \\
\text { WA } \\
\text { IL } \\
\text { NJ } \\
\text { PA }\end{array}$ & $\begin{array}{l}38,678,923 \\
37,126,334 \\
35,923,015 \\
35,915,640 \\
33,609,893\end{array}$ & $\begin{array}{l}6.79 \\
5.88 \\
5.35 \\
8.38 \\
7.48\end{array}$ \\
\hline 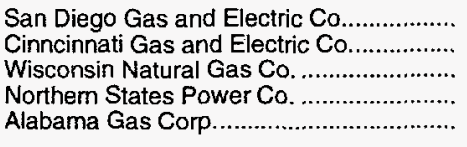 & $\begin{array}{l}\text { CA } \\
\text { OH } \\
\text { WI } \\
\text { MN } \\
\text { AL }\end{array}$ & $\begin{array}{l}33,236,117 \\
32,127,247 \\
31,892,881 \\
31,869,803 \\
30,063,247\end{array}$ & $\begin{array}{l}6.67 \\
6.10 \\
6.30 \\
5.22 \\
7.48\end{array}$ \\
\hline 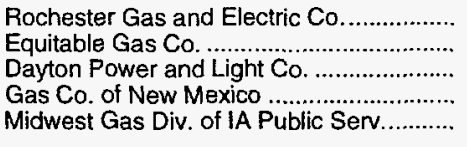 & $\begin{array}{l}\text { NY } \\
\text { PA } \\
\text { OH } \\
\text { NM } \\
\text { IA }\end{array}$ & $\begin{array}{l}28,376,190 \\
28,092,356 \\
27,759,663 \\
26,475,669 \\
26,403,244\end{array}$ & $\begin{array}{l}7.84 \\
9.01 \\
5.66 \\
5.65 \\
5.45\end{array}$ \\
\hline 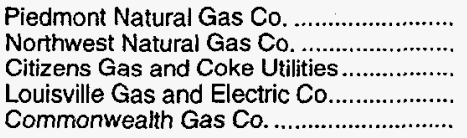 & $\begin{array}{l}\text { NC,SC } \\
\text { OR,WA } \\
\text { IN } \\
\text { KY } \\
\text { MA }\end{array}$ & $\begin{array}{l}25,624,215 \\
25,089,702 \\
25,016,071 \\
22,934,883 \\
21,555,195\end{array}$ & $\begin{array}{l}7.04 \\
7.04 \\
5.93 \\
4.82 \\
9.31\end{array}$ \\
\hline
\end{tabular}

Notes: Natural gas companies with large amounts of on-system sales to residential consumers were selected from the respondents to the Form ElA-176, "Annual Report of Natural and Supplemental Gas Supply and Disposition." Prices are calculated from the company total reported volumes of on-system sales to residential consumers and the total reported associated revenue. When more than one State of operation is indicated, the price has been calculated from the sum of the volumes and revenues reported by the company for all of the States shown.

Source: Energy Information Administration (EIA), Forn EIA-176, "Annual Report of Natural and Supplemental Gas Supply and Disposition." 
Table 31. Selected Volumes and Prices of Natural Gas Sold to Commercial Consumers in the United States, 1994

(Volumes in Thousand Cubic Feet, Prices in Dollars per Thousand Cubic Feet)

\begin{tabular}{|c|c|c|c|}
\hline Company & State & Volume & Price \\
\hline $\begin{array}{l}\text { Public Serv. Elec. and Gas Co.................. } \\
\text { Columbia Gas..................................... } \\
\text { Southern California Gas Co. ................... } \\
\text { Con Edison Co of New York, Inc.............. } \\
\text { Consumers Power Co.............................. }\end{array}$ & $\begin{array}{l}\text { NJ } \\
\text { OH,PA } \\
\text { CA } \\
\text { NY } \\
\text { MI }\end{array}$ & $\begin{array}{l}91,075,256 \\
64,757,126 \\
60,461,429 \\
60,205,645 \\
54,894,076\end{array}$ & $\begin{array}{l}5.66 \\
5.77 \\
6.63 \\
5.68 \\
4.38\end{array}$ \\
\hline 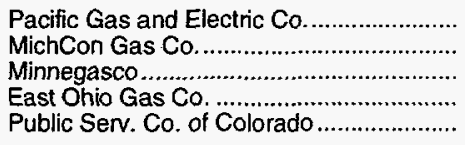 & $\begin{array}{l}\mathrm{CA} \\
\mathrm{MI} \\
\mathrm{MN}, \mathrm{SD} \\
\mathrm{OH} \\
\mathrm{CO}\end{array}$ & $\begin{array}{l}50,790,539 \\
49,042,476 \\
48,505,594 \\
46,831,374 \\
46,178,587\end{array}$ & $\begin{array}{l}7.84 \\
5.04 \\
4.29 \\
5.12 \\
4.24\end{array}$ \\
\hline 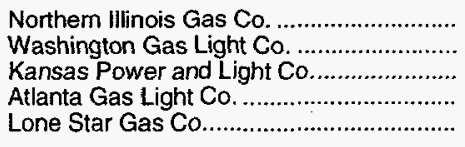 & $\begin{array}{l}\text { IL } \\
\text { MD, VA,DC } \\
\text { KS,MO } \\
\text { GA } \\
\text { TX }\end{array}$ & $\begin{array}{l}45,122,157 \\
43,260,632 \\
41,262,511 \\
41,005,160 \\
37,558,976\end{array}$ & $\begin{array}{l}4.71 \\
5.64 \\
4.68 \\
6.24 \\
5.09\end{array}$ \\
\hline 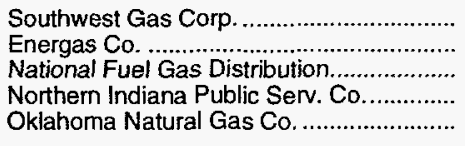 & $\begin{array}{l}\text { AZ,CA,NV } \\
\text { TX } \\
\text { NY,PA } \\
\text { IN } \\
\text { OK }\end{array}$ & $\begin{array}{l}30,880,855 \\
27,705,843 \\
25,814,409 \\
25,658,354 \\
24,942,436\end{array}$ & $\begin{array}{l}5.38 \\
3.57 \\
6.59 \\
5.52 \\
4.69\end{array}$ \\
\hline 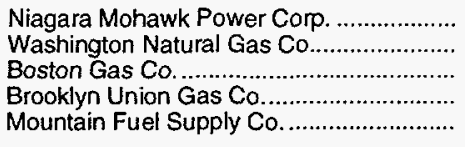 & $\begin{array}{l}\text { NY } \\
\text { WA } \\
\text { MA } \\
\text { NY } \\
\text { UT }\end{array}$ & $\begin{array}{l}24,160,263 \\
24,123,339 \\
23,520,197 \\
21,713,647 \\
21,704,235\end{array}$ & $\begin{array}{l}6.39 \\
5.06 \\
6.13 \\
7.74 \\
3.81\end{array}$ \\
\hline 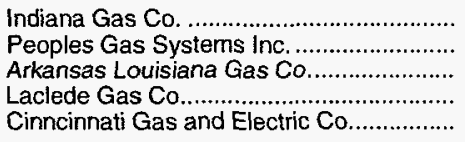 & $\begin{array}{l}\text { IN } \\
\text { FL } \\
\text { AR,LA } \\
\mathrm{MO} \\
\mathrm{OH}\end{array}$ & $\begin{array}{l}21,515,658 \\
21,409,173 \\
21,101,792 \\
21,097,416 \\
20,872,343\end{array}$ & $\begin{array}{l}5.57 \\
5.65 \\
5.15 \\
5.07 \\
5.55\end{array}$ \\
\hline 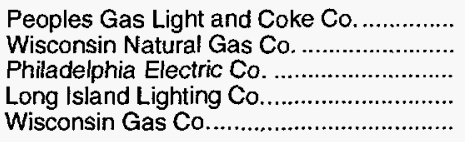 & $\begin{array}{l}\text { IL } \\
\text { WI } \\
\text { PA } \\
\text { NY } \\
\text { WI }\end{array}$ & $\begin{array}{l}20,850,965 \\
19,614,060 \\
19,206,464 \\
19,125,843 \\
18,598,131\end{array}$ & $\begin{array}{l}6.22 \\
5.23 \\
6.49 \\
7.59 \\
5.20\end{array}$ \\
\hline 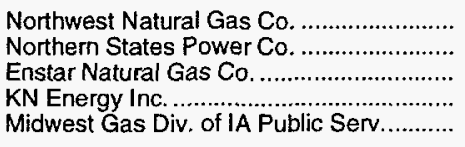 & $\begin{array}{l}\text { OR,WA } \\
\text { MN } \\
\text { AK } \\
\text { CO } \\
\text { IA }\end{array}$ & $\begin{array}{l}18,164,384 \\
16,850,091 \\
16,481,155 \\
14,720,916 \\
14,659,969\end{array}$ & $\begin{array}{l}5.55 \\
4.54 \\
2.85 \\
4.30 \\
4.47\end{array}$ \\
\hline 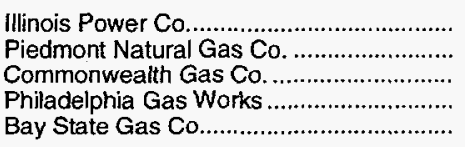 & $\begin{array}{l}\text { IL } \\
\text { NC,SC } \\
\text { MA } \\
\text { PA } \\
\text { MA }\end{array}$ & $\begin{array}{l}14,539,773 \\
14,262,613 \\
13,907,079 \\
13,857,754 \\
13,521,004\end{array}$ & $\begin{array}{l}4.51 \\
5.90 \\
6.80 \\
6.90 \\
7.15\end{array}$ \\
\hline 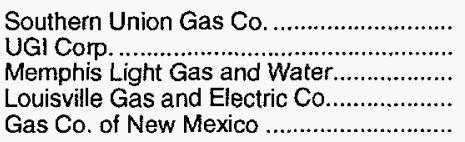 & $\begin{array}{l}\mathrm{AZ} \\
\mathrm{PA} \\
\mathrm{TN} \\
\mathrm{KY} \\
\mathrm{NM}\end{array}$ & $\begin{array}{l}13,221,645 \\
13,155,849 \\
13,115,610 \\
12,717,985 \\
11,756,120\end{array}$ & $\begin{array}{l}4.36 \\
7.15 \\
4.50 \\
4.20 \\
4.47\end{array}$ \\
\hline
\end{tabular}

Notes: Natural gas companies with large amounts of on-system sales to commercial consumers were selected from the respondents to the Form ElA-176, "Annual Report of Natural and Supplemental Gas Supply and Disposition." Prices are calculated from the company total reported volumes of on-system sales to commercial consumers and the total reported associated revenue. When more than one State of operation is indicated, the price has been calculated from the sum of the volumes and revenues reported by the company for all of the States shown.

Source: Energy Information Administration (EIA), Form ElA-176, "Annual Report of Natural and Supplemental Gas Supply and Disposition." 
Table 32. Leading Suppliers of Natural Gas to End Users in the United States, 1994 (Thousand Cubic Feet)

\begin{tabular}{|c|c|c|c|c|c|c|}
\hline Name & Residential & Commercial & Industrial & Vehicle Fuel & Electric Utilities & Total \\
\hline 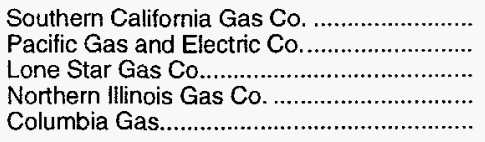 & $\begin{array}{r}256,9199,669 \\
215,8144,272 \\
91,267,209 \\
231, ; 85,861 \\
164,442,595\end{array}$ & $\begin{array}{r}102,559,294 \\
140,821,781 \\
38,974,817 \\
89,599,846 \\
85,969,244\end{array}$ & $\begin{array}{l}244,645,266 \\
163,895,662 \\
323,279,123 \\
136,543,347 \\
152,613,093\end{array}$ & $\begin{array}{r}214,754 \\
284,477 \\
23,027 \\
2,041 \\
7,356\end{array}$ & $\begin{array}{r}260,289,594 \\
272,811,818 \\
85,013,396 \\
30,738,584 \\
235,137\end{array}$ & $\begin{array}{l}864,108,577 \\
793,658,010 \\
538,557,572 \\
488,269,679 \\
403,267,425\end{array}$ \\
\hline 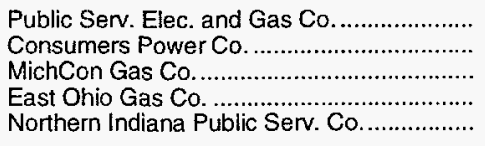 & $\begin{array}{r}138,930,616 \\
172,714,580 \\
151,2441,414 \\
138,679,151 \\
65,885,605\end{array}$ & $\begin{array}{l}99,657,642 \\
70,555,843 \\
93,745,120 \\
55,573,508 \\
25,658,354\end{array}$ & $\begin{array}{r}131,011,967 \\
144,914,905 \\
91,829,832 \\
81,762,960 \\
174,699,560\end{array}$ & $\begin{array}{r}0 \\
401 \\
0 \\
41,630 \\
50,239\end{array}$ & $\begin{array}{r}25,555,657 \\
1,320,114 \\
2,831,198 \\
362,575 \\
8,343,281\end{array}$ & $\begin{array}{l}395,155,882 \\
389,505,843 \\
339,647,564 \\
276,419,824 \\
274,637,039\end{array}$ \\
\hline $\begin{array}{l}\text { Oklahoma Natural Gas Co.......................... } \\
\text { Atlanta Gas Light Co................................. } \\
\text { Peoples Gas Light and Coke Co................ } \\
\text { Con Edison Co of New York, Inc................ } \\
\text { Kansas Power and Light Co........................ }\end{array}$ & $\begin{array}{r}54,420,719 \\
87,-112,759 \\
135,407,045 \\
52,944,060 \\
94,328,824\end{array}$ & $\begin{array}{l}27,051,118 \\
45,309,222 \\
55,050,741 \\
60,205,645 \\
54,690,694\end{array}$ & $\begin{array}{r}161,455,601 \\
95,135,839 \\
33,101,452 \\
8,143,485 \\
58,209,923\end{array}$ & $\begin{array}{r}154,450 \\
317 \\
20,043 \\
4,491 \\
2,235\end{array}$ & $\begin{array}{r}4,457,172 \\
1,136,986 \\
985,307 \\
91,196,465 \\
3,983,800\end{array}$ & $\begin{array}{l}247,539,060 \\
229,295,123 \\
224,564,588 \\
212,494,146 \\
211,215,476\end{array}$ \\
\hline 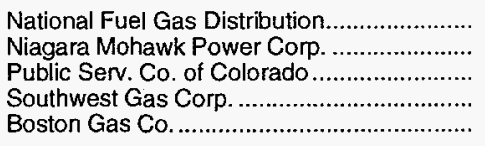 & $\begin{array}{l}87,926,986 \\
54,551,040 \\
75,000,910 \\
44,\{03,552 \\
50,276,879\end{array}$ & $\begin{array}{l}36,192,855 \\
47,953,290 \\
46,178,587 \\
37,974,120 \\
42,774,246\end{array}$ & $\begin{array}{r}47,939,015 \\
54,707,730 \\
40,738,495 \\
42,822,600 \\
8,920,770\end{array}$ & $\begin{array}{r}7,656 \\
24,859 \\
39,857 \\
80,577 \\
1,075\end{array}$ & $\begin{array}{r}0 \\
5,397,336 \\
106,324 \\
34,940,001 \\
32,688,050\end{array}$ & $\begin{array}{l}172,066,512 \\
162,634,255 \\
162,064,173 \\
160,620,850 \\
134,661,020\end{array}$ \\
\hline 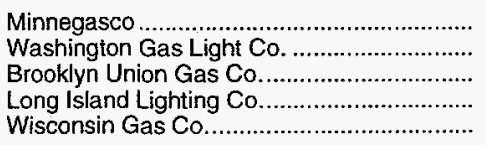 & $\begin{array}{l}65,420,459 \\
75,812,140 \\
98,774,226 \\
39,648,794 \\
46,368,613\end{array}$ & $\begin{array}{l}48,821,594 \\
46,217,192 \\
22,658,547 \\
19,601,979 \\
18,598,131\end{array}$ & $\begin{array}{r}19,711,359 \\
0 \\
6,000,310 \\
19,087,769 \\
54,451,764\end{array}$ & $\begin{array}{r}27,531 \\
11,748 \\
6,751 \\
0 \\
25,063\end{array}$ & $\begin{array}{r}534,070 \\
8,607,799 \\
0 \\
43,202,950 \\
192,621\end{array}$ & $\begin{array}{l}134,515,013 \\
130,648,879 \\
127,439,834 \\
121,541,492 \\
119,636,192\end{array}$ \\
\hline 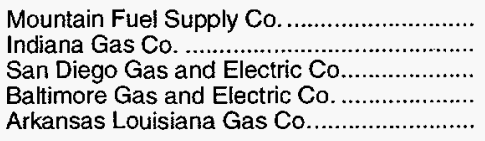 & $\begin{array}{l}48,529,451 \\
43,524,355 \\
33,641,526 \\
38,678,923 \\
47,397,072\end{array}$ & $\begin{array}{l}26,142,554 \\
22,968,194 \\
12,377,852 \\
22,637,894 \\
28,779,652\end{array}$ & $\begin{array}{l}36,452,976 \\
44,141,418 \\
22,360,991 \\
43,175,634 \\
26,222,788\end{array}$ & $\begin{array}{r}49,181 \\
0 \\
36,496 \\
24,158 \\
0\end{array}$ & $\begin{array}{r}7,956,295 \\
611,349 \\
40,171,310 \\
2,436,036 \\
0\end{array}$ & $\begin{array}{l}119,130,457 \\
111,245,316 \\
108,588,175 \\
106,952,645 \\
102,399,512\end{array}$ \\
\hline 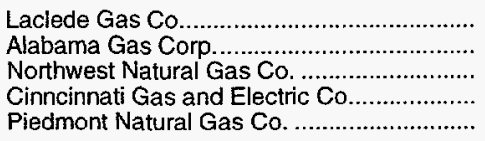 & $\begin{array}{l}56,036,170 \\
30,(063,247 \\
25,089,702 \\
32,127,247 \\
25,1524,215\end{array}$ & $\begin{array}{l}25,285,735 \\
14,580,746 \\
19,518,273 \\
22,444,388 \\
19,786,572\end{array}$ & $\begin{array}{l}18,444,597 \\
48,961,728 \\
51,481,530 \\
36,051,693 \\
45,570,253\end{array}$ & $\begin{array}{r}141 \\
0 \\
17,412 \\
0 \\
5,340\end{array}$ & $\begin{array}{r}0 \\
2,798,541 \\
0 \\
1,530,237 \\
151,866\end{array}$ & $\begin{array}{l}99,766,643 \\
96,404,262 \\
96,106,917 \\
92,153,565 \\
91,138,246\end{array}$ \\
\hline 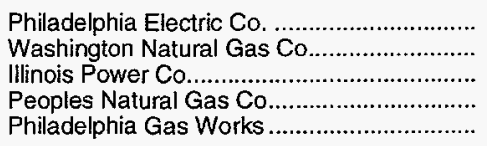 & $\begin{array}{l}33,1509,893 \\
37,126,334 \\
35,1923,015 \\
40,448,314 \\
50,1314,576\end{array}$ & $\begin{array}{l}22,992,231 \\
25,377,527 \\
15,189,038 \\
15,991,732 \\
13,952,228\end{array}$ & $\begin{array}{l}20,500,025 \\
24,292,701 \\
33,580,799 \\
19,972,959 \\
12,176,160\end{array}$ & $\begin{array}{r}799 \\
152,427 \\
1,409 \\
61 \\
2,618\end{array}$ & $\begin{array}{r}12,907,899 \\
0 \\
475,430 \\
734,350 \\
0\end{array}$ & $\begin{array}{l}90,010,847 \\
86,948,989 \\
85,169,691 \\
77,147,416 \\
76,945,582\end{array}$ \\
\hline 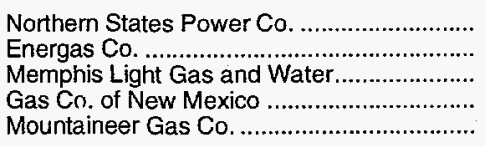 & $\begin{array}{l}31,1369,803 \\
20,1563,685 \\
20,298,638 \\
26,475,755 \\
18,489,640\end{array}$ & $\begin{array}{l}18,090,120 \\
27,705,843 \\
13,614,797 \\
21,151,240 \\
16,150,339\end{array}$ & $\begin{array}{l}19,349,961 \\
20,395,735 \\
31,214,710 \\
12,801,813 \\
25,899,298\end{array}$ & $\begin{array}{r}0 \\
16,031 \\
1,162 \\
60,511 \\
0\end{array}$ & $\begin{array}{r}536,912 \\
0 \\
971,362 \\
2,761,167 \\
0\end{array}$ & $\begin{array}{l}69,846,796 \\
68,781,294 \\
66,100,669 \\
63,250,486 \\
60,539,277\end{array}$ \\
\hline $\begin{array}{l}\text { Midwest Gas Div. of IA Public Serv............... } \\
\text { Dayton Power and Light Co......................... } \\
\text { New Jersey Natural Gas Co........................ } \\
\text { Citizens Gas and Coke Utilities .................... } \\
\text { Rochester Gas and Electric Co................... }\end{array}$ & $\begin{array}{l}26,403,244 \\
27,759,663 \\
35,915,640 \\
25,116,071 \\
28,376,190\end{array}$ & $\begin{array}{l}16,796,626 \\
13,656,236 \\
11,147,768 \\
15,533,480 \\
13,810,888\end{array}$ & $\begin{array}{r}15,237,579 \\
15,663,630 \\
4,870,440 \\
11,205,605 \\
8,843,937\end{array}$ & $\begin{array}{r}3,129 \\
0 \\
4,261 \\
4,347 \\
5,932\end{array}$ & $\begin{array}{r}1,224,922 \\
574,277 \\
2,213,695 \\
0 \\
0\end{array}$ & $\begin{array}{l}59,665,500 \\
57,653,806 \\
54,151,804 \\
51,759,503 \\
51,036,947\end{array}$ \\
\hline
\end{tabular}

Notes: The natural gas companies with the 50 largest amounts of total deliveries were selected from the respondents to the Form ElA-176, "Annual Report of Natural and Supplemental Gas Supply and Disposition." For each company, the total deliveries include deliveries to residential, commercial, industrial, and electric utility consumers, deliveries of natural gas for vehicle fuel; and all volumes transported to consumers for the account of others. The totals are summed from each company's reports in all States of operations. Number of vehicle fuel consumers generally refers to the number of fueling stations. Totals may not equal sum of components due to independent rounding. Source: Energy Information Administration (EIA), FormI EIA-176, "Annual Report of Natural and Supplemental Gas Supply and Disposition." 
Table 33. Average Residential Annual Consumption and Cost of Natural Gas per Consumer by State, 1990-1994

\begin{tabular}{|c|c|c|c|c|c|c|c|c|c|c|}
\hline \multirow[b]{2}{*}{ State } & \multicolumn{2}{|c|}{1990} & \multicolumn{2}{|c|}{1991} & \multicolumn{2}{|c|}{1992} & \multicolumn{2}{|c|}{1993} & \multicolumn{2}{|c|}{1994} \\
\hline & $\begin{array}{l}\text { Volume } \\
\text { (thousand } \\
\text { cubic feet) }\end{array}$ & $\begin{array}{c}\text { Cost } \\
\text { (dollars) }\end{array}$ & $\begin{array}{l}\text { Volume } \\
\text { (thousand } \\
\text { cubic feet) }\end{array}$ & $\begin{array}{c}\text { Cost } \\
\text { (dollars) }\end{array}$ & $\begin{array}{l}\text { Volume } \\
\text { (thousand } \\
\text { cubic feet) }\end{array}$ & $\begin{array}{c}\text { Cost } \\
\text { (dollars) }\end{array}$ & $\begin{array}{l}\text { Volume } \\
\text { (thousand } \\
\text { cubic feet) }\end{array}$ & $\underset{\text { (dollars) }}{\text { Cost }}$ & $\begin{array}{l}\text { Volume } \\
\text { (thousand } \\
\text { cubic feet) }\end{array}$ & $\begin{array}{c}\text { Cost } \\
\text { (dollars) }\end{array}$ \\
\hline $\begin{array}{l}\text { Alabama ............. } \\
\text { Alaska.................. } \\
\text { Arizona ............... } \\
\text { Arkansas ............. } \\
\text { California ............ }\end{array}$ & $\begin{array}{r}66 \\
200 \\
52 \\
80 \\
61\end{array}$ & $\begin{array}{l}435 \\
759 \\
354 \\
409 \\
350\end{array}$ & $\begin{array}{r}66 \\
187 \\
49 \\
82 \\
59\end{array}$ & $\begin{array}{l}464 \\
781 \\
341 \\
408 \\
369\end{array}$ & $\begin{array}{r}70 \\
193 \\
47 \\
78 \\
55\end{array}$ & $\begin{array}{l}471 \\
732 \\
340 \\
399 \\
330\end{array}$ & $\begin{array}{r}70 \\
183 \\
46 \\
89 \\
57\end{array}$ & $\begin{array}{l}499 \\
724 \\
332 \\
477 \\
358\end{array}$ & $\begin{array}{r}67 \\
192 \\
47 \\
80 \\
59\end{array}$ & $\begin{array}{l}495 \\
690 \\
352 \\
455 \\
378\end{array}$ \\
\hline $\begin{array}{l}\text { Colorado.............. } \\
\text { Connecticut ......... } \\
\text { D.C. ....................... } \\
\text { Delaware .............. } \\
\text { Florida ................... }\end{array}$ & $\begin{array}{r}93 \\
87 \\
111 \\
82 \\
28\end{array}$ & $\begin{array}{l}427 \\
749 \\
798 \\
501 \\
240\end{array}$ & $\begin{array}{r}97 \\
86 \\
112 \\
79 \\
28\end{array}$ & $\begin{array}{l}446 \\
756 \\
791 \\
460 \\
248\end{array}$ & $\begin{array}{r}93 \\
98 \\
122 \\
87 \\
30\end{array}$ & $\begin{array}{l}422 \\
879 \\
925 \\
535 \\
277\end{array}$ & $\begin{array}{r}102 \\
99 \\
122 \\
86 \\
R 29\end{array}$ & $\begin{array}{r}460 \\
931 \\
1,017 \\
573 \\
288\end{array}$ & $\begin{array}{r}93 \\
97 \\
117 \\
85 \\
28\end{array}$ & $\begin{array}{l}456 \\
985 \\
974 \\
633 \\
278\end{array}$ \\
\hline $\begin{array}{l}\text { Georgia................ } \\
\text { Hawaii................... } \\
\text { Idaho ................... } \\
\text { Illinois ................... } \\
\text { Indiana................. }\end{array}$ & $\begin{array}{r}68 \\
19 \\
75 \\
135 \\
106\end{array}$ & $\begin{array}{l}461 \\
319 \\
379 \\
681 \\
570\end{array}$ & $\begin{array}{r}71 \\
18 \\
81 \\
141 \\
108\end{array}$ & $\begin{array}{l}475 \\
421 \\
420 \\
696 \\
588\end{array}$ & $\begin{array}{r}77 \\
18 \\
71 \\
142 \\
111\end{array}$ & $\begin{array}{l}499 \\
334 \\
371 \\
721 \\
602\end{array}$ & $\begin{array}{r}81 \\
19 \\
85 \\
146 \\
117\end{array}$ & $\begin{array}{l}550 \\
326 \\
455 \\
807 \\
673\end{array}$ & $\begin{array}{r}72 \\
19 \\
75 \\
139 \\
109\end{array}$ & $\begin{array}{l}529 \\
318 \\
399 \\
763 \\
683\end{array}$ \\
\hline 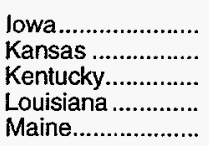 & $\begin{array}{r}101 \\
95 \\
90 \\
57 \\
54\end{array}$ & $\begin{array}{l}504 \\
428 \\
442 \\
348 \\
411\end{array}$ & $\begin{array}{r}110 \\
99 \\
94 \\
58 \\
58\end{array}$ & $\begin{array}{l}530 \\
435 \\
457 \\
336 \\
399\end{array}$ & $\begin{array}{r}103 \\
94 \\
96 \\
59 \\
63\end{array}$ & $\begin{array}{l}537 \\
441 \\
481 \\
329 \\
440\end{array}$ & $\begin{array}{r}R_{113} \\
109 \\
102 \\
60 \\
65\end{array}$ & $\begin{array}{l}617 \\
536 \\
536 \\
367 \\
485\end{array}$ & $\begin{array}{r}104 \\
96 \\
94 \\
56 \\
63\end{array}$ & $\begin{array}{l}563 \\
490 \\
511 \\
349 \\
496\end{array}$ \\
\hline $\begin{array}{l}\text { Maryland............. } \\
\text { Massachusetts .... } \\
\text { Michigan .............. } \\
\text { Minnesota............ } \\
\text { Mississippi ........... }\end{array}$ & $\begin{array}{r}86 \\
95 \\
127 \\
113 \\
66\end{array}$ & $\begin{array}{l}553 \\
746 \\
639 \\
523 \\
349\end{array}$ & $\begin{array}{r}88 \\
91 \\
129 \\
121 \\
67\end{array}$ & $\begin{array}{l}545 \\
741 \\
655 \\
545 \\
348\end{array}$ & $\begin{array}{r}84 \\
105 \\
136 \\
114 \\
68\end{array}$ & $\begin{array}{l}540 \\
833 \\
686 \\
552 \\
335\end{array}$ & $\begin{array}{r}95 \\
105 \\
138 \\
115 \\
71\end{array}$ & $\begin{array}{l}675 \\
874 \\
696 \\
610 \\
372\end{array}$ & $\begin{array}{r}93 \\
101 \\
134 \\
117 \\
67\end{array}$ & $\begin{array}{l}647 \\
907 \\
669 \\
604 \\
365\end{array}$ \\
\hline $\begin{array}{l}\text { Missouri................ } \\
\text { Montana .............. } \\
\text { Nebraska .............. } \\
\text { Nevada ................ } \\
\text { New Hampshire... }\end{array}$ & $\begin{array}{r}96 \\
97 \\
102 \\
67 \\
90\end{array}$ & $\begin{array}{l}498 \\
444 \\
469 \\
379 \\
670\end{array}$ & $\begin{array}{r}100 \\
104 \\
108 \\
68 \\
82\end{array}$ & $\begin{array}{l}512 \\
468 \\
502 \\
379 \\
589\end{array}$ & $\begin{array}{l}96 \\
91 \\
99 \\
61 \\
93\end{array}$ & $\begin{array}{l}488 \\
439 \\
477 \\
344 \\
702\end{array}$ & $\begin{array}{r}109 \\
108 \\
117 \\
68 \\
92\end{array}$ & $\begin{array}{l}587 \\
530 \\
579 \\
385 \\
701\end{array}$ & $\begin{array}{r}96 \\
96 \\
104 \\
63 \\
90\end{array}$ & $\begin{array}{l}519 \\
503 \\
520 \\
421 \\
720\end{array}$ \\
\hline $\begin{array}{l}\text { New Jersey.......... } \\
\text { New MexicG ........ } \\
\text { New York............ } \\
\text { North Carolina ..... } \\
\text { North Dakota ....... }\end{array}$ & $\begin{array}{r}87 \\
76 \\
86 \\
67 \\
107\end{array}$ & $\begin{array}{l}571 \\
432 \\
639 \\
415 \\
504\end{array}$ & $\begin{array}{r}88 \\
78 \\
76 \\
70 \\
118\end{array}$ & $\begin{array}{l}593 \\
424 \\
557 \\
439 \\
568\end{array}$ & $\begin{array}{r}98 \\
81 \\
84 \\
74 \\
108\end{array}$ & $\begin{array}{l}678 \\
383 \\
635 \\
489 \\
541\end{array}$ & $\begin{array}{r}95 \\
80 \\
96 \\
78 \\
117\end{array}$ & $\begin{array}{l}664 \\
437 \\
784 \\
542 \\
614\end{array}$ & $\begin{array}{r}104 \\
75 \\
96 \\
73 \\
114\end{array}$ & $\begin{array}{l}738 \\
423 \\
841 \\
531 \\
592\end{array}$ \\
\hline $\begin{array}{l}\text { Ohio } \\
\text { Oklahoma ............... } \\
\text { Oregon .............. } \\
\text { Pennsylvania ....... } \\
\text { Rhode Island ....... }\end{array}$ & $\begin{array}{r}111 \\
81 \\
72 \\
104 \\
91\end{array}$ & $\begin{array}{l}590 \\
387 \\
449 \\
686 \\
656\end{array}$ & $\begin{array}{r}115 \\
84 \\
70 \\
104 \\
88\end{array}$ & $\begin{array}{l}606 \\
396 \\
429 \\
703 \\
675\end{array}$ & $\begin{array}{r}121 \\
79 \\
65 \\
113 \\
101\end{array}$ & $\begin{array}{l}627 \\
392 \\
403 \\
745 \\
776\end{array}$ & $\begin{array}{r}123 \\
93 \\
80 \\
113 \\
99\end{array}$ & $\begin{array}{l}705 \\
459 \\
515 \\
771 \\
811\end{array}$ & $\begin{array}{r}118 \\
82 \\
74 \\
112 \\
87\end{array}$ & $\begin{array}{l}691 \\
450 \\
515 \\
835 \\
789\end{array}$ \\
\hline $\begin{array}{l}\text { South Carolina..... } \\
\text { South Dakota....... } \\
\text { Tennessee........... } \\
\text { Texas.................... } \\
\text { Utah.................... }\end{array}$ & $\begin{array}{l}54 \\
97 \\
74 \\
65 \\
96\end{array}$ & $\begin{array}{l}388 \\
498 \\
378 \\
376 \\
506\end{array}$ & $\begin{array}{r}57 \\
104 \\
75 \\
68 \\
111\end{array}$ & $\begin{array}{l}397 \\
514 \\
388 \\
388 \\
603\end{array}$ & $\begin{array}{l}63 \\
98 \\
75 \\
65 \\
96\end{array}$ & $\begin{array}{l}440 \\
503 \\
412 \\
377 \\
520\end{array}$ & $\begin{array}{r}66 \\
97 \\
80 \\
69 \\
107\end{array}$ & $\begin{array}{l}469 \\
515 \\
457 \\
409 \\
549\end{array}$ & $\begin{array}{r}56 \\
101 \\
75 \\
64 \\
97\end{array}$ & $\begin{array}{l}431 \\
532 \\
458 \\
382 \\
482\end{array}$ \\
\hline $\begin{array}{l}\text { Vermont............... } \\
\text { Virginia ................. } \\
\text { Washington ......... } \\
\text { West Virginia ....... } \\
\text { Wisconsin .............. } \\
\text { Wyoming .............. }\end{array}$ & $\begin{array}{r}117 \\
83 \\
88 \\
93 \\
102 \\
101\end{array}$ & $\begin{array}{l}680 \\
557 \\
442 \\
602 \\
582 \\
489\end{array}$ & $\begin{array}{r}111 \\
83 \\
94 \\
93 \\
108 \\
106\end{array}$ & $\begin{array}{l}691 \\
566 \\
440 \\
605 \\
605 \\
501\end{array}$ & $\begin{array}{r}123 \\
94 \\
81 \\
100 \\
104 \\
95\end{array}$ & $\begin{array}{l}825 \\
628 \\
407 \\
632 \\
613 \\
448\end{array}$ & $\begin{array}{r}117 \\
95 \\
94 \\
100 \\
107 \\
109\end{array}$ & $\begin{array}{l}727 \\
713 \\
492 \\
643 \\
676 \\
521\end{array}$ & $\begin{array}{r}108 \\
90 \\
88 \\
100 \\
102 \\
99\end{array}$ & $\begin{array}{l}751 \\
690 \\
501 \\
664 \\
642 \\
503\end{array}$ \\
\hline Average ................ & 87 & 507 & 88 & 514 & 90 & 528 & 94 & 582 & 91 & 582 \\
\hline
\end{tabular}

$\mathrm{R}$ = Revised data.

Source: Energy Information Administration (EIA), Form EIA-176, "Annual Report of Natural and Supplemental Gas Supply and Disposition." 



\section{Census Division Summary}

The West South Central Census Division produced 13.6 trillion cubic feet of natural gas during 1994. This was 69 percent of the marketed production of natural gas in the United States, and the region showed an increase in production of 1 percent from the previous year, compared to the 2 percent increase in production nationally. This census division is comprised of the three States with the largest amounts of gas production: Louisiana, Oklahoma, and Texas. In the Mountain Census Division, the second leading producing area, production held at 3.0 trillion cubic feet. New Mexico and Wyoming, ranked fourth and sixth in natural gas production, are located in this census division.
During 1994 as in previous years, the West South Central Census Division continued to lead in consumption of natural gas at 5.3 trillion cubic feet, 28 percent of the Nation's consumption. Industrial consumers used 59 percent of this gas. The East North Central Census Division was the second leading consuming region among all census divisions. The use of natural gas in residences was the largest part of its consumption.

Detailed information on natural gas supply, disposition, and prices for each census division for 1990 to 1994 is presented in Tables 34 through 45.

Figure 19. Marketed Production of Natural Gas by Census Division, 1994 (Million Cubic Feet)
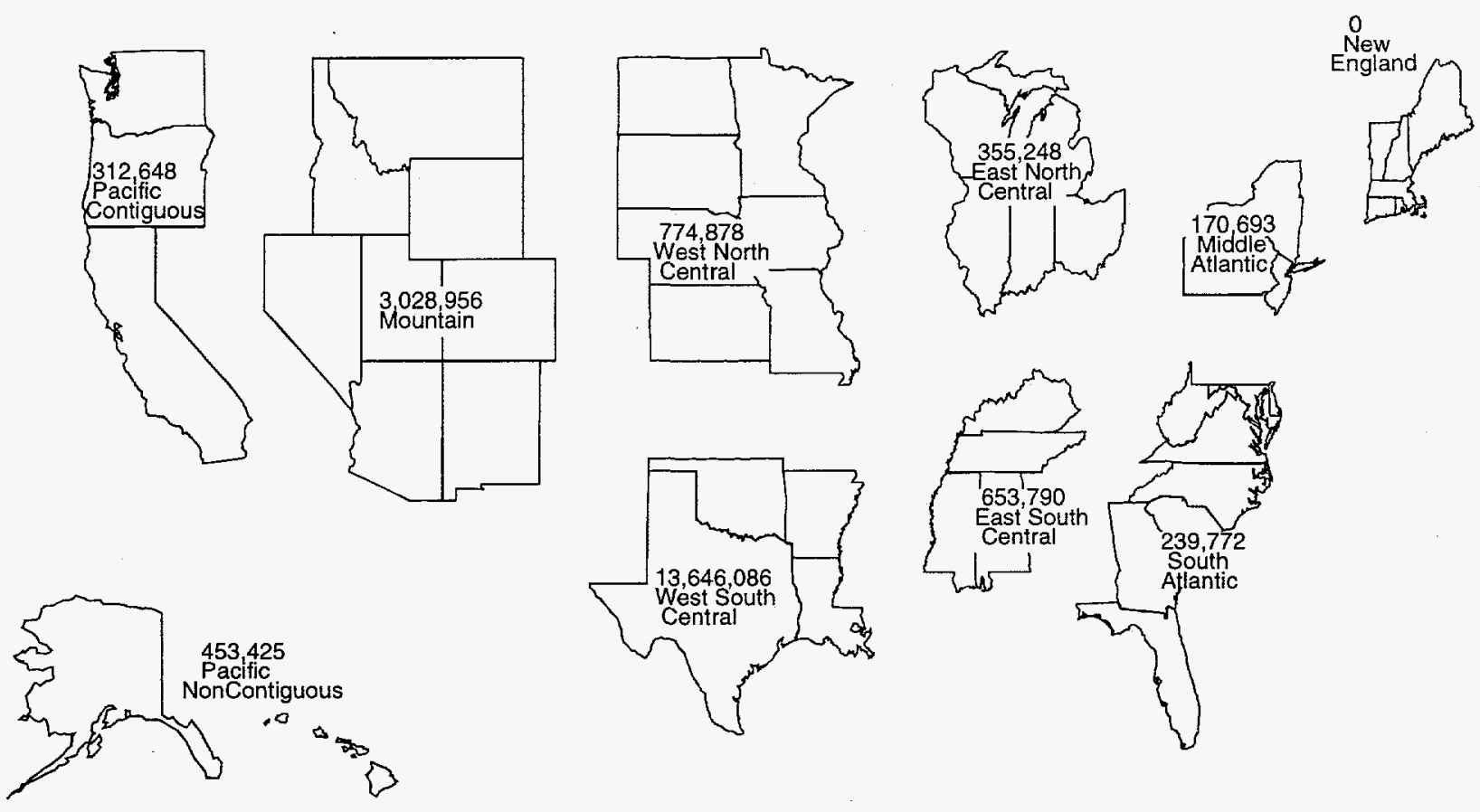

Sources: Illinois, Lotisiana, Pennsylvania, South Dakota, and West Virginia, 1994: Energy Information Administration (EIA), U.S. Crude Oil, Natural Gas, and Natural Gas Liquids Reserves, 1994 Annual Report, DOE/EIA-0216(94); and EIA computations. All other data: Form EIA-627, "Annual Quantity and Value of Natural Gas Report" and the United States Minerals Management Service. 
Table 34. Natural Gas Delivered to Consumers by Census Division, 1994

\begin{tabular}{|c|c|c|c|c|c|c|}
\hline \multirow[b]{2}{*}{ Census Division } & \multicolumn{2}{|c|}{ Residential } & \multicolumn{2}{|c|}{ Commercial } & \multicolumn{2}{|r|}{ Industrial } \\
\hline & $\begin{array}{c}\text { Quantity } \\
\text { (million cubic } \\
\text { feet) }\end{array}$ & Consumers & $\begin{array}{c}\text { Quantity } \\
\text { (million cubic } \\
\text { feet) }\end{array}$ & Consumers & $\begin{array}{c}\text { Quantity } \\
\text { (million cubic } \\
\text { feet }\end{array}$ & Consumers \\
\hline 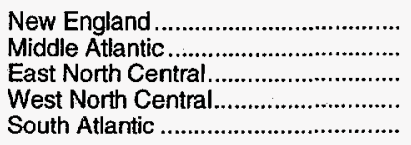 & $\begin{array}{r}188,530 \\
870,685 \\
1,467,349 \\
464,344 \\
391,714\end{array}$ & $\begin{array}{r}1,918,291 \\
8,491,832 \\
11,749,087 \\
4,495,448 \\
5,161,109\end{array}$ & $\begin{array}{l}147,127 \\
493,738 \\
701,870 \\
310,307 \\
293,056\end{array}$ & $\begin{array}{l}180,043 \\
727,372 \\
969,251 \\
499,138 \\
482,552\end{array}$ & $\begin{array}{r}172,631 \\
641,701 \\
1,349,297 \\
511,094 \\
690,558\end{array}$ & $\begin{array}{l}13,274 \\
38,829 \\
59,407 \\
12,557 \\
12,786\end{array}$ \\
\hline $\begin{array}{l}\text { East South Central .............................. } \\
\text { West South Central ............................ } \\
\text { Mountain......................................... } \\
\text { Pacific Contiguous............................ } \\
\text { Pacific Noncontiguous..................... }\end{array}$ & $\begin{array}{r}196,700 \\
377,151 \\
272,805 \\
602,951 \\
15,473\end{array}$ & $\begin{array}{r}2,586,801 \\
5,663,010 \\
3,432,387 \\
9,786,893 \\
108,284\end{array}$ & $\begin{array}{r}132,262 \\
268,330 \\
197,515 \\
327,931 \\
22,898\end{array}$ & $\begin{array}{r}275,528 \\
523,570 \\
331,808 \\
532,958 \\
15,280\end{array}$ & $\begin{array}{r}480,551 \\
3,158,342 \\
285,475 \\
826,923 \\
61,404\end{array}$ & $\begin{array}{r}7,864 \\
11,229 \\
3,895 \\
43,101 \\
11\end{array}$ \\
\hline \multirow[t]{3}{*}{ Total } & $4,847,702$ & $53,393,142$ & $2,895,034$ & $4,537,500$ & $8,177,975$ & 202,953 \\
\hline & Vehicle Fuel & \multicolumn{2}{|r|}{ Electric Utilities } & $\begin{array}{l}\text { Delivered to } \\
\text { Consumers }\end{array}$ & \multirow{2}{*}{(} & \multirow{2}{*}{$\begin{array}{c}\text { Heating Value } \\
\text { (Btu per cubic foot) }\end{array}$} \\
\hline & $\begin{array}{c}\text { Quantity } \\
\text { (milliorı cubic } \\
\text { feat) }\end{array}$ & \multicolumn{2}{|r|}{$\begin{array}{c}\text { Quantity } \\
\text { (million cubic } \\
\text { feet) }\end{array}$} & $\begin{array}{c}\text { Quantity } \\
\text { (million cubic } \\
\text { feet) }\end{array}$ & & \\
\hline 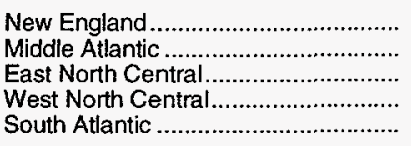 & $\begin{array}{r}6 \\
67 \\
185 \\
67 \\
126\end{array}$ & \multicolumn{2}{|r|}{$\begin{array}{r}48,558 \\
237,862 \\
68,371 \\
43,374 \\
235,179\end{array}$} & $\begin{array}{r}556,852 \\
2,244,053 \\
3,587,073 \\
1,329,186 \\
1,610,633\end{array}$ & \multicolumn{2}{|r|}{$\begin{array}{l}1,026 \\
1,033 \\
1,022 \\
1,004 \\
1,042\end{array}$} \\
\hline 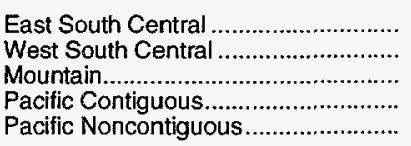 & $\begin{array}{r}19 \\
224 \\
312 \\
723 \\
-\end{array}$ & \multicolumn{2}{|r|}{$\begin{array}{r}87,745 \\
1,504,407 \\
102,719 \\
629,883 \\
29,048\end{array}$} & $\begin{array}{r}897,277 \\
5,308,454 \\
858,826 \\
2,388,411 \\
128,823\end{array}$ & \multicolumn{2}{|r|}{$\begin{array}{l}1,038 \\
1,036 \\
1,027 \\
1,025 \\
1,002\end{array}$} \\
\hline 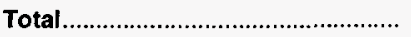 & 1,730 & \multicolumn{2}{|r|}{$2,987,146$} & $18,909,587$ & \multicolumn{2}{|r|}{1,028} \\
\hline
\end{tabular}

Notes: Number of vehicle fuel consumers generally refers to the number of fueling stations. Totals may not equal sum of components due to independent rounding.

Sources: Energy Information Administration (EIA), Forrn EIA.759, "Monthly Power Plant Report" and Form ElA-176, "Annual Report of Natural and Supplemental Gas Supply and Disposition." 
Table 35. Average Price of Natural Gas Delivered to Consumers by Census Division, 1993-1994 (Dollars per Thousand Cubic Feet)

\begin{tabular}{|c|c|c|c|c|c|c|}
\hline \multirow{2}{*}{ Census Division } & \multicolumn{3}{|c|}{ Residential } & \multicolumn{3}{|c|}{ Commercial } \\
\hline & \multicolumn{2}{|c|}{1993} & 1994 & \multicolumn{2}{|c|}{1993} & 1994 \\
\hline 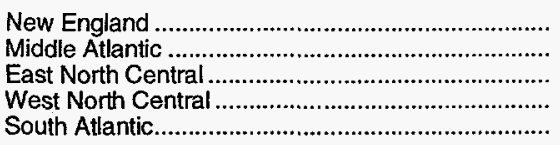 & \multicolumn{2}{|c|}{$\begin{array}{r}8.50 \\
7.46 \\
5.54 \\
5.25 \\
\mathbf{R} 7.15\end{array}$} & $\begin{array}{l}9.16 \\
7.94 \\
5.61 \\
5.26 \\
7.39\end{array}$ & \multicolumn{2}{|c|}{$\begin{array}{l}6.43 \\
5.95 \\
5.03 \\
4.47 \\
5.72\end{array}$} & $\begin{array}{l}7.04 \\
6.37 \\
5.08 \\
4.44 \\
5.77\end{array}$ \\
\hline \multirow[t]{3}{*}{ 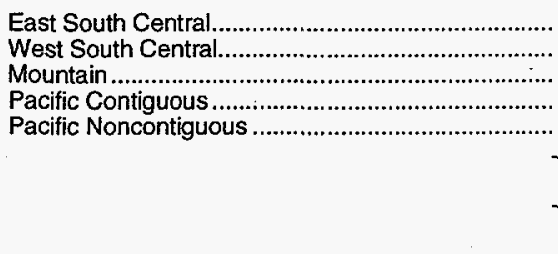 } & \multicolumn{2}{|c|}{$\begin{array}{l}5.84 \\
5.69 \\
5.17 \\
6.15 \\
4.49\end{array}$} & $\begin{array}{l}6.15 \\
5.90 \\
5.47 \\
6.35 \\
4.09\end{array}$ & \multicolumn{2}{|c|}{$\begin{array}{r}5.16 \\
R_{4.54} \\
4.33 \\
5.70 \\
3.75\end{array}$} & $\begin{array}{l}5.38 \\
4.53 \\
4.61 \\
6.46 \\
3.43\end{array}$ \\
\hline & \multicolumn{2}{|c|}{ Industrial } & \multicolumn{2}{|c|}{ Vehicle Fuel } & \multicolumn{2}{|c|}{ Electric Utilities } \\
\hline & 1993 & 1994 & 1993 & 1994 & 1993 & 1994 \\
\hline 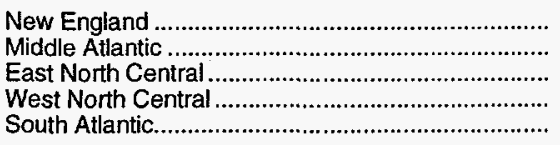 & $\begin{array}{r}4.88 \\
4.00 \\
3.95 \\
3.33 \\
\text { P }_{3} .70\end{array}$ & $\begin{array}{l}4.75 \\
4.02 \\
4.03 \\
3.27 \\
3.56\end{array}$ & $\begin{array}{l}6.27 \\
5.06 \\
4.62 \\
4.35 \\
4.05\end{array}$ & $\begin{array}{l}6.63 \\
4.11 \\
4.28 \\
2.99 \\
3.94\end{array}$ & $\begin{array}{l}2.73 \\
2.67 \\
1.92 \\
2.41 \\
2.48\end{array}$ & $\begin{array}{l}2.26 \\
2.28 \\
1.95 \\
2.02 \\
2.26\end{array}$ \\
\hline 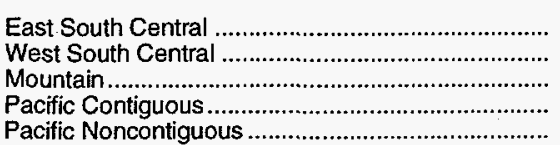 & $\begin{array}{r}\mathbf{R}_{3.47} \\
\mathbf{R}_{2.44} \\
2.84 \\
2.93 \\
1.29\end{array}$ & $\begin{array}{l}3.44 \\
2.21 \\
2.88 \\
3.21 \\
1.42\end{array}$ & $\begin{array}{r}4.70 \\
2.83 \\
4.11 \\
4.63 \\
--\end{array}$ & $\begin{array}{r}4.00 \\
2.57 \\
4.26 \\
4.85 \\
--\end{array}$ & $\begin{array}{r}R_{2.45} \\
2.55 \\
2.48 \\
3.03 \\
0.64\end{array}$ & $\begin{array}{l}2.01 \\
2.25 \\
2.09 \\
2.54 \\
0.72\end{array}$ \\
\hline
\end{tabular}

R = Revised data.

Note: All average prices are volume weighted.

Sources: Energy information Administration (EIA), Form FERC-423, "Monthly Report of Cost and Quality of Fuels for Electric Plants" and Form ElA-176, "Annual Report of Natural and Supplemental Gas Supply and Disposition."

Figure 20. Natural Gas Delivered to Consumers by Census Division, 1994

(Million Cubic Feet)
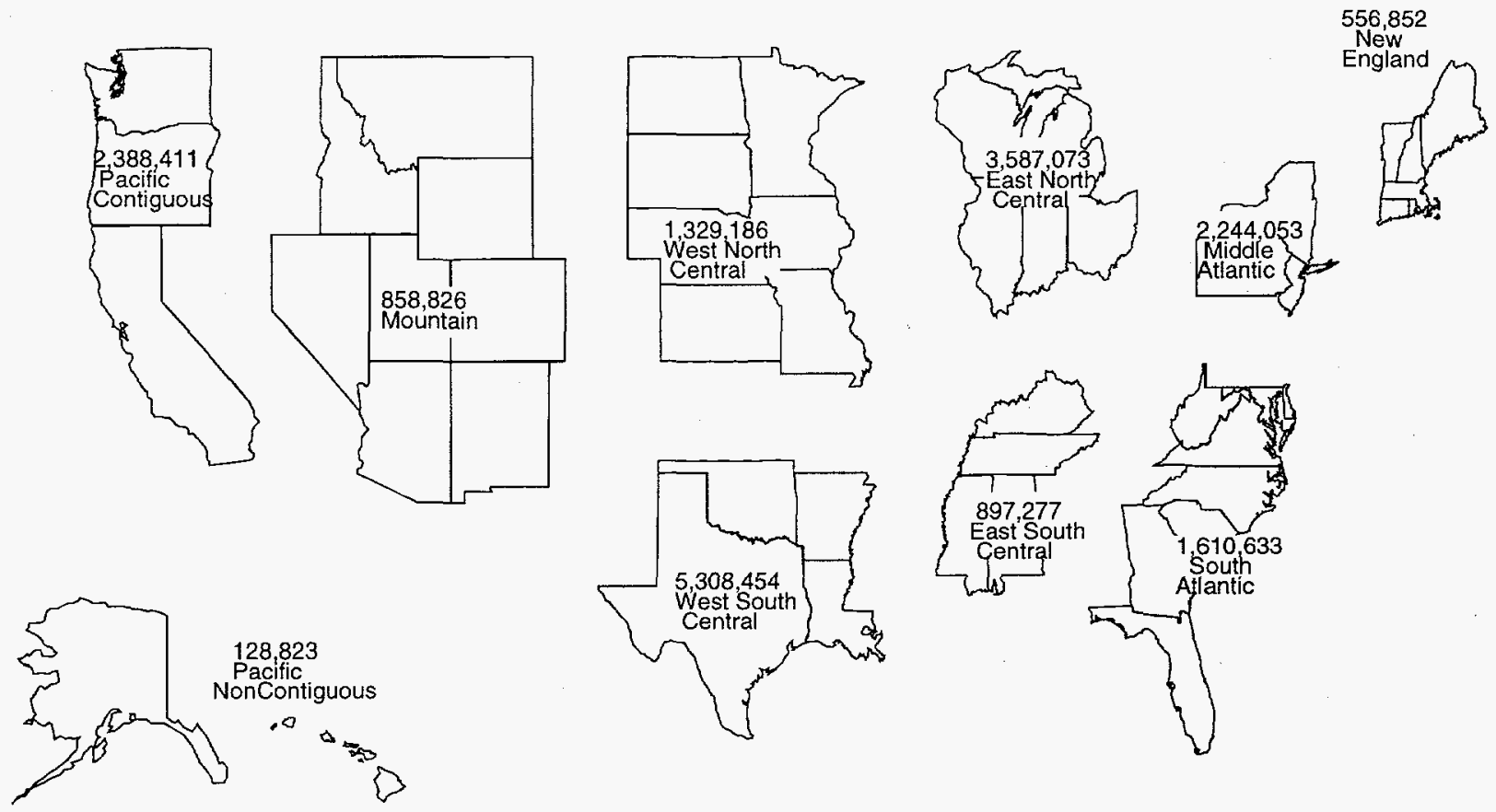

Sources: Energy Information Administration (EIA), Form ElA-759, "Monthly Power Plant Peport" and Form ElA-176, "Annual Report of Natural and Supplemental Gas Supply and Disposition." 


\section{New England - Natural Gas 1994}

$\begin{array}{llll}\text { Million } & \text { Percent of } & \text { Million } & \text { Percent of } \\ \text { Cu. Feet } & \text { National Total } & \text { Cu. Feet } & \text { National Total }\end{array}$

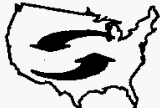

Net Interregion

Movements:

473,595

Marketed

Production:

0

0.00

Deliveries to Consumers:

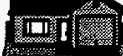

Residential:

188,530

3.89

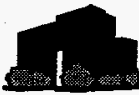

Total:

Ja. Industrial:

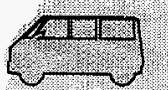

Vehicle Fuel:

6

0.35

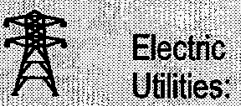

48,558

Table 36. Summary Statistics for Natural Gas - Now England, 1990-1994

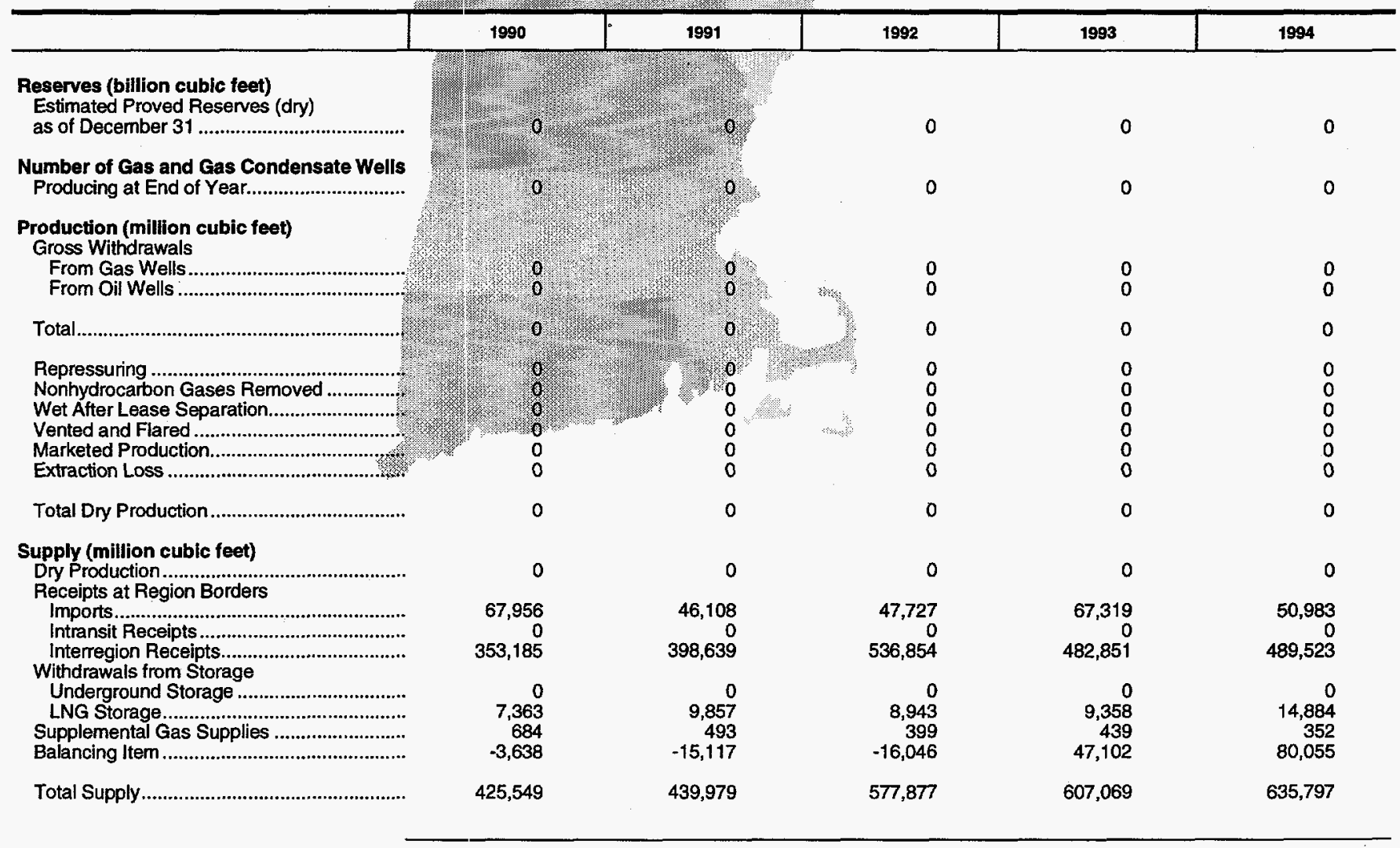

See footnotes at end of table. 
Table 36. Summary Statistics for Natural Gas - New England, 1990-1994 (Continued)

\begin{tabular}{|c|c|c|c|c|c|}
\hline & 1990 & 1991 & 1992 & 1993 & 1994 \\
\hline \multicolumn{6}{|l|}{ Disposition (million cubic feet) } \\
\hline $\begin{array}{l}\text { Consumption.................................................. } \\
\text { Deliveries at Region Borders }\end{array}$ & 417,029 & 433,744 & 513,723 & 528,517 & 560,718 \\
\hline 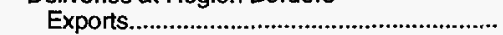 & 0 & 0 & 0 & 0 & 0 \\
\hline 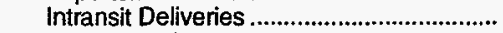 & 0 & 0 & 0 & 0 & 0 \\
\hline Interregion Deliveries...................................... & 78 & 53 & 57,826 & 71,931 & 66,911 \\
\hline \multicolumn{6}{|l|}{ Additions to Storage } \\
\hline 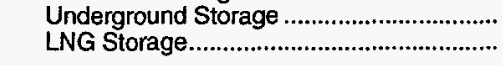 & $\begin{array}{r}0 \\
8,443\end{array}$ & $\begin{array}{r}0 \\
6,182\end{array}$ & $\begin{array}{r}0 \\
6,328\end{array}$ & $\begin{array}{r}0 \\
6,621\end{array}$ & 8,168 \\
\hline Total Disposition & 425,549 & 439,979 & 577,877 & 607,069 & 635,797 \\
\hline \multicolumn{6}{|l|}{ Consumption (million cubic feet) } \\
\hline 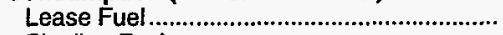 & 0 & 0 & 0 & 0 & 0 \\
\hline 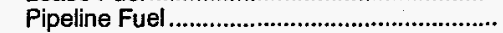 & 1,872 & 2,235 & 2,796 & 3,253 & 3,866 \\
\hline 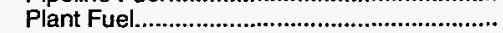 & 0 & 0 & 0 & 0 & 0 \\
\hline \multicolumn{6}{|l|}{ Delivered to Consumers } \\
\hline 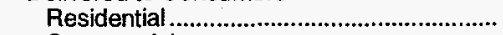 & 170,679 & 166,036 & 191,906 & 193,088 & 188,530 \\
\hline 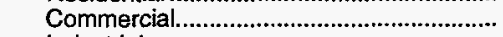 & 96,899 & 97,242 & 113,660 & $\uparrow 16,897$ & 147,127 \\
\hline 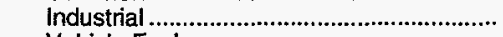 & 81,334 & 121,658 & 163,006 & 185,135 & 172,631 \\
\hline Vehicle Fuel & 8 & 9 & 12 & 3 & 6 \\
\hline 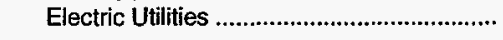 & 66,238 & 46,564 & 42,343 & 30,141 & 48,558 \\
\hline Total Delivered to Consumers .......................... & 415,157 & 431,509 & 510,928 & 525,264 & 556,852 \\
\hline Total Consumption & 417,029 & 433,744 & 513,723 & 528,517 & 560,718 \\
\hline \multicolumn{6}{|l|}{$\begin{array}{l}\text { Delivered for the Account of Others } \\
\text { (million cubic feet) }\end{array}$} \\
\hline 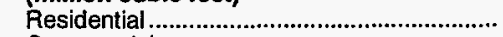 & 0 & 0 & 0 & 0 & 0 \\
\hline 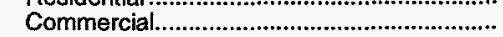 & 1,531 & 1,983 & 1,177 & 2,226 & 27,587 \\
\hline 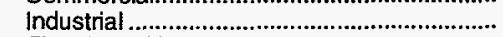 & 8,587 & 49,551 & 93,656 & 117,146 & 105,989 \\
\hline 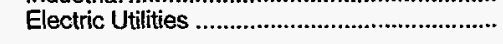 & 24,157 & 23,121 & 25,019 & 9,866 & 36,933 \\
\hline \multicolumn{6}{|l|}{$\begin{array}{l}\text { Firm Deliveries } \\
\text { (million cubic feet) }\end{array}$} \\
\hline 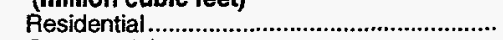 & NA & NA & NA & 193,088 & 188,194 \\
\hline 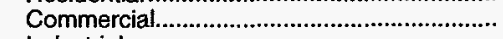 & NA & NA & NA & 99,399 & 110,544 \\
\hline 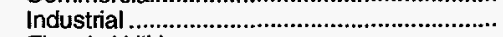 & NA & NA & NA & 42,800 & 46,191 \\
\hline Electric Utilities ................................................. & NA & NA & NA & 12,439 & 28,168 \\
\hline 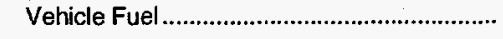 & NA & NA & NA & 3 & \\
\hline \multicolumn{6}{|l|}{$\begin{array}{l}\text { Interruptible Deliveries } \\
\text { (million cubic feet) }\end{array}$} \\
\hline 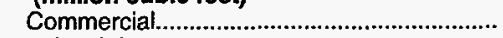 & NA & NA & NA & 17,497 & 36,583 \\
\hline 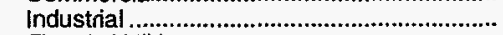 & NA & NA & NA & 42,800 & 46,191 \\
\hline 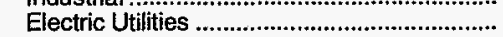 & NA & NA & NA & 10,855 & 22,465 \\
\hline 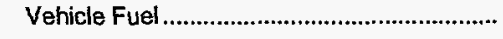 & NA & NA & NA & -- & 2 \\
\hline \multicolumn{6}{|l|}{ Number of Consumers } \\
\hline 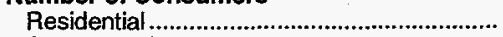 & $1,838,051$ & $1,854,346$ & $1,871,671$ & $1,888,138$ & $1,918,291$ \\
\hline 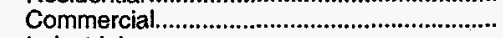 & 166,624 & 171,798 & 170,758 & 188,913 & 180,043 \\
\hline 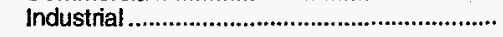 & 11,194 & 9,489 & 13,184 & 11,721 & 13,274 \\
\hline \multicolumn{6}{|l|}{$\begin{array}{l}\text { Average Annual Consumption per Consumer } \\
\text { (thousand cubic feet) }\end{array}$} \\
\hline 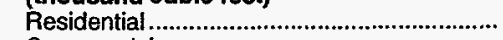 & 93 & 90 & 103 & 102 & 98 \\
\hline Commercial. & 582 & 566 & 666 & 619 & 817 \\
\hline 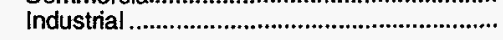 & 7,266 & 12,821 & 12,364 & 15,795 & 13,005 \\
\hline \multicolumn{6}{|l|}{$\begin{array}{l}\text { Average Prices for Natural Gas } \\
\text { (dollars per thousand cubic feet) } \\
\text { Wellhead (Marketed Production) }\end{array}$} \\
\hline 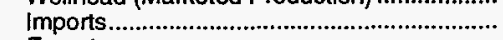 & 2.85 & 2.98 & 2.87 & 2.41 & 2.43 \\
\hline 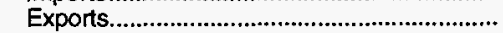 & 0 & & & & \\
\hline 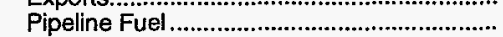 & 3.02 & 2.91 & 2.95 & 2.98 & 2.72 \\
\hline 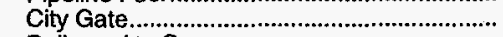 & 3.45 & 3.42 & 3.59 & 3.95 & 4.00 \\
\hline Delivered to Consumers & & & & & \\
\hline 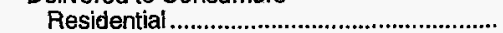 & 7.88 & 8.14 & 8.09 & 8.50 & 9.16 \\
\hline 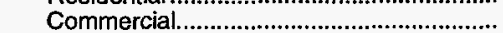 & 6.32 & 6.34 & 6.29 & 6.43 & 7.04 \\
\hline 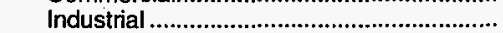 & 4.42 & 4.34 & 4.44 & 4.88 & 4.75 \\
\hline 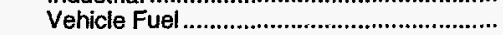 & 3.86 & 3.78 & 4.06 & 6.27 & 6.63 \\
\hline Electric Utilities ............................................. & 2.53 & 2.24 & 2.66 & 2.73 & 2.26 \\
\hline
\end{tabular}

NA $=$ Not Available.

Notes: Deliveries to electric utilities (consumption) are reported on the Form EIA-759, "Monthly Power Plant Report." Deliveries to electric utilities for the account of others are reported on the Form EIA-176, "Annual Report of Natural and Supplemental Gas Supply and Disposition." See the discussion on electric utility data and Table A1 in Appendix A for a comparison of reporting to these two forms. Totals may not add due to independent rounding. Census Division includes Connecticut, Maine, Massachusetts, Appendix A for a comparison of reporting to

Sources: Energy Information Administration (EIA), Form ElA-176, "Annual Report of Natural and Supplemental Gas Supply and Disposition"; Form ElA-627, "Annual

Sources: Energy Information Administration (EIA), Form ElA-176, "Annual Peport of Natural and Supplemental Gas Supply and Disposition"; Form ElA-627, "Annual
Quantity and Value of Natural Gas Report"; Form EIA-857, "Monthly Report of Natural Gas Purchases and Deliveries to Consumers"; Form ElA-816, "Monthly Natural Gas

Quantity and Value of Natural Gas Report"; Form ElA-857, "Monthly Report of Natural Gas Purchases and Deliveries to Consumers"; Form EIA-816, "Monthly Natural Gas

Liquids Report"; Form ElA-759, "Monthly Power Plant Report"; Form FERC-423, "Monthly Report of Cost and Quality of Fuels for Electric Plants"; Form ElA-191, "Underground Gas Storage Report"; Form FPC-14, "Annual Report for Importers and Expd 
-

\begin{tabular}{|c|c|c|c|c|c|}
\hline$\angle \forall L^{\prime \prime} 68 Z^{\prime} \varepsilon$ & 991'\&ZZ'E & $G \angle \varepsilon^{\prime} \sqcup 6 己^{t} \varepsilon$ & OLZ'ZSZ'E & 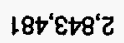 & 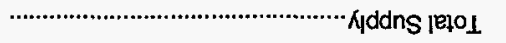 \\
\hline 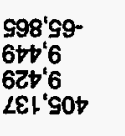 & $\begin{array}{l}6 \angle \varepsilon^{\prime} Z \varepsilon \downarrow- \\
869^{\prime} 9 ! \\
\angle 8 Z^{\prime} \angle \\
80 \angle L^{\prime} 8+b\end{array}$ & 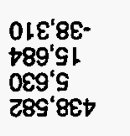 & $\begin{array}{l}669^{\prime} 08 \\
689^{\prime} 91 \\
276^{\prime} y \\
86 t^{\prime} 0 \angle L\end{array}$ & $\begin{array}{l}+166^{\prime} 9 t- \\
\angle 9 S^{\prime} 01 \\
182^{\prime} t \\
\text { S68' }\end{array}$ & 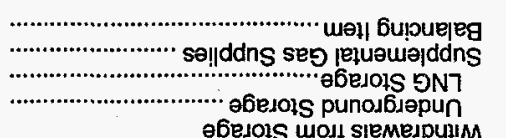 \\
\hline $\begin{array}{l}\forall \varepsilon t^{\circ} Z \angle I^{\prime} Z \\
0 \\
\varepsilon 86^{\prime} 889\end{array}$ & $\begin{array}{l}t S \varepsilon^{\prime} \angle Z Z^{\prime} Z \\
0 \\
10 L^{\prime} Z 0 S\end{array}$ & $\begin{array}{l}6 \varepsilon L^{\prime} G \angle Z^{\prime} Z \\
0 \\
0 \angle \nabla^{\prime} G \& \emptyset\end{array}$ & $\begin{array}{l}8 Z \varepsilon^{\prime} \angle L 0^{\prime} Z \\
0 \\
\varepsilon \varepsilon Z^{\prime} 8 B !\end{array}$ & $\begin{array}{l}\varepsilon 0 L^{4} 8 \varepsilon Z^{\prime} Z \\
0 \\
\angle L Z^{\prime} 86\end{array}$ & - stdiasay uọ6analu| \\
\hline $08 \mathrm{I}^{\circ} 0 \angle \mathrm{L}$ & 008 'ZSL & $6 \angle S^{\prime}|9|$ & $z 88^{6} \nabla \angle L$ & 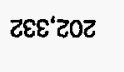 & (pəə) ग!qno uo!!!!w) R/ddns \\
\hline $081^{\circ} 0 \angle L$ & ০08'Zs। & $6 \angle G^{\prime}|9|$ & $288^{\prime}+\angle t$ & 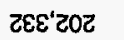 & 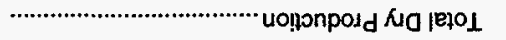 \\
\hline $\begin{array}{l}\text { ELS } \\
869^{\prime} 0 \angle L \\
1 ! \\
+0 L^{\prime} 0 \angle L \\
0 \\
0\end{array}$ & 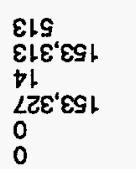 & & $\begin{array}{l}96 \varepsilon \\
L L Z^{\prime} G \angle L \\
b \\
8 \angle Z^{\prime} G \angle L \\
0 \\
0\end{array}$ & $\begin{array}{l}00 \varepsilon \\
Z \varepsilon g^{\prime} Z 0 z \\
\text { SLE } \\
\angle 00^{\prime} \varepsilon 0 Z \\
0 \\
0\end{array}$ & 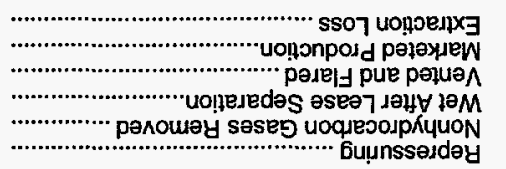 \\
\hline$\$ 0 \angle{ }^{\prime} 0 \angle L$ & $\angle Z \varepsilon^{\prime} \varepsilon S$ & & $8 \angle Z " S \angle L$ & $\angle 00^{\prime} \mathrm{EOZ}$ & 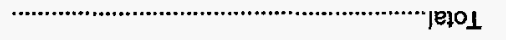 \\
\hline $\begin{array}{l}+00^{\circ} Z \\
00 L^{\prime} 891\end{array}$ & $\begin{array}{l}\text { oL9 } \\
\angle L L ' Z S \downarrow\end{array}$ & & 106\% & 61212 & 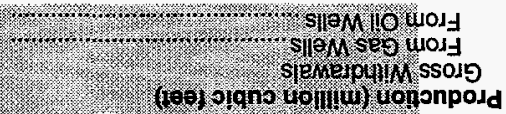 \\
\hline$\forall E O^{\prime} \angle \varepsilon$ & $\angle S 8^{\prime} 9 \varepsilon$ & & 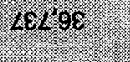 & & 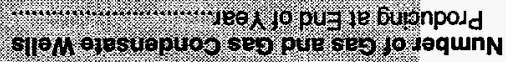 \\
\hline $2+0^{\prime} z$ & & & $090 \%$ & & 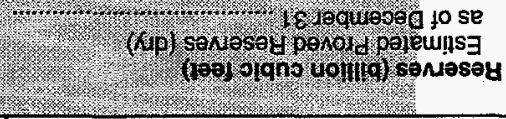 \\
\hline \$66t & 8661 & 2661 & 1661 & 0661 & \\
\hline
\end{tabular}

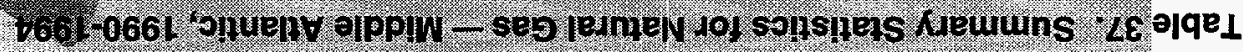

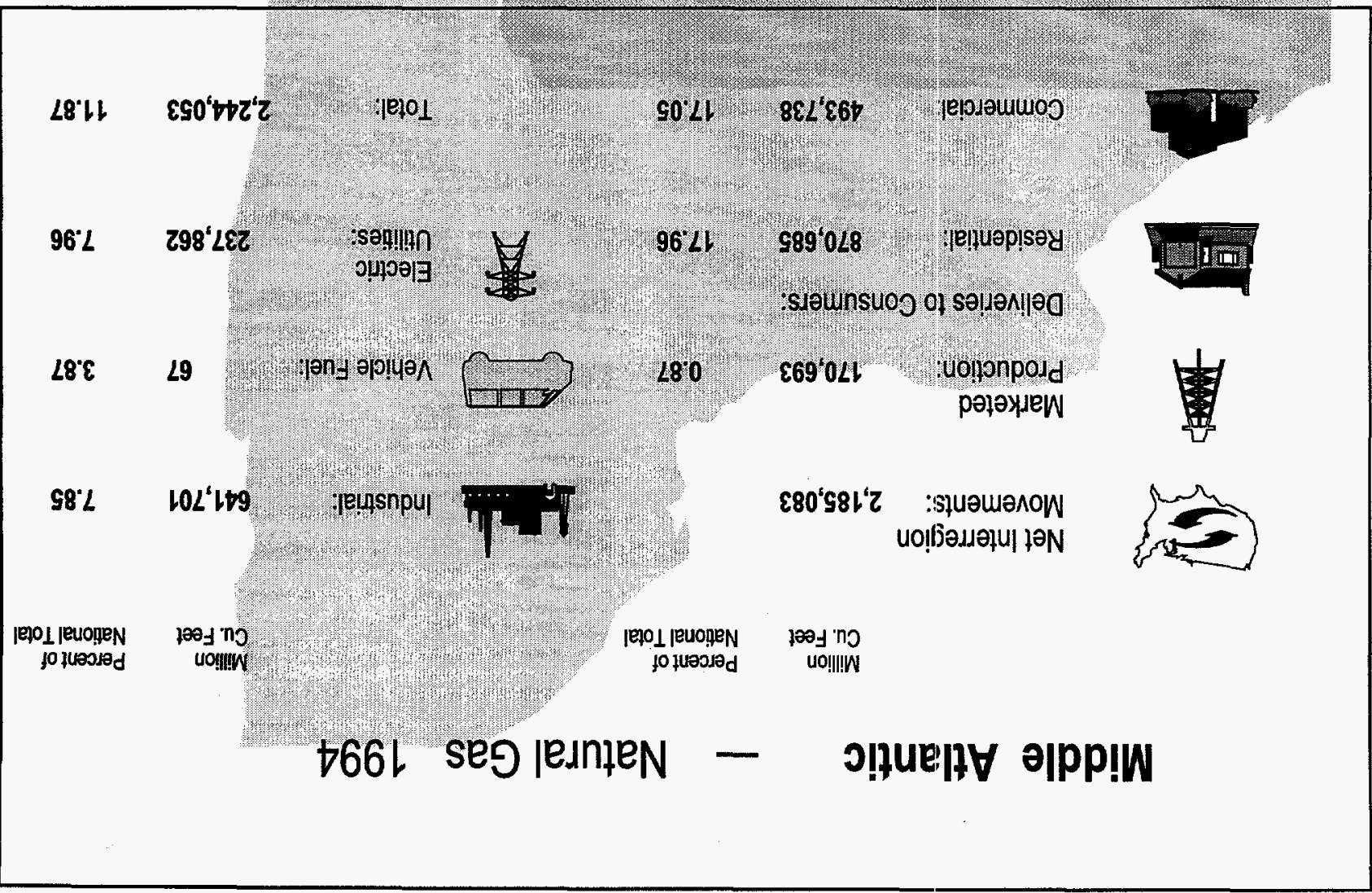


Table 37. Summary Statistics for Natural Gas - Middle Atlantic, 1990-1994 (Continued)

\begin{tabular}{|c|c|c|c|c|c|}
\hline & 1990 & 1991 & 1992 & 1993 & 1994 \\
\hline \multicolumn{6}{|l|}{ Disposition (million cubic feet) } \\
\hline $\begin{array}{l}\text { Consumption............................................... } \\
\text { Deliveries at Region Borders }\end{array}$ & $1,934,761$ & $1,977,940$ & $2,187,785$ & $2,186,786$ & $2,295,488$ \\
\hline 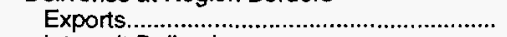 & 0 & 0 & 0 & 0 & 0 \\
\hline 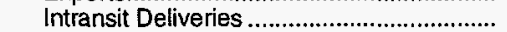 & 0 & 0 & 0 & 0 & 0 \\
\hline Interregion Deliveries.................................... & 447,207 & 497,916 & 654,539 & 578,376 & 576,334 \\
\hline \multicolumn{6}{|l|}{ Additions to Storage } \\
\hline 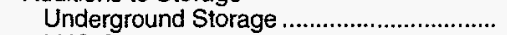 & 452,258 & 770,782 & 446,027 & 450,242 & 406,139 \\
\hline 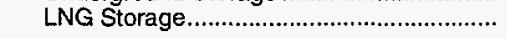 & 9,256 & 5,632 & 6,023 & 7,760 & 11,786 \\
\hline 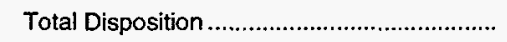 & $2,843,481$ & $3,252,270$ & $3,294,375$ & $3,223,165$ & $3,289,747$ \\
\hline \multicolumn{6}{|l|}{ Consumption (million cubic feet) } \\
\hline 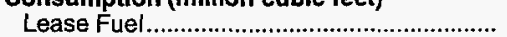 & 6,715 & 4,416 & 4,005 & 4,473 & 4,823 \\
\hline 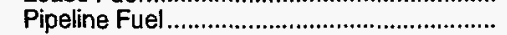 & 41,752 & 42,057 & 47,991 & 45,287 & 46,496 \\
\hline 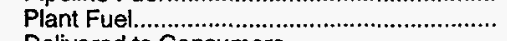 & 156 & 159 & 341 & 235 & 116 \\
\hline \multicolumn{6}{|l|}{ Delivered to Consumers } \\
\hline 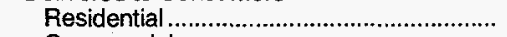 & 749,663 & 758,259 & 843,680 & 848,781 & 870,685 \\
\hline 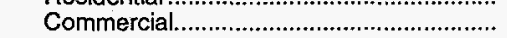 & 436,254 & 446,384 & 482,360 & 481,446 & 493,738 \\
\hline 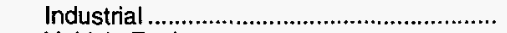 & 427,009 & 451,087 & 558,797 & 590,803 & 641,701 \\
\hline 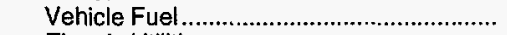 & & 27 & 9 & 24 & 67 \\
\hline Electric Utilities ....................................... & 273,208 & 275,551 & 250,602 & 215,737 & 237,862 \\
\hline Total Delivered to Consumers ....................... & $1,886,138$ & $1,931,309$ & $2,135,448$ & $2,136,791$ & $2,244,053$ \\
\hline Total Consumption & $1,934,761$ & $1,977,940$ & $2,187,785$ & $2,186,786$ & $2,295,488$ \\
\hline \multicolumn{6}{|l|}{$\begin{array}{l}\text { Delivered for the Account of Others } \\
\text { (million cubic teet) }\end{array}$} \\
\hline 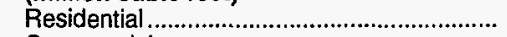 & 3,666 & 3,769 & 3,815 & 4,433 & 3,366 \\
\hline 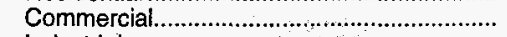 & 65,026 & 74,485 & 91,044 & 90,938 & 92,186 \\
\hline 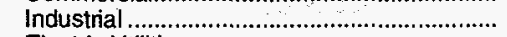 & 276,641 & 311,449 & 372,873 & 392,264 & 449,203 \\
\hline 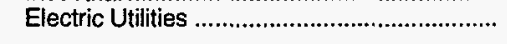 & 24,003 & 27,342 & 23,768 & 22,638 & 24,666 \\
\hline \multicolumn{6}{|l|}{ Firm Deliveries } \\
\hline 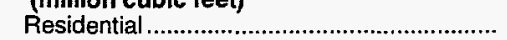 & NA & NA & NA & 848,781 & 867,990 \\
\hline 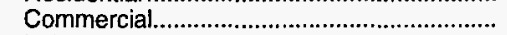 & NA & NA & NA & 407,353 & 429,236 \\
\hline 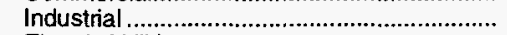 & NA & NA & NA & $R_{259,982}$ & 258,876 \\
\hline 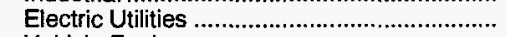 & NA & NA & NA & 61,638 & 69,631 \\
\hline 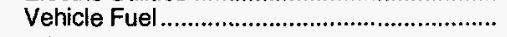 & NA & NA & NA & 22 & 40 \\
\hline \multicolumn{6}{|l|}{$\begin{array}{l}\text { Interruptible Deliveries } \\
\text { (million cubic feet) }\end{array}$} \\
\hline 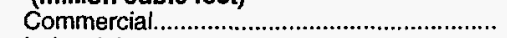 & NA & NA & NA & 74,093 & 64,501 \\
\hline 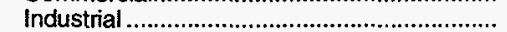 & NA & NA & NA & $\mathrm{A}_{259,982}$ & 258,876 \\
\hline 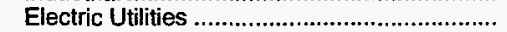 & NA & NA & NA & 155,188 & 149,733 \\
\hline 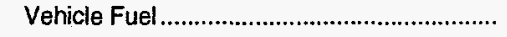 & NA & NA & NA & 2 & 28 \\
\hline \multicolumn{6}{|l|}{ Number of Consumers } \\
\hline 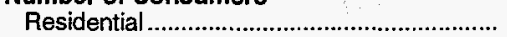 & $8,211,285$ & $8,810,402$ & $8,917,964$ & $8,437,324$ & $8,491,832$ \\
\hline 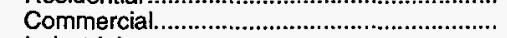 & 686,037 & 717,452 & 722,563 & 712,267 & 727,372 \\
\hline 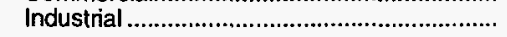 & 37,222 & 43,625 & 46,168 & 41,464 & 38,829 \\
\hline \multicolumn{6}{|l|}{$\begin{array}{l}\text { Average Annual Consumption per Consumer } \\
\text { (thousand cubic feet) }\end{array}$} \\
\hline 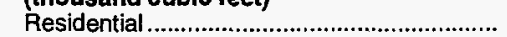 & 91 & 86 & 95 & 101 & 103 \\
\hline 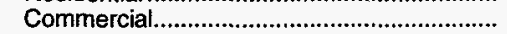 & 636 & 622 & 668 & 676 & 679 \\
\hline 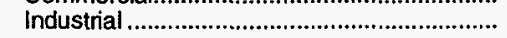 & 11,472 & 10,340 & 12,104 & 14,249 & 16,526 \\
\hline \multicolumn{6}{|l|}{$\begin{array}{l}\text { Average Prices for Natural Gas } \\
\text { (dollars per thousand cubic feet) }\end{array}$} \\
\hline Wellhead (Marketed Production) ................... & 2.33 & 2.19 & 1.99 & 2.67 & 2.71 \\
\hline 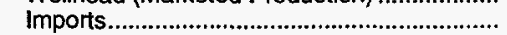 & 2.83 & 2.63 & 2.48 & 2.49 & 2.44 \\
\hline 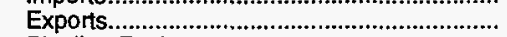 & 0 & & & & \\
\hline 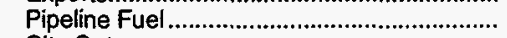 & 2.57 & 2.45 & 2.48 & 2.88 & 2.45 \\
\hline 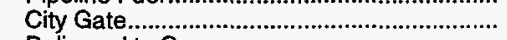 & 3.23 & 3.08 & 3.17 & 3.41 & 3.22 \\
\hline \multicolumn{6}{|l|}{ Delivered to Consumers } \\
\hline 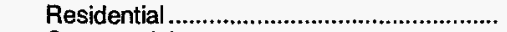 & 6.96 & 7.02 & 7.12 & 7.46 & 7.94 \\
\hline 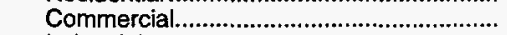 & 5.58 & 5.53 & 5.72 & 5.95 & 6.37 \\
\hline Industrial & 4.28 & 4.04 & 3.80 & 4.00 & 4.02 \\
\hline Vehicle Fuel & 4.80 & 4.74 & 5.65 & 5.06 & 4.11 \\
\hline Electric Utilities .......................................... & 2.43 & 2.24 & 2.44 & 2.67 & 2.28 \\
\hline
\end{tabular}

R $=$ Revised data:

NA = Not Available. Notes: Deliveries to electric utilities (consumption) are reported on the Form ElA-759, "Monthly Power Plant Report." Deliveries to electric utilities for the account of others
are reported on the Form ElA-176, "Annual Report of Natural and Supplemental Gas Supply and Disposition." See the discussion on electric utility data and Table A1 in are reported on the Form EIA-176, "Annual Report of Natural and Supplemental Gas Supply and Disposition." See the discussion on electric utility data and Table A1 in
Appendix A for a comparison of reporting to these two forms. Totals may not add due to independent rounding. The Middle Atlantic Census Division includes New Jersey,
New York and Pennsylvania. Sources: Energy Information Administration (EIA), Form ElA-176, "Annual Report of Natural and Supplemental Gas Supply and Disposition"; Form ElA-627, "Annual Quantity and Value of Natural Gas Report"; Form EIA-857, "Monthly Report of Natural Gas Purchases and Deliveries to Consumers"; Form EIA-816, "Monthly Natural Gas Liquids Report"; Form EIA-759, "Monthly Power Plant Report"; Form FERC-423, "Monthly Report of Cost and Quality of Fuels for Electric Plants"; Form EIA-191, "Underground Gas Storage Report"; Form FPC-14, "Annual Report for Importers and Exporters of Natural Gas"; U.S. Crude Oil, Natural Gas, and Natural Gas Liquids Reserves, 1994 Annual Report, DOEJEIA-0216(94); and the U.S. Minerals Management Service. 


\section{East North Central}

\section{- Natural Gas 1994}

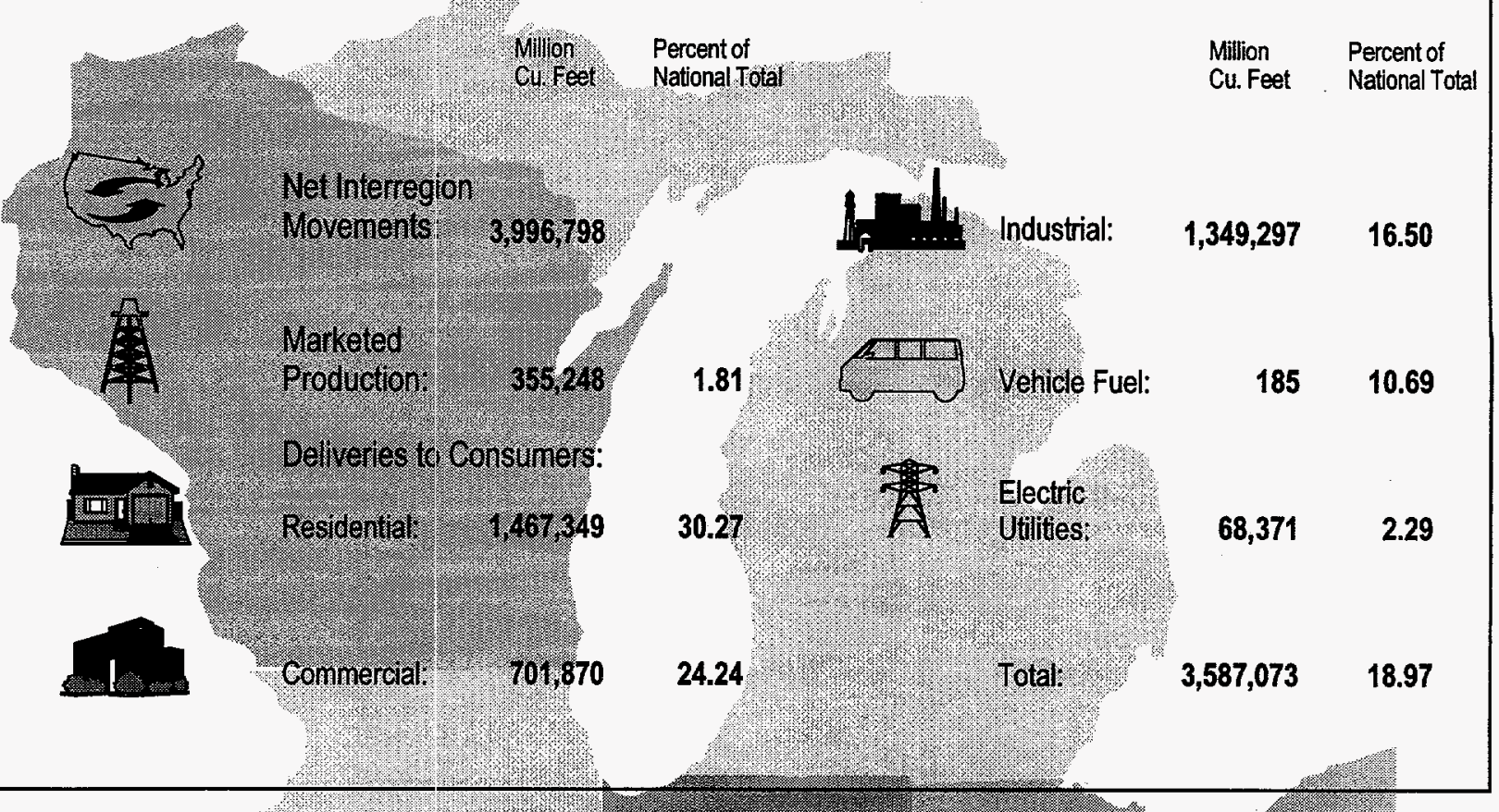

Table 38. Summary Statistics for Natural Gas - East Nortin Eonul, 1 1990-1994

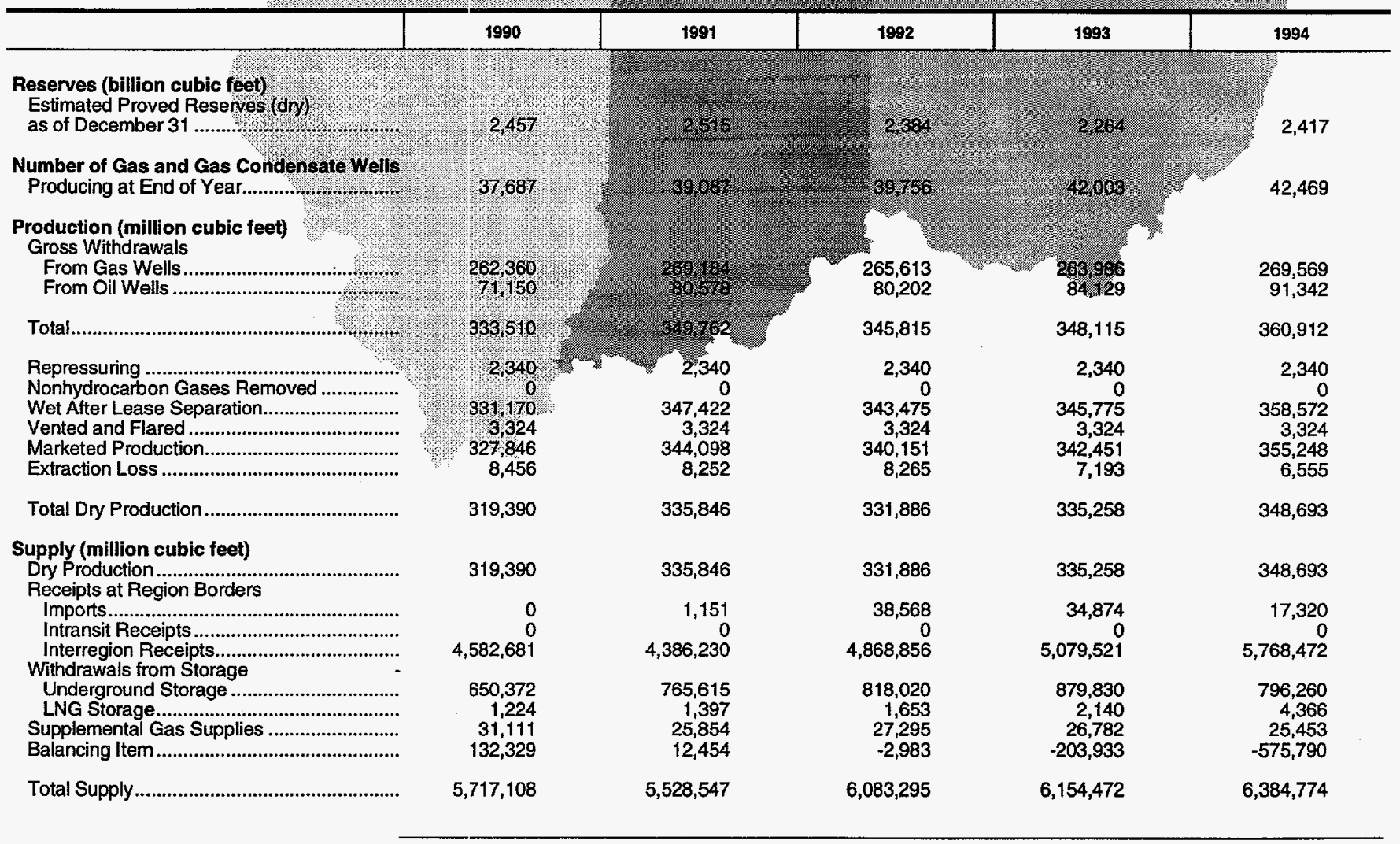

See footnotes at end of table. 
Table 38. Summary Statistics for Natural Gas - East North Central, 1990-1994 (Continued)

\begin{tabular}{|c|c|c|c|c|c|}
\hline & 1990 & 1991 & 1992 & 1993 & 1994 \\
\hline $\begin{array}{l}\text { Disposition (million cubic feet) } \\
\text { Consumption................................................ } \\
\text { Deliveries at Region Borders }\end{array}$ & $3,263,719$ & $3,369,738$ & $3,509,808$ & $3,643,905$ & $3,669,234$ \\
\hline 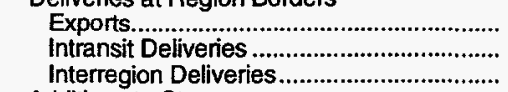 & $\begin{array}{r}17,284 \\
331,862 \\
1,296,327\end{array}$ & $\begin{array}{r}14,751 \\
346,290 \\
1,071,028\end{array}$ & $\begin{array}{r}67,763 \\
471,606 \\
1,241,993\end{array}$ & $\begin{array}{r}44,413 \\
324,093 \\
1,220,828\end{array}$ & $\begin{array}{r}65,797 \\
472,499 \\
1,250,698\end{array}$ \\
\hline 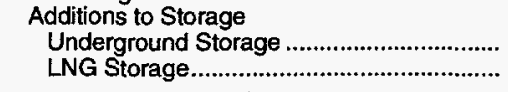 & $\begin{array}{r}805,891 \\
2,025\end{array}$ & $\begin{array}{r}724,941 \\
1,798\end{array}$ & $\begin{array}{r}790,648 \\
1,477\end{array}$ & $\begin{array}{r}918,386 \\
2,849\end{array}$ & $\begin{array}{r}922,315 \\
4,230\end{array}$ \\
\hline 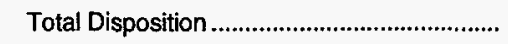 & $5,717,108$ & $5,528,547$ & $6,083,295$ & $6,154,472$ & $6,384,774$ \\
\hline 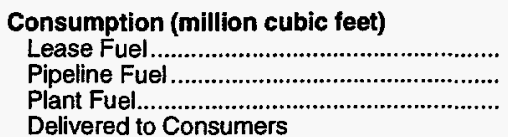 & $\begin{array}{r}11,032 \\
52,841 \\
3,335\end{array}$ & $\begin{array}{r}7,729 \\
48,723 \\
3,489\end{array}$ & $\begin{array}{r}5,626 \\
51,307 \\
3,210\end{array}$ & $\begin{array}{r}6,251 \\
56,167 \\
3,408\end{array}$ & $\begin{array}{r}7,707 \\
70,950 \\
3,504\end{array}$ \\
\hline 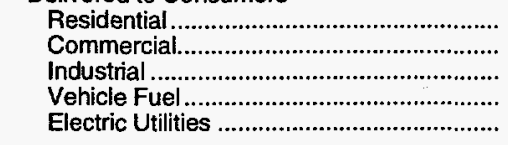 & $\begin{array}{r}1,332,422 \\
636,759 \\
1,184,725 \\
142 \\
42,463\end{array}$ & $\begin{array}{r}1,396,426 \\
649,640 \\
1,210,995 \\
149 \\
52,588\end{array}$ & $\begin{array}{r}1,450,173 \\
675,446 \\
1,276,376 \\
158 \\
47,513\end{array}$ & $\begin{array}{r}1,513,299 \\
702,557 \\
1,315,680 \\
149 \\
46,394\end{array}$ & $\begin{array}{r}1,467,349 \\
701,870 \\
1,349,297 \\
185 \\
68,371\end{array}$ \\
\hline Total Delivered to Consumers ....................... & $3,196,511$ & $3,309,797$ & $3,449,665$ & $3,578,079$ & $3,587,073$ \\
\hline 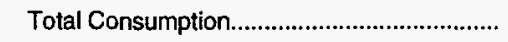 & $3,263,719$ & $3,369,738$ & $3,509,808$ & $3,643,905$ & $3,669,234$ \\
\hline 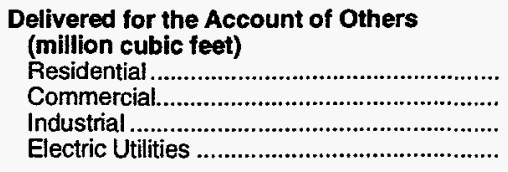 & $\begin{array}{r}25,409 \\
160,350 \\
955,856 \\
35,982\end{array}$ & $\begin{array}{r}30,953 \\
162,350 \\
997,618 \\
41,815\end{array}$ & $\begin{array}{r}33,985 \\
169,248 \\
1,058,204 \\
39,062\end{array}$ & $\begin{array}{r}36,078 \\
180,740 \\
1,102,744 \\
37,463\end{array}$ & $\begin{array}{r}35,364 \\
197,813 \\
1,144,409 \\
59,337\end{array}$ \\
\hline 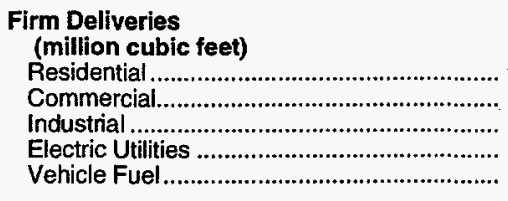 & $\begin{array}{l}\text { NA } \\
\text { NA } \\
\text { NA } \\
\text { NA } \\
\text { NA }\end{array}$ & $\begin{array}{l}\text { NA } \\
\text { NA } \\
\text { NA } \\
\text { NA } \\
\text { NA }\end{array}$ & $\begin{array}{l}\text { NA } \\
\text { NA } \\
\text { NA } \\
\text { NA } \\
\text { NA }\end{array}$ & $\begin{array}{r}1,513,299 \\
682,660 \\
381,835 \\
34,582 \\
126\end{array}$ & $\begin{array}{r}1,467,338 \\
683,452 \\
412,504 \\
58,370 \\
159\end{array}$ \\
\hline 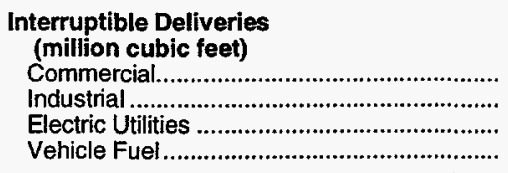 & $\begin{array}{l}\text { NA } \\
\text { NA } \\
\text { NA } \\
\text { NA }\end{array}$ & $\begin{array}{l}\text { NA } \\
\text { NA } \\
\text { NA } \\
\text { NA }\end{array}$ & $\begin{array}{l}\text { NA } \\
\text { NA } \\
\text { NA } \\
\text { NA }\end{array}$ & $\begin{array}{r}19,897 \\
381,835 \\
8,953 \\
23\end{array}$ & $\begin{array}{r}18,418 \\
412,504 \\
10,847 \\
26\end{array}$ \\
\hline 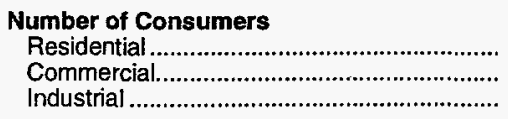 & $\begin{array}{r}11,078,902 \\
917,654 \\
59,385\end{array}$ & $\begin{array}{r}11,242,141 \\
934,355 \\
60,001\end{array}$ & $\begin{array}{r}11,381,928 \\
946,144 \\
60,965\end{array}$ & $\begin{array}{r}11,557,297 \\
R_{961,127} \\
62,397\end{array}$ & $\begin{array}{r}11,749,087 \\
969,251 \\
59,407\end{array}$ \\
\hline 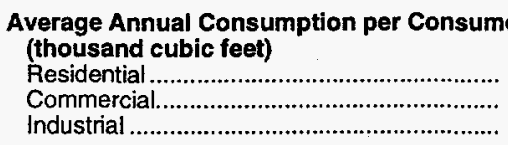 & $\begin{array}{r}120 \\
694 \\
19,950\end{array}$ & $\begin{array}{r}124 \\
695 \\
20,183\end{array}$ & $\begin{array}{r}127 \\
714 \\
20,936\end{array}$ & $\begin{array}{r}131 \\
731 \\
21,086\end{array}$ & $\begin{array}{r}125 \\
724 \\
22,713\end{array}$ \\
\hline $\begin{array}{l}\text { Average Prices for Natural Gas } \\
\text { (dollars per thousand cubic feet) }\end{array}$ & & & & & \\
\hline 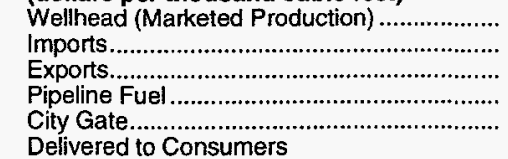 & $\begin{array}{r}2.78 \\
0 \\
\mathrm{~A}_{2.70} \\
2.12 \\
3.13\end{array}$ & $\begin{array}{r}2.61 \\
1.70 \\
\mathbf{R}_{1.91} \\
1.56 \\
3.03\end{array}$ & $\begin{array}{r}2.56 \\
1.60 \\
\mathbf{R}_{1.83} \\
1.64 \\
3.18\end{array}$ & $\begin{array}{r}2.41 \\
2.02 \\
\mathrm{R}_{2.14} \\
1.21 \\
3.29\end{array}$ & $\begin{array}{l}2.13 \\
2.16 \\
2.49 \\
1.35 \\
3.10\end{array}$ \\
\hline 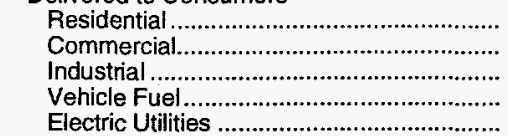 & $\begin{array}{l}5.20 \\
4.66 \\
3.80 \\
3.10 \\
1.49\end{array}$ & $\begin{array}{l}5.17 \\
4.66 \\
3.64 \\
3.62 \\
1.60\end{array}$ & $\begin{array}{l}5.22 \\
4.68 \\
3.65 \\
3.63 \\
1.54\end{array}$ & $\begin{array}{l}5.54 \\
5.03 \\
3.95 \\
4.62 \\
1.92\end{array}$ & $\begin{array}{l}5.61 \\
5.08 \\
4.03 \\
4.28 \\
1.95\end{array}$ \\
\hline
\end{tabular}

\section{$R=$ Revised data.}

NA = Not Available. Notes: Deliveries to electric utilities (consumption) are reported on the Form ElA-759, "Monthly Power Plant Report." Deliveries to electric utilities for the account of others
are reported on the Form EIA-176, "Annual Report of Natural and Supplemental Gas Supply and Disposition." See the discussion on electric utility data and Table A1 in Appendix A for a comparison of reporting to these two forms. Totals may not add due to independent rounding. The East North Central Census Division includes lilinois, Indiana, Michigan, Ohio, and Wisconsin.

Sources: Energy Information Administration (ElA), Form ElA-176, "Annual Report of Natural and Supplemental Gas Supply and Disposition"; Form ElA-627, "Annual Quantity and Value of Natural Gas Report"; Form ElA-857, "Monthly Report of Natural Gas Purchases and Deliveries to Consumers"; Form EIA-816, "Monthly Natural Gas Liquids Report"; Form EIA-759, "Monthly Power Plant Report"; Form FEAC-423, "Monthly Report of Cost and Quality of Fuels for Electric Plants"; Form EIA-191, "Underground Gas Storage Report"; Form FPC-14, "Annual Report for Importers and Exporters of Natural Gas"; U.S. Crude Oil, Natural Gas, and Natural Gas Liquid's Reserves, 1994 Annual Report, DOE/EIA-0216(94); and the U.S. Minerals Management Service. 


\section{West North Central - Natural Gas 1994}

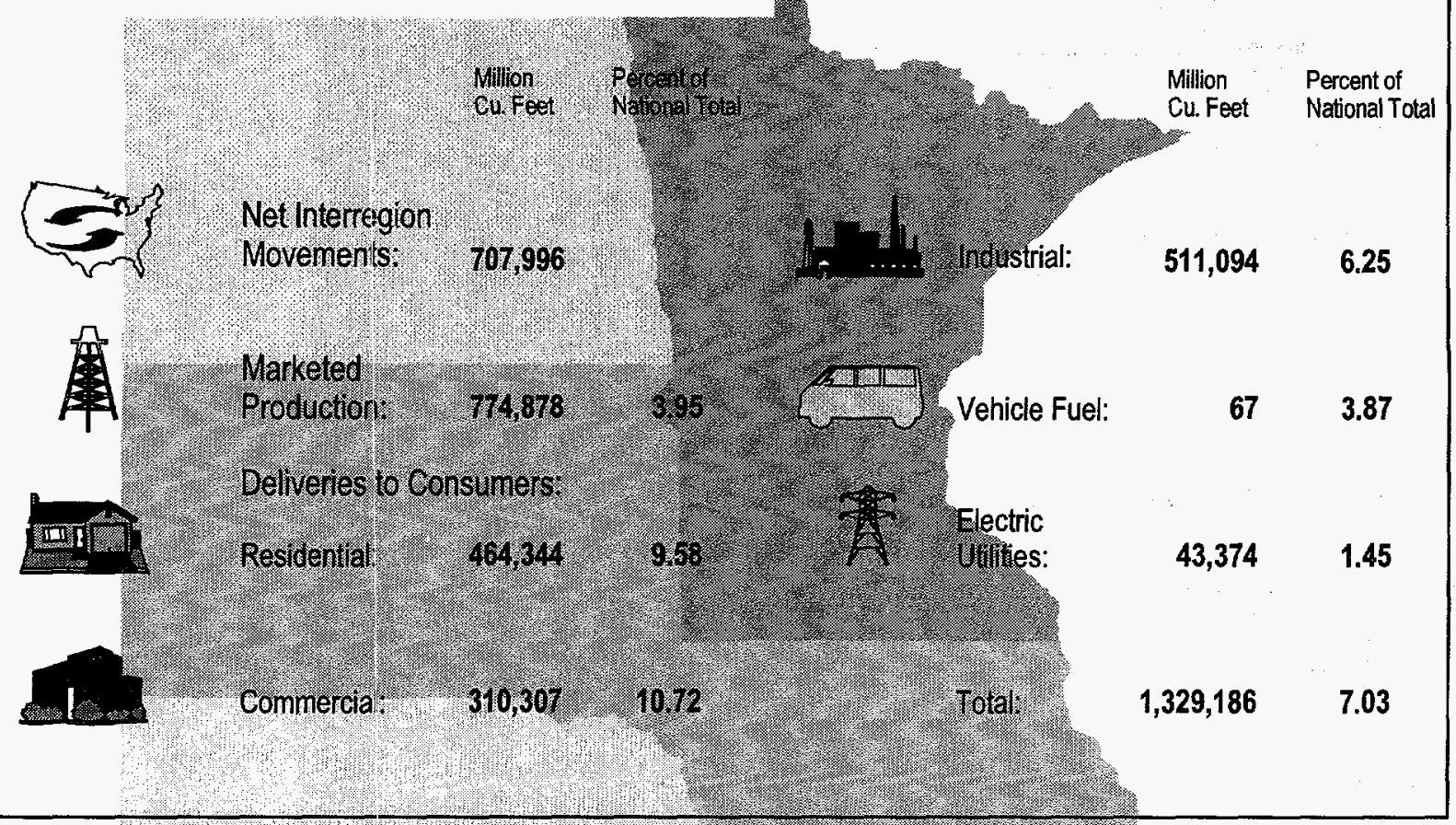

Table 39. Summary Statistics for Natural Gas - West North Central, 1990-1994

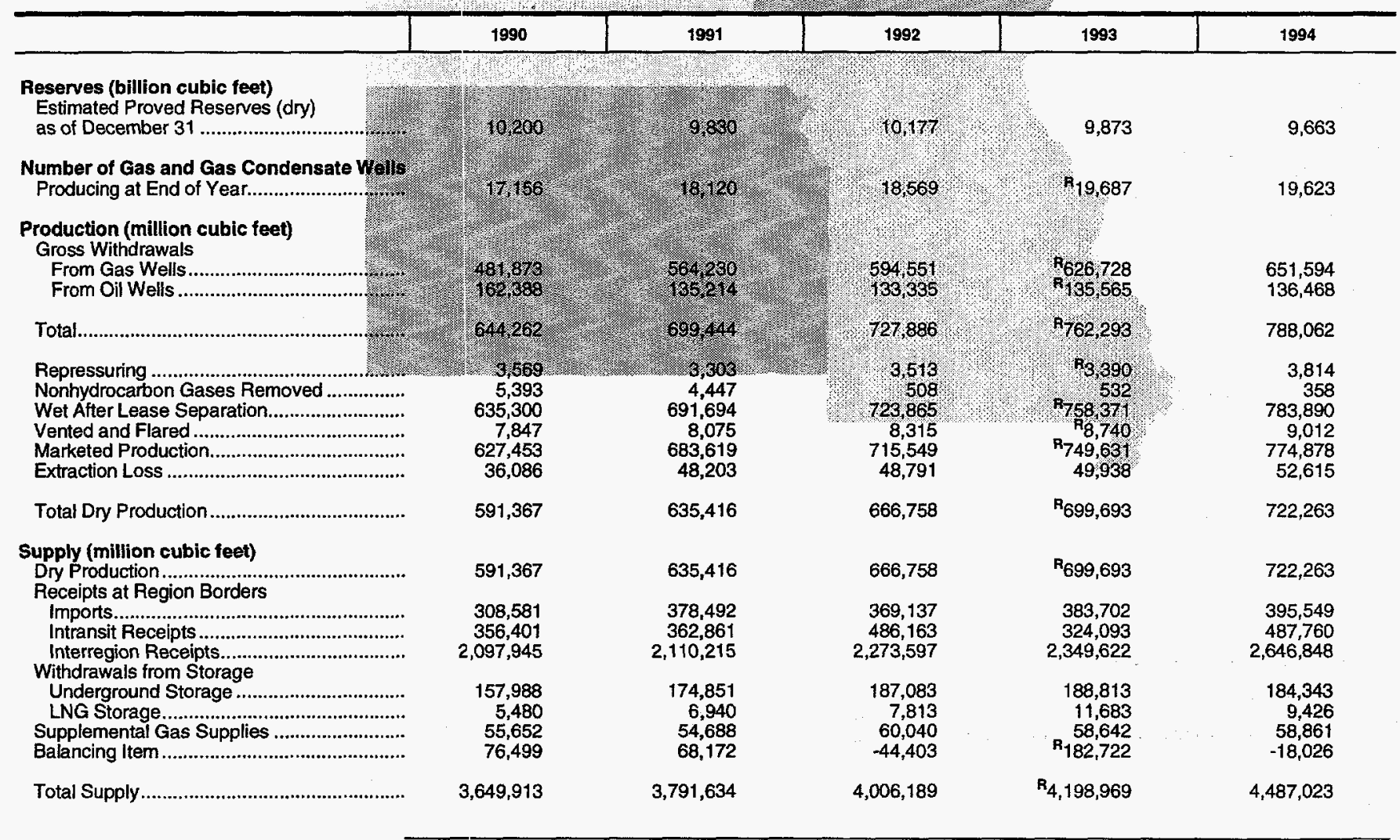

See footnotes at end of table. 
Table 39. Summary Statistics for Natural Gas - West North Central, 1990-1994 (Continued)

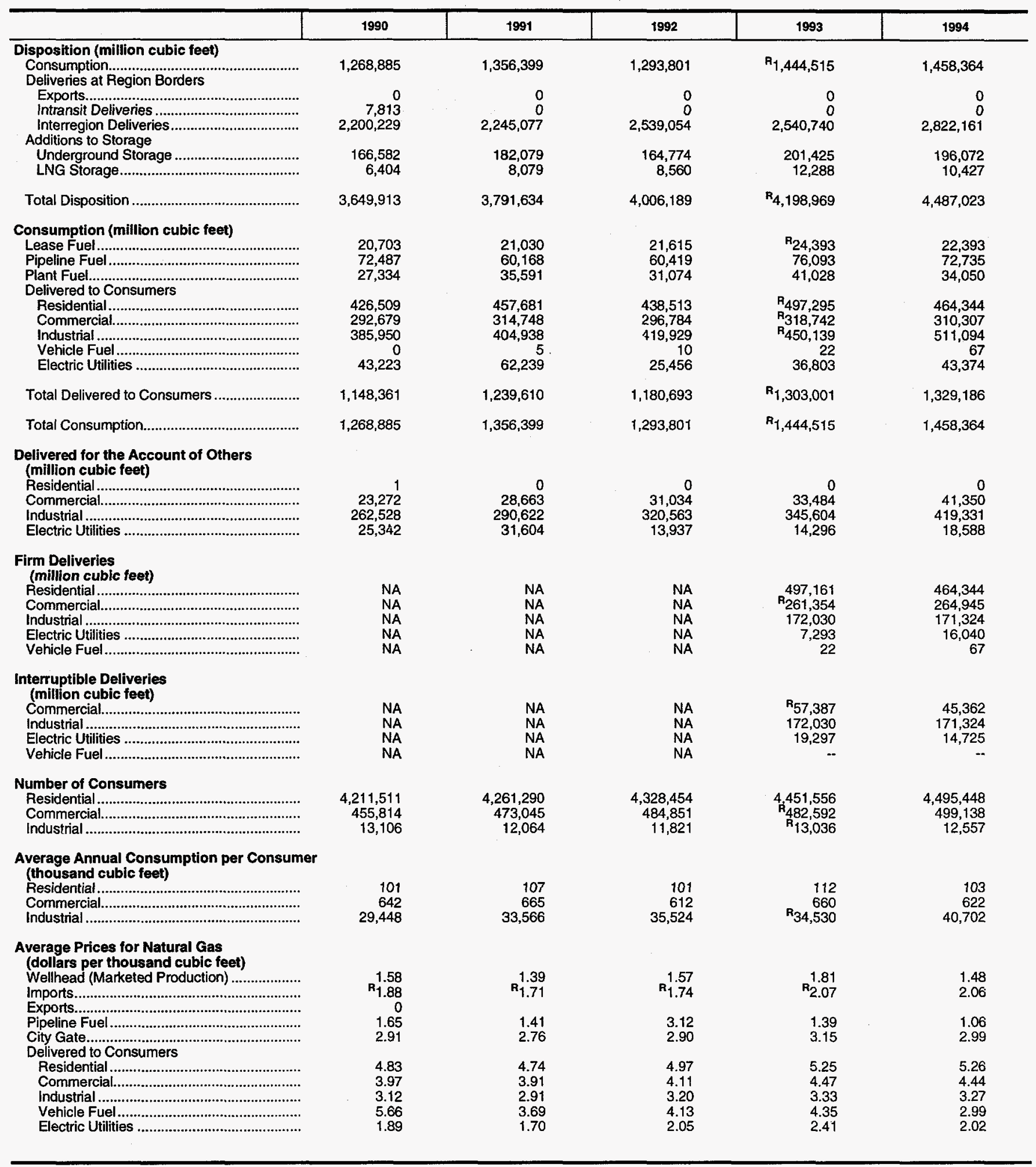

$R=$ Revised data.

NA $=$ Not Available.
Notes: Deliveries to electric utilities (consumption) are reported on the Form ElA-759, "Monthly Power Plant Report." Deliveries to electric utilities for the account of others are reported on the Form EIA-176, "Annual Report of Natural and Supplemental Gas Supply and Disposition." See the discussion on electric utility data and Table A1 in Appendix A for a comparison of reporting to these two forms. Totals may not add due to independent rounding. The West North Central Census Division includes lowa, Appendix A for a comparison of reporting to these two forms. Totals may not

Kansas, Minnesota, Missouri, Nebraska, North Dakota, and South Dakota.
Sources: Energy Information Administration (EIA), Form EIA-176, "Annual Report of Natural and Supplemental Gas Supply and Disposition*; Form ElA-627, "Annual Sources: Energy Information Administration (EIA), Form EIA-176, "Annual Report of Natural and Supplemental Gas Supply and Disposition"; Form ElA-627, "Annual
Quantity and Value of Natural Gas Report"; Form ElA-857, "Monthly Report of Natural Gas Purchases and Deliveries to Consumers"; Form EIA-816, "Monthly Natural Gas Quantity and Value of Natural Gas Report"; Form ElA-857, "Monthly Report of Natural Gas Purchases and Deliveries to Consumers"; Form ElA-816, "Monthly Natural Gas
Liquids Report"; Form EIA-759, "Monthly Power Plant Report"; Form FERC-423, "Monthly Report of Cost and Quality of Fuels for Electric Plants"; Form EIA-191, "Underground Gas Storage Report"; Form FPC-14, "Annual Report for Importers and Exporters of Natural Gas"; U.S. Crude Oil, Natural Gas, and Natural Gas Liquids Resenves, 1994 Annual Report, DOEEJEIA-0216(94); and the U.S. Minerals Management Services. 
South Atlantic - Natural Gas 1994

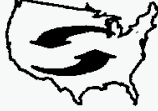

捅
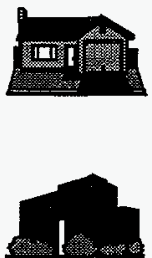

Net Interregion

Movements:

Marketed

Production:
Million

Cu. Feet

Percent of Million
Cu. Feet
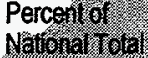

\section{Deliveries to Consumers:}

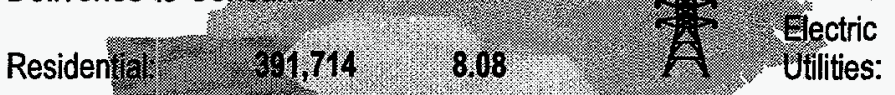

235,179

Commerclet $203.056 \div 10.12$

Total:

$1,610,633$

8.52

Table 40. Summary Statistics for Matural Cas - South Atlantic, 1990-1994

\begin{tabular}{|c|c|c|c|c|c|}
\hline & 1990 & 1991 & 1992 & 1993 & 1994 \\
\hline 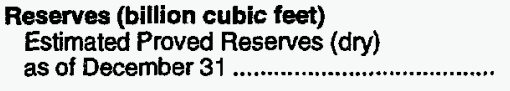 & 2,390 & 791 & 3,307 & 3,811 & 4,496 \\
\hline 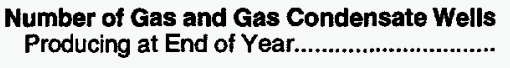 & 38,326 & & 39,412 & $\mathbf{R}_{35,149}$ & 36,727 \\
\hline 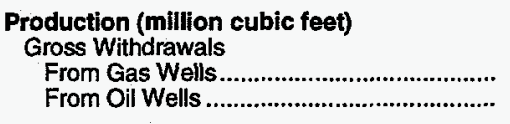 & $\begin{array}{r}192,796 \\
7,566\end{array}$ & 213,840 & $\begin{array}{r}206,766 \\
7,584\end{array}$ & $\begin{array}{r}R_{208,892} \\
8,011\end{array}$ & $\begin{array}{r}232,286 \\
8,468\end{array}$ \\
\hline 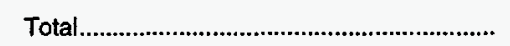 & 200,362 & 2,0498 & 214,349 & $R_{216,903}$ & 240,754 \\
\hline 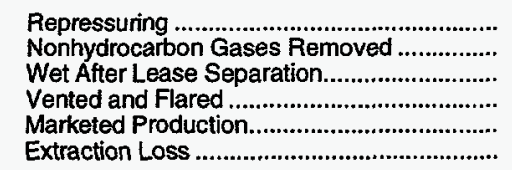 & $\begin{array}{r}115 \\
681 \\
199,565 \\
286 \\
199,279 \\
11,939\end{array}$ & 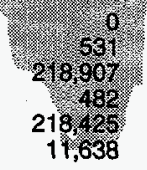 & $\begin{array}{r}0 \\
682 \\
213,667 \\
245 \\
213,422 \\
11,999\end{array}$ & $\begin{array}{r}0 \\
721 \\
\mathrm{R}_{216,182} \\
205 \\
\mathrm{R}_{215,977} \\
13,387\end{array}$ & $\begin{array}{r}0 \\
762 \\
239,992 \\
220 \\
239,772 \\
12,690\end{array}$ \\
\hline Total Dry Production & 187,340 & 206,787 & 201,423 & $R_{202,590}$ & 227,082 \\
\hline $\begin{array}{l}\text { Supply (million cubic feet) } \\
\text { Dry Production................................................... } \\
\text { Receipts at Region Borders }\end{array}$ & 187,340 & 206,787 & 201,423 & $\mathbf{R}_{202,590}$ & 227,082 \\
\hline 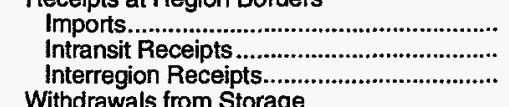 & $\begin{array}{r}0 \\
0 \\
3,219,869\end{array}$ & $\begin{array}{r}0 \\
0 \\
3,125,183\end{array}$ & $\begin{array}{r}0 \\
0 \\
3,309,501\end{array}$ & $\begin{array}{r}0 \\
0 \\
3,290,584\end{array}$ & $\begin{array}{r}0 \\
0 \\
3,415,392\end{array}$ \\
\hline 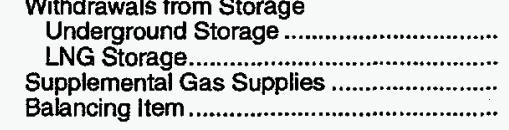 & $\begin{array}{r}96,632 \\
2,860 \\
4,719 \\
80,021\end{array}$ & $\begin{array}{r}141,795 \\
4,053 \\
4,805 \\
-84,508\end{array}$ & $\begin{array}{r}165,996 \\
3,951 \\
4,230 \\
12,279\end{array}$ & $\begin{array}{r}172,256 \\
5,452 \\
4,791 \\
R_{143,864}\end{array}$ & $\begin{array}{r}173,690 \\
7,333 \\
5,494 \\
100,679\end{array}$ \\
\hline Total Supply & $3,591,441$ & $3,398,116$ & $3,697,380$ & $R_{3,819,537}$ & $3,929,670$ \\
\hline
\end{tabular}


Table 40. Summary Statistics for Natural Gas - South Atlantic, 1990-1994 (Continued)

\begin{tabular}{|c|c|c|c|c|c|}
\hline & 1990 & 1991 & 1992 & 1993 & 1994 \\
\hline $\begin{array}{l}\text { Disposition (million cubic feet) } \\
\text { Consumption............................................ } \\
\text { Deliveries at Region Borders }\end{array}$ & $1,471,278$ & $1,498,898$ & $1,596,374$ & $\mathrm{H}_{1,622,052}$ & $1,680,512$ \\
\hline 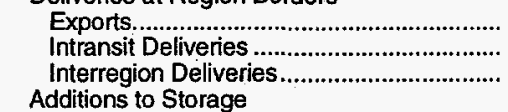 & $\begin{array}{r}0 \\
0 \\
1,968,272\end{array}$ & $\begin{array}{r}0 \\
0 \\
1,768,014\end{array}$ & $\begin{array}{r}0 \\
0 \\
1,938,877\end{array}$ & $\begin{array}{r}0 \\
0 \\
2,015,987\end{array}$ & $\begin{array}{r}0 \\
0 \\
2,054,593\end{array}$ \\
\hline 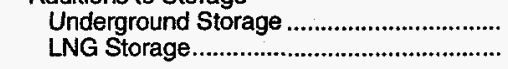 & $\begin{array}{r}145,648 \\
6,243\end{array}$ & $\begin{array}{r}126,760 \\
4,443\end{array}$ & $\begin{array}{r}158,149 \\
3,980\end{array}$ & $\begin{array}{r}175,763 \\
5,734\end{array}$ & $\begin{array}{r}186,532 \\
8,033\end{array}$ \\
\hline 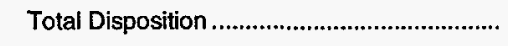 & $3,591,441$ & $3,398,116$ & $3,697,380$ & $\mathbf{R}_{3,819,537}$ & $3,929,670$ \\
\hline 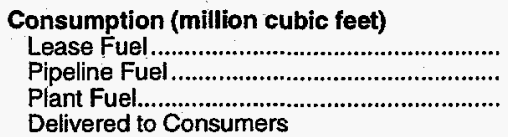 & $\begin{array}{r}7,167 \\
37,572 \\
4,670\end{array}$ & $\begin{array}{r}7,093 \\
37,646 \\
4,729\end{array}$ & $\begin{array}{r}9,279 \\
46,996 \\
4,605\end{array}$ & $\begin{array}{r}R_{5,346} \\
49,731 \\
4,557\end{array}$ & $\begin{array}{r}6,529 \\
59,911 \\
3,439\end{array}$ \\
\hline 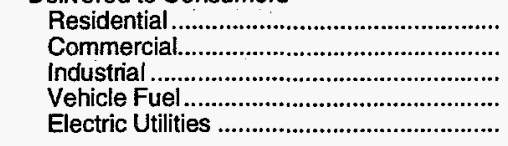 & $\begin{array}{r}329,510 \\
236,458 \\
621,487 \\
2 \\
234,412\end{array}$ & $\begin{array}{r}345,654 \\
263,448 \\
585,297 \\
8 \\
255,024\end{array}$ & $\begin{array}{r}385,200 \\
287,357 \\
623,133 \\
16 \\
239,787\end{array}$ & $\begin{array}{r}R_{403,479} \\
R_{295,379} \\
R_{644,009} \\
53 \\
219,499\end{array}$ & $\begin{array}{r}391,714 \\
293,056 \\
690,558 \\
126 \\
235,179\end{array}$ \\
\hline Total Delivered to Consumers ............................ & $1,421,869$ & $1,449,430$ & $1,535,494$ & $\mathbf{R}_{1,562,419}$ & $1,610,633$ \\
\hline 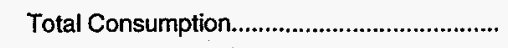 & $1,471,278$ & $1,498,898$ & $1,596,374$ & $\mathbf{R}_{1,622,052}$ & $1,680,512$ \\
\hline 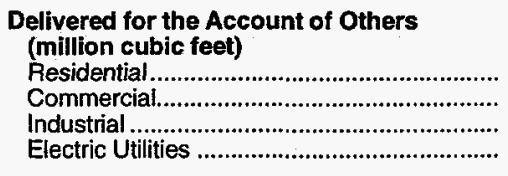 & $\begin{array}{r}87 \\
21,450 \\
315,938 \\
84,394\end{array}$ & $\begin{array}{r}85 \\
23,450 \\
323,793 \\
186,587\end{array}$ & $\begin{array}{r}89 \\
26,192 \\
351,672 \\
202,596\end{array}$ & $\begin{array}{r}99 \\
\mathrm{R}_{25,958} \\
379,240 \\
208,839\end{array}$ & $\begin{array}{r}100 \\
27,611 \\
430,040 \\
230,067\end{array}$ \\
\hline 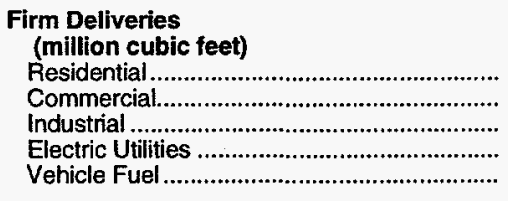 & $\begin{array}{l}\text { NA } \\
\text { NA } \\
\text { NA } \\
\text { NA } \\
\text { NA }\end{array}$ & $\begin{array}{l}\text { NA } \\
\text { NA } \\
\text { NA } \\
\text { NA }\end{array}$ & $\begin{array}{l}\text { NA } \\
\text { NA } \\
\text { NA } \\
\text { NA } \\
\text { NA }\end{array}$ & $\begin{array}{r}404,376 \\
R_{227,176} \\
R_{472,888} \\
174,890 \\
49\end{array}$ & $\begin{array}{r}391,699 \\
226,856 \\
466,809 \\
207,925 \\
122\end{array}$ \\
\hline 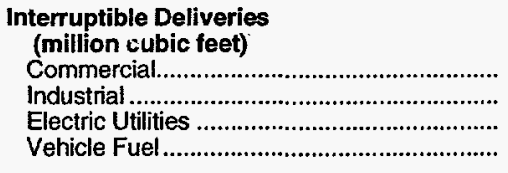 & $\begin{array}{l}\text { NA } \\
\text { NA } \\
\text { NA } \\
\text { NA }\end{array}$ & $\begin{array}{l}\text { NA } \\
\text { NA } \\
\text { NA } \\
\text { NA }\end{array}$ & $\begin{array}{l}\text { NA } \\
\text { NA } \\
\text { NA } \\
\text { NA }\end{array}$ & $\begin{array}{r}\mathrm{R}_{68,203} \\
\mathrm{R}_{472,888} \\
55,781 \\
4\end{array}$ & $\begin{array}{r}66,200 \\
466,809 \\
38,825 \\
5\end{array}$ \\
\hline 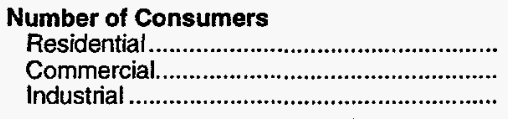 & $\begin{array}{r}4,624,549 \\
430,880 \\
14,788\end{array}$ & $\begin{array}{r}4,727,189 \\
446,720 \\
10,524\end{array}$ & $\begin{array}{r}4,943,742 \\
455,316 \\
10,283\end{array}$ & $\begin{array}{r}4,976,403 \\
R_{467,891} \\
R_{11,672}\end{array}$ & $\begin{array}{r}5,161,109 \\
482,552 \\
12,786\end{array}$ \\
\hline 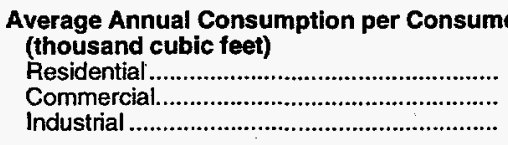 & $\begin{array}{r}71 \\
549 \\
42,026\end{array}$ & $\begin{array}{r}73 \\
590 \\
55,615\end{array}$ & $\begin{array}{r}78 \\
631 \\
60,598\end{array}$ & $\begin{array}{r}81 \\
\mathbf{R}_{631} \\
\mathbf{R}_{55,176}\end{array}$ & $\begin{array}{r}76 \\
607 \\
54,009\end{array}$ \\
\hline 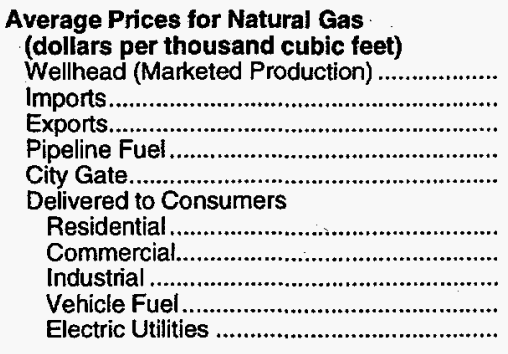 & $\begin{array}{l}6.71 \\
5.29 \\
3.56 \\
4.47 \\
2.56\end{array}$ & $\begin{array}{l}2.07 \\
2.97 \\
\\
6.64 \\
5.13 \\
3.21 \\
4.72 \\
2.18\end{array}$ & $\begin{array}{l}6.66 \\
5.19 \\
3.33 \\
4.67 \\
2.35\end{array}$ & $\begin{array}{r}2.83 \\
3.40 \\
\mathbf{R}_{7.15} \\
5.72 \\
\mathrm{R}_{3.70} \\
4.05 \\
2.48\end{array}$ & $\begin{array}{l}2.77 \\
3.36 \\
\\
7.39 \\
5.77 \\
3.56 \\
3.94 \\
2.26\end{array}$ \\
\hline
\end{tabular}

R $=$ Revised data.

Notes: Deliveries to electric utilities (consumption) are reported on the Form EIA-759, "Monthly Power Plant Report." Deliveries to electric utilities for the account of others are reported on the Form ElA-176, "Annual Report of Natural and Supplemental Gas Supply and Disposition." See the discussion on electric utility data and Table A1 in Appendix A for a comparison of reporting to these two forms. Totals may not add due to independent rounding. The South Atlantic Census Division includes Delaware, District Appendix A for a comparison of reporting to these two forms. Totals may not add due to independent

of Columbia, Florida, Georgia, Maryland, North Carolina, South Carolina, Virginia, and West Virginia.
Sources: Energy Information Administration (EIA), Form EIA-176, "Annual Report of Natural and Supplemental Gas Supply and Disposition*, Form ElA-627, "Annual

Sources: Energy Information Administration (EIA), Form ElA-176, "Annual Report of Natural and Supplemental Gas Supply and Disposition", Form ElA-627, "Annual
Quantity and Value of Natural Gas Report"; Form ElA-857, "Monthly Report of Natural Gas Purchases and Deliveries to Consumers"; Form ElA-816, "Monthly Natural Gas

Quantity and Value of Natural Gas Report"; Form ElA-857, "Monthly Report of Natural Gas Purchases and Deliveries to Consumers"; Form ElA-816, "Monthly Natural Gas
Liquids Report"; Form EIA-759, "Monthly Power Plant Report"; Form FERC-423, "Monthly Peport of Cost and Quality of Fuels for Electric Plants"; Form EIA-191, "Underground Gas Storage Report"; Form FPC-14, "Annual Report for importers and Exporters of Natural Gas"; U.S. Crude Oil, Natural Gas, and Natural Gas Liquids Reserves, 1994 Annual Report, DOE/EIA-0216(94); and the U.S. Minerals Management Service. 


\section{East South Central - Natural Gas 1994}

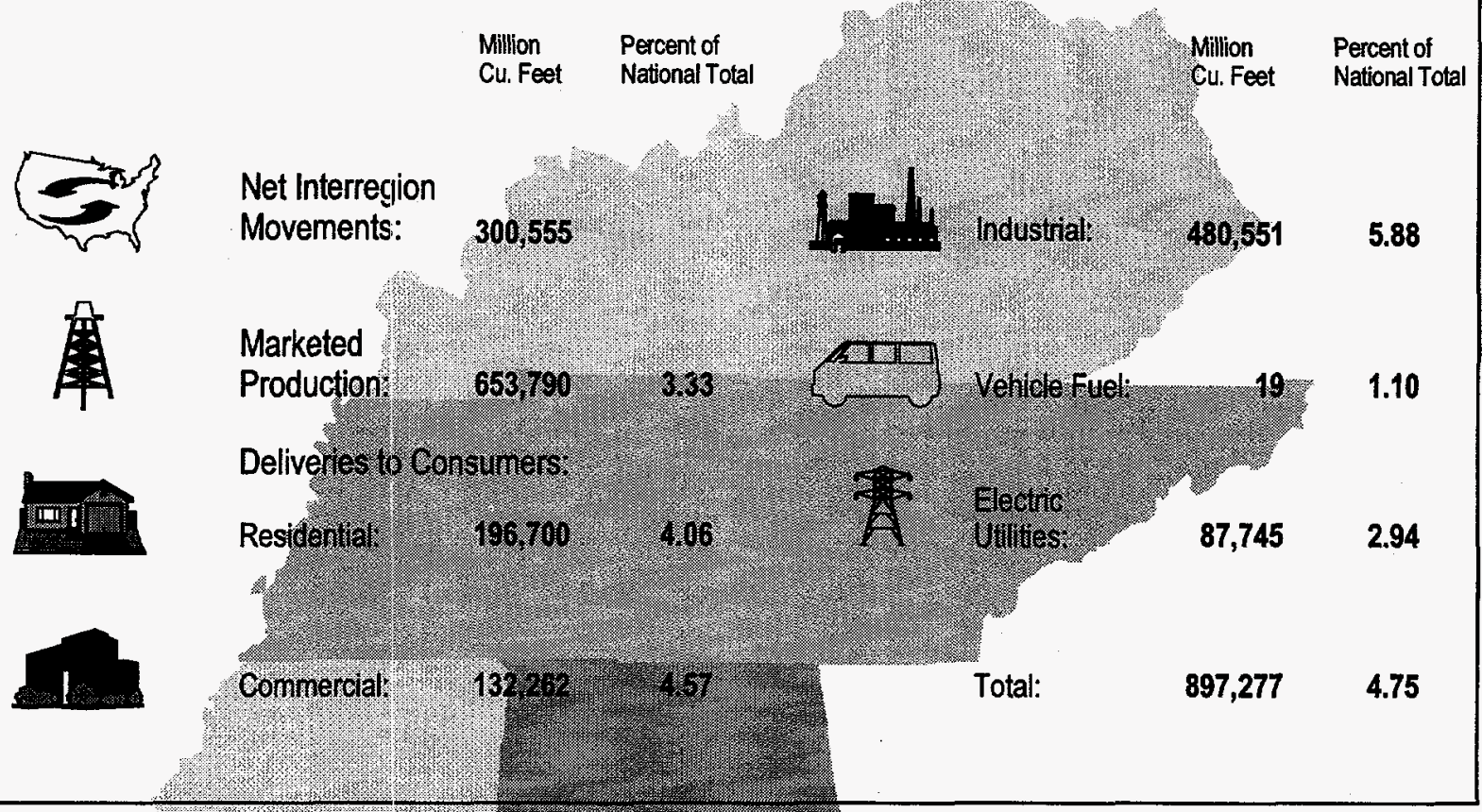

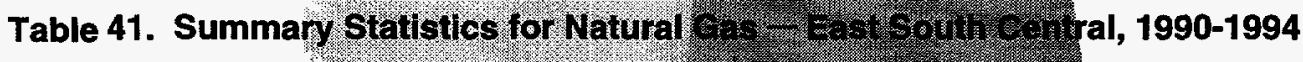

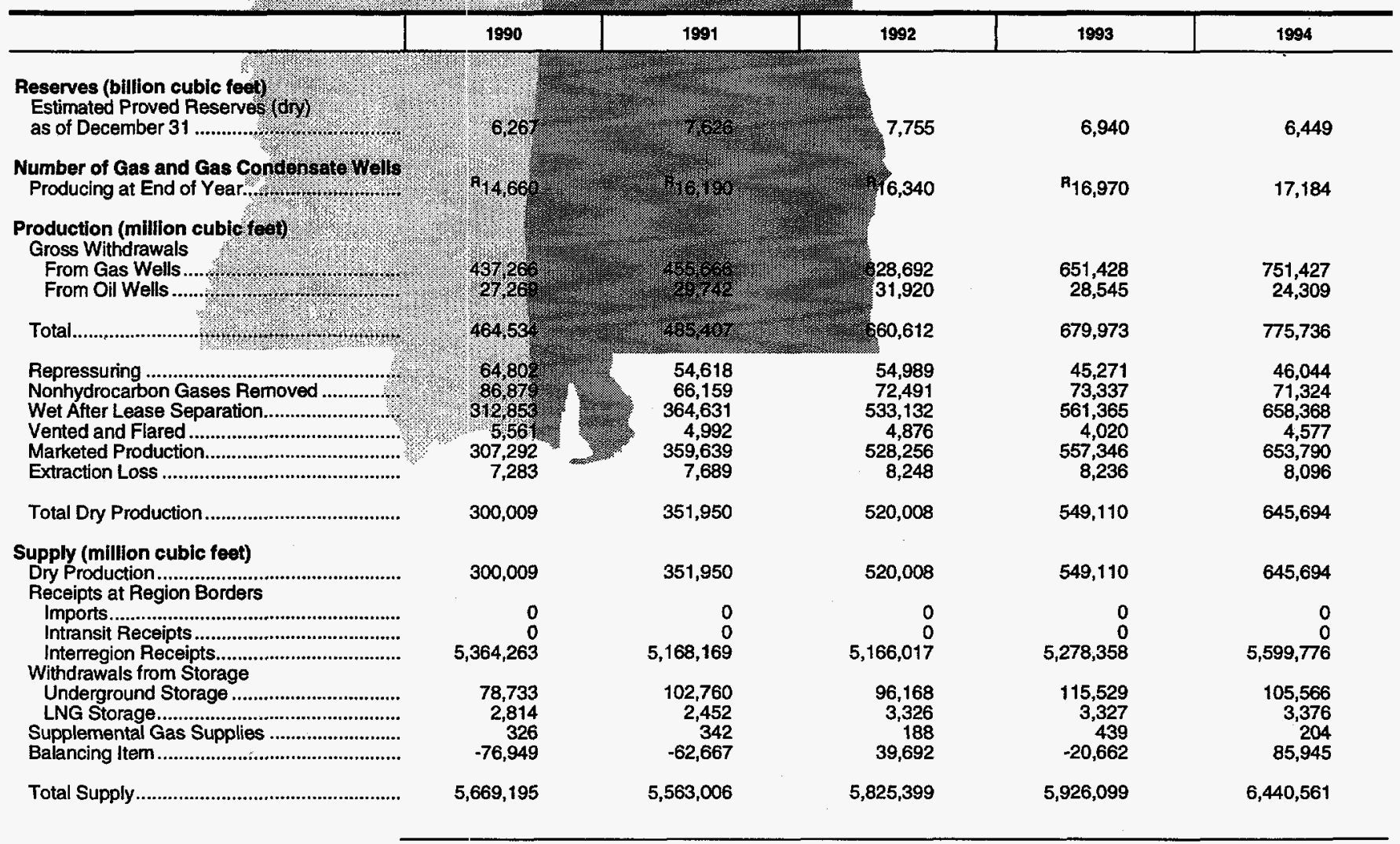

See footnotes at end of table. 
Table 41. Summary Statistics for Natural Gas - East South Central, 1990-1994 (Continued)

\begin{tabular}{|c|c|c|c|c|c|}
\hline & 1990 & 1991 & 1992 & 1993 & 1994 \\
\hline $\begin{array}{l}\text { Disposition (million cubic feet) } \\
\text { Consumption .................................................... } \\
\text { Deliveries at Region Borders }\end{array}$ & 901,658 & 918,186 & 949,815 & 979,800 & $1,011,915$ \\
\hline 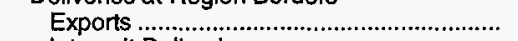 & 0 & 0 & 0 & 0 & 0 \\
\hline 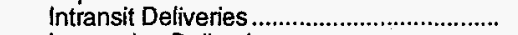 & 0 & 0 & 0 & 0 & 0 \\
\hline Interregion Deliveries........................................ & $4,644,280$ & $4,547,308$ & $4,767,852$ & $4,839,004$ & $5,299,221$ \\
\hline 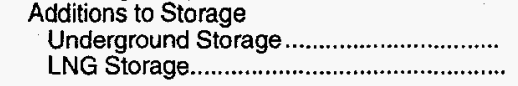 & $\begin{array}{r}119,355 \\
3,901\end{array}$ & $\begin{array}{r}95,480 \\
2,032\end{array}$ & $\begin{array}{r}104,473 \\
3,259\end{array}$ & $\begin{array}{r}104,020 \\
3,275\end{array}$ & $\begin{array}{r}125,496 \\
3,929\end{array}$ \\
\hline 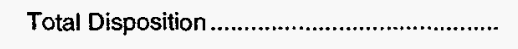 & $5,669,195$ & $5,563,006$ & $5,825,399$ & $5,926,099$ & $6,440,561$ \\
\hline 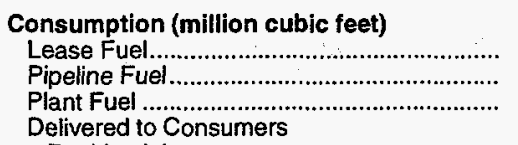 & $\begin{array}{r}15,367 \\
96,590 \\
4,994\end{array}$ & $\begin{array}{r}10,555 \\
86,865 \\
5,133\end{array}$ & $\begin{array}{r}14,906 \\
84,142 \\
5,757\end{array}$ & $\begin{array}{r}15,826 \\
90,732 \\
5,680\end{array}$ & $\begin{array}{r}14,757 \\
95,060 \\
4,821\end{array}$ \\
\hline 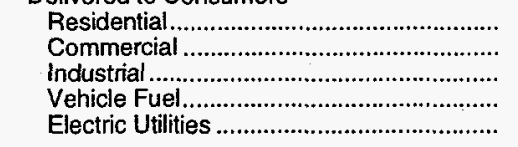 & $\begin{array}{r}172,859 \\
117,193 \\
424,540 \\
4 \\
70,110\end{array}$ & $\begin{array}{r}180,726 \\
121,107 \\
446,870 \\
1 \\
66,928\end{array}$ & $\begin{array}{r}190,262 \\
125,125 \\
471,509 \\
5 \\
58,109\end{array}$ & $\begin{array}{r}205,541 \\
133,493 \\
482,184 \\
10 \\
46,335\end{array}$ & $\begin{array}{r}196,700 \\
132,262 \\
480,551 \\
19 \\
87,745\end{array}$ \\
\hline Total Delivered to Consumers ............................. & 784,707 & 815,633 & 845,009 & 867,563 & 897,277 \\
\hline 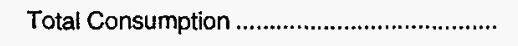 & 901,658 & 918,186 & 949,815 & 979,800 & $1,011,915$ \\
\hline 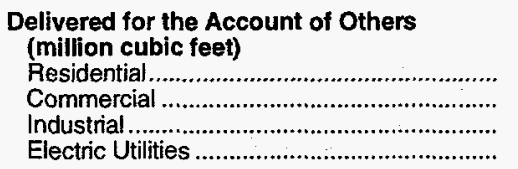 & $\begin{array}{r}0 \\
8,411 \\
242,861 \\
65,263\end{array}$ & $\begin{array}{r}0 \\
8,839 \\
264,556 \\
60,518\end{array}$ & $\begin{array}{r}0 \\
9,643 \\
285,872 \\
44,262\end{array}$ & $\begin{array}{r}2 \\
10,536 \\
312,266 \\
30,039\end{array}$ & $\begin{array}{r}0 \\
11,854 \\
312,869 \\
35,696\end{array}$ \\
\hline 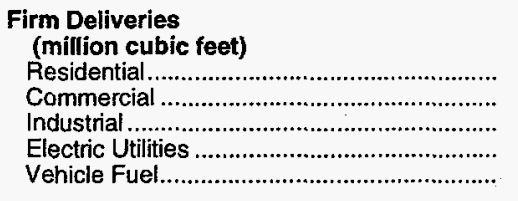 & $\begin{array}{l}\text { NA } \\
\text { NA } \\
\text { NA } \\
\text { NA } \\
\text { NA }\end{array}$ & $\begin{array}{l}\text { NA } \\
\text { NA } \\
\text { NA } \\
\text { NA } \\
\text { NA }\end{array}$ & $\begin{array}{l}\text { NA } \\
\text { NA } \\
\text { NA } \\
\text { NA } \\
\text { NA }\end{array}$ & $\begin{array}{r}205,541 \\
\mathrm{R}_{122,676} \\
\mathrm{R}_{317,640} \\
30,870 \\
10\end{array}$ & $\begin{array}{r}196,342 \\
120,154 \\
261,446 \\
2,051 \\
10\end{array}$ \\
\hline 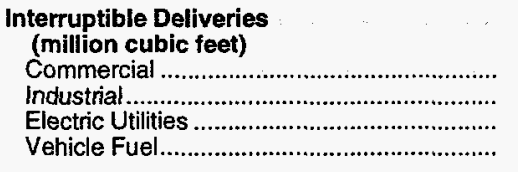 & $\begin{array}{l}\text { NA } \\
\text { NA } \\
\text { NA } \\
\text { NA }\end{array}$ & $\begin{array}{l}\text { NA } \\
\text { NA } \\
\text { NA } \\
\text { NA }\end{array}$ & $\begin{array}{l}\text { NA } \\
\text { NA } \\
\text { NA } \\
\text { NA }\end{array}$ & $\begin{array}{r}R_{10,817} \\
R_{317,640} \\
16,926\end{array}$ & $\begin{array}{r}12,108 \\
261,446 \\
36,486 \\
9\end{array}$ \\
\hline 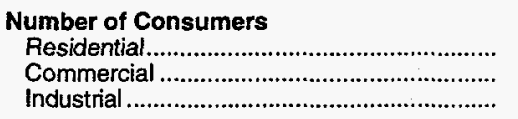 & $\begin{array}{r}2,319,908 \\
255,926 \\
7,653\end{array}$ & $\begin{array}{r}2,381,506 \\
259,282 \\
7,793\end{array}$ & $\begin{array}{r}2,443,619 \\
263,846 \\
7,869\end{array}$ & $\begin{array}{r}2,516,613 \\
269,995 \\
7,879\end{array}$ & $\begin{array}{r}2,586,801 \\
275,528 \\
7,864\end{array}$ \\
\hline 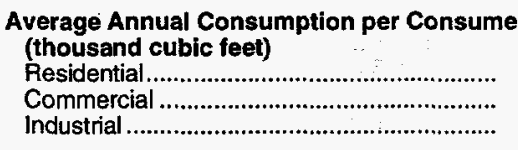 & $\begin{array}{r}75 \\
458 \\
55,474\end{array}$ & $\begin{array}{r}76 \\
467 \\
57,343\end{array}$ & $\begin{array}{r}78 \\
474 \\
59,920\end{array}$ & $\begin{array}{r}82 \\
494 \\
61,199\end{array}$ & $\begin{array}{r}76 \\
480 \\
61,108\end{array}$ \\
\hline 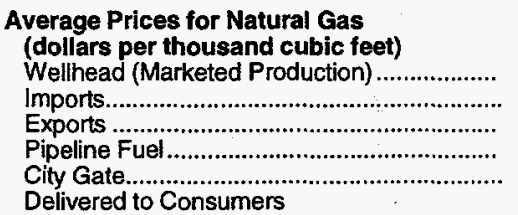 & $\begin{array}{r}2.32 \\
0 \\
0 \\
2.09 \\
3.00\end{array}$ & $\begin{array}{l}2.51 \\
2.82\end{array}$ & $\begin{array}{l}2.37 \\
2.95\end{array}$ & $\begin{array}{l}2.40 \\
3.13\end{array}$ & $\begin{array}{l}1.98 \\
3.01\end{array}$ \\
\hline 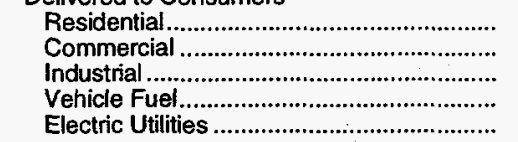 & $\begin{array}{l}5.47 \\
4.78 \\
3.14 \\
2.23 \\
1.86\end{array}$ & $\begin{array}{l}5.56 \\
4.77 \\
2.92 \\
4.11 \\
1.63\end{array}$ & $\begin{array}{l}5.59 \\
4.87 \\
3.08 \\
5.56 \\
1.88\end{array}$ & $\begin{array}{r}5.84 \\
5.16 \\
\mathbf{R}_{3.47} \\
4.70 \\
\mathbf{R}_{2.45}\end{array}$ & $\begin{array}{l}6.15 \\
5.38 \\
3.44 \\
4.00 \\
2.01\end{array}$ \\
\hline
\end{tabular}

$R=$ Revised data.
NA $=$ Not Available.

Notes: Deliveries to electric utilities (consumption) are reported on the Form EIA-759, "Monthly Power Plant Report." Deliveries to electric utilities for the account of others are reported on the Form EIA-176, "Annual Report of Natural and Supplemental Gas Supply and Disposition." See the discussion on electric utility data and Table A1 in Appendix A for a comparison of reporting to these two forms. Totals may not add due to independent rounding. The East South Central Census Division includes Alabama, Appendix A for a comparison of reporting

Sources: Energy Information Administration, (EIA), Form EIA-176, "Annual Report of Natural and Supplemental Gas Supply and Disposition"; Form EIA-627, "Annual Sources: Energy Information Administration, (EIA), Form EIA-176, "Annual Report of Natural and Supplemental Gas Supply and Disposition"; Form EIA-627, "Annual
Quantity and Value of Natural Gas Report"; Form EIA-857, "Monthly Report of Natural Gas Purchases and Deliveries to Consumers"; Form EIA-816, "Monthly Natural Gas Quantity and Value of Natural Gas Report"; Form ElA-857, "Monthly Report of Natural Gas Purchases and Deliveries to Consumers"; Form ElA-816, "Monthly Natural Gas Liquids Report"; Form ElA-759, "Monthly Power Plant Report"; Form FERC-423, "Monthly Report of Cost and Quality of Fuels for Electric Plants"; Form ElA-191, "Underground
Gas Storage Report"; Form FPC-14, "Annuai Report for Importers and Exporters of Natural Gas"; U.S. Crude Oil, Natural Gas, and Natural Gas Liquids Reserves, 1994 Annual Report, DOE/EIA-0216(94); and the U.S. Minerals Management Services. 


\section{West South Central - Natural Gas 1994}

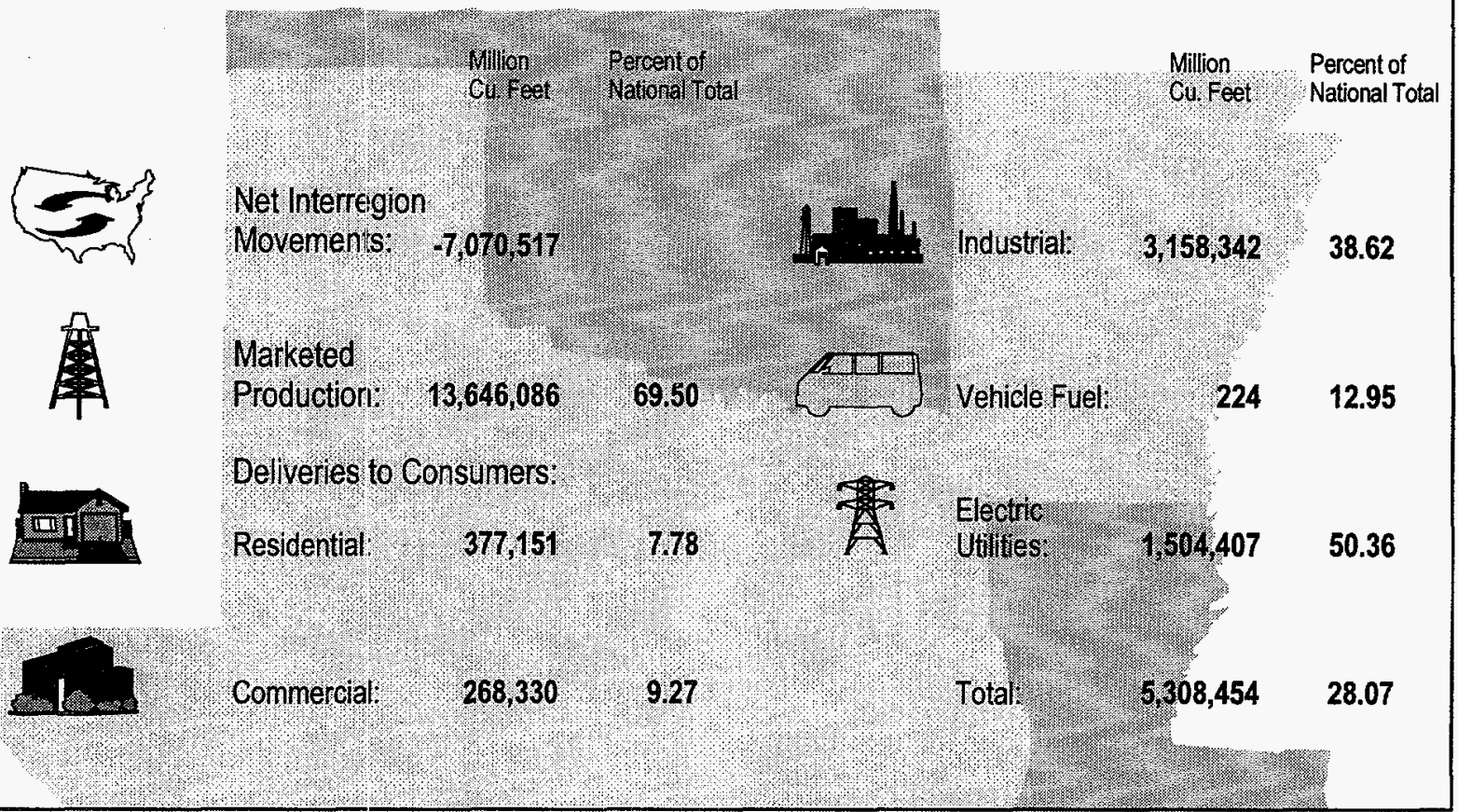

Table 42. Summary Statistics for Natural Gas - West South Central, 1990-1994

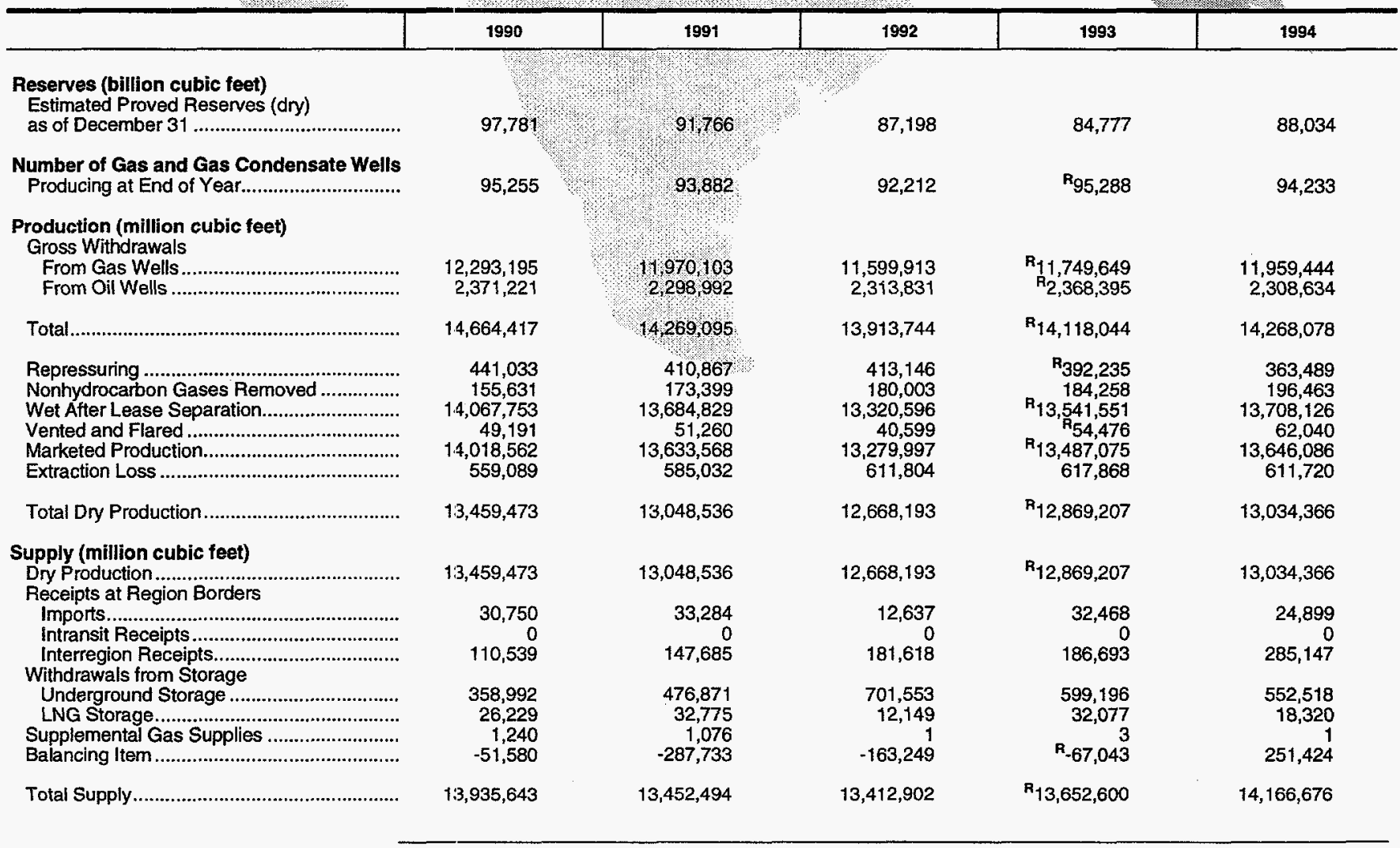


Table 42. Summary Statistics for Natural Gas - West South Central, 1990-1994 (Continued)

\begin{tabular}{|c|c|c|c|c|c|}
\hline & 1990 & 1991 & 1992 & 1993 & 1994 \\
\hline $\begin{array}{l}\text { Disposition (million cubic feet) } \\
\text { Consumption..................................................... } \\
\text { Deliveries at Region Borders }\end{array}$ & $6,009,616$ & $5,847,498$ & $5,790,481$ & ${ }^{P_{6,129}, 673}$ & $6,106,594$ \\
\hline 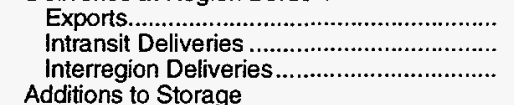 & $\begin{array}{r}13,983 \\
0 \\
7,411,578\end{array}$ & $\begin{array}{r}58,851 \\
0 \\
7,068,648\end{array}$ & $\begin{array}{r}93,408 \\
0 \\
6,882,836\end{array}$ & $\begin{array}{r}36,423 \\
0 \\
6,853,273\end{array}$ & $\begin{array}{r}44,041 \\
0 \\
7,336,522\end{array}$ \\
\hline 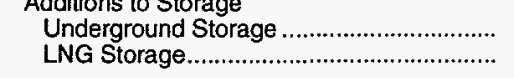 & $\begin{array}{r}472,434 \\
28,031\end{array}$ & $\begin{array}{r}444,174 \\
33,322\end{array}$ & $\begin{array}{r}633,582 \\
12,595\end{array}$ & $\begin{array}{r}602,502 \\
30,730\end{array}$ & $\begin{array}{r}661,623 \\
17,895\end{array}$ \\
\hline 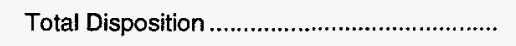 & $13,935,643$ & $13,452,494$ & $13,412,902$ & $\mathbf{R}_{13,652,600}$ & $14,166,676$ \\
\hline 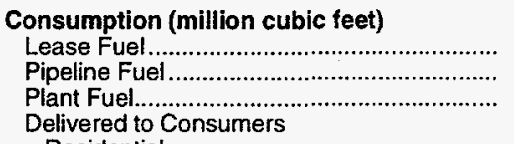 & $\begin{array}{l}482,706 \\
196,455 \\
288,834\end{array}$ & $\begin{array}{l}284,409 \\
169,007 \\
298,122\end{array}$ & $\begin{array}{l}316,047 \\
168,671 \\
297,325\end{array}$ & $\begin{array}{r}R_{345,514} \\
174,630 \\
279,995\end{array}$ & $\begin{array}{l}339,889 \\
196,927 \\
261,325\end{array}$ \\
\hline 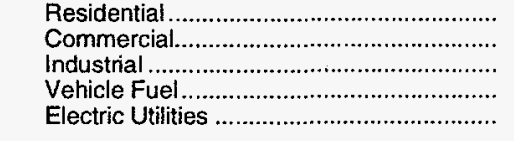 & $\begin{array}{r}368,853 \\
259,606 \\
2,935,890 \\
44 \\
1,477,228\end{array}$ & $\begin{array}{r}386,631 \\
271,999 \\
2,982,897 \\
19 \\
1,454,413\end{array}$ & $\begin{array}{r}375,189 \\
273,622 \\
2,960,486 \\
58 \\
1,399,083\end{array}$ & $\begin{array}{r}412,313 \\
R_{270,908} \\
R_{3,154,777} \\
189 \\
1,491,347\end{array}$ & $\begin{array}{r}377,151 \\
268,330 \\
3,158,342 \\
224 \\
1,504,407\end{array}$ \\
\hline Total Delivered to Consumers ......................... & $5,041,621$ & $5,095,960$ & $5,008,438$ & $\mathbf{R}_{5,329,533}$ & $5,308,454$ \\
\hline 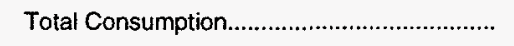 & $6,009,616$ & $5,847,498$ & $5,790,481$ & $\mathbf{R}_{6,129,673}$ & $6,106,594$ \\
\hline 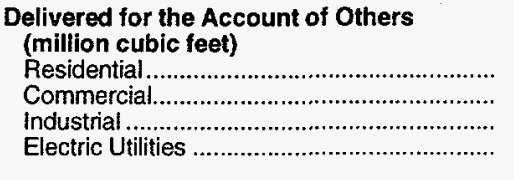 & $\begin{array}{r}1,976 \\
22,528 \\
1,887,178 \\
936,643\end{array}$ & $\begin{array}{r}43 \\
25,163 \\
1,922,857 \\
899,941\end{array}$ & $\begin{array}{r}43 \\
47,391 \\
2,009,082 \\
821,127\end{array}$ & $\begin{array}{r}2 \\
R_{35,390} \\
R_{2,157,426} \\
888,534\end{array}$ & $\begin{array}{r}0 \\
37,812 \\
2,344,856 \\
992,094\end{array}$ \\
\hline 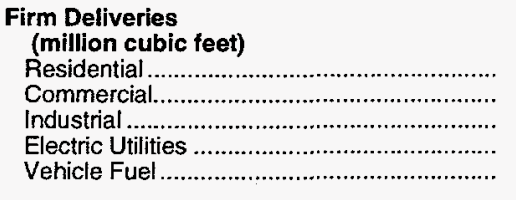 & $\begin{array}{l}\text { NA } \\
\text { NA } \\
\text { NA } \\
\text { NA } \\
\text { NA }\end{array}$ & $\begin{array}{l}\text { NA } \\
\text { NA } \\
\text { NA } \\
\text { NA } \\
\text { NA }\end{array}$ & $\begin{array}{l}\text { NA } \\
\text { NA } \\
\text { NA } \\
\text { NA } \\
\text { NA }\end{array}$ & $\begin{array}{r}412,313 \\
\mathbf{R}_{241,098} \\
\mathbf{R}_{1,405,833} \\
\mathbf{R}_{681,236} \\
83\end{array}$ & $\begin{array}{r}376,859 \\
241,730 \\
1,295,779 \\
767,092 \\
30\end{array}$ \\
\hline 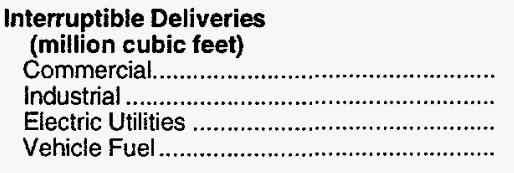 & $\begin{array}{l}\text { NA } \\
\text { NA } \\
\text { NA } \\
\text { NA }\end{array}$ & $\begin{array}{l}\text { NA } \\
\text { NA } \\
\text { NA } \\
\text { NA }\end{array}$ & $\begin{array}{l}\text { NA } \\
\text { NA } \\
\text { NA }\end{array}$ & $\begin{array}{r}R_{29,810} \\
R_{1,405,833} \\
587,119 \\
106\end{array}$ & $\begin{array}{r}26,600 \\
1,295,779 \\
610,088 \\
194\end{array}$ \\
\hline 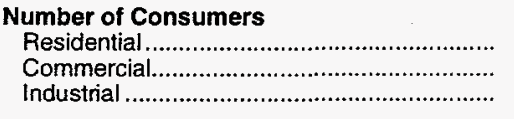 & $\begin{array}{r}5,470,002 \\
478,271 \\
19,448\end{array}$ & $\begin{array}{r}5,530,225 \\
479,460 \\
19,446\end{array}$ & $\begin{array}{r}5,562,827 \\
503,361 \\
11,111\end{array}$ & $\begin{array}{r}5,643,699 \\
R_{510,704} \\
\mathbf{R}_{11,691}\end{array}$ & $\begin{array}{r}5,663,010 \\
523,570 \\
11,229\end{array}$ \\
\hline 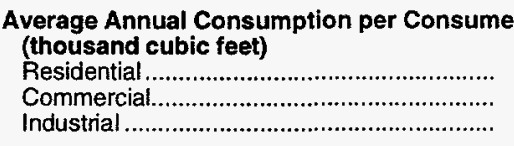 & $\begin{array}{r}67 \\
543 \\
150,961\end{array}$ & $\begin{array}{r}70 \\
567 \\
153,394\end{array}$ & $\begin{array}{r}67 \\
544 \\
266,446\end{array}$ & $\begin{array}{r}73 \\
R_{530} \\
R_{269,847}\end{array}$ & $\begin{array}{r}67 \\
512 \\
281,267\end{array}$ \\
\hline 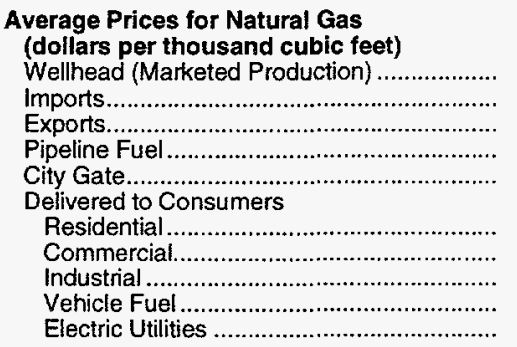 & $\begin{array}{l}1.67 \\
1.88 \\
1.82 \\
1.84 \\
2.80\end{array}$ & $\begin{array}{l}1.63 \\
1.70 \\
1.75 \\
1.84 \\
2.64\end{array}$ & $\begin{array}{l}1.75 \\
1.73 \\
1.92 \\
1.88 \\
2.81\end{array}$ & $\begin{array}{r}5.69 \\
\mathbf{R}_{4.54} \\
\mathbf{R}_{2.44} \\
2.83 \\
2.55\end{array}$ & $\begin{array}{l}1.94 \\
1.79 \\
1.68 \\
1.57 \\
2.82\end{array}$ \\
\hline
\end{tabular}

$\mathrm{R}=$ Revised data.

NA = Not Available.

Notes: Deliveries to electric utilities (consumption) are reported on the Form ElA-759, "Monthly Power Plant Report." Deliveries to electric utilities for the account of others are reported on the Form ElA-176, "Annual Report of Natural and Supplemental Gas Supply and Disposition." See the discussion on electric utility data and Table A1 in Appendix A for a comparison of reporting to these two forms. Totals may not add due to independent rounding. The West South Central Census Division includes Arkansas, Louisiana, Oklahoma, and Texas.

Sources: Energy information Administration (EIA), Form EIA-176, "Annual Report of Natural and Supplemental Gas Supply and Disposition"; Form ElA-627, "Annual Quantity and Value of Natural Gas Report"; Form ElA-857, "Monthly Report of Natural Gas Purchases and Deliveries to Consumers"; Form ElA-816, "Monthly' Natural Gas Liquids Report"; Form EIA-759, "Monthly Power Plant Report"; Form FERC-423, "Monthly Report of Cost and Quality of Fuels for Electric Plants"; Form EIA-191, "Underground Gas Storage Report"; Form FPC-14, "Annual Report for Importers and Exporters of Natural Gas"; U.S. Crude Oil, Natural Gas, and Natural Gas Liquids Reserves, 1994 Annual Report, DOE/EIA-0216(94); and the U.S. Minerals Management Service. 
Mountain

\section{Wilion 2 Percent of}

Cu Feat Y NationalTotal
Million

Cu. Feet
Percent of National Total

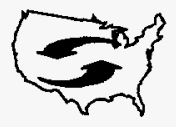

Net Interregion

Movements:

$-1,689,015$

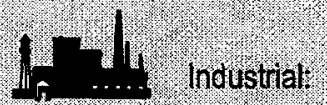

285,475

Marketed

Production

$3,028,956$

15.4

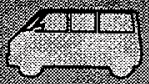

Vehiche Fuel:

312

18.03

Deliveries to Consumers:

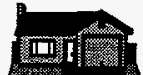

Residentia

272,805

5.63

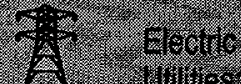

Whilli:e:

102,719

3.44

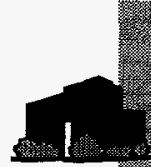

connenet:

197.515

Total:

858,826

Table 43. Summary Stattstres tor Nowral Gas - Mountain, 1990-1994

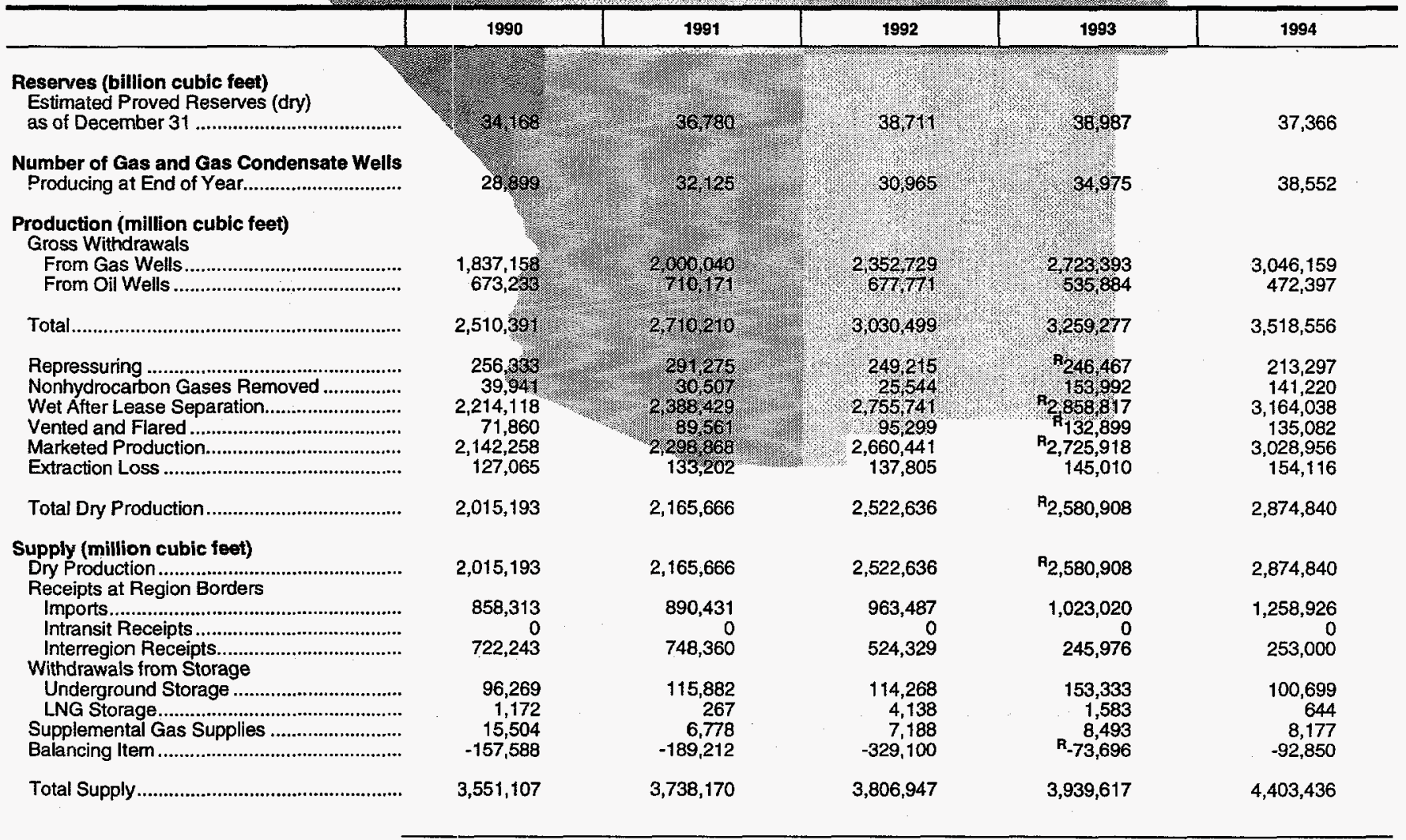

See footnotes at end of table. 
Table 43. Summary Statistics for Natural Gas - Mountain, 1990-1994 (Continued)

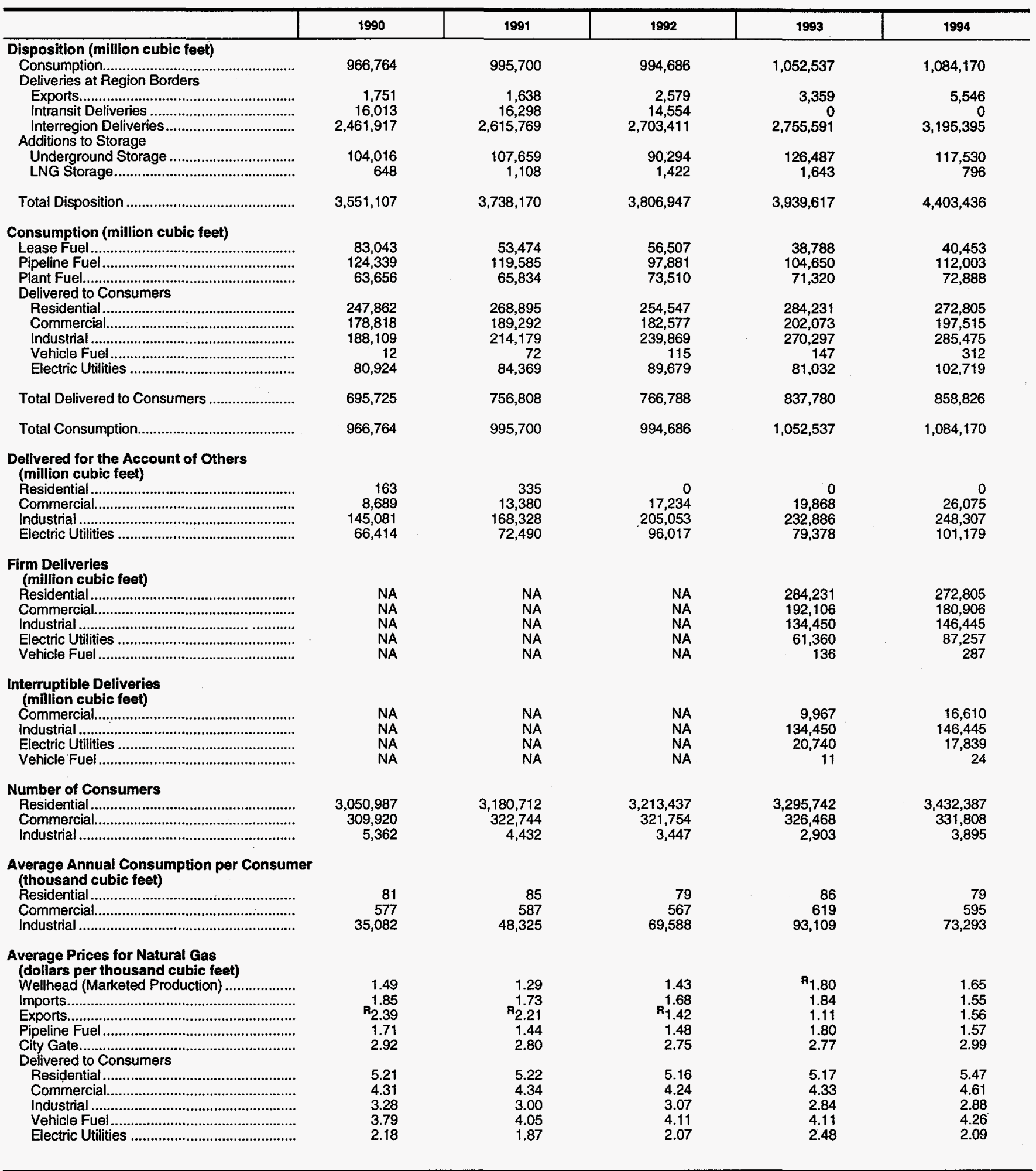

$\mathbf{R}=$ Revised data.

NA = Not Available.

Notes: Deliveries to electric utilities (consumption) are reported on the Form ElA-759, "Monthly Power Plant Report." Deliveries to electric utilities for the account of others are reported on the Form EIA-176, "Annual Report of Natural and Supplemental Gas Supply and Disposition." See the discussion on electric utility data and Table A1 in Appendix A for a comparison of reporting to these two forms. Totals may not add due to independent rounding. The Mountain Census Division includes Arizona, Colorado, idaho, Montana, Nevada, New Mexico, Utah, and Wyoming.

Sources: Energy Information Administration (EIA), Form EIA-176, "Annual Report of Natural and Supplemental Gas Supply and Disposition"; Form EIA-627, "Annual Quantity and Value of Natural Gas Report"; Form EIA-857, "Monthly Report of Natural Gas Purchases and Deliveries to Consumers"; Form EIA-816, "Monthly Natural Gas Liquids Report"; Form EIA-759, "Monthly Power Plant Report"; Form FERC-423, "Monthly Report of Cost and Quality of Fuels for Electric Plants". Form EIA-191, "Underground Gas Storage Report". Form FPC-14. "Annual Report for Importers and Exporters of Natural Gas"; US. Crude Oil, Natural Gas, and Natural Gas Liquids Fesenves, 1994 Annual Report, DOE/EIA-0216(94); and the U.S. Minerals Management Service. 


\section{Pacific Contiguous}
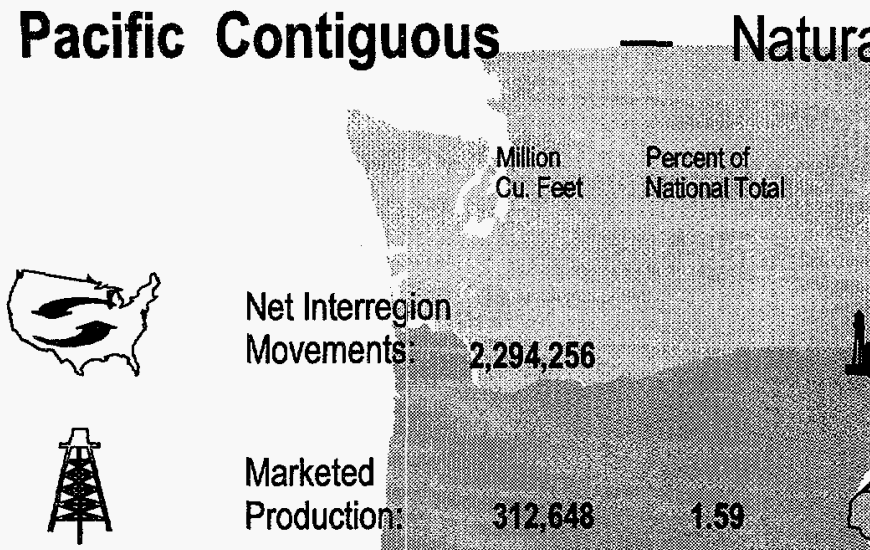

Net Interregion

Movements:

$2,294,256$

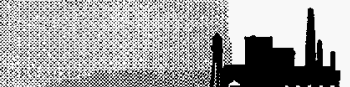

Industrial:

826,923

Marketed

Production:

312,648

1.59

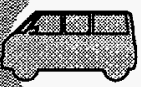

Vehicle Fuel:

723

41.79

Deliveries to Consumers:

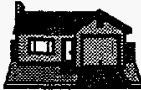

Residential:

602,951

1244

Electric

Utilities:

629,883

21.09

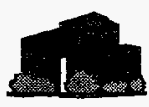

Commercial:

327,931

11.33

Total:

$2,388,411$

12.63

Table 44. Summary Statistics for Natural Gas - Pacific Contiguous, 1990-1994

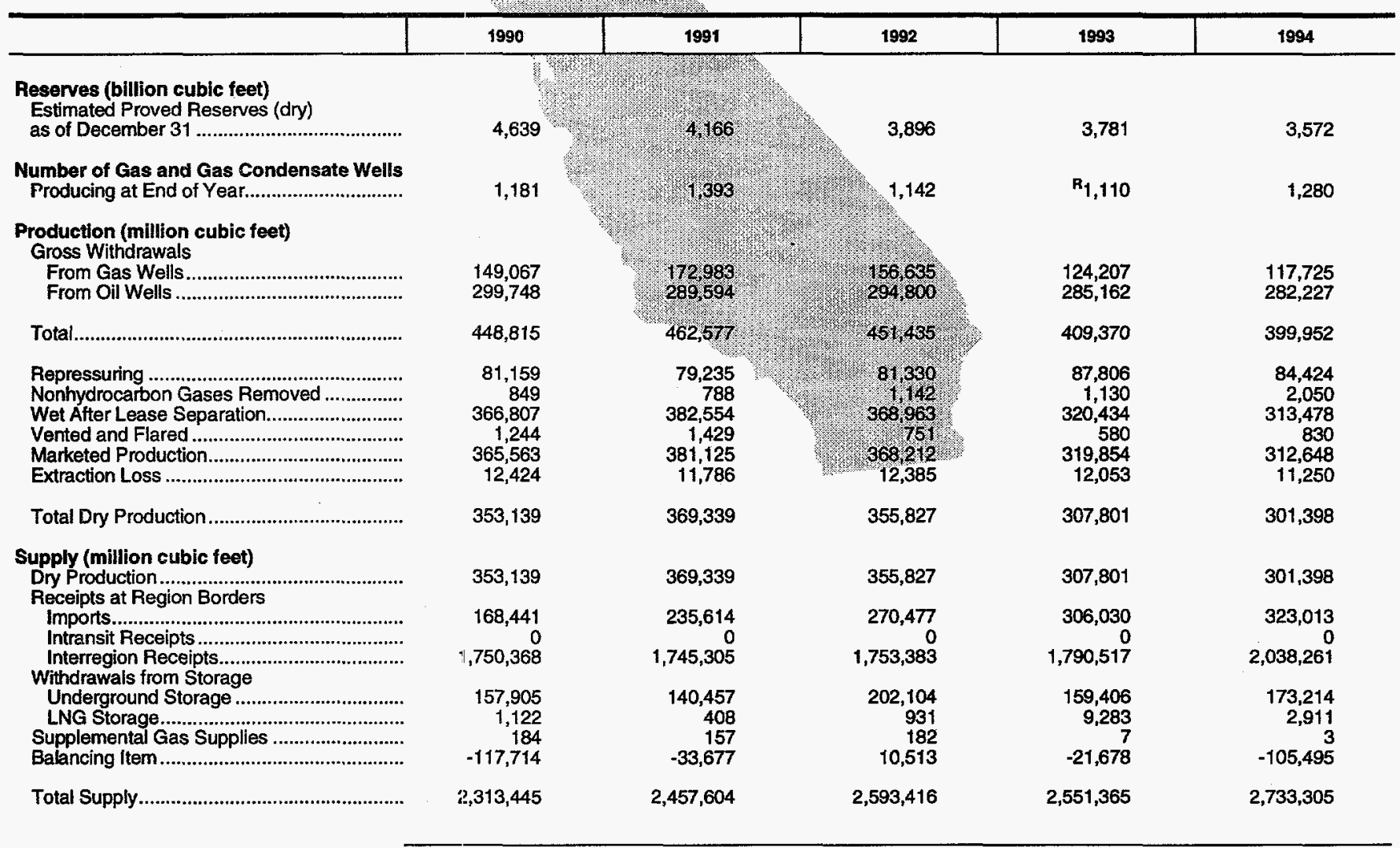

See footnotes at end of table. 
Table 44. Summary Statistics for Natural Gas - Pacific Contiguous, 1990-1994 (Continued)

\begin{tabular}{|c|c|c|c|c|c|}
\hline & 1990 & 1994 & 1992 & 1993 & 1994 \\
\hline $\begin{array}{l}\text { Disposition (million cubic feet) } \\
\text { Consumption............................................................ } \\
\text { Deliveries at Region Borders }\end{array}$ & $2,136,008$ & $2,267,140$ & $2,322,074$ & $2,310,388$ & $2,482,502$ \\
\hline 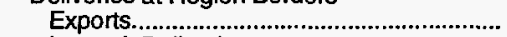 & 0 & 0 & 0 & 0 & 0 \\
\hline 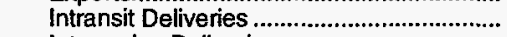 & 0 & 0 & 0 & 0 & 0 \\
\hline \multirow{2}{*}{\multicolumn{6}{|c|}{ Additions to Storage }} \\
\hline & & & & & \\
\hline 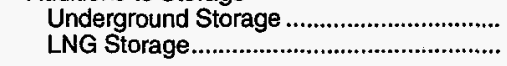 & $\begin{array}{r}167,265 \\
864\end{array}$ & $\begin{array}{r}156,500 \\
662\end{array}$ & $\begin{array}{r}167,446 \\
389\end{array}$ & $\begin{array}{r}180,913 \\
4,318\end{array}$ & $\begin{array}{r}180,572 \\
3,213\end{array}$ \\
\hline 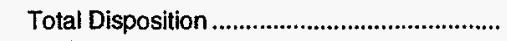 & $2,313,445$ & $2,457,604$ & $2,593,416$ & $2,551,365$ & $2,733,305$ \\
\hline 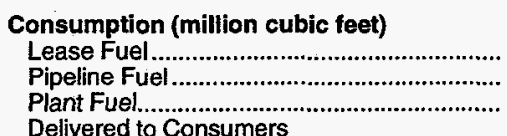 & $\begin{array}{r}14,847 \\
34,200 \\
7,958\end{array}$ & $\begin{array}{r}96,502 \\
32,422 \\
7,809\end{array}$ & $\begin{array}{r}84,288 \\
24,644 \\
8,008\end{array}$ & $\begin{array}{r}80,329 \\
20,906 \\
7,096\end{array}$ & $\begin{array}{r}63,347 \\
24,357 \\
6,388\end{array}$ \\
\hline \multicolumn{6}{|l|}{ Delivered to Consumers } \\
\hline 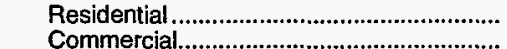 & 578,235 & 581,243 & 545,694 & 584,003 & 602,951 \\
\hline 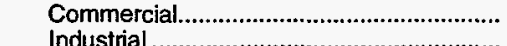 & 344,210 & 351,675 & 342,378 & 317,950 & 327,931 \\
\hline 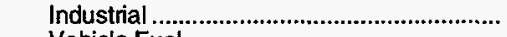 & 692,520 & 737,404 & 732,854 & 812,614 & 826,923 \\
\hline 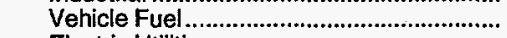 & & & 127 & 363 & \\
\hline 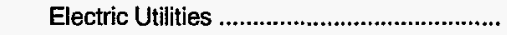 & 463,982 & 460,009 & 584,082 & 487,127 & 629,883 \\
\hline Total Delivered to Consumers........................ & $2,079,002$ & $2,130,407$ & $2,205,134$ & $2,202,057$ & $2,388,411$ \\
\hline 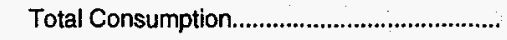 & $2,136,008$ & $2,267,140$ & $2,322,074$ & $2,310,388$ & $2,482,502$ \\
\hline \multicolumn{6}{|l|}{$\begin{array}{l}\text { Delivered for the Account of Others } \\
\text { (million cubic feet) }\end{array}$} \\
\hline 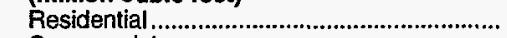 & 0 & 1,256 & 3,502 & 3,700 & 3,507 \\
\hline 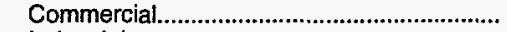 & 41,263 & 67,606 & 78,045 & 60,956 & 136,770 \\
\hline 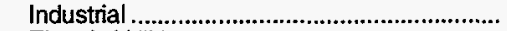 & 421,700 & 511,785 & 527,181 & 583,061 & 643,271 \\
\hline 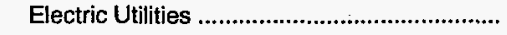 & 121,648 & 236,659 & 422,650 & 359,692 & 585,194 \\
\hline \multicolumn{6}{|l|}{$\begin{array}{l}\text { Firm Deliveries } \\
\text { (million cubic feet) }\end{array}$} \\
\hline 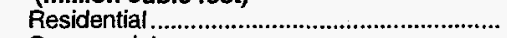 & NA & NA & NA & 584,003 & 602,951 \\
\hline 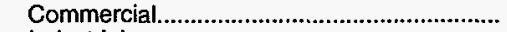 & NA & NA & NA & 298,075 & 279,164 \\
\hline 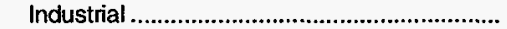 & NA & NA & NA & 166,902 & 359,909 \\
\hline 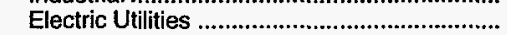 & NA & NA & NA & 431,820 & 316,671 \\
\hline 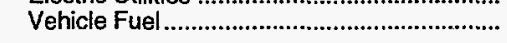 & NA & NA & NA & 340 & 607 \\
\hline \multicolumn{6}{|l|}{$\begin{array}{l}\text { Interruptible Deliveries } \\
\text { (million cubic feet) }\end{array}$} \\
\hline 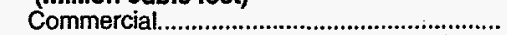 & NA & NA & NA & 19,875 & 48,767 \\
\hline 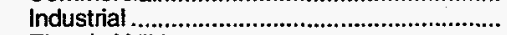 & NA & NA & NA & 166,902 & 359,909 \\
\hline Electric Utilities ............................................. & NA & NA & NA & 47,427 & 308,741 \\
\hline 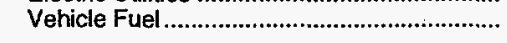 & NA & NA & NA & 23 & 116 \\
\hline \multicolumn{6}{|l|}{ Number of Consumers } \\
\hline 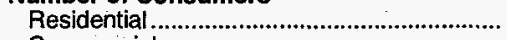 & $9,282,038$ & $9,503,129$ & $9,563,782$ & $9,662,813$ & $9,786,893$ \\
\hline 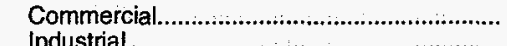 & 520,396 & 537,539 & 526,109 & 529,368 & 532,958 \\
\hline 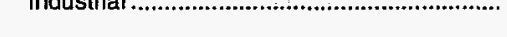 & $30,1 / 5$ & $49,14 r$ & 44,358 & 40,092 & 40,101 \\
\hline \multicolumn{6}{|l|}{$\begin{array}{l}\text { Average Annual Consumption per Consumer } \\
\text { (thousand cubic feet) }\end{array}$} \\
\hline & 62 & 61 & 57 & 60 & 62 \\
\hline 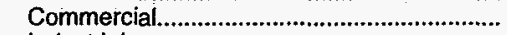 & 661 & 654 & 651 & 601 & 615 \\
\hline 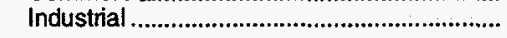 & 13,802 & 15,004 & 16,374 & 17,329 & 19,186 \\
\hline \multicolumn{6}{|l|}{$\begin{array}{l}\text { Average Prices for Natural Gas } \\
\text { (dollars per thousand cubic feet) }\end{array}$} \\
\hline Wellhead (Marketed Production) .................... & 2.36 & 2.45 & 2.33 & 2.37 & 3.03 \\
\hline 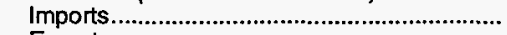 & 1.61 & 1.51 & 1.47 & 1.81 & 1.79 \\
\hline 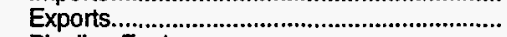 & 0 & & & & \\
\hline Pipeline Fuel .......................................................... & 2.28 & 2.19 & 1.67 & 0.97 & 1.09 \\
\hline 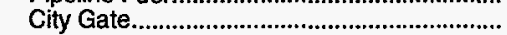 & 2.80 & 2.69 & 2.62 & 2.77 & 2.58 \\
\hline \multicolumn{6}{|l|}{ Delivered to Consumers } \\
\hline 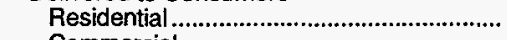 & 5.74 & 6.14 & 5.90 & 6.15 & 6.35 \\
\hline 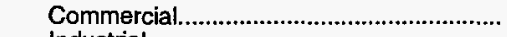 & 4.99 & 5.25 & 5.01 & 5.70 & 6.46 \\
\hline 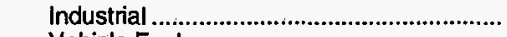 & 3.71 & 3.76 & 3.54 & 2.93 & 3.21 \\
\hline 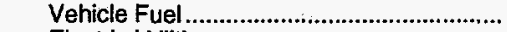 & 4.11 & 4.27 & 4.58 & 4.63 & 4.85 \\
\hline 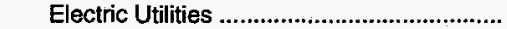 & 3.09 & 2.92 & 2.79 & 3.03 & 2.54 \\
\hline
\end{tabular}

$R=$ Revised data.
NA $=$ Not Available.

Notes: Deliveries to electric utilities (consumption) are reported on the Form ElA-759, "Monthly Power Plant Report." Deliveries to electric utilities for the account of others are reported on the Form EIA-176, "Annual Report of Natural and Supplemental Gas Supply and Disposition." See the discussion on electric utility data and Table A1 in Appendix A for a comparison of reporting to these two forms. Totals may not add due to independent rounding. The Pacific Contiguous Census Division includes Califomia, Oregon, and Washington.

Sources: Energy Information Administration (EIA), Form EIA-176, "Annual Report of Natural and Supplemental Gas Supply and Disposition"; Form EIA-627, "Annual Quantity and Value of Natural Gas Report"; Form EIA-857, "Monthly Report of Natural Gas Purchases and Deliveries to Consumers"; Form ElA-816, "Monthly Natural Gas Liquids Report"; Form EIA-759, "Monthly Power Plant Report"; Form FERC-423, "Monthly Report of Cost and Quality of Fuels for Electric Plants"; Form EIA-191, "Underground Gas Storage Report"; Form FPC-14, "Annual Report for Importers and Exporters of Natural Gas"; U.S. Crude Oil, Natural Gas, and Natural Gas Liquids Reserves, 1994 Annual Report, DOE/EIA-0216(94); and the U.S. Minerals Management Service. 


\section{Pacific Noncontiguous}

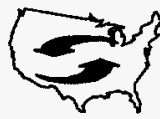

Net Interreglon

Movements:

$-62,682$

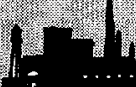

Industrial:

61,404

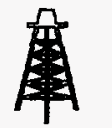

Marketed

Production: $\quad 453,425$

2.31

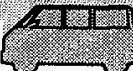

Vehicle Fuel:

0

Deliveries to Consumers:

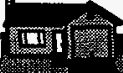

Residential:

15,473

0.32

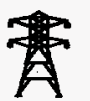

Electric

Utilities:

Commercial:

22,898

0.79

Total:

128,823

Table 45. Summary Statistics for Natural Gas - Pacific Noncontiguous, 1990-1994

\begin{tabular}{|c|c|c|c|c|c|}
\hline & 1990 & 1991 & 1992 & 1993 & 1994 \\
\hline 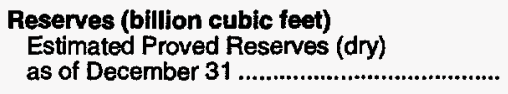 & 9,300 & 9,553 & 9,638 & 9,907 & 9,733 \\
\hline 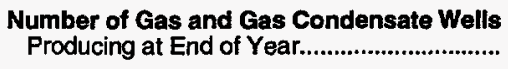 & 111 & 110 & 112 & 113 & 104 \\
\hline 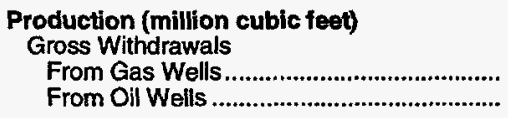 & $\begin{array}{r}204,093 \\
1,849,231\end{array}$ & $\begin{array}{r}197,502 \\
2,181,393\end{array}$ & $\begin{array}{r}198,603 \\
2,427,110\end{array}$ & $\begin{array}{r}190,139 \\
2,588,202\end{array}$ & $\begin{array}{r}180,639 \\
2,905,261\end{array}$ \\
\hline Total & $2,053,324$ & $2,378,896$ & $2,625,713$ & $2,778,341$ & $3,085,900$ \\
\hline 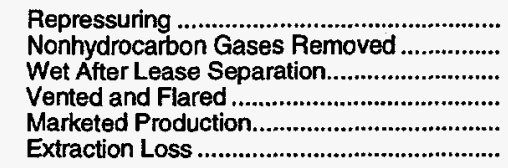 & $\begin{array}{r}1,639,689 \\
0 \\
413,634 \\
10,727 \\
402,907 \\
21,476\end{array}$ & $\begin{array}{r}1,930,290 \\
0 \\
448,605 \\
10,784 \\
437,822 \\
28,440\end{array}$ & $\begin{array}{r}2,168,019 \\
0 \\
457,694 \\
14,097 \\
443,597 \\
32,004\end{array}$ & $\begin{array}{r}2,325,506 \\
0 \\
452,835 \\
22,485 \\
430,350 \\
32,257\end{array}$ & $\begin{array}{r}2,619,236 \\
0 \\
466,665 \\
13,240 \\
453,425 \\
30,945\end{array}$ \\
\hline 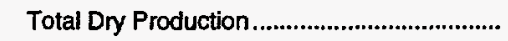 & 381,431 & 409,382 & 411,593 & 398,093 & 422,480 \\
\hline $\begin{array}{l}\text { Supply (million cubic feet) } \\
\text { Dry Production ............................................. } \\
\text { Receipts at Region Borders }\end{array}$ & 381,431 & 409,382 & 411,593 & 398,093 & 422,480 \\
\hline 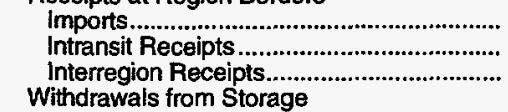 & $\begin{array}{l}0 \\
0 \\
0\end{array}$ & $\begin{array}{l}0 \\
0 \\
0\end{array}$ & $\begin{array}{l}0 \\
0 \\
0\end{array}$ & $\begin{array}{l}0 \\
0 \\
0\end{array}$ & $\begin{array}{l}0 \\
0 \\
0\end{array}$ \\
\hline 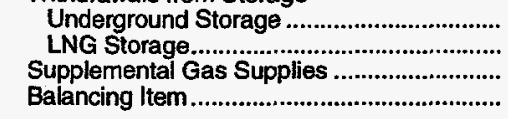 & $\begin{array}{r}0 \\
0 \\
2,817 \\
13,671\end{array}$ & $\begin{array}{r}0 \\
0 \\
2,725 \\
11,810\end{array}$ & $\begin{array}{r}0 \\
0 \\
2,711 \\
24,044\end{array}$ & $\begin{array}{r}0 \\
0 \\
2,705 \\
36,112\end{array}$ & $\begin{array}{r}0 \\
0 \\
2,831 \\
43,345\end{array}$ \\
\hline 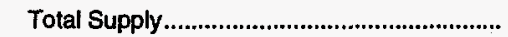 & 397,920 & 423,916 & 438,348 & 436,910 & 468,656 \\
\hline
\end{tabular}

See footnotes at end of table. 
Table 45. Summary Statistics for Natural Gas - Pacific Noncontiguous, 1990-1994 (Continued)

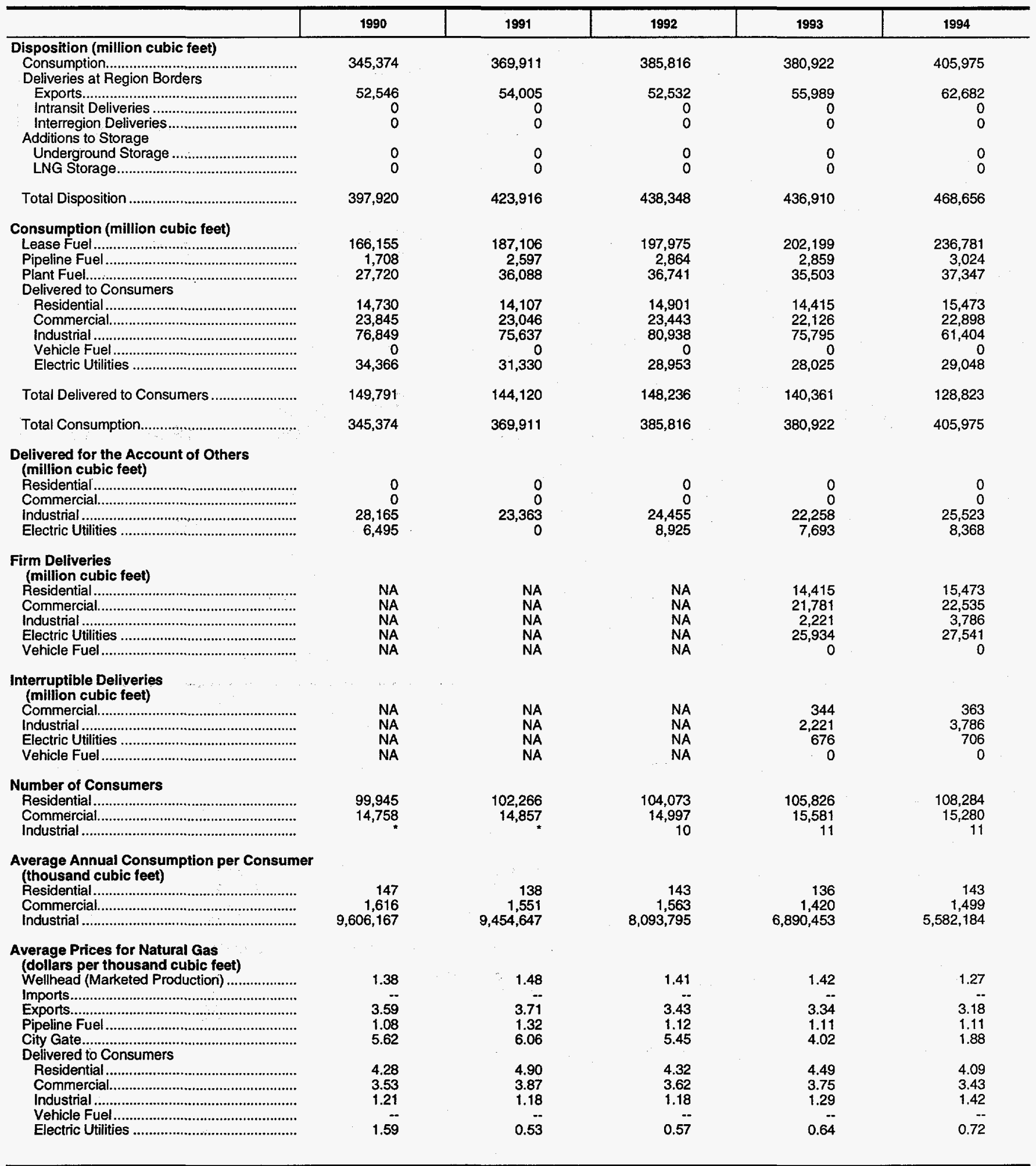

NA = Not Available.

Notes: Deliveries to electric utilities (consumption) are reported on the Form EIA-759, "Monthly Power Plant Peport." Deliveries to electric utilities for the account of others are reported on the Form EIA-176, "Annual Report of Natural and Supplemental Gas Supply and Disposition." See the discussion on electric utility data and Table A1 in Appendix A for a comparison of reporting to these two forms. Totals may not add due to independent rounding. The Pacific Noncontiguous Census Division includes Alaska and Hawaii.

Sources: Energy Information Administration (EIA), Form EIA-176, "Annual Report of Natural and Supplemental Gas Supply and Disposition"; Form ElA-627, "Annual Quantity and Value of Natural Gas Report"; Form EIA-857, "Monthly Report of Natural Gas Purchases and Deliveries to Consumers"; Form EIA-816, "Monthly Natural Gas Liquids Report"; Form EIA-759, "Monthly Power Plant Report"; Form FERC-423, "Monthly Report of Cost and Quality of Fuels for Electric Plants"; Form EIA-191, "Underground Gas Storage Report"; Form FPC-14, "Annual Report for Importers and Exporters of Natural Gas"; U.S. Crude Oil, Natural Gas, and Natural Gas Liquids Reserves, 1994 Annual Gas Storage Report"; Form FPC-14, "Annual Report for Importers and Expo 



\section{State Summary}

Texas and Louisiana continue to lead the Nation as the major producers of natural gas, followed by Oklahoma. These three States together accounted for 13.5 trillion cubic feet or 69 percent of the U. S. total marketed production in 1994.

The Nation's offshore natural gas was produced by Alabama, Alaska, California, Louisiana, and Texas, and together they produced nearly one-fourth of the total U. S. gross withdrawals. Louisiana continues to be by far the largest contributor (64 percent) to the total U. S. offshore withdrawals. Two-thirds of Louisiana's total gross with- drawals are offshore, while Texas' offshore gross withdrawals are about one-fifth of its total.

Texas and Louisiana, the major producing States, together with California are the leading consumers of natural gas, accounting for 36 percent of the total U. S. consumption of natural gas in 1994. Consumption of natural gas in California increased 7 percent, to 2.1 trillion cubic feet in 1994.

Detailed information on natural gas supply, disposition, and prices for each State for 1990 to 1994 are presented in Tables 48 through 98.

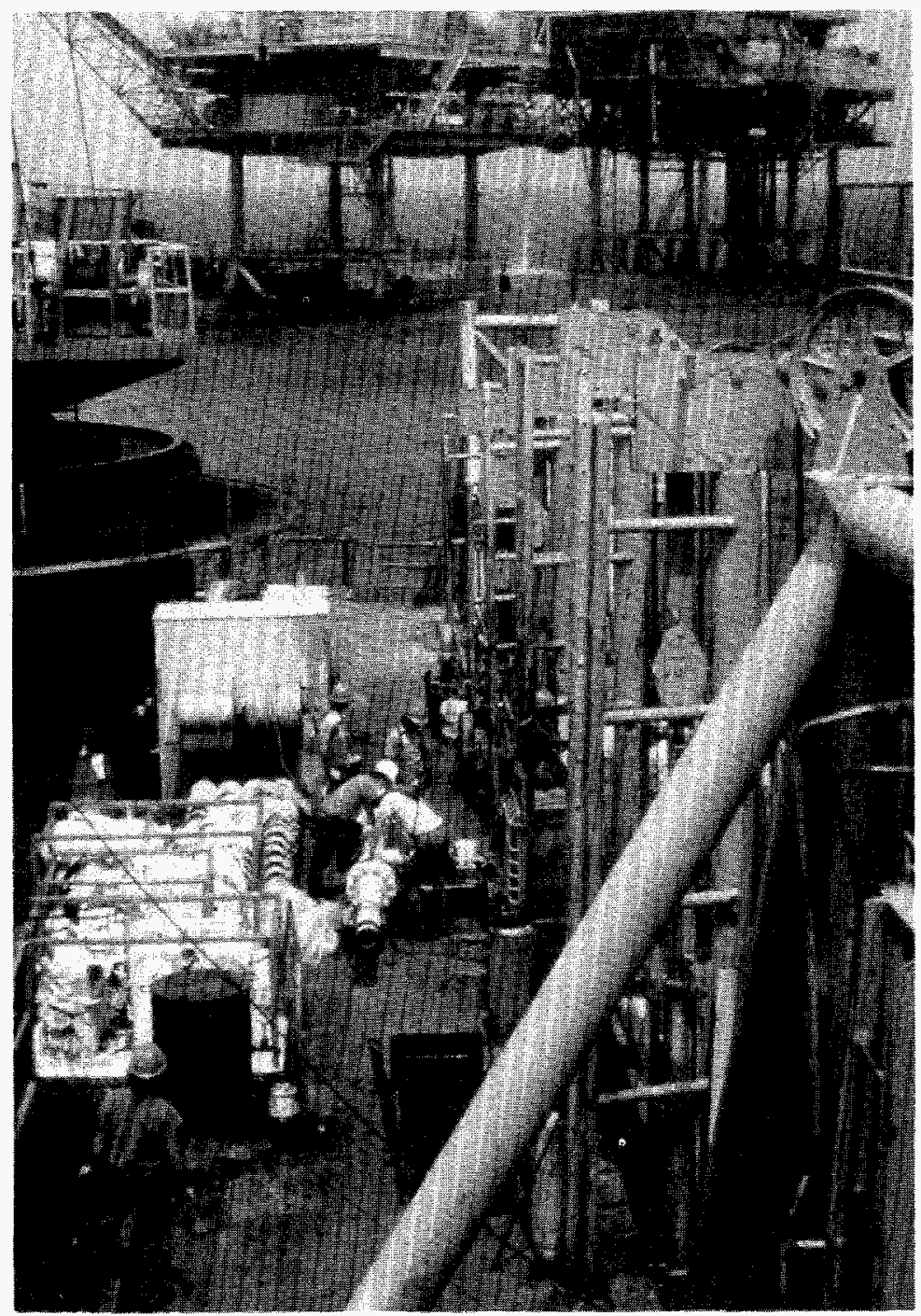

Work continues around the clock on an offshore drilling platform. 
Table 46. Percent Distribution of Natural Gas Supply and Disposition by State, 1994

\begin{tabular}{|c|c|c|c|}
\hline State & $\begin{array}{c}\text { Estimaled } \\
\text { Proved Reserves (dry) }\end{array}$ & Marketed Production & Total Consumption \\
\hline 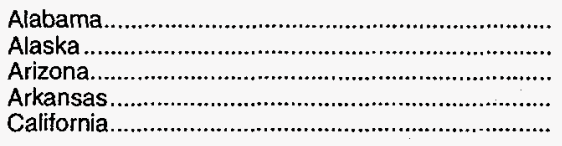 & $\begin{array}{r}2.95 \\
5.94 \\
\text { NA } \\
0.98 \\
2.18\end{array}$ & $\begin{array}{r}2.62 \\
2.31 \\
* \\
0.96 \\
1.58\end{array}$ & $\begin{array}{r}1.39 \\
1.94 \\
0.64 \\
1.18 \\
10.23\end{array}$ \\
\hline 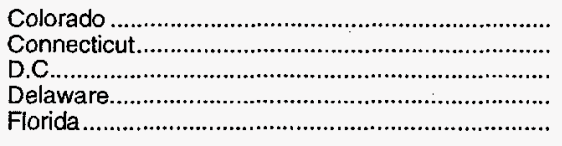 & $\begin{array}{r}4.12 \\
0 \\
0 \\
0 \\
0.06\end{array}$ & $\begin{array}{r}2.31 \\
0 \\
0 \\
0 \\
0.04\end{array}$ & $\begin{array}{l}1.33 \\
0.58 \\
0.15 \\
0.23 \\
1.77\end{array}$ \\
\hline 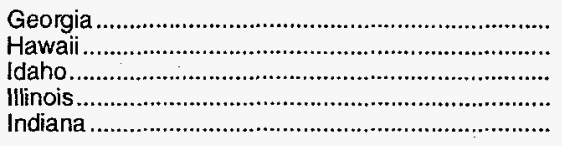 & $\begin{array}{r}0 \\
0 \\
0 \\
\text { NA } \\
\text { NA }\end{array}$ & $\begin{array}{l}0 \\
0 \\
0 \\
* \\
*\end{array}$ & $\begin{array}{l}1.65 \\
0.01 \\
0.27 \\
4.94 \\
2.50\end{array}$ \\
\hline 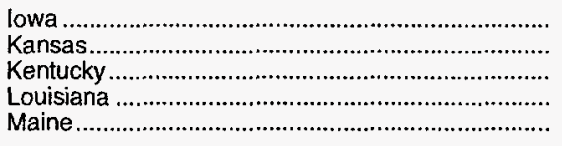 & $\begin{array}{r}0 \\
5.59 \\
0.59 \\
18.67 \\
0\end{array}$ & $\begin{array}{r}0 \\
3.63 \\
0.37 \\
26.33 \\
0\end{array}$ & $\begin{array}{l}1.20 \\
2.01 \\
1.00 \\
7.82 \\
0.02\end{array}$ \\
\hline 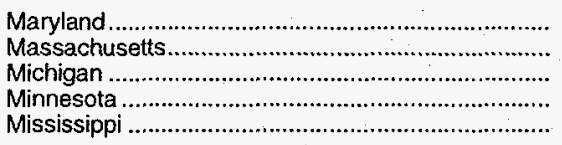 & $\begin{array}{r}\text { NA } \\
0 \\
0.81 \\
0 \\
0.40\end{array}$ & $\begin{array}{r}* \\
0 \\
1.13 \\
0 \\
0.32\end{array}$ & $\begin{array}{l}0.89 \\
1.63 \\
4.46 \\
1.56 \\
1.29\end{array}$ \\
\hline 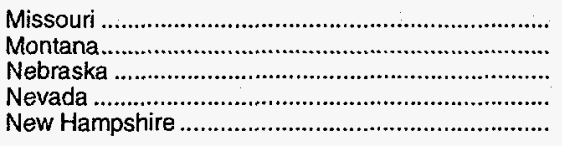 & $\begin{array}{r}\text { NA } \\
0.44 \\
\text { NA } \\
0 \\
0\end{array}$ & $\begin{array}{r}0.26 \\
0.02 \\
\star \\
0\end{array}$ & $\begin{array}{l}1.29 \\
0.25 \\
0.61 \\
0.49 \\
0.10\end{array}$ \\
\hline 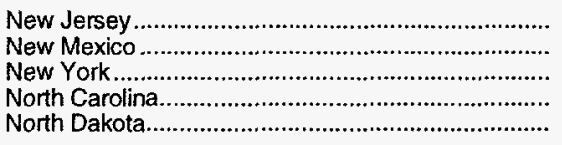 & $\begin{array}{r}0 \\
10.52 \\
0.15 \\
0 \\
0.31\end{array}$ & $\begin{array}{r}0 \\
7.93 \\
0.10 \\
0 \\
0.29\end{array}$ & $\begin{array}{l}2.82 \\
1.06 \\
4.88 \\
0.91 \\
0.21\end{array}$ \\
\hline 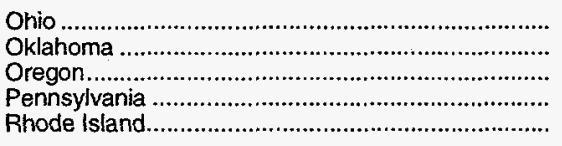 & $\begin{array}{r}0.67 \\
8.23 \\
N A \\
1.10 \\
0\end{array}$ & $\begin{array}{r}0.67 \\
9.85 \\
0.02 \\
0.77 \\
0\end{array}$ & $\begin{array}{l}4.06 \\
2.76 \\
0.71 \\
3.36 \\
0.34\end{array}$ \\
\hline $\begin{array}{l}\text { South Carolina } \\
\text { South Dakota } \\
\text { Tennessee } \\
\text { Texas } \\
\text { Utah }\end{array}$ & $\begin{array}{r}0 \\
N A \\
N A \\
25.85 \\
1.09\end{array}$ & $\begin{array}{r}0 \\
0.01 \\
0.01 \\
32.36 \\
1.38\end{array}$ & $\begin{array}{r}0.70 \\
0.15 \\
1.19 \\
17.66 \\
0.66\end{array}$ \\
\hline 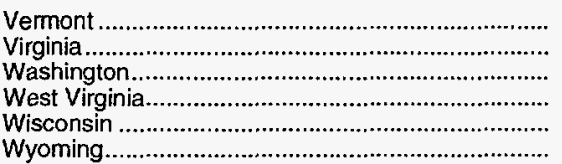 & $\begin{array}{r}0 \\
1.12 \\
0 \\
1.57 \\
0 \\
6.64\end{array}$ & $\begin{array}{r}0 \\
0.26 \\
0 \\
0.93 \\
0 \\
3.55\end{array}$ & $\begin{array}{l}0.04 \\
1.11 \\
1.03 \\
0.70 \\
1.71 \\
0.51\end{array}$ \\
\hline
\end{tabular}

NA = Not Available.

" = Volume is less than .01 percent.

Sources: Energy Information Administration (ElA), Form ElA-176, "Annual Report of Natural and Supplemental Gas Supply and Disposition"; Form EIA-627, "Annual Quantity and Value of Natural Gas Report"; and EIA Report, U.S. Crude Oil, Natural Gas, and Natural Gas Liquids Reserves, 1994 Annual Report, DOE/EIA-0216(94). 
Table 47. Percent Distribution of Natural Gas Delivered to Consumers by State, 1994

\begin{tabular}{|c|c|c|c|c|c|}
\hline State & Residential & Commercial & Industrial & Vehicle Fuel & Electric Utilities \\
\hline 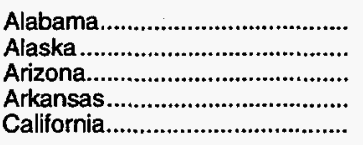 & $\begin{array}{r}1.03 \\
0.31 \\
0.61 \\
0.86 \\
10.75\end{array}$ & $\begin{array}{l}0.88 \\
0.72 \\
1.01 \\
0.95 \\
\dot{9} .05\end{array}$ & $\begin{array}{l}2.22 \\
0.75 \\
0.32 \\
1.64 \\
8.03\end{array}$ & $\begin{array}{r}0.20 \\
-\overline{-} \\
3.51 \\
-- \\
31.82\end{array}$ & $\begin{array}{r}0.13 \\
0.97 \\
0.79 \\
0.84 \\
20.13\end{array}$ \\
\hline 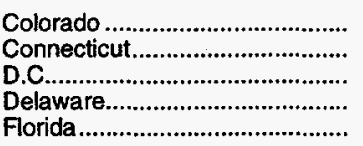 & $\begin{array}{l}2.05 \\
0.86 \\
0.33 \\
0.18 \\
0.29\end{array}$ & $\begin{array}{l}2.28 \\
1.35 \\
0.51 \\
0.19 \\
1.38\end{array}$ & $\begin{array}{r}0.87 \\
0.38 \\
- \\
0.21 \\
1.55\end{array}$ & $\begin{array}{r}3.93 \\
0.10 \\
0 . \\
0.04 \\
3.92\end{array}$ & $\begin{array}{r}0.16 \\
0.27 \\
-- \\
0.58 \\
6.05\end{array}$ \\
\hline 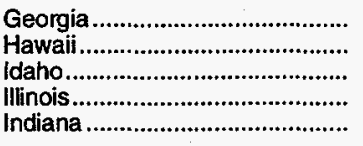 & $\begin{array}{l}2.18 \\
0.01 \\
0.25 \\
9.77 \\
3.25\end{array}$ & $\begin{array}{l}1.87 \\
0.08 \\
0.35 \\
6.83 \\
2.62\end{array}$ & $\begin{array}{r}2.13 \\
\cdots \\
0.36 \\
3.73 \\
3.30\end{array}$ & $\begin{array}{r}0.16 \\
-- \\
0.57 \\
1.62 \\
3.42\end{array}$ & $\begin{array}{r}0.03 \\
- \\
1.16 \\
0.30\end{array}$ \\
\hline 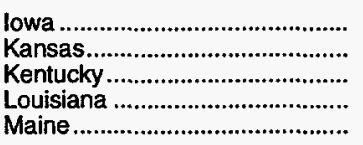 & $\begin{array}{l}1.61 \\
1.53 \\
1.29 \\
1.09 \\
0.02\end{array}$ & $\begin{array}{l}1.66 \\
1.81 \\
1.27 \\
0.84 \\
0.08\end{array}$ & $\begin{array}{r}1.33 \\
2.30 \\
1.02 \\
12.22 \\
0.02\end{array}$ & $\begin{array}{l}0.29 \\
0.58 \\
0.11 \\
1.30 \\
-\end{array}$ & $\begin{array}{r}0.09 \\
0.91 \\
0.01 \\
9.28 \\
--\end{array}$ \\
\hline 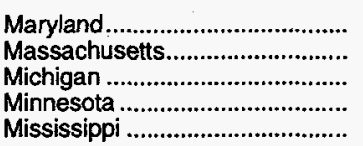 & $\begin{array}{l}1.58 \\
2.47 \\
7.52 \\
2.52 \\
0.56\end{array}$ & $\begin{array}{l}1.53 \\
2.92 \\
6.32 \\
2.90 \\
0.66\end{array}$ & $\begin{array}{l}0.58 \\
1.14 \\
4.01 \\
1.16 \\
1.18\end{array}$ & $\begin{array}{l}1.47 \\
0.15 \\
0.79 \\
1.66 \\
0.52\end{array}$ & $\begin{array}{l}0.43 \\
1.29 \\
0.61 \\
0.20 \\
2.76\end{array}$ \\
\hline 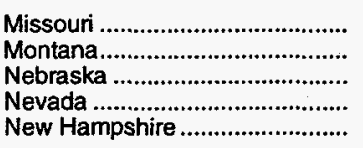 & $\begin{array}{l}2.53 \\
0.39 \\
0.92 \\
0.44 \\
0.14\end{array}$ & $\begin{array}{l}2.29 \\
0.45 \\
1.35 \\
0.65 \\
0.22\end{array}$ & $\begin{array}{l}0.88 \\
0.17 \\
0.45 \\
0.35 \\
0.06\end{array}$ & $\begin{array}{l}0.04 \\
0.32 \\
0.51 \\
2.08\end{array}$ & $\begin{array}{l}0.15 \\
0.02 \\
0.10 \\
1.08 \\
0.04\end{array}$ \\
\hline 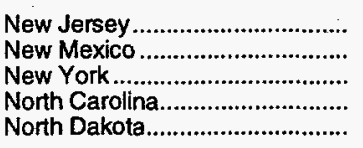 & $\begin{array}{l}4.47 \\
0.64 \\
7.95 \\
0.98 \\
0.22\end{array}$ & $\begin{array}{l}4.56 \\
0.86 \\
7.71 \\
1.35 \\
0.37\end{array}$ & $\begin{array}{l}2.33 \\
0.23 \\
2.62 \\
1.16 \\
0.07\end{array}$ & $\begin{array}{l}0.25 \\
3.57 \\
3.05 \\
0.45 \\
0.49\end{array}$ & $\begin{array}{r}1.43 \\
1.08 \\
6.11 \\
0.03\end{array}$ \\
\hline 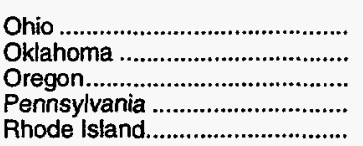 & $\begin{array}{l}7.08 \\
1.43 \\
0.60 \\
5.54 \\
0.36\end{array}$ & $\begin{array}{l}5.76 \\
1.26 \\
0.79 \\
4.78 \\
0.42\end{array}$ & $\begin{array}{l}3.80 \\
2.40 \\
0.77 \\
2.89 \\
0.50\end{array}$ & $\begin{array}{l}2.79 \\
9.05 \\
1.01 \\
0.59 \\
0.08\end{array}$ & $\begin{array}{l}0.09 \\
5.13 \\
0.88 \\
0.43 \\
0.02\end{array}$ \\
\hline 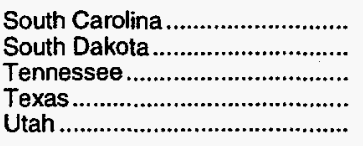 & $\begin{array}{l}0.48 \\
0.25 \\
1.18 \\
4.40 \\
1.01\end{array}$ & $\begin{array}{l}0.62 \\
0.36 \\
1.75 \\
6.23 \\
0.92\end{array}$ & $\begin{array}{r}1.19 \\
0.07 \\
1.45 \\
22.37 \\
0.45\end{array}$ & $\begin{array}{l}0.12 \\
0.32 \\
0.30 \\
2.62 \\
3.02\end{array}$ & $\begin{array}{r}0.10 \\
0.01 \\
0.03 \\
35.12 \\
0.30\end{array}$ \\
\hline 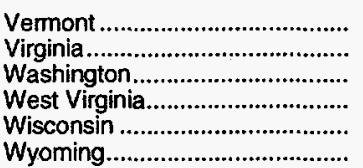 & $\begin{array}{l}0.05 \\
1.34 \\
1.10 \\
0.73 \\
2.64 \\
0.24\end{array}$ & $\begin{array}{l}0.09 \\
1.83 \\
1.49 \\
0.86 \\
2.72 \\
0.32\end{array}$ & $\begin{array}{l}0.03 \\
1.05 \\
1.32 \\
0.57 \\
1.65 \\
0.74\end{array}$ & $\begin{array}{l}1 . \overline{14} \\
8.96 \\
0.02 \\
2.10 \\
1.02\end{array}$ & $\begin{array}{l}0.01 \\
0.64 \\
0.08 \\
0.01 \\
0.13\end{array}$ \\
\hline
\end{tabular}

* Volume is less than .01 percent.

Sources: Energy Information Administration, Form EIA-176, "Annual Report of Natural and Supplemental Gas Supply and Disposition" and Form EIA-759, "Monthly Power Plant Report." 


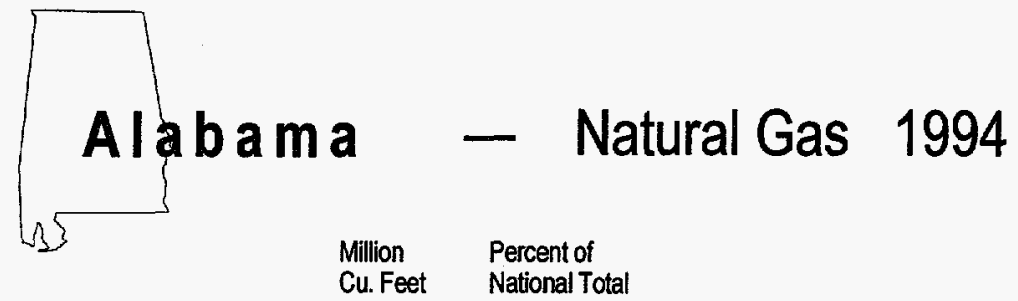

Million Percent of

Cu. Feet National Total

Cu. Feet

National Total

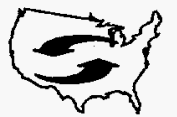

Net Interstate

Movements:

$-173,113$

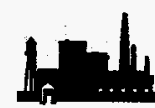

Industrial:

181,718

2.22

Marketed

Production: $\quad 515,271$

2.62

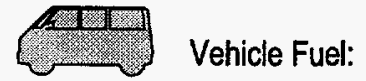

3

0.17

Deliveries to Consumers:

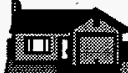

Residential:

49,748

1.03

Electric

3,834

0.13

Commercial:

25,526

0.88

Total:

260,830

1.38

Table 48. Summary Statistics for Natural Gas - Alabama, 1990-1994

\begin{tabular}{|c|c|c|c|c|c|}
\hline & 1990 & 1991 & 1992 & 1993 & 1994 \\
\hline 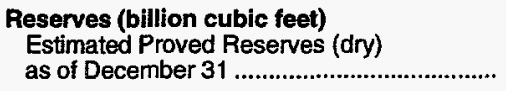 & 4,125 & 5,414 & 5,802 & 5,140 & 4,830 \\
\hline $\begin{array}{l}\text { Number of Gas and Gas Condensate Wells } \\
\text { Producing at End of Year........................................ }\end{array}$ & 2,362 & 3,392 & 3,350 & 3,514 & 3,565 \\
\hline 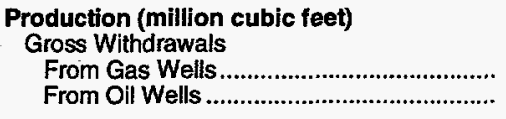 & $\begin{array}{r}181,324 \\
5,219\end{array}$ & $\begin{array}{r}218,145 \\
5,731\end{array}$ & $\begin{array}{r}403,848 \\
9,766\end{array}$ & $\begin{array}{r}435,066 \\
11,255\end{array}$ & $\begin{array}{r}566,140 \\
12,722\end{array}$ \\
\hline 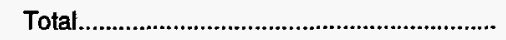 & 186,542 & 223,876 & 413,614 & 446,321 & 578,862 \\
\hline 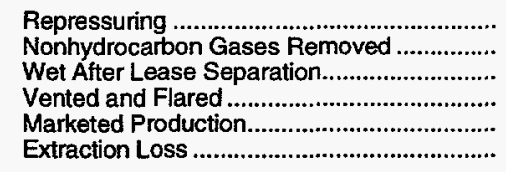 & $\begin{array}{r}28,540 \\
20,792 \\
137,209 \\
1,933 \\
135,276 \\
4,939\end{array}$ & $\begin{array}{r}30,689 \\
20,146 \\
173,040 \\
2,193 \\
170,847 \\
4,997\end{array}$ & $\begin{array}{r}29,996 \\
26,719 \\
356,899 \\
1,799 \\
355,099 \\
5,490\end{array}$ & $\begin{array}{r}31,179 \\
25,320 \\
389,822 \\
1,798 \\
388,024 \\
5,589\end{array}$ & $\begin{array}{r}33,961 \\
26,980 \\
517,921 \\
2,650 \\
515,271 \\
5,647\end{array}$ \\
\hline 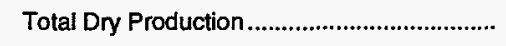 & 130,337 & 165,850 & 349,609 & 382,435 & 509,624 \\
\hline 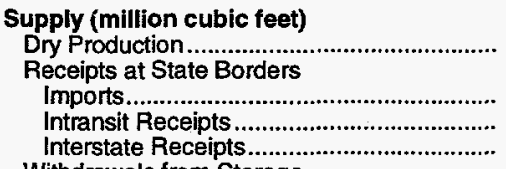 & $\begin{array}{r}130,337 \\
0 \\
0 \\
0 \\
2,818,949\end{array}$ & $\begin{array}{r}0 \\
0 \\
2,782,487\end{array}$ & $\begin{array}{r}0 \\
0 \\
2,792,910\end{array}$ & $\begin{array}{r}0 \\
0 \\
2,849,645\end{array}$ & $\begin{array}{r}509,624 \\
0 \\
0 \\
2,715,849\end{array}$ \\
\hline 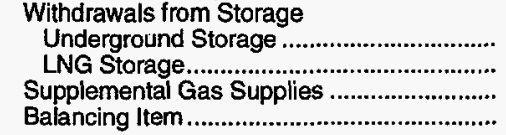 & $\begin{array}{r}0 \\
437 \\
320 \\
-16,872\end{array}$ & $\begin{array}{r}0 \\
530 \\
332 \\
-19,986\end{array}$ & $\begin{array}{r}0 \\
262 \\
171 \\
-30,213\end{array}$ & $\begin{array}{r}0 \\
396 \\
410 \\
-35,767\end{array}$ & $\begin{array}{r}55 \\
497 \\
69 \\
-46,632\end{array}$ \\
\hline 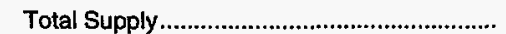 & $2,933,171$ & $2,929,212$ & $3,112,739$ & $3,197,119$ & $3,179,462$ \\
\hline
\end{tabular}

See footnotes at end of table. 
Table 48. Summary Statistics for Natural Gas - Alabama, 1990-1994 (Continued)

\begin{tabular}{|c|c|c|c|c|c|}
\hline & 1990 & 1991 & 1992 & 1993 & 1994 \\
\hline $\begin{array}{l}\text { Disposition (million cubic feet) } \\
\text { Consumption........................................... } \\
\text { Deliveries at State Borders }\end{array}$ & 243,803 & 253,874 & 278,794 & 292,346 & 288,863 \\
\hline 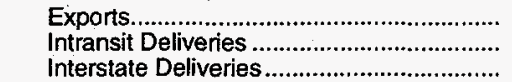 & $\begin{array}{r}0 \\
0 \\
2,688,474\end{array}$ & $\begin{array}{r}0 \\
0 \\
2,674,828\end{array}$ & $\begin{array}{r}0 \\
0 \\
2,833,444\end{array}$ & $\begin{array}{r}0 \\
0 \\
2,904,162\end{array}$ & $\begin{array}{r}0 \\
\mathrm{G} \\
2,888,962\end{array}$ \\
\hline 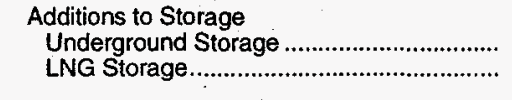 & $\begin{array}{r}0 \\
893\end{array}$ & $\begin{array}{r}0 \\
511\end{array}$ & $\begin{array}{r}0 \\
501\end{array}$ & $\begin{array}{r}0 \\
612\end{array}$ & $\begin{array}{l}694 \\
944\end{array}$ \\
\hline 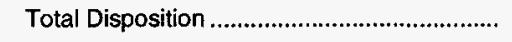 & $2,933,171$ & $2,929,212$ & $3,112,739$ & $3,197,119$ & $3,179,462$ \\
\hline 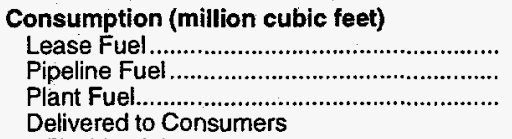 & $\begin{array}{r}6,171 \\
14,636 \\
3,710\end{array}$ & $\begin{array}{r}4,907 \\
16,482 \\
3,720\end{array}$ & $\begin{array}{r}8,391 \\
18,631 \\
4,477\end{array}$ & $\begin{array}{r}8,912 \\
15,559 \\
4,453\end{array}$ & $\begin{array}{r}9,381 \\
14,904 \\
3,747\end{array}$ \\
\hline 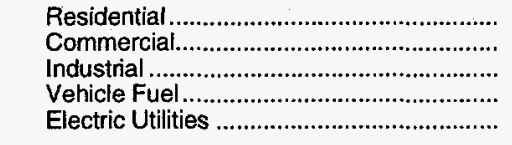 & $\begin{array}{r}45,411 \\
24,287 \\
145,628 \\
3 \\
3,958\end{array}$ & $\begin{array}{r}46,149 \\
23,711 \\
154,825 \\
0 \\
4,081\end{array}$ & $\begin{array}{r}49,644 \\
25,232 \\
169,049 \\
3 \\
3,368\end{array}$ & $\begin{array}{r}51,366 \\
25,723 \\
181,692 \\
4 \\
4,636\end{array}$ & $\begin{array}{r}49,748 \\
25,526 \\
181,718 \\
3 \\
3,834\end{array}$ \\
\hline Total Delivered to Consumers ............................ & 219,286 & 228,765 & 247,295 & 263,421 & 260,830 \\
\hline 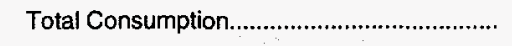 & 243,803 & 253,874 & 278,794 & 292,346 & 288,863 \\
\hline 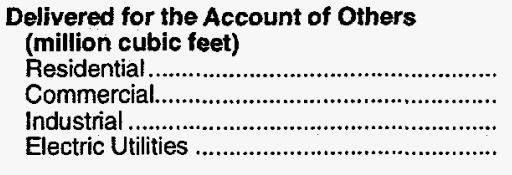 & $\begin{array}{r}0 \\
4,967 \\
96,889 \\
2,287\end{array}$ & $\begin{array}{r}0 \\
4,112 \\
106,274 \\
3,045\end{array}$ & $\begin{array}{r}0 \\
4,868 \\
118,457 \\
2,739\end{array}$ & $\begin{array}{r}2 \\
4,950 \\
131,817 \\
2,753\end{array}$ & $\begin{array}{r}0 \\
5,043 \\
132,073 \\
3,271\end{array}$ \\
\hline 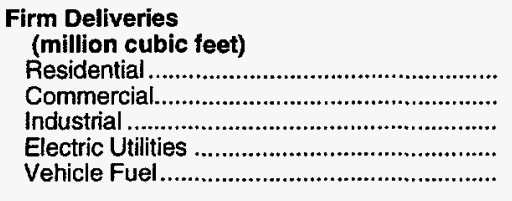 & $\begin{array}{l}\text { NA } \\
\text { NA } \\
\text { NA } \\
\text { NA } \\
\text { NA }\end{array}$ & $\begin{array}{l}\text { NA } \\
\text { NA } \\
\text { NA } \\
\text { NA } \\
\text { NA }\end{array}$ & $\begin{array}{l}\text { NA } \\
\text { NA } \\
\text { NA } \\
\text { NA } \\
\text { NA }\end{array}$ & $\begin{array}{r}51,366 \\
21,280 \\
125,313 \\
33 \\
4\end{array}$ & $\begin{array}{r}49,748 \\
21,008 \\
110,374 \\
83 \\
3\end{array}$ \\
\hline 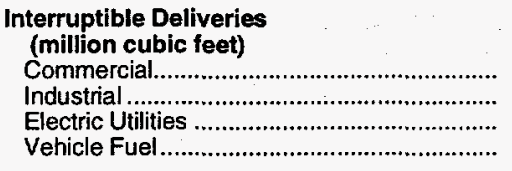 & $\begin{array}{l}\text { NA } \\
\text { NA } \\
\text { NA } \\
\text { NA }\end{array}$ & $\begin{array}{l}\text { NA } \\
\text { NA } \\
\text { NA } \\
\text { NA }\end{array}$ & $\begin{array}{l}\text { NA } \\
\text { NA } \\
\text { NA } \\
\text { NA }\end{array}$ & $\begin{array}{r}4,443 \\
125,313 \\
4,483 \\
--\end{array}$ & $\begin{array}{r}4,518 \\
110,374 \\
3,569 \\
--\end{array}$ \\
\hline 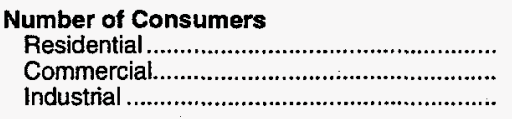 & $\begin{array}{r}686,149 \\
56,903 \\
2,431\end{array}$ & $\begin{array}{r}700,195 \\
57,265 \\
2,523\end{array}$ & $\begin{array}{r}711,043 \\
58,068 \\
2,509\end{array}$ & $\begin{array}{r}730,114 \\
57,827 \\
2,458\end{array}$ & $\begin{array}{r}744,294 \\
60,320 \\
2,477\end{array}$ \\
\hline 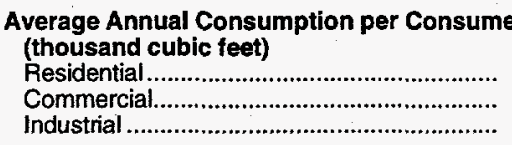 & $\begin{array}{r}66 \\
427 \\
59,904\end{array}$ & $\begin{array}{r}66 \\
414 \\
61,365\end{array}$ & $\begin{array}{r}70 \\
435 \\
67,377\end{array}$ & $\begin{array}{r}70 \\
445 \\
73,919\end{array}$ & $\begin{array}{r}67 \\
423 \\
73,362\end{array}$ \\
\hline 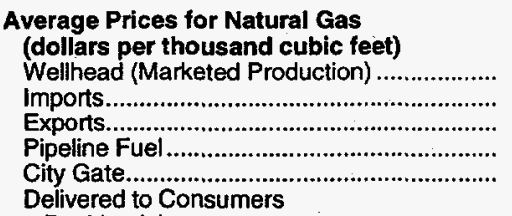 & $\begin{array}{r}2.75 \\
0 \\
0 \\
2.17 \\
3.13\end{array}$ & $\begin{array}{r}2.33 \\
0 \\
0 \\
3.02 \\
3.11\end{array}$ & $\begin{array}{r}2.29 \\
0 \\
0 \\
2.24 \\
3.21\end{array}$ & $\begin{array}{r}2.46 \\
0 \\
0 \\
2.34 \\
3.51\end{array}$ & $\begin{array}{r}2.17 \\
0 \\
0 \\
2.13 \\
3.44\end{array}$ \\
\hline 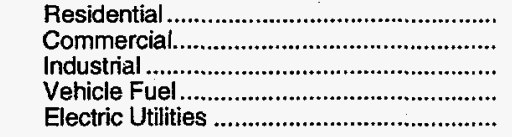 & $\begin{array}{l}6.57 \\
5.43 \\
3.16 \\
0.74 \\
2.22\end{array}$ & $\begin{array}{r}7.05 \\
5.74 \\
3.00 \\
0 \\
1.91\end{array}$ & $\begin{array}{l}6.74 \\
5.71 \\
3.07 \\
6.46 \\
2.28\end{array}$ & $\begin{array}{r}7.10 \\
6.19 \\
\mathrm{R}_{3.28} \\
4.60 \\
2.65\end{array}$ & $\begin{array}{l}7.41 \\
6.38 \\
3.26 \\
4.24 \\
2.37\end{array}$ \\
\hline
\end{tabular}

$R$ = Revised data.

NA $=$ Not Available.

Notes: Deliveries to electric utilities (consumption) are reported on the Form ElA-176, "Annual Report of Natural and Supplemental Gas Supply and Disposition." See the discussion on electric utility data and Table At in Appendix A for a comparison of reporting to these two forms. Totals may not add due to independent rounding.

discussion on electric utility data and Table At in Appendix A for a comparison of reporting to these two forms. Totals may not add due to independent rounding.

Quantity and Value of Natural Gas Report'; Form EIA-857, "Monthly Report of Natural Gas Purchases and Deliveries to Consumers"; Form EIA-816, "Monthly Natural Gas

Quantity and Value of Natural Gas Report'; Form EiA-857, "Monthly Report of Natural Gas Purchases and Deliveries to Consumers"; Form EIA-816, "Monthly Natural Gas

Liquids Report"; Form ElA-759, "Monthly Power Plant Report"; Form FERC-423, "Monthly Report of Cost and Quality of Fuels for Electric Plants"; Form ElA-191, "Underground

Geport, DOEJEIA-0216(94); and the U.S. Minerals Management Service. 


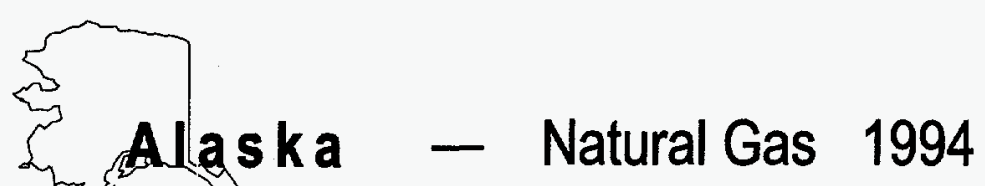

Million Percent of

Cu. Feet National Total

Million

Percent of

Cu. Feet National Total

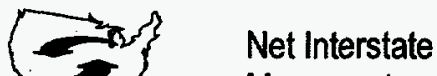

Movements:

$-62,682$

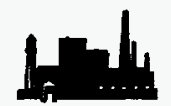

Industrial:

61,404

0.75

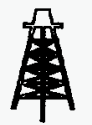

Marketed

Production:

453,425

2.31

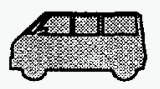

Vehicle Fuel:

0.00

Deliveries to Consumers:

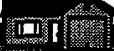

Residential:

14,895

0.31

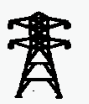

Electric

Utilities:

29,048

0.97

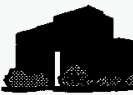

Commercial: $\quad 20,698$

0.71

Total:

126,045

0.67

Table 49. Summary Statistics for Natural Gas - Alaska, 1990-1994

\begin{tabular}{|c|c|c|c|c|c|}
\hline & 1990 & 1991 & 1992 & 1993 & 1994 \\
\hline 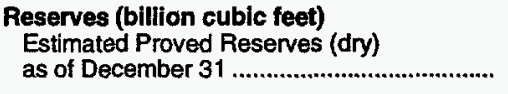 & 9,300 & 9,553 & 9,638 & 9,907 & 9,733 \\
\hline $\begin{array}{l}\text { Number of Gas and Gas Condensate Wells } \\
\text { Producing at End of Year................................. }\end{array}$ & 111 & 110 & 112 & 113 & 104 \\
\hline 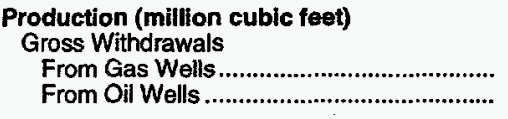 & $\begin{array}{r}204,093 \\
1,849,231\end{array}$ & $\begin{array}{r}197,502 \\
2,181,393\end{array}$ & $\begin{array}{r}198,603 \\
2,427,110\end{array}$ & $\begin{array}{r}190,139 \\
2,588,202\end{array}$ & $\begin{array}{r}180,639 \\
2,905,261\end{array}$ \\
\hline 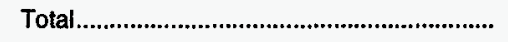 & $?, 053,324$ & $2,378,896$ & $2,625,713$ & $2,778,341$ & $3,085,900$ \\
\hline 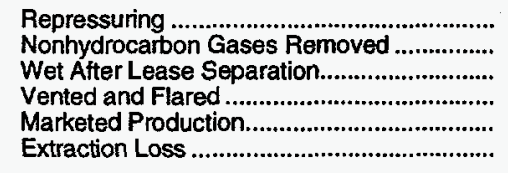 & $\begin{array}{r}1,639,689 \\
0 \\
413,634 \\
10,727 \\
402,907 \\
21,476\end{array}$ & $\begin{array}{r}1,930,290 \\
0 \\
448,605 \\
10,784 \\
437,822 \\
28,440\end{array}$ & $\begin{array}{r}2,168,019 \\
0 \\
457,694 \\
14,097 \\
443,597 \\
32,004\end{array}$ & $\begin{array}{r}2,325,506 \\
0 \\
452,835 \\
22,485 \\
430,350 \\
32,257\end{array}$ & $\begin{array}{r}2,619,236 \\
0 \\
466,665 \\
13,240 \\
453,425 \\
30,945\end{array}$ \\
\hline 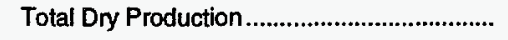 & 381,431 & 409,382 & 411,593 & 398,093 & 422,480 \\
\hline 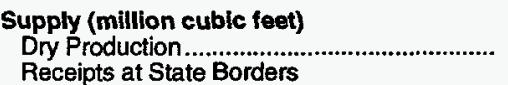 & 381,431 & 409,382 & 411,593 & 398,093 & 422,480 \\
\hline 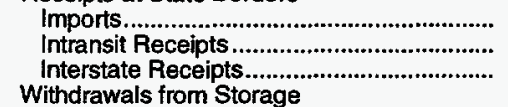 & $\begin{array}{l}0 \\
0 \\
0\end{array}$ & $\begin{array}{l}0 \\
0 \\
0\end{array}$ & $\begin{array}{l}0 \\
0 \\
0\end{array}$ & $\begin{array}{l}0 \\
0 \\
0\end{array}$ & $\begin{array}{l}0 \\
0 \\
0\end{array}$ \\
\hline 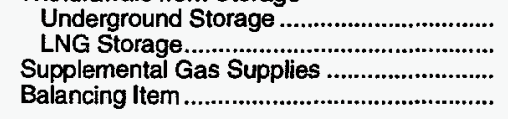 & $\begin{array}{r}0 \\
0 \\
0 \\
13,700\end{array}$ & $\begin{array}{r}0 \\
0 \\
0 \\
11,841\end{array}$ & $\begin{array}{r}0 \\
0 \\
0 \\
24,060\end{array}$ & $\begin{array}{r}0 \\
0 \\
0 \\
36,136\end{array}$ & $\begin{array}{r}0 \\
0 \\
0 \\
43,398\end{array}$ \\
\hline Total Supply & 395,131 & 421,223 & 435,652 & 434,229 & 465,878 \\
\hline
\end{tabular}

See footnotes at end of table. 
Table 49. Summary Statistics for Natural Gas - Alaska, 1990-1994 (Continued)

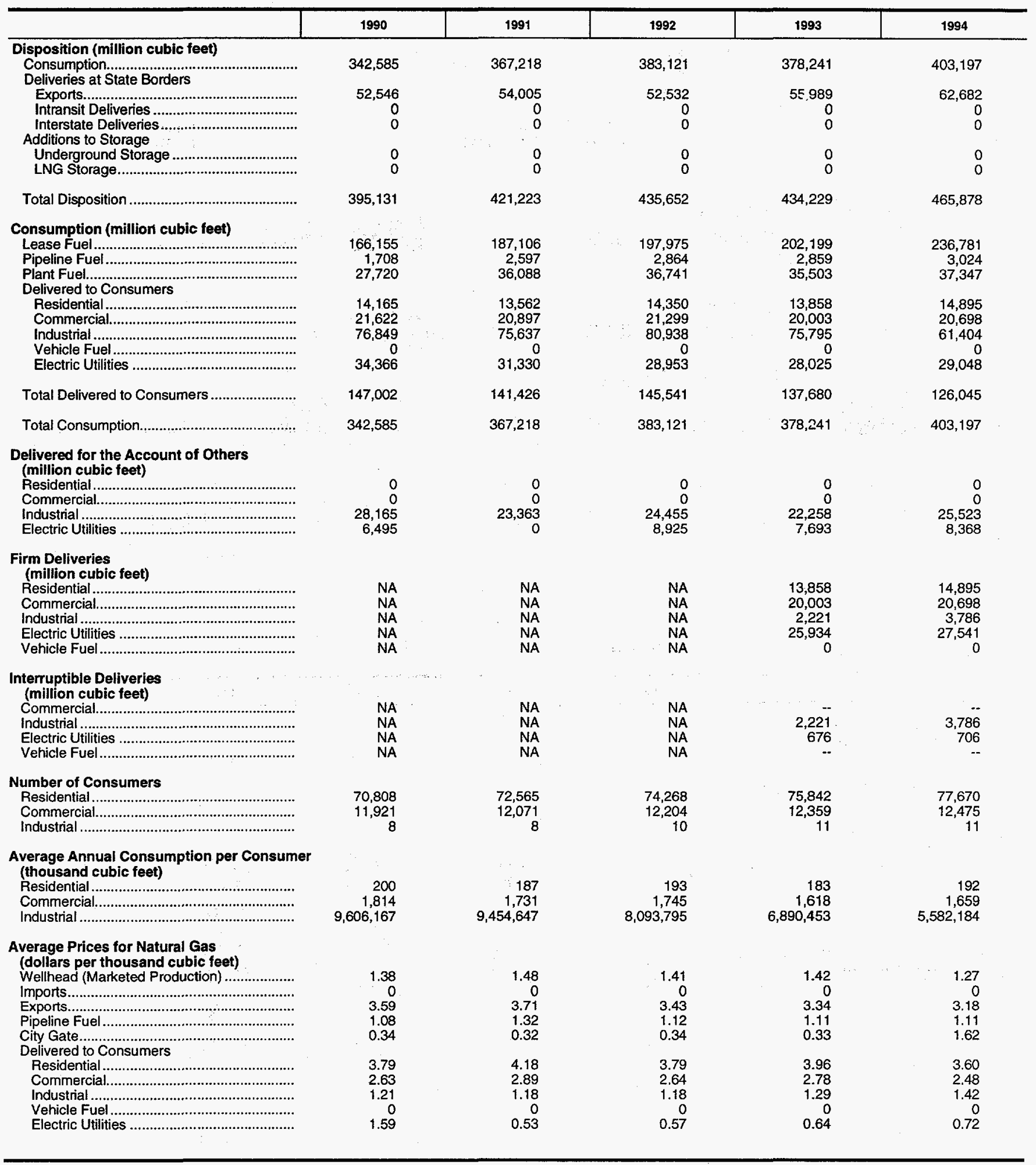

NA = Not Available.

NA = Not Available.
Notes: Deliveries to electric utilities (consumption) are reported on the Form ElA-176, "Annual Report of Natural and Supplemental Gas Supply and Disposition." See the discussion on electric utility data and Table A1 in Appendix A for a comparison of reporting to these two forms. Totals may not add due to independent rounding.

discussion on electric utility data and Table A1 in Appendix A for a comparison of reporting to these two forms. Totals may not add due to independent rounding.

Sources: Energy Information Administration (EIA), Form ElA-176, "Annual Feport of Natural and Supplemental Gas Supply and Disposition"; Form ElA-627, "Annual
Quantity and Value of Natural Gas Report"; Form ElA-857, "Monthly Report of Natural Gas Purchases and Deliveries to Consumers"; Form ElA-816, "Monthly Natural Gas

Quantity and Value of Natural Gas Report"; Form EIA-857, "Monthly Report of Natural Gas Purchases and Deliveries to Consumers"; Form EIA-816, "Monthly Natural Gas
Liquids Report"; Form EIA-759, "Monthly Power Plant Report"; Form FERC-423, "Monthly Report of Cost and Quality of Fuels for Electric Plants"; Form EIA-191, "Underground

Liquids Report"; Form EIA-759, "Monthly Power Plant Report"; Form FERC-423, "Monthly Report of Cost and Quality of Fuels for Electric Plants"; Form ElA-191, "Underground Report, DOE/EIA-0216(94); and the U.S. Minerals Management Service. 


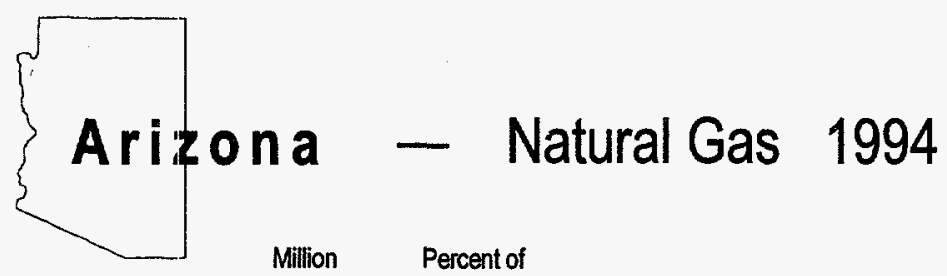

Cu. Feet National Total

Million

Cu. Feet

Percent of

National Total

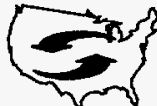

Net interstate

Movements:

112,688

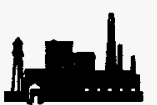

Industrial:

25,869

0.32

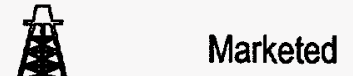

Production:

752

0.00

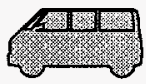

Vehicle Fuel:

61

3.53

Deliveries to Consumers:

Residential:

29,684

0.61

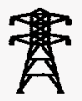

Electric

Utilities:

23,716

0.79

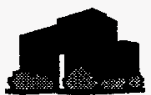

Commercial:

29,187

1.01

Total:

108,517

0.57

Table 50. Summary Statistics for Natural Gas - Arizona, 1990-1994

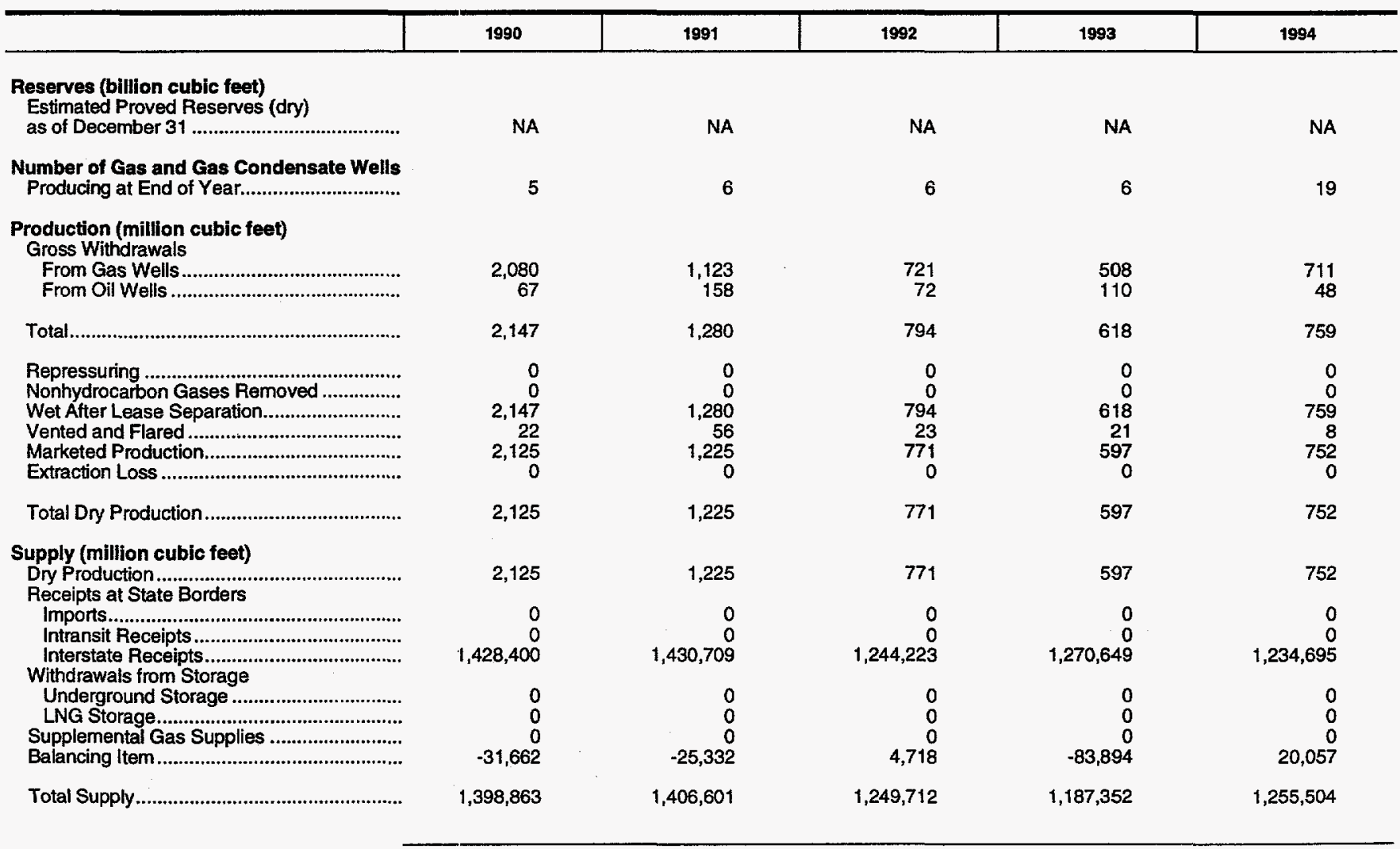

See footnotes at end of table. 
Table 50. Summary Statistics for Natural Gas - Arizona, 1990-1994 (Continued)

\begin{tabular}{|c|c|c|c|c|c|}
\hline & 1990 & 1991 & 1992 & 1993 & 1994 \\
\hline \multicolumn{6}{|l|}{ Disposition (million cubic feet) } \\
\hline $\begin{array}{l}\text { Consumption. } \\
\text { Deliveries at State Borders }\end{array}$ & \multicolumn{3}{|c|}{ Deliveries at State Borders } & 114,806 & 133,497 \\
\hline 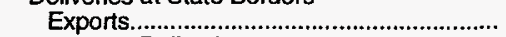 & 1,676 & 1,597 & 2,565 & 3,253 & 2,459 \\
\hline 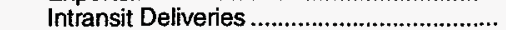 & & & 0 & & 0 \\
\hline 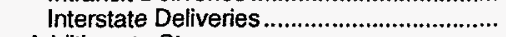 & $1,270,461$ & $1,279,952$ & $1,117,497$ & $1,069,293$ & $1,119,548$ \\
\hline \multicolumn{6}{|l|}{ Additions to Storage } \\
\hline Underground Storage ................................ & 0 & 0 & 0 & 0 & 0 \\
\hline 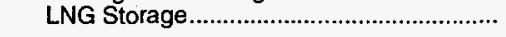 & 0 & 0 & 0 & 0 & 0 \\
\hline 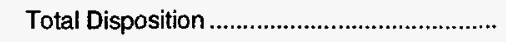 & $1,398,863$ & $1,406,601$ & $1,249,712$ & $1,187,352$ & $1,255,504$ \\
\hline \multicolumn{6}{|l|}{ Consumption (million cubic feet) } \\
\hline 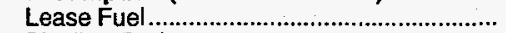 & 71 & 45 & 41 & 49 & 61 \\
\hline 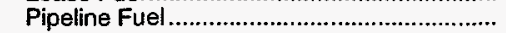 & 25,277 & 23,534 & 23,376 & 17,339 & 24,919 \\
\hline 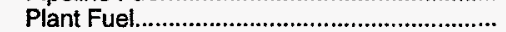 & 0 & 0 & 0 & 0 & 0 \\
\hline \multicolumn{6}{|l|}{ Delivered to Consumers } \\
\hline 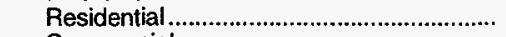 & 30,320 & 31,353 & 28,386 & 28,161 & 29,684 \\
\hline 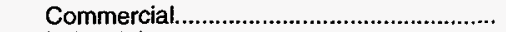 & 28,401 & 27,597 & 27,089 & 27,568 & 29,187 \\
\hline 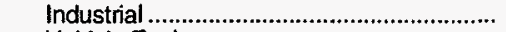 & 18,379 & 19,204 & 19,774 & 21,164 & 25,869 \\
\hline 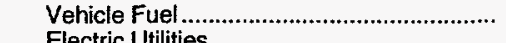 & $\begin{array}{r}0 \\
24278\end{array}$ & $\begin{array}{r}37 \\
23282\end{array}$ & $\begin{array}{r}46 \\
2039\end{array}$ & 44 & 61 \\
\hline & & & & & \\
\hline Total Delivered to Consumers ........................... & 101,378 & 101,473 & 106,234 & 97,417 & 108,517 \\
\hline 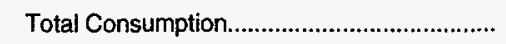 & 126,726 & 125,052 & 129,650 & 114,806 & 133,497 \\
\hline \multicolumn{6}{|l|}{$\begin{array}{l}\text { Delivered for the Account of Others } \\
\text { (million cubic feet) }\end{array}$} \\
\hline 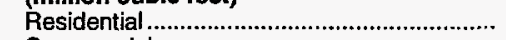 & 0 & 0 & 0 & 0 & 0 \\
\hline 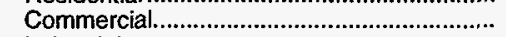 & 1,219 & 1,876 & 2,021 & 2,336 & 2,709 \\
\hline 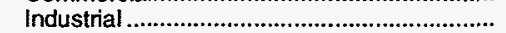 & 10,421 & 9,966 & 13,925 & 15,868 & 18,035 \\
\hline 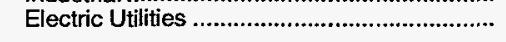 & 14,222 & 13,831 & 40,553 & 29,866 & 33,188 \\
\hline \multicolumn{6}{|l|}{$\begin{array}{l}\text { Firm Deliveries } \\
\text { (million cubic feet) }\end{array}$} \\
\hline 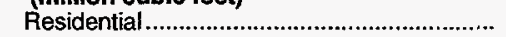 & NA & NA & NA & 28,161 & 29,684 \\
\hline 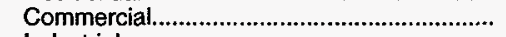 & NA & NA & NA & 27,132 & 28,829 \\
\hline 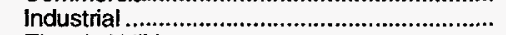 & NA & NA & NA & 1,043 & 971 \\
\hline 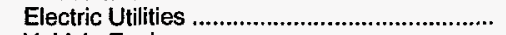 & NA & NA & NA & 27,219 & 32,636 \\
\hline 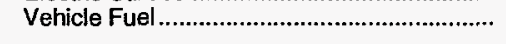 & NA & NA & NA & 44 & 61 \\
\hline \multirow{2}{*}{\multicolumn{6}{|c|}{$\begin{array}{l}\text { Interruptible Deliveries } \\
\text { (million cubic feet) }\end{array}$}} \\
\hline & & & & & \\
\hline 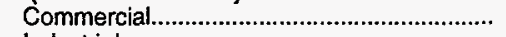 & NA & NA & NA & 436 & 357 \\
\hline 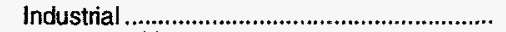 & NA & NA & NA & 1,043 & 971 \\
\hline 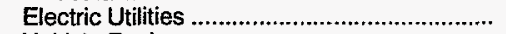 & NA & NA & NA & 2,678 & 594 \\
\hline 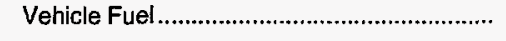 & NA & NA & NA & - & -- \\
\hline \multicolumn{6}{|l|}{ Number of Consumers } \\
\hline 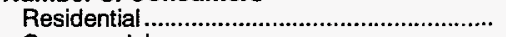 & 586,866 & 642,659 & 604,899 & 610,337 & 635,335 \\
\hline 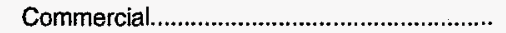 & 47,292 & 53,982 & 47,781 & 47,678 & 48,568 \\
\hline 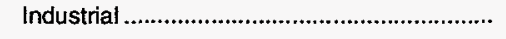 & 526 & 532 & 532 & 526 & 519 \\
\hline \multirow{2}{*}{\multicolumn{6}{|c|}{$\begin{array}{l}\text { Average Annual Consumption per Consumer } \\
\text { (thousand cubic feet) }\end{array}$}} \\
\hline & & & & & \\
\hline 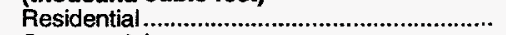 & 52 & 49 & 47 & 46 & 47 \\
\hline 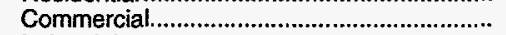 & 601 & 511 & 567 & 578 & 601 \\
\hline 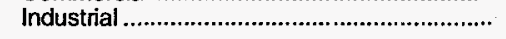 & 34,941 & 36,098 & 37,169 & 40,236 & 49,844 \\
\hline \multicolumn{6}{|l|}{$\begin{array}{l}\text { Average Prices for Natural Gas } \\
\text { (dollars per thousand cubic feet) }\end{array}$} \\
\hline Wellhead (Marketed Production) ................... & 1.20 & 1.50 & 1.85 & 1.30 & 1.40 \\
\hline 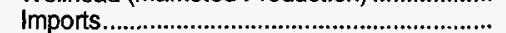 & 0 & 0 & 0 & 0 & 0 \\
\hline 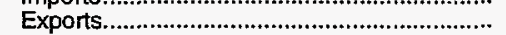 & 2.42 & 2.22 & 1.42 & 1.08 & 1.73 \\
\hline Pipeline Fuel & 1.65 & 1.26 & 1.25 & 1.68 & 1.28 \\
\hline 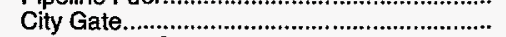 & 2.73 & 2.45 & 2.33 & 2.62 & 2.53 \\
\hline \multicolumn{6}{|l|}{ Delivered to Consumers } \\
\hline 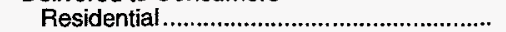 & 6.85 & 6.99 & 7.24 & 7.20 & 7.54 \\
\hline 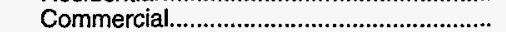 & 4.79 & 5.07 & 5.20 & 5.06 & 5.27 \\
\hline & 3.71 & 3.51 & 4.16 & 4.02 & 3.57 \\
\hline 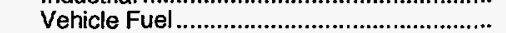 & 0 & 3.82 & 3.63 & 3.57 & 3.93 \\
\hline 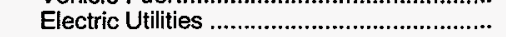 & 2.45 & 2.06 & 2.28 & 2.88 & 2.23 \\
\hline
\end{tabular}

NA = Not Available.

Notes: Deliveries to electric utilities (consumption) are reported on the Form EIA-176, "Annual Report of Natural and Supplemental Gas Supply and Disposition." See the discussion on electric utility data and Table A1 in Appendix A for a comparison of reporting to these two forms. Totals may not add due to independent rounding.

Sources: Energy Information Administration (EIA), Form EIA-176, "Annual Feport of Natural and Supplemental Gas Supply and Disposition"; Form ElA-627, "Annual Quantity and Value of Natural Gas Report"; Form EIA-857, "Monthly Report of Natural Gas Purchases and Deliveries to Consumers"; Form EIA-816, "Monthly Natural Gas Liquids Report"; Form EIA-759, "Monthly Power Plant Report"; Form FERC-423, "Monthly Report of Cost and Quality of Fuels for Electric Plants"; Form EIA-191, "Underground Gas Storage Report"; Form FPC-14, "Annual Report for Importers and Exporters of Natural Gas"; U.S. Crude Oil, Natural Gas, and Natural Gas Liquids Reserves, 1994 Annual Report, DOE/EIA-0216(94); and the U.S. Minerals Management Service. 


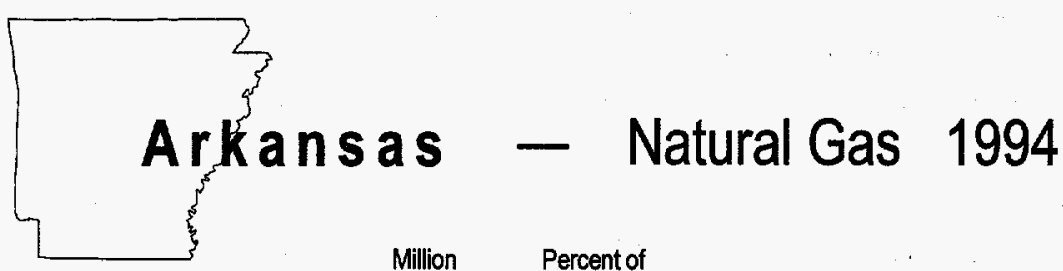

$\begin{array}{lll}\text { Cu. Feet National Total } & \text { Million } & \text { Percent of } \\ \text { Cu. Feet } & \text { National Total }\end{array}$

Net Interstate

Movements:

63,881

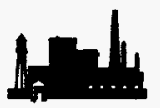

Industrial:

133,921

1.64

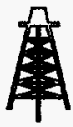

Marketed

Production:

187,673

0.96

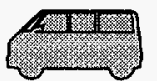

Vehicle Fuel:

0

0.00

Deliveries to Consumers:

III]

Residential:

41,527

0.86

$\$$

Electric

Utilities:

24,977

0.84

Commercial: $\quad 27,410 \quad 0.95$
Total:

227,835

Table 51. Summary Statistics for Natural Gas - Arkansas, 1990-1994

\begin{tabular}{|c|c|c|c|c|c|}
\hline & 1990 & 1991 & 1992 & 1993 & 1994 \\
\hline 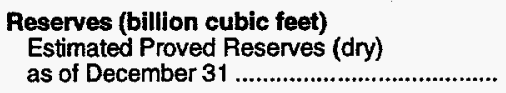 & 1,731 & 1,669 & 1,750 & 1,552 & 1,607 \\
\hline $\begin{array}{l}\text { Number of Gas and Gas Condensate Wells } \\
\text { Producing at End of Year.................................... }\end{array}$ & 2,952 & 2,780 & 3,500 & 3,500 & 3,500 \\
\hline 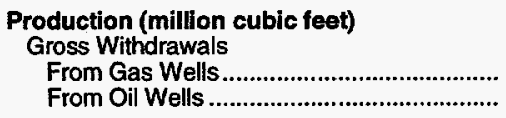 & $\begin{array}{r}161,148 \\
34,256\end{array}$ & $\begin{array}{r}153,583 \\
16,049\end{array}$ & $\begin{array}{r}171,543 \\
39,364\end{array}$ & $\begin{array}{r}166,273 \\
38,279\end{array}$ & $\begin{array}{r}161,967 \\
33,446\end{array}$ \\
\hline 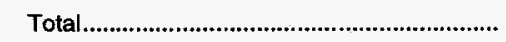 & 195,405 & 169,632 & 210,906 & 204,552 & 195,413 \\
\hline 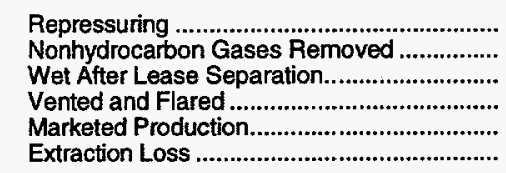 & $\begin{array}{r}20,165 \\
0 \\
175,240 \\
284 \\
174,956 \\
800\end{array}$ & $\begin{array}{r}4,722 \\
0 \\
164,910 \\
208 \\
164,702 \\
290\end{array}$ & $\begin{array}{r}8,056 \\
0 \\
202,850 \\
371 \\
202,479 \\
413\end{array}$ & $\begin{array}{r}7,773 \\
0 \\
196,779 \\
409 \\
196,370 \\
507\end{array}$ & $\begin{array}{r}7,426 \\
0 \\
187,986 \\
313 \\
187,673 \\
553\end{array}$ \\
\hline 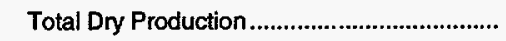 & 174,156 & 164,412 & 202,066 & 195,863 & 187,120 \\
\hline 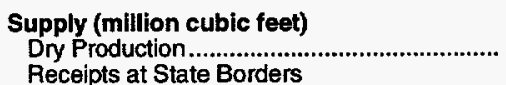 & 174,156 & 164,412 & 202,066 & 195,863 & 187,120 \\
\hline 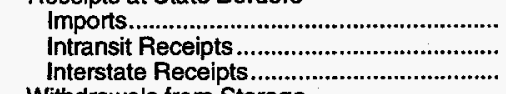 & $\begin{array}{r}0 \\
0 \\
2,223,388\end{array}$ & $\begin{array}{r}0 \\
0 \\
2,263,622\end{array}$ & $\begin{array}{r}0 \\
0 \\
2,235,546\end{array}$ & $\begin{array}{r}0 \\
0 \\
2,384,562\end{array}$ & $\begin{array}{r}0 \\
0 \\
2,865,799\end{array}$ \\
\hline 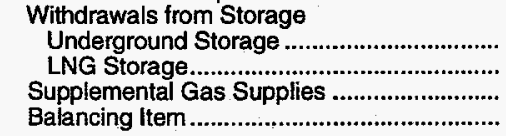 & $\begin{array}{r}472 \\
23 \\
0 \\
16,237\end{array}$ & $\begin{array}{r}3,010 \\
49 \\
0 \\
-21,688\end{array}$ & $\begin{array}{r}2,975 \\
51 \\
0 \\
-20,293\end{array}$ & $\begin{array}{r}8,481 \\
44 \\
0 \\
-4,044\end{array}$ & $\begin{array}{r}5,731 \\
68 \\
0 \\
-9,054\end{array}$ \\
\hline 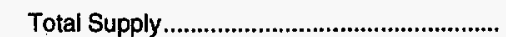 & $2,414,276$ & $2,409,405$ & $2,420,345$ & $2,584,905$ & $3,049,665$ \\
\hline
\end{tabular}

See footnotes at end of table. 
Table 51. Summary Statistics for Natural Gas - Arkansas, 1990-1994 (Continued)

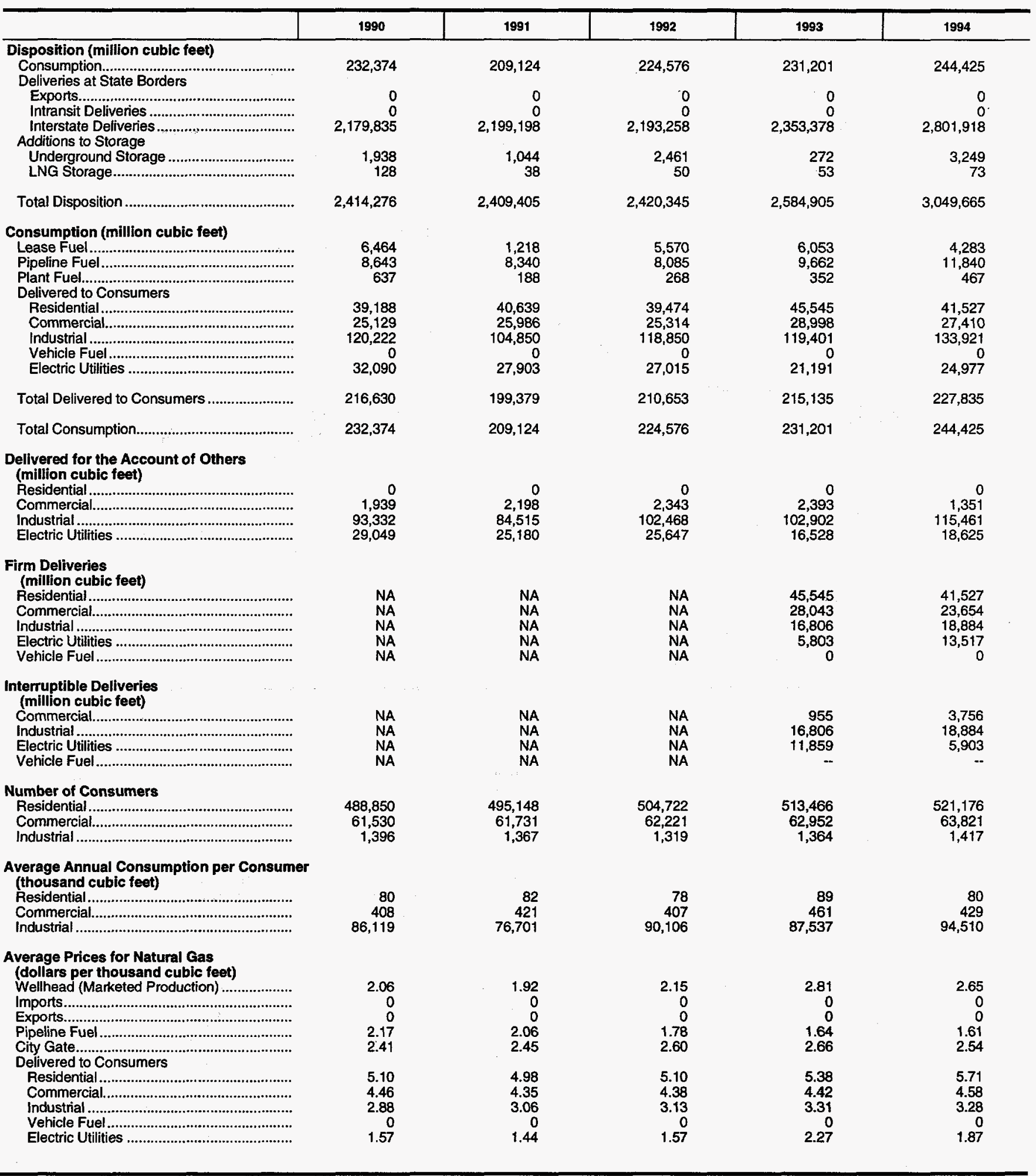

NA $=$ Not Available.

Notes: Deliveries to electric utilities (consumption) are reported on the Form ElA-176, "Annual Report of Natural and Supplemental Gas Supply and Disposition." See the discussion on electric utility data and Table A1 in Appendix A for a comparison of reporting to these two forms. Totals may not add due to independent rounding.

Sources: Energy Information Administration (EIA), Form EIA-176, "Annual Report of Natural and Supplemental Gas Supply and Disposition"; Form EIA-627, Annual

Quantity and Value of Natural Gas Report'; Form ElA-857, "Monthly Report of Natural Gas Purchases and Deliveries to Consumers"; Form ElA-816, "Monthly Natural Gas

Liquids Report"; Form ElA-759, "Monthly Power Plant Report"; Form FERC-423, "Monthly Report of Cost and Quality of Fuels for Electric Plants"; Form ElA-191, "Underground

Gas Storage Report"; Fom FPC-14, "Annual Report for importers and Exporters of Natural Gas"; U.S. Crude Oil, Natural Gas, and Natural Gas Liquids Reserves, t994 Annual

Report, DOEE/EIA-02 i6(94); and the U.S. Minerals Management Service. 


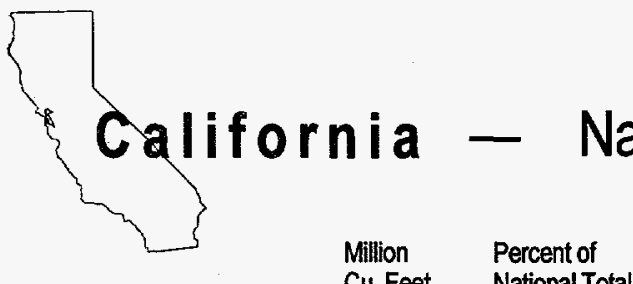

Million Percent of

Cu. Feet National Total

Cu. Feet National Total

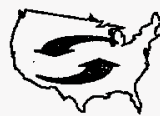

Net Interstate

Movements:

$1,887,149$

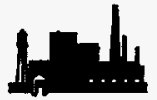

Industrial:

656,751

8.03

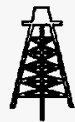

Marketed

Production

309,427

1.58

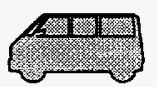

Vehicle Fuel:

550

31.79

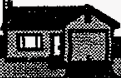

Deliveries to Consumers:

Residential: $\quad \mathbf{5 2 0 , 9 5 9}$

10.75

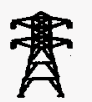

Electric

Utilities:

601,290

20.13

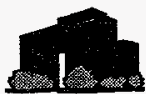

Commercial: $\quad 261,989$

9.05

Total:

$2,041,539$

10.80

Table 52. Summary Statistics for Natural Gas - California, 1990-1994

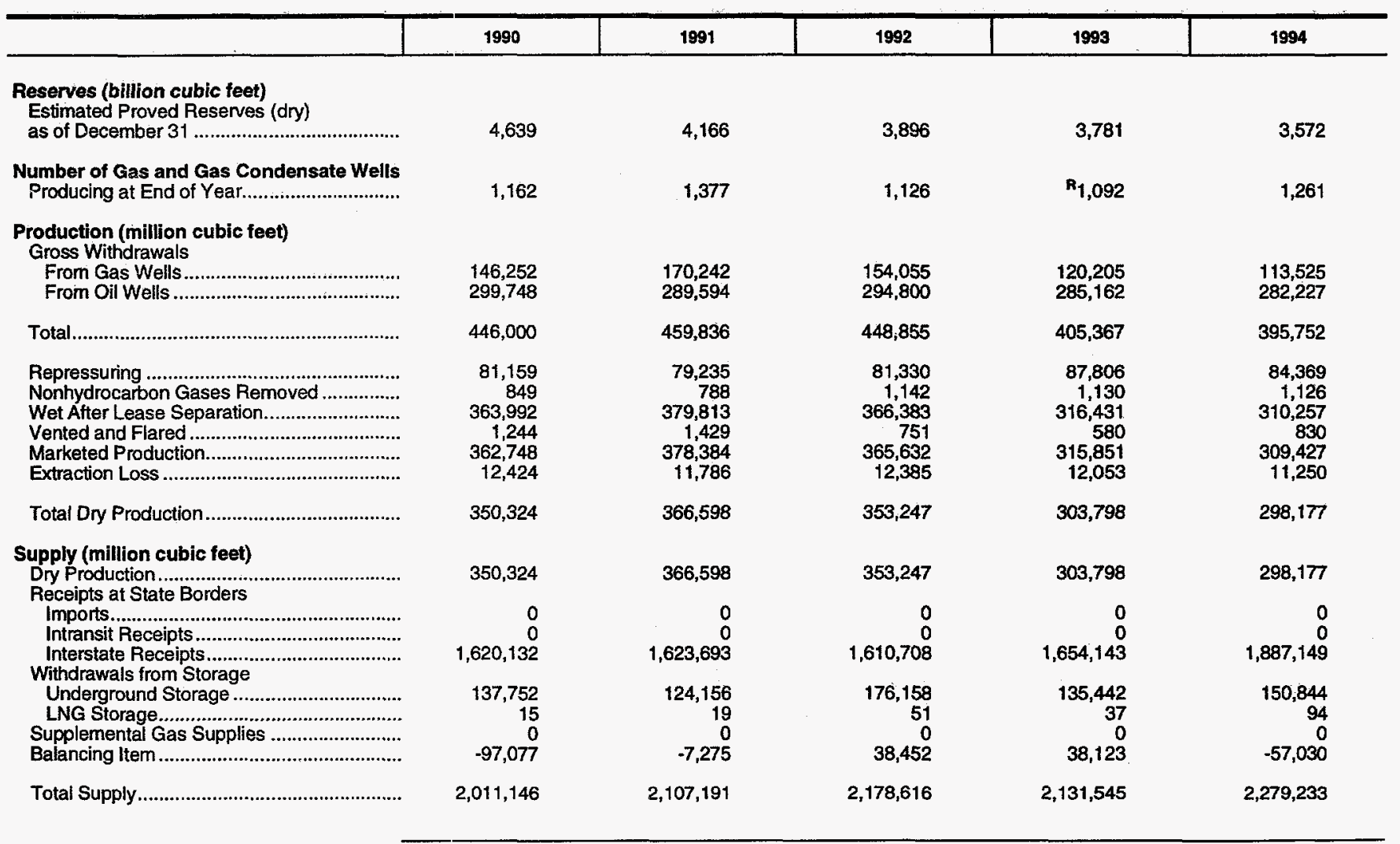

See footnotes at end of table. 


\begin{tabular}{|c|c|c|c|c|c|}
\hline & 1990 & 1991 & 1992 & 1993 & 1994 \\
\hline \multicolumn{6}{|l|}{ Disposition (million cubic feet) } \\
\hline \multicolumn{6}{|l|}{ Deliveries at State Borders } \\
\hline 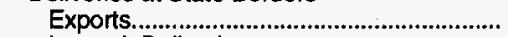 & 0 & 0 & 0 & 0 & 0 \\
\hline Intransit Deliveries & 0 & 0 & 0 & 0 & 0 \\
\hline Interstate Deliveries ......................................... & 0 & 0 & 0 & 0 & 0 \\
\hline \multicolumn{6}{|l|}{ Additions to Storage } \\
\hline 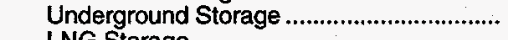 & 147,074 & 136,433 & 148,039 & 155,135 & 155,910 \\
\hline 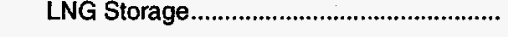 & & 50 & 13 & 13 & \\
\hline Total Disposition ................................................ & $2,011,146$ & $2,107,191$ & $2,178,616$ & $2,131,545$ & $2,279,233$ \\
\hline \multicolumn{6}{|l|}{ Consumption (million cubic feet) } \\
\hline 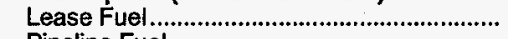 & 14,754 & 96,442 & 84,220 & 80,210 & 63,251 \\
\hline 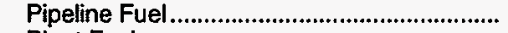 & 20,132 & 18,509 & 14,763 & 11,800 & 12,132 \\
\hline \multicolumn{5}{|l|}{ Delivered to Consumers } & 6,388 \\
\hline 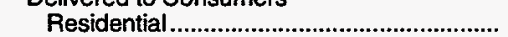 & 514,507 & 508,697 & 479,537 & 500,968 & 520,959 \\
\hline 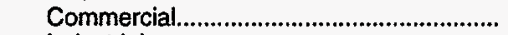 & 285,090 & 287,608 & 285,008 & 250,283 & 261,989 \\
\hline 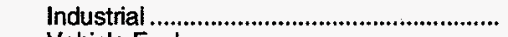 & 565,206 & 602,619 & 594,569 & 659,723 & 656,751 \\
\hline 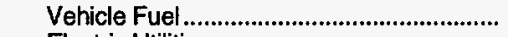 & 4 & & 27 & 255 & 550 \\
\hline 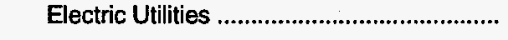 & 456,406 & 449,014 & 564,432 & 466,061 & 601,290 \\
\hline Total Delivered to Consumers .......................... & $1,821,213$ & $1,847,949$ & $1,923,573$ & $1,877,290$ & $2,041,539$ \\
\hline 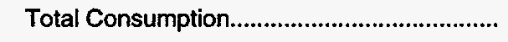 & $1,864,057$ & $1,970,709$ & $2,030,564$ & $1,976,397$ & $2,123,310$ \\
\hline \multicolumn{6}{|l|}{$\begin{array}{l}\text { Delivered for the Account of Others } \\
\text { (million cubic feet) }\end{array}$} \\
\hline 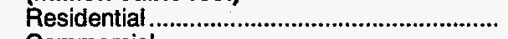 & 0 & 1,256 & 3,502 & 3,700 & 3,507 \\
\hline 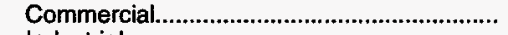 & 38,337 & 63,882 & 72,782 & 57,781 & 134,346 \\
\hline 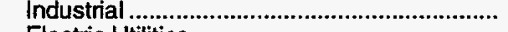 & 346,643 & 422,738 & 430,836 & 485,326 & 537,503 \\
\hline 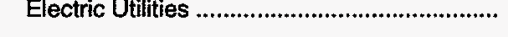 & 114,142 & 225,773 & 406,235 & 342,457 & 556,557 \\
\hline \multicolumn{6}{|l|}{$\begin{array}{l}\text { Firm Deliveries } \\
\text { (million cubic feet) }\end{array}$} \\
\hline 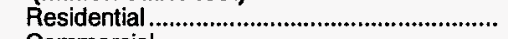 & NA & NA & NA & 500,968 & 520,959 \\
\hline 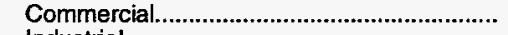 & NA & NA & NA & 240,998 & 222,004 \\
\hline 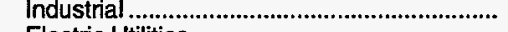 & NA & NA & NA & 77,232 & 257,015 \\
\hline 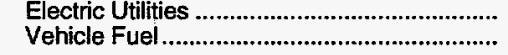 & $\begin{array}{l}\text { NA } \\
\text { NA }\end{array}$ & $\begin{array}{l}\text { NA } \\
\text { NA }\end{array}$ & $\begin{array}{l}\text { NA } \\
\text { NA }\end{array}$ & $\begin{array}{r}416,941 \\
255\end{array}$ & $\begin{array}{r}288,054 \\
529\end{array}$ \\
\hline \multicolumn{6}{|l|}{$\begin{array}{l}\text { Interruptible Deliveries } \\
\text { (million cubic feet) }\end{array}$} \\
\hline 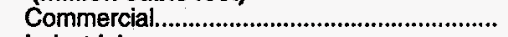 & NA & NA & NA & 9,284 & 39,985 \\
\hline 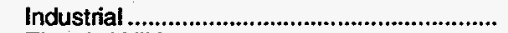 & NA & NA & NA & 77,232 & 257,015 \\
\hline 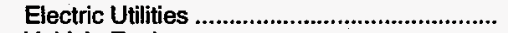 & NA & NA & NA & 45,071 & 308,720 \\
\hline 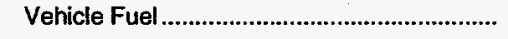 & NA & NA & NA & -- & 21 \\
\hline \multicolumn{6}{|l|}{ Number of Consumers } \\
\hline 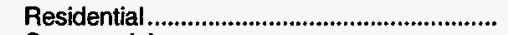 & $8,497,848$ & $8,634,774$ & $8,680,613$ & $8,726,187$ & $8,790,733$ \\
\hline 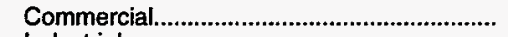 & 415,073 & 421,278 & 412,467 & 411,648 & 411,140 \\
\hline 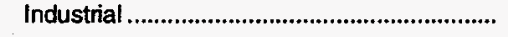 & 46,048 & 44,865 & 40,528 & 42,748 & 38,750 \\
\hline \multicolumn{6}{|l|}{$\begin{array}{l}\text { Average Annual Consumption per Consumer } \\
\text { (thousand cubic feet) }\end{array}$} \\
\hline 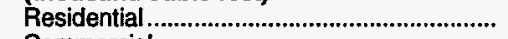 & 61 & 59 & 55 & 57 & \\
\hline 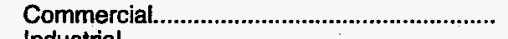 & 687 & 683 & 691 & 608 & 637 \\
\hline 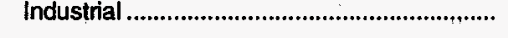 & 12,274 & 13,432 & 14,671 & 15,433 & 16,948 \\
\hline \multicolumn{6}{|l|}{$\begin{array}{l}\text { Average Prices for Natural Gas } \\
\text { (dollars per thousand cubic feet) }\end{array}$} \\
\hline Wellhead (Marketed Production) .................... & 2.36 & 2.46 & 2.34 & 2.38 & 3.04 \\
\hline 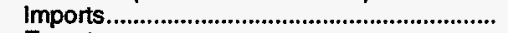 & 0 & 0 & 0 & 0 & 0 \\
\hline 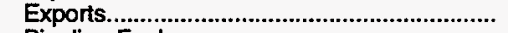 & & 0 & 0 & & \\
\hline 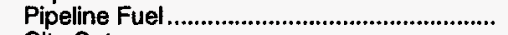 & 2.40 & 2.19 & 1.40 & 0.53 & 0.33 \\
\hline 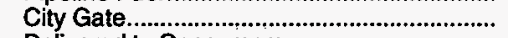 & 2.90 & 2.80 & 2.72 & 2.85 & 2.57 \\
\hline \multicolumn{6}{|l|}{ Delivered to Consumers } \\
\hline 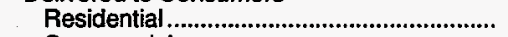 & 5.78 & 6.27 & 5.97 & 6.23 & 6.39 \\
\hline 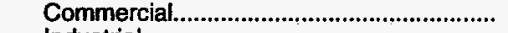 & 5.12 & 5.50 & 5.15 & 6.03 & 7.12 \\
\hline 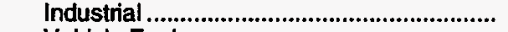 & 3.91 & 3.96 & 3.67 & 2.82 & 3.25 \\
\hline Vehicle Fuel................................................ & 4.84 & 5.77 & 6.43 & 4.76 & 5.09 \\
\hline Electric Utilities ............................................... & 3.13 & 2.95 & 2.81 & 3.05 & 2.56 \\
\hline
\end{tabular}

R $=$ Revised data.

NA $=$ Not Available.
Notes: Deliveries to electric utilities (consumption) are reported on the Form ElA-176, "Annual Report of Natural and Supplemental Gas Supply and Disposition." See the

Notes: Deliveries to electric utilities (consumption) are reported on the Form ElA-176, "Annual Report of Natural and Supplemental Gas Supply and Disposition.
discussion on electric utility data and Table A1 in Appendix A for a comparison of reporting to these two forms. Totals may not add due to independent rounding.

discussion on electric utility data and Table A1 in Appendix A for a comparison of reporting to these two forms. Totals may not add due to independent rounding.

Sources: Energy Information Administration (EIA), Form ElA-176, "Annual Report of Natural and Supplemental Gas Supply and Disposition"; Form ElA-627, "Annual
Quantity and Value of Natural Gas Report"; Form EIA-857, "Monthly Report of Natural Gas Purchases and Deliveries to Consumers"; Form EIA-816, "Monthly Natural Gas

Quantity and Value of Natural Gas Report"; Form ElA-857, "Monthly Report of Natural Gas Purchases and Deliveries to Consumers"; Form ElA-816, "Monthly Natural Gas
Liquids Report"; Form ElA-759, "Monthly Power Plant Report"; Form FERC-423, "Monthly Report of Cost and Quality of Fuels for Electric Plants"; Form ElA-191, "Underground Liquids Report"; Form ElA-759, "Monthly Power Plant Report"; Form FERC-423, "Monthly Report of Cost and Quality of Fuels for Electric Plants"; Form ElA-191, "Underground Report, DOE/EIA-0216(94); and the U.S. Minerals Management Service. 


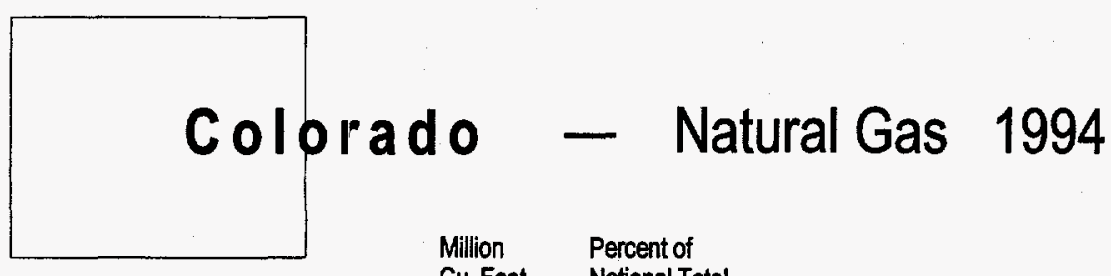

Cu. Feet National Total

Million

Percent of

Cu. Feet

National Total

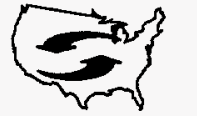

Net Interstate

Movements:

$-213,230$

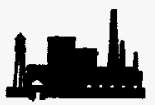

Industrial:

71,093

0.87

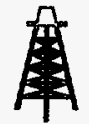

Marketed

Production:

453,207

2.31

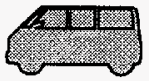

Vehicle Fuel:

68

3.93

Deliveries to Consumers:

$10 \pi$

Residential:

99,504

2.05

Electric

4,881

0.16

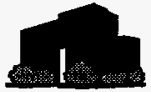

Commercial:

65,870

2.28

Total:

241,416

1.28

Table 53. Summary Statistics for Natural Gas - Colorado, 1990-1994

\begin{tabular}{|c|c|c|c|c|c|}
\hline & 1990 & 1991 & 1992 & 1993 & 1994 \\
\hline $\begin{array}{l}\text { Reserves (billion cubic feet) } \\
\text { Estimated Proved Reserves (dry) } \\
\text { as of December } 31 \text {.......................................... }\end{array}$ & 4,555 & 5,767 & 6,198 & 6,722 & 6,753 \\
\hline $\begin{array}{l}\text { Number of Gas and Gas Condensate Wells } \\
\text { Producing at End of Year..................................... }\end{array}$ & 5,741 & 5,562 & 5,912 & 6,372 & 7,056 \\
\hline 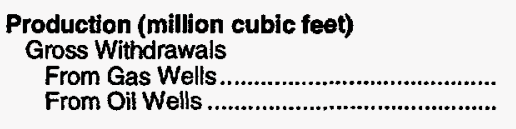 & $\begin{array}{r}199,805 \\
68,878\end{array}$ & $\begin{array}{r}222,909 \\
72,209\end{array}$ & $\begin{array}{r}256,426 \\
77,568\end{array}$ & $\begin{array}{r}320,849 \\
93,155\end{array}$ & $\begin{array}{l}365,651 \\
101,379\end{array}$ \\
\hline Total & 268,683 & 295,118 & 333,994 & 414,004 & 467,031 \\
\hline 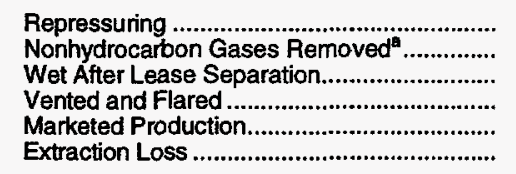 & $\begin{array}{r}10,986 \\
9,981 \\
247,716 \\
4,719 \\
242,997 \\
13,178\end{array}$ & $\begin{array}{r}6,267 \\
0 \\
288,851 \\
2,890 \\
285,961 \\
15,822\end{array}$ & $\begin{array}{r}9,085 \\
0 \\
324,909 \\
1,868 \\
323,041 \\
18,149\end{array}$ & $\begin{array}{r}10,995 \\
0 \\
403,009 \\
2,024 \\
400,985 \\
18,658\end{array}$ & $\begin{array}{r}11,347 \\
0 \\
455,683 \\
2,476 \\
453,207 \\
19,612\end{array}$ \\
\hline Total Dry Production & 229,819 & 270,139 & 304,892 & 382,327 & 433,595 \\
\hline 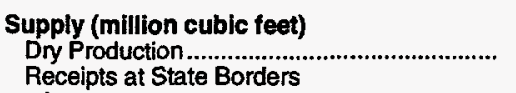 & 229,819 & 270,139 & 304,892 & 382,327 & 433,595 \\
\hline 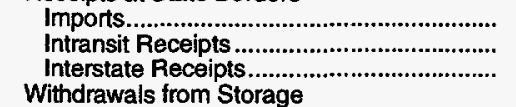 & $\begin{array}{r}0 \\
0 \\
497,558\end{array}$ & $\begin{array}{r}0 \\
0 \\
576,054\end{array}$ & $\begin{array}{r}0 \\
0 \\
523,124\end{array}$ & $\begin{array}{r}0 \\
0 \\
503,217\end{array}$ & $\begin{array}{r}0 \\
0 \\
585,207\end{array}$ \\
\hline 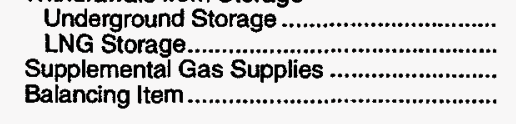 & $\begin{array}{r}31,174 \\
0 \\
15,379 \\
-16,583\end{array}$ & $\begin{array}{r}23,214 \\
0 \\
6,778 \\
-4,943\end{array}$ & $\begin{array}{r}27,921 \\
0 \\
7,158 \\
-13,191\end{array}$ & $\begin{array}{r}54,294 \\
0 \\
8,456 \\
23,747\end{array}$ & $\begin{array}{r}30,085 \\
0 \\
8,168 \\
48,328\end{array}$ \\
\hline Total Supply & 757,347 & 871,241 & 849,905 & 972,040 & $1,105,383$ \\
\hline
\end{tabular}

See footnotes at end of table. 
Table 53. Summary Statistics for Natural Gas - Colorado, 1990-1994 (Continued)

\begin{tabular}{|c|c|c|c|c|c|}
\hline & 1990 & 1991 & 1992 & 1993 & 1994 \\
\hline $\begin{array}{l}\text { Disposition (million cubic feet) } \\
\text { Consumption........................................ } \\
\text { Deliveries at State Borders }\end{array}$ & 239,112 & 260,513 & 253,073 & 284,185 & 275,761 \\
\hline \multicolumn{6}{|l|}{ 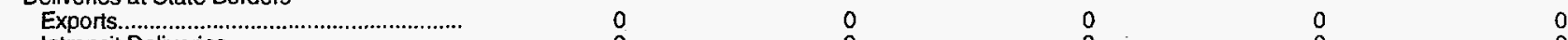 } \\
\hline 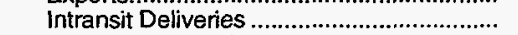 & 0 & 0 & 0 & 0 & 0 \\
\hline Interstate Deliveries ............................................. & 490,561 & 580,145 & 573,771 & 636,724 & 798,437 \\
\hline \multicolumn{6}{|l|}{ Additions to Storage } \\
\hline 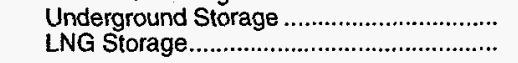 & $\begin{array}{r}27,674 \\
0\end{array}$ & $\begin{array}{r}30,584 \\
0\end{array}$ & $\begin{array}{r}23,061 \\
0\end{array}$ & $\begin{array}{r}51,132 \\
0\end{array}$ & $\begin{array}{r}31,185 \\
0\end{array}$ \\
\hline 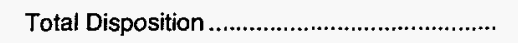 & 757,347 & 871,241 & 849,905 & 972,040 & $1,105,383$ \\
\hline \multicolumn{6}{|l|}{ Consumption (million cubic feet) } \\
\hline 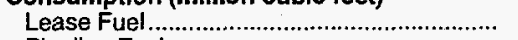 & 8,888 & 14,802 & 8,936 & 12,969 & 11,865 \\
\hline Pipeline Fuel ........................................................... & 9,114 & 8,346 & 8,248 & 7,617 & 9,998 \\
\hline \multirow{2}{*}{\multicolumn{6}{|c|}{ Delivered to Consumers }} \\
\hline & & & & & \\
\hline 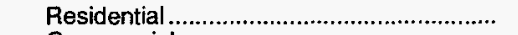 & 91,916 & 97.440 & 94,614 & 106,187 & 99,504 \\
\hline 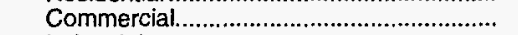 & 66,290 & 68,938 & 66,420 & 71,647 & 65,870 \\
\hline 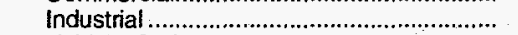 & 48,952 & 56,176 & 57,579 & 69,024 & 71,093 \\
\hline Vehicle Fuel........................................... & 11 & 15 & & & 68 \\
\hline Electric Utilities ................................................ & 5,485 & 5,715 & 5,019 & 4,860 & 4,881 \\
\hline Total Delivered to Consumers ......................... & 212,655 & 228,283 & 223,656 & 251,736 & 241,416 \\
\hline Total Consumption ....................................................... & 239,112 & 260,513 & 253,073 & $284,: 85$ & 275,761 \\
\hline \multicolumn{6}{|l|}{$\begin{array}{l}\text { Delivered for the Account of Others } \\
\text { (million cubic feet) }\end{array}$} \\
\hline 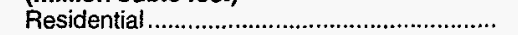 & 163 & 335 & 0 & 0 & 0 \\
\hline 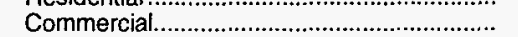 & 1,800 & 2,763 & 2,993 & 3,241 & 3,403 \\
\hline 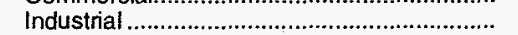 & 33,799 & 36,685 & 39,040 & 44,465 & 51,292 \\
\hline Electric Utilities ............................................... & 4,904 & 6,097 & 3,931 & 3,165 & 2,685 \\
\hline \multicolumn{6}{|l|}{$\begin{array}{l}\text { Firm Deliveries } \\
\text { (million cubic feet) }\end{array}$} \\
\hline 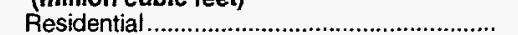 & NA & NA & NA & 106,187 & 99,504 \\
\hline Commercial........................................................ & NA & NA & NA & 68,725 & 60,755 \\
\hline 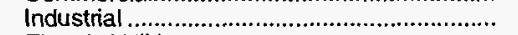 & NA & NA & NA & 38,315 & 48,468 \\
\hline 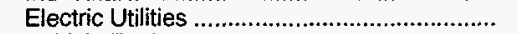 & NA & NA & NA & 2,034 & 1,532 \\
\hline 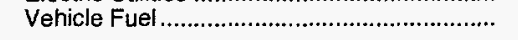 & NA & NA & NA & 7 & 60 \\
\hline \multicolumn{6}{|l|}{$\begin{array}{l}\text { Interruptible Deliveries } \\
\text { (million cubic feet) }\end{array}$} \\
\hline 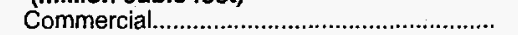 & NA & NA & NA & 2,922 & 5,115 \\
\hline Industrial & NA & NA & NA & 38,315 & 48,468 \\
\hline 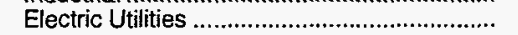 & NA & NA & NA & 2,736 & 2,687 \\
\hline 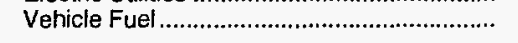 & NA & NA & NA & 11 & 8 \\
\hline \multicolumn{6}{|l|}{ Number of Consumers } \\
\hline 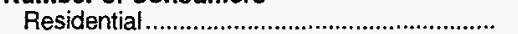 & 983,592 & $1,002,154$ & $1,022,542$ & $1,044,699$ & $1,073,308$ \\
\hline 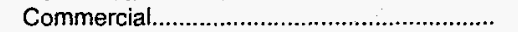 & 112,661 & 113,945 & 114,898 & 115,924 & 115,994 \\
\hline 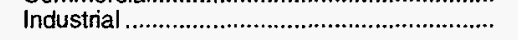 & 1,018 & 1,074 & 1,108 & 1,032 & 1,176 \\
\hline \multirow{2}{*}{\multicolumn{6}{|c|}{$\begin{array}{l}\text { Average Annual Consumption per Consumer } \\
\text { (thousand cubic feet) }\end{array}$}} \\
\hline & 93 & 97 & 93 & 102 & 93 \\
\hline 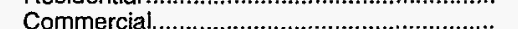 & 588 & 605 & 578 & 618 & 568 \\
\hline 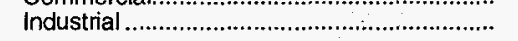 & 48,087 & 52,305 & 51,967 & 66,884 & 60,453 \\
\hline \multirow{2}{*}{\multicolumn{6}{|c|}{$\begin{array}{l}\text { Average Prices for Natural Gas } \\
\text { (dollars per thousand cubic feet) }\end{array}$}} \\
\hline & & & 1.37 & 1.61 & 1.39 \\
\hline imports . ( & 0 & 0 & 0 & 0 & 1.390 \\
\hline 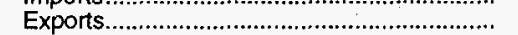 & 0 & 0 & 0 & 0 & 0 \\
\hline 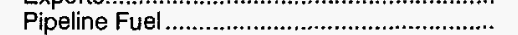 & 1.75 & 1.79 & 1.89 & 1.86 & 1.78 \\
\hline City Gate & 2.94 & 2.85 & 2.85 & 2.95 & 3.31 \\
\hline \multicolumn{6}{|l|}{ Delivered to Consumers } \\
\hline 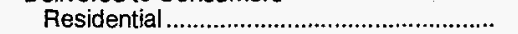 & 4.57 & 4.59 & 4.56 & 4.52 & 4.92 \\
\hline 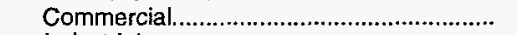 & 3.99 & 4.04 & 4.00 & 4.04 & 4.37 \\
\hline 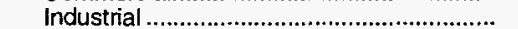 & 2.78 & 2.34 & 2.20 & 2.35 & 2.38 \\
\hline 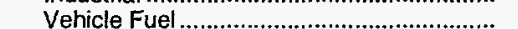 & 3.48 & 3.44 & 3.45 & 2.70 & 3.55 \\
\hline 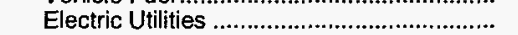 & 2.15 & 2.14 & 2.14 & 2.53 & 2.21 \\
\hline
\end{tabular}

a Beginning in 1991, Carbon Dioxide is no longer a component of gross withdrawals and nonhydrocarbon gases removed.

$\mathrm{NA}=$ Not Available.

Notes: Deliveries to electric utilities (consumption) are reported on the Form ElA-176, "Annual Peport of Natural and Supplemental Gas Supply and Disposition." See the discussion on electric utility data and Table A1 in Appendix A for a comparison of reporting to these two forms. Totals may not add due to independent rounding.

Sources: Energy Information Administration (EIA), Form EIA-176, "Annual Report of Natural and Supplemental Gas Supply and Disposition"; Form EIA-627, "Annual

Quantity and Value of Natural Gas Feport"; Form ElA-857, "Monthly Report of Natural Gas Purchases and Deliveries to Consumers"; Form ElA-816, "Monthly Natural Gas

Liquids Report"; Form ElA-759, "Monthly Power Plant Report"; Form FERC-423, "Monthly Report of Cost and Quality of Fuels for Electric Plants"; Form EIA-191, "Underground Gas Storage Report"; Form FPC-14, "Annual Report for importers and Exporters of Natural Gas"; U.S. Crude Oil, Natural Gas, and Natural Gas Liquids Reserves, 1994 Annual Report, DOE/EIA-0216(94); and the U.S. Minerals Management Service. 


\section{Connecticut - Natural Gas 1994 \\ Million Percent of}

Cu. Feet National Total

Net Interstate

Movements:

110,478

Marketed

Production:

0

0.00

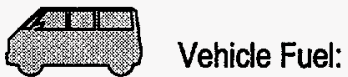

2

0.12

Deliveries to Consumers:

Residential: $\quad 41,600$

0.86

$\$$

Electric

Utilities:

8,002

0.27

Commercial:

39,082

1.35
Million

Cu. Feet

Percent of National Total

\section{Total:}

119,334

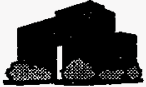

Commercial:

39,082

1.35

Table 54. Summary Statistics for Natural Gas - Connecticut, 1990-1994

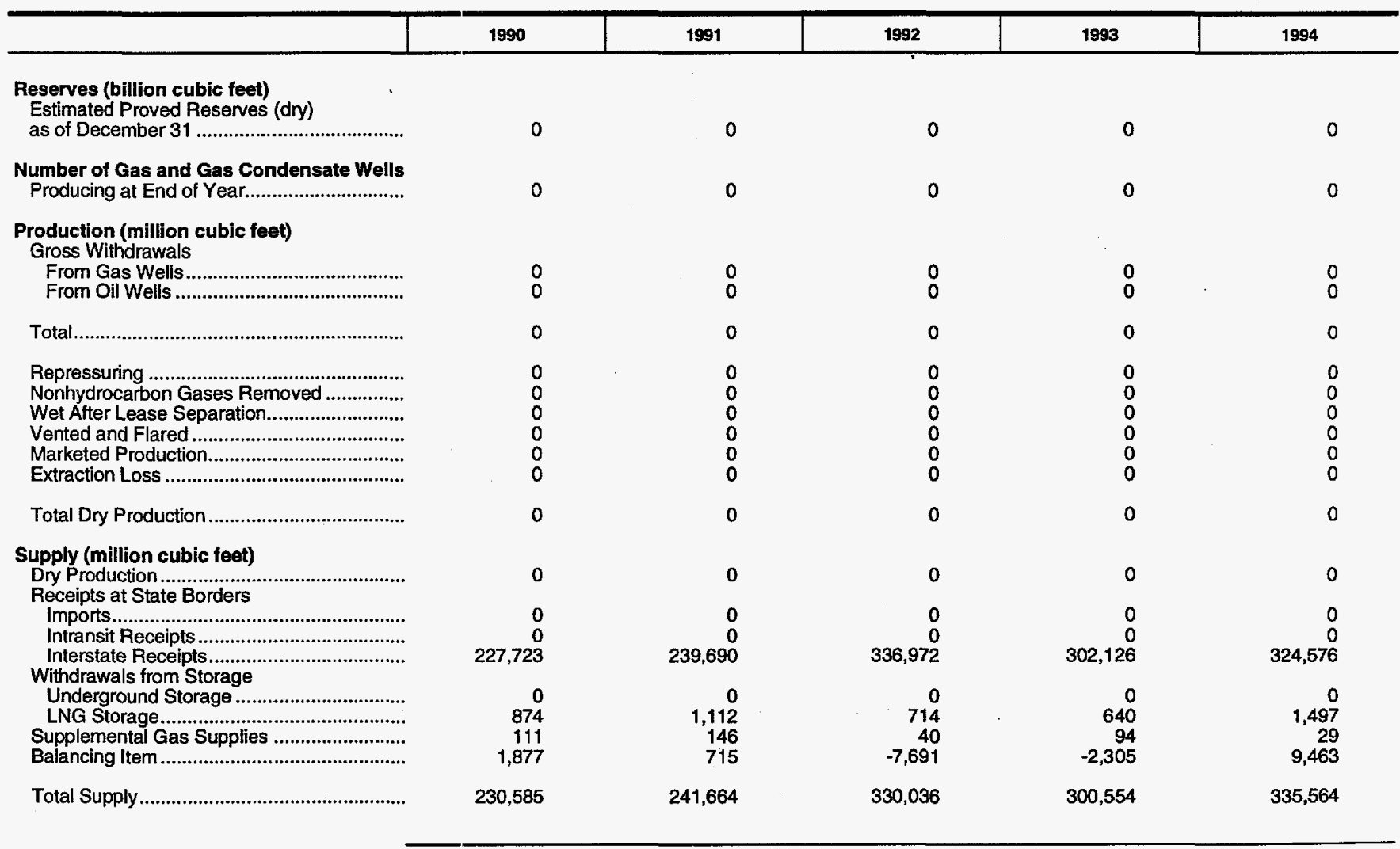

See footnotes at end of table. 
Table 54. Summary Statistics for Natural Gas - Connecticut, 1990-1994 (Continued)

\begin{tabular}{|c|c|c|c|c|c|}
\hline & 1990 & 1991 & 1992 & 1993 & 1994 \\
\hline $\begin{array}{l}\text { Disposition (million cubic feet) } \\
\text { Consumption........................................... } \\
\text { Deliveries at State Borders }\end{array}$ & 97,634 & 101,904 & 111,305 & 111,501 & 120,031 \\
\hline 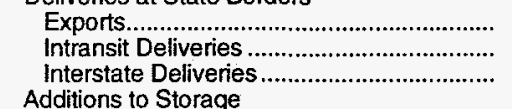 & $\begin{array}{r}0 \\
0 \\
131,255\end{array}$ & $\begin{array}{r}0 \\
0 \\
138,751\end{array}$ & $\begin{array}{r}0 \\
0 \\
218,371\end{array}$ & $\begin{array}{r}0 \\
0 \\
188,443\end{array}$ & $\begin{array}{r}0 \\
0 \\
214,098\end{array}$ \\
\hline $\begin{array}{l}\text { Underground Storage } \\
\text { LNG Storage }\end{array}$ & $\begin{array}{r}0 \\
1,696\end{array}$ & $\begin{array}{r}0 \\
1,010\end{array}$ & $\begin{array}{r}0 \\
359\end{array}$ & $\begin{array}{r}0 \\
610\end{array}$ & $\begin{array}{r}0 \\
1,435\end{array}$ \\
\hline Total Disposition & 230,585 & 241,664 & 330,036 & 300,554 & 335,564 \\
\hline 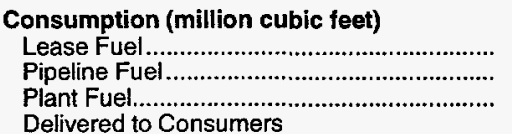 & $\begin{array}{r}0 \\
486 \\
0\end{array}$ & $\begin{array}{r}0 \\
523 \\
0\end{array}$ & $\begin{array}{r}0 \\
590 \\
0\end{array}$ & $\begin{array}{r}0 \\
460 \\
0\end{array}$ & $\begin{array}{r}0 \\
697 \\
0\end{array}$ \\
\hline 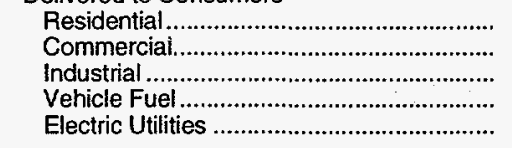 & $\begin{array}{r}37,446 \\
29,410 \\
25,448 \\
0 \\
4,843\end{array}$ & $\begin{array}{r}37,181 \\
26,838 \\
32,660 \\
0 \\
4,702\end{array}$ & $\begin{array}{r}42,394 \\
29,838 \\
36,383 \\
2,100\end{array}$ & $\begin{array}{r}42,213 \\
31,427 \\
36,842 \\
\\
557\end{array}$ & $\begin{array}{r}41,600 \\
39,082 \\
30,647 \\
2 \\
8,002\end{array}$ \\
\hline Total Delivered to Consumers ........................ & 97,147 & 101,380 & 110,715 & 111,040 & 119,334 \\
\hline Total Consumption & 97,634 & 101,904 & 111,305 & 111,501 & 120,031 \\
\hline 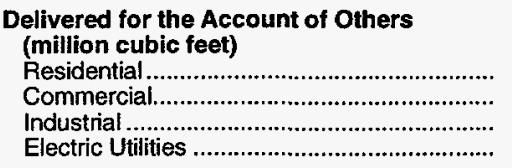 & $\begin{array}{r}0 \\
1,169 \\
3,572 \\
4,796\end{array}$ & $\begin{array}{r}0 \\
1,887 \\
10,370 \\
4,518\end{array}$ & $\begin{array}{r}0 \\
1,037 \\
12,514 \\
1,881\end{array}$ & $\begin{array}{r}0 \\
602 \\
10,164 \\
140\end{array}$ & $\begin{array}{r}0 \\
7,455 \\
1,435 \\
6,941\end{array}$ \\
\hline 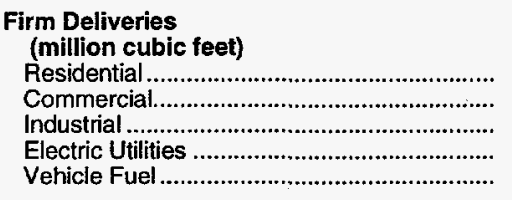 & $\begin{array}{l}\text { NA } \\
\text { NA } \\
\text { NA } \\
\text { NA } \\
\text { NA }\end{array}$ & $\begin{array}{l}\text { NA } \\
\text { NA } \\
\text { NA } \\
\text { NA } \\
\text { NA }\end{array}$ & $\begin{array}{l}\text { NA } \\
\text { NA } \\
\text { NA } \\
\text { NA } \\
\text { NA }\end{array}$ & $\begin{array}{r}42,213 \\
22,635 \\
18,655 \\
108 \\
0\end{array}$ & $\begin{array}{r}41,265 \\
27,680 \\
16,358 \\
6,807 \\
1\end{array}$ \\
\hline 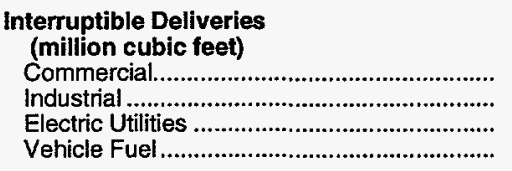 & $\begin{array}{l}\text { NA } \\
\text { NA } \\
\text { NA } \\
\text { NA }\end{array}$ & $\begin{array}{l}\text { NA } \\
\text { NA } \\
\text { NA } \\
\text { NA }\end{array}$ & $\begin{array}{l}\text { NA } \\
\text { NA } \\
\text { NA } \\
\text { NA }\end{array}$ & $\begin{array}{r}8,792 \\
18,655 \\
276 \\
--\end{array}$ & $\begin{array}{r}11,402 \\
16,358 \\
1,047 \\
1\end{array}$ \\
\hline 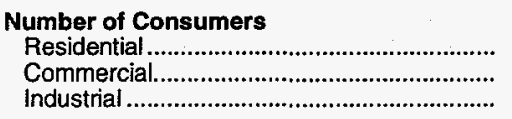 & $\begin{array}{r}428,912 \\
45,364 \\
3,061\end{array}$ & $\begin{array}{r}430,078 \\
45,925 \\
2,921\end{array}$ & $\begin{array}{r}432,244 \\
46,859 \\
2,923\end{array}$ & $\begin{array}{r}427,761 \\
45,529 \\
2,952\end{array}$ & $\begin{array}{r}428,157 \\
45,042 \\
3,754\end{array}$ \\
\hline 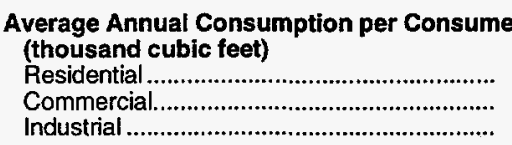 & $\begin{array}{r}87 \\
648 \\
8,314\end{array}$ & $\begin{array}{r}86 \\
584 \\
11,181\end{array}$ & $\begin{array}{r}98 \\
637 \\
12,447\end{array}$ & $\begin{array}{r}99 \\
690 \\
12,480\end{array}$ & $\begin{array}{r}97 \\
868 \\
8,164\end{array}$ \\
\hline $\begin{array}{l}\text { Average Prices for Natural Gas } \\
\text { (dollars per thousand cubic feet) } \\
\text { Wellhead (Marketed Production) }\end{array}$ & & & & & \\
\hline 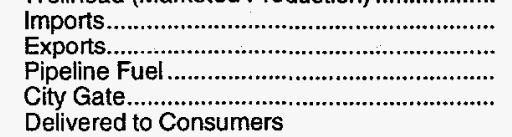 & $\begin{array}{r}0 \\
0 \\
3.68 \\
3.66\end{array}$ & $\begin{array}{r}0 \\
0 \\
3.08 \\
3.50\end{array}$ & $\begin{array}{r}0 \\
0 \\
2.95 \\
3.73\end{array}$ & $\begin{array}{r}0 \\
0 \\
3.53 \\
3.87\end{array}$ & $\begin{array}{r}0 \\
0 \\
2.62 \\
4.17\end{array}$ \\
\hline 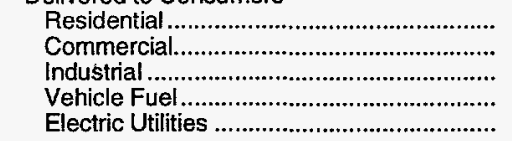 & $\begin{array}{r}8.58 \\
6.30 \\
4.80 \\
0 \\
2.81\end{array}$ & $\begin{array}{r}8.74 \\
6.90 \\
4.84 \\
0 \\
2.16\end{array}$ & $\begin{array}{r}8.96 \\
7.20 \\
4.92 \\
12.45 \\
2.74\end{array}$ & $\begin{array}{l}9.43 \\
7.02 \\
4.77 \\
8.97 \\
3.90\end{array}$ & $\begin{array}{r}10.14 \\
7.39 \\
4.49 \\
7.74 \\
1.99\end{array}$ \\
\hline
\end{tabular}

NA $=$ Not Available.

Notes: Deliveries to electric utilities (consumption) are reported on the Form ElA-176, "Annual Report of Natural and Supplemental Gas Supply and Disposition." See the

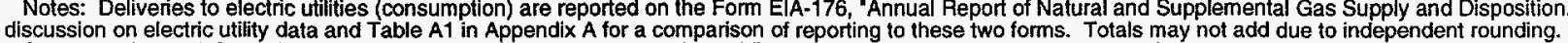

discussion on electric utility data and Table A1 in Appendix A for a comparison of reporting to these two forms. Totals may not add due to independent rounding.
Sources: Energy information Administration (EIA), Form ElA-176, "Annual Report of Natural and Supplemental Gas Supply and Disposition"; Form EIA-627, "Annual

Sources: Energy Information Administration (EIA), Form EIA-176, "Annual Report of Natural and Supplemental Gas Supply and Disposition"; Form EIA-627, "Annual
Quantity and Value of Natural Gas Report"; Form EIA-857, "Monthly Report of Natural Gas Purchases and Deliveries to Consumers"; Form ElA-816, "Monthly Natural Gas

Liquids Report"; Form EIA-759, "Monthly Power Plant Report"; Form FERC-423, "Monthly Report of Cost and Quality of Fuels for Electric Plants"; Form EIA-191, "Underground Liquids Report"; Form ElA-759, "Monthly Power Plant Report"; Form FERC-423, "Monthly Report of Cost and Quality of Fuels for Electric Plants"; Form ElA-191, "Underground
Gas Storage Report"; Form FPC-14, "Annual Report for Importers and Exporters of Natural Gas"; U.S. Crude Oil, Natural Gas, and Natural Gas Liquids Reserves, 1994 Annual Gas Storage Report"; Form FPC-14, "Annual Report for importers and Expor
Report, DOE/EIA-0216(94); and the U.S. Minerals Management Service. 


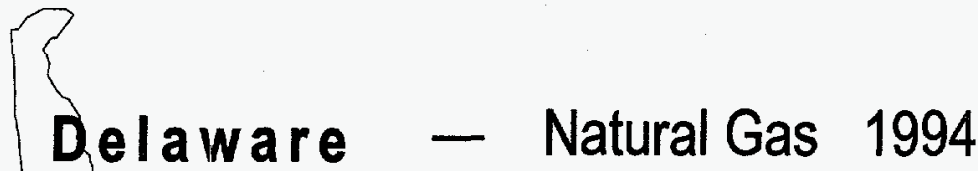

Million Percent of

Cu. Feet National Total

Million

Percent of

National Total $\quad \mathrm{Cu}$. Feet

National Tota

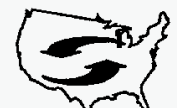

Net Interstate

Movements:

49,408

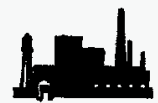

Industrial:

17,216

0.21

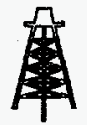

Marketed

Production:

0

0.00

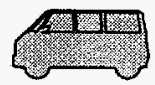

Vehicle Fuel:

Deliveries to Consumers:

Residential:

8,557

0.18

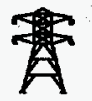

Electric

Utilities:

17,399

0.58

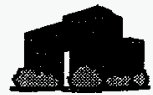

Commercial:

5,459

0.19

Total:

48,632

0.26

Table 55. Summary Statistics for Natural Gas — Delaware, 1990-1994

\begin{tabular}{|c|c|c|c|c|c|}
\hline & 1990 & 1991 & 1992 & 1993 & 1994 \\
\hline 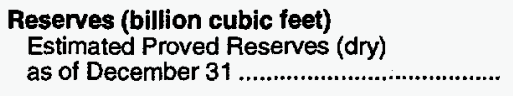 & 0 & 0 & 0 & 0 & 0 \\
\hline 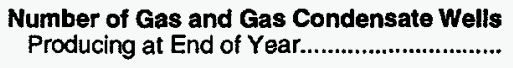 & 0 & 0 & 0 & 0 & 0 \\
\hline 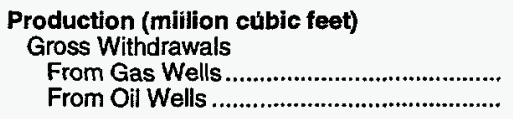 & $\begin{array}{l}0 \\
0\end{array}$ & $\begin{array}{l}0 \\
0\end{array}$ & $\begin{array}{l}0 \\
0\end{array}$ & $\begin{array}{l}0 \\
0\end{array}$ & $\begin{array}{l}0 \\
0\end{array}$ \\
\hline 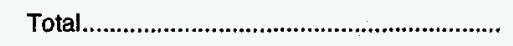 & 0 & 0 & 0 & 0 & 0 \\
\hline 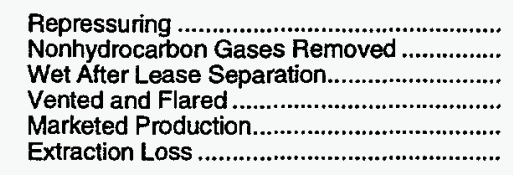 & $\begin{array}{l}0 \\
0 \\
0 \\
0 \\
0 \\
0\end{array}$ & $\begin{array}{l}0 \\
0 \\
0 \\
0 \\
0 \\
0\end{array}$ & $\begin{array}{l}0 \\
0 \\
0 \\
0 \\
0 \\
0\end{array}$ & $\begin{array}{l}0 \\
0 \\
0 \\
0 \\
0 \\
0\end{array}$ & $\begin{array}{l}0 \\
0 \\
0 \\
0 \\
0 \\
0\end{array}$ \\
\hline 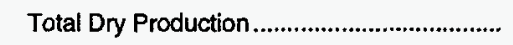 & 0 & 0 & 0 & 0 & 0 \\
\hline 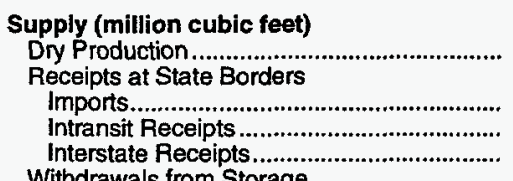 & $\begin{array}{r}0 \\
0 \\
37,742\end{array}$ & $\begin{array}{r}0 \\
0 \\
41,760\end{array}$ & $\begin{array}{r}0 \\
0 \\
40,864\end{array}$ & $\begin{array}{r}0 \\
0 \\
43,788\end{array}$ & $\begin{array}{r}0 \\
0 \\
52,304\end{array}$ \\
\hline 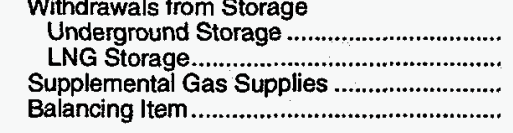 & $\begin{array}{r}0 \\
81 \\
4,410 \\
-288\end{array}$ & $\begin{array}{r}0 \\
72 \\
4,262 \\
-1,172\end{array}$ & $\begin{array}{r}0 \\
66 \\
3,665 \\
-1,696\end{array}$ & $\begin{array}{r}0 \\
95 \\
3,597 \\
-2,807\end{array}$ & $\begin{array}{r}0 \\
202 \\
3,032 \\
-3,781\end{array}$ \\
\hline Total Supply & 41,945 & 44,921 & 42,898 & 44,674 & 51,758 \\
\hline
\end{tabular}

See footnotes at end of table. 
Table 55. Summary Statistics for Natural Gas - Delaware, 1990-1994 (Continued)

\begin{tabular}{|c|c|c|c|c|c|}
\hline & 1990 & 1991 & 1992 & 1993 & 1994 \\
\hline $\begin{array}{l}\text { Disposition (million cubic feet) } \\
\text { Consumption............................................... } \\
\text { Deliveries at State Borders }\end{array}$ & 39,127 & 41,948 & 39,608 & 41,612 & 48,636 \\
\hline 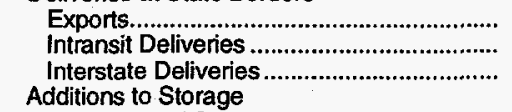 & $\begin{array}{r}0 \\
0 \\
2,730\end{array}$ & $\begin{array}{r}0 \\
0 \\
2,894\end{array}$ & $\begin{array}{r}0 \\
0 \\
3,230\end{array}$ & $\begin{array}{r}0 \\
0 \\
2,963\end{array}$ & $\begin{array}{r}0 \\
0 \\
2,896\end{array}$ \\
\hline 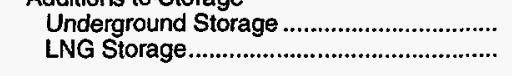 & $\begin{array}{r}0 \\
88\end{array}$ & $\begin{array}{r}0 \\
79\end{array}$ & $\begin{array}{r}0 \\
61\end{array}$ & $\begin{array}{r}0 \\
99\end{array}$ & $\begin{array}{r}0 \\
225\end{array}$ \\
\hline 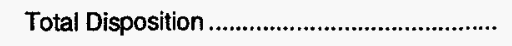 & 41,945 & 44,921 & 42,898 & 44,674 & 51,758 \\
\hline 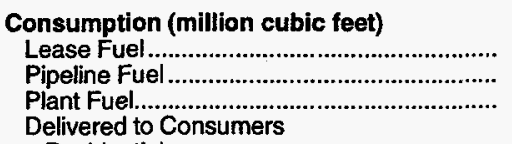 & $\begin{array}{l}0 \\
4 \\
0\end{array}$ & $\begin{array}{l}0 \\
4 \\
0\end{array}$ & $\begin{array}{l}0 \\
4 \\
0\end{array}$ & $\begin{array}{l}0 \\
4 \\
0\end{array}$ & $\begin{array}{l}0 \\
4 \\
0\end{array}$ \\
\hline 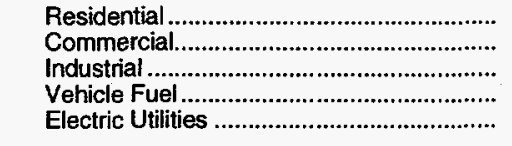 & $\begin{array}{r}7,270 \\
4,042 \\
17,036 \\
0 \\
10,776\end{array}$ & $\begin{array}{r}7,189 \\
4,253 \\
16,147 \\
0 \\
14,354\end{array}$ & $\begin{array}{r}8,194 \\
4,965 \\
18,060 \\
0 \\
8,384\end{array}$ & $\begin{array}{r}8,295 \\
5,195 \\
19,453 \\
0 \\
8,665\end{array}$ & $\begin{array}{r}8,557 \\
5,459 \\
17,216 \\
1 \\
17,399\end{array}$ \\
\hline Total Delivered to Consumers ......................... & 39,124 & 41,944 & 39,604 & 41,608 & 48,632 \\
\hline 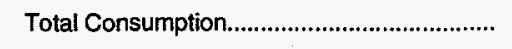 & 39,127 & 41,948 & 39,608 & 41,612 & 48,636 \\
\hline 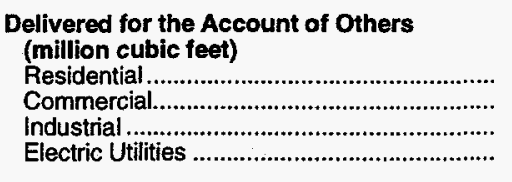 & $\begin{array}{r}0 \\
0 \\
4,847 \\
35\end{array}$ & $\begin{array}{r}0 \\
0 \\
5,509 \\
39\end{array}$ & $\begin{array}{r}0 \\
0 \\
6,316 \\
48\end{array}$ & $\begin{array}{r}0 \\
0 \\
4,922 \\
58\end{array}$ & $\begin{array}{r}0 \\
0 \\
5,631 \\
16,120\end{array}$ \\
\hline 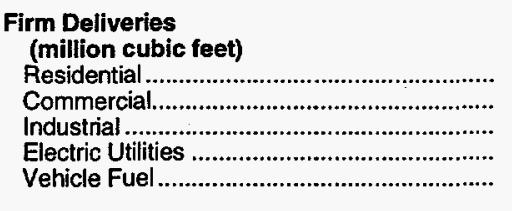 & $\begin{array}{l}\text { NA } \\
\text { NA } \\
\text { NA } \\
\text { NA } \\
\text { NA }\end{array}$ & $\begin{array}{l}\text { NA } \\
\text { NA } \\
\text { NA } \\
\text { NA } \\
\text { NA }\end{array}$ & $\begin{array}{l}\text { NA } \\
\text { NA } \\
\text { NA } \\
\text { NA } \\
\text { NA }\end{array}$ & $\begin{array}{r}8,295 \\
5,195 \\
6,309 \\
8,322 \\
0\end{array}$ & $\begin{array}{r}8,557 \\
5,459 \\
6,880 \\
16,187 \\
1\end{array}$ \\
\hline 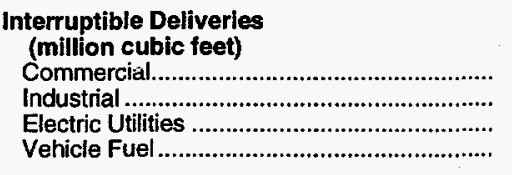 & $\begin{array}{l}\text { NA } \\
\text { NA } \\
\text { NA } \\
\text { NA }\end{array}$ & $\begin{array}{l}\text { NA } \\
\text { NA } \\
\text { NA } \\
\text { NA }\end{array}$ & $\begin{array}{l}\text { NA } \\
\text { NA } \\
\text { NA } \\
\text { NA }\end{array}$ & $\begin{array}{r}6,309 \\
141 \\
-\end{array}$ & $\begin{array}{r}-- \\
6,880 \\
1,281 \\
--\end{array}$ \\
\hline 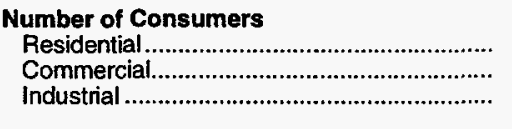 & $\begin{array}{r}88,894 \\
7,485 \\
240\end{array}$ & $\begin{array}{r}91,467 \\
7,895 \\
243\end{array}$ & $\begin{array}{r}94,027 \\
8,173 \\
248\end{array}$ & $\begin{array}{r}96,914 \\
8,409 \\
249\end{array}$ & $\begin{array}{r}100,431 \\
8,721 \\
252\end{array}$ \\
\hline 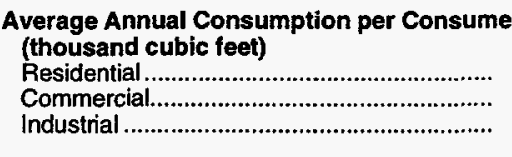 & $\begin{array}{r}82 \\
540 \\
70,984\end{array}$ & $\begin{array}{r}79 \\
539 \\
66,449\end{array}$ & $\begin{array}{r}87 \\
607 \\
72,823\end{array}$ & $\begin{array}{r}86 \\
618 \\
78,126\end{array}$ & $\begin{array}{r}85 \\
626 \\
68,319\end{array}$ \\
\hline 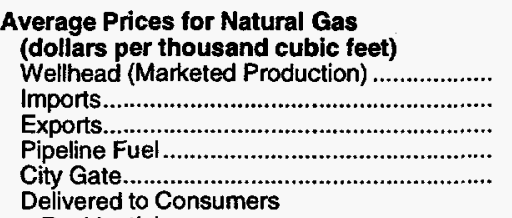 & $\begin{array}{r}0 \\
0 \\
3.10 \\
2.76\end{array}$ & $\begin{array}{r}0 \\
0 \\
2.88 \\
2.54\end{array}$ & $\begin{array}{r}0 \\
0 \\
3.01 \\
2.83\end{array}$ & $\begin{array}{r}0 \\
0 \\
3.19 \\
3.24\end{array}$ & $\begin{array}{r}0 \\
0 \\
3.02 \\
2.95\end{array}$ \\
\hline 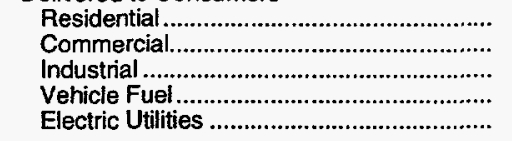 & $\begin{array}{r}6.13 \\
5.12 \\
3.44 \\
0 \\
2.72\end{array}$ & $\begin{array}{r}5.86 \\
4.81 \\
3.09 \\
0 \\
2.49\end{array}$ & $\begin{array}{r}6.13 \\
4.94 \\
3.25 \\
0 \\
2.70\end{array}$ & $\begin{array}{r}6.70 \\
5.46 \\
3.40 \\
0 \\
2.69\end{array}$ & $\begin{array}{r}7.43 \\
6.17 \\
3.43 \\
0 \\
2.43\end{array}$ \\
\hline
\end{tabular}

NA = Not Available.

Notes: Deliveries to electric utilities (consumption) are reported on the Form EIA-176, "Annual Report of Natural and Supplemental Gas Supply and Disposition." See the discussion on electric utility data and Table A1 in Appendix A for a comparison of reporting to these two forms. Totals may not add due to independent rounding

Sources: Energy Information Administration (EIA). Form EIA-176, "Annual Report of Natural and Supplemental Gas Supply and Disposition"; Form EIA-627, "Annual

Quantity and Value of Natural Gas Report"; Form ElA-857, "Monthly Report of Natural Gas Purchases and Deliveries to Consumers"; Form EIA-816, "Monthly Natural Gas

Liquids Report"; Form EIA-759, "Monthly Power Plant Report"; Form FERC-423, "Monthly Report of Cost and Quality of Fuels for Electric Plants"; Form ElA-191, "Underground

Gas Storage Report"; Form FPC-14, "Annual Report for Importers and Exporters of Natural Gas"; U.S. Crude Oil, Natural Gas, and Natural Gas Liquids Reserves, 1994 Annual Report, DOE/EIA-0216(94); and the U.S. Minerals Management Service. 


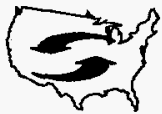

Net Interstate

Movements:

29,806

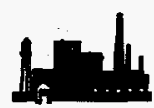

Industrial:

0

0.00

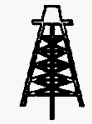

Marketed

Production:

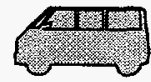

Vehicle Fuel:

0

0.00

Deliveries to Consumers:

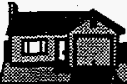

Residential:

15,865

0.33

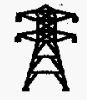

Electric

Utilities:

0

0.00

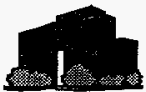

Commercial:

14,742

0.51

Total:

30,607

0.16

Table 56. Summary Statistics for Natural Gas - District of Columbia, 1990-1994

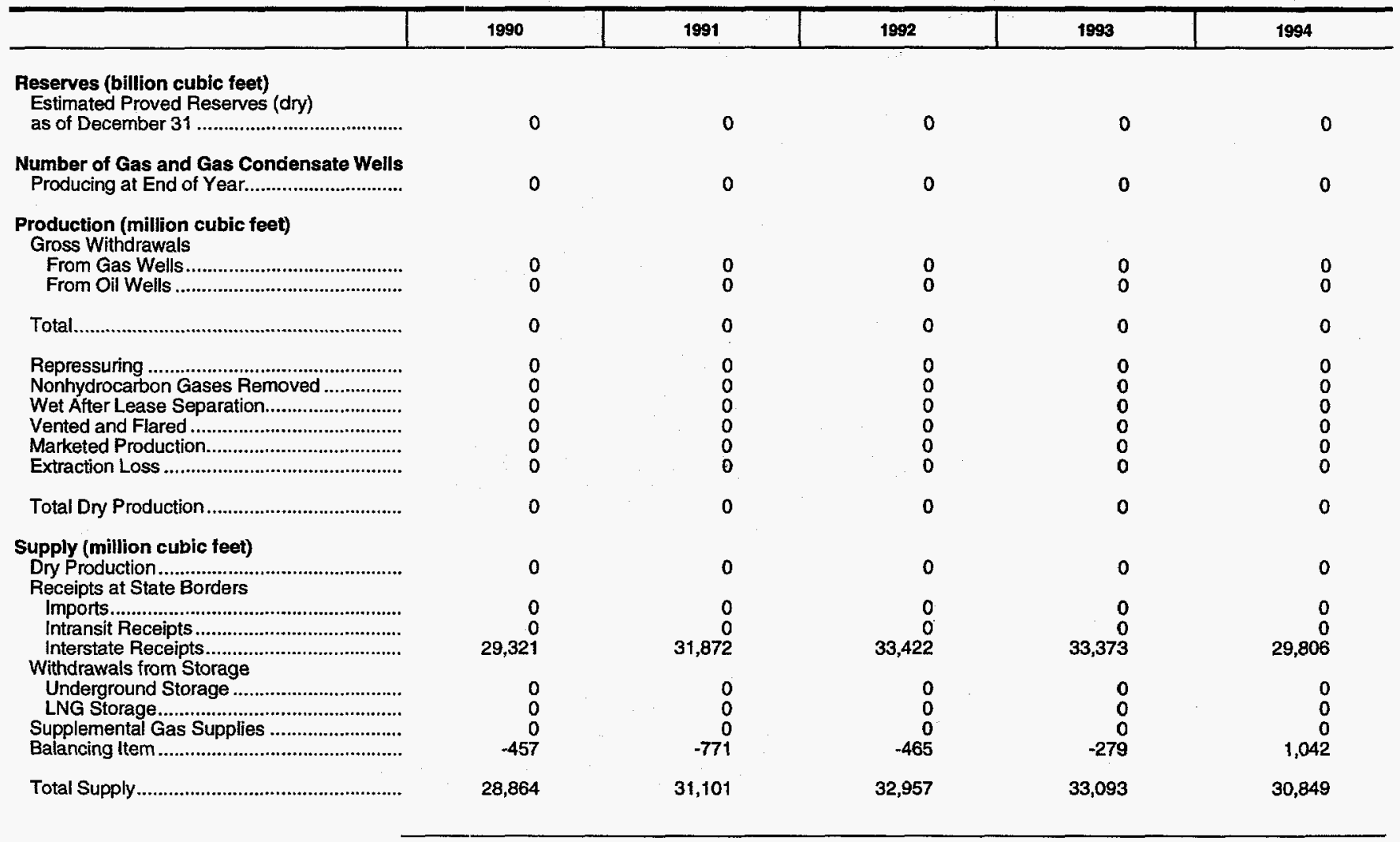


Table 56. Summary Statistics for Natural Gas - District of Columbia, 1990-1994 (Continued)

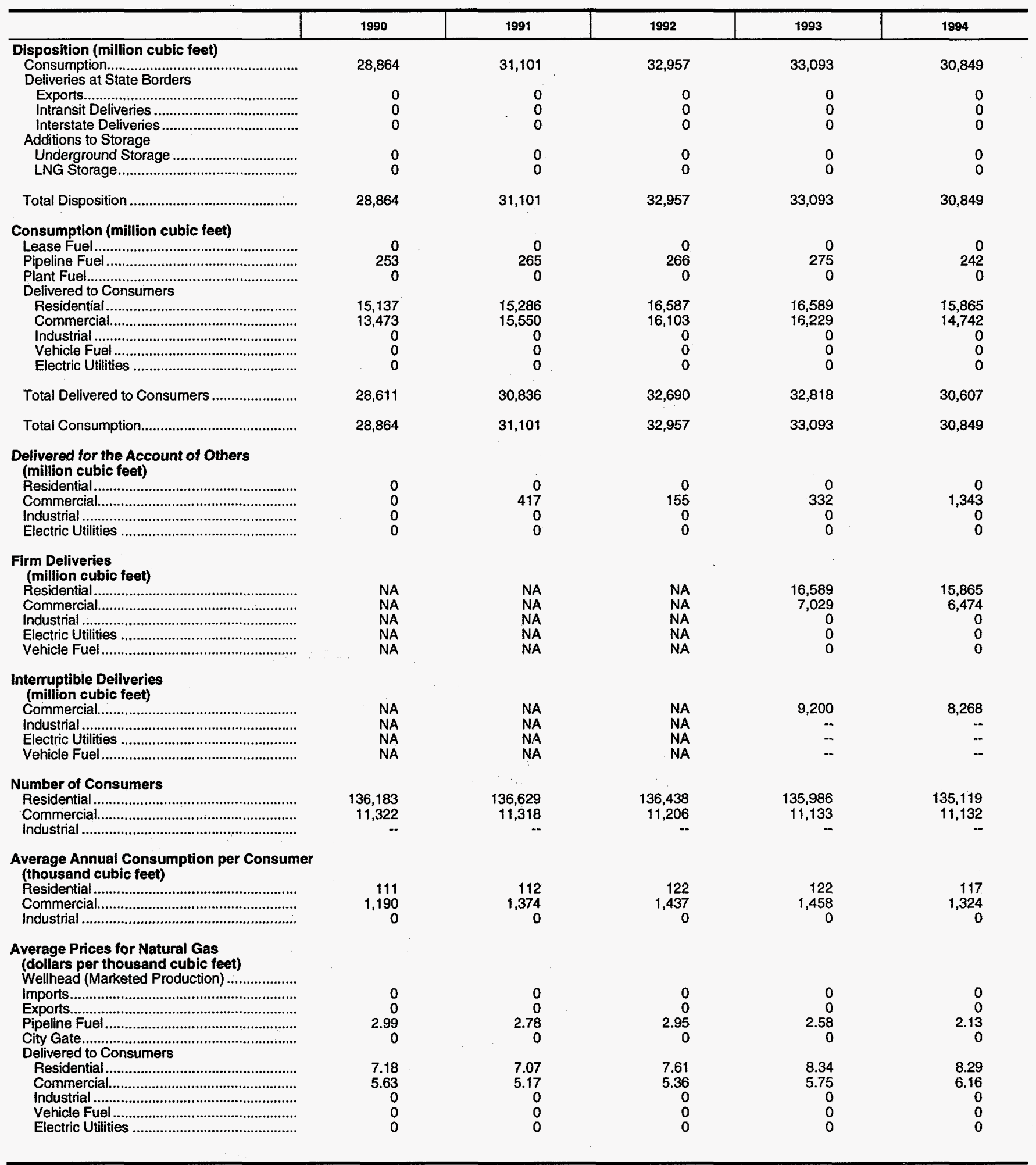

NA $=$ Not Available.

Notes: Deliveries to electric utilities (consumption) are reported on the Form ElA-176, "Annual Report of Natural and Supplemental Gas Supply and Disposition." See the discussion on electric utility data and Table A1 in Appendix A for a comparison of reporting to these two forms. Totals may not add due to independent rounding.

discussion on electric utility data and Table A1 in Appendix A for a comparison of reporting to these two forms. Totals may not add due to independent rounding.

Sources: Energy Information Administration (EIA), Form ElA-176, "Annual Report of Natural and Supplemental Gas Supply and Disposition"; Form ElA-627, "Annual
Quantity and Value of Natural Gas Report"; Form ElA-857, "Monthly Peport of Natural Gas Purchases and Deliveries to Consumers"; Form ElA-816, "Monthly Natural Gas

Quantity and Value of Natural Gas Report"; Form ElA-857, "Monthly Report of Natural Gas Purchases and Deliveries to Consumers"; Form EIA-816, "Monthly Natural Gas

Gas Storage Report"; Form FPC-14, "Annual Report for Importers and Exporters of Natural Gas"; U.S. Crude Oil, Natural Gas, and Natural Gas Liquids Reserves, 1994 Annual Report, DOE/EIA-0216(94); and the U.S. Minerals Management Service. 


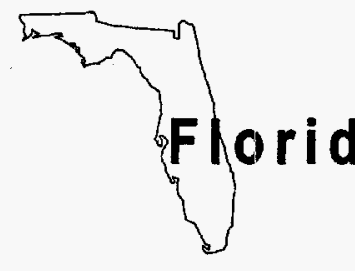

Million Percent of

Cu. Feet National Total

Million

Cu. Feet

Percent of

National Total

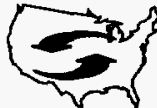

Net Interstate

Movements:

359,142

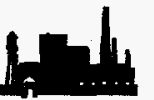

Industrial:

126,873

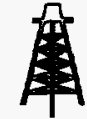

Marketed

Production:

7,486

0.04

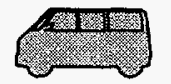

Vehicle Fuel:

68

Deliveries to Consumers:

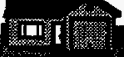

Residential:

13,855

0.29

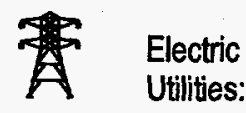

180,697

6.05

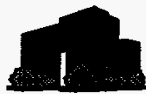

Commercial:

39,935

1.38

Total:

361,428

Table 57. Summary Statistics for Natural Gas - Florida, 1990-1994

\begin{tabular}{|c|c|c|c|c|c|}
\hline & 1990 & 1991 & 1992 & 1993 & 1994 \\
\hline 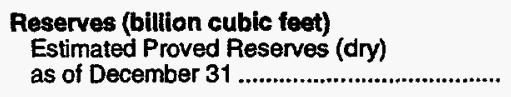 & 45 & 38 & 47 & 50 & 98 \\
\hline 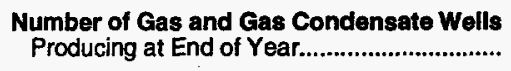 & 0 & 0 & 0 & 0 & 0 \\
\hline 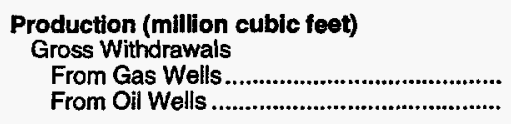 & $\begin{array}{r}0 \\
7,566\end{array}$ & $\begin{array}{r}0 \\
5,898\end{array}$ & $\begin{array}{r}0 \\
7,584\end{array}$ & $\begin{array}{r}0 \\
8,011\end{array}$ & $\begin{array}{r}0 \\
8,468\end{array}$ \\
\hline Total & 7,566 & 5,898 & 7,584 & 8,011 & 8,468 \\
\hline 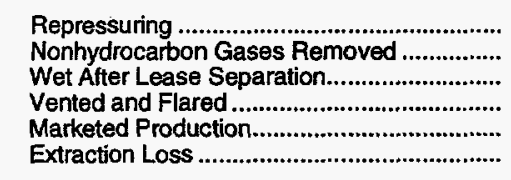 & $\begin{array}{r}115 \\
681 \\
6,769 \\
286 \\
6,483 \\
2,831\end{array}$ & $\begin{array}{r}0 \\
531 \\
5,367 \\
482 \\
4,884 \\
1,893\end{array}$ & $\begin{array}{r}0 \\
682 \\
6,901 \\
245 \\
6,657 \\
2,563\end{array}$ & $\begin{array}{r}0 \\
721 \\
7,290 \\
205 \\
7,085 \\
2,557\end{array}$ & $\begin{array}{r}0 \\
762 \\
7,706 \\
220 \\
7,486 \\
1,789\end{array}$ \\
\hline Total Dry Production & 3,652 & 2,991 & 4,094 & 4,528 & 5,697 \\
\hline 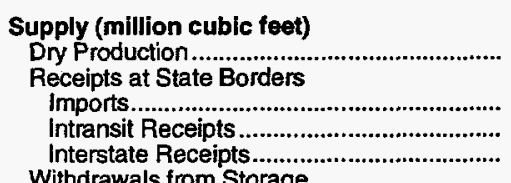 & $\begin{array}{r}0 \\
0 \\
325,897\end{array}$ & $\begin{array}{r}0 \\
0 \\
335,763\end{array}$ & $\begin{array}{r}0 \\
0 \\
341,354\end{array}$ & $\begin{array}{r}0 \\
0 \\
336,185\end{array}$ & $\begin{array}{r}0 \\
0 \\
359,172\end{array}$ \\
\hline 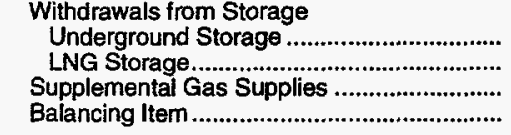 & $\begin{array}{r}0 \\
0 \\
0 \\
-2,492\end{array}$ & $\begin{array}{r}0 \\
0 \\
0 \\
5,391\end{array}$ & $\begin{array}{r}0 \\
0 \\
0 \\
7,573\end{array}$ & $\begin{array}{r}0 \\
0 \\
0 \\
\mathrm{~F}_{-4,795}\end{array}$ & $\begin{array}{r}0 \\
0 \\
0 \\
2,713\end{array}$ \\
\hline Total Supply & 327,057 & 344,145 & 353,021 & $R_{335,918}$ & 367,582 \\
\hline
\end{tabular}

See footnotes at end of table. 
Table 57. Summary Statistics for Natural Gas - Florida, 1990-1994 (Continued)

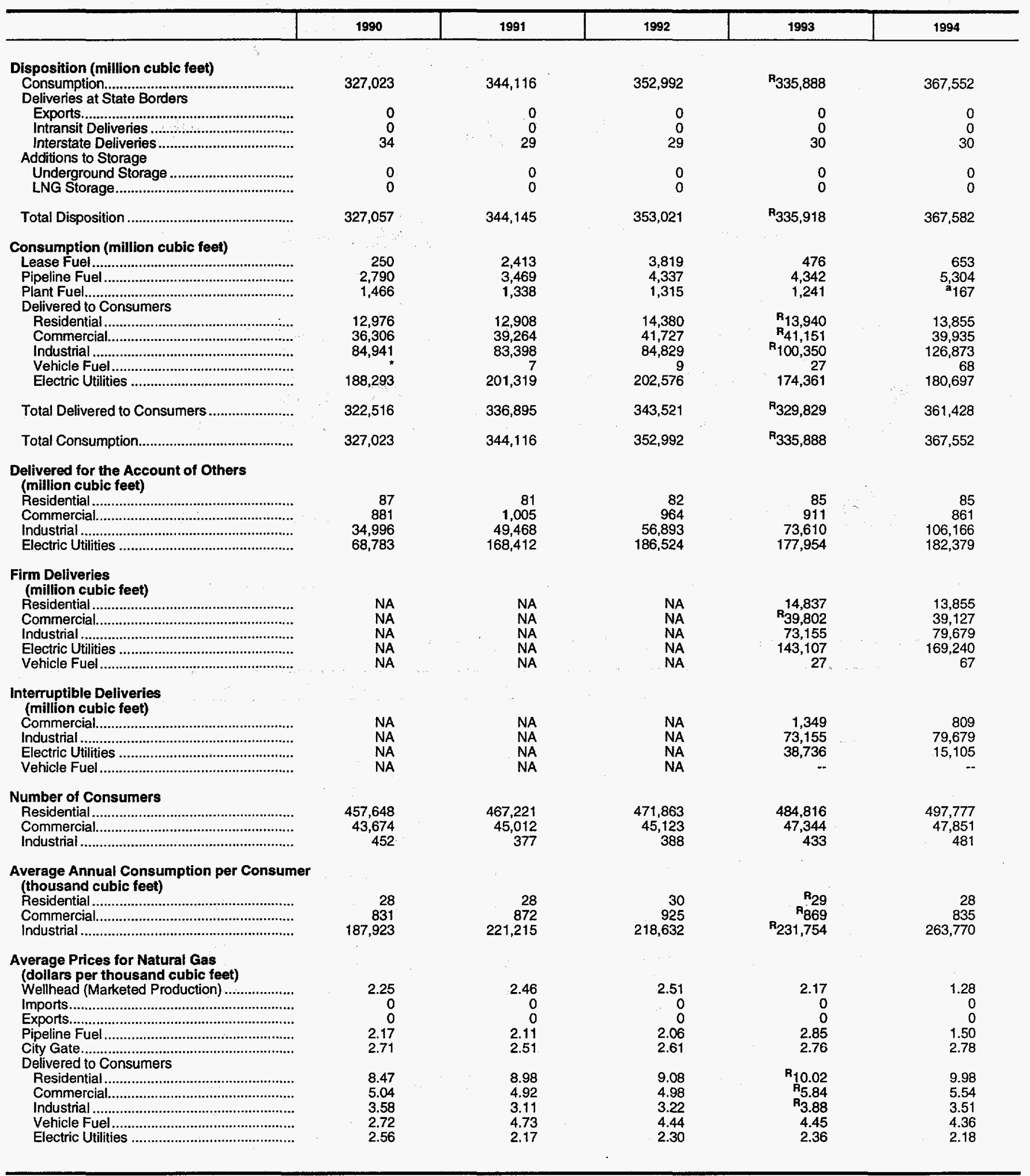

a In previous years, Florida had two plants reporting. One of these shut down in October 1993, leaving one plant reporting in 1994.

$R=$ Revised data.

NA $=$ Not Available.
$=$ Volume is less than 500,000 cubic feet

Notes: Deliveries to electric utilities (consumption) are reported on the Form ElA-176, "Annual Report of Natural and Supplemental Gas Supply and Disposition." See the discussion on electric utility data and Table A1 in Appendix A for a comparison of reporting to these two forms. Totals may not add due to independent rounding.

Sources: Energy Information Administration (EIA), Form EIA-176, "Annual Report of Natural and Supplemental Gas Supply and Disposition"; Form EIA-627, "Annual

Quantity and Value of Natural Gas Report"; Form ElA-857, "Monthly Report of Natural Gas Purchases and Deliveries to Consumers"; Form EIA-816, "Monthly Natural Gas

Liquids Report"; Form EIA-759, "Monthly Power Plant Report"; Form FERC-423, "Monthly Report of Cost and Quality of Fuels for Electric Plants"; Form EIA-191, "Underground

Gas Storage Report"; Form FPC-14, "Annual Report for Importers and Exporters of Natural Gas"; U.S. Crude Oit, Natural Gas, and Natural Gas Liquids Reserves, 1994 Annual Report, DOE/EIA-0216(94); and the U.S. Minerals Management Service. 


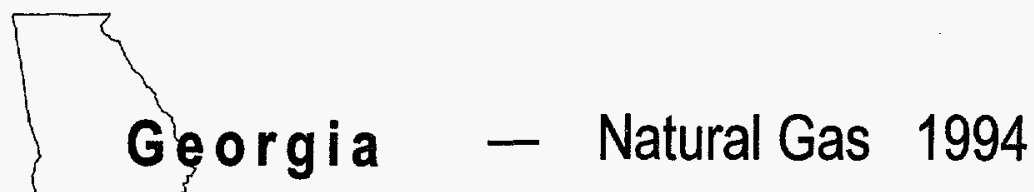

Million Percent of

Cu. Feet National Total

Million

Cu. Feet

Percent of National Total

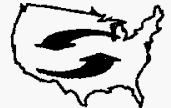

Net Interstate

Movements:

331,812

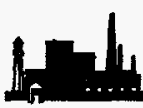

Industrial:

173,901

2.13

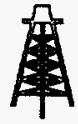

Marketed

Production:

0

0.00

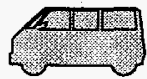

Vehicle Fuel:

3

0.17

Deliveries to Consumers:

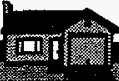

Residentia:

105,436

2.17

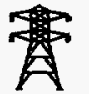

Electric

Utilities:

1,028

Commercial:

54,051

1.87

Total:

334,418

1.77

Table 58. Summary Statistics for Natural Gas - Georgia, 1990-1994

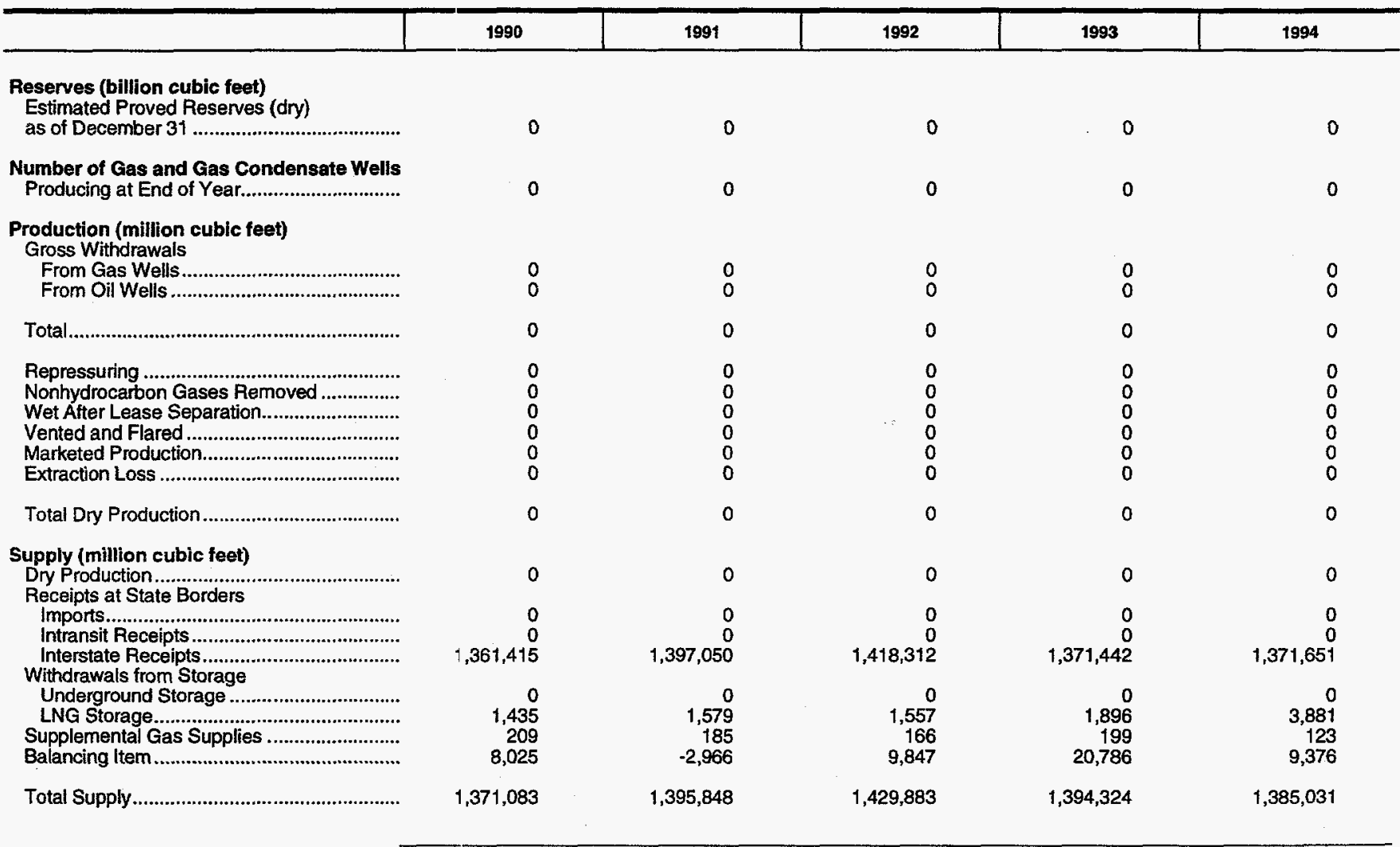

See footnotes at end of table. 
Table 58. Summary Statistics for Natural Gas - Georgia, 1990-1994 (Continued)

\begin{tabular}{|c|c|c|c|c|c|}
\hline & 1990 & 1991 & 1992 & 1993 & 1994 \\
\hline \multicolumn{6}{|l|}{ Disposition (million cubic feet) } \\
\hline \multicolumn{5}{|l|}{ Deliveries at State Borders } & 341,405 \\
\hline \multicolumn{6}{|l|}{ 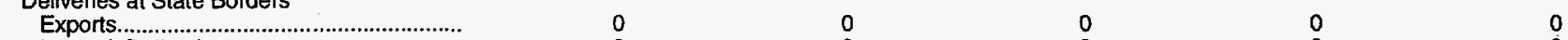 } \\
\hline Intransit Deliveries ............................................... & & 0 & 0 & 0 & 0 \\
\hline \multirow{2}{*}{\multicolumn{6}{|c|}{ Additions to Storage }} \\
\hline & & & & & \\
\hline 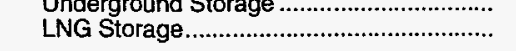 & $\begin{array}{r}0 \\
2,779\end{array}$ & 1,969 & 1,573 & 1,855 & 3,788 \\
\hline 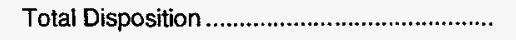 & $1,371,083$ & $1,395,848$ & $1,429,883$ & $1,394,324$ & $1,385,031$ \\
\hline \multicolumn{6}{|l|}{ Consumption (million cubic feet) } \\
\hline 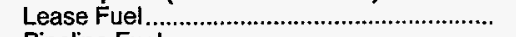 & 0 & 0 & 0 & 0 & 0 \\
\hline 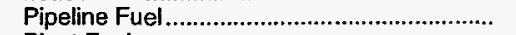 & 7,342 & 7,432 & 7,501 & 7,020 & 6,987 \\
\hline Plant Fuel.......................................................... & 0 & 0 & 0 & 0 & 0 \\
\hline \multicolumn{6}{|l|}{ Delivered to Consumers } \\
\hline 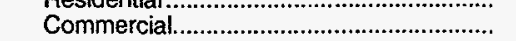 & 49,486 & $\begin{array}{l}90,002 \\
51,036\end{array}$ & 53,861 & 57,525 & 54,051 \\
\hline 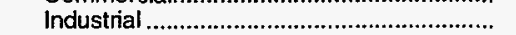 & 161,992 & 167,098 & 172,227 & 167,388 & 173,901 \\
\hline 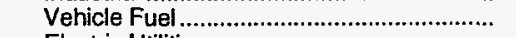 & & & & 4 & 3 \\
\hline Electric Utilities ............................................... & 1,932 & 838 & 1,162 & 3,026 & 1,028 \\
\hline Total Delivered to Consumers ......................... & 303,674 & 315,634 & 335,464 & 343,598 & 334,418 \\
\hline 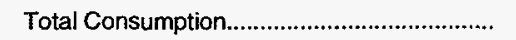 & 311,015 & 323,066 & 342,965 & 350,618 & 341,405 \\
\hline \multicolumn{6}{|l|}{$\begin{array}{l}\text { Delivered for the Account of Others } \\
\text { (million cubic feet) }\end{array}$} \\
\hline 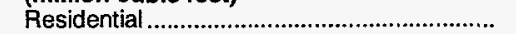 & 0 & 0 & 0 & 0 & 0 \\
\hline 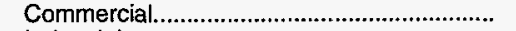 & 5,721 & 6,395 & 6,389 & 5,487 & 4,304 \\
\hline 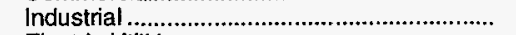 & 101,295 & 105,563 & 111,987 & 112,491 & 108,859 \\
\hline 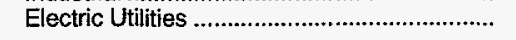 & 0 & 0 & 0 & 0 & 0 \\
\hline \multicolumn{6}{|l|}{$\begin{array}{l}\text { Firm Deliveries } \\
\text { (million cubic feet) }\end{array}$} \\
\hline 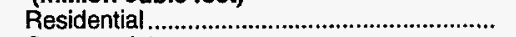 & NA & NA & NA & 115,655 & 105,436 \\
\hline 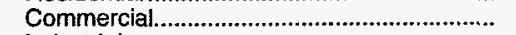 & NA & NA & NA & 44,652 & 42,537 \\
\hline Industrial ...................................................... & NA & NA & NA & 138,617 & 127,590 \\
\hline 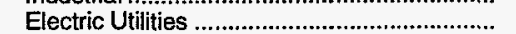 & NA & NA & NA & 3,080 & 1,137 \\
\hline 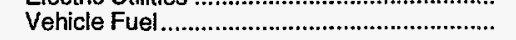 & NA & NA & NA & 0 & 0 \\
\hline \multicolumn{6}{|l|}{$\begin{array}{l}\text { Interruptible Deliveries } \\
\text { (million cubic feet) }\end{array}$} \\
\hline 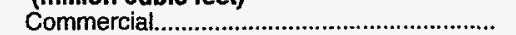 & NA & NA & NA & 12,873 & 11,514 \\
\hline 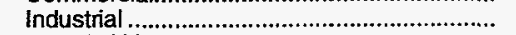 & NA & NA & NA & 138,617 & 127,590 \\
\hline 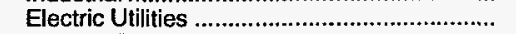 & NA & NA & NA & - & - \\
\hline 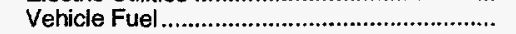 & NA & NA & NA & 4 & 2 \\
\hline \multicolumn{6}{|l|}{ Number of Consumers } \\
\hline 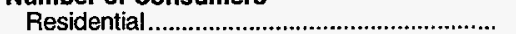 & $1,334,935$ & $1,363,723$ & $1,396,860$ & $1,430,626$ & $1,460,141$ \\
\hline 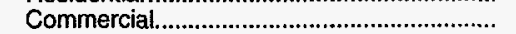 & 108,295 & 109,659 & 111,423 & 114,889 & 117,980 \\
\hline 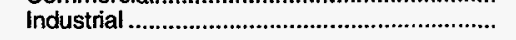 & 3,153 & 3,124 & 3,186 & 3,302 & 3,277 \\
\hline \multicolumn{6}{|l|}{$\begin{array}{l}\text { Average Annual Consumption per Consumer } \\
\text { (thousand cubic feet) }\end{array}$} \\
\hline 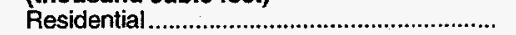 & 68 & 71 & 77 & 81 & 72 \\
\hline 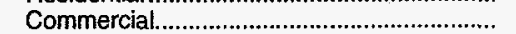 & 457 & 465 & 483 & 501 & 458 \\
\hline 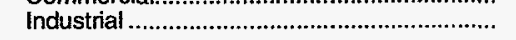 & 51,377 & 53,489 & 54,057 & 50,693 & 53,067 \\
\hline \multirow{2}{*}{\multicolumn{6}{|c|}{$\begin{array}{l}\text { Average Prices for Natural Gas } \\
\text { (dollars per thousand cubic feet) } \\
\text { Wellhead (Marketed Production) ...................... }\end{array}$}} \\
\hline & & & & & \\
\hline Exports. & 0 & 0 & 0 & 0 & 0 \\
\hline 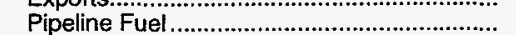 & 2.19 & 2.08 & 2.08 & 2.24 & 2.14 \\
\hline 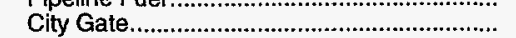 & 3.43 & 3.38 & 3.28 & 3.77 & 3.54 \\
\hline \multicolumn{6}{|l|}{ Delivered to Consumers } \\
\hline 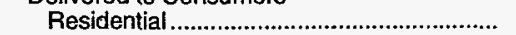 & 6.82 & 6.70 & 6.44 & 6.80 & 7.32 \\
\hline 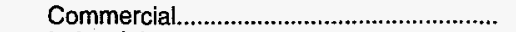 & 5.76 & 5.67 & 5.55 & 5.83 & 6.18 \\
\hline 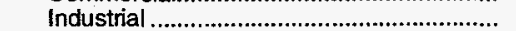 & 3.59 & 3.34 & 3.50 & 4.10 & 3.90 \\
\hline Vehicle Fuel.............................................. & 0 & 0 & 0 & 3.54 & 4.07 \\
\hline 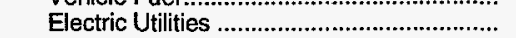 & 3.04 & 2.83 & 2.89 & 3.31 & 3.29 \\
\hline
\end{tabular}

NA $=$ Not Available.

Notes: Deliveries to electric utilities (consumption) are reported on the Form ElA-176, "Annual Report of Natural and Supplemental Gas Supply and Disposition." See the discussion on electric utility data and Table At in Appendix A for a comparison of reporting to these two forms. Totals may not add due to independent rounding.

Sources: Energy Information Administration (EIA), Form EIA-176. "Annual Report of Natural and Supplemental Gas Supply and Disposition"; Form EIA-627, "Annual Quantity and Value of Natural Gas Report"; Form EIA-857, "Monthly Report of Natural Gas Purchases and Deliveries to Consumers'; Form EIA-816, "Monthly Natural Gas Liquids Report"; Form EIA-759, "Monthly Power Plant Report"; Form FERC-423, "Monthly Report of Cost and Quality of Fuels for Electric Plants"; Form EIA-191, "Underground Gas Storage Report"; Form FPC-14, "Annual Report for Importers and Exporters of Natural Gas"; U.S. Crude Oil, Natural Gas, and Natural Gas Liquids Reserves, 1994 Annual Report, DOE/EIA-0216(94); and the U.S. Minerals Management Service. 


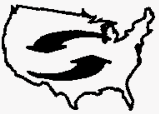

Net Interstate

Movements:

0

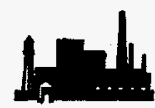

Industrial:

0

Marketed

Production:

0

0.00

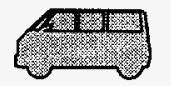

Vehicle Fuel:

0

0.00

Deliveries to Consumers:

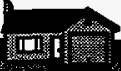

Residential:

578

0.01

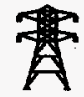

Electric

Utilities:

0

Commercial:

2,200

0.08

Total:

2,778

Table 59. Summary Statistics for Natural Gas - Hawaii, 1990-1994

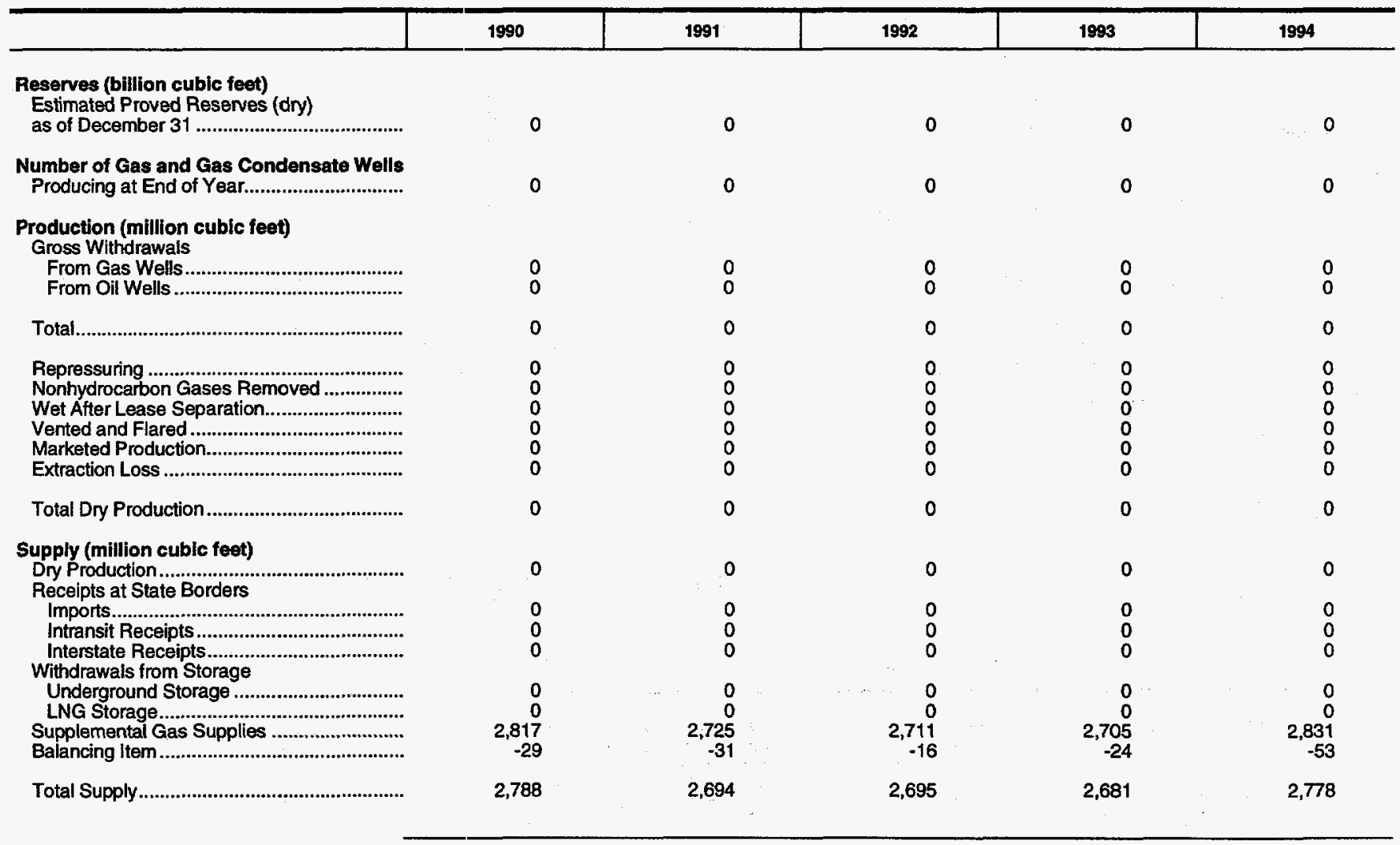


Table 59. Summary Statistics for Natural Gas - Hawaii, 1990-1994 (Continued)

\begin{tabular}{|c|c|c|c|c|c|}
\hline & 1990 & 1991 & i992 & 1993 & 1994 \\
\hline \multicolumn{6}{|l|}{ Disposition (million cubic feet) } \\
\hline \multicolumn{6}{|l|}{$\begin{array}{l}\text { Deliveries at State Borders } \\
\text { Den }\end{array}$} \\
\hline 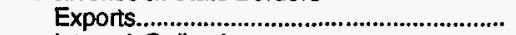 & 0 & 0 & 0 & 0 & 0 \\
\hline Intransit Deliveries .................................... & 0 & 0 & 0 & 0 & 0 \\
\hline 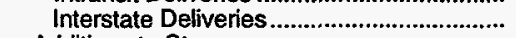 & 0 & 0 & 0 & 0 & 0 \\
\hline \multicolumn{6}{|l|}{ Additions to Storage } \\
\hline Underground Storage ........................................ & 0 & 0 & 0 & 0 & 0 \\
\hline 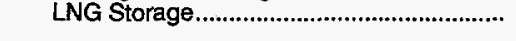 & & 0 & 0 & 0 & 0 \\
\hline 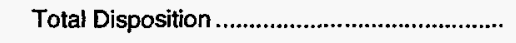 & 2,788 & 2,694 & 2,695 & 2,681 & 2,778 \\
\hline \multicolumn{6}{|l|}{ Consumption (million cubic feet) } \\
\hline 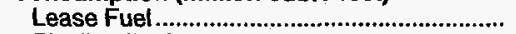 & 0 & 0 & 0 & 0 & 0 \\
\hline Pipeline Fuel............................................. & 0 & 0 & 0 & 0 & 0 \\
\hline 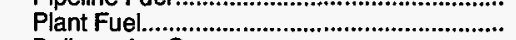 & 0 & 0 & 0 & 0 & 0 \\
\hline \multicolumn{6}{|l|}{ Delivered to Consumers } \\
\hline 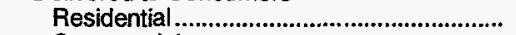 & 565 & 545 & 551 & 558 & 578 \\
\hline 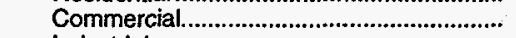 & 2,223 & 2,148 & 2,144 & 2,123 & 2,200 \\
\hline Industrial & 0 & 0 & 0 & 0 & 0 \\
\hline 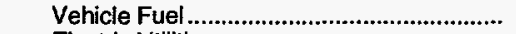 & 0 & 0 & 0 & 0 & 0 \\
\hline 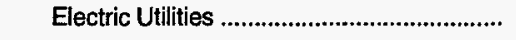 & 0 & 0 & 0 & 0 & 0 \\
\hline Total Delivered to Consumers ............................ & 2,788 & 2,694 & 2,695 & 2,681 & 2,778 \\
\hline 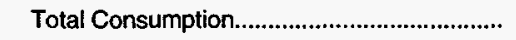 & 2,788 & 2,694 & 2,695 & 2,681 & 2,778 \\
\hline \multicolumn{6}{|l|}{$\begin{array}{l}\text { Delivered for the Account of Others } \\
\text { (million cubic feet) }\end{array}$} \\
\hline Residential .................................................... & 0 & 0 & 0 & 0 & 0 \\
\hline 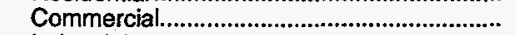 & 0 & 0 & 0 & 0 & 0 \\
\hline 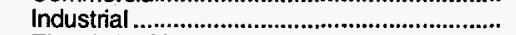 & 0 & 0 & 0 & 0 & 0 \\
\hline 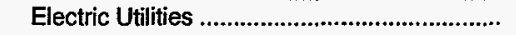 & 0 & 0 & 0 & 0 & 0 \\
\hline \multicolumn{6}{|l|}{$\begin{array}{l}\text { Firm Deliveries } \\
\text { (million cubic feet) }\end{array}$} \\
\hline 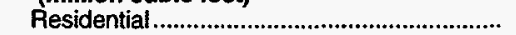 & NA & NA & NA & 558 & 578 \\
\hline 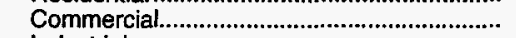 & NA & NA & NA & 1,779 & 1,837 \\
\hline 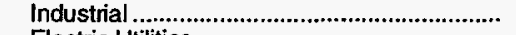 & NA & NA & NA & 0 & \\
\hline 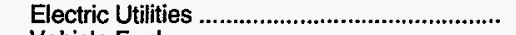 & NA & NA & NA & 0 & 0 \\
\hline 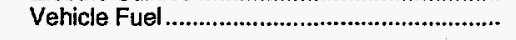 & NA & NA & NA & 0 & 0 \\
\hline \multicolumn{6}{|l|}{$\begin{array}{l}\text { Interruptible Deliveries } \\
\text { (million cubic feet) }\end{array}$} \\
\hline 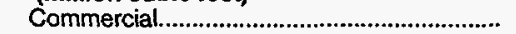 & NA & NA & NA & 344 & 363 \\
\hline Industrial & NA & NA & NA & - & - \\
\hline 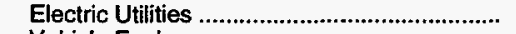 & NA & NA & NA & -- & - \\
\hline 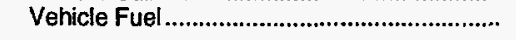 & NA & NA & NA & -- & -- \\
\hline \multicolumn{6}{|l|}{ Number of Consumers } \\
\hline 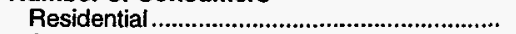 & 29,137 & 29,701 & 29,805 & 29,984 & 30,614 \\
\hline 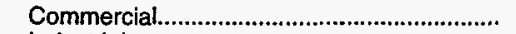 & 2,837 & 2,786 & 2,793 & 3,222 & 2,805 \\
\hline 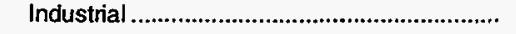 & -. & -- & -- & -- & - \\
\hline \multicolumn{6}{|l|}{$\begin{array}{l}\text { Average Annual Consumption per Consumer } \\
\text { (thousand cubic feet) }\end{array}$} \\
\hline 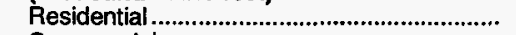 & & 18 & 18 & 19 & 19 \\
\hline 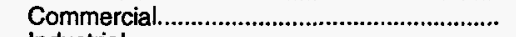 & 784 & 771 & 768 & 659 & 784 \\
\hline 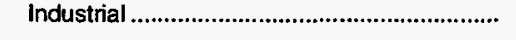 & 0 & 0 & 0 & 0 & 0 \\
\hline \multirow{4}{*}{\multicolumn{6}{|c|}{ 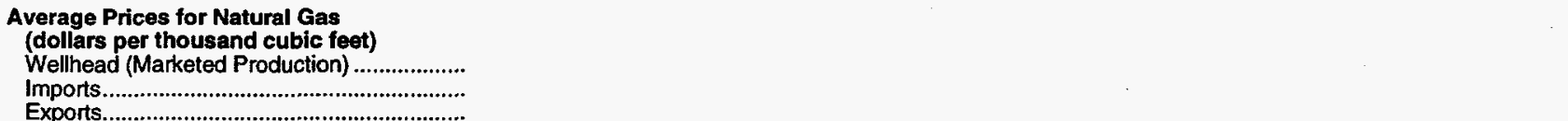 }} \\
\hline & & & & & \\
\hline & & & & & \\
\hline & & & & & \\
\hline 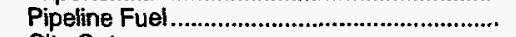 & 0 & 0 & 0 & 0 & 0 \\
\hline 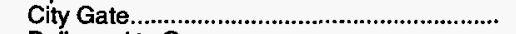 & 7.67 & 8.74 & 7.72 & 5.61 & 4.94 \\
\hline \multicolumn{6}{|l|}{ Delivered to Consumers } \\
\hline 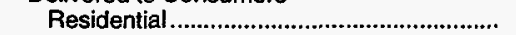 & 16.45 & 22.93 & 18.03 & 17.51 & 16.83 \\
\hline 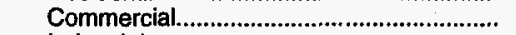 & 12.25 & 13.36 & 13.34 & 12.90 & 12.40 \\
\hline 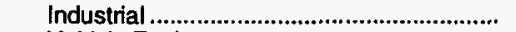 & 0 & 0 & 0 & 0 & 0 \\
\hline 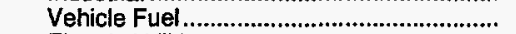 & 0 & 0 & 0 & 0 & 0 \\
\hline Electric Utilities .......................................... & 0 & 0 & 0 & 0 & 0 \\
\hline
\end{tabular}

NA = Not Available.

Notes: Deliveries to electric utilities (consumption) are reported on the Form ElA-176, "Annual Report of Natural and Supplemental Gas Supply and Disposition." See the discussion on electric utility data and Table A1 in Appendix A for a comparison of reporting to these two forms. Totals may not add due to independent rounding.

Sources: Energy Information Administration (EIA), Form EIA-176, "Annual Report of Natural and Supplemental Gas Supply and Disposition"; Form EIA-627, "Annual Quantity and Value of Natural Gas Report"; Fom ElA-857, "Monthly Report of Natural Gas Purchases and Deliveries to Consumers"; Form EIA-816, "Monthly' Natural Gas

Liquids Report"; Form ElA-759, "Monthly Power Plant Report"; Form FERC-423, "Monthly Report of Cost and Quality of Fuels for Electric Plants"; Form ElA-191, "Underground Gas Storage Report"; Form FPC-14, "Annual Report for Importers and Exporters of Natural Gas"; U.S. Crude Oil, Natural Gas, and Natural Gas Liquids Reserves, 1994 Annual Report, DOEEJEIA-0216(94); and the U.S. Minerals Management Service. 


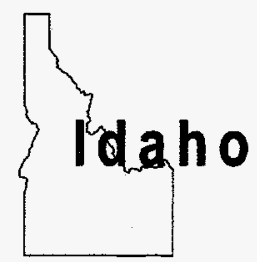

\section{- Natural Gas 1994}

$\begin{array}{llll}\text { Million } & \text { Percent of } & \text { Million } & \text { Percent of } \\ \text { Cu. Feet } & \text { National Total } & \text { Cu. Feet } & \text { National Total }\end{array}$

Net Interstate

Movements:

61,584

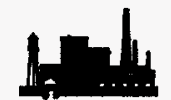

Industrial:

29,781

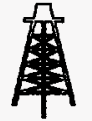

Marketed

Production:

0

0.00

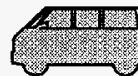

Vehicle Fuel:

10

0.58

Deliveries to Consumers:

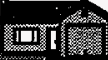

Residential:

12,285

0.25

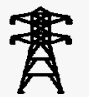

Electric

Utilities:

0

0.00

Commercial: $\quad \mathbf{1 0 , 0 8 8} \quad 0.35$

Total:

52,164

Table 60. Summary Statistics for Natural Gas — Idaho, 1990-1994

\begin{tabular}{|c|c|c|c|c|c|}
\hline & 1990 & 1991 & 1992 & 1993 & 1994 \\
\hline 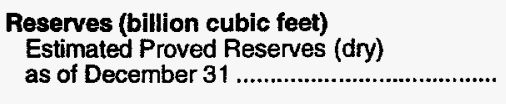 & 0 & 0 & 0 & 0 & 0 \\
\hline 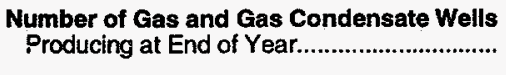 & 0 & 0 & 0 & 0 & 0 \\
\hline 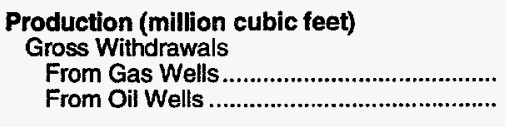 & $\begin{array}{l}0 \\
0\end{array}$ & $\begin{array}{l}0 \\
0\end{array}$ & $\begin{array}{l}0 \\
0\end{array}$ & $\begin{array}{l}0 \\
0\end{array}$ & $\begin{array}{l}0 \\
0\end{array}$ \\
\hline 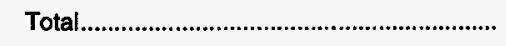 & 0 & 0 & 0 & 0 & 0 \\
\hline 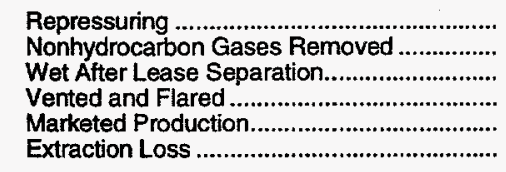 & $\begin{array}{l}0 \\
0 \\
0 \\
0 \\
0 \\
0\end{array}$ & $\begin{array}{l}0 \\
0 \\
0 \\
0 \\
0 \\
0\end{array}$ & $\begin{array}{l}0 \\
0 \\
0 \\
0 \\
0 \\
0\end{array}$ & $\begin{array}{l}0 \\
0 \\
0 \\
0 \\
0 \\
0\end{array}$ & $\begin{array}{l}0 \\
0 \\
0 \\
0 \\
0 \\
0\end{array}$ \\
\hline 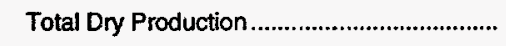 & 0 & 0 & 0 & 0 & 0 \\
\hline $\begin{array}{l}\text { Supply (million cubic teet) } \\
\text { Dry Production ............................................ } \\
\text { Receipts at State Borders }\end{array}$ & 0 & 0 & 0 & 0 & 0 \\
\hline 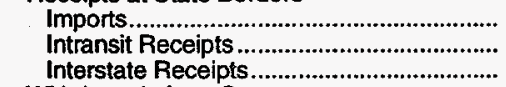 & $\begin{array}{r}514,597 \\
0 \\
80,926\end{array}$ & $\begin{array}{r}496,967 \\
0 \\
87,575\end{array}$ & $\begin{array}{r}496,451 \\
0 \\
103,589\end{array}$ & $\begin{array}{r}511,727 \\
0 \\
91,967\end{array}$ & $\begin{array}{r}723,071 \\
0 \\
98,230\end{array}$ \\
\hline 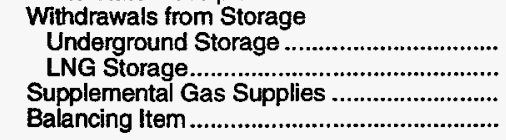 & $\begin{array}{r}0 \\
268 \\
0 \\
-3,649\end{array}$ & $\begin{array}{r}0 \\
227 \\
0 \\
-1,097\end{array}$ & $\begin{array}{r}0 \\
1,385 \\
0 \\
-7,330\end{array}$ & $\begin{array}{r}0 \\
1,339 \\
0 \\
7,351\end{array}$ & $\begin{array}{r}0 \\
373 \\
0 \\
-4,498\end{array}$ \\
\hline 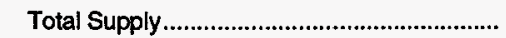 & 592,142 & 583,673 & 594,096 & 612,383 & 817,176 \\
\hline
\end{tabular}

See footnotes at end of table. 
Table 60. Summary Statistics for Natural Gas - Idaho, 1990-1994 (Continued)

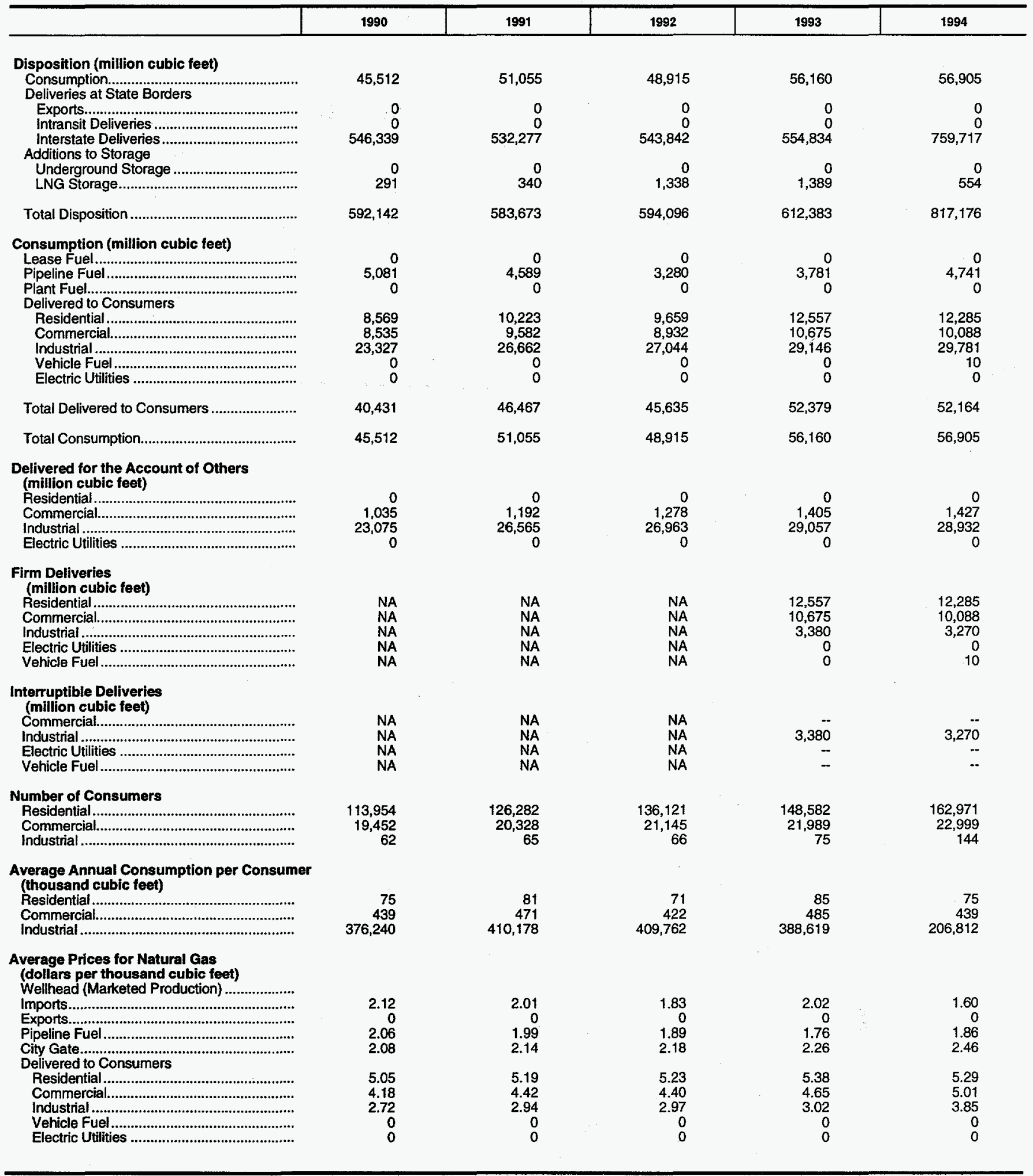

NA = Not Available.

Notes: Deliveries to electric utilities (consumption) are reported on the Form ElA-176, "Annual Report of Natural and Supplemental Gas Supply and Disposition." See the discussion on electric utility data and Table A1 in Appendix A for a comparison of reporting to these two forms. Totals may not add due to independent rounding.

Sources: Energy Information Administration (EIA), Form EIA-176, "Annual Report of Natural and Supplemental Gas Supply and Disposition"; Form EIA-627, "Annual Quantity and Value of Natural Gas Report"; Form EIA-857, "Monthly Report of Natural Gas Purchases and Deliveries to Consumers"; Form EIA-816, "Monthly Natural Gas

Liquids Report"; Form EIA-759, "Monthly Power Plant Report"; Form FERC-423, "Monthly Report of Cost and Quality of Fuels for Electric Plants"; Form ElA-191, "Underground Gas Storage Report"; Form FPC-14, "Annual Report for Importers and Exporters of Natural Gas"; U.S. Crude Oil, Natural Gas, and Natural Gas Liquids Reserves, 1994 Annual Report, DOEJEIA-0216(94); and the U.S. Minerals Management Service. 


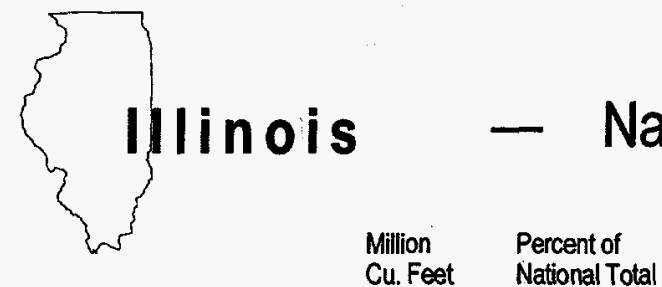

Million

Percent of

Cu. Feet National Total

Cu. Feet

National Total

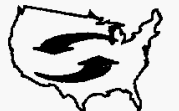

Net Interstate

Movements:

$1,161,327$

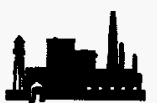

Industrial:

305,092

3.73

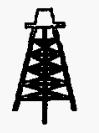

Marketed

Production:

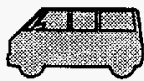

Vehicle Fuel:

Deliveries to Consumers:

Residential:

473,788

9.77

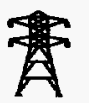

Electric

Utilities:

Commercial:

197,576

6.82

Total:

$1,010,989$

5.35

Table 61. Summary Statistics for Natural Gas - Illinois, 1990-1994

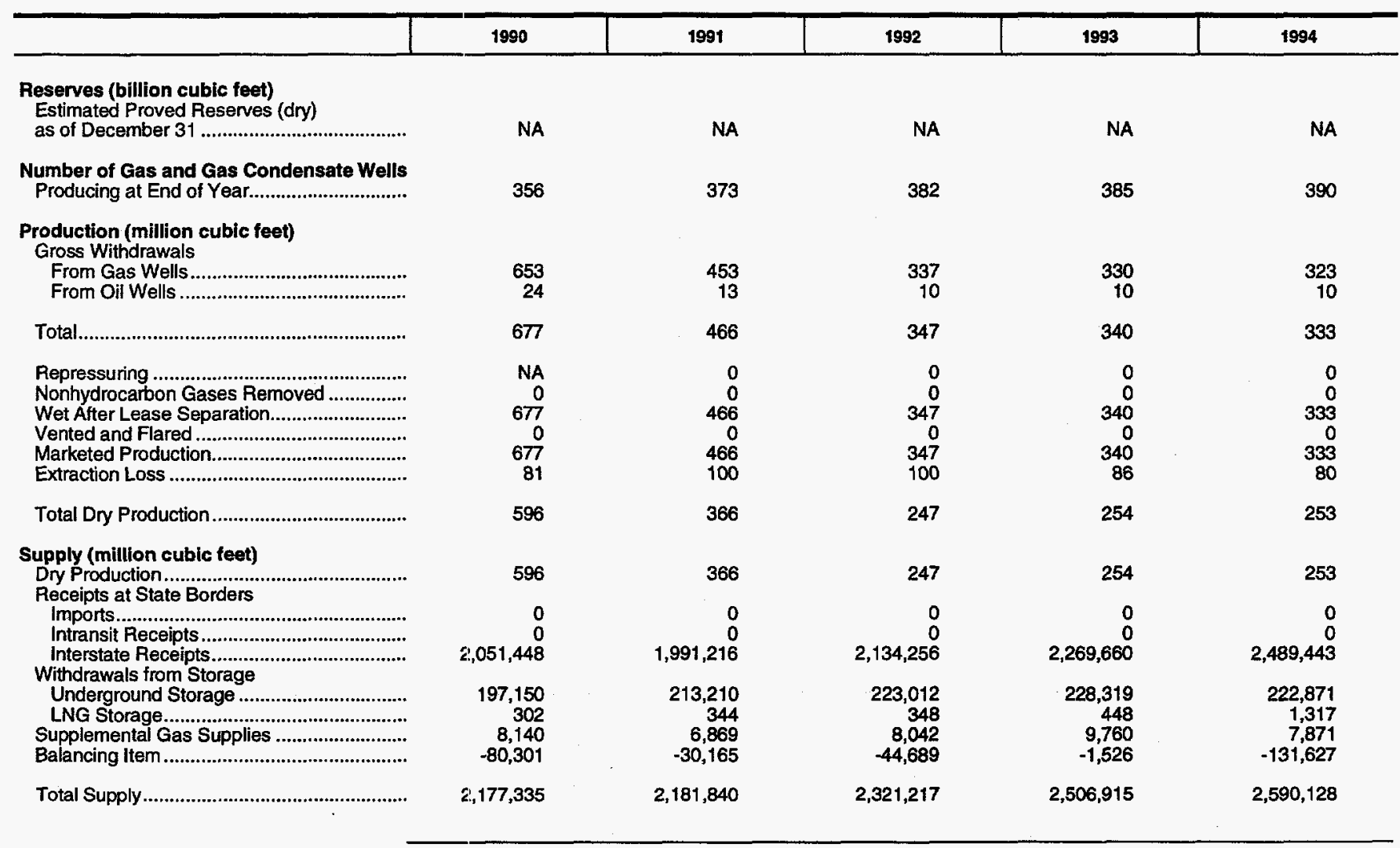

See footnotes at end of table. 
Table 61. Summary Statistics for Natural Gas - Illinois, 1990-1994 (Continued)

\begin{tabular}{|c|c|c|c|c|c|}
\hline & 1990 & 1991 & 1992 & 1993 & 1994 \\
\hline \multicolumn{6}{|l|}{ Disposition (million cubic feet) } \\
\hline Deliveries at State Borders & 939,502 & 987,589 & 993,428 & $1,031,253$ & $1,024,908$ \\
\hline 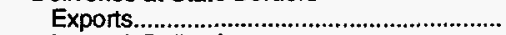 & 0 & 0 & 0 & 0 & 0 \\
\hline 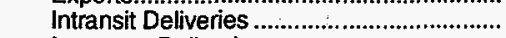 & 0 & 0 & 0 & 0 & 0 \\
\hline Interstate Deliveries .................................... & $1,023,874$ & 981,904 & $1,112,815$ & $1,234,731$ & $1,328,116$ \\
\hline \multicolumn{6}{|l|}{ Additions to Storage } \\
\hline 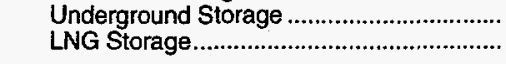 & $\begin{array}{r}213,076 \\
883\end{array}$ & $\begin{array}{r}212,232 \\
115\end{array}$ & $\begin{array}{r}214,404 \\
570\end{array}$ & $\begin{array}{r}240,515 \\
417\end{array}$ & $\begin{array}{r}235,778 \\
1,326\end{array}$ \\
\hline 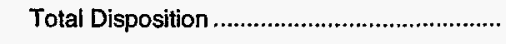 & $2,177,335$ & $2,181,840$ & $2,321,217$ & $2,506,915$ & $2,590,128$ \\
\hline \multicolumn{6}{|l|}{ Consumption (million cubic feet) } \\
\hline 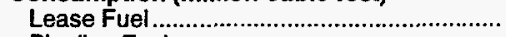 & 22 & 10 & 9 & 10 & 10 \\
\hline Pipeline Fuel ................................................ & 12,111 & 11,070 & 11,330 & 11,620 & 13,808 \\
\hline 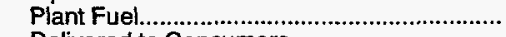 & 109 & 132 & 98 & 106 & 101 \\
\hline \multicolumn{6}{|l|}{ Delivered to Consumers } \\
\hline 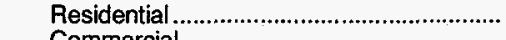 & 442,163 & 466,970 & 475,360 & 495,311 & 473,788 \\
\hline 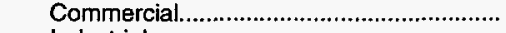 & 200,267 & 193,844 & 196,964 & 203,157 & 197,576 \\
\hline 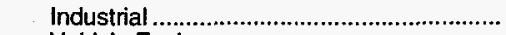 & 275,630 & 302,691 & 300,366 & 305,014 & 305,092 \\
\hline 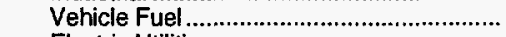 & & & & 12 & 28 \\
\hline Electric Utilities ......................................... & $9 ; 195$ & 12,865 & 9,293 & 16,022 & 34,505 \\
\hline Total Delivered to Consumers ........................ & 927,261 & 976,377 & 981,991 & $1,019,517$ & $1,010,989$ \\
\hline Total Consumption & 939,502 & 987,589 & 993,428 & $1,031,253$ & $1,024,908$ \\
\hline \multicolumn{6}{|l|}{$\begin{array}{l}\text { Delivered for the Account of Others } \\
\text { (million cubic feet) }\end{array}$} \\
\hline 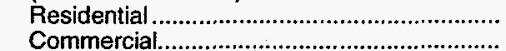 & 22,412 & $\begin{array}{l}27,326 \\
79,512\end{array}$ & $\begin{array}{l}29,783 \\
83,264\end{array}$ & & 31,119 \\
\hline 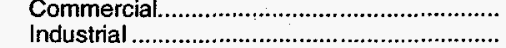 & $\begin{array}{r}84,936 \\
221,564\end{array}$ & $\begin{array}{r}79,512 \\
256,186\end{array}$ & $\begin{array}{r}83,264 \\
255,365\end{array}$ & $\begin{array}{r}90,812 \\
265,110\end{array}$ & $\begin{array}{r}93,206 \\
267,348\end{array}$ \\
\hline 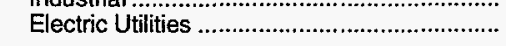 & 6,682 & 9,150 & $\begin{array}{r}200,300 \\
9,726\end{array}$ & 14,227 & 32,064 \\
\hline \multicolumn{6}{|l|}{$\begin{array}{l}\text { Firm Deliveries } \\
\text { (milion cubic feet) }\end{array}$} \\
\hline 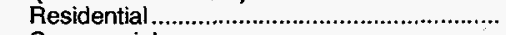 & NA & NA & NA & 495,311 & 473,788 \\
\hline 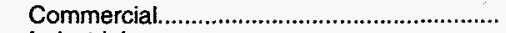 & NA & NA & NA & 200,101 & 197,467 \\
\hline Industrial & NA & NA & NA & 24,640 & 21,916 \\
\hline 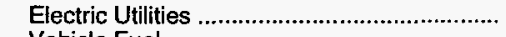 & NA & NA & NA & 15,077 & 33,596 \\
\hline Vehicle Fuel.................................................... & NA & NA & NA & 12 & 28 \\
\hline \multicolumn{6}{|l|}{$\begin{array}{l}\text { Interruptible Deliveries } \\
\text { (million cubic feet) }\end{array}$} \\
\hline 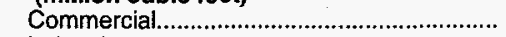 & NA & NA & NA & 3,056 & 108 \\
\hline 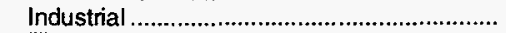 & NA & NA & NA & 24,640 & 21,916 \\
\hline 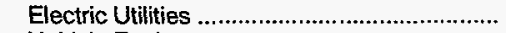 & NA & NA & NA & 489 & 2 \\
\hline 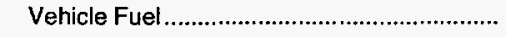 & NA & NA & NA & -- & -- \\
\hline \multicolumn{6}{|l|}{ Number of Consumers } \\
\hline 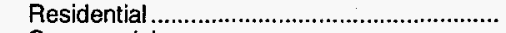 & $3,287,091$ & $3,320,285$ & $3,354,679$ & $3,388,983$ & $3,418,052$ \\
\hline 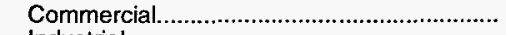 & 257,851 & 261,107 & 263,988 & $\mathrm{R}_{268,104}$ & 262,308 \\
\hline 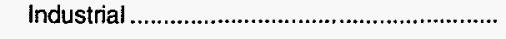 & 25,991 & 26,489 & 27,178 & 27,807 & 25,788 \\
\hline \multicolumn{6}{|l|}{$\begin{array}{l}\text { Average Annual Consumption per Consumer } \\
\text { (thousand cubic feet) }\end{array}$} \\
\hline 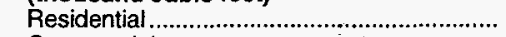 & 135 & 141 & 142 & 146 & 139 \\
\hline 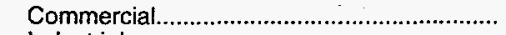 & 777 & 742 & 746 & $R_{758}$ & 753 \\
\hline 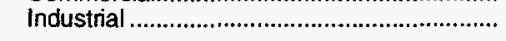 & 10,605 & 11,427 & 11,052 & 10,969 & 11,831 \\
\hline \multicolumn{6}{|l|}{$\begin{array}{l}\text { Average Prices for Natural Gas } \\
\text { (dollars per thousand cubic feet) }\end{array}$} \\
\hline Wellhead (Marketed Production) .................... & 2.11 & 2.17 & 2.15 & $\mathbf{R}_{2.30}$ & 2.40 \\
\hline 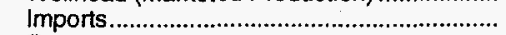 & 0 & 0 & 0 & 0 & 0 \\
\hline 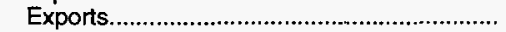 & 0 & 0 & 0 & 0 & 0 \\
\hline 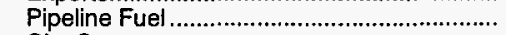 & 2.06 & 2.29 & 2.44 & 1.97 & 1.88 \\
\hline 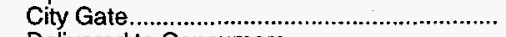 & 3.09 & 2.91 & 3.20 & 3.30 & 3.02 \\
\hline Delivered to Consumers & & & & & \\
\hline 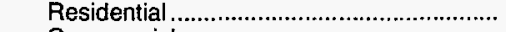 & 5.06 & 4.95 & 5.09 & 5.52 & 5.50 \\
\hline 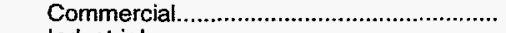 & 4.64 & 4.56 & 4.65 & 5.10 & 5.12 \\
\hline 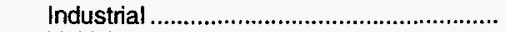 & 4.10 & 3.77 & 3.75 & 4.44 & 4.39 \\
\hline Vehicle Fuel ................................................... & 4.50 & 3.41 & 3.80 & 4.04 & 3.26 \\
\hline Electric Utilities ....................................... & 2.73 & 2.14 & 2.24 & 2.48 & 2.04 \\
\hline
\end{tabular}

$R=$ Revised data.

NA $=$ Not Available.
Notes: Deliveries to electric utilities (consumption) are reported on the Form ElA-176, "Annual Repart of Natural and Supplemental Gas Supply and Disposition." See the Notes: Deliveries to electric utilities (consumption) are reported on the Form ElA-176, "Annual Report of Natural and Supplemental Gas Supply and Disposition.
discussion on electric utility data and Table A1 in Appendix A for a comparison of reporting to these two forms. Totals may not add due to independent rounding.

discussion on electric utility data and Table A1 in Appendix A for a comparison of reporting to these two forms. Totals may not add due to independent rounding.
Sources: Energy Information Administration (EIA), Form EIA-176, "Annual Report of Natural and Supplemental Gas Supply and Disposition": Form EIA-627, "Annual

Sources: Energy Information Administration (EIA), Form ElA-176, "Annual Report of Natural and Supplemental Gas Supply and Disposition"; Form ElA-627, "Annual
Quantity and Value of Natural Gas Feport"; Form ElA-857, "Monthly Report of Natural Gas Purchases and Deliveries to Consumers"; Form EIA-816, "Monthly Natural Gas

Quantity and Value of Natural Gas Feport"; Form EIA-857, "Monthly Report of Natural Gas Purchases and Deliveries to Consumers"; Form EIA-816, "Monthly Natural Gas
Liquids Report"; Form EIA-759, "Monthly Power Plant Report"; Form FERC-423, "Monthly Report of Cost and Quality of Fuels for Electric Plants"; Form EIA-191, "Underground Liquids Report"; Form ElA-759, "Monthly Power Plant Report"; Form FERC-423, "Monthly Report of Cost and Quality of Fuels for Electric Plants"; Form ElA-191, "Underground
Gas Storage Report"; Form FPC-14, "Annual Report for Importers and Exporters of Natural Gas"; U.S. Crude Oil, Natural Gas, and Natural Gas Liquids Reserves, 1994 Annual Gas Storage Report"; Form FPC-14, "Annual Report for Importers and Expo
Report, DOE/EIA-0216(94); and the U.S. Minerals Management Service. 


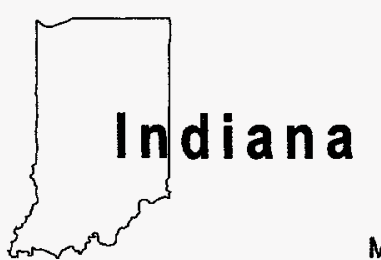

Million Percent of

Million

Percent of

Cu. Feet National Total

Cu. Feet National Total

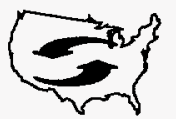

Net Interstate

Movements:

600,860

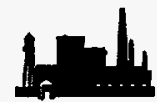

Industrial:

270,128

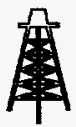

Marketed

Production:

107

0.00

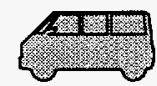

Vehicle Fuel:

59

Deliveries to Consumers:

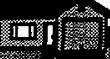

Residential:

157,467

3.25

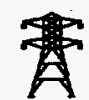

Electric

Utilities:

9,009

0.30

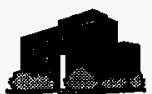

Commercial:

75,819

2.62

Total:

512,482

2.71

Table 62. Summary Statistics for Natural Gas — Indiana, 1990-1994

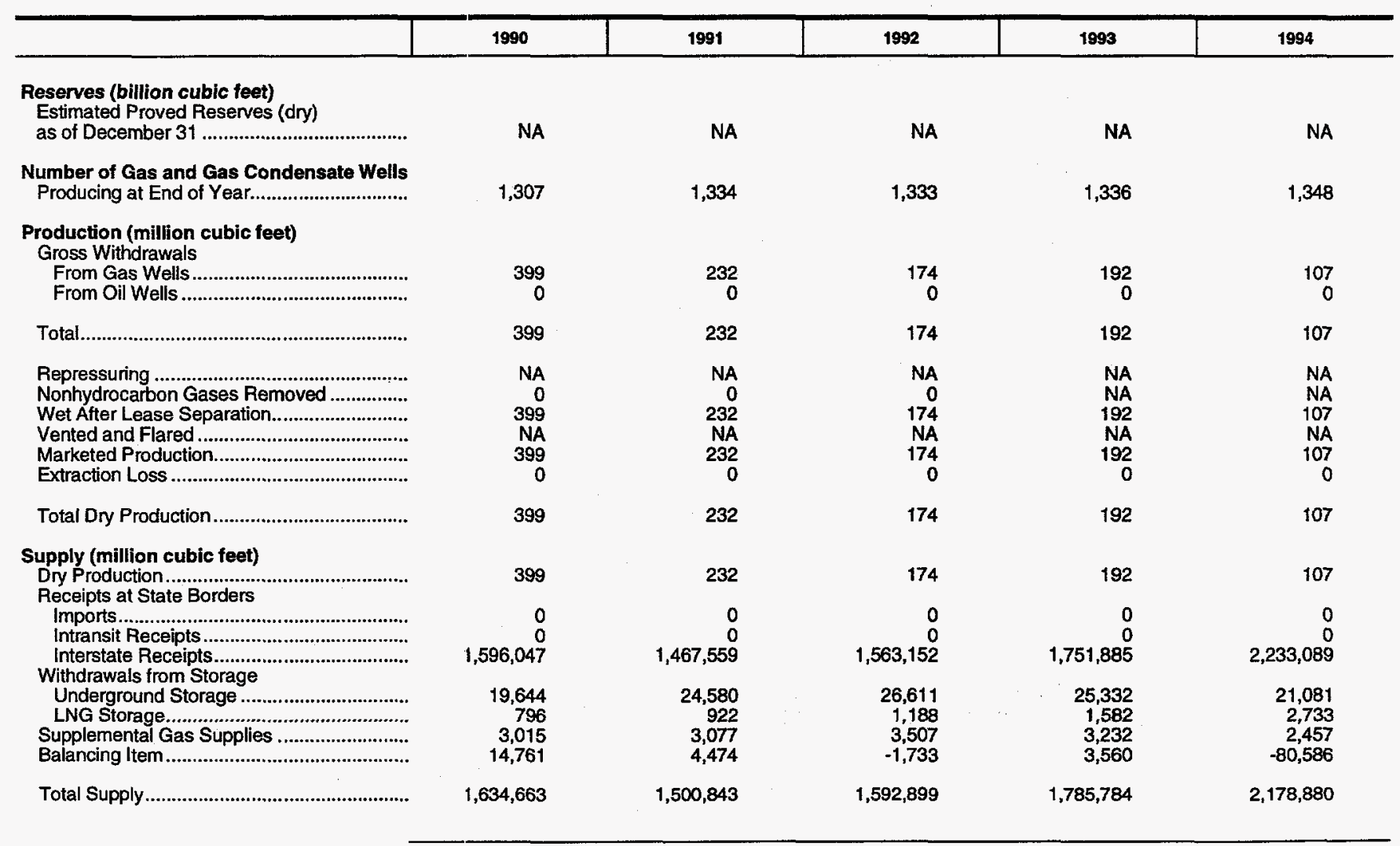

See footnotes at end of table. 
Table 62. Summary Statistics for Natural Gas - Indiana, 1990-1994 (Continued)

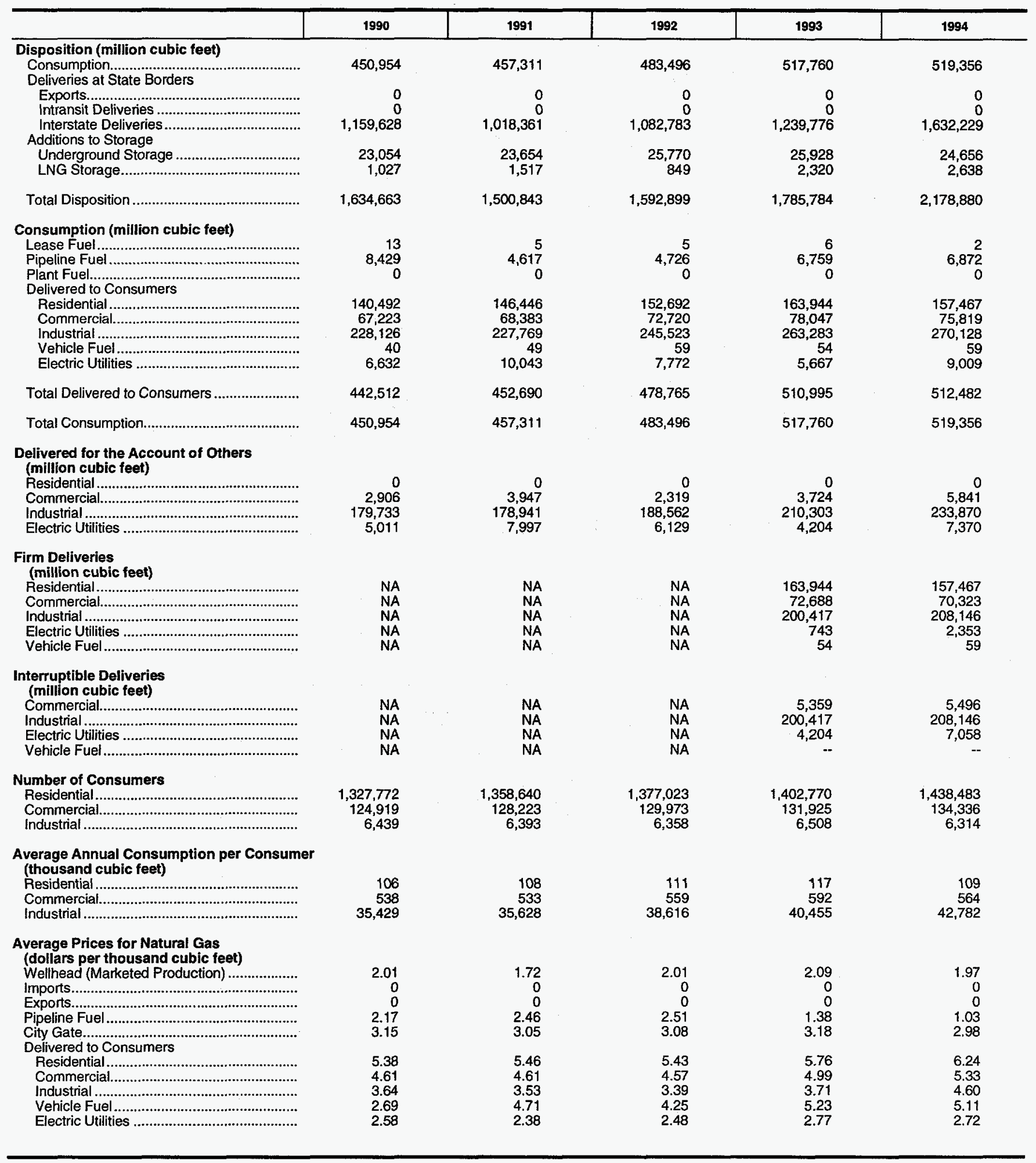

NA = Not Available.

Notes: Deliveries to electric utilities (consumption) are reported on the Form EIA-176, "Annual Report of Natural and Supplemental Gas Supply and Disposition." See the discussion on electric utility data and Table A1 in Appendix A for a comparison of reporting to these two forms. Totals may not add due to independent rounding.

Sources: Energy Information Administration (EIA), Form EIA-176, "Annual Report of Natural and Supplemental Gas Supply and Disposition"; Form EIA-627, "Annual

Quantity and Value of Natural Gas Report"; Form ElA-857, "Monthly Report of Natural Gas Purchases and Deliveries to Consumers"; Form ElA-816, "Monthly Natural Gas

Liquids Report"; Form EIA-759, "Monthly Power Plant Report"; Form FERC-423. "Monthly Report of Cost and Quality of Fuels for Electric Plants"; Form EIA-191, "Underground

Gas Storage Report"; Form FPC-14, "Annual Report for Importers and Exporters of Natural Gas"; U.S. Crude Oil, Natural Gas, and Natural Gas Liquids Reserves, 1994 Annual Report, DOE/EIA-0216(94); and the U.S. Minerals Management Service. 


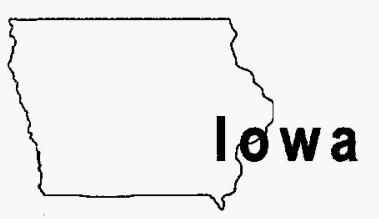

Million Percent of

Cu. Feet National Total

Million

Cu. Feet

Percent of National Total

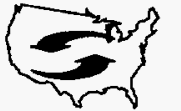

Net Interstate

Movements:

287,281

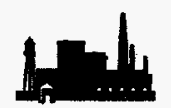

Industrial:

108,731

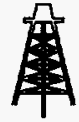

Marketed

Production:

0

0.00

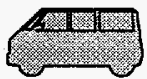

Vehicle Fuel:

5

0.29

Deliveries to Consumers:

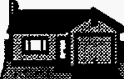

Residential:

78,260

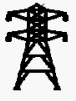

Electric

Utilities:

2,696

Commercial:

47,922

Total:

237,614

1.26

Table 63. Summary Statistics for Natural Gas — lowa, 1990-1994

\begin{tabular}{|c|c|c|c|c|c|}
\hline & 1990 & 1991 & 1992 & 1993 & 1994 \\
\hline $\begin{array}{l}\text { Reserves (billion cubic feet) } \\
\text { Estimated Proved Reserves (dry) } \\
\text { as of December } 31\end{array}$ & 0 & 0 & 0 & 0 & 0 \\
\hline 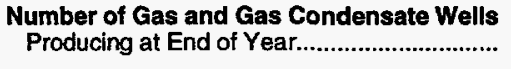 & 0 & 0 & 0 & 0 & 0 \\
\hline 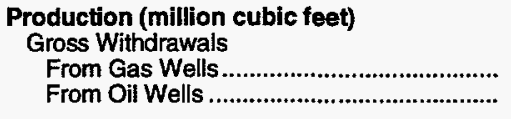 & $\begin{array}{l}0 \\
0\end{array}$ & $\begin{array}{l}0 \\
0\end{array}$ & $\begin{array}{l}0 \\
0\end{array}$ & $\begin{array}{l}0 \\
0\end{array}$ & $\begin{array}{l}0 \\
0\end{array}$ \\
\hline 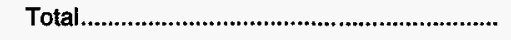 & 0 & 0 & 0 & 0 & 0 \\
\hline 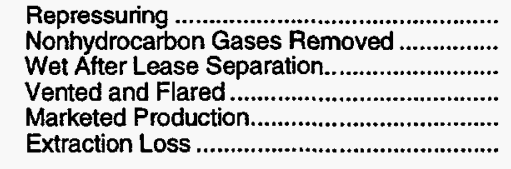 & $\begin{array}{l}0 \\
0 \\
0 \\
0 \\
0 \\
0\end{array}$ & $\begin{array}{l}0 \\
0 \\
0 \\
0 \\
0 \\
0\end{array}$ & $\begin{array}{l}0 \\
0 \\
0 \\
0 \\
0 \\
0\end{array}$ & $\begin{array}{l}0 \\
0 \\
0 \\
0 \\
0 \\
0\end{array}$ & $\begin{array}{l}0 \\
0 \\
0 \\
0 \\
0 \\
0\end{array}$ \\
\hline Total Dry Production & 0 & 0 & 0 & 0 & 0 \\
\hline 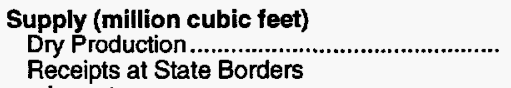 & 0 & 0 & 0 & 0 & 0 \\
\hline 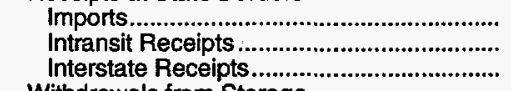 & $\begin{array}{r}0 \\
0 \\
1,135,682\end{array}$ & $\begin{array}{r}0 \\
0 \\
1,075,376\end{array}$ & $\begin{array}{r}0 \\
0 \\
1,270,083\end{array}$ & $\begin{array}{r}0 \\
0 \\
1,232,578\end{array}$ & $\begin{array}{r}0 \\
0 \\
1,443,469\end{array}$ \\
\hline 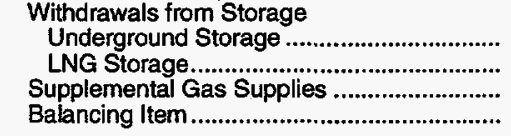 & $\begin{array}{r}47,770 \\
655 \\
81 \\
-25,267\end{array}$ & $\begin{array}{r}62,486 \\
669 \\
46 \\
20,993\end{array}$ & $\begin{array}{r}70,077 \\
4,247 \\
45 \\
-15,634\end{array}$ & $\begin{array}{r}61,007 \\
5,597 \\
84 \\
R_{-45,975}\end{array}$ & $\begin{array}{r}68,162 \\
3,521 \\
123 \\
-35,943\end{array}$ \\
\hline 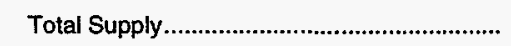 & $1,158,921$ & $1,159,570$ & $1,328,817$ & $R_{1,253,291}$ & $1,479,332$ \\
\hline
\end{tabular}

See footnotes at end of table. 
Table 63. Summary Statistics for Natural Gas - lowa, 1990-1994 (Continued)

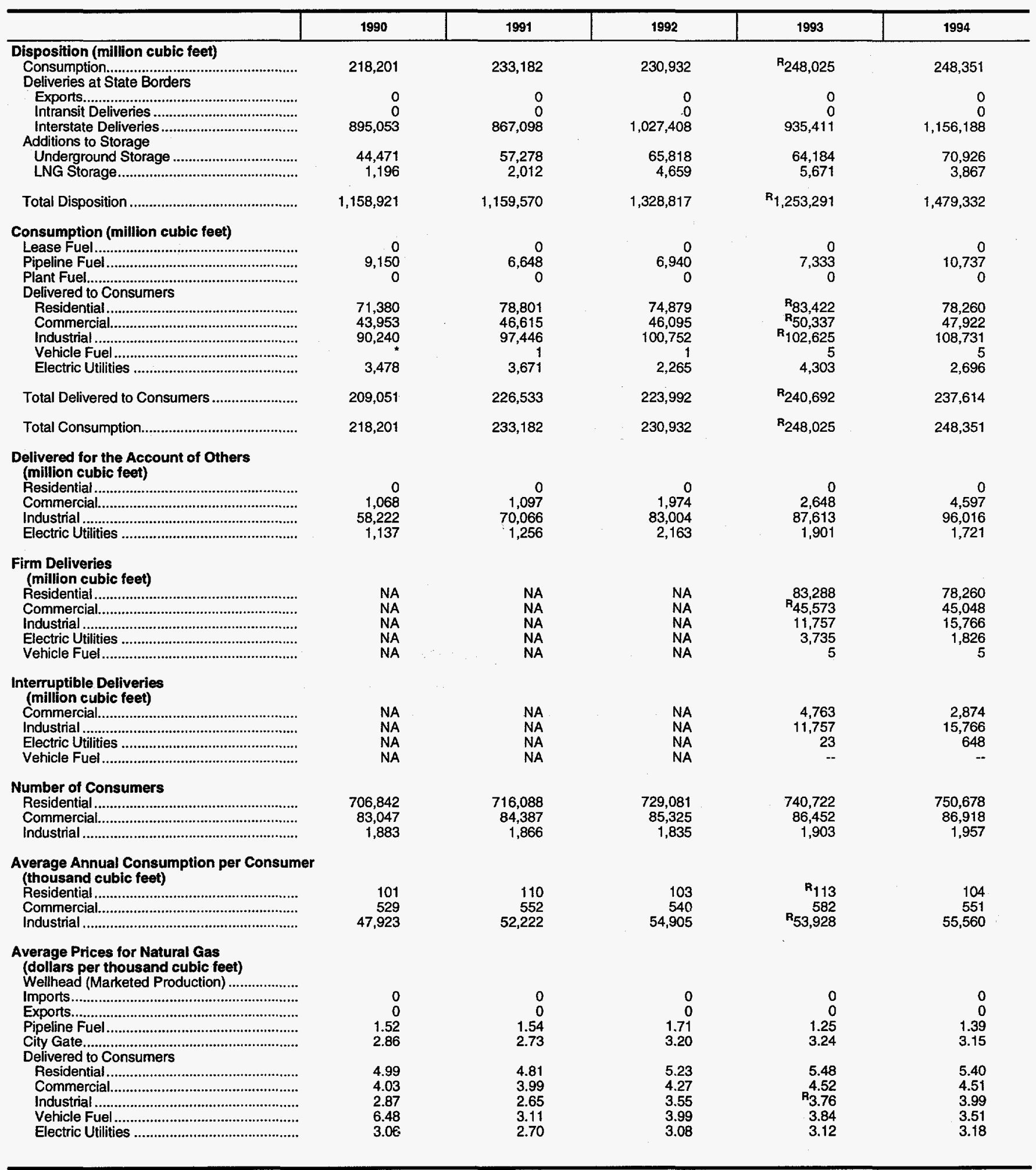

$R=$ Revised data.

NA = Not Available.

Notes: Deliveries to electric utilities (consumption) are reported on the Form ElA-176, "Annual Report of Natural and Supplemental Gas Supply and Disposition." See the discussion on electric utitity data and Table A1 in Appendix A for a comparison of reporting to these two forms. Totals may not add due to independent rounding.

discussion on electric utility data and Table A1 in Appendix A for a comparison of reporting to these two forms. Totals may not add due to independent rounding.

Sources: Energy Information Administration (EIA), Form EIA-176, "Annual Report of Natural and Supplemental Gas Supply and Disposition"; Form EIA-627, "Annual
Quantity and Value of Natural Gas Report"; Form ElA-857, "Monthly Report of Natural Gas Purchases and Deliveries to Consumers"; Form EIA-816, "Monthly Natural Gas

Quantity and Value of Natural Gas Report"; Form ElA-857, "Monthly Report of Natural Gas Purchases and Deliveries to Consumers"; Form EIA-816, "Monthly Natural Gas

Liquids Report"; Form EIA-759, "Monthly Power Plant Report"; Form FERC-423, "Monthly Report of Cost and Quality of Fuels for Electric Plants"; Form EIA-191, "Underground Report, DOE/EIA-0216(94); and the U.S. Minerals Management Service. 


\title{
Kansas - Natural Gas 1994
}

\author{
Million Percent of \\ Cu. Feet National Total \\ Million Percent of \\ Cu. Feet National Tota
}

Net Interstate

Movements:

$-262,252$

Marketed

Production: $\quad \mathbf{7 1 2 , 7 3 0}$

3.63

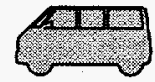

Vehicle Fuel:

10

0.58

Deliveries to Consumers:

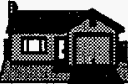

Residential:

74,156

1.53

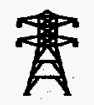

Electric

Utilities:

27,279

0.91

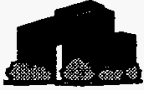

Commercial:

52,253

1.80

Total:

341,677

2.30

187,979

Table 64. Summary Statistics for Natural Gas - Kansas, 1990-1994

\begin{tabular}{|c|c|c|c|c|c|}
\hline & 1990 & 1991 & 1992 & 1993 & 1994 \\
\hline $\begin{array}{l}\text { Reserves (billion cubic feet) } \\
\text { Estimated Proved Reserves (dry) } \\
\text { as of December } 31 \text {.......................................... }\end{array}$ & 9,614 & 9,358 & 9,681 & 9,348 & 9,156 \\
\hline 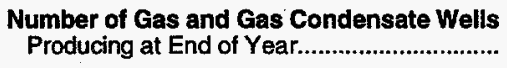 & 16,980 & 17,948 & 18,400 & 19,472 & 19,365 \\
\hline 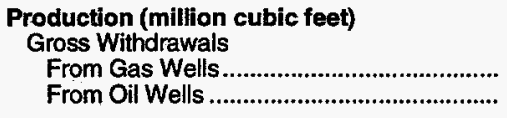 & $\begin{array}{l}467,467 \\
107,622\end{array}$ & $\begin{array}{r}550,241 \\
79,943\end{array}$ & $\begin{array}{r}580,572 \\
79,169\end{array}$ & $\begin{array}{r}605,578 \\
82,579\end{array}$ & $\begin{array}{r}628,900 \\
85,759\end{array}$ \\
\hline 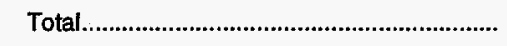 & 575,090 & 630,185 & 659,741 & 688,157 & 714,659 \\
\hline 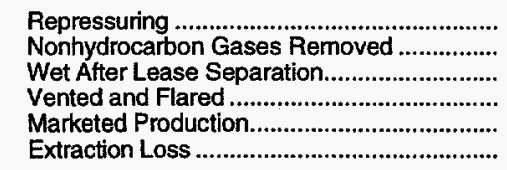 & $\begin{array}{r}930 \\
\mathrm{NA} \\
574,160 \\
557 \\
573,603 \\
29,642\end{array}$ & $\begin{array}{r}1,098 \\
N A \\
629,087 \\
628 \\
628,459 \\
41,848\end{array}$ & $\begin{array}{r}1,092 \\
\mathrm{NA} \\
658,649 \\
642 \\
658,007 \\
42,733\end{array}$ & $\begin{array}{r}1,140 \\
\text { NA } \\
687,017 \\
670 \\
686,347 \\
44,014\end{array}$ & $\begin{array}{r}1,215 \\
\mathrm{NA} \\
713,444 \\
715 \\
712,730 \\
46,936\end{array}$ \\
\hline 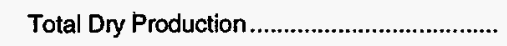 & 543,961 & 586,611 & 615,274 & 642,333 & 665,794 \\
\hline 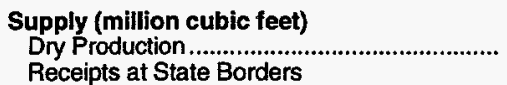 & 543,961 & 586,611 & 615,274 & 642,333 & 665,794 \\
\hline 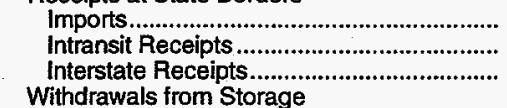 & $\begin{array}{r}0 \\
0 \\
1,036,161\end{array}$ & $\begin{array}{r}0 \\
0 \\
906,394\end{array}$ & $\begin{array}{r}0 \\
0 \\
1,047,474\end{array}$ & $\begin{array}{r}0 \\
0 \\
974,939\end{array}$ & $\begin{array}{r}0 \\
0 \\
1,127,799\end{array}$ \\
\hline 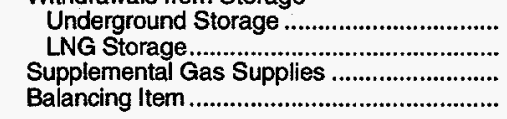 & $\begin{array}{r}95,377 \\
0 \\
0 \\
115,792\end{array}$ & $\begin{array}{r}98,805 \\
0 \\
0 \\
71,146\end{array}$ & $\begin{array}{r}102,735 \\
0 \\
0 \\
-32,874\end{array}$ & $\begin{array}{r}109,475 \\
0 \\
0 \\
27,185\end{array}$ & $\begin{array}{r}99,854 \\
0 \\
0 \\
20,700\end{array}$ \\
\hline 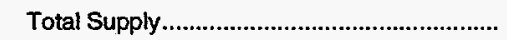 & $1,791,291$ & $1,662,957$ & $1,732,609$ & $1,753,932$ & $1,914,147$ \\
\hline
\end{tabular}

See footnotes at end of table. 
Table 64. Summary Statistics for Natural Gas - Kansas, 1990-1994 (Continued)

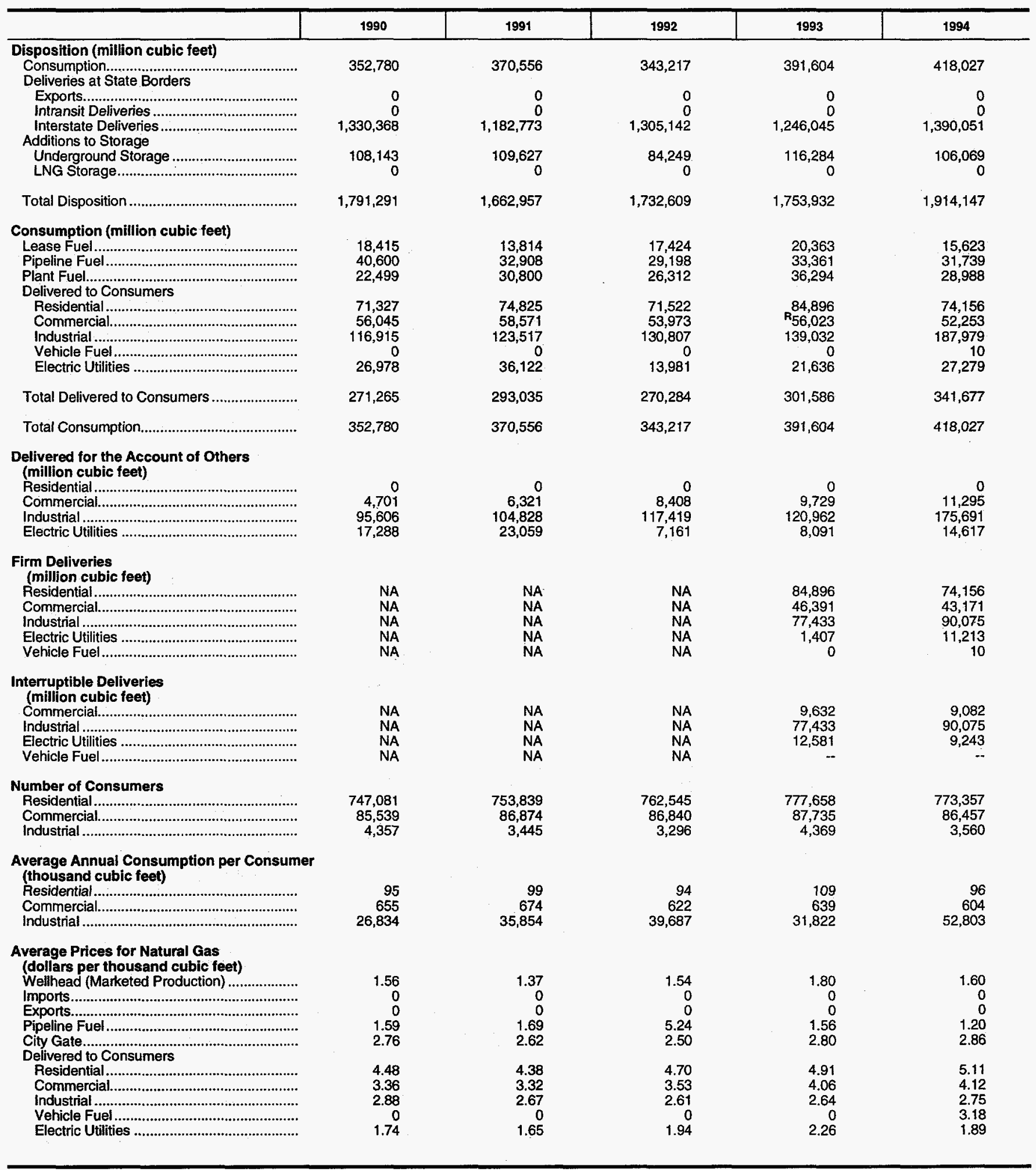

R = Revised data.

NA $=$ Not Available.
Notes: Deliveries to electric utilities (consumption) are reported on the Form ElA-176, "Annual Report of Natural and Supplemental Gas Supply and Disposition." See the

discussion on electric utility data and Table A1 in Appendix A for a comparison of reporting to these two forms. Totals may not add due to independent rounding.

Sources: Energy Information Administration (EIA), Form ElA-176, "Annual Report of Natural and Supplemental Gas Supply and Disposition"; Form ElA-627, "Annual
Quantity and Value of Natural Gas Report"; Form EIA-857, "Monthly Report of Natural Gas Purchases and Deliveries to Consumers"; Form EIA-816, "Monthly Natural Gas

Luantity and Value of Natural Gas Report'; Form ElA-857, "Monthly Report of Natural Gas Purchases and Deliveries to Consumers"; Form EIA-816, "Monthly Natural Gas Liquids Report; Form ElA-759, "Monthly Power Plant Report"; Form FERC-423, "Monthly Report of Cost and Quality of Fuels for Electric Plants"; Form ElA-191, "Underground Gas Storage Report"; Form FPC-14, "Annual Report for Importers and Expo 


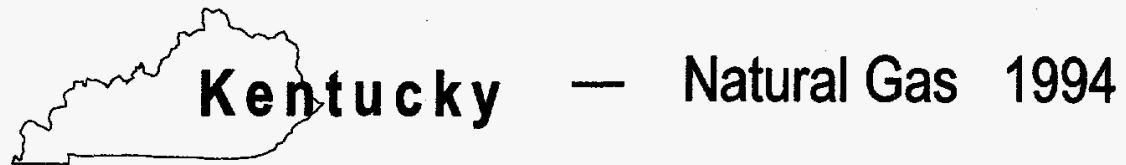

$\begin{array}{llll}\text { Million } & \text { Percent of } & \text { Million } & \text { Percent of } \\ \text { Cu. Feet } & \text { National Total } & \text { Cu. Feet } & \text { National Total }\end{array}$

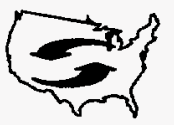

Net Interstiate

Movements:

85,965

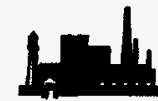

Industrial:

83,081

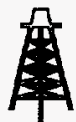

Marketed

Production:

73,081

0.37

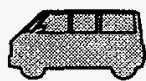

Vehicle Fuel:

2

0.12

Deliveries to Consumers:

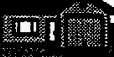

Residential:

62,533

1.29

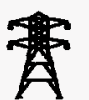

Electric

Utilities:

Total:

182,710

0.97

Table 65. Summary Statistics for Natural Gas - Kentucky, 1990-1994

\begin{tabular}{|c|c|c|c|c|c|}
\hline & 1990 & 1991 & 1992 & 1993 & 1994 \\
\hline 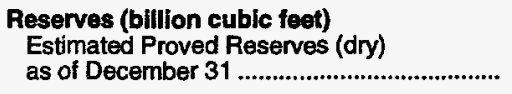 & 1,016 & 1,155 & 1,084 & 1,003 & 969 \\
\hline $\begin{array}{c}\text { Number of Gas and Gas Condensate Wells } \\
\text { Producing at End of Year................................... }\end{array}$ & 11,713 & 12,169 & 12,483 & 12,836 & 13,036 \\
\hline 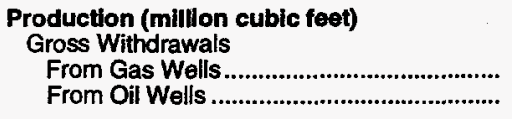 & $\begin{array}{r}75,333 \\
0\end{array}$ & $\begin{array}{r}78,904 \\
0\end{array}$ & $\begin{array}{r}79,690 \\
0\end{array}$ & $\begin{array}{r}86,966 \\
0\end{array}$ & $\begin{array}{r}73,081 \\
0\end{array}$ \\
\hline Total & 75,333 & 78,904 & 79,690 & 86,966 & 73,081 \\
\hline 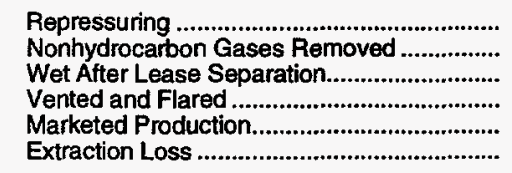 & $\begin{array}{r}* \\
75,333 \\
75,333 \\
1,899\end{array}$ & $\begin{array}{r}* \\
78,904 \\
78,904 \\
2,181\end{array}$ & $\begin{array}{r}\text { * } \\
79,690 \\
79,690 \\
2,342\end{array}$ & $\begin{array}{r}* \\
86,966 \\
86,966 \\
2,252\end{array}$ & $\begin{array}{r}* \\
73,081 \\
73,081 \\
2,024\end{array}$ \\
\hline 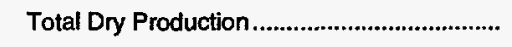 & 73,434 & 76,723 & 77,348 & 84,714 & 71,057 \\
\hline 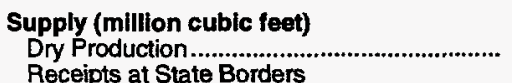 & 73,434 & 76,723 & 77,348 & 84,714 & 71,057 \\
\hline 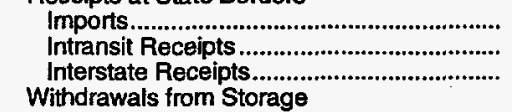 & $\begin{array}{r}0 \\
0 \\
91,303,547\end{array}$ & $\begin{array}{r}0 \\
0 \\
3,030,350\end{array}$ & $\begin{array}{r}0 \\
0 \\
3,197,882\end{array}$ & $\begin{array}{r}0 \\
0 \\
3,314,559\end{array}$ & $\begin{array}{r}0 \\
0 \\
3,663,838\end{array}$ \\
\hline 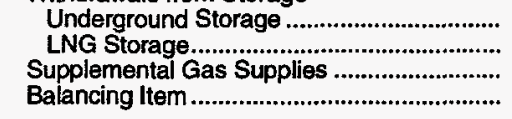 & $\begin{array}{r}45,078 \\
0 \\
2 \\
-207,989\end{array}$ & $\begin{array}{r}48,822 \\
0 \\
2 \\
-94,227\end{array}$ & $\begin{array}{r}42,795 \\
0 \\
5 \\
-55,636\end{array}$ & $\begin{array}{r}59,742 \\
0 \\
16 \\
-63,628\end{array}$ & $\begin{array}{r}54,986 \\
0 \\
50 \\
56,159\end{array}$ \\
\hline 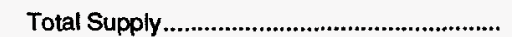 & $3,214,073$ & $3,061,670$ & $3,262,394$ & $3,395,404$ & $3,846,090$ \\
\hline
\end{tabular}

See footnotes at end of table. 
Table 65. Summary Statistics for Natural Gas - Kentucky, 1990-1994 (Continued)

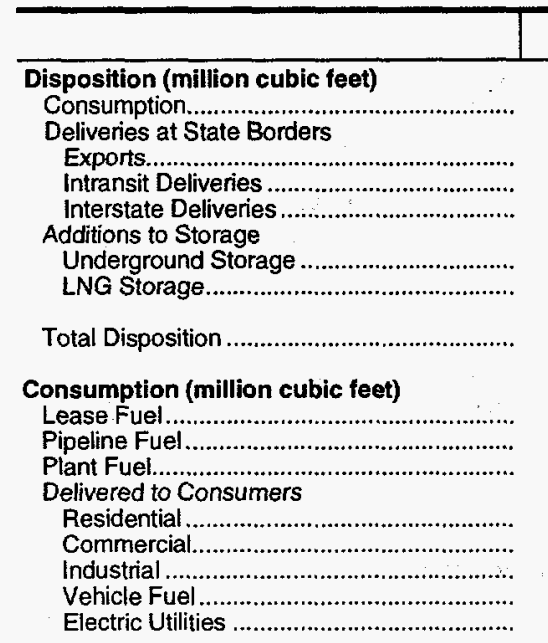

Total Delivered to Consumers .

Total Consumption.

Delivered for the Account of Others

(million cubic feet)

Residential.

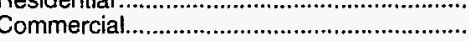

Industrial.

Electric Utilities

irm Deliveries

(million cubic feet)

Residential.

Commercial.

Industrial.

Electric Utilities

Vehicle Fuel

Interruptible Deliveries

(million cubic feet)

Commercial.

Industrial.

Electric Utilities

Vehicle Fuel..

Number of Consumers

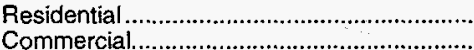

Industrial.

Average Annual Consumption per Consumer (thousand cubic feet)

Residential.

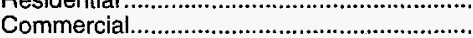

Industrial...

Average Prices for Natural Gas

(dollars per thousand cubic feet)

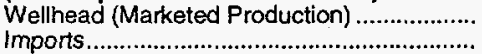

Exports.

Pipeline Fuel.

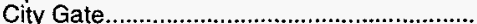

Delivered to Consumers

Residential...

Industrial.

Vehicle Fuel

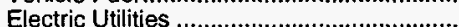

\section{0}

184,333

0
0
$2,963,545$

66,195

195
0

$3,214,073$

2,492

24,624

657

56,064

31,806

68,408

0
283

156,560

184,333

0
1,575

50,673

673
0

NA
NA
NA
NA
NA

NA
NA
NA

NA

624,477

67,086

1,544

90
474

474
44,305

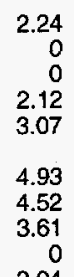

2.24
0
0
2.12
3.07

4.93
4.52
3.61
0
3.04 \begin{tabular}{l|l}
1991 & 1992 \\
\hline
\end{tabular}

187,443

189,877

0
0
$3,023,149$

49,367

47,425

$3,061,670$

$3,262,394$

2, 105

15,847
707

1,730
19,983

702

59,465

33,700

71,637

0
227

165,028

187,443

61,911

35,419

73,619

269

171,218

189,877

0
2,451
47,776
0

2,035
52,422

32

NA
NA
NA
NA
NA

NA

NA

NA
NA

633,942

68,461

68,461
1,587

94
492
45,140

2.03

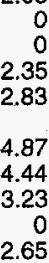

NA

NA

NA
NA
NA

NA
NA

69,466
1,608

510
45,783

1.92

0
0
251

2.51
3.02

5.01

4.47
3.23

3.23

3.78
2.77
1993

203,295

208,386

0
0
$3,143,992$

48,117
0

$3,395,404$

$3,846,090$

2,573
19,017

689

66,909

37,817

76,020

269

181,016

203,295

0
0

$3,577,873$

59,831
0

2,162

22,903

62,533

36,744

83,081

2
350

182,710

208,386

0
3,171
57,149

2,809
53,163

66,909
$\mathbf{R}_{36,334}$
$37,872,451$

37,872

38,900
267

1

$\mathbf{R}_{1,484}$

37,872

22

3,900
3

654,664

71,998

1,585

668,774

73,462

1,621

102

525

47,962

94

500
51,253

2.28

2.24

0
0
2.67

0
0
1.95

$R=$ Revised data.

NA $=$ Not Available.
$*=$ Volume is less than 500,000 cubic feet

Notes: Deliveries to electric utilities (consumption) are reported on the Form ElA-176, "Annual Report of Natural and Supplemental Gas Supply and Disposition. " See the

discussion on electric utility data and Table A1 in Appendix A for a comparison of reporting to these two forms. Totals may not add due to independent rounding.
Sources: Energy Information Administration (EIA), Form ElA-176, "Annual Report of Natural and Supplemental Gas Supply and Disposition"; Form EIA-627, "Annual

Quantity and Value of Natural Gas Report"; Form ElA-857, "Monthly Report of Natural Gas Purchases and Deliveries to Consumers"; Form ElA-816, "Monthly' Natural Gas

Liquids Report"; Form EIA-759, "Monthly Power Plant Report"; Form FERC-423, "Monthly Report of Cost and Quality of Fuels for Electric Plants"; Form EIA-191, "Underground

Gas Storage Report"; Form FPC-14, "Annual Report for Importers and Exporters of Natural Gas"; U.S. Crude Oil, Natural Gas, and Natural Gas Liquids Reserves, 1994 Annual

Report, DOEJEIA-0216(94); and the U.S. Minerals Management Service. 


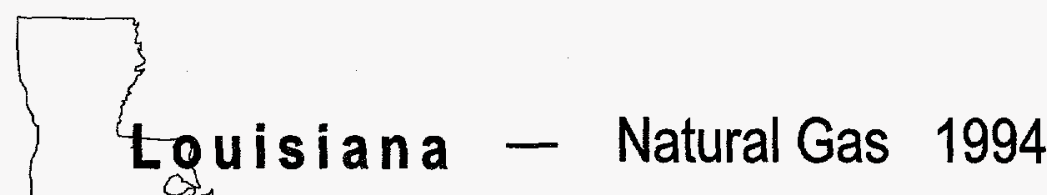

Million Percent of

Cu. Feet National Total

Million

National Tota

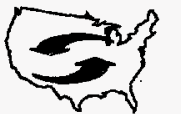

Net Interstate

Movements:

$-3,787,013$

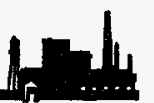

Industrial:

999,034

12.22

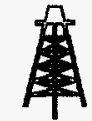

Marketed

Production: $\quad \mathbf{5 , 1 6 9 , 7 0 5}$

26.33

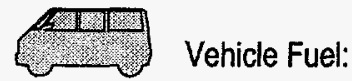

22

Deliveries to Consumers:

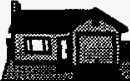

Residential:

52,981

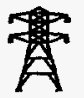

Electric

Utilities:

277,116

9.28

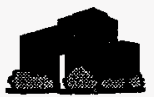

Commercial:

24,184

0.84

Total:

$1,353,337$

7.16

Table 66. Summary Statistics for Natural Gas - Louisiana, 1990-1994

\begin{tabular}{|c|c|c|c|c|c|}
\hline & 1990 & 1991 & 1992 & 1993 & 1994 \\
\hline 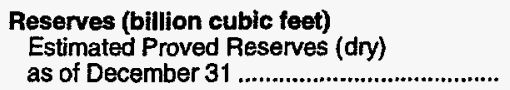 & 34,407 & 32,523 & 29,433 & 28,557 & 30,583 \\
\hline 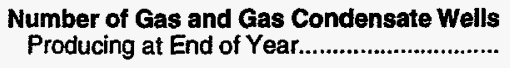 & 16,889 & 15,271 & 13,512 & $\mathbf{P}_{15,569}$ & 12,958 \\
\hline 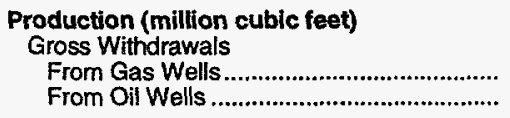 & $\begin{array}{r}4,726,927 \\
576,558\end{array}$ & $\begin{array}{r}4,483,691 \\
616,378\end{array}$ & $\begin{array}{r}4,347,709 \\
629,760\end{array}$ & $\begin{array}{r}R_{4,375,820} \\
{ }_{670,803}\end{array}$ & $\begin{array}{r}4,527,042 \\
699,055\end{array}$ \\
\hline Total & $5,303,485$ & $5,100,068$ & $4,977,470$ & $R_{5,046,623}$ & $5,226,097$ \\
\hline 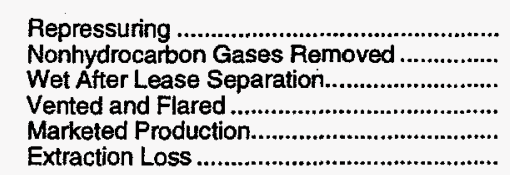 & $\begin{array}{r}40,836 \\
N A \\
5,262,649 \\
20,660 \\
5,241,989 \\
119,405\end{array}$ & $\begin{array}{r}45,292 \\
N A \\
5,054,776 \\
20,415 \\
5,034,361 \\
129,154\end{array}$ & $\begin{array}{r}42,631 \\
N A \\
4,934,839 \\
20,538 \\
4,914,300 \\
132,656\end{array}$ & $\begin{array}{r}R_{35,904} \\
N A \\
R_{5,010,719} \\
R_{19,580} \\
R_{4,991,138} \\
130,336\end{array}$ & $\begin{array}{r}36,703 \\
N A \\
5,189,394 \\
19,689 \\
5,169,705 \\
128,583\end{array}$ \\
\hline 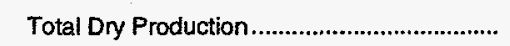 & $5,122,584$ & $4,905,207$ & $4,781,644$ & $R_{4,860,802}$ & $5,041,122$ \\
\hline 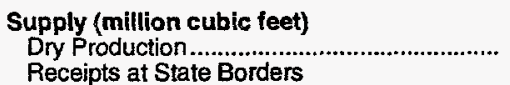 & $5,122,584$ & $4,905,207$ & $4,781,644$ & $R_{4,860,802}$ & $5,041,122$ \\
\hline 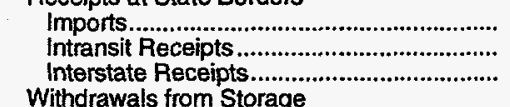 & $\begin{array}{r}30,750 \\
0 \\
1,718,218\end{array}$ & $\begin{array}{r}33,284 \\
0 \\
1,654,594\end{array}$ & $\begin{array}{r}12,637 \\
0 \\
1,870,183\end{array}$ & $\begin{array}{r}30,790 \\
0 \\
1,831,797\end{array}$ & $\begin{array}{r}17,887 \\
0 \\
1,844,714\end{array}$ \\
\hline 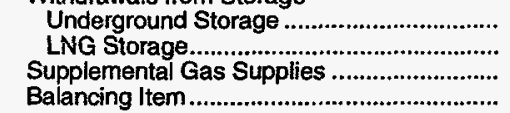 & $\begin{array}{r}127,294 \\
26,206 \\
0 \\
162,707\end{array}$ & $\begin{array}{r}202,991 \\
32,726 \\
0 \\
72,065\end{array}$ & $\begin{array}{r}207,010 \\
12,097 \\
0 \\
33,706\end{array}$ & $\begin{array}{r}221,931 \\
32,033 \\
0 \\
R_{197,928}\end{array}$ & $\begin{array}{r}184,731 \\
18,252 \\
0 \\
409,022\end{array}$ \\
\hline Total Supply & $7,187,758$ & $6,900,868$ & $6,917,278$ & $R_{7,175,281}$ & $7,515,728$ \\
\hline
\end{tabular}


Table 66. Summary Statistics for Natural Gas - Louisiana, 1990-1994 (Continued)

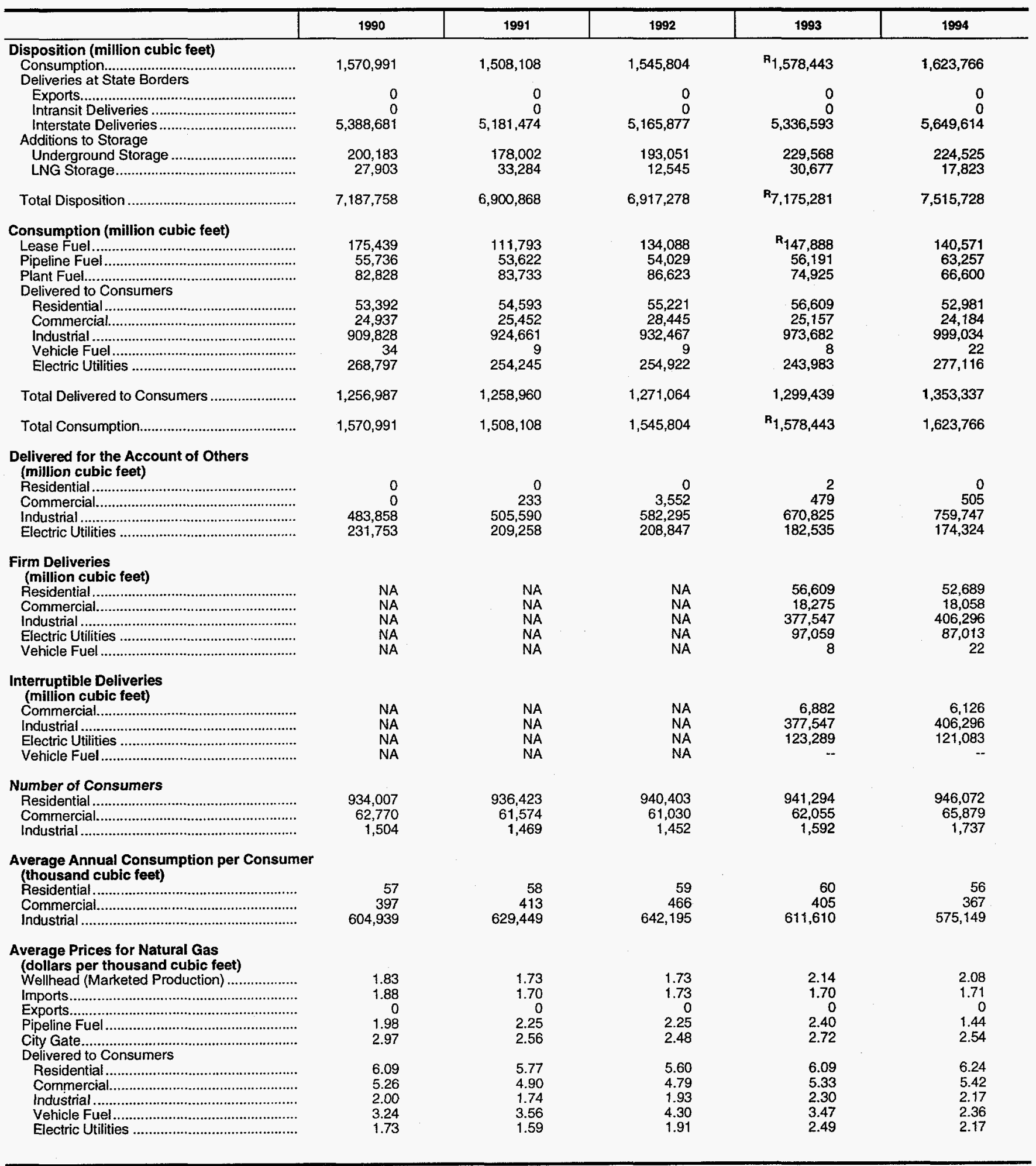

$R=$ Revised data.

NA = Not Available.

Notes: Deliveries to electric utilities (consumption) are reported on the Form E1A-176, "Annual Report of Natural and Supplemental Gas Supply and Disposition." See the discussion on electric utility data and Table A1 in Appendix A for a comparison of reporting to these two forms. Totals may not add due to independent rounding.

Sources: Energy Information Administration (EIA), Form ElA-176, "Annual Report of Natural and Supplemental Gas Supply and Disposition"; Form ElA-627, "Annual Quantity and Value of Natural Gas Report"; Form ElA-857, "Monthly Report of Natural Gas Purchases and Deliveries to Consumers"; Form EIA-816, "Monthly Natural Gas Liquids Report"; Form EIA-759, "Monthly Power Plant Report"; Form FERC-423, "Monthly Report of Cost and Quality of Fuels for Electric Plants"; Form EIA-191, "Underground Gas Storage Report"; Form FPC-14, "Annual Report for Importers and Exporters of Natural Gas"; U.S. Crude Oil, Natural Gas, and Natural Gas Liquids Reserves, 1994 Annual Report, DOEJEIA-0216(94); and the U.S. Minerals Management Service. 


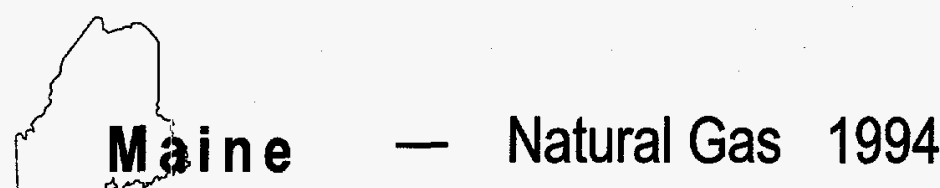

Million Percent of

Cu. Feet National Total

Million Percent of

Cu. Feet National Total

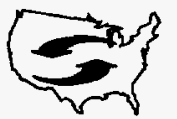

Net Interstate

Movements:

4,928

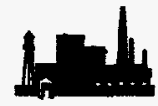

Industrial:

1,771

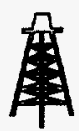

Marketed

Production:

0

0.00

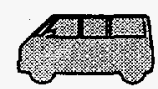

Vehicle Fuel:

Deliveries to Consumers:

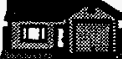

Residential:

894

0.02

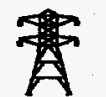

Electric

Utilities:

0.00

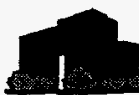

Commercial:

2,381

0.08

Total:

5,045

0.03

Table 67. Summary Statistics for Natural Gas - Maine, 1990-1994

\begin{tabular}{|c|c|c|c|c|c|}
\hline & 1990 & 1991 & 1992 & 1993 & 1994 \\
\hline $\begin{array}{l}\text { Reserves (billion cubic feet) } \\
\text { Estimated Proved Reserves (dry) } \\
\text { as of December } 31\end{array}$ & 0 & 0 & 0 & 0 & 0 \\
\hline 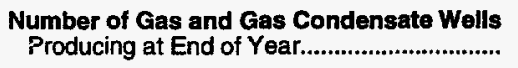 & 0 & 0 & 0 & 0 & 0 \\
\hline 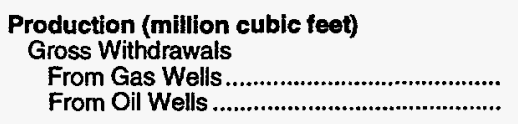 & $\begin{array}{l}0 \\
0\end{array}$ & $\begin{array}{l}0 \\
0\end{array}$ & $\begin{array}{l}0 \\
0\end{array}$ & $\begin{array}{l}0 \\
0\end{array}$ & $\begin{array}{l}0 \\
0\end{array}$ \\
\hline Total & 0 & 0 & 0 & 0 & 0 \\
\hline 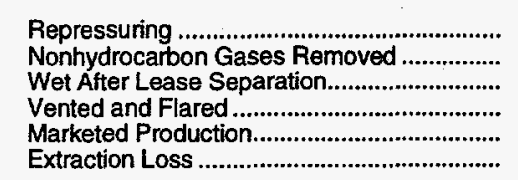 & $\begin{array}{l}0 \\
0 \\
0 \\
0 \\
0 \\
0\end{array}$ & $\begin{array}{l}0 \\
0 \\
0 \\
0 \\
0 \\
0\end{array}$ & $\begin{array}{l}0 \\
0 \\
0 \\
0 \\
0 \\
0\end{array}$ & $\begin{array}{l}0 \\
0 \\
0 \\
0 \\
0 \\
0\end{array}$ & $\begin{array}{l}0 \\
0 \\
0 \\
0 \\
0 \\
0\end{array}$ \\
\hline 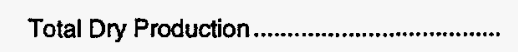 & 0 & 0 & 0 & 0 & 0 \\
\hline 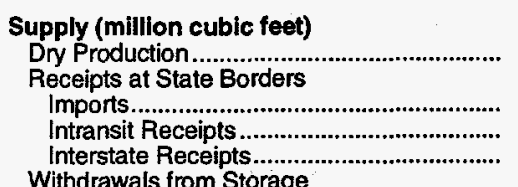 & $\begin{array}{r}0 \\
0 \\
4,471\end{array}$ & $\begin{array}{r}0 \\
0 \\
4,839\end{array}$ & $\begin{array}{r}0 \\
0 \\
5,161\end{array}$ & $\begin{array}{r}0 \\
0 \\
4,989\end{array}$ & $\begin{array}{r}0 \\
0 \\
4,928\end{array}$ \\
\hline 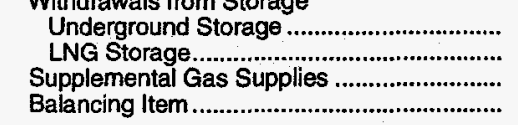 & $\begin{array}{r}0 \\
22 \\
0 \\
-120\end{array}$ & $\begin{array}{r}0 \\
23 \\
0 \\
-45\end{array}$ & $\begin{array}{r}0 \\
22 \\
0 \\
-26\end{array}$ & $\begin{array}{r}0 \\
19 \\
0 \\
-24\end{array}$ & $\begin{array}{r}0 \\
22 \\
0 \\
131\end{array}$ \\
\hline 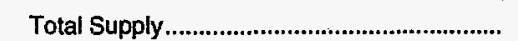 & 4,372 & 4,817 & 5,157 & 4,985 & 5,082 \\
\hline
\end{tabular}

See footnotes at end of table. 
Table 67. Summary Statistics for Natural Gas - Maine, 1990-1994 (Continued)

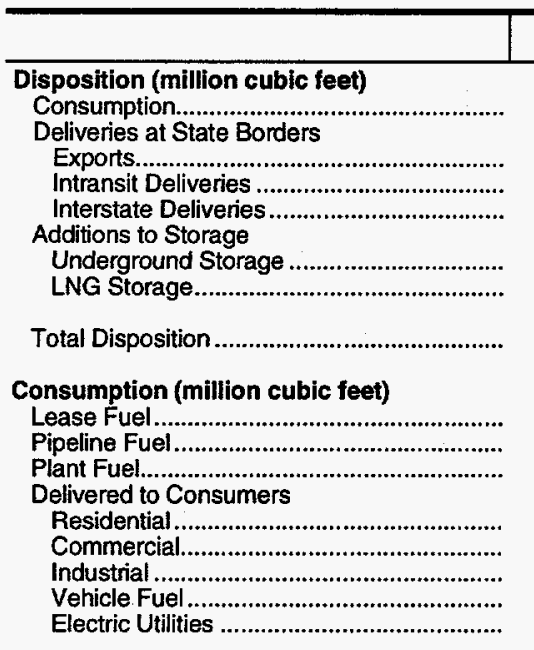

Total Delivered to Consumers...

Total Consumption.

Delivered for the Account of Others

(million cubic feet)

Residential...

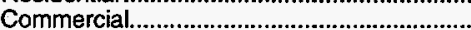

Industrial

Electric Utilities

Firm Deliveries

(million cubic feet)

Residential

Commercial

Industrial.

Electric Utilities

Vehicle Fuel.

Interruptible Deliveries

(million cubic feet)

Commercial..

Industrial

Utilities

Number of Consumers

Residential.

Commercial

Industrial

Average Annual Consumption per Consumer (thousand cubic feet)

Residential

Commercial

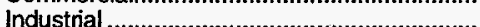

Average Prices for Natural Gas

(dollars per thousand cubic feet)

Wellhead (Marketed Production).

Imports.

Exports

Pipeline Fuel

City Gate.

Delivered to Consumers

Residential.

Commerci

Industrial.

Vehicle Fuel.

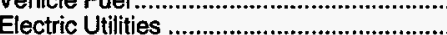

\begin{tabular}{|l|l|l|l|l|}
\hline 1990 & 1991 & 1992 & 1993 & 1994 \\
\hline
\end{tabular}

4,354

4,792

5,131

4,968

5,055

0
0

0
18

4,372

0
0
0

0

0
25

4,817

0

0

0
26

5,157

0
0

0

0
16

4,985

0
27

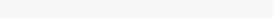

648
1,678
2,024
0

4,350

4,354

0
3
0

722

722
1,860

2,207

0
0

4,789

4,792

\section{(}

872

2,209

2,048

0
0

5,129

5,131

0

901

2,311

1,753

0
0

4,966

4,968

0

0
0
0

5,082

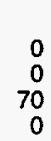

0
0
91
0

0
0
0
0

0
0
0
0

0
0
0
0

$\begin{array}{lll}\text { NA } & \text { NA } & \text { NA } \\ \text { NA } & \text { NA } & \text { NA } \\ \text { NA } & \text { NA } & \text { NA } \\ \text { NA } & \text { NA } & \text { NA } \\ \text { NA } & \text { NA } & \text { NA }\end{array}$

$\begin{array}{rr}901 & 894 \\ 2,311 & 2,381 \\ 0 & 604 \\ 0 & 0 \\ 0 & 0\end{array}$

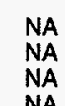

NA
NA
NA
NA
NA

NA

12,000

4,250

12,424
4,455

4,455
81

13,766

4,838

13,880
4,979

66

14,104
5,297

89

54
395
25,297

58

27,248

63
457

25,595

65
464

464
26,564

63

449
19,895

NA = Not Available.

Notes: Deliveries to electric utilities (consumption) are reported on the Form ElA-176, "Annual Report of Natural and Supplemental Gas Supply and Disposition." See the discussion on electric utility data and Table A1 in Appendix A for a comparison of reporting to these two forms. Totals may not add due to independent rounding.

Sources: Energy Information Administration (EIA), Form EIA-176, "Annual Report of Natural and Supplemental Gas Supply and Disposition"; Form EIA-627, "Annual

Quantity and Value of Natural Gas Report"; Form ElA-857, "Monthly Report of Natural Gas Purchases and Deliveries to Consumers"; Form EIA-816, "Monthly Natural Gas

Liquids Report"; Form ElA-759, "Monthly Power Plant Report"; Form FERC-423, "Monthly Report of Cost and Quality of Fuels for Electric Plants"; Form ElA-191, "Underground Gas Storage Report"; Form FPC-14, "Annual Report for Imponters and Exporters of Natural Gas"; U.S. Cnude Oil, Natural Gas, and Natural Gas Liquids Reserves, 1994 Annual Report, DOE/EIA-0216(94); and the U.S. Minerals Management Service. 


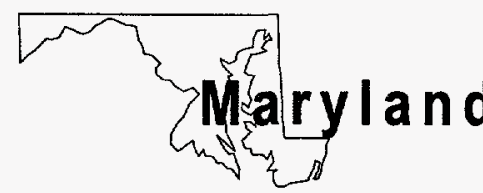

Mallion

Nationt of

Million

Cu. Feet

Percent of

Cu. Feet National Tota

National Total

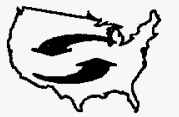

Net Interstate

Movements:

226,594

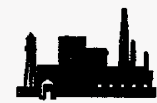

industrial:

47,691

0.58

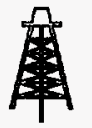

Marketed

Production:

26

0.00

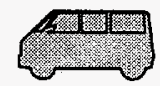

Vehicle Fuel:

25

Deliveries to Consumers:

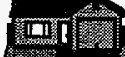

Residential:

76,688

1.58

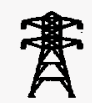

Electric

Utilities:

12,718

0.43

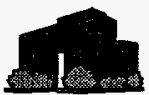

Commercial: $\quad 44,136$

1.52

Total:

181,259

0.96

Table 68. Summary Statistics for Natural Gas - Maryland, 1990-1994

\begin{tabular}{|c|c|c|c|c|c|}
\hline & 1990 & 1991 & 1992 & 1993 & 1994 \\
\hline $\begin{array}{l}\text { Reserves (billion cubic feet) } \\
\text { Estimated Proved Reserves (dry) } \\
\text { as of December } 31\end{array}$ & NA & NA & NA & NA & NA \\
\hline 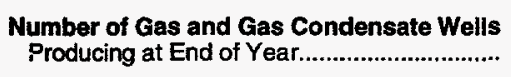 & 7 & 7 & 9 & 7 & 7 \\
\hline 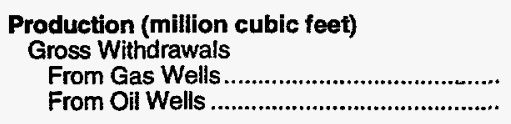 & $\begin{array}{r}22 \\
0\end{array}$ & $\begin{array}{r}29 \\
0\end{array}$ & $\begin{array}{r}33 \\
0\end{array}$ & $\begin{array}{r}28 \\
0\end{array}$ & $\begin{array}{r}26 \\
0\end{array}$ \\
\hline 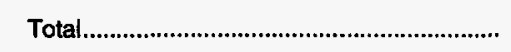 & 22 & 29 & 33 & 28 & 26 \\
\hline 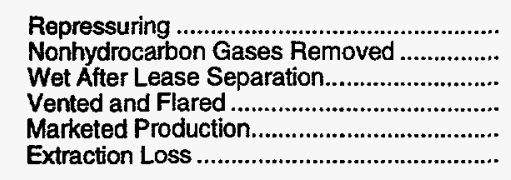 & $\begin{array}{r}0 \\
0 \\
22 \\
0 \\
22 \\
0\end{array}$ & $\begin{array}{r}0 \\
0 \\
29 \\
0 \\
29 \\
0\end{array}$ & $\begin{array}{r}0 \\
0 \\
33 \\
0 \\
33 \\
0\end{array}$ & $\begin{array}{r}0 \\
0 \\
28 \\
0 \\
28 \\
0\end{array}$ & $\begin{array}{r}0 \\
0 \\
26 \\
0 \\
26 \\
0\end{array}$ \\
\hline Total Dry Production & 22 & 29 & 33 & 28 & 26 \\
\hline Supply (million cubic feet) & 22 & 29 & 33 & 28 & 26 \\
\hline 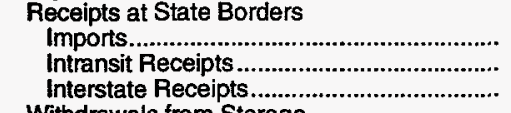 & $\begin{array}{r}0 \\
0 \\
931,632\end{array}$ & $\begin{array}{r}0 \\
0 \\
931,110\end{array}$ & $\begin{array}{r}0 \\
0 \\
959,084\end{array}$ & $\begin{array}{r}0 \\
0 \\
855,001\end{array}$ & $\begin{array}{r}0 \\
0 \\
883,179\end{array}$ \\
\hline 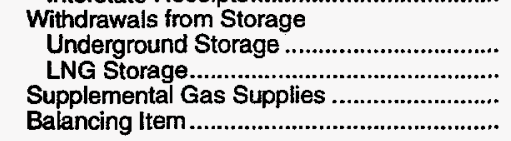 & $\begin{array}{r}14,074 \\
377 \\
24 \\
-3,827\end{array}$ & $\begin{array}{r}20,656 \\
531 \\
72 \\
-17,242\end{array}$ & $\begin{array}{r}19,169 \\
715 \\
126 \\
-10,859\end{array}$ & $\begin{array}{r}20,420 \\
610 \\
418 \\
18,085\end{array}$ & $\begin{array}{r}17,406 \\
529 \\
987 \\
-45,552\end{array}$ \\
\hline Total Supply & 942,303 & 935,157 & 968,267 & 894,562 & 856,574 \\
\hline
\end{tabular}

See footnotes at end of table. 
Table 68. Summary Statistics for Natural Gas - Maryland, 1990-1994 (Continued)

\begin{tabular}{|c|c|c|c|c|c|}
\hline & 1990 & 1991 & 1992 & 1993 & 1994 \\
\hline $\begin{array}{l}\text { Disposition (million cubic feet) } \\
\text { Consumption.............................................. } \\
\text { Deliveries at State Borders }\end{array}$ & 172,267 & 173,081 & 181,300 & 180,614 & 183,734 \\
\hline 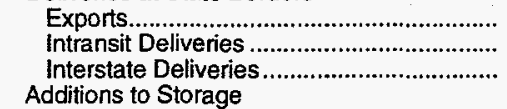 & $\begin{array}{r}0 \\
0 \\
749,836\end{array}$ & $\begin{array}{r}0 \\
0 \\
738,910\end{array}$ & $\begin{array}{r}0 \\
0 \\
766,661\end{array}$ & $\begin{array}{r}0 \\
0 \\
697,939\end{array}$ & $\begin{array}{r}0 \\
0 \\
656,585\end{array}$ \\
\hline 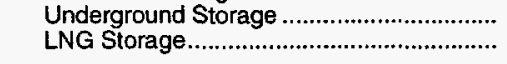 & $\begin{array}{r}19,431 \\
769\end{array}$ & $\begin{array}{r}22,508 \\
657\end{array}$ & $\begin{array}{r}19,502 \\
804\end{array}$ & $\begin{array}{r}15,314 \\
696\end{array}$ & $\begin{array}{r}15,316 \\
939\end{array}$ \\
\hline 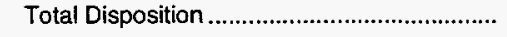 & 942,303 & 935,157 & 968,267 & 894,562 & 856,574 \\
\hline 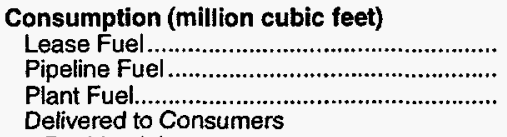 & $\begin{array}{r}1 \\
2,403 \\
0\end{array}$ & $\begin{array}{r}0 \\
2,519 \\
0\end{array}$ & $\begin{array}{r}0 \\
2,419 \\
0\end{array}$ & $\begin{array}{r}1 \\
2,429 \\
0\end{array}$ & $\begin{array}{r}1 \\
2,475 \\
0\end{array}$ \\
\hline 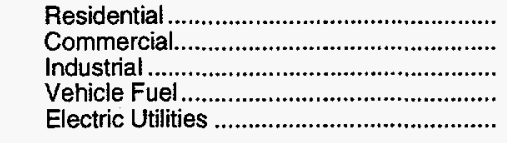 & $\begin{array}{r}66,428 \\
24,051 \\
61,848 \\
0 \\
17,536\end{array}$ & $\begin{array}{r}69,235 \\
38,117 \\
47,150 \\
0 \\
16,059\end{array}$ & $\begin{array}{r}75,122 \\
42,464 \\
49,720 \\
0 \\
11,575\end{array}$ & $\begin{array}{r}76,871 \\
43,635 \\
48,842 \\
19 \\
8,817\end{array}$ & $\begin{array}{r}76,688 \\
44,136 \\
47,691 \\
25 \\
12,718\end{array}$ \\
\hline Total Delivered to Consumers ........................ & 169,863 & 170,561 & 178,881 & 178,185 & 181,259 \\
\hline 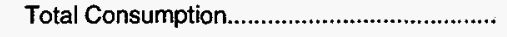 & 172,267 & 173,081 & 181,300 & 180,614 & 183,734 \\
\hline 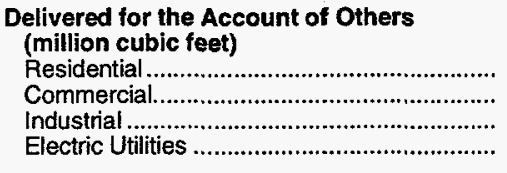 & $\begin{array}{r}0 \\
1,052 \\
39,245 \\
8,483\end{array}$ & $\begin{array}{r}0 \\
1,308 \\
39,686 \\
6,430\end{array}$ & $\begin{array}{r}0 \\
1,692 \\
39,740 \\
3,691\end{array}$ & $\begin{array}{r}0 \\
1,497 \\
38,989 \\
3,034\end{array}$ & $\begin{array}{r}0 \\
1,291 \\
39,697 \\
4,186\end{array}$ \\
\hline 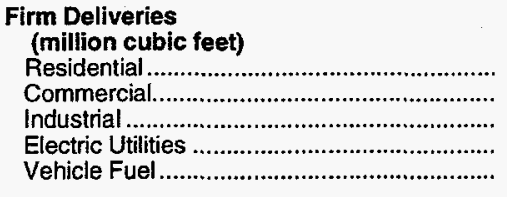 & $\begin{array}{l}\text { NA } \\
\text { NA } \\
\text { NA } \\
\text { NA } \\
\text { NA }\end{array}$ & $\begin{array}{l}\text { NA } \\
\text { NA } \\
\text { NA } \\
\text { NA } \\
\text { NA }\end{array}$ & $\begin{array}{l}\text { NA } \\
\text { NA } \\
\text { NA } \\
\text { NA } \\
\text { NA }\end{array}$ & $\begin{array}{r}76,871 \\
35,491 \\
36,307 \\
1,231 \\
19\end{array}$ & $\begin{array}{r}76,688 \\
36,183 \\
35,856 \\
2,977 \\
25\end{array}$ \\
\hline 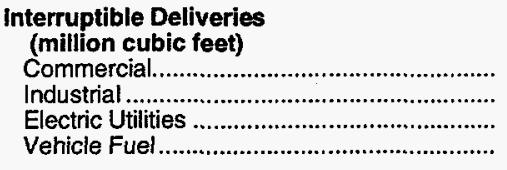 & $\begin{array}{l}\text { NA } \\
\text { NA } \\
\text { NA } \\
\text { NA }\end{array}$ & $\begin{array}{l}\text { NA } \\
\text { NA } \\
\text { NA } \\
\text { NA }\end{array}$ & $\begin{array}{l}\text { NA } \\
\text { NA } \\
\text { NA } \\
\text { NA }\end{array}$ & $\begin{array}{r}8,144 \\
36,307 \\
5,770 \\
\ldots\end{array}$ & $\begin{array}{r}7,953 \\
35,856 \\
9,910 \\
--\end{array}$ \\
\hline 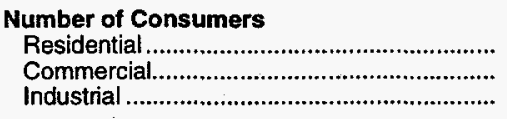 & $\begin{array}{r}774,707 \\
55,576 \\
5,646\end{array}$ & $\begin{array}{r}782,373 \\
61,878 \\
520\end{array}$ & $\begin{array}{r}894,677 \\
62,858 \\
514\end{array}$ & $\begin{array}{r}807,204 \\
63,767 \\
496\end{array}$ & $\begin{array}{r}824,137 \\
64,698 \\
516\end{array}$ \\
\hline 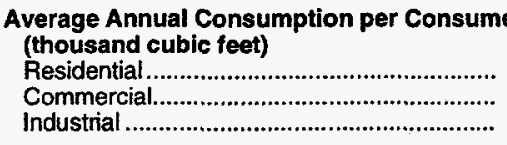 & $\begin{array}{r}86 \\
433 \\
10,954\end{array}$ & $\begin{array}{r}88 \\
616 \\
90,673\end{array}$ & $\begin{array}{r}84 \\
676 \\
96,731\end{array}$ & $\begin{array}{r}95 \\
684 \\
98,472\end{array}$ & $\begin{array}{r}93 \\
682 \\
92,425\end{array}$ \\
\hline 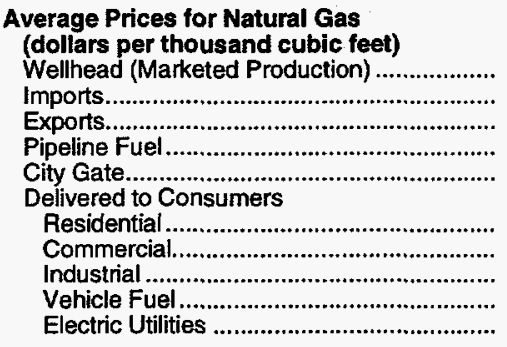 & $\begin{array}{r}1.14 \\
0 \\
0 \\
2.15 \\
3.16\end{array}$ & $\begin{array}{r}1.55 \\
0 \\
0 \\
1.94 \\
3.05\end{array}$ & $\begin{array}{r}6.43 \\
5.24 \\
3.56 \\
0 \\
2.66\end{array}$ & $\begin{array}{r}2.44 \\
0 \\
0 \\
2.08 \\
3.53\end{array}$ & $\begin{array}{r}1.37 \\
0 \\
0 \\
2.01 \\
3.38\end{array}$ \\
\hline
\end{tabular}

NA $=$ Not Available.

Notes: Deliveries to electric utilities (consumption) are reported on the Form ElA-176, "Annual Report of Natural and Supplemental Gas Supply and Disposition." See the

discussion on electric utility data and Table A1 in Appendix A for a comparison of reporting to these two forms. Totals may not add due to independent rounding.

Sources: Energy Information Administration (EIA), Form EIA-176, "Annual Report of Nafural and Supplemental Gas Supply and Disposition"; Form EIA-627, "Annual

Quantity and Value of Natural Gas Feport"; Form ElA-857, "Monthly Report of Natural Gas Purchases and Deliveries to Consumers"; Form ElA-816, "Monthly Natural Gas

Liquids Report"; Form ElA-759, "Monthly Power Plant Report"; Form FERC-423, "Monthly Report of Cost and Quality of Fuels for Electric Plants"; Form EIA-191, "Underground Gas Storage Report"; Form FPC-14, "Annual Report for Importers and Exporters of Natural Gas"; U.S. Crude Oil, Natural Gas, and Natural Gas Liquids Reserves, 1994 Annual Report, DOOE/EIA-0216(94); and the U.S. Minerals Management Service. 


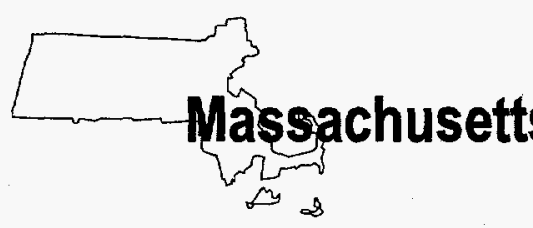

Million Percent of

Cu. Feet National Total

Million

Percent of

National Total $\quad$ Cu. Feet

National Total

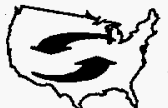

Net Interstate

Movements:

276,771

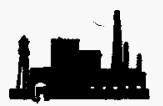

Industrial:

92,798

1.13

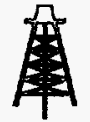

Marketed

Production:

0.00

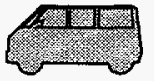

Vehicle Fuel:

3

0.17

Deliveries to Consumers:

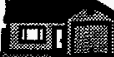

Residential:

119,642

2.47

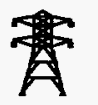

Electric

Utilities:

38,567

Commercial:

84,534

2.92

Total:

335,544

1.77

Table 69. Summary Statistics for Natural Gas - Massachusetts, 1990-1994

\begin{tabular}{|c|c|c|c|c|c|}
\hline & 1990 & 1991 & 1992 & 1993 & 1994 \\
\hline 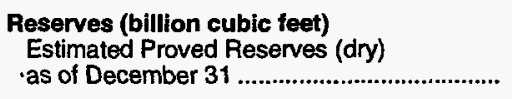 & 0 & 0 & 0 & 0 & 0 \\
\hline 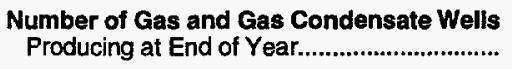 & 0 & 0 & 0 & 0 & 0 \\
\hline 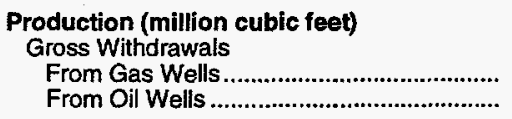 & $\begin{array}{l}0 \\
0\end{array}$ & $\begin{array}{l}0 \\
0\end{array}$ & $\begin{array}{l}0 \\
0\end{array}$ & $\begin{array}{l}0 \\
0\end{array}$ & $\begin{array}{l}0 \\
0\end{array}$ \\
\hline 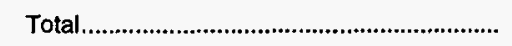 & 0 & 0 & 0 & 0 & 0 \\
\hline 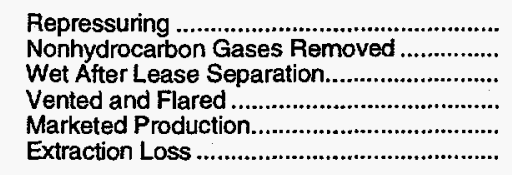 & $\begin{array}{l}0 \\
0 \\
0 \\
0 \\
0 \\
0\end{array}$ & $\begin{array}{l}0 \\
0 \\
0 \\
0 \\
0 \\
0\end{array}$ & $\begin{array}{l}0 \\
0 \\
0 \\
0 \\
0 \\
0\end{array}$ & $\begin{array}{l}0 \\
0 \\
0 \\
0 \\
0 \\
0\end{array}$ & $\begin{array}{l}0 \\
0 \\
0 \\
0 \\
0 \\
0\end{array}$ \\
\hline 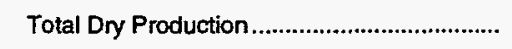 & 0 & 0 & 0 & 0 & 0 \\
\hline 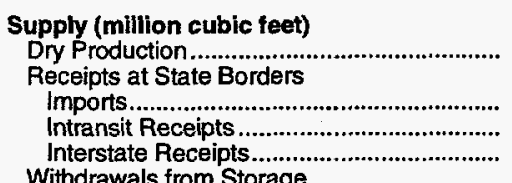 & $\begin{array}{r}53,443 \\
0 \\
232,459\end{array}$ & $\begin{array}{r}30,312 \\
0 \\
272,029\end{array}$ & $\begin{array}{r}30,479 \\
0 \\
334,816\end{array}$ & $\begin{array}{r}50,895 \\
0 \\
278,925\end{array}$ & $\begin{array}{r}32,891 \\
0 \\
292,161\end{array}$ \\
\hline 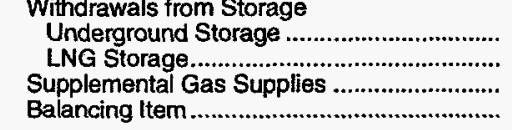 & $\begin{array}{r}0 \\
6,279 \\
317 \\
-4,748\end{array}$ & $\begin{array}{r}0 \\
8,366 \\
120 \\
-13,536\end{array}$ & $\begin{array}{r}0 \\
6,990 \\
105 \\
-10,049\end{array}$ & $\begin{array}{r}0 \\
7,900 \\
61 \\
40,758\end{array}$ & $\begin{array}{r}0 \\
12,369 \\
154 \\
53,615\end{array}$ \\
\hline 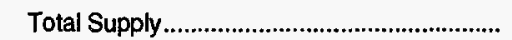 & 287,750 & 297,291 & 362,342 & 378,539 & 391,191 \\
\hline
\end{tabular}

See footnotes at end of table. 
Table 69. Summary Statistics for Natural Gas - Massachusetts, 1990-1994 (Continued)

\begin{tabular}{|c|c|c|c|c|c|}
\hline & 1990 & 1991 & 1992 & 1993 & 1994 \\
\hline $\begin{array}{l}\text { Disposition (million cubic feet) } \\
\text { Consumption } \\
\text { Deliveries at State Borders }\end{array}$ & 258,214 & 251,539 & 295,001 & 312,343 & 337,361 \\
\hline 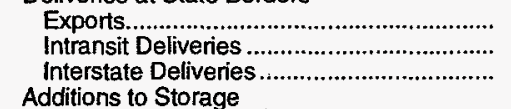 & $\begin{array}{r}0 \\
0 \\
22,950\end{array}$ & $\begin{array}{r}0 \\
0 \\
41,353\end{array}$ & $\begin{array}{r}0 \\
0 \\
62,194\end{array}$ & $\begin{array}{r}0 \\
0 \\
60,665\end{array}$ & $\begin{array}{r}0 \\
0 \\
48,281\end{array}$ \\
\hline 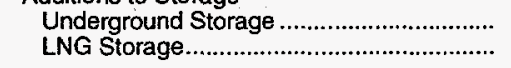 & $\begin{array}{r}0 \\
6,586\end{array}$ & $\begin{array}{r}0 \\
4,399\end{array}$ & $\begin{array}{r}0 \\
5,147\end{array}$ & $\begin{array}{r}0 \\
5,531\end{array}$ & $\begin{array}{r}0 \\
5,550\end{array}$ \\
\hline 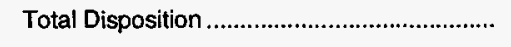 & 287,750 & 297,291 & 362,342 & 378,539 & 391,191 \\
\hline 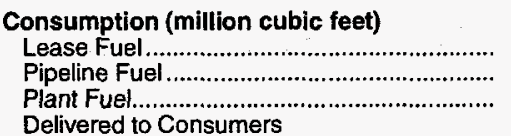 & $\begin{array}{r}0 \\
1,235 \\
0\end{array}$ & $\begin{array}{r}0 \\
1,511 \\
0\end{array}$ & $\begin{array}{r}0 \\
1,763 \\
0\end{array}$ & $\begin{array}{r}0 \\
2,234 \\
0\end{array}$ & $\begin{array}{r}0 \\
1,817 \\
0\end{array}$ \\
\hline 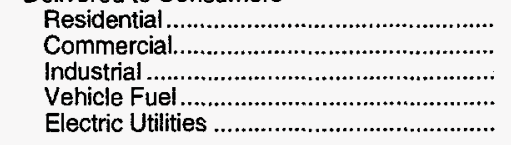 & $\begin{array}{r}106,809 \\
50,618 \\
44,326 \\
55,226\end{array}$ & $\begin{array}{r}102,955 \\
53,188 \\
54,750 \\
1 \\
39,133\end{array}$ & $\begin{array}{r}119,670 \\
64,352 \\
70,872 \\
2 \\
38,341\end{array}$ & $\begin{array}{r}121,228 \\
65,429 \\
94,658 \\
2 \\
28,793\end{array}$ & $\begin{array}{r}119,642 \\
84,534 \\
92,798 \\
3 \\
38,567\end{array}$ \\
\hline Total Delivered to Consumers ........................ & 256,979 & 250,028 & 293,238 & 310,109 & 335,544 \\
\hline 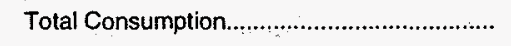 & 258,214 & 251,539 & 295,001 & 312,343 & 337,361 \\
\hline 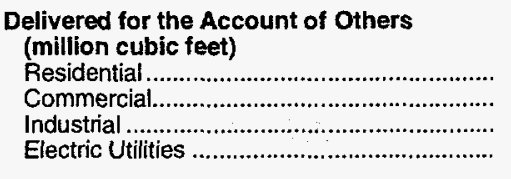 & $\begin{array}{r}0 \\
32 \\
4,309 \\
18,328\end{array}$ & $\begin{array}{r}0 \\
96 \\
15,856 \\
16,832\end{array}$ & $\begin{array}{r}0 \\
140 \\
38,685 \\
22,672\end{array}$ & $\begin{array}{r}0 \\
1,625 \\
65,739 \\
9,725\end{array}$ & $\begin{array}{r}0 \\
20,132 \\
67,072 \\
29,992\end{array}$ \\
\hline 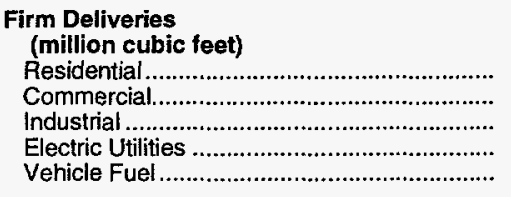 & $\begin{array}{l}\text { NA } \\
\text { NA } \\
\text { NA } \\
\text { NA } \\
\text { NA }\end{array}$ & $\begin{array}{l}\text { NA } \\
\text { NA } \\
\text { NA } \\
\text { NA } \\
\text { NA }\end{array}$ & $\begin{array}{l}\text { NA } \\
\text { NA } \\
\text { NA } \\
\text { NA } \\
\text { NA }\end{array}$ & $\begin{array}{r}121,228 \\
58,104 \\
19,562 \\
12,331 \\
2\end{array}$ & $\begin{array}{r}119,642 \\
60,879 \\
25,286 \\
21,361 \\
1\end{array}$ \\
\hline 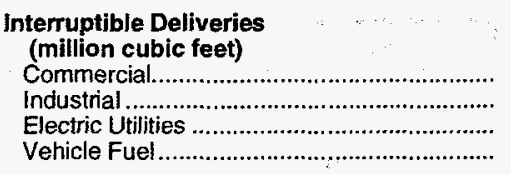 & $\begin{array}{l}\text { NA } \\
\text { NA } \\
\text { NA } \\
\text { NA }\end{array}$ & $\begin{array}{l}\text { NA } \\
\text { NA } \\
\text { NA } \\
\text { NA }\end{array}$ & $\begin{array}{l}\text { NA } \\
\text { NA } \\
\text { NA } \\
\text { NA }\end{array}$ & $\begin{array}{r}7,325 \\
19,562 \\
10,311 \\
-\end{array}$ & $\begin{array}{r}23,656 \\
25,286 \\
19,983 \\
1\end{array}$ \\
\hline 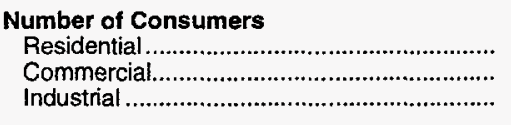 & $\begin{array}{r}1,118,429 \\
85,775 \\
6,539\end{array}$ & $\begin{array}{r}1,127,536 \\
88,746 \\
5,006\end{array}$ & $\begin{array}{r}1,137,911 \\
85,873 \\
8,723\end{array}$ & $\begin{array}{r}1,155,443 \\
102,187 \\
7,283\end{array}$ & $\begin{array}{r}1,179,869 \\
92,744 \\
8,019\end{array}$ \\
\hline 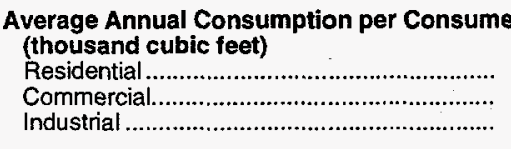 & $\begin{array}{r}95 \\
590 \\
6,779\end{array}$ & $\begin{array}{r}91 \\
599 \\
10,937\end{array}$ & $\begin{array}{r}105 \\
749 \\
8,125\end{array}$ & $\begin{array}{r}105 \\
640 \\
12,997\end{array}$ & $\begin{array}{r}101 \\
911 \\
11,572\end{array}$ \\
\hline 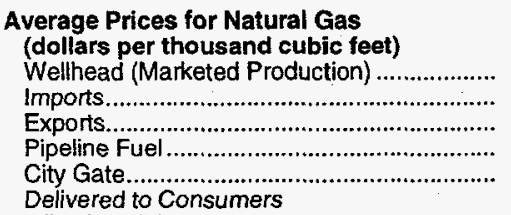 & $\begin{array}{r}2.80 \\
0 \\
2.67 \\
3.34\end{array}$ & $\begin{array}{r}3.08 \\
0 \\
2.79 \\
3.37\end{array}$ & $\begin{array}{r}2.88 \\
0 \\
2.91 \\
3.52\end{array}$ & $\begin{array}{r}2.50 \\
0 \\
2.71 \\
3.98\end{array}$ & $\begin{array}{r}2.59 \\
0 \\
2.13 \\
3.98\end{array}$ \\
\hline 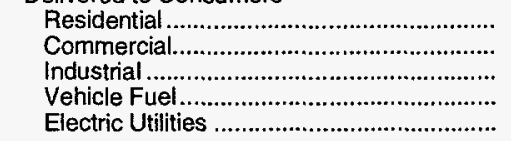 & $\begin{array}{l}7.82 \\
6.35 \\
4.14 \\
3.59 \\
2.53\end{array}$ & $\begin{array}{l}8.11 \\
6.17 \\
3.99 \\
3.90 \\
2.27\end{array}$ & $\begin{array}{l}7.92 \\
5.86 \\
4.14 \\
3.65 \\
2.68\end{array}$ & $\begin{array}{l}8.33 \\
6.04 \\
5.08 \\
4.97 \\
2.72\end{array}$ & $\begin{array}{l}8.94 \\
6.82 \\
5.25 \\
5.66 \\
2.32\end{array}$ \\
\hline
\end{tabular}

NA = Not Available.

Notes: Deliveries to electric utilities (consumption) are reported on the Form ElA-176, "Annual Report of Natural and Supplemental Gas Supply and Disposition." See the discussion on electric utility data and Table A1 in Appendix A for a comparison of reporting to these two forms. Totals may not add due to independent rounding.

discussion on electric utility data and Table A1 in Appendix A for a comparison of reporting to these two foms. Totals may not add due to independent rounding.
Sources: Energy Information Administration (EIA), Form EIA-176, "Annual Report of Natural and Supplemental Gas Supply and Disposition"; Form EIA-627, "Annual

Sources: Energy Information Administration (EIA), Form EIA-176, "Annual Report of Natural and Supplemental Gas Supply and Disposition"; Form EIA-627, "Annual
Quantity and Value of Natural Gas Report"; Form EIA-857, "Monthly Report of Natural Gas Purchases and Deliveries to Consumers"; Form EIA-816, "Monthly Natural Gas

Quantity and Value of Natural Gas Report"; Form ElA-857, "Monthly Report of Natural Gas Purchases and Deliveries to Consumers"; Form ElA-816, "Monthly Natural Gas
Liquids Report"; Form ElA-759, "Monthly Power Plant Report"; Form FERC-423, "Monthly Report of Cost and Quality of Fuels for Electric Plants"; Form ElA-191, "Underground

Gas Storage Report"; Form FP'-14, "Annual Report for Importers and Exporters of Natural Gas"; U.S. Crude Oil, Natural Gas, and Natural Gas Liquids Reserves, 1994 Annual Report, DOE/EIA-0216(94); and the U.S. Minerals Management Service. 


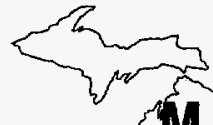

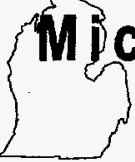

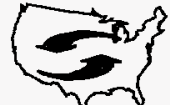

Net Interstate

Movements:

974,941

Marketed

Production:

222,657

Deliveries to Consumers:

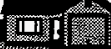

Residential:

364,588

7.52

Commercial:

183,068

6.32
Million

Cu. Feet

Percent of

Percent of

National Total

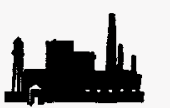

Industrial:

327,848

4.01
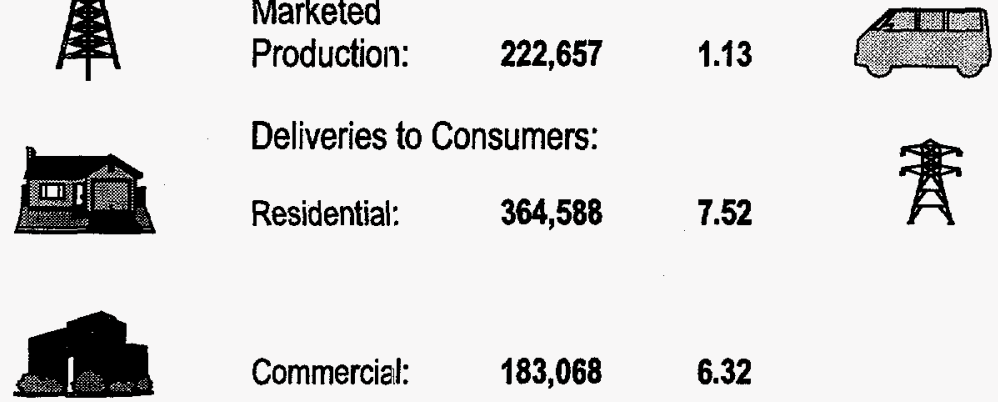

Vehicle Fuel:

14

0.81

Electric

18,218

0.61

Total:

893,735

4.73

Table 70. Summary Statistics for Natural Gas - Michigan, 1990-1994

\begin{tabular}{|c|c|c|c|c|c|}
\hline & 1990 & 1991 & 1992 & 1993 & 1994 \\
\hline 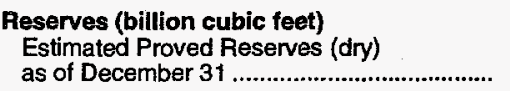 & 1,243 & 1,334 & 1,223 & 1,160 & 1,323 \\
\hline $\begin{array}{c}\text { Number of Gas and Gas Condensate Wells } \\
\text { Producing at End of Year................................... }\end{array}$ & 1,438 & 2,620 & 3,257 & 5,500 & 6,000 \\
\hline 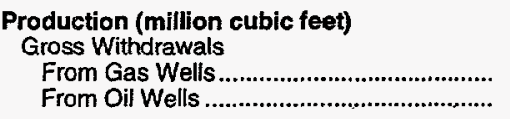 & $\begin{array}{r}106,689 \\
71,126\end{array}$ & $\begin{array}{r}120,848 \\
80,565\end{array}$ & $\begin{array}{r}120,287 \\
80,192\end{array}$ & $\begin{array}{r}126,179 \\
84,119\end{array}$ & $\begin{array}{r}136,989 \\
91,332\end{array}$ \\
\hline 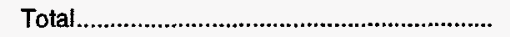 & 177,815 & 201,413 & 200,479 & 210,299 & 228,321 \\
\hline 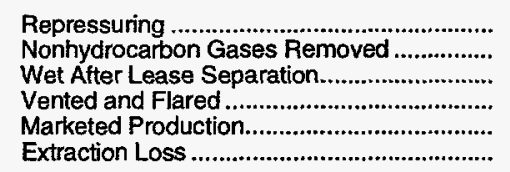 & $\begin{array}{r}2,340 \\
\text { NA } \\
175,475 \\
3,324 \\
172,151 \\
8,317\end{array}$ & $\begin{array}{r}2,340 \\
\text { NA } \\
199,073 \\
3,324 \\
195,749 \\
8,103\end{array}$ & $\begin{array}{r}2,340 \\
N A \\
198,139 \\
3,324 \\
194,815 \\
8,093\end{array}$ & $\begin{array}{r}2,340 \\
\text { NA } \\
207,959 \\
3,324 \\
204,635 \\
7,012\end{array}$ & $\begin{array}{r}2,340 \\
N A \\
225,981 \\
3,324 \\
222,657 \\
6,371\end{array}$ \\
\hline 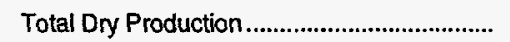 & 163,834 & 187,646 & 186,722 & 197,623 & 216,286 \\
\hline $\begin{array}{l}\text { Supply (million cubic feet) } \\
\text { Dry Production }\end{array}$ & 163,834 & 187,646 & 186,722 & 197,623 & 216,286 \\
\hline 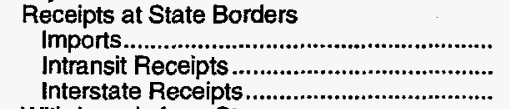 & $\begin{array}{r}0 \\
0 \\
993,264\end{array}$ & $\begin{array}{r}1,151 \\
0 \\
942,159\end{array}$ & $\begin{array}{r}38,568 \\
0 \\
1,135,780\end{array}$ & $\begin{array}{r}34,874 \\
0 \\
1,042,778\end{array}$ & $\begin{array}{r}17,320 \\
0 \\
1,495,917\end{array}$ \\
\hline 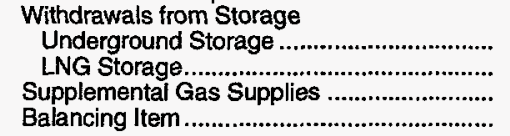 & $\begin{array}{r}314,461 \\
0 \\
19,106 \\
94,341\end{array}$ & $\begin{array}{r}394,196 \\
0 \\
15,016 \\
6,050\end{array}$ & $\begin{array}{r}392,716 \\
0 \\
14,694 \\
58,803\end{array}$ & $\begin{array}{r}437,280 \\
0 \\
12,795 \\
32,932\end{array}$ & $\begin{array}{r}389,224 \\
0 \\
13,688 \\
-197,896\end{array}$ \\
\hline 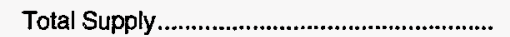 & $1,585,006$ & $1,546,217$ & $1,827,283$ & $1,758,282$ & $1,934,539$ \\
\hline
\end{tabular}

See footnotes at end of table. 
Table 70. Summary Statistics for Natural Gas - Michigan, 1990-1994 (Continued)

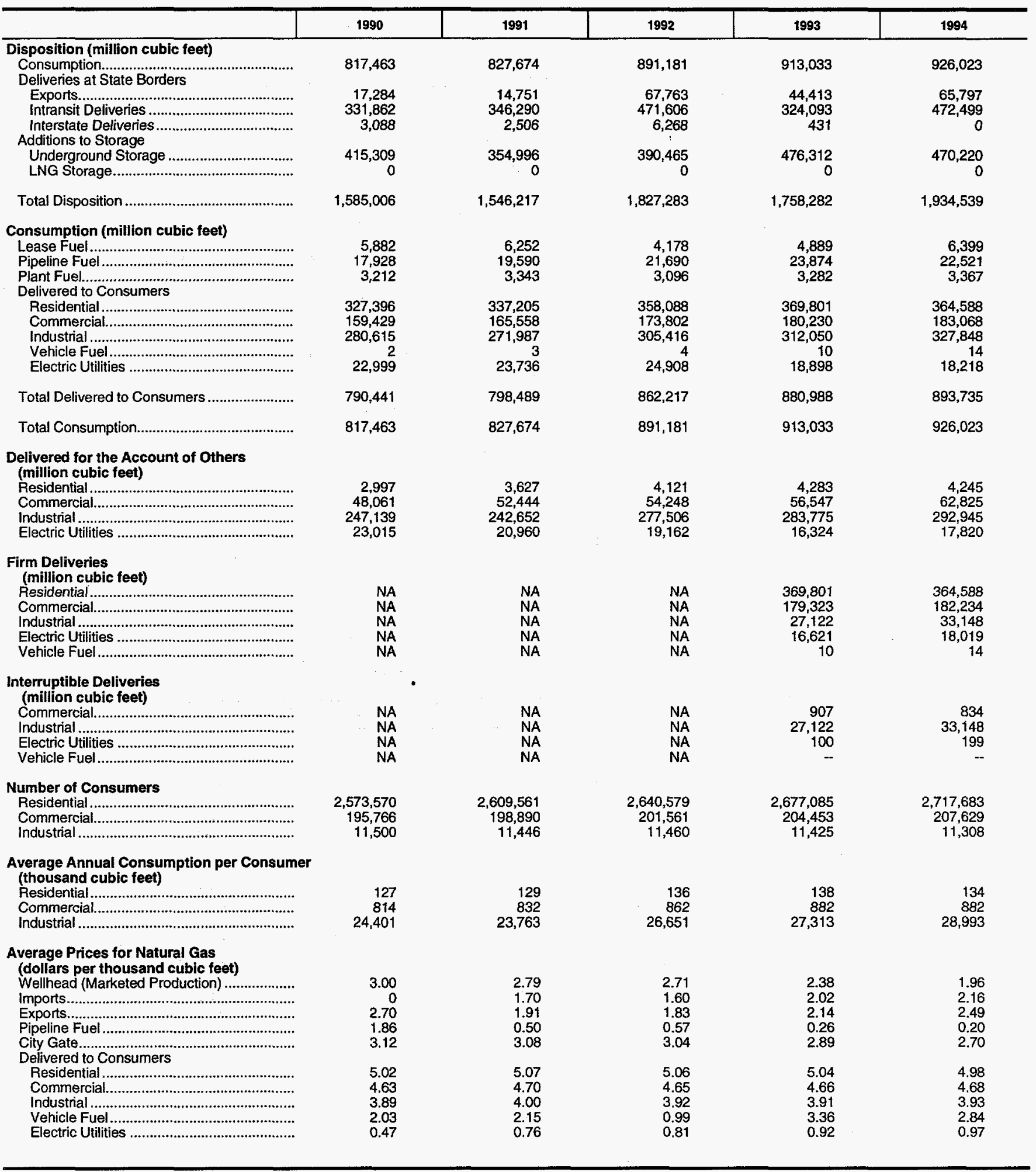

NA $=$ Not Available.
Notes: Deliveries to electric utilities (consumption) are reported on the Form ElA-176, "Annual Report of Natural and Supplemental Gas Supply and Disposition." See the discussion on electric utility data and Table A1 in Appendix A for a comparison of reporting to these two forms. Totals may not add due to independent rounding.

Sources: Energy Information Administration (EIA), Form EIA-176, "Annual Report of Natural and Supplemental Gas Supply and Disposition"; Form EIA-627, "Annual Quantity and Value of Natural Gas Report"; Form ElA-857, "Monthly Report of Natural Gas Purctiases and Deliveries to Consumers"; Form EIA-816, "Monthly Natural Gas Quantity and Value of Natural Gas Report"; Form ElA-857, "Monthly Report of Natural Gas Purchases and Deliveries to Consumers"; Form EIA-816, "Monthly Natural Gas Liquids Report"; Form ElA-759, "Monthly Power Plant Report"; Form FERC-423, "Monthly Peport of Cost and Quality of Fuels for Electric Plants"; Form ElA-191, "Underground Report, DOE/EIA-0216(94); and the U.S. Minerals Management Service. 


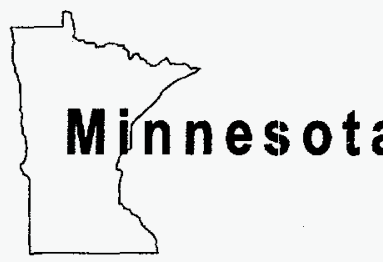

Million Percent of

Million

Percent of

Cu. Feet National Total

Cu. Feet

National Total

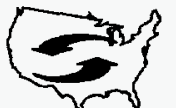

Net Interstate

Movements:

318,356

Industrial:

94,468

1.16

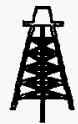

Marketed

Production:

0

0.00

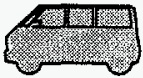

Vehicle Fuel:

29

Deliveries to Consumers:

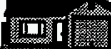

Residential:

122,249

2.52

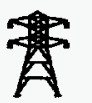

Electric

Utilities:

5,826

0.20

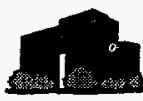

Commercial:

83,933

2.90

Total:

306,505

Table 71. Summary Statistics for Natural Gas - Minnesota, 1990-1994

\begin{tabular}{|c|c|c|c|c|c|}
\hline & 1990 & 1991 & 1992 & 1993 & 1994 \\
\hline 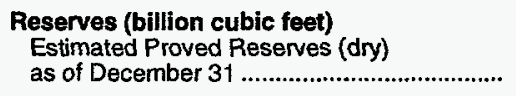 & 0 & 0 & 0 & 0 & 0 \\
\hline 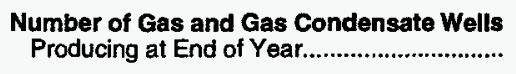 & 0 & 0 & 0 & 0 & 0 \\
\hline 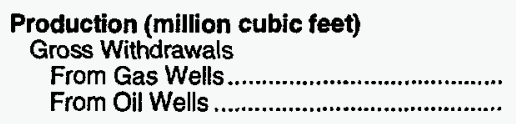 & $\begin{array}{l}0 \\
0\end{array}$ & $\begin{array}{l}0 \\
0\end{array}$ & $\begin{array}{l}0 \\
0\end{array}$ & $\begin{array}{l}0 \\
0\end{array}$ & $\begin{array}{l}0 \\
0\end{array}$ \\
\hline 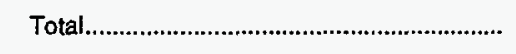 & 0 & 0 & 0 & 0 & 0 \\
\hline 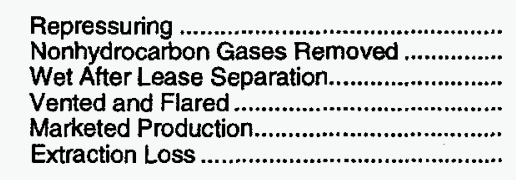 & $\begin{array}{l}0 \\
0 \\
0 \\
0 \\
0 \\
0\end{array}$ & $\begin{array}{l}0 \\
0 \\
0 \\
0 \\
0 \\
0\end{array}$ & $\begin{array}{l}0 \\
0 \\
0 \\
0 \\
0 \\
0\end{array}$ & $\begin{array}{l}0 \\
0 \\
0 \\
0 \\
0 \\
0\end{array}$ & $\begin{array}{l}0 \\
0 \\
0 \\
0 \\
0 \\
0\end{array}$ \\
\hline Total Dry Production & 0 & 0 & 0 & 0 & 0 \\
\hline 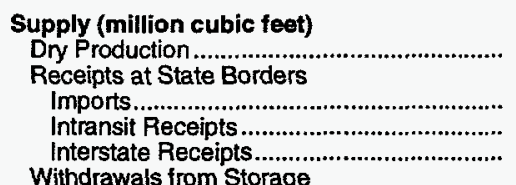 & $\begin{array}{l}308,581 \\
356,401 \\
584,815\end{array}$ & $\begin{array}{l}378,492 \\
362,861 \\
638,534\end{array}$ & $\begin{array}{l}369,137 \\
486,163 \\
706,629\end{array}$ & $\begin{array}{l}383,702 \\
324,093 \\
756,282\end{array}$ & $\begin{array}{l}393,932 \\
487,760 \\
814,096\end{array}$ \\
\hline 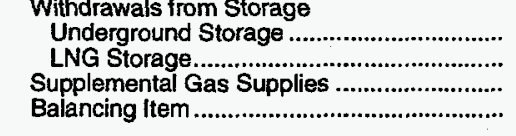 & $\begin{array}{r}1,077 \\
4,670 \\
56 \\
7,160\end{array}$ & $\begin{array}{r}1,018 \\
6,044 \\
49 \\
102\end{array}$ & $\begin{array}{r}1,329 \\
3,380 \\
52 \\
35,488\end{array}$ & $\begin{array}{r}1,834 \\
5,864 \\
78 \\
199,493\end{array}$ & $\begin{array}{l}1,192 \\
5,537 \\
289 \\
6,163\end{array}$ \\
\hline Total Supply & $1,262,760$ & $1,387,101$ & $1,602,179$ & $1,671,346$ & $1,708,970$ \\
\hline
\end{tabular}

See footnotes at end of table. 
Table 71. Summary Statistics for Natural Gas - Minnesota, 1990-1994 (Continued)

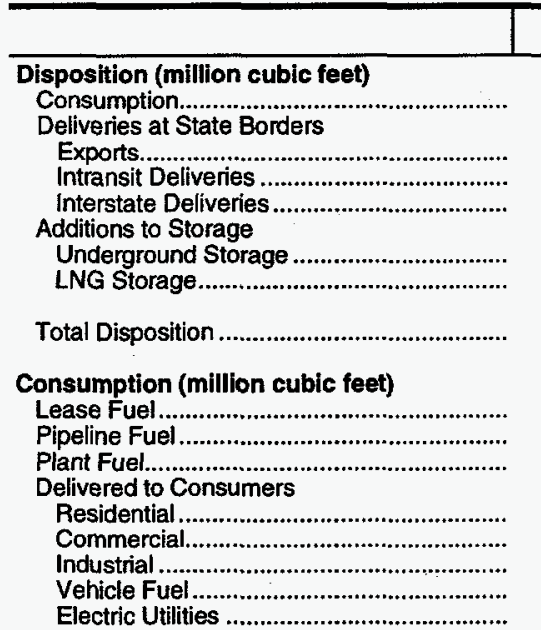

Total Delivered to Consumers...

Total Consumption,

Delivered for the Account of Others

(million cubic feet)

Residential..

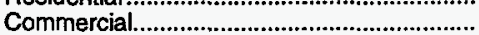

Industrial .......

Firm Deliveries

(million cubic feet)

Residential......

Industrial.....

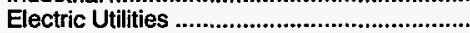

Vehicle Fuel.

Interruptible Deliveries

(million cubic feet)

Commercial.

Industrial.

Vehicle Futi.

Number of Consumers

Residential

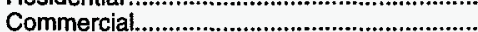

Industrial .

Average Annual Consumption per Consumer (thousand cubic feet)

Residential

Commercial...........

Industrial.

Average Prices for Natural Gas

(dollars per thousand cubic feet)

Wellhead (Marketed Production)

Imports..

Pipeline Fuel .............................................

City Gate .

Delivered to Consumers

Residential

………………………

Industrial.

Electric Utilities

\section{0}

290,587

0
7,813

958,389

1,256

4,714

$1,262,760$

0
12,017
0

106,966

78,015

88,359

5,231

278,570

290,587

0
3,220
51,265

$\begin{array}{r}51,970 \\ \hline\end{array}$

4,094
53,727

53,727

1,657

NA

NA

NA

NA

NA

NA

NA

946,107

95,474

2,574

113
817

34,327

1.90
0
1.89
2.83

4.63
3.98
2.97
0
1.93

1991

314,476

0
0
$1,065,366$

1,285

$1,387,101$

0
13,332
0

117,148

85,875

92,251

5,870

301,144

314,476

NA
NA
NA
NA
NA

NA

NA

NA

970,941

97,388

2,486

121
882

882
37,108

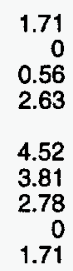

0

74

1

1992

308,821

0
0
$1,288,392$

1372

1,372
3,594

$1,602,179$

0
14,948
0

113,560

82,381

93,025

4,906

293,873

308,821

0
3,134
53,665
1,443

$\begin{array}{r}5,443 \\ \hline\end{array}$

NA

NA
NA
NA

NA

NA
NA
NA

998,201

99,707

2,515

114
826

826
36,988
1993

327,939

0
0
$1,335,315$

1,762

6,330

$1,671,346$

0
16,227
0

123,401

123,401
86,629

97,771

3,910

311,712

327,939

1994

323,815

0
0
$1,377,432$

1,556

6,167

$1,708,970$

0
17,310
0

122,249

83,933

94,468

29
5,826

306,505

323,815

$\begin{array}{rr}0 & 0 \\ 2,623 & 3,336 \\ 55,355 & 53,928 \\ 1,071 & 906\end{array}$

123,401

62,193

32,680

106
1

122,249

62,668

36,503

1,701
29

24,436

32,680

2,201

21,265

36,503

2,296

$1,074,631$
93,062

93,062
2,477

$1,049,263$

102,857

2,592

115
931
39.471

117
816

816
36,446

NA $=$ Not Available.

Notes: Deliveries to electric utilities (consumption) are reported on the Form ElA-176, "Annual Report of Natural and Supplemental Gas Supply and Disposition." See the discussion on electric utility data and Table A1 in Appendix A for a comparison of reporting to these two forms. Totals may not add due to independent rounding.

Sources: Energy Information Administration (EIA), Form EIA- 76 , "Annual Report of Natural and Supplemental Gas Supply and Disposition"; Form EIA-627, "Annual

Quantity and Value of Natural Gas Report"; Form EIA-857, "Monthly Report of Natural Gas Purchases and Deliveries to Consumers"; Form EIA-816, "Monthly Natural Gas

Liquids Report"; Form EIA-759, "Monthly Power Plant Report"; Form FERC-423, "Monthly Report of Cost and Quality of Fuels for Electric Plants"; Form EIA-191, "Underground

Gas Storage Report"; Form FPC-14, "Annual Report for Importers and Exporters of Natural Gas"; U.S. Crude Oil, Natural Gas, and Natural Gas Liquids Reserves, 1994 Annual Report, DÓÉEIA-0216(94); and the U.S. Minerals Management Service. 


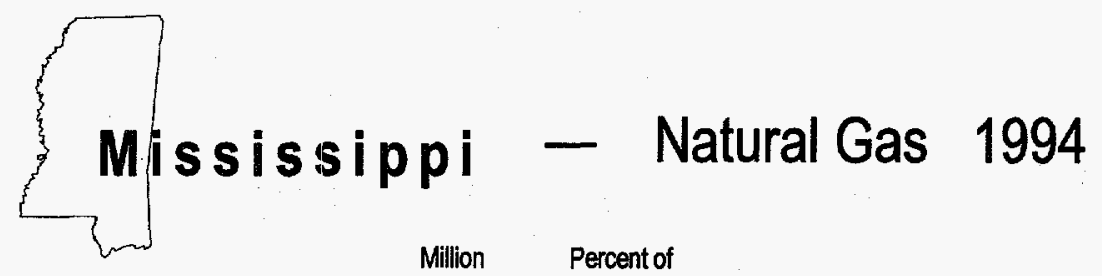

Cu. Feet National Total

Million Percent of Cu. Feet National Tota

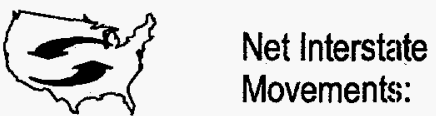

Movements:

167,621

Marketed

Production:

63,448

0.32

Deliveries to Consumers:

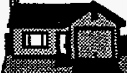

Residential:

27,086

0.56<smiles>C1=C[As][Al]1</smiles>

Industrial:

96,863

1.18

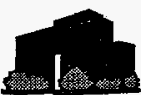

Commercial:

19,232

0.66

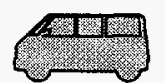

Vehicle Fuel:

9

0.52

Electric

Utilities:

82,541

2.76

Total:

225,730

Tàble 72. Summary Statistics for Natural Gas — Mississippi, 1990-1994

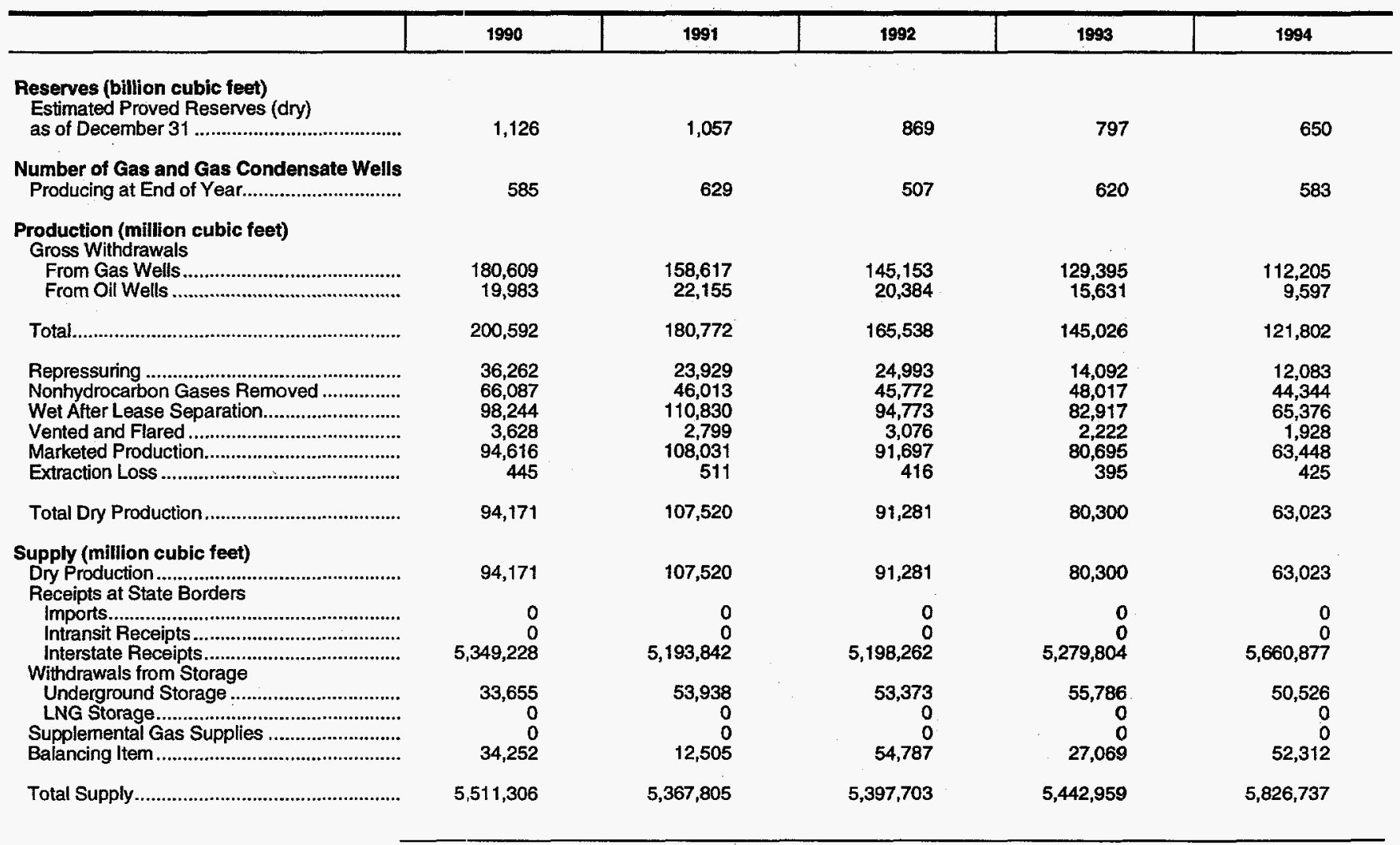

See footnotes at end of table. 
Table 72. Summary Statistics for Natural Gas - Mississippi, 1990-1994 (Continued)

\begin{tabular}{|c|c|c|c|c|c|}
\hline & 1990 & 1991 & 1992 & 1993 & 1994 \\
\hline 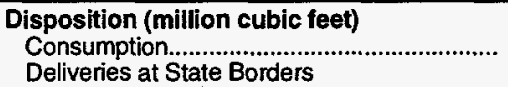 & 253,690 & 249,761 & 239,442 & 229,941 & 268,510 \\
\hline 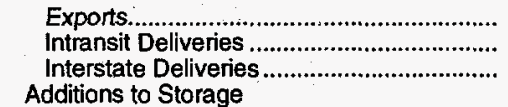 & $\begin{array}{r}0 \\
0 \\
5,204,455\end{array}$ & $\begin{array}{r}0 \\
0 \\
5,069,990\end{array}$ & $\begin{array}{r}0 \\
0 \\
5,103,156\end{array}$ & $\begin{array}{r}0 \\
0 \\
5,157,115\end{array}$ & $\begin{array}{r}0 \\
0 \\
5,493,256\end{array}$ \\
\hline $\begin{array}{l}\text { Underground Storage } \\
\text { LNG Storage }\end{array}$ & $\begin{array}{r}53,161 \\
0\end{array}$ & $\begin{array}{r}48,054 \\
0\end{array}$ & $\begin{array}{r}55,105 \\
0\end{array}$ & $\begin{array}{r}55,903 \\
0\end{array}$ & $\begin{array}{r}64,972 \\
0\end{array}$ \\
\hline 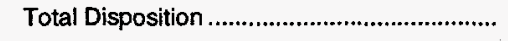 & $5,511,306$ & $5,367,805$ & $5,397,703$ & $5,442,959$ & $5,826,737$ \\
\hline 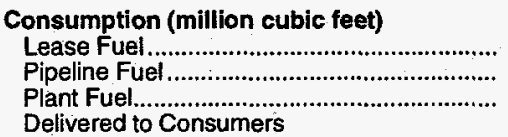 & $\begin{array}{r}6,636 \\
37,735 \\
621\end{array}$ & $\begin{array}{r}3,877 \\
34,645 \\
708\end{array}$ & $\begin{array}{r}4,372 \\
33,276 \\
573\end{array}$ & $\begin{array}{r}4,291 \\
37,500 \\
538\end{array}$ & $\begin{array}{r}3,169 \\
39,147 \\
463\end{array}$ \\
\hline 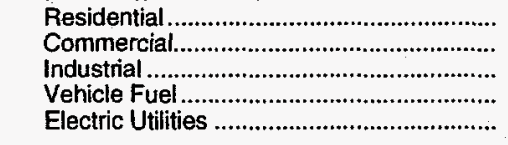 & $\begin{array}{r}25,045 \\
17,548 \\
100,801 \\
0 \\
65,304\end{array}$ & $\begin{array}{r}25,756 \\
17,743 \\
104,622 \\
0 \\
62,409\end{array}$ & $\begin{array}{r}26,487 \\
17,942 \\
102,612 \\
0 \\
54,180\end{array}$ & $\begin{array}{r}28,347 \\
19,199 \\
100,166 \\
0 \\
39,900\end{array}$ & $\begin{array}{r}27,086 \\
19,232 \\
96,863 \\
9 \\
82,541\end{array}$ \\
\hline Total Delivered to Consumers ........................... & 208,699 & 210,531 & 201,221 & 187,611 & 225,730 \\
\hline 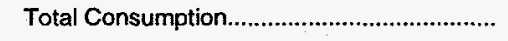 & 253,690 & 249,761 & 239,442 & 229,941 & 268,510 \\
\hline 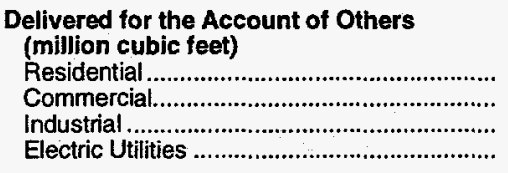 & $\begin{array}{r}0 \\
777 \\
51,694 \\
62,412\end{array}$ & $\begin{array}{r}0 \\
731 \\
54,398 \\
57,297\end{array}$ & $\begin{array}{r}0 \\
645 \\
55,973 \\
41,304\end{array}$ & $\begin{array}{r}0 \\
647 \\
60,506 \\
26,270\end{array}$ & $\begin{array}{r}0 \\
647 \\
59,032 \\
31,927\end{array}$ \\
\hline 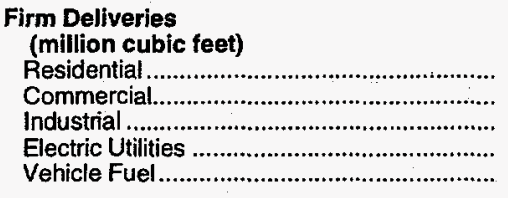 & $\begin{array}{l}\text { NA } \\
\text { NA } \\
\text { NA } \\
\text { NA } \\
\text { NA }\end{array}$ & $\begin{array}{l}\text { NA } \\
\text { NA } \\
\text { NA } \\
\text { NA } \\
\text { NA }\end{array}$ & $\begin{array}{l}\text { NA } \\
\text { NA } \\
\text { NA } \\
\text { NA } \\
\text { NA }\end{array}$ & $\begin{array}{r}28,347 \\
18,060 \\
68,299 \\
30,631 \\
0\end{array}$ & $\begin{array}{r}27,086 \\
17,748 \\
37,783 \\
1,701 \\
0\end{array}$ \\
\hline 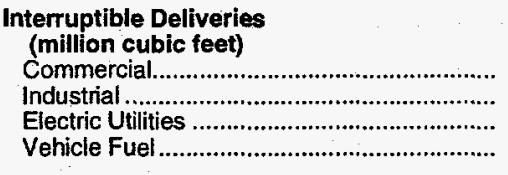 & $\begin{array}{l}\text { NA } \\
\text { NA } \\
\text { NA } \\
\text { NA }\end{array}$ & $\begin{array}{l}\text { NA } \\
\text { NA } \\
\text { NA } \\
\text { NA }\end{array}$ & $\begin{array}{l}\text { NA } \\
\text { NA } \\
\text { NA } \\
\text { NA }\end{array}$ & $\begin{array}{r}1,139 \\
68,299 \\
10,797 \\
--\end{array}$ & $\begin{array}{r}1,484 \\
37,783 \\
31,943 \\
9\end{array}$ \\
\hline 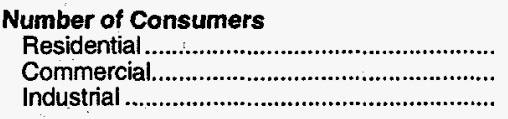 & $\begin{array}{r}382,251 \\
43,184 \\
1,317\end{array}$ & $\begin{array}{r}386,264 \\
43,693 \\
1,314\end{array}$ & $\begin{array}{r}392,155 \\
44,313 \\
1,327\end{array}$ & $\begin{array}{r}398,472 \\
45,310 \\
1,324\end{array}$ & $\begin{array}{r}405,312 \\
43,803 \\
1,326\end{array}$ \\
\hline 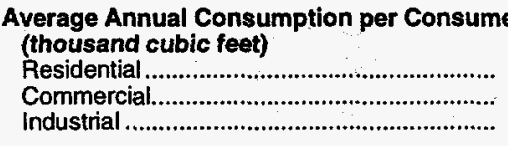 & $\begin{array}{r}66 \\
406 \\
76,539\end{array}$ & $\begin{array}{r}67 \\
406 \\
79,621\end{array}$ & $\begin{array}{r}68 \\
405 \\
77,326\end{array}$ & $\begin{array}{r}71 \\
424 \\
75,654\end{array}$ & $\begin{array}{r}67 \\
439 \\
73,049\end{array}$ \\
\hline 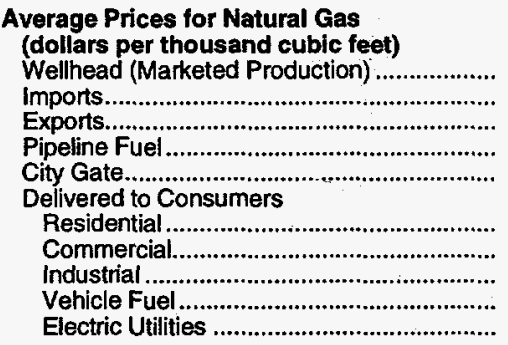 & $\begin{array}{r}5.33 \\
4.48 \\
2.57 \\
0 \\
1.83\end{array}$ & $\begin{array}{r}1.66 \\
0 \\
0 \\
2.33 \\
2.55\end{array}$ & $\begin{array}{r}1.64 \\
0 \\
0 \\
2.34 \\
2.62 \\
\\
4.95 \\
4.13 \\
2.53 \\
0 \\
1.85\end{array}$ & $\begin{array}{r}1.73 \\
0 \\
0 \\
2.37 \\
2.90\end{array}$ & $\begin{array}{r}1.49 \\
0 \\
0 \\
1.98 \\
2.83 \\
\\
5.46 \\
4.56 \\
2.98 \\
2.82 \\
1.98\end{array}$ \\
\hline
\end{tabular}

NA $=$ Not Available.

Notes: Deliveries to electric utilities (consumption) are reported on the Form ElA-176, "Annual Report of Natural and Supplemental Gas Supply and Disposition." See the discussion on electric utility data and Table A1 in Appendix A for a comparison of reporting to these two forms. Totals may not add due to independent rounding.

Sources: Energy Information Administration (EIA), Form EIA-176, "Annual Report of Natural and Supplemental Gas Supply and Disposition"; Form EIA-627, "Annual Quantity and Value of Natural Gas Report"; Form ElA-857, "Monthly Report of Natural Gas Purchases and Deliveries to Consumers"; Fom EIA-816, "Monthly Natural Gas

Liquids Report"; Form EIA-759, "Monthly Power Plant Report"; Form FERC-423, "Monthly Report of Cost and Quality of Fuels for Electric Plants"; Form EIA-191, "Underground Gas Storage Report"; Form FPC-14, "Annual Report for Importers and Exporters of Natural Gas"; U.S. Crude Oil, Natural Gas, and Natural Gas Liquids Reserves, 1994 Annual Report, DOEJEIA-0216(94); and the U.S. Minerais Management Service. 


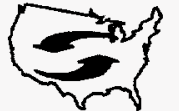

Net Interstate

Movements:

245,986

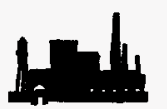

Industrial:

71,602

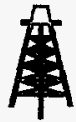

Marketed

Production:

8

0.00

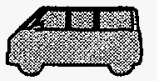

Vehicle Fuel:

1

Deliveries to Consumers:

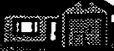

Residential:

122,566

2.53

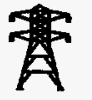

Electric

Utilities:

4,351

0.15

Commercial: $\quad 66,196$

2.29

Total:

264,715

1.40

Table 73. Summary Statistics for Natural Gas — Missouri, 1990-1994

\begin{tabular}{|c|c|c|c|c|c|}
\hline & 1990 & 1991 & 1992 & 1993 & 1994 \\
\hline $\begin{array}{l}\text { Reserves (billion cubic feet) } \\
\text { Estimated Proved Reserves (dry) } \\
\text { as of December } 31\end{array}$ & NA & NA & NA & NA & NA \\
\hline 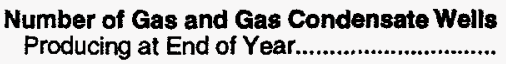 & 8 & 6 & 5 & 8 & 12 \\
\hline 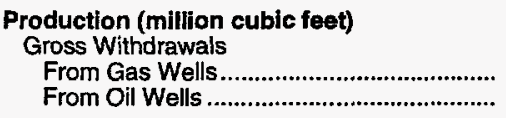 & $\begin{array}{l}7 \\
0\end{array}$ & $\begin{array}{r}15 \\
0\end{array}$ & $\begin{array}{r}27 \\
0\end{array}$ & $\begin{array}{r}14 \\
0\end{array}$ & $\begin{array}{l}8 \\
0\end{array}$ \\
\hline 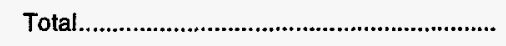 & 7 & 15 & 27 & 14 & 8 \\
\hline 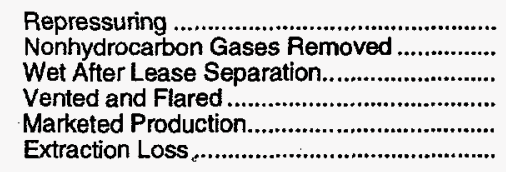 & $\begin{array}{l}0 \\
0 \\
7 \\
0 \\
7 \\
0\end{array}$ & $\begin{array}{r}0 \\
0 \\
15 \\
0 \\
15 \\
0\end{array}$ & $\begin{array}{r}0 \\
0 \\
27 \\
0 \\
27 \\
0\end{array}$ & $\begin{array}{r}0 \\
0 \\
14 \\
0 \\
14 \\
0\end{array}$ & $\begin{array}{l}0 \\
0 \\
8 \\
0 \\
8 \\
0\end{array}$ \\
\hline 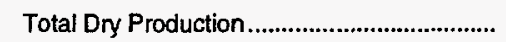 & 7 & 15 & 27 & 14 & 8 \\
\hline $\begin{array}{l}\text { Supply (million cubic teet) } \\
\text { Dry Production ........................................... }\end{array}$ & 7 & 15 & 27 & 14 & 8 \\
\hline Imports & $\begin{array}{r}0 \\
0 \\
1,236,536\end{array}$ & $\begin{array}{r}0 \\
0 \\
1,221,675\end{array}$ & $\begin{array}{r}0 \\
0 \\
1,219,024\end{array}$ & $\begin{array}{r}0 \\
0 \\
1,306,977\end{array}$ & $\begin{array}{r}0 \\
0 \\
1,442,661\end{array}$ \\
\hline 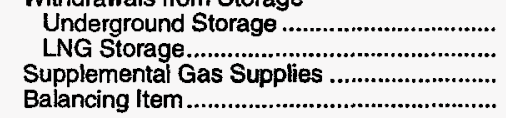 & $\begin{array}{r}3,992 \\
0 \\
0 \\
-375\end{array}$ & $\begin{array}{r}3,855 \\
0 \\
0 \\
1,099\end{array}$ & $\begin{array}{r}3,094 \\
0 \\
0 \\
-3,927\end{array}$ & $\begin{array}{r}4,779 \\
0 \\
0 \\
8,729\end{array}$ & $\begin{array}{r}5,082 \\
0 \\
371 \\
21,145\end{array}$ \\
\hline 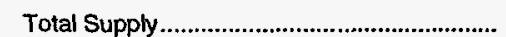 & $1,240,160$ & $1,226,644$ & $1,218,219$ & $1,320,498$ & $1,469,266$ \\
\hline
\end{tabular}

See footnotes at end of table. 
Table 73. Summary Statistics for Natural Gas - Missouri, 1990-1994 (Continued)

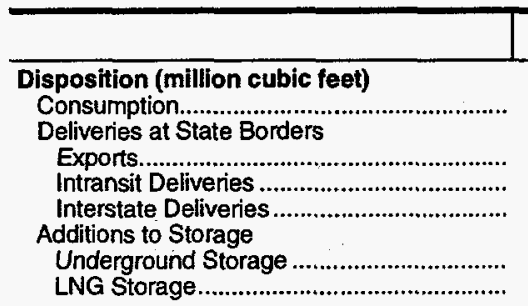

Total Disposition.

Consumption (million cubic feet)

Lease Fuel..

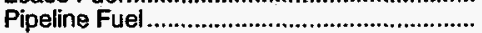

Plant Fuel..

Delivered to Consumers

Residential

Commercial.

Industrial........

Vehicle Fuel...

Total Delivered to Consumers

Total Consumption.

Delivered for the Account of Others

(million cubic feet)

Residential.

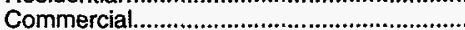

Industrial

Electric Utilities

\section{Firm Deliveries}

(million cubic feet)

Residential.

Industrial...

Electric Utilities

Vehicle Fuel.

Interruptible Deliveries

(million cubic feet)

Commercial.

Industrial

Electric Utilities

Vehicle Fuel....

Number of Consumers

Residential

Commercia

Industrial

Average Annual Consumption per Consumer (thousand cubic feet)

Residential

Commercial

Industrial

Average Prices for Natural Gas

(dollars per thousand cubic feet)

Wellhead (Marketed Production)

Imports.

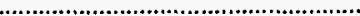

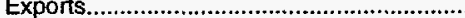

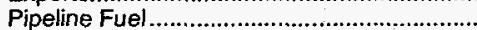

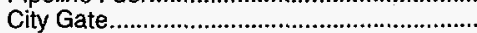

Delivered to Consumers

Residential.

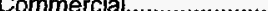

Industrial.

Vehicle Fuel

Electric Utilities

1990

238,709

0
0
0

96,135

5,316
0

$1,240,160$

0
5,302
0

115,950

59,387
54,538

54,538

3,532

233,408

238,709

1
8,306

36,711

2,015

NA

NA

NA

NA

NA

NA

NA

NA

$1,213,305$

105,164

3,140

117,675
3,096

0
8,910
42,745

42,745
2,869

120,680
63,191

57,186

12,709

253,765

256,328

1992

NA

NA

NA

NA

3,096

96
565
17.369

100
537

18,471

1.57
0
0
1.77
3.14

5.21
4.53
4.19
0
1.75

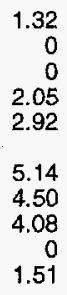

240,745

0
0
974,394

3,080
0

$1,218,219$

1
2,309
0

116,655

60,963

58,466

2,351

238,435

240,745

0
8,817

44,131

$\begin{array}{r}1,418 \\ \hline\end{array}$

NA

NA

NA

NA

1993

279,562

267,594

$1,036,22$

4,711
0

4,997
0

$1,320,498$

$1,469,266$

0
2,879
0

122,566

66,196

71,602

4,351

264,715

269,716

267,594

279,562

0
11,072

56,873

46,608
2,607

1,246

134,172

61,652

31,101

1,374
0

122,566

63,080

13,635

1,243

8,018

31,101

3,723

3,116

13,635

1,911

NA

$1,220,203$

125,174

$\begin{array}{r}2,989 \\ \hline\end{array}$

$1,225,921$

125,571

3,040

$1,281,007$

132,378

3,115

96
487

487
19,560

109

555
20,060

96
500

22,986

NA $=$ Not Available.

Notes: Deliveries to electric utilities (consumption) are reported on the Form ElA-176, "Annual Report of Natural and Supplemental Gas Supply and Disposition." See the

discussion on electric utility data and Table A1 in Appendix A for a comparison of reporting to these two forms. Totals may nof add due to independent rounding.

Sources: Energy Information Administration (EIA), Form ElA-176, "Annual Report of Natural and Supplemental Gas Supply and Disposition"; Form EIA-627, "Annual

Quantity and Value of Natural Gas Report"; Form EiA-857, "Monthly Report of Natural Gas Purchases and Deliveries to Consumers"; Form EIA-816, "Monthly Natural Gas

Liquids Report"; Form EIA-759, "Monthly Power Plant Report"; Form FERC-423, "Monthly Report of Cost and Quality of Fuels for Electric Plants"; Form EIA-191, "Underground

Gas Storage Report"; Form FPC-14, "Annual Report for Importers and Exporters of Natural Gas"; U.S. Crude Oil, Natural Gas, and Natural Gas Liquids Reserves, 1994 Annual

Report, DOE/EIA-0216(94); and the U.S. Minerals Management Service. 


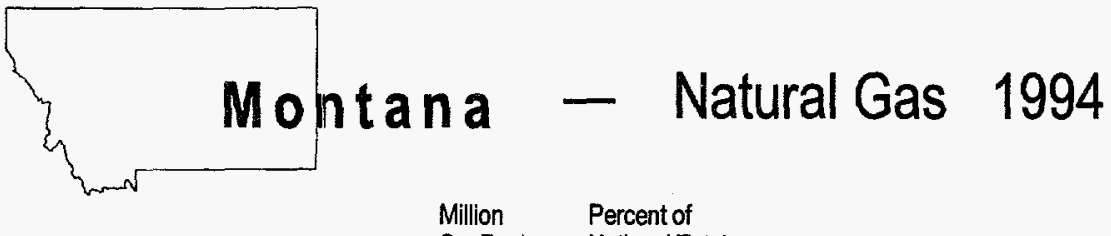

Cu. Feet National Total

Million

Cu. Feet

Percent of National Total

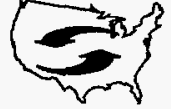

Net Interstate

Movements:

1,516

Marketed

Production:

50,416

0.26

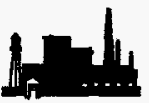

Industrial:

13,940

0.17
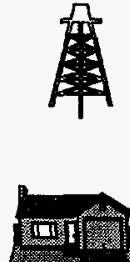

Deliveries to Consumers:

Residential:

18,714

0.39

Commercial:

12,981

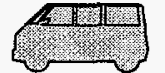

Vehicle Fuel:

6

0.35

Commercia:

12,981

\section{Table 74. Summary Statistics for Natural Gas - Montana, 1990-1994}

\begin{tabular}{|c|c|c|c|c|c|}
\hline & 1990 & 1991 & 1992 & 1993 & 1994 \\
\hline 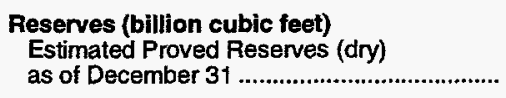 & 899 & 831 & 859 & 673 & 717 \\
\hline $\begin{array}{l}\text { Number of Gas and Gas Condensate Wells } \\
\text { Producing at End of Year.................................... }\end{array}$ & 2,607 & 2,802 & 2,890 & 3,075 & 2,940 \\
\hline 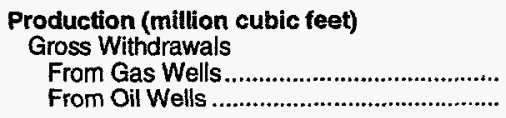 & $\begin{array}{r}42,369 \\
9,168\end{array}$ & $\begin{array}{r}44,449 \\
8,553\end{array}$ & $\begin{array}{r}46,918 \\
7,892\end{array}$ & $\begin{array}{r}48,187 \\
7,330\end{array}$ & $\begin{array}{r}44,350 \\
6,722\end{array}$ \\
\hline 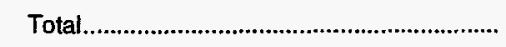 & 51,537 & 53,002 & 54,810 & 55,517 & 51,072 \\
\hline 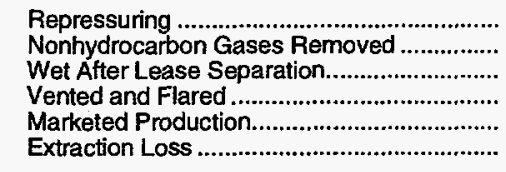 & $\begin{array}{r}222 \\
\mathrm{NA} \\
51,315 \\
886 \\
50,429 \\
1,091\end{array}$ & $\begin{array}{r}231 \\
\mathrm{NA} \\
52,771 \\
772 \\
51,999 \\
1,055\end{array}$ & $\begin{array}{r}180 \\
\text { NA } \\
54,630 \\
763 \\
53,867 \\
907\end{array}$ & $\begin{array}{r}231 \\
N A \\
55,286 \\
758 \\
54,528 \\
741\end{array}$ & $\begin{array}{r}105 \\
N A \\
50,967 \\
551 \\
50,416 \\
631\end{array}$ \\
\hline 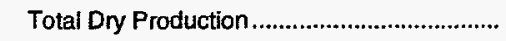 & 49,338 & 50,944 & 52,960 & 53,787 & 49,785 \\
\hline 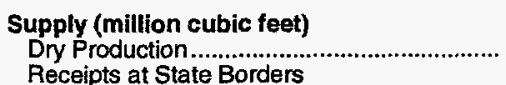 & 49,338 & 50,944 & 52,960 & 53,787 & 49,785 \\
\hline 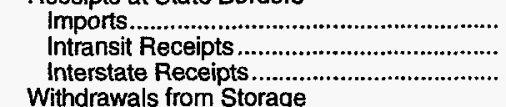 & $\begin{array}{r}343,716 \\
0 \\
28,628\end{array}$ & $\begin{array}{r}393,463 \\
0 \\
22,128\end{array}$ & $\begin{array}{r}467,036 \\
0 \\
21,791\end{array}$ & $\begin{array}{r}511,294 \\
0 \\
19,293\end{array}$ & $\begin{array}{r}535,855 \\
0 \\
20,408\end{array}$ \\
\hline 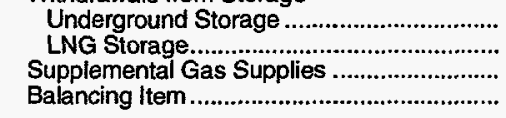 & $\begin{array}{r}21,786 \\
0 \\
0 \\
-1,241\end{array}$ & $\begin{array}{r}22,129 \\
0 \\
0 \\
-6,025\end{array}$ & $\begin{array}{r}24,310 \\
0 \\
0 \\
-15,444\end{array}$ & $\begin{array}{r}32,202 \\
0 \\
0 \\
-22,077\end{array}$ & $\begin{array}{r}22,509 \\
0 \\
0 \\
-7,062\end{array}$ \\
\hline 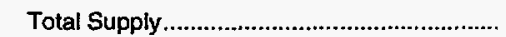 & 442,227 & 482,639 & 550,653 & 594,498 & 621,495 \\
\hline
\end{tabular}

See footnotes at end of table. 
Table 74. Summary Statistics for Natural Gas - Montana, 1990-1994 (Continued)

\begin{tabular}{|c|c|c|c|c|c|}
\hline & 1990 & 1991 & 1992 & 1993 & 1994 \\
\hline \multicolumn{6}{|l|}{ Disposition (million cubic feet) } \\
\hline \multicolumn{5}{|l|}{ Deliveries at State Borders } & 52,058 \\
\hline 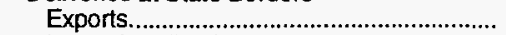 & 75 & 41 & 14 & 106 & 3,087 \\
\hline 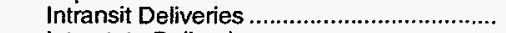 & 16,013 & 16,298 & 14,554 & 0 & 0 \\
\hline Interstate Deliveries ......................................... & 368,321 & 408,507 & 478,815 & 530,201 & 551,660 \\
\hline \multicolumn{6}{|l|}{ Additions to Storage } \\
\hline Underground Storage ................................. & 14,648 & 12,392 & 11,708 & 10,894 & 14,690 \\
\hline 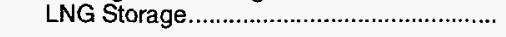 & & 0 & 0 & 0 & \\
\hline 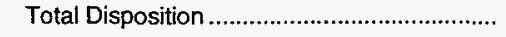 & 442,227 & 482,639 & 550,653 & 594,498 & 621,495 \\
\hline \multicolumn{6}{|l|}{ Consumption (million cubic feet) } \\
\hline 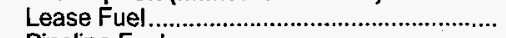 & 1,705 & 1,162 & 1,448 & 2,084 & 2,037 \\
\hline 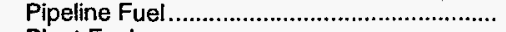 & 2,051 & 2,321 & 3,019 & 3,715 & 3,542 \\
\hline 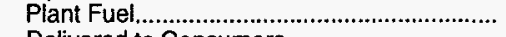 & 557 & 518 & 423 & 295 & 206 \\
\hline \multicolumn{6}{|l|}{ Delivered to Consumers } \\
\hline Residential & 16,850 & 18,413 & 16,673 & 20,360 & 18,714 \\
\hline 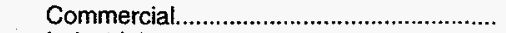 & 12,164 & 12,846 & 11,557 & 13,880 & 12,981 \\
\hline 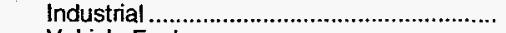 & 9,424 & 9,873 & 12,218 & 12,690 & 13,940 \\
\hline 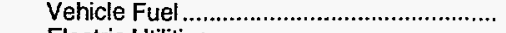 & & 2 & 2 & 4 & 6 \\
\hline 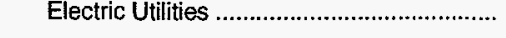 & 418 & 268 & 220 & 270 & 632 \\
\hline Total Delivered to Consumers ....................... & 38,856 & 41,401 & 40,671 & 47,204 & 46,274 \\
\hline 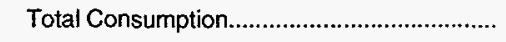 & 43,169 & 45,402 & 45,561 & 53,298 & 52,058 \\
\hline \multicolumn{6}{|l|}{$\begin{array}{l}\text { Delivered for the Account of Others } \\
\text { (million cubic feet) }\end{array}$} \\
\hline 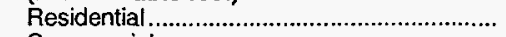 & 0 & 0 & 0 & 0 & 0 \\
\hline 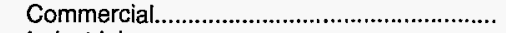 & 261 & 327 & 533 & 939 & 1,070 \\
\hline 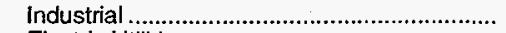 & 3,306 & 4,493 & 10,078 & 11,407 & 13,395 \\
\hline 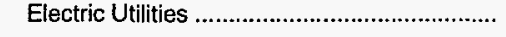 & 118 & 178 & 99 & 155 & 139 \\
\hline \multicolumn{6}{|l|}{$\begin{array}{l}\text { Firm Deliveries } \\
\text { (million cubic feet) }\end{array}$} \\
\hline 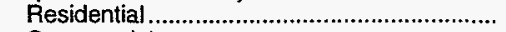 & NA & NA & NA & 20,360 & 18,714 \\
\hline 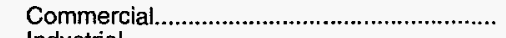 & NA & NA & NA & 13,576 & 12,678 \\
\hline 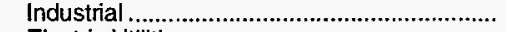 & NA & NA & NA & 7,431 & 8,360 \\
\hline Electric Utilities .............................................. & NA & NA & NA & 115 & 523 \\
\hline 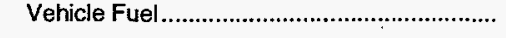 & NA & NA & NA & 4 & 6 \\
\hline \multirow{2}{*}{\multicolumn{6}{|c|}{$\begin{array}{l}\text { Interruptible Deliveries } \\
\text { (million cubic feet) }\end{array}$}} \\
\hline & & & & & \\
\hline 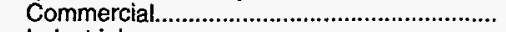 & NA & NA & NA & 304 & 303 \\
\hline 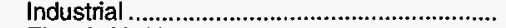 & NA & NA & NA & 7,431 & 8,360 \\
\hline 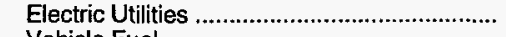 & NA & NA & NA & 155 & 139 \\
\hline 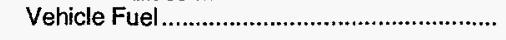 & NA & NA & NA & -. & $\cdot \cdots$ \\
\hline \multicolumn{6}{|l|}{ Number of Consumers } \\
\hline 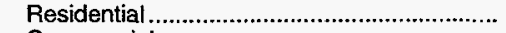 & 174,384 & 177,726 & 182,641 & 188,879 & 194,357 \\
\hline Commercial. & 23,331 & 23,185 & 23,610 & 24,373 & 25,349 \\
\hline 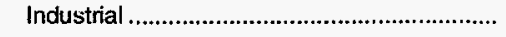 & 457 & 452 & 459 & 462 & 453 \\
\hline \multicolumn{6}{|l|}{$\begin{array}{l}\text { Average Annual Consumption per Consumer } \\
\text { (thousand cubic feet) }\end{array}$} \\
\hline 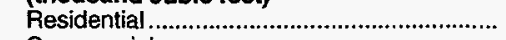 & 97 & 104 & 91 & 108 & 96 \\
\hline 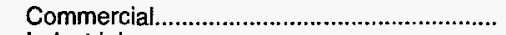 & 521 & 554 & 490 & 569 & 512 \\
\hline 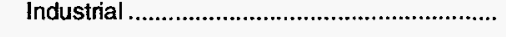 & 20,622 & 21,842 & 26,620 & 27,469 & 30,773 \\
\hline \multicolumn{6}{|l|}{$\begin{array}{l}\text { Average Prices for Natural Gas } \\
\text { (dollars per thousand cubic feet) }\end{array}$} \\
\hline Wellhead (Marketed Production) ..................... & 1.79 & 1.66 & 1.62 & 1.55 & 1.46 \\
\hline 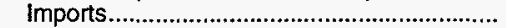 & 1.44 & 1.38 & 1.52 & 1.66 & 1.47 \\
\hline 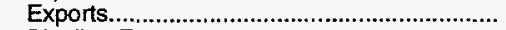 & 1.69 & 1.72 & 1.66 & 1.93 & 1.43 \\
\hline Pipeline Fuel & 1.75 & 1.76 & 1.63 & 2.15 & 1.53 \\
\hline 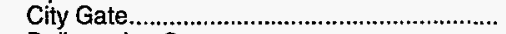 & 3.30 & 3.69 & 3.45 & 3.29 & 3.49 \\
\hline \multicolumn{6}{|l|}{ Delivered to Consumers } \\
\hline 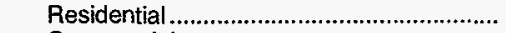 & 4.59 & 4.52 & 4.80 & 4.92 & 5.23 \\
\hline Commercial. & 4.64 & 4.35 & 4.46 & 4.67 & 4.91 \\
\hline 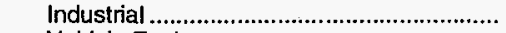 & 3.27 & 3.22 & 4.19 & 2.76 & 4.91 \\
\hline Vehicle Fuel ............................................... & 4.59 & 4.50 & 4.51 & 5.17 & 4.34 \\
\hline 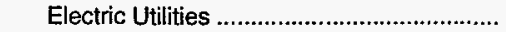 & 1.77 & 4.33 & 3.30 & 2.83 & 1.21 \\
\hline
\end{tabular}

NA = Not Available.

Notes: Deliveries to electric utilities (consumption) are reported on the Form ElA-176, "Annual Report of Natural and Supplemental Gas Supply and Disposition." See the Notes: Deliveries to electric utilities (consumption) are reported on the Form ElA-176, "Annual Report of Natural and Supplemental Gas Supply and Dispositon."
discussion on electric utility data and Table A1 in Appendix A for a comparison of reporting to these two forms. Totals may not add due to independent rounding

discussion on electric utility data and Table A1 in Appendix A for a comparison of reporting to these two forms. Totals may not add due to independent rounding.
Sources: Energy Information Administration (EIA). Form ElA-176, "Annual Report of Natural and Supplemental Gas Supply and Disposition"; Form ElA-627, "Annual

Sources: Energy Information Administration (EIA), Form EIA-176, "Annual Report of Natural and Supplemental Gas Supply and Disposition"; Form ElA-627, "Annual
Quantity and Value of Natural Gas Report"; Form ElA-857, "Monthly Report of Natural Gas Purchases and Deliveries to Consumers"; Form ElA-816, "Monthly Natural Gas

Quantity and Value of Natural Gas Report"; Form ElA-857, "Monthly Report of Natural Gas Purchases and Deliveries to Consumers"; Form EIA-816, "Monthly Natural Gas
Liquids Report"; Form EIA-759, "Monthly Power Plant Report"; Form FERC-423, "Monthly Report of Cost and Quality of Fuels for Electric Plants"; Form EIA-191, "Underground

Giquids Report"; Form ElA-759, "Monthly Power Plant Report"; Form FERC-423, "Monthly Report of Cost and Quality of Fuels for Electric Plants"; Form ElA-191, "Underground

Report, DOE/EIA-0216(94); and the U.S. Minerals Management Service. 


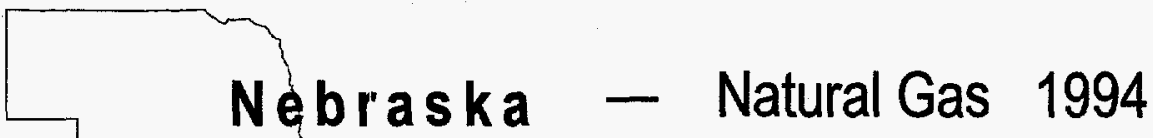

Million Percent of

Million

Cu. Feet

Percent of

Cu. Feet National Total

National Total

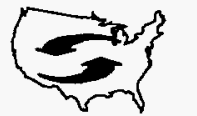

Net Interstate

Movements:

150,340

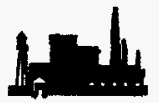

Industrial:

36,960

0.45

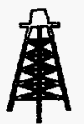

Marketed

Production:

2,898

0.01

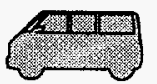

Vehicle Fuel:

9

0.52

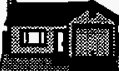

Deliveries to Consumers:

Residential

44,397

0.92

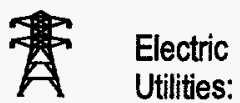

Commercial:

38,946

1.35

Total:

123,373

0.65

Table 75. Summary Statistics for Natural Gas - Nebraska, 1990-1994

\begin{tabular}{|c|c|c|c|c|c|}
\hline & 1990 & 1991 & 1992 & 1993 & 1994 \\
\hline $\begin{array}{l}\text { Peserves (billion cubic feet) } \\
\text { Estimated Proved Reserves (dry) } \\
\text { as of December } 31\end{array}$ & NA & NA & NA & NA & NA \\
\hline 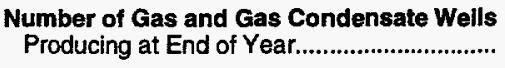 & 11 & 12 & 22 & 59 & 87 \\
\hline 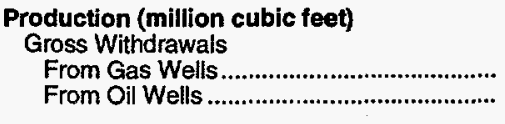 & $\begin{array}{l}114 \\
679\end{array}$ & $\begin{array}{l}126 \\
657\end{array}$ & $\begin{array}{l}486 \\
691\end{array}$ & $\begin{array}{r}1,391 \\
723\end{array}$ & $\begin{array}{r}2,093 \\
805\end{array}$ \\
\hline 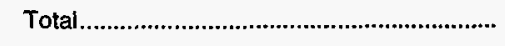 & 793 & 784 & 1,177 & 2,114 & 2,898 \\
\hline 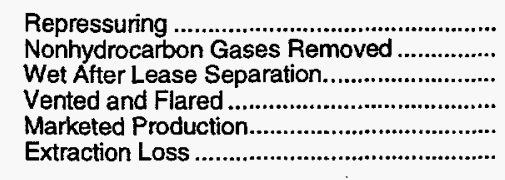 & $\begin{array}{r}* \\
793 \\
793 \\
*\end{array}$ & $\begin{array}{r}* \\
784 \\
784 \\
13\end{array}$ & $\begin{array}{r}* \\
\text { * } \\
1,177 \\
0 \\
1,177 \\
3\end{array}$ & $\begin{array}{r}* \\
2,114 \\
2,114 \\
0\end{array}$ & $\begin{array}{r}* \\
2,898 \\
2,898 \\
8\end{array}$ \\
\hline 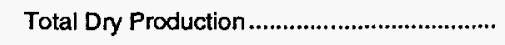 & 793 & 771 & 1,174 & 2,114 & 2,890 \\
\hline 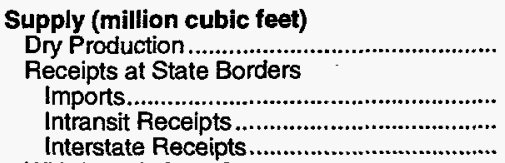 & $\begin{array}{r}0 \\
0 \\
970,896\end{array}$ & $\begin{array}{r}0 \\
0 \\
924,993\end{array}$ & $\begin{array}{r}0 \\
0 \\
1,021,817\end{array}$ & $\begin{array}{r}0 \\
0 \\
933,217\end{array}$ & $\begin{array}{r}0 \\
0 \\
1,170,211\end{array}$ \\
\hline 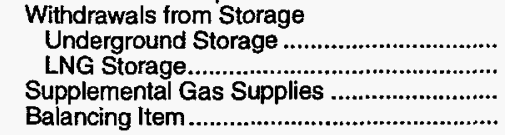 & $\begin{array}{r}9,772 \\
155 \\
2,361 \\
-19,191\end{array}$ & $\begin{array}{r}8,687 \\
211 \\
2,032 \\
-25,323\end{array}$ & $\begin{array}{r}9,848 \\
173 \\
1,437 \\
-18,422\end{array}$ & $\begin{array}{r}11,718 \\
222 \\
791 \\
2,039\end{array}$ & $\begin{array}{r}10,052 \\
367 \\
890 \\
-24,879\end{array}$ \\
\hline 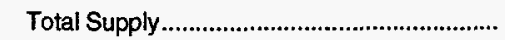 & 964,786 & 911,371 & $1,016,025$ & 950,100 & $1,159,532$ \\
\hline
\end{tabular}

See footnotes at end of table. 
Table 75. Summary Statistics for Natural Gas - Nebraska, 1990-1994 (Continued)

\begin{tabular}{|c|c|c|c|c|c|}
\hline & 1990 & 1991 & 1992 & 1993 & 1994 \\
\hline $\begin{array}{l}\text { Disposition (million cubic feet) } \\
\text { Consumption............................................ } \\
\text { Deliveries at State Borders }\end{array}$ & 111,063 & 115,808 & 106,853 & 126,112 & 126,744 \\
\hline 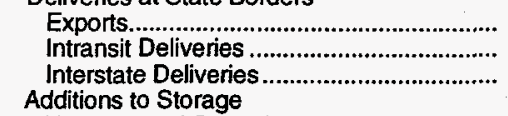 & $\begin{array}{r}0 \\
0 \\
845,834\end{array}$ & $\begin{array}{r}0 \\
0 \\
786,554\end{array}$ & $\begin{array}{r}0 \\
0 \\
898,635\end{array}$ & $\begin{array}{r}0 \\
0 \\
809,216\end{array}$ & $\begin{array}{r}0 \\
0 \\
1,019,871\end{array}$ \\
\hline 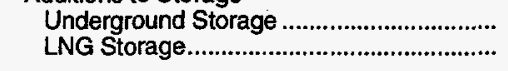 & $\begin{array}{r}7,395 \\
493\end{array}$ & $\begin{array}{r}8,916 \\
92\end{array}$ & $\begin{array}{r}10,254 \\
283\end{array}$ & $\begin{array}{r}14,485 \\
287\end{array}$ & $\begin{array}{r}12,524 \\
393\end{array}$ \\
\hline 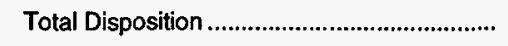 & 964,786 & 911,371 & $1,016,025$ & 950,100 & $1,159,532$ \\
\hline 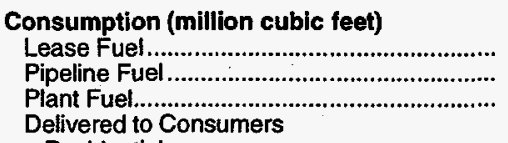 & $\begin{array}{r}26 \\
3,536 \\
0\end{array}$ & $\begin{array}{r}17 \\
2,368 \\
14\end{array}$ & $\begin{array}{r}31 \\
2,555 \\
9\end{array}$ & $\begin{array}{r}56 \\
2,520 \\
0\end{array}$ & $\begin{array}{r}86 \\
3,282 \\
3\end{array}$ \\
\hline 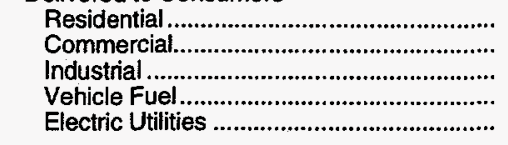 & $\begin{array}{r}41,499 \\
36,489 \\
25,746 \\
0 \\
3,766\end{array}$ & $\begin{array}{r}44,671 \\
40,291 \\
24,758 \\
0 \\
3,689\end{array}$ & $\begin{array}{r}41,414 \\
34,490 \\
26,451 \\
\star \\
1,903\end{array}$ & $\begin{array}{r}48,256 \\
\mathbf{R}_{34,745} \\
\mathbf{R}_{38,658} \\
1 \\
1,876\end{array}$ & $\begin{array}{r}44,397 \\
38,946 \\
36,960 \\
9 \\
3,061\end{array}$ \\
\hline Total Delivered to Consumers ......................... & 107,500 & 113,409 & 104,258 & 123,537 & 123,373 \\
\hline 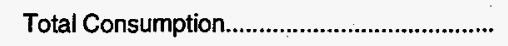 & 111,063 & 115,808 & 106,853 & 126,112 & 126,744 \\
\hline 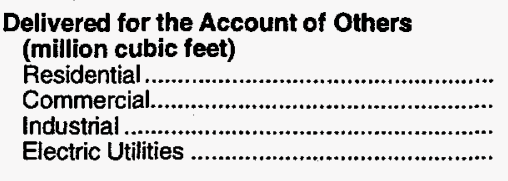 & $\begin{array}{r}0 \\
2,231 \\
14,304 \\
2,793\end{array}$ & $\begin{array}{r}0 \\
3,294 \\
13,266 \\
2,612\end{array}$ & $\begin{array}{r}0 \\
4,063 \\
15,558 \\
1,038\end{array}$ & $\begin{array}{r}0 \\
3,142 \\
28,068 \\
484\end{array}$ & $\begin{array}{r}0 \\
7,726 \\
28,971 \\
34\end{array}$ \\
\hline 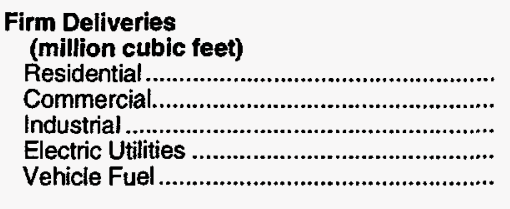 & $\begin{array}{l}\text { NA } \\
\text { NA } \\
\text { NA } \\
\text { NA } \\
\text { NA }\end{array}$ & $\begin{array}{l}\text { NA } \\
\text { NA } \\
\text { NA } \\
\text { NA } \\
\text { NA }\end{array}$ & $\begin{array}{l}\text { NA } \\
\text { NA } \\
\text { NA } \\
\text { NA } \\
\text { NA }\end{array}$ & $\begin{array}{r}48,256 \\
28,822 \\
10,428 \\
668 \\
1\end{array}$ & $\begin{array}{r}44,397 \\
33,550 \\
7,069 \\
55 \\
9\end{array}$ \\
\hline 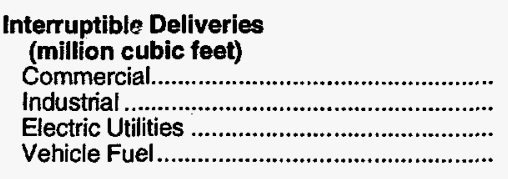 & $\begin{array}{l}\text { NA } \\
\text { NA } \\
\text { NA } \\
\text { NA }\end{array}$ & $\begin{array}{l}\text { NA } \\
\text { NA } \\
\text { NA } \\
\text { NA }\end{array}$ & $\begin{array}{l}\text { NA } \\
\text { NA } \\
\text { NA } \\
\text { NA }\end{array}$ & $\begin{array}{r}R_{5,923} \\
10,428 \\
615 \\
--\end{array}$ & $\begin{array}{r}5,396 \\
7,069 \\
563 \\
--\end{array}$ \\
\hline 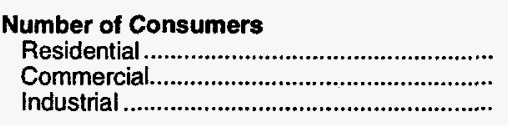 & $\begin{array}{r}407,094 \\
60,405 \\
712\end{array}$ & $\begin{array}{r}413,354 \\
60,947 \\
718\end{array}$ & $\begin{array}{r}418,611 \\
61,319 \\
696\end{array}$ & $\begin{array}{r}413,358 \\
R_{60,599} \\
R_{718}\end{array}$ & $\begin{array}{r}428,201 \\
62,045 \\
766\end{array}$ \\
\hline 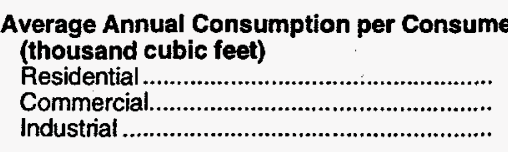 & $\begin{array}{r}102 \\
604 \\
36,160\end{array}$ & $\begin{array}{r}108 \\
661 \\
34,482\end{array}$ & $\begin{array}{r}99 \\
562 \\
38,005\end{array}$ & $\begin{array}{r}117 \\
573 \\
R_{53,842}\end{array}$ & $\begin{array}{r}104 \\
628 \\
48,251\end{array}$ \\
\hline 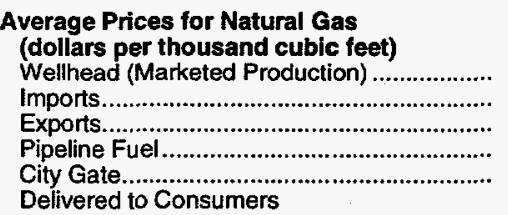 & $\begin{array}{r}2.26 \\
0 \\
0 \\
1.70 \\
2.95\end{array}$ & $\begin{array}{r}2.06 \\
0 \\
0 \\
1.43 \\
2.75\end{array}$ & $\begin{array}{r}1.78 \\
0 \\
0 \\
1.54 \\
2.91\end{array}$ & $\begin{array}{r}1.81 \\
0 \\
0 \\
1.79 \\
3.46\end{array}$ & $\begin{array}{r}1.60 \\
0 \\
0 \\
1.34 \\
2.98\end{array}$ \\
\hline 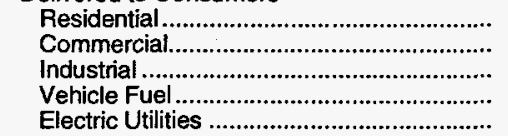 & $\begin{array}{r}4.60 \\
3.86 \\
2.97 \\
0 \\
1.90\end{array}$ & $\begin{array}{r}4.64 \\
3.87 \\
2.76 \\
0 \\
1.85\end{array}$ & $\begin{array}{r}4.82 \\
3.99 \\
2.92 \\
0 \\
2.28\end{array}$ & $\begin{array}{r}4.96 \\
4.27 \\
3.09 \\
0 \\
2.66\end{array}$ & $\begin{array}{l}5.01 \\
4.24 \\
3.12 \\
4.67 \\
2.02\end{array}$ \\
\hline
\end{tabular}

$R=$ Revised data.

NA = Not Available

$*$ Volume is less than 500,000 cubic feet.

Notes: Deliveries to electric utilities (consumption) are reported on the Form ElA-176, "Annual Report of Natural and Supplemental Gas Supply and Disposition." See the discussion on electric utility data and Table A1 in Appendix A for a comparison of reporting to these two forms. Totals may not add due to independent rounding.

Sources: Energy information Administration (EIA), Form EIA-176, "Annual Report of Natural and Supplemental Gas Supply and Disposition"; Form EIA-627, "Annual

Quantity and Value of Natural Gas Report"; Form EIA-857, "Monthly Report of Natural Gas Purchases and Deliveries to Consumers"; Form ElA-816, "Monthly Natural Gas

Liquids Report"; Form EIA-759, "Monthly Power Plant Peport"; Form FERC-423, "Monthly Report of Cost and Quality of Fuels for Electric Plants"; Form EIA-191, "Underground

Gas Storage Report"; Form FPC-14, "Annual Report for Importers and Exporters of Natural Gas"; U.S. Crude Oil, Natural Gas, and Natural Gas Liquids Reserves, 1994 Annual Report, DOE/EIA-0216(94); and the U.S. Minerals Management Service. 


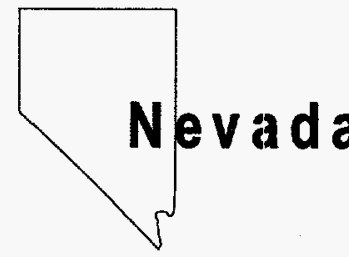

\section{- Natural Gas 1994}

Million Percent of

Cu. Feet National Total

Million

Cu. Feet

Percent of

National Total
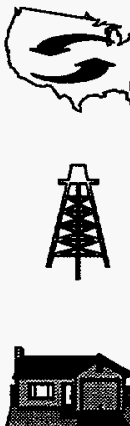

Net Interstate

Movements:

102,628

Marketed

Production:

Deliveries to Consumers:

Residential:

21,263

0.44

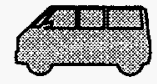

Vehicle Fuel:

36

Commercial:

18,694

0.65

Total:

101,105

Table 76. Summary Statistics for Natural Gas - Nevada, 1990-1994

\begin{tabular}{|c|c|c|c|c|c|}
\hline & 1990 & 1991 & 1992 & 1993 & 1994 \\
\hline 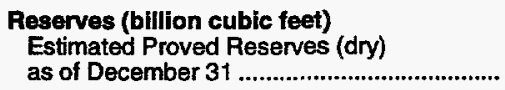 & 0 & 0 & 0 & 0 & 0 \\
\hline 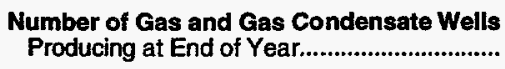 & 0 & 0 & 0 & 0 & 0 \\
\hline 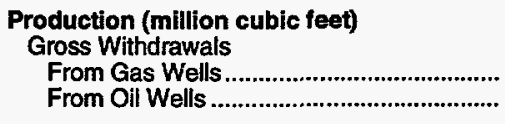 & $\begin{array}{l}0 \\
0\end{array}$ & $\begin{array}{r}0 \\
53\end{array}$ & $\begin{array}{r}0 \\
30\end{array}$ & $\begin{array}{r}0 \\
21\end{array}$ & $\begin{array}{r}0 \\
16\end{array}$ \\
\hline 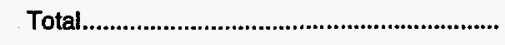 & 0 & 53 & 30 & 21 & 16 \\
\hline 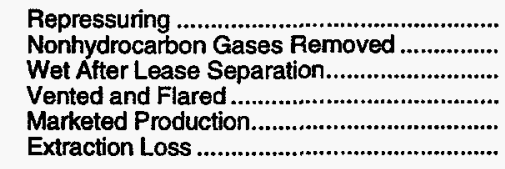 & $\begin{array}{l}0 \\
0 \\
0 \\
0 \\
0 \\
0\end{array}$ & $\begin{array}{r}0 \\
0 \\
53 \\
0 \\
53 \\
0\end{array}$ & $\begin{array}{r}0 \\
0 \\
30 \\
0 \\
30 \\
0\end{array}$ & $\begin{array}{r}0 \\
0 \\
21 \\
0 \\
21 \\
0\end{array}$ & $\begin{array}{r}0 \\
0 \\
16 \\
0 \\
16 \\
0\end{array}$ \\
\hline 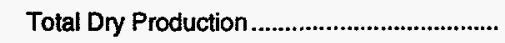 & 0 & 53 & 30 & 21 & 16 \\
\hline 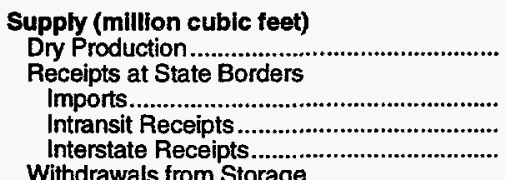 & $\begin{array}{r}0 \\
0 \\
68,994\end{array}$ & $\begin{array}{r}0 \\
0 \\
69,164\end{array}$ & $\begin{array}{r}0 \\
0 \\
259,899\end{array}$ & $\begin{array}{r}0 \\
0 \\
329,846\end{array}$ & $\begin{array}{r}0 \\
0 \\
348,147\end{array}$ \\
\hline 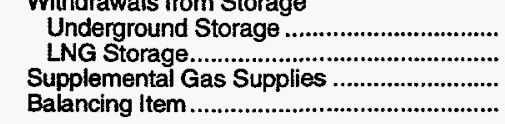 & $\begin{array}{r}0 \\
904 \\
125 \\
-2,243\end{array}$ & $\begin{array}{r}0 \\
39 \\
0 \\
-1,400\end{array}$ & $\begin{array}{r}0 \\
155 \\
30 \\
-10,782\end{array}$ & $\begin{array}{r}0 \\
244 \\
38 \\
-3,470\end{array}$ & $\begin{array}{r}0 \\
271 \\
9 \\
-893\end{array}$ \\
\hline Total Supply & 67,780 & 67,856 & 249,332 & 326,679 & 347,550 \\
\hline
\end{tabular}


Table 76. Summary Statistics for Natural Gas - Nevada, 1990-1994 (Continued)

\begin{tabular}{|c|c|c|c|c|c|}
\hline & 1990 & 1991 & 1992 & 1993 & 1994 \\
\hline 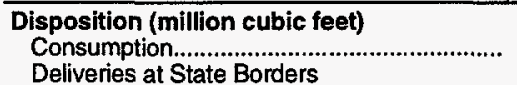 & 64,876 & 64,859 & 68,416 & 84,955 & 101,790 \\
\hline 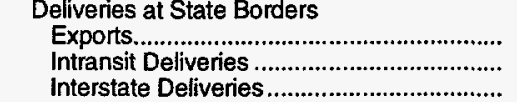 & $\begin{array}{r}0 \\
0 \\
2,548\end{array}$ & $\begin{array}{r}0 \\
0 \\
2,230\end{array}$ & $\begin{array}{r}0 \\
0 \\
180,832\end{array}$ & $\begin{array}{r}0 \\
0 \\
241,471\end{array}$ & $\begin{array}{r}0 \\
0 \\
245,519\end{array}$ \\
\hline 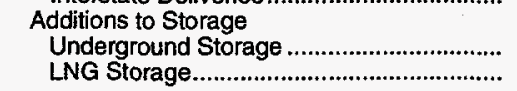 & $\begin{array}{r}0 \\
356\end{array}$ & $\begin{array}{r}0 \\
767\end{array}$ & $\begin{array}{r}0 \\
83\end{array}$ & $\begin{array}{r}0 \\
253\end{array}$ & $\begin{array}{r}0 \\
241\end{array}$ \\
\hline 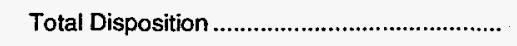 & 67,780 & 67,856 & 249,332 & 326,679 & 347,550 \\
\hline 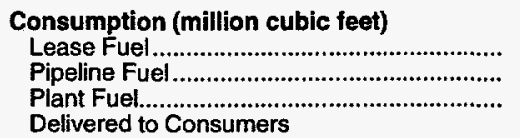 & $\begin{array}{r}0 \\
791 \\
0\end{array}$ & $\begin{array}{r}53 \\
341 \\
0\end{array}$ & $\begin{array}{r}30 \\
474 \\
0\end{array}$ & $\begin{array}{r}21 \\
641 \\
0\end{array}$ & $\begin{array}{r}16 \\
669 \\
0\end{array}$ \\
\hline 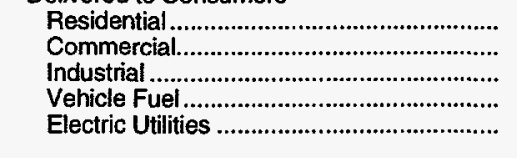 & $\begin{array}{r}17,153 \\
15,073 \\
7,511 \\
0 \\
24,348\end{array}$ & $\begin{array}{r}19,135 \\
16,960 \\
6,624 \\
8 \\
21,738\end{array}$ & $\begin{array}{r}18,184 \\
16,101 \\
9,259 \\
12 \\
24,355\end{array}$ & $\begin{array}{r}20,683 \\
17,549 \\
24,734 \\
21 \\
21,305\end{array}$ & $\begin{array}{r}21,263 \\
18,694 \\
28,867 \\
36 \\
32,246\end{array}$ \\
\hline Total Delivered to Consumers ............................. & 64,086 & 64,465 & 67,912 & 84,293 & 101,105 \\
\hline 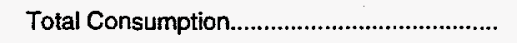 & 64,876 & 64,859 & 68,416 & 84,955 & 101,790 \\
\hline 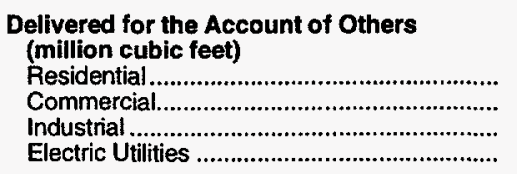 & $\begin{array}{r}0 \\
346 \\
6,457 \\
23,193\end{array}$ & $\begin{array}{r}0 \\
1,563 \\
5,687 \\
21,403\end{array}$ & $\begin{array}{r}0 \\
1,889 \\
8,569 \\
28,675\end{array}$ & $\begin{array}{r}0 \\
1,283 \\
23,660 \\
21,066\end{array}$ & $\begin{array}{r}0 \\
3,276 \\
28,312 \\
32,377\end{array}$ \\
\hline 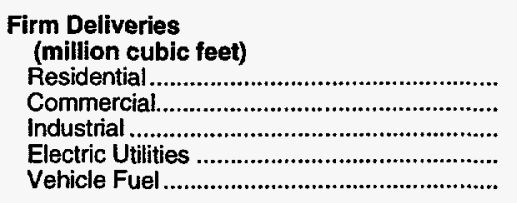 & $\begin{array}{l}\text { NA } \\
\text { NA } \\
\text { NA } \\
\text { NA } \\
\text { NA }\end{array}$ & $\begin{array}{l}\text { NA } \\
\text { NA } \\
\text { NA } \\
\text { NA } \\
\text { NA }\end{array}$ & $\begin{array}{l}\text { NA } \\
\text { NA } \\
\text { NA } \\
\text { NA } \\
\text { NA }\end{array}$ & $\begin{array}{r}20,683 \\
17,305 \\
0 \\
19,155 \\
21\end{array}$ & $\begin{array}{r}21,263 \\
18,407 \\
95 \\
31,074 \\
20\end{array}$ \\
\hline 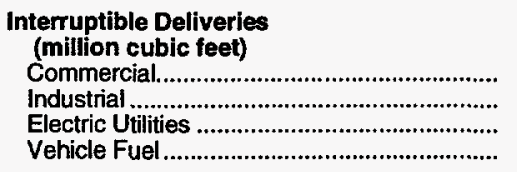 & $\begin{array}{l}\text { NA } \\
\text { NA } \\
\text { NA } \\
\text { NA }\end{array}$ & $\begin{array}{l}\text { NA } \\
\text { NA } \\
\text { NA } \\
\text { NA }\end{array}$ & $\begin{array}{l}\text { NA } \\
\text { NA } \\
\text { NA } \\
\text { NA }\end{array}$ & $\begin{array}{r}245 \\
1,923 \\
-\end{array}$ & $\begin{array}{r}286 \\
95 \\
1,303 \\
16\end{array}$ \\
\hline 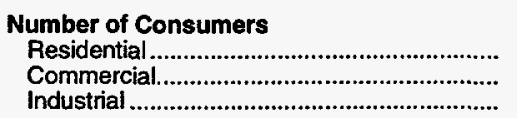 & $\begin{array}{r}256,119 \\
20,694 \\
100\end{array}$ & $\begin{array}{r}283,307 \\
22,124 \\
113\end{array}$ & $\begin{array}{r}295,714 \\
22,799 \\
114\end{array}$ & $\begin{array}{r}305,099 \\
23,207 \\
117\end{array}$ & $\begin{array}{r}336,353 \\
24,521 \\
119\end{array}$ \\
\hline 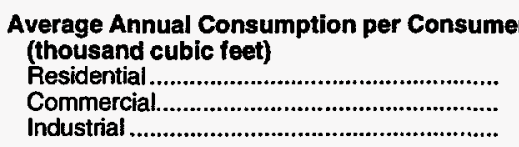 & $\begin{array}{r}67 \\
728 \\
75,114\end{array}$ & $\begin{array}{r}68 \\
767 \\
58,621\end{array}$ & $\begin{array}{r}61 \\
706 \\
81,222\end{array}$ & $\begin{array}{r}68 \\
756 \\
211,398\end{array}$ & $\begin{array}{r}63 \\
762 \\
242,577\end{array}$ \\
\hline 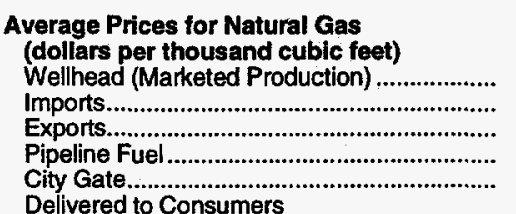 & $\begin{array}{r}0 \\
0 \\
1.70 \\
2.75\end{array}$ & $\begin{array}{r}0 \\
0 \\
0 \\
1.74 \\
2.33\end{array}$ & $\begin{array}{r}0 \\
0 \\
0 \\
1.77 \\
2.37\end{array}$ & $\begin{array}{r}0 \\
0 \\
0 \\
1.79 \\
3.03\end{array}$ & $\begin{array}{r}0 \\
0 \\
0 \\
1.87 \\
3.18\end{array}$ \\
\hline 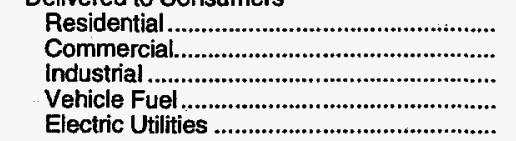 & $\begin{array}{r}5.66 \\
4.38 \\
4.10 \\
0 \\
2.02\end{array}$ & $\begin{array}{l}5.61 \\
4.34 \\
4.21 \\
3.72 \\
1.78\end{array}$ & $\begin{array}{l}5.59 \\
4.33 \\
4.07 \\
3.45 \\
1.91\end{array}$ & $\begin{array}{l}5.69 \\
4.40 \\
4.30 \\
3.34 \\
2.45\end{array}$ & $\begin{array}{l}6.66 \\
5.36 \\
5.67 \\
3.81 \\
1.99\end{array}$ \\
\hline
\end{tabular}

NA = Not Available.

Notes: Deliveries to electric utilities (consumption) are reported on the Form ElA-176, "Annual Report of Natural and Supplemental Gas Supply and Disposition." See the discussion on electric utility data and Table A1 in Appendix A for a comparison of reporting to these two forms. Totals may not add due to independent rounding.

Sources: Energy Information Administration (EIA), Form EIA-176, "Annual Report of Natural and Supplemental Gas Supply and Disposition"; Form EIA-627, "Annual Quantity and Value of Natural Gas Report"; Form EiA-857, "Monthly Report of Natural Gas Purchases and Dellveries to Consumers"; Form ElA-816, "Monthly Natural Gas Liquids Report"; Form ElA-759, "Monthly Power Plant Report"; Form FERC-423, "Monthly Report of Cost and Quality of Fuels for Electric Plants"; Form ElA-191, "Underground Gas Storage Report"; Form FPC-14, "Annual Report for Importers and Exporters of Natural Gas"; U.S. Cnude Oil, Natural Gas, and Natural Gas Liquids Reserves, 1994 Annual Report, DOOE/EIA-0216(94); and the U.S. Minerals Management Service. 


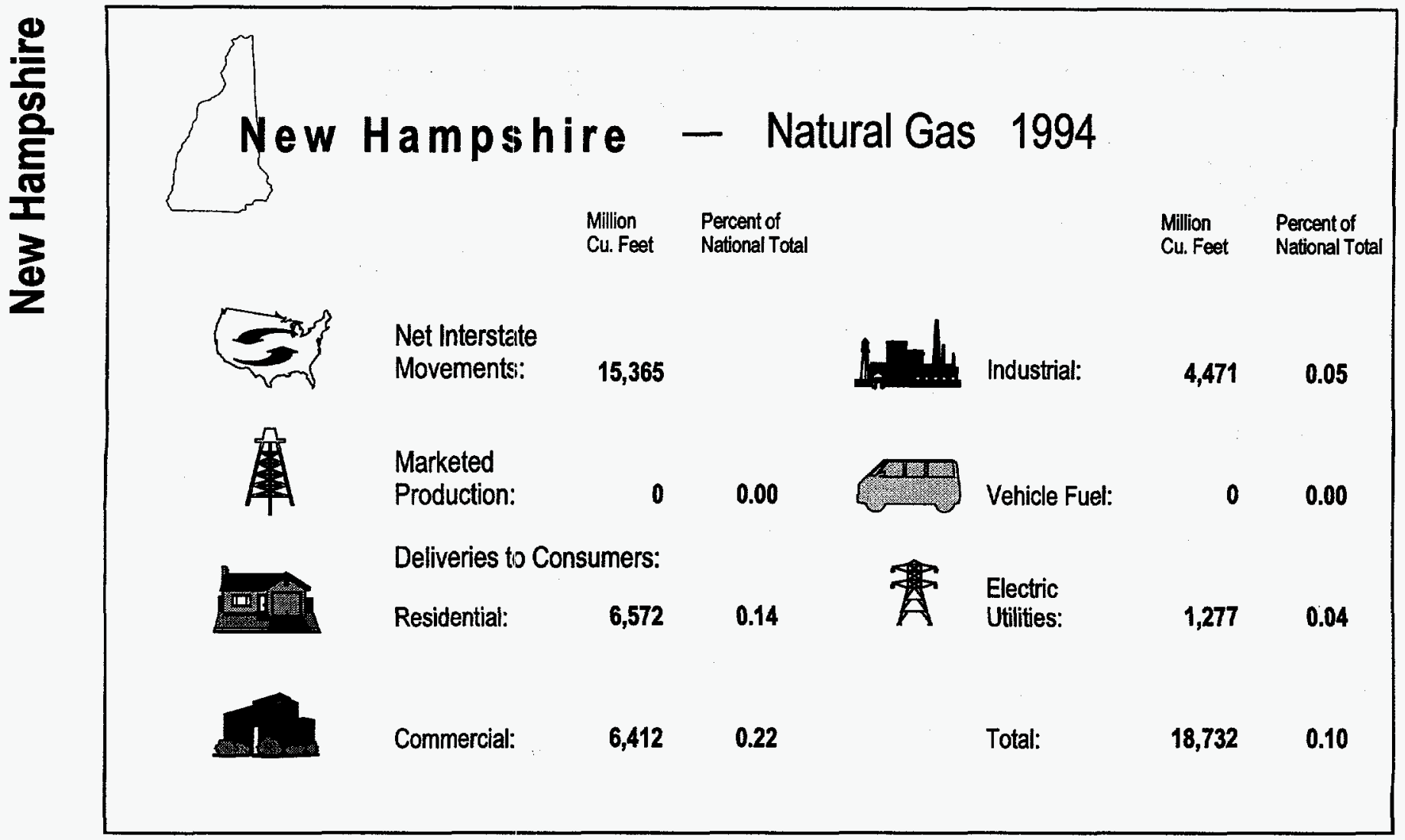

Table 77. Summary Statistics for Natural Gas - New Hampshire, 1990-1994

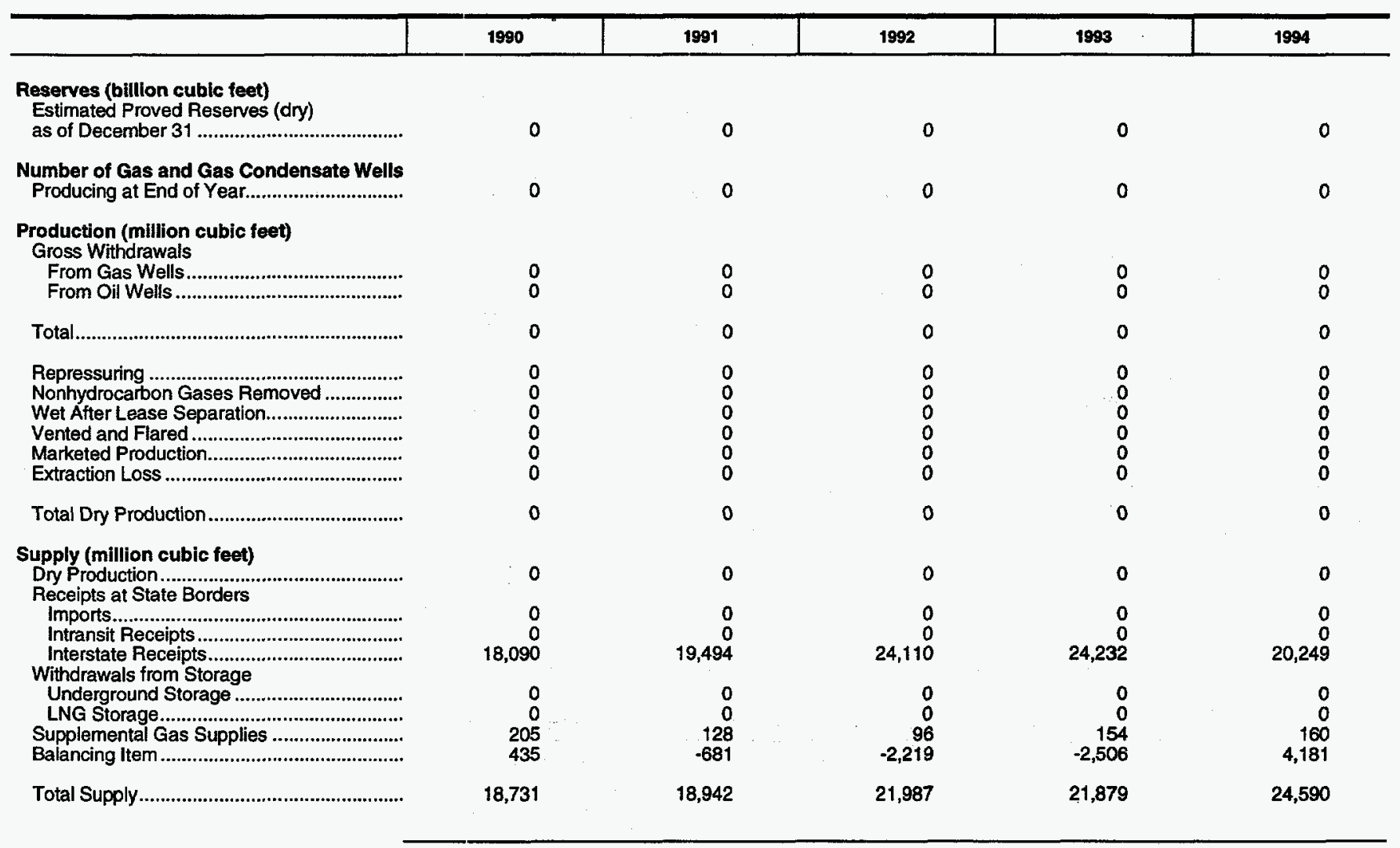

See footnotes at end of table. 
Table 77. Summary Statistics for Natural Gas - New Hampshire, 1990-1994 (Continued)

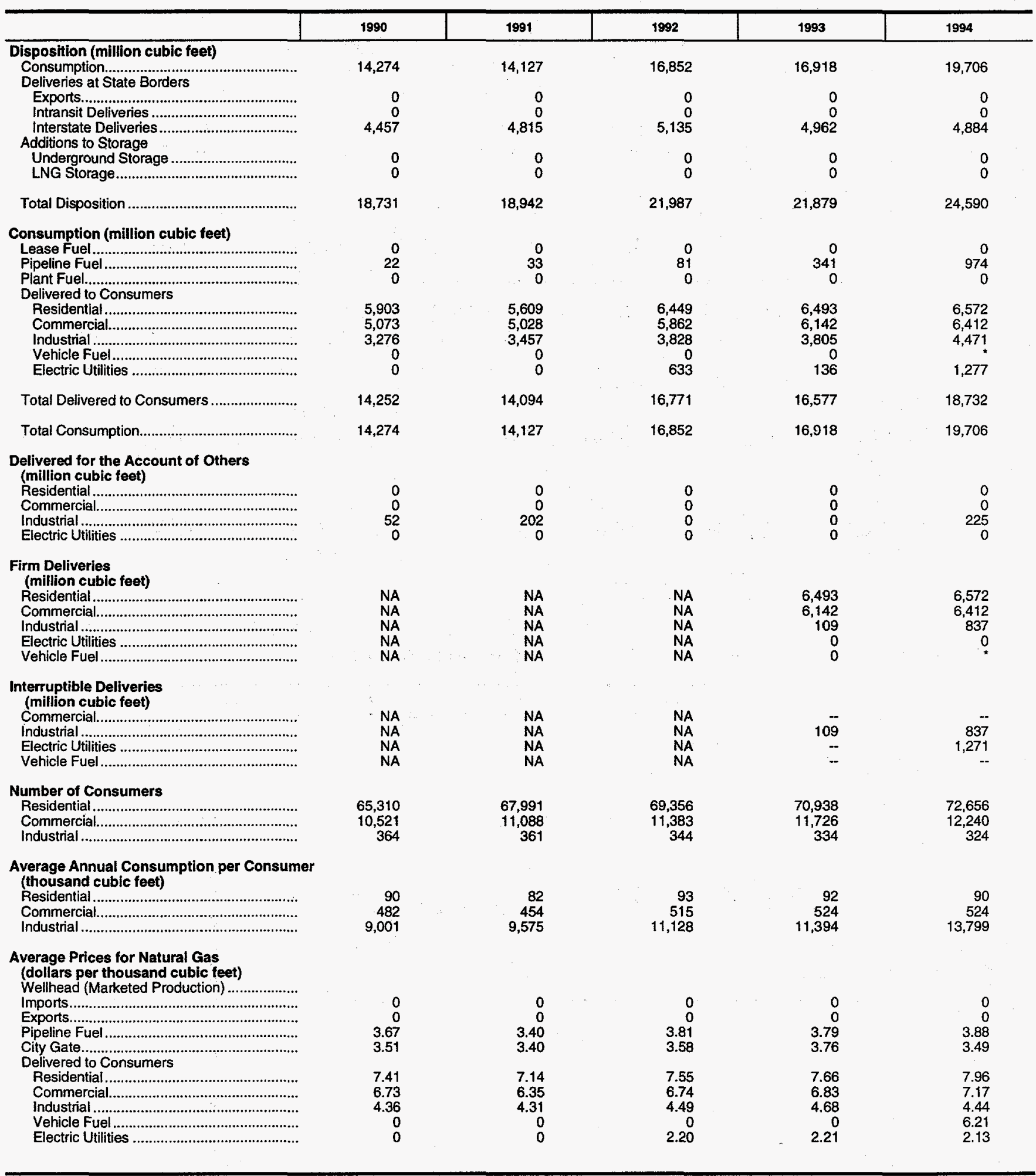

NA = Not Available.

Notes: Deliveries to electric utilities (consumption) are reported on the Form ElA-176, "Annual Report of Natural and Supplemental Gas Supply and Disposition." See the discussion on electric utility data and Table A1 in Appendix A for a comparison of reporting to these two forms. Totals may not add due to independent rounding.

Sources: Energy Information Administration (EIA). Form EIA-176, "Annual Report of Natural and Supplemental Gas Supply and Disposition"; Form EIA-627, "Annual

Quantity and Value of Natural Gas Report"; Form EIA-857, "Monthly Report of Natural Gas Purchases and Deliveries to Consumers"; Form EIA-816, "Monthly Natural Gas

Liquids Report"; Form ElA-759, "Monthly Power Plant Report"; Form FERC-423, "Monthly Report of Cost and Quality of Fuels for Electric Plants"; Form EIA-191, "Underground

Gas Storage Report"; Form FPC-14, "Annual Report for Importers and Exporters of Natural Gas"; U.S. Crude Oil, Natural Gas, and Natural Gas Liquids Reserves, 1994 Annual

Report, DOE/EIA-0216(94); and the U.S. Minerals Management Service. 


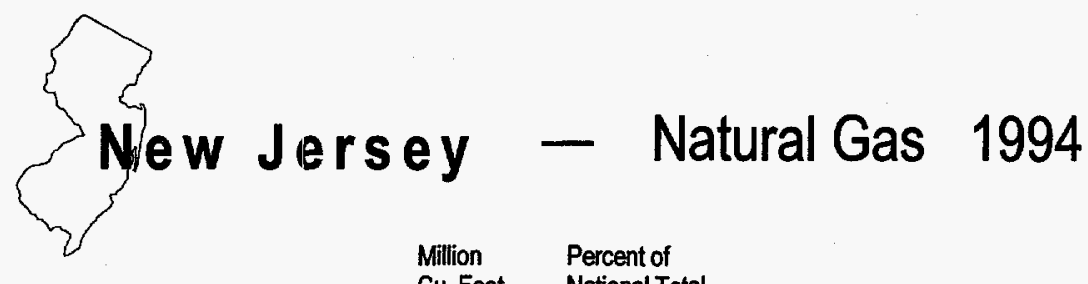

Million

Percent of

Cu. Feet National Total

Cu. Feet

National Total

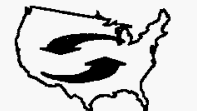

Net Interstate

Movements:

530,317

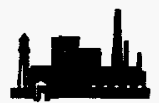

Industrial:

190,845

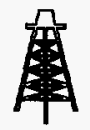

Marketed

Production:

0.00

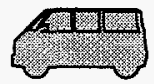

Vehicle Fuel:

4

0.23

Deliveries to Consumers:

TाTा)

Residential:

216,873

4.47

Electric

42,625

1.43

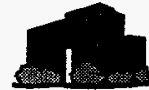

Commercial: $\quad 132,008$

4.56

Total:

582,356

3.08

Table 78. Summary Statistics for Natural Gas - New Jersey, 1990-1994

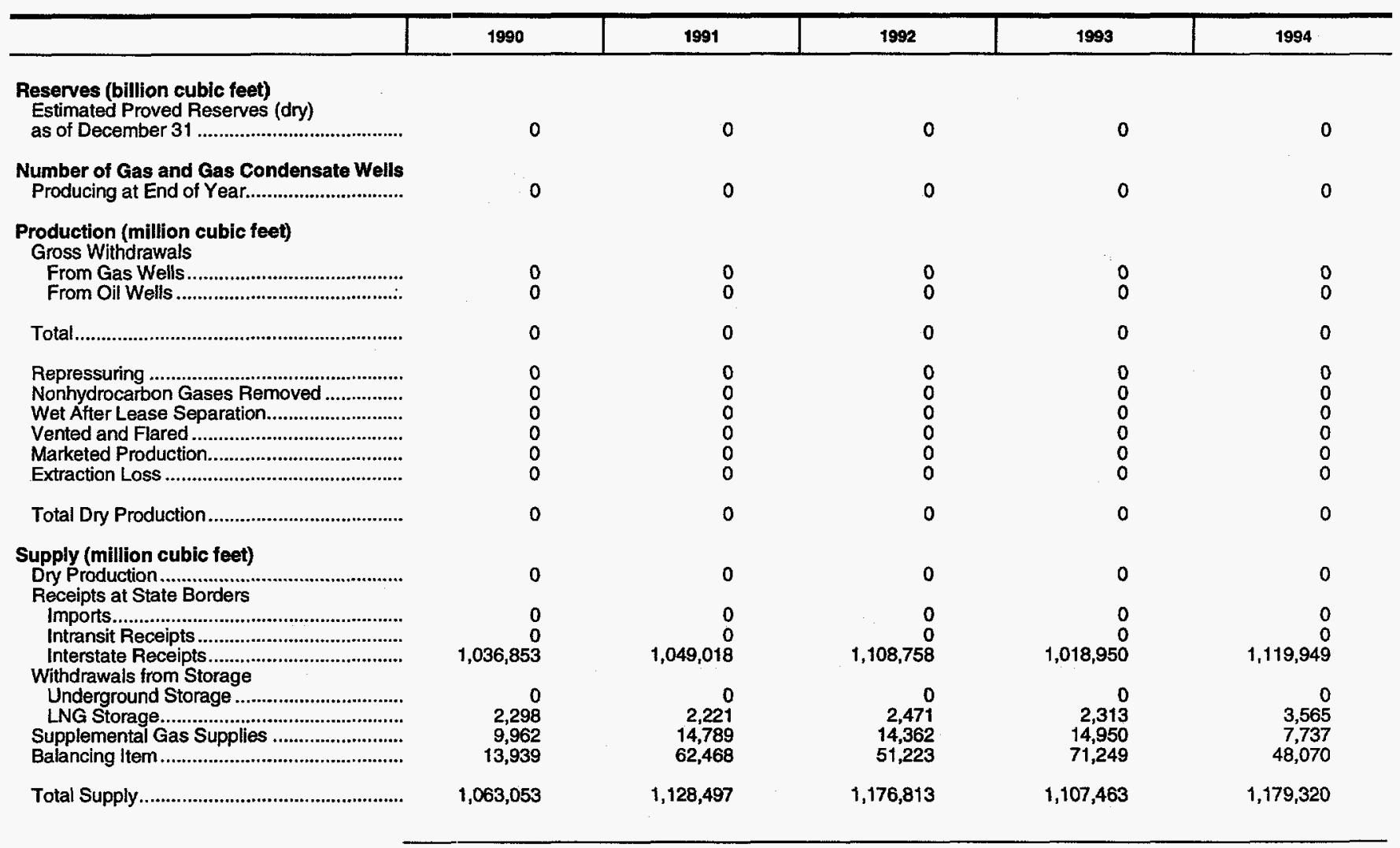

See footnotes at end of table. 
Table 78. Summary Statistics for Natural Gas - New Jersey, 1990-1994 (Continued)

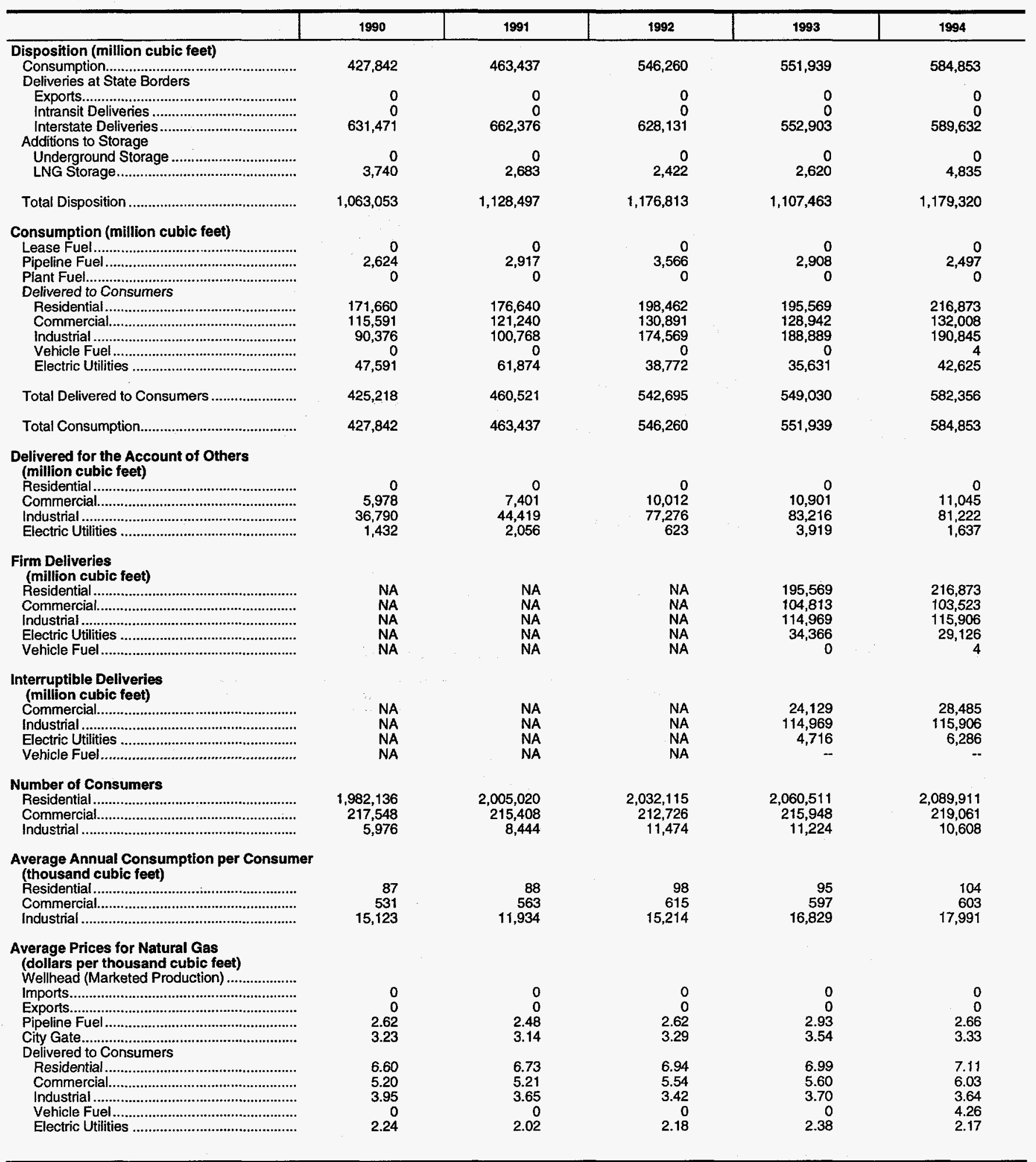

NA $=$ Not Available.

Notes: Deliveries to electric utilities (consumption) are reported on the Form ElA-176, "Annual Report of Natural and Supplemental Gas Supply and Disposition." See the discussion on electric utility data and Table A1 in Appendix A for a comparison of reporting to these two forms. Totals may not add due to independent rounding.

Sources: Energy Information Administration (EIA), Form EIA-176, "Annual Report of Natural and Supplemental Gas Supply and Disposition"; Form EIA-627, "Annual. Quantity and Value of Natural Gas Report"; Form ElA-857, "Monthly Report of Natural Gas Purchases and Deliveries to Consumers"; Form EIA-816, "Monthly Nafural Gas Liquids Report"; Form EIA-759, "Monthly Power Plant Report"; Form FERC-423, "Monthly Report of Cost and Quality of Fuels for Electric Plants"; Form EIA-191, "Underground Gas Storage Report"; Form FPC-14, "Annual Report for Importers and Exporters of Natural Gas"; U.S. Crude Oil, Natural Gas, and Natural Gas Liquids Reserves, 1994 Annual Report, DOE/EIA-0216(94); and the U.S. Minerals Management Service. 


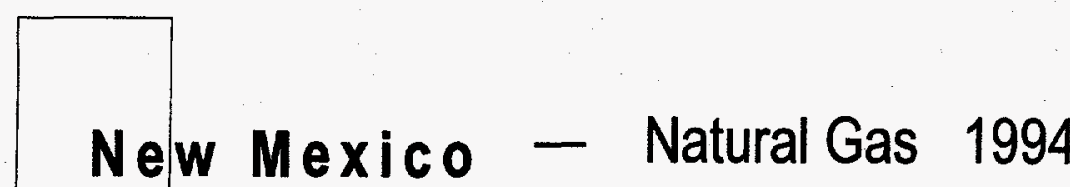

Million Percent of

Cu. Feet National Total

Million

Percent of

Cu. Feet

National Total

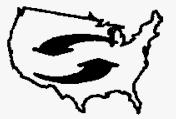

Net Interstate

Movements:

$-1,082,958$

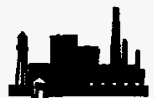

Industrial:

18,741

0.23

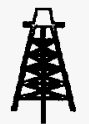

Marketed

Production

$1,557,689$

7.93

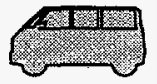

Vehicle Fuel:

62

Deliveries to Consumers:

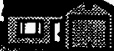

Residential:

30,868

0.64

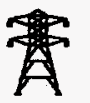

Electric

Utilities:

32,214

1.08

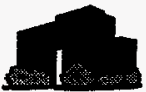

Commercial:

24,964

0.86

Total:

106,849

0.57

Table 79. Summary Statistics for Natural Gas - New Mexico, 1990-1994

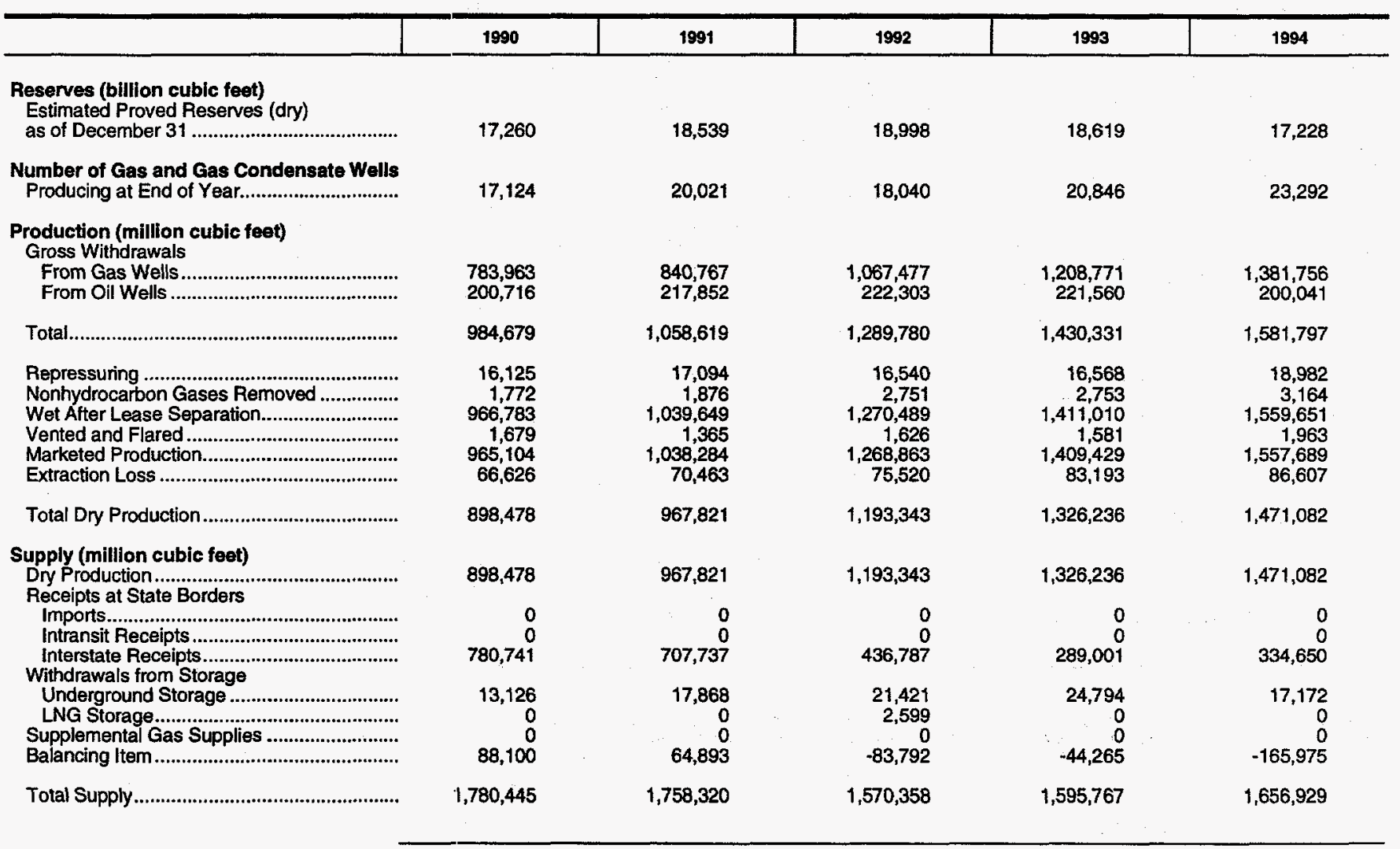

See footnotes at end of table. 
Table 79. Summary Statistics for Natural Gas - New Mexico, 1990-1994 (Continued)

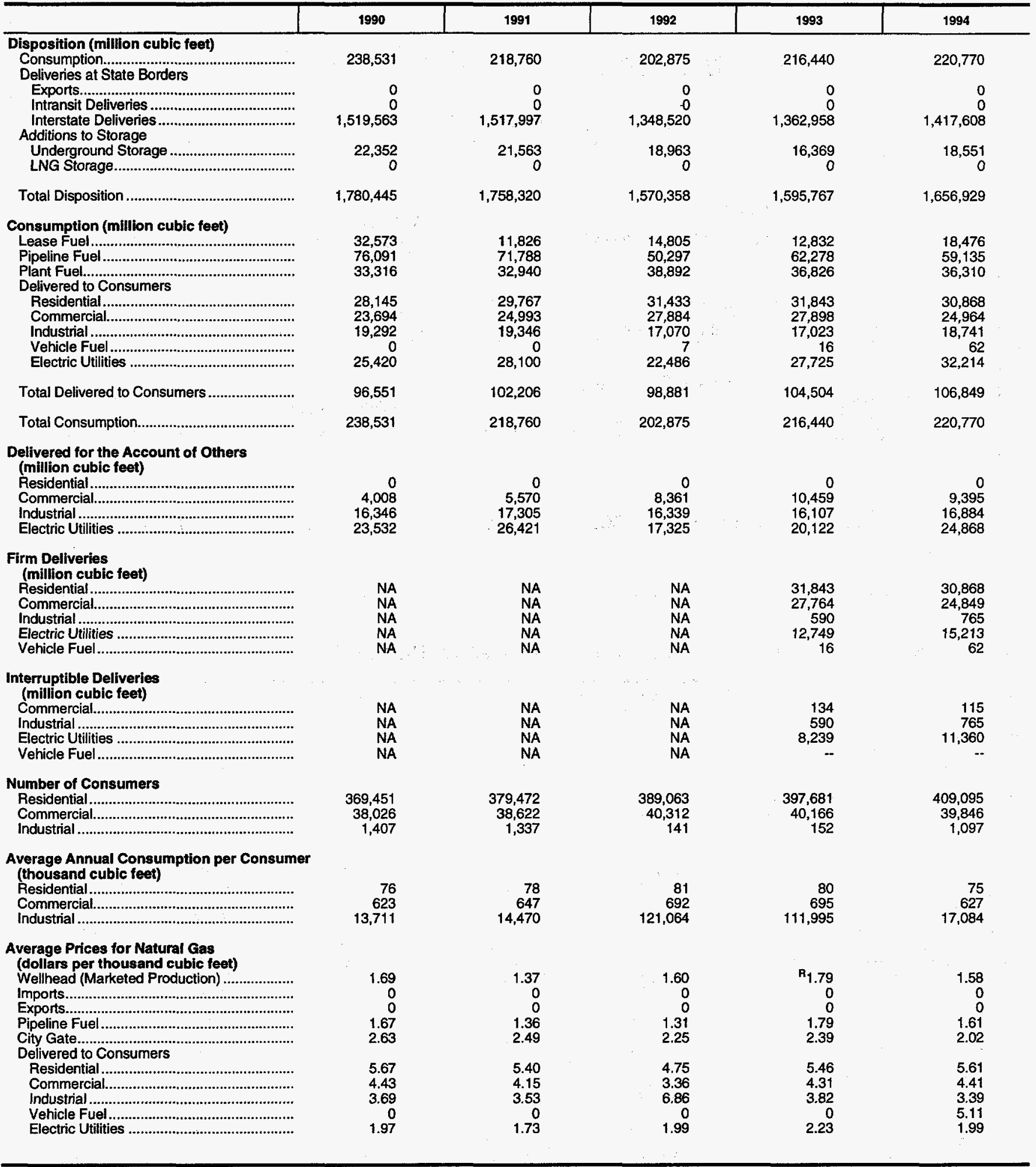

R $=$ Revised data.
NA $=$ Not Available.

Notes: Deliveries to electric utilities (consumption) are reported on the Form ElA-176, "Annual Report of Natural and Supplemental Gas Supply and Disposition." See the discussion on electric utility data and Table A1 in Appendix A for a comparison of reporting to these two forms. Tolals may not add due to independent rounding.

Sources: Energy Information Administration (EIA), Form EIA-176. "Annual Report of Natural and Supplemental Gas Supply and Disposition"; Form ElA-627, "Annual

Quantity and Value of Natural Gas Report"; Form ElA-857, "Monthly Report of Natural Gas Purchases and Deliveries to Consumers"; Form EIÁ-816, "Monthly Natural Gas

Liquids Report"; Form EIA-759, "Monthly Power Plant Report"; Form FERC-423, "Monthly Report of Cost and Quality of Fuels for Electric Plants"; Form EIA-191, "Underground Gas Storage Report"; Form FPC-14, "Annual Report for Importers and Exporters of Natural Gas"; U.S. Crude Oil, Natural Gas, and Natural Gas Liquids Reserves, 1994 Annual Report, DOEJEIA-0216(94); and the U.S. Minerals Management Service. 


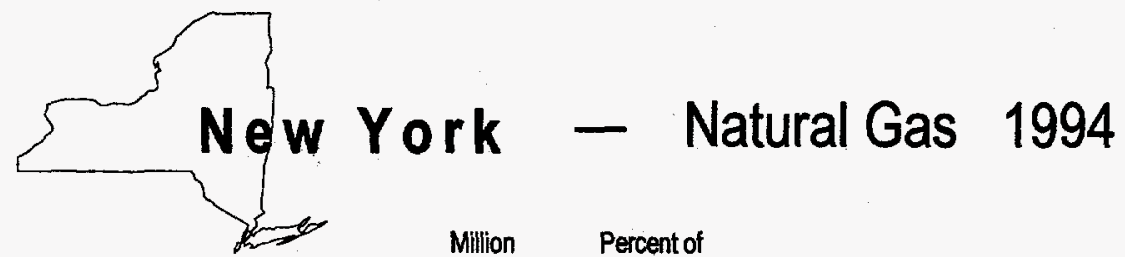

Million

Percent of

Cu. Feet National Total

Cu. Feet

National Total

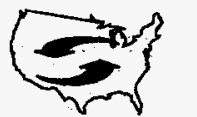

Net Interstate

Movements:

965,848

Industrial:

214,438

2.62

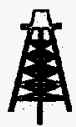

Marketed

Production:

20,465

0.10

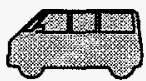

Vehicle Fuel:

53

Deliveries to Consumers:

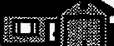

Residential:

385,408

7.95

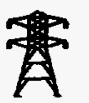

Electric

Utilities:

182,521

6.11

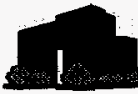

Commercial:

223,256

7.71

Total:

$1,005,676$

5.32

Table 80. Summary Statistics for Natural Gas - New York, 1990-1994

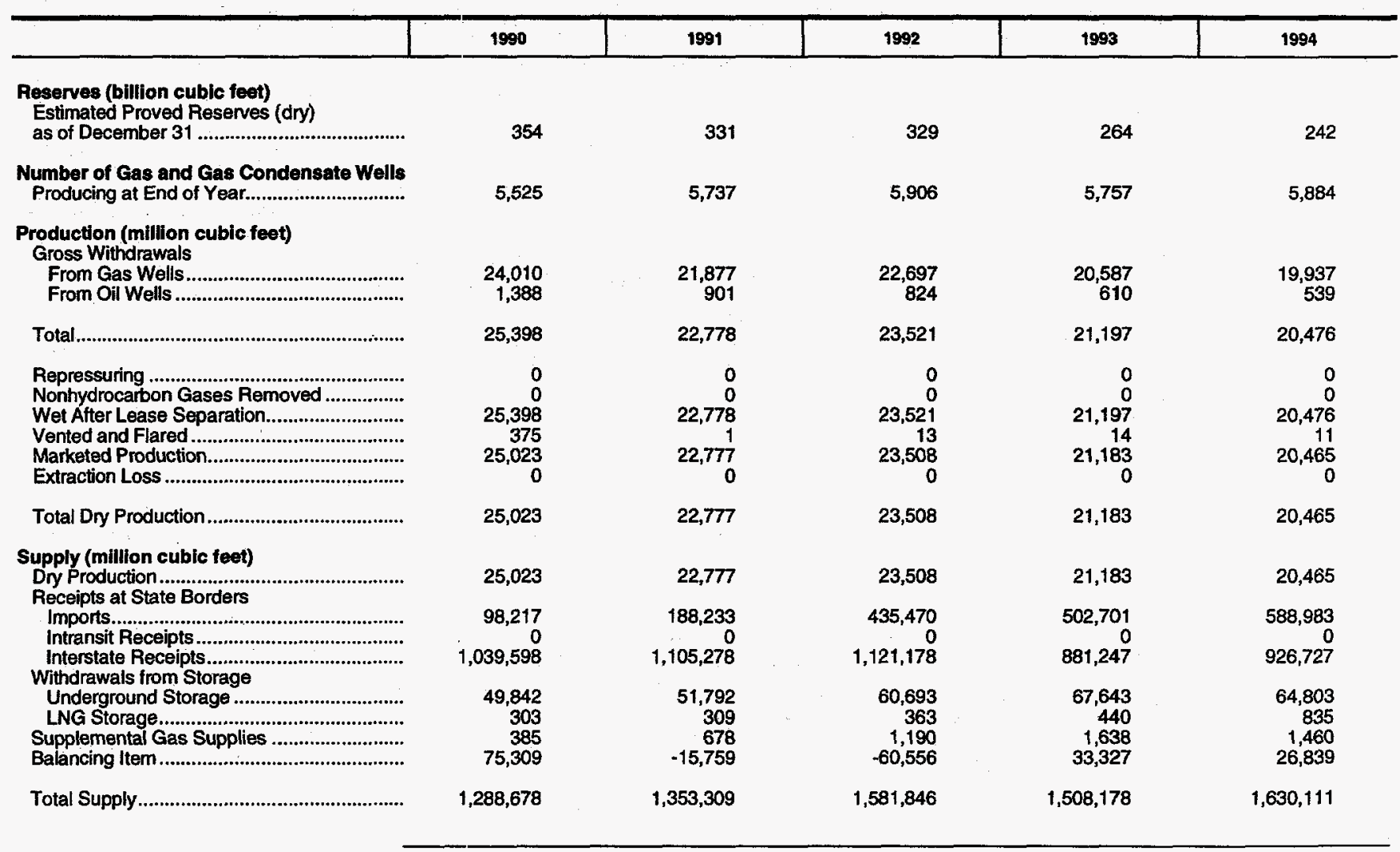

See footnotes at end of table. 
Table 80. Summary Statistics for Natural Gas - New York, 1990-1994 (Continued)

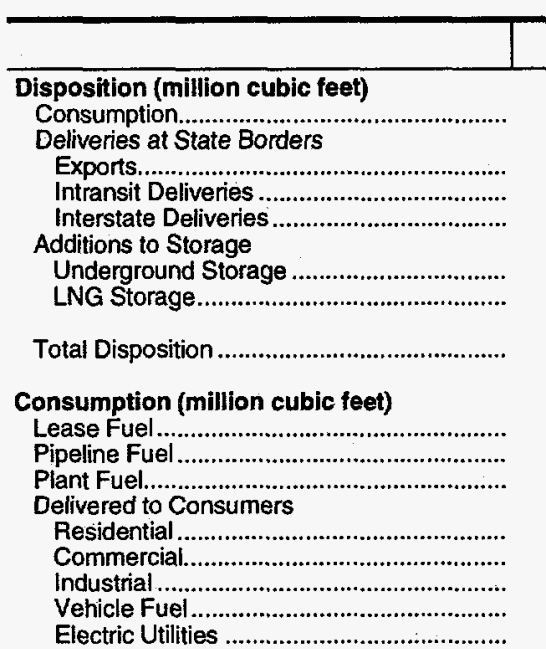

Total Delivered to Consumers .......................

Total Consumption...

Delivered for the Account of Others

(million cubic feet)

Residential

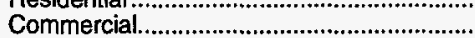

Industrial.

Electric Utilities

Firm Deliveries

(miltion cubic feet)

Residential

Commercial.................

industrial

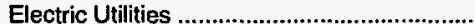

Vehicle Fuet.

Interruptible Deliveries

(million cubic feet)

Commercial.

Industrial

Electric Utilities

Vehicle Fuel.

Number of Consumers

Residential $\quad 3,917,354$

Commercial....................................................... $\quad 281,717$

Industrial...

281,717
25,008

NA
NA
NA
NA
NA

2,660
31,904

31,904
60,359

21,280

38,556

83,934

23,485

338,892

199,598

118,944

211,640

869,100

875,184

1992

959,004

0
0
559,844

62,265

732

$1,581,846$

965

5,878

378,689

217,214

147,520

208,731

952,161

959,004

2,413

48,552

113,074

19,893

3,036
50,279

125,916

15,455

0
0
495,010

68,532

810

$1,508,178$

563

6,089
0

384,216

220,729

160,406

171,803

937,173

943,826

1994

$1,012,497$

0
0
549,862

66,627

1,125

$1,630,111$

781

6,039
0

385,408

223,256

214,438

53
182,521

$1,005,676$

$1,012,497$

2,369

45,626

180,002

14,082

384,216

184,795

55,969

21,288

19

382,713

201,478

78,967

34,310

310
28

35,934

55,969

147,451

21,779

78,967

135,488

488
25

$4,522,274$

315,974

28,198

$3,990,564$

298,020

23,833

$4,008,868$

301,499

21,833

Average Annual Consumption per Consumer (thousand cublc feet)

Residential

Commercial.

Industrial

86
692
4,050

76

642
4,125

84

687
5,232

96

741
6,730

96
740

9,822

Average Prices for Natural Gas

(dollars per thousand cubic feet)

Wellhead (Marketed Production) ....................

Imports.

Exports.

Pipeline Fuel

City Gate.

Delivered to Consumers

Residential

Commercial.

Industrial.....

Vehicle Fuel.

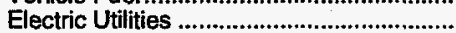

2.20

2.83

259

3.05

7.40

5.59

4.86

4.70

2.46

2.15

2.63

2.25

2.48

2.86

3.01

7.35

5.47
4.72

2.30

7.58
5.75

5.75

4.93
5.47

5.47
2.48

2.35

2.40
2.49

0

3.15

2.44
0

2.21

3.32

3.02

8.15

6.16

4.45
2.73

8.75

6.51

5.22

3.64
2.30

NA = Not Available.

Notes: Deliveries to electric utilities (consumption) are reported on the Form ElA-176, "Annual Report of Natural and Supplemental Gas Supply and Disposition." See the discussion on electric utility data and Table A1 in Appendix A for a comparison of reporting to these two forms. Totals may not add due to independent rounding.

Sources: Energy Information Administration (EIA), Form EJA-176, "Annual Report of Natural and Supplemental Gas Supply and Disposition"; Form EIA-627, "Annual

Quantity and Value of Natural Gas Report"; Form EiA-857, "Monthly Report of Natural Gas Purchases and Deliveries to Consumers"; Form EIA-816, "Monthly Natural Gas

Liquids Report"; Form EIA-759, "Monthly Power Plant Report"; Form FERC-423, "Monthly Report of Cost and Quality of Fuels for Electric Plants"; Form EIA-191, "Underground

Gas Storage Report"; Form FPC-14, "Annual Report for importers and Exporters of Natural Gas"; U.S. Cnude Oil, Natural Gas, and Natural Gas Liquids Reserves, 1994 Annual Report, DOEJEIA-0216(94); and the U.S. Minerals Management Service. 
Million Percent of

Cu. Feet National Total

Net Interstate

Movements:

198,606

Marketed

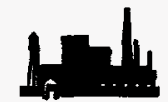

Industrial:

94,838

1.16

Million

Cu. Feet

Percent of

National Total
Production:

0

0.00

Deliveries to Consumers:

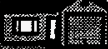

Residential:

47,451

0.98

Commercial:

38,940

1.35

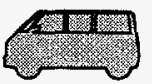

Vehicle Fuel:

8

0.46

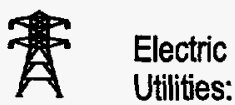

871

0.03

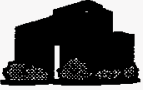

Total:

182,107

Table 81. Summary Statistics for Natural Gas - North Carolina, 1990-1994

\begin{tabular}{|c|c|c|c|c|c|}
\hline & 1990 & 1991 & 1992 & 1993 & 1994 \\
\hline $\begin{array}{l}\text { Reserves (billion cubic feet) } \\
\text { Estimated Proved Reserves (dry) } \\
\text { as of December } 31\end{array}$ & 0 & 0 & 0 & 0 & 0 \\
\hline 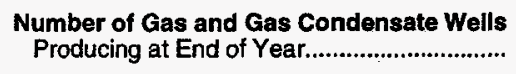 & 0 & 0 & 0 & 0 . & 0 \\
\hline 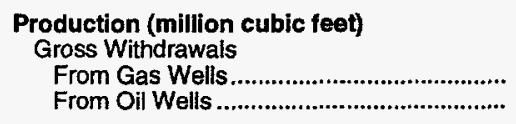 & $\begin{array}{l}0 \\
0\end{array}$ & $\begin{array}{l}0 \\
0\end{array}$ & $\stackrel{0}{0}$ & $\begin{array}{l}0 \\
0\end{array}$ & $\stackrel{0}{0}$ \\
\hline Total & 0 & 0 & 0 & 0 & 0 \\
\hline 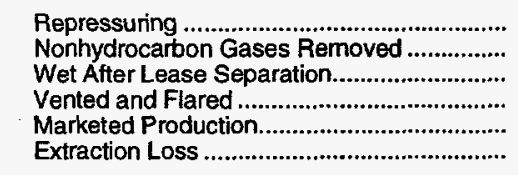 & $\begin{array}{l}0 \\
0 \\
0 \\
0 \\
0 \\
0\end{array}$ & $\begin{array}{l}0 \\
0 \\
0 \\
0 \\
0 \\
0\end{array}$ & $\begin{array}{l}0 \\
0 \\
0 \\
0 \\
0 \\
0\end{array}$ & $\begin{array}{l}0 \\
0 \\
0 \\
0 \\
0 \\
0\end{array}$ & $\begin{array}{l}0 \\
0 \\
0 \\
0 \\
0 \\
0\end{array}$ \\
\hline Total Dry Production & 0 & 0 & 0 & 0 & 0 \\
\hline 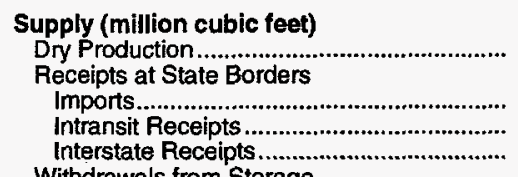 & $\begin{array}{r}0 \\
0 \\
931,485\end{array}$ & $\begin{array}{r}0 \\
0 \\
928,505\end{array}$ & $\begin{array}{r}0 \\
0 \\
943,723\end{array}$ & $\begin{array}{r}0 \\
0 \\
902,203\end{array}$ & $\begin{array}{r}0 \\
0 \\
901,123\end{array}$ \\
\hline 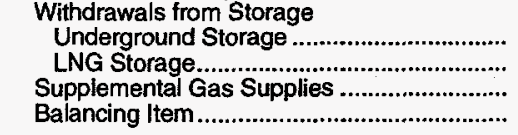 & $\begin{array}{r}0 \\
578 \\
0 \\
-3,141\end{array}$ & $\begin{array}{r}0 \\
1,232 \\
0 \\
-10,276\end{array}$ & $\begin{array}{r}0 \\
1,122 \\
-11,985\end{array}$ & $\begin{array}{r}0 \\
1,823 \\
4 \\
-16,686\end{array}$ & $\begin{array}{r}0 \\
1,802 \\
3 \\
-10,433\end{array}$ \\
\hline 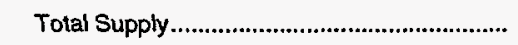 & 928,922 & 919,461 & 932,862 & 887,344 & 892,496 \\
\hline
\end{tabular}

See footnotes at end of table. 
Table 81. Summary Statistics for Natural Gas - North Carolina, 1990-1994 (Continued)

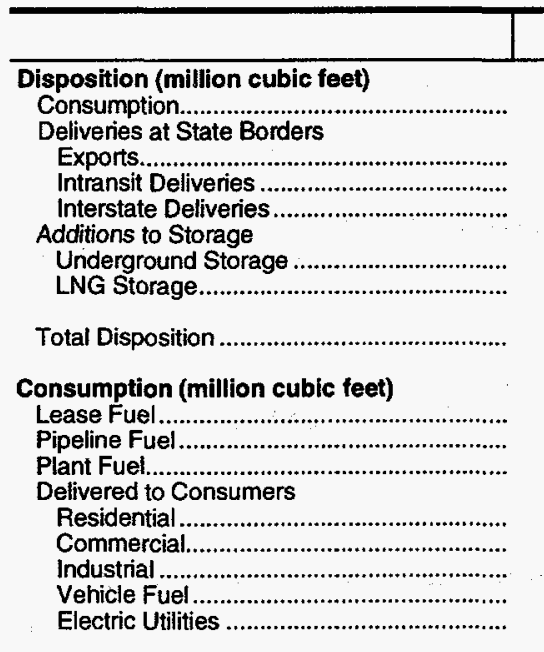

Total Delivered to Consumers ......................

Total Consumption

Delivered for the Account of Others

(million cubic feet)

Residential.

Commercial.

Industrial.

Electric Utilities ...

Firm Deliveries

(million cubic feet)

Residential...

Industrial.

Electric Utilities

Vehicle Fuel

Interruptible Deliveries

(million cubjc feet)

Commercial

Industrial.

Electric Utilities

Vehicle Fuel.

Number of Consumers

Residential.

Commercial

Industrial .

Average Annual Consumption per Consumer (thousand cubic feet)

Residential

Commercial........

ndustrial

Average Prices for Natural Gas

(dollars per thousand cubic feet)

Wellhead (Marketed Production)

Imports..

Pipeline Fuel

City Gate

Delivered to Consumers

Residential...

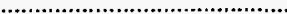

Industrial

Vehicle Fuel

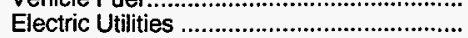

\begin{tabular}{|l|l|l|l}
\hline 1990 & 1991 & 1992 & 1993 \\
\hline
\end{tabular}

161,208

0
0
765,691

2,023

928,922

6,281

35,003

31,277

86,184

2,461

154,927

161,208

0
1,696

24,962

2,149

NA

NA

NA

NA

NA

NA

520,140

68,088

2,802

67
459

459
30,758
166,380

0
0
751,862

751,862

0
1,219

919,461

0
6,207
0

37,976

34,313

84,863

3,020

160,172

166,380

0
1,725
23,348
2,106

NA
NA
NA
NA
NA

NA

NA

NA

539,321

70,207

3,506

70
489

24,205

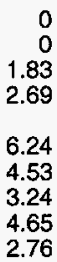

179,595

0
0
752237

0
1,030

932,862

0
6,439

42,588

36,418

90,984
7

3,159

173,156

179,595

185,648

0
0

700,015

0
1,681

887,344

0
5,953

47,104

37,370

92,309

2,911

179,694

185,648

1994

187,861

0
0
702517

0
2,118

892,496

0
5,753

47,451

38,940

94,838
8

871

182,107

187,861

0
1,497

17,302

461

0
561
20,068

20,068
1,692

1,314

38,907

47, 104

35,630

80,933

98
1

47,451

36,567

76,023

149
8

1,740

80,933

2,644

2,374

76,023

703

NA

575,096

72,647

3,119

607,388

76,386

2,664

652,307

80,739

3,401

74
501

501
29,171

78
489
34650

73
482
27,885

NA $=$ Not Available.

Notes: Deliveries to electric utilities (consumption) are reported on the Form ElA-176, "Annual Report of Natural and Supplemental Gas Supply and Disposition." See the discussion on electric utility data and Table A1 in Appendix A for a comparison of reporting to these two forms. Totals may not add due to independent rounding

Sources: Energy Information Administration (EIA), Form EIA-176, "Annual Report of Natural and Supplemental Gas Supply and Disposition"; Form EIA-627, "Annual

Quantity and Value of Natural Gas Report"; Form ElA-857, "Monthly Report of Natural Gas Purchases and Deliveries to Consumers"; Form ElA-816, "Monthly Natural Gas

Liquids Report"; Form EIA-759, "Monthly Power Plant Report"; Form FERC-423, "Monthly Report of Cost and Quality of Fuels for Electric Plants"; Form EIA-191, "Underground

Gas Storage Report"; Form FPC-14, "Annual Report for Importers and Exporters of Natural Gas"; U.S. Crude Oil, Natural Gas, and' Natural Gas Liquid's Reserves, 1994 Annual

Report, DOE/EIA-0216(94); and the U.S. Minerals Management Service. 


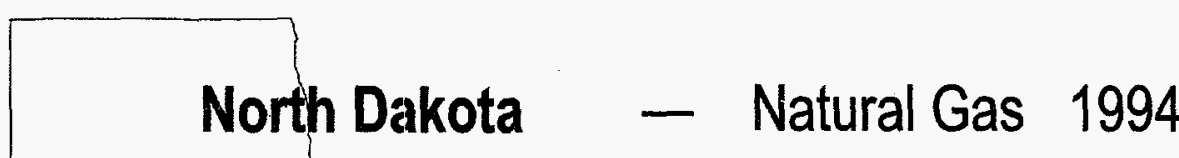

$\begin{array}{llll}\text { Million } & \text { Percent of } & \text { Million } & \text { Percent of } \\ \text { Cu. Feet } & \text { National Total } & \text { Cu. Feet } & \text { National Total }\end{array}$

Net Interstate

Movements:

$-61,969$

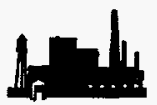

Industrial:

5,846

Marketed

Production:

57,805

0.29

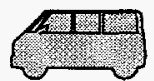

Vehicle Fuel:

8

0.46

Deliveries to Consumers:

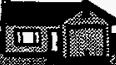

Residential:"

10,661

0.22

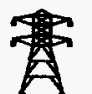

Electric

Utilities:

3

0.00

Commercia: $\quad \mathbf{1 0 , 7 8 3} \quad 0.37$

Total:

27,301

0.14

Table 82. Summary Statistics for Natural Gas - North Dakota, 1990-1994

\begin{tabular}{|c|c|c|c|c|c|}
\hline & 1990 & 1991 & 1992 & 1993 & 1994 \\
\hline 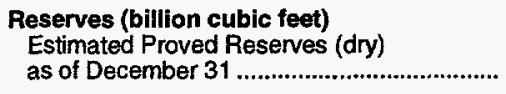 & 586 & 472 & 496 & 525 & 507 \\
\hline $\begin{array}{l}\text { Number of Gas and Gas Condensate Wells } \\
\text { Producing at End of Year................................ }\end{array}$ & 103 & 100 & 104 & 101 & 104 \\
\hline 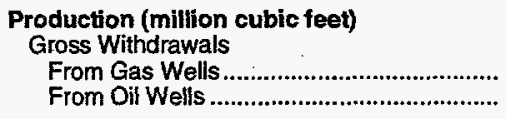 & $\begin{array}{l}13,736 \\
49,854\end{array}$ & $\begin{array}{l}13,359 \\
49,298\end{array}$ & $\begin{array}{l}12,461 \\
47,518\end{array}$ & $\begin{array}{l}18,892 \\
46,059\end{array}$ & $\begin{array}{r}19,592 \\
43,640\end{array}$ \\
\hline 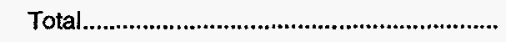 & 63,590 & 62,657 & 59,979 & 64,951 & 63,232 \\
\hline 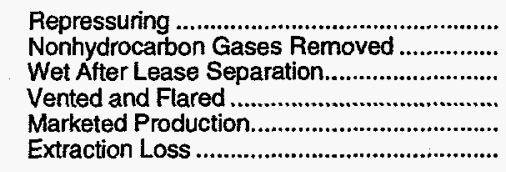 & $\begin{array}{r}2,386 \\
5,393 \\
55,811 \\
3,642 \\
52,169 \\
6,444\end{array}$ & $\begin{array}{r}2,128 \\
4,447 \\
56,082 \\
2,603 \\
53,479 \\
6,342\end{array}$ & $\begin{array}{r}2,391 \\
508 \\
57,080 \\
2,197 \\
54,883 \\
6,055\end{array}$ & $\begin{array}{r}2,231 \\
532 \\
62,188 \\
2,337 \\
59,851 \\
5,924\end{array}$ & $\begin{array}{r}2,577 \\
358 \\
60,297 \\
2,492 \\
57,805 \\
5,671\end{array}$ \\
\hline 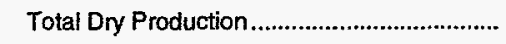 & 45,725 & 47,137 & 48,828 & 53,927 & 52,134 \\
\hline 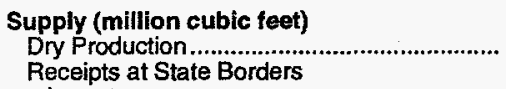 & 45,725 & 47,137 & 48,828 & 53,927 & 52,134 \\
\hline 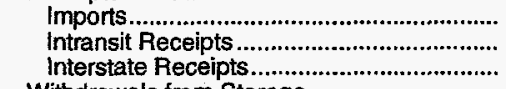 & $\begin{array}{r}0 \\
0 \\
351,741\end{array}$ & $\begin{array}{r}0 \\
0 \\
394,319\end{array}$ & $\begin{array}{r}0 \\
0 \\
465,592\end{array}$ & $\begin{array}{r}0 \\
0 \\
517,897\end{array}$ & $\begin{array}{r}1,617 \\
0 \\
543,543\end{array}$ \\
\hline 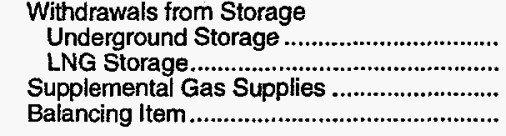 & $\begin{array}{r}0 \\
0 \\
53,144 \\
-1,693\end{array}$ & $\begin{array}{r}0 \\
0 \\
52,557 \\
-211\end{array}$ & $\begin{array}{r}0 \\
0 \\
58,496 \\
-7,998\end{array}$ & $\begin{array}{r}0 \\
0 \\
57,680 \\
-8,099\end{array}$ & $\begin{array}{r}0 \\
0 \\
57,127 \\
-4,439\end{array}$ \\
\hline 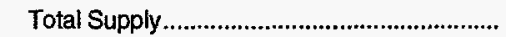 & 448,918 & 493,803 & 564,918 & 621,405 & 649,983 \\
\hline
\end{tabular}

See footnotes at end of table. 
Table 82. Summary Statistics for Natural Gas - North Dakota, 1990-1994 (Continued)

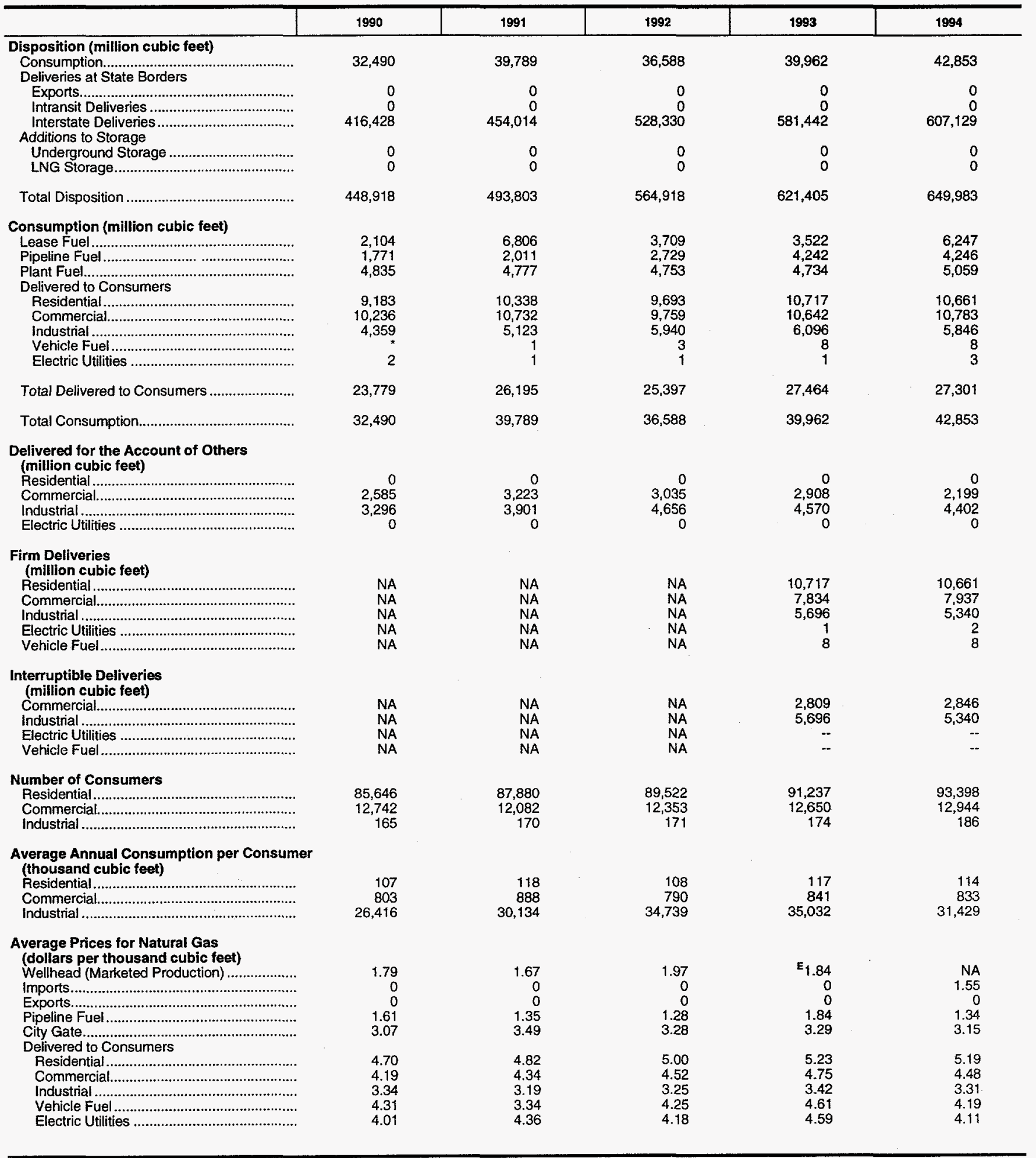

$E=$ Estimated Data.

NA = Not Available.

* = Volume is less than 500,000 cubic feet

Notes: Deliveries to electric utilities (consumption) are reported on the Form ElA-176, "Annual Report of Natural and Supplemental Gas Supply and Disposition." See the discussion on electric utility data and Table A1 in Appendix A for a comparison of reporting to these two forms. Totals may not add due to independent rounding.

Sources: Energy Information Administration (EIA), Form EIA-176, "Annual Report of Natural and Supplemental Gas Supply and Disposition"; Form EIA-627, "Annual Quantity and Value of Natural Gas Report"; Form ElA-857, "Monthly Report of Natural Gas Purchases and Deliveries to Consumers"; Form EIA-816, "Monthly' Natural Gas Liquids Feport"; Form EIA-759, "Monthly Power Plant Report"; Form FERC-423, "Monthly Report of Cost and Quality of Fuels for Electric Plants"; Form EIA-191, "Underground Gas Storage Report"; Form FPC-14, "Annual Report for Importers and Exporters of Natural Gas"; U.S. Crude Oii, Natural Gas, and Natural Gas Liquids Reserves, 1994 Annual Report, DOE/EIA-0216(94); and the U.S. Minerals Management Service. 


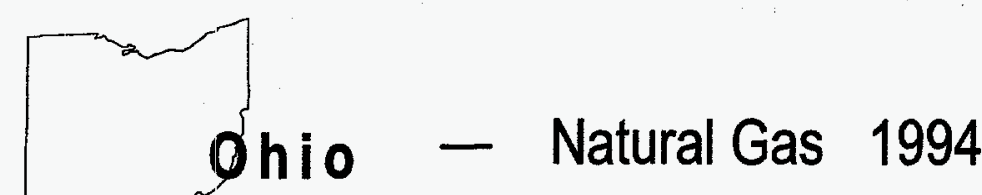

Million Percent of Million Percent of

Cu. Feet National Total Nu. Feet National Total

Net Interstate

Movements:

779,246

Marketed

Production:

132,151

0.67

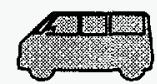

Vehicle Fuel:

48

2.77

Deliveries to Consumers:

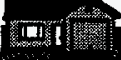

Residential:

343,331

7.08

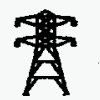

Electric

Utilities:

2,818

0.09

Commercial: $\quad 166,798 \quad 5.76$

Total:

824,119

Table 83. Summary Statistics for Natural Gas - Ohio, 1990-1994

\begin{tabular}{|c|c|c|c|c|c|}
\hline & 1990 & 1991 & 1992 & 1993 & 1994 \\
\hline 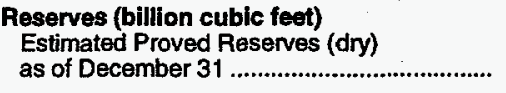 & 1,214 & 1,181 & 1,161 & 1,104 & 1,094 \\
\hline $\begin{array}{l}\text { Number of Gas and Gas Condensate Wells } \\
\text { Producing at End of Year.................................... }\end{array}$ & 34,586 & 34,760 & 34,784 & 34,782 & 34,731 \\
\hline 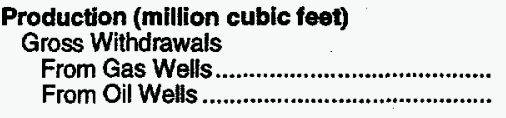 & $\begin{array}{r}154,619 \\
0\end{array}$ & $\begin{array}{r}147,651 \\
0\end{array}$ & $\begin{array}{r}144,815 \\
0\end{array}$ & $\begin{array}{r}137,285 \\
0\end{array}$ & $\begin{array}{r}132,151 \\
0\end{array}$ \\
\hline 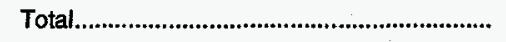 & 154,619 & 147,651 & $\$ 44,815$ & 137,285 & 132,151 \\
\hline 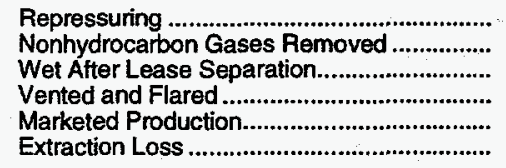 & $\begin{array}{r}\text { NA } \\
\text { NA } \\
154,619 \\
\text { NA } \\
154,619 \\
58\end{array}$ & $\begin{array}{r}N A \\
N A \\
147,651 \\
N A \\
147,651 \\
49\end{array}$ & $\begin{array}{r}\text { NA } \\
\text { NA } \\
144,815 \\
\text { NA } \\
144,815 \\
72\end{array}$ & $\begin{array}{r}\text { NA } \\
\text { NA } \\
137,285 \\
\text { NA } \\
137,285 \\
95\end{array}$ & $\begin{array}{r}\text { NA } \\
\text { NA } \\
132,151 \\
\text { NA } \\
132,151 \\
104\end{array}$ \\
\hline 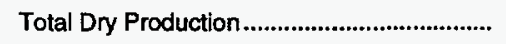 & 154,561 & 147,602 & 144,743 & 137,190 & 132,047 \\
\hline $\begin{array}{l}\text { Supply (million cubic feet) } \\
\text { Dry Production } \\
\text { Receipts at State Borders }\end{array}$ & 154,561 & 147,602 & 144,743 & 137,190 & 132,047 \\
\hline 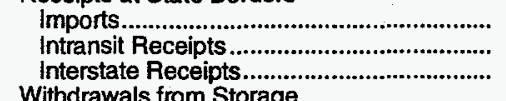 & $\begin{array}{r}0 \\
0 \\
2 ., 154,654\end{array}$ & $\begin{array}{r}0 \\
0 \\
1,889,579\end{array}$ & $\begin{array}{r}0 \\
0 \\
2,125,670\end{array}$ & $\begin{array}{r}0 \\
0 \\
2,226,113\end{array}$ & $\begin{array}{r}0 \\
0 \\
2,617,648\end{array}$ \\
\hline 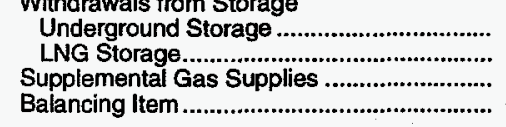 & $\begin{array}{r}119,117 \\
0 \\
849 \\
94,065\end{array}$ & $\begin{array}{r}133,629 \\
0 \\
891 \\
23,978\end{array}$ & $\begin{array}{r}175,682 \\
0 \\
1,051 \\
4,785\end{array}$ & $\begin{array}{r}188,899 \\
0 \\
992 \\
-174,022\end{array}$ & $\begin{array}{r}163,084 \\
0 \\
1,432 \\
-40,803\end{array}$ \\
\hline Total Supply & $2,523,246$ & $2,195,679$ & $2,451,931$ & $2,379,173$ & $2,873,408$ \\
\hline
\end{tabular}

See footnotes at end of table. 
Table 83. Summary Statistics for Natural Gas - Ohio, 1990-1994 (Continued)

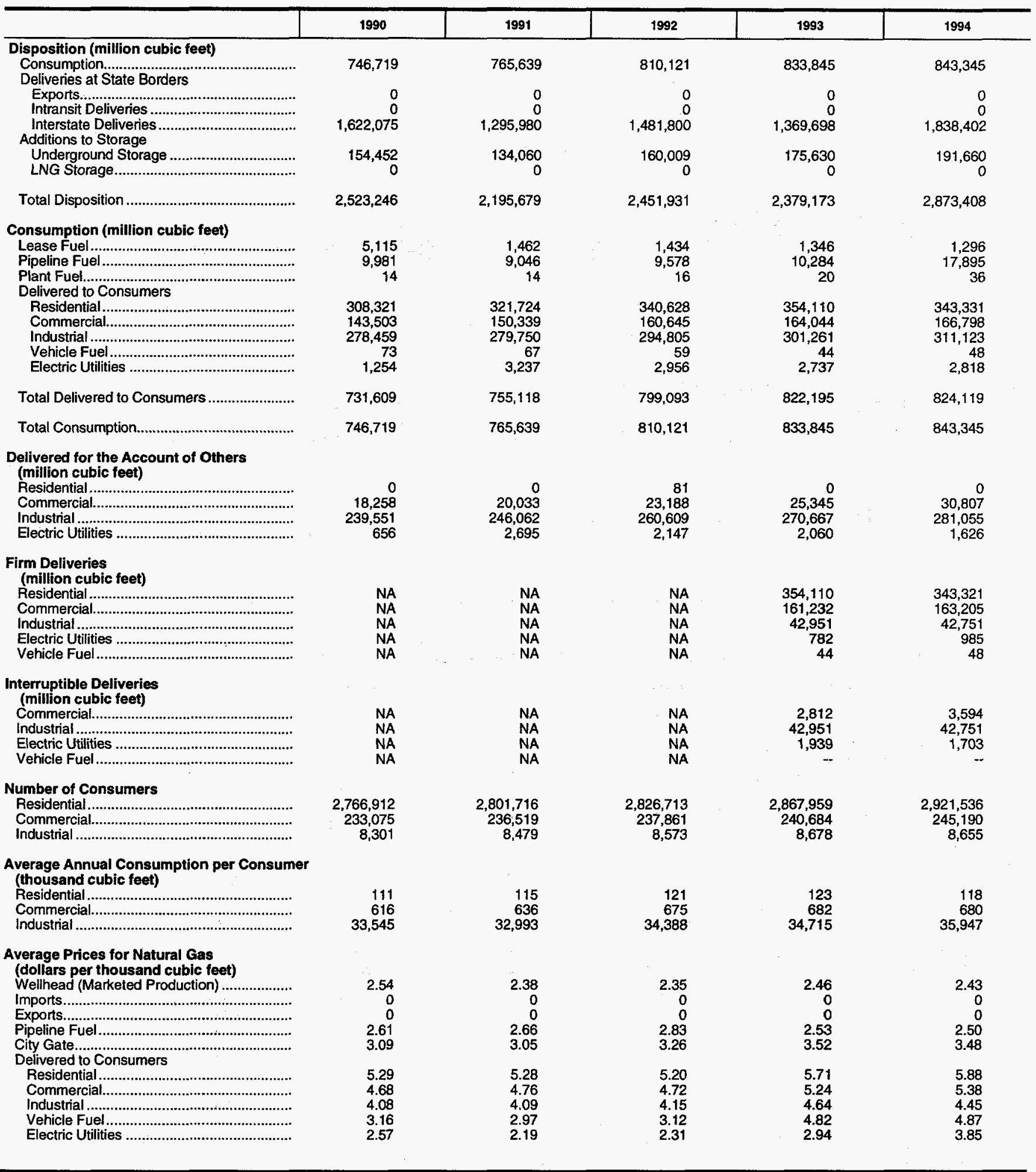

NA = Not Available.

Notes: Deliveries to electric utilities (consumption) are reported on the Form ElA-176, "Annual Report of Natural and Supplemental Gas Supply and Disposition." See the discussion on electric utility data and Table A1 in Appendix A for a comparison of reporting to these two forms. Totals may not add due to independent rounding.

Sources: Energy Information Administration (EIA), Form EIA-176, "Annual Report of Natural and Supplemental Gas Supply and Disposition"; Form EIA-627, "Annual Quantity and Value of Natural Gas Report"; Form ElA-857, "Monthly Report of Natural Gas Purchases and Deliveries to Consumers"; Form EIA-816, "Monthly Natural Gas Liquids Report"; Form EIA-759, "Monthly Power Plant Report"; Form FERC-423, "Monthly Report of Cost and Quality of Fuels for Electric Plants"; Form EIA-191, "Underground Gas Storage Report"; Form FPC-14, "Annual Report for Importers and Exporters of Natural Gas"; U.S. Crude Oil, Natural Gas, and Natural Gas Liquids Reserves, 1994 Annual Report, DOE/EIA-0216(94); and the U.S. Minerals Management Service. 


\section{Oklahoma - Natural Gas 1994 \\ Million Percent of}

Cu. Feet National Total

Million Percent of Cu. Feet National Total

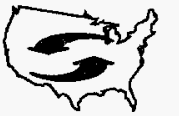

Net Interslate

Movements:

$-1,242,085$

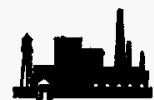

Industrial:

195,909

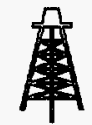

Marketed

Production: $\quad 1,934,864$

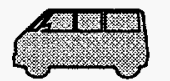

Vehicle Fuel:

157

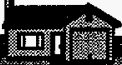

Deliveries to Consumers:

Residential:

69,211

1.43

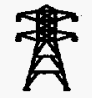

Electric

Utilities:

153,109

5.13

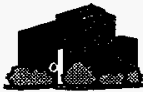

Commercial:

36,504

1.26

Total:

454,889

Table 84. Summary Statistics for Natural Gas - Oklahoma, 1990-1994

\begin{tabular}{|c|c|c|c|c|c|}
\hline & 1990 & 1991 & 1992 & 1993 & 1994 \\
\hline 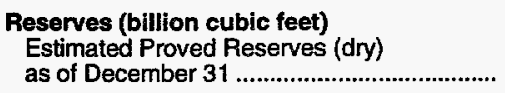 & 16,151 & 14,725 & 13,926 & 13,289 & 13,487 \\
\hline $\begin{array}{l}\text { Number of Gas and Gas Condensate Wells } \\
\text { Producing at End of Year.................................... }\end{array}$ & 24,547 & 28,216 & 28,902 & 29,118 & 29,121 \\
\hline 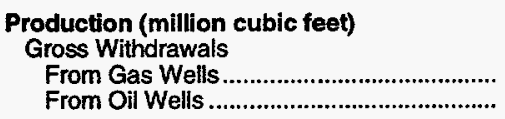 & $\begin{array}{r}1,830,380 \\
428,091\end{array}$ & $\begin{array}{r}1,794,138 \\
359,714\end{array}$ & $\begin{array}{r}1,674,405 \\
342,950\end{array}$ & $\begin{array}{r}1,732,997 \\
316,945\end{array}$ & $\begin{array}{r}1,626,858 \\
308,006\end{array}$ \\
\hline 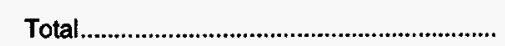 & $2,258,471$ & $2,153,852$ & $2,017,356$ & $2,049,942$ & $1,934,864$ \\
\hline 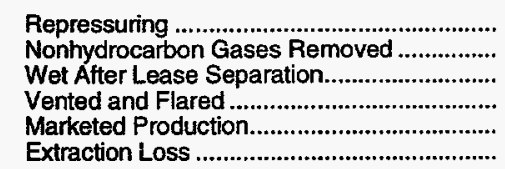 & $\begin{array}{r}N A \\
N A \\
2,258,471 \\
N A \\
2,258,471 \\
96,698\end{array}$ & $\begin{array}{r}N A \\
N A \\
2,153,852 \\
N A \\
2,153,852 \\
101,851\end{array}$ & $\begin{array}{r}\text { NA } \\
\text { NA } \\
2,017,356 \\
\text { NA } \\
2,017,356 \\
104,609\end{array}$ & $\begin{array}{r}\text { NA } \\
\text { NA } \\
2,049,942 \\
\text { NA } \\
2,049,942 \\
101,962\end{array}$ & $\begin{array}{r}\text { NA } \\
\text { NA } \\
1,934,864 \\
\text { NA } \\
1,934,864 \\
101,564\end{array}$ \\
\hline Total Dry Production & $2,161,773$ & $2,052,001$ & $1,912,747$ & $1,947,980$ & $1,833,300$ \\
\hline 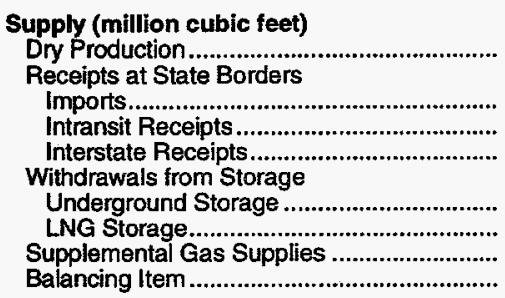 & $\begin{array}{r}80,976 \\
0 \\
0 \\
-98,666\end{array}$ & $\begin{array}{r}106,360 \\
0 \\
0 \\
-67,805\end{array}$ & $\begin{array}{r}107,526 \\
0 \\
0 \\
-112,462\end{array}$ & $\begin{array}{r}136,715 \\
0 \\
0 \\
-195,446\end{array}$ & $\begin{array}{r}104,738 \\
0 \\
0 \\
-303\end{array}$ \\
\hline 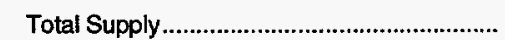 & $2,457,967$ & $2,421,086$ & $2,453,674$ & $2,400,678$ & $2,481,414$ \\
\hline
\end{tabular}

See footnotes at end of table. 
Table 84. Summary Statistics for Natural Gas - Oklahoma, 1990-1994 (Continued)

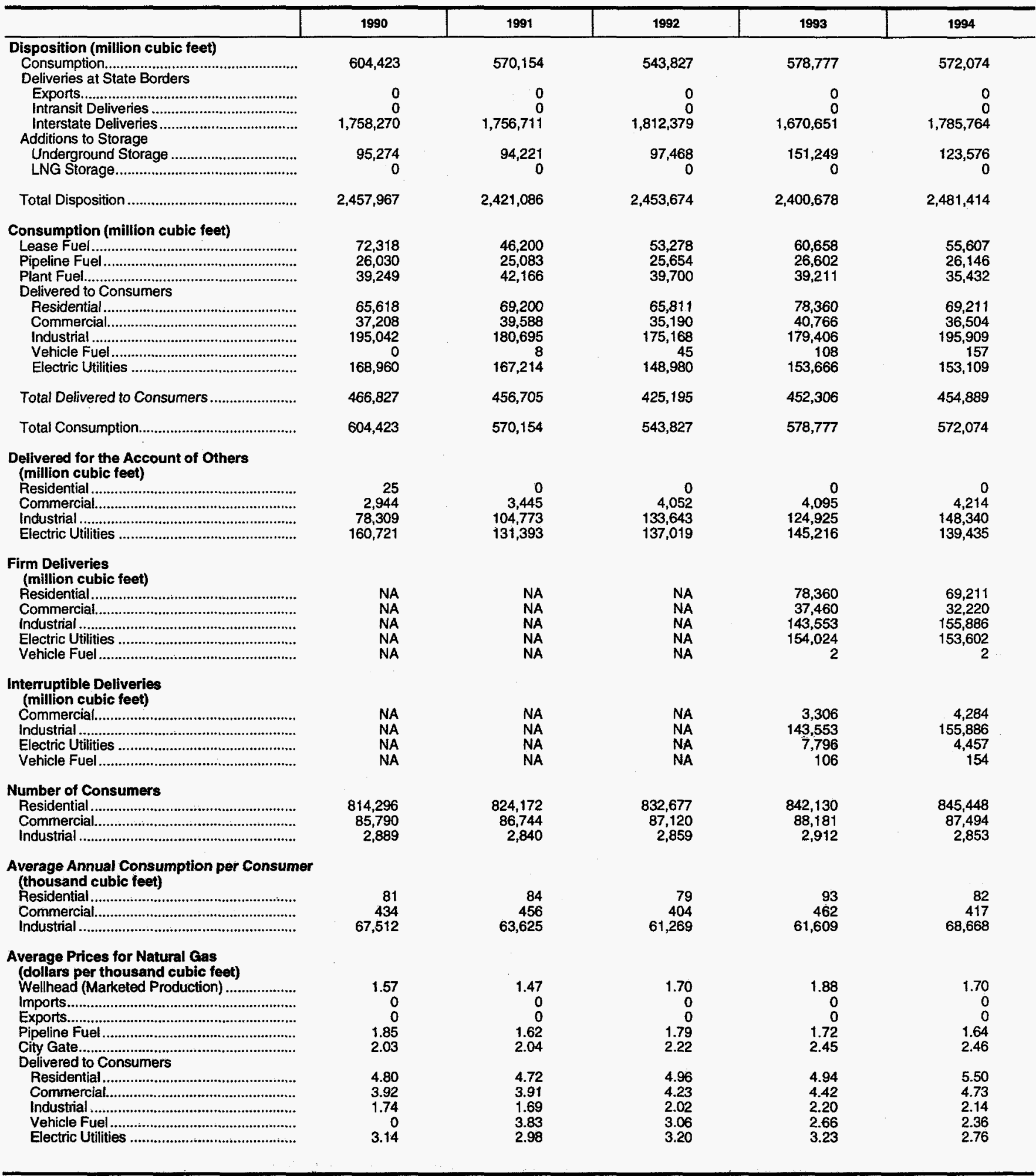

NA $=$ Not Available.

Notes: Deliveries to electric utilities (consumption) are reported on the Form EIA-176, "Annual Report of Natural and Supplemental Gas Supply and Disposition." See the discussion on electric utility data and Table A1 in Appendix A for a comparison of reporting to these two forms. Totals may not add due to independent rounding.

Sources: Energy Information Administration (EIA), Form EIA-176, "Annual Report of Nalural and Supplemental Gas Supply and Disposition"; Form EIA-627, "Annual Quantity and Value of Natural Gas Report"; Form ElA-857, "Monthly Report of Natural Gas Purchases and Deliveries to Consumers"; Form EIA-816, "Monthly' Natural Gas Liquids Report"; Form EIA-759, Monthly Power Plant Report"; Form FERC-423, "Monthly Report of Cost and Ouality of Fuels for Electric Plants"; Form ElA-191, "Underground Gas Storage Report"; Form FPC-14, "Annual Report for Importers and Exporters of Natural Gas"; U.S. Crude Oil, Natural Gas, and Natural Gas Liquids Reserves, 1994 Annual Report, DOE/EIA-0216(94); and the U.S. Minerals Management Service. 


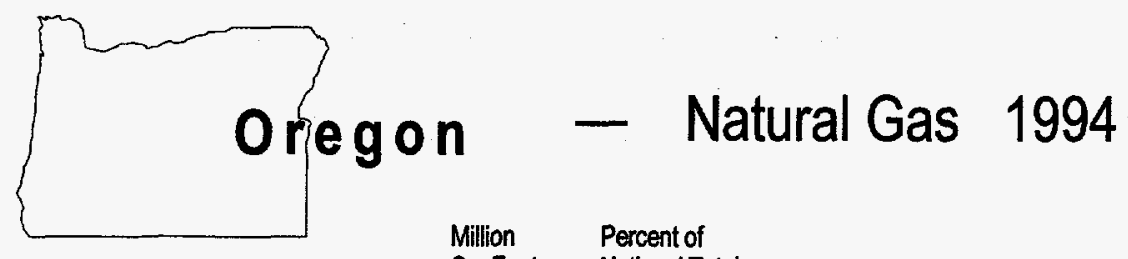

Cu. Feet National Total

Million Percent of Cu. Feet National Total

Net Interstate
Movements:

136,833

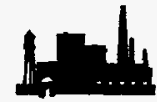

Industrial:

62,569

0.77<smiles></smiles>

Marketed

Production:

3,221

0.02

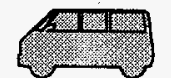

Vehicle Fuel:

17

0.98

Deliveries to Consumers:

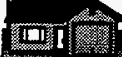

Residential:

28,848

0.60

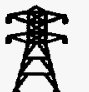

Electric

Utilities:

26,132

0.87

Commercial: $\quad 22,960$

0.79

Total:

140,526

Table 85. Summary Statistics for Natural Gas - Oregon, 1990-1994

\begin{tabular}{|c|c|c|c|c|c|}
\hline & 1990 & 1991 & 1992 & 1993 & 1994 \\
\hline 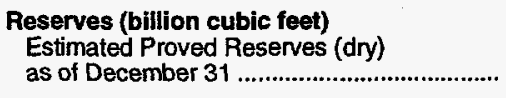 & NA & NA & NA & NA & NA \\
\hline 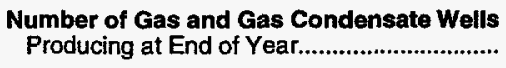 & 19 & $\therefore 16$ & 16 & 18 & 19 \\
\hline 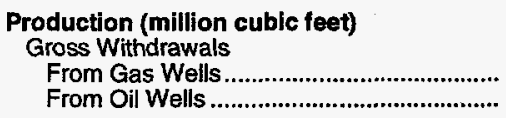 & $\begin{array}{r}2,815 \\
0\end{array}$ & $\begin{array}{r}2,741 \\
0\end{array}$ & $\begin{array}{r}2,580 \\
0\end{array}$ & $\begin{array}{r}4,003 \\
0\end{array}$ & $\begin{array}{r}4,200 \\
0\end{array}$ \\
\hline 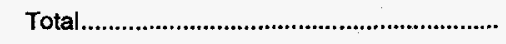 & 2,815 & 2,741 & 2,580 & 4,003 & 4,200 \\
\hline 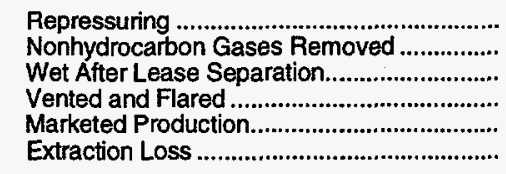 & $\begin{array}{r}0 \\
0 \\
2,815 \\
0 \\
2,815 \\
0\end{array}$ & $\begin{array}{r}0 \\
0 \\
2,741 \\
0 \\
2,741 \\
0\end{array}$ & $\begin{array}{r}0 \\
0 \\
2,580 \\
0 \\
2,580 \\
0\end{array}$ & $\begin{array}{r}0 \\
0 \\
4,003 \\
0 \\
4,003 \\
0\end{array}$ & $\begin{array}{r}55 \\
924 \\
3,221 \\
0 \\
3,221 \\
0\end{array}$ \\
\hline 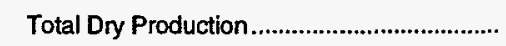 & 2,815 & 2,741 & 2,580 & 4,003 & 3,221 \\
\hline 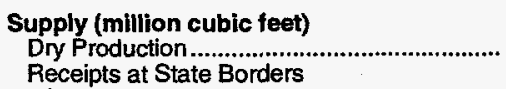 & 2,815 & 2,741 & 2,580 & 4,003 & 3,221 \\
\hline 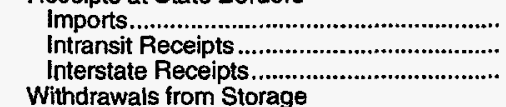 & $\begin{array}{r}0 \\
0 \\
500,269\end{array}$ & $\begin{array}{r}0 \\
0 \\
488,666\end{array}$ & $\begin{array}{r}0 \\
0 \\
541,201\end{array}$ & $\begin{array}{r}0 \\
0 \\
540,702\end{array}$ & $\begin{array}{r}0 \\
0 \\
765,764\end{array}$ \\
\hline 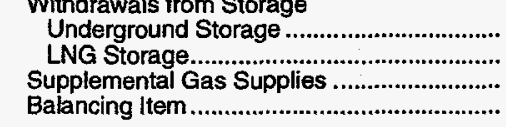 & $\begin{array}{r}4,531 \\
1,003 \\
3 \\
-4,980\end{array}$ & $\begin{array}{r}5,288 \\
389 \\
4 \\
37,975\end{array}$ & $\begin{array}{r}6,985 \\
409 \\
2 \\
27,923\end{array}$ & $\begin{array}{r}6,302 \\
1,360 \\
3 \\
25,583\end{array}$ & $\begin{array}{r}5,237 \\
1,117 \\
2 \\
6,816\end{array}$ \\
\hline 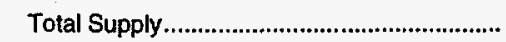 & 503,641 & 535,062 & 579,101 & 577,952 & 782,157 \\
\hline
\end{tabular}

See footnotes at end of table. 
Table 85. Summary Statistics for Natural Gas - Oregon, 1990-1994 (Continued)

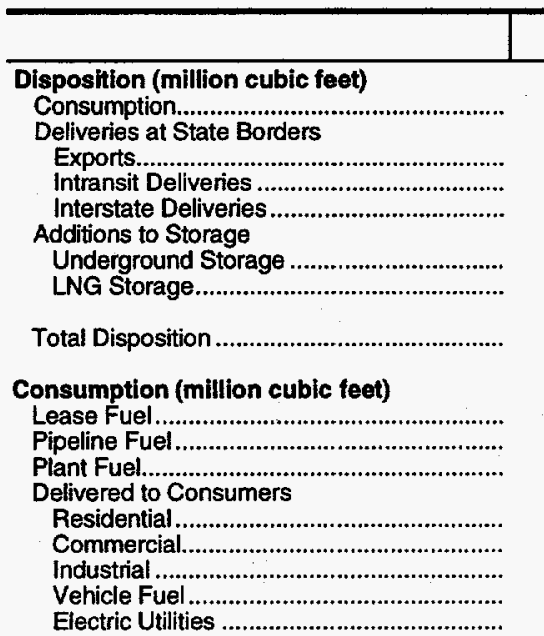

Total Delivered to Consumers.

Total Consumption.

Delivered for the Account of Others

(million cubic feet)

Residential...

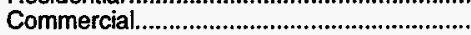

Industrial..

Electric Utilities

Firm Deliveries

(million cubic feet)

Residential

Commercial....................

Industrial

Electric Utilities

Vehicle Fuel...

Interruptible Deliveries

(million cubic feet)

Commercial...

Industrial .................................................

Electric Utilities

Vehicle Fuel

Number of Consumers

Residential.

Commercial.....................................................

Industrial..

Average Annual Consumption per Consumer (thousand cubic feet)

Residential

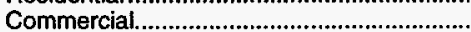

Industrial.

Average Prices for Natural Gas

(dollars per thousand cubic feet)

Wellhead (Marketed Production)...

Imports.

Exports..

Pipeline Fuel.

City Gate..

Residential

Commercial

Industrial ...

Electric Utilities

\section{0}

109,215

0
0
387,778

\section{5,894}

754

503,641

93
9,014

0

23,383

20,449

48,890

7,386

100,108

109,215

0
464

38,128

7,458

NA

NA

NA

NA

NA

NA

NA

326,177

47,175

699

72

433
69,943

72
33
43

3

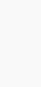

1.39

1.39
0
0
2.16
2.47
6.27
4.85
3.47
0
0

\section{1}

123,436

0
0
405,163

5,853

609

535,062

60
8,819
0

0

26,324

22,328

55,049

10,856

114,557

123,436

0
477
44,521

10,798

\section{NA \\ NA \\ NA}

NA

NA
NA
NA
NA

376,166

55,374

787

70
403

69,949

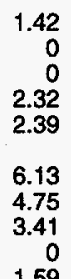

\section{2}

122,350

0
0
450,261

6,114

376

579,101

68
6,812

0

23,109

19,570

58,519

6
14,264

115,469

122,350

0
433
46,434

46,434
12,818

504
43,558

16,041

0
0
435,002

6,202

577,952

118
4,877
0

29,777

24,047

60,617

8
16,167

130,615

135,611

1994

146,409

0

628,931

5,956

860

782,157

95
5,788

28,848

22,960

62,569

17
26,132

140,526

146,409

0
430
42,891

26,310

28,848

22,529

43,835

26,310

44,549

14,879

NA

NA

NA

NA

NA

354,256

50,251

740

51,910

696

504

44,549

1,162

430

43,835

--

991,845

53,700

765

\section{5}

389
79,080

80

463
87,093

74
428

81,789

NA = Not Available.

Notes: Deliveries to electric utilities (consumption) are reported on the Form ElA-176, "Annual Report of Natural and Supplemental Gas Supply and Disposition." See the discussion on electric utility data and Table A1 in Appendix A for a comparison of reporting to these two forms. Totals may not add due to independent rounding.

Sources: Energy Information Administration (EIA), Form EIA-176, "Annual Report of Natural and Supplemental Gas Supply and Disposition"; Form EIA-627, "Annual

Quantity and Value of Natural Gas Report"; Form EIA-857, "Monthly Report of Natural Gas Purchases and Deliveries to Consumers"; Form ElA-816, "Monthly Natural Gas

Liquids Report"; Form EIA-759, "Monthly Power Plant Report"; Form FERC-423, "Monthly Report of Cost and Quality of Fuels for Electric Plants"; Form EIA-191, "Underground

Gas Storage Report"; Form FPC-14, "Annual Report for Importers and Exporters of Natural Gas"; U.S. Crude Oil, Natural Gas, and Natural Gas Liquids Reserves, 1994 Annual Report, DDEIEIA-0216(94); and the U.S. Minerals Management Service. 


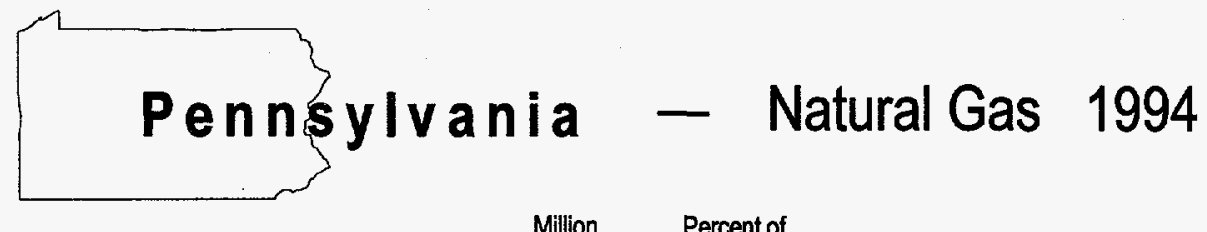

Cu. Feet National Total

Million

Cu. Feet

Percent of National Total<smiles>c1ccc(C2CC3CCCCC3C2)cc1</smiles>

Net Interstate

Movements:

688,918

Marketed

Production:

150,228

0.77

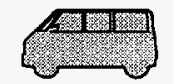

Vehicle Fuel:

10

0.58

Deliveries to Consumers:

Residential:

268,405

5.54

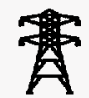

Electric

Utilities:

12,716

0.43

Commercial: $\quad 138,473 \quad 4.78$

Table 86. Summary Statistics for Natural Gas - Pennsylvania, 1990-1994

\begin{tabular}{|c|c|c|c|c|c|}
\hline & 1990 & 1991 & 1992 & 1993 & 1994 \\
\hline $\begin{array}{l}\text { Reserves (billion cubic feet) } \\
\text { Estimated Proved Reserves (dry) } \\
\text { as of December } 31\end{array}$ & 1,720 & 1,629 & 1,528 & 1,717 & 1,800 \\
\hline 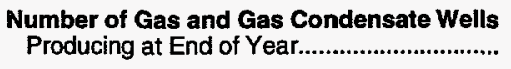 & 30,300 & 31,000 & 31,000 & 31,100 & 31,150 \\
\hline 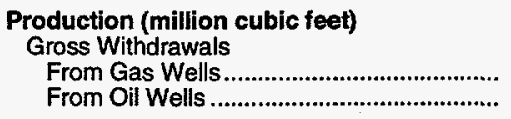 & $\begin{array}{r}171,748 \\
5,861\end{array}$ & $\begin{array}{r}152,500 \\
0\end{array}$ & $\begin{array}{r}138,675 \\
0\end{array}$ & $\begin{array}{r}132,130 \\
0\end{array}$ & $\begin{array}{r}148,763 \\
1,465\end{array}$ \\
\hline 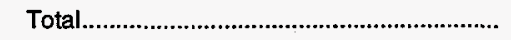 & 177,609 & 152,500 & 138,675 & 132,130 & 150,228 \\
\hline 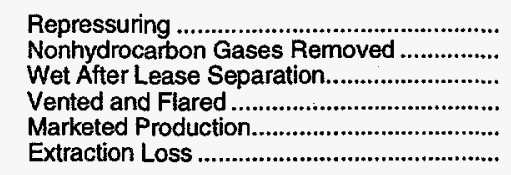 & $\begin{array}{r}\text { NA } \\
\text { NA } \\
177,609 \\
\text { NA } \\
177,609 \\
300\end{array}$ & $\begin{array}{r}\text { NA } \\
\text { NA } \\
152,500 \\
\text { NA } \\
152,500 \\
395\end{array}$ & $\begin{array}{r}\text { NA } \\
\text { NA } \\
138,675 \\
\text { NA } \\
138,675 \\
604\end{array}$ & $\begin{array}{r}\text { NA } \\
N A \\
132,130 \\
N A \\
132,130 \\
513\end{array}$ & $\begin{array}{r}\text { NA } \\
\text { NA } \\
150,228 \\
\text { NA } \\
150,228 \\
513\end{array}$ \\
\hline Total Dry Production & 177,309 & 152,105 & 138,071 & 131,617 & 149,715 \\
\hline 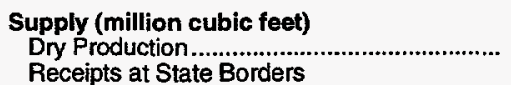 & 177,309 & 152,105 & 138,071 & 131,617 & 149,715 \\
\hline 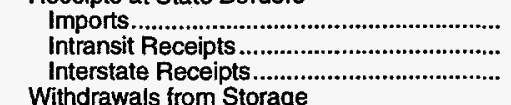 & $\begin{array}{r}0 \\
0 \\
2,245,534\end{array}$ & $\begin{array}{r}0 \\
0 \\
2,032,791\end{array}$ & $\begin{array}{r}0 \\
0 \\
2,240,904\end{array}$ & $\begin{array}{r}0 \\
0 \\
2,167,583\end{array}$ & $\begin{array}{r}0 \\
0 \\
2,165,861\end{array}$ \\
\hline 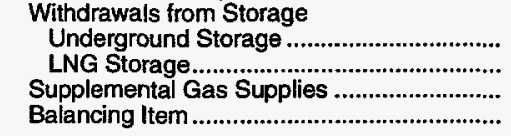 & $\begin{array}{r}287,053 \\
1,679 \\
220 \\
-136,163\end{array}$ & $\begin{array}{r}718,706 \\
2,412 \\
222 \\
33,989\end{array}$ & $\begin{array}{r}377,889 \\
2,797 \\
132 \\
-28,977\end{array}$ & $\begin{array}{r}381,060 \\
4,534 \\
110 \\
-236,955\end{array}$ & $\begin{array}{r}340,334 \\
5,030 \\
252 \\
-140,774\end{array}$ \\
\hline Total Supply & $2,575,632$ & $2,940,224$ & $2,730,816$ & $2,447,949$ & $2,520,419$ \\
\hline
\end{tabular}

See footnotes at end of table. 
Table 86. Summary Statistics for Natural Gas - Pennsylvania, 1990-1994 (Continued)

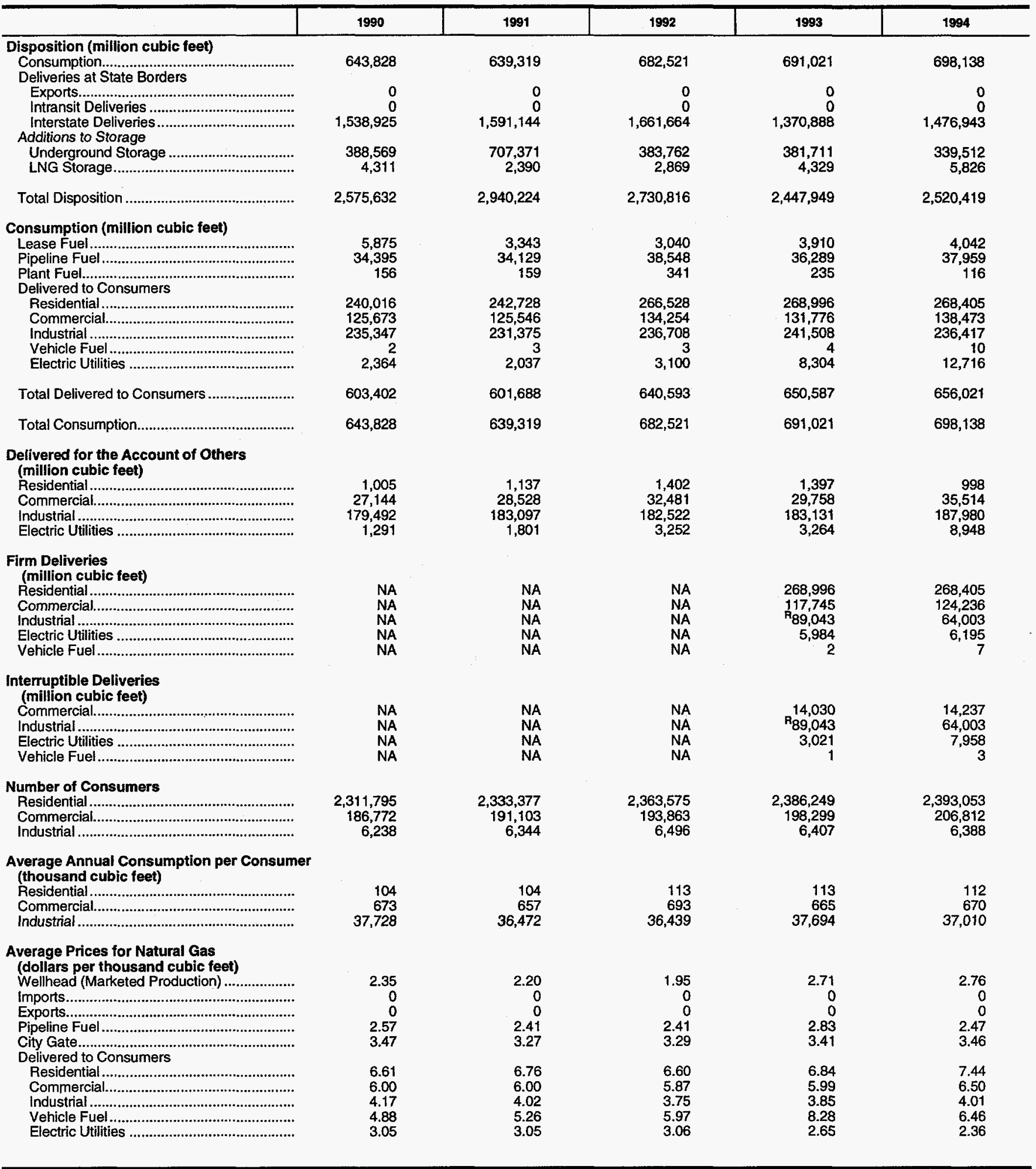

$R=$ Revised data.

$N A=$ Not Available.
Notes: Deliveries to electric utilities (consumption) are reported on the Form ElA-176, "Annual Report of Natural and Supplemental Gas Supply and Disposition." See the Notes: Deliveries to electric utilities (consumption) are reported on the Form ElA-176, "Annual Report of Natural and Supplemental Gas Supply and Disposition.
discussion on electric utility data and

discussion on electric utility data and Table A1 in Appendix A for a comparison of reporting to these two forms. Totals may not add due to independent rounding.
Sources: Energy information Administration (EIA). Form EIA-176. "Annual Report of Natural and Supplemental Gas Supply and Disposition": Form EIA-627, "Annual Sources: Energy Information Administration (ElA), Form EIA-176, "Annual Report of Natural and Supplemental Gas Supply and Disposition"; Form EIA-627, "Annual
Quantity and Value of Natural Gas Report"; Form ElA-857, "Monthly Report of Natural Gas Purchases and Deliveries to Consumers"; Form ElA-816, "Monthly Natural Gas

Quantity and Value of Natural Gas Report"; Form ElA-857, "Monthly Report of Natural Gas Purchases and Deliveries to Consumers"; Form ElA-816, "Monthly Natural Gas Liquids Report"; Form ElA-759, "Monthly Power Plant Report"; Form FERC-423, "Monthly Report of Cost and Quality of Fuels for Electric Plants"; Form ElA-191, "Underground Gas Storage Report"; Fom FPC-14, "Annual Report for Importers and Expor

Report, DOE/EIA-0216(94); and the U.S. Minerals Management Service. 


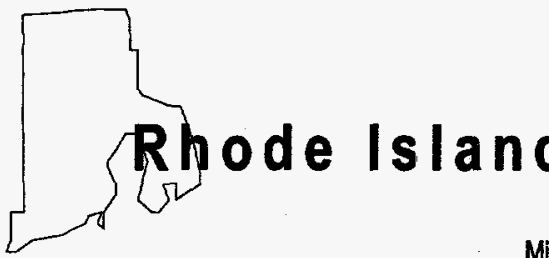

Million

Percent of

Cu. Feet National Total

Million

Cu. Feet

Percent of

National Total

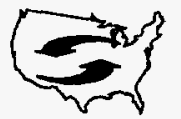

Net Interstate

Movements:

58,750

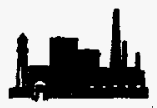

Industrial:

40,921

0.50

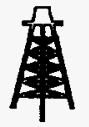

Marketed

Production:

0

0.00

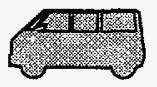

Vehicle Fuel:

1

0.06

Deliveries to Consumers:

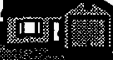

Residential:

17,384

0.36

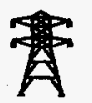

Electric

Utilities:

546

0.02

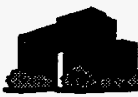

Commercial:

12,049

0.42

Total:

70,901

0.37

Table 87. Summary Statistics for Natural Gas — Rhode Island, 1990-1994

\begin{tabular}{|c|c|c|c|c|c|}
\hline & 1990 & 1991 & 1992 & 1993 & 1994 \\
\hline $\begin{array}{l}\text { Reserves (billion cubic feet) } \\
\text { Estimated Proved Reserves (dry) } \\
\text { as of December } 31\end{array}$ & 0 & 0 & 0 & 0 & 0 \\
\hline 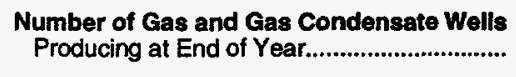 & 0 & 0 & 0 & 0 & 0 \\
\hline 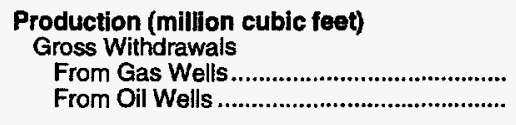 & $\begin{array}{l}0 \\
0\end{array}$ & $\begin{array}{l}0 \\
0\end{array}$ & $\begin{array}{l}0 \\
0\end{array}$ & $\begin{array}{l}0 \\
0\end{array}$ & $\stackrel{0}{0}$ \\
\hline Total. & 0 & 0 & 0 & 0 & 0 \\
\hline 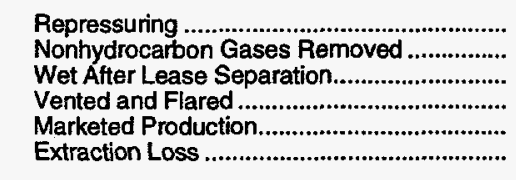 & $\begin{array}{l}0 \\
0 \\
0 \\
0 \\
0 \\
0\end{array}$ & $\begin{array}{l}0 \\
0 \\
0 \\
0 \\
0 \\
0\end{array}$ & $\begin{array}{l}0 \\
0 \\
0 \\
0 \\
0 \\
0\end{array}$ & $\begin{array}{l}0 \\
0 \\
0 \\
0 \\
0 \\
0\end{array}$ & $\begin{array}{l}0 \\
0 \\
0 \\
0 \\
0 \\
0\end{array}$ \\
\hline 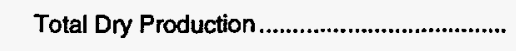 & 0 & 0 & 0 & 0 & 0 \\
\hline 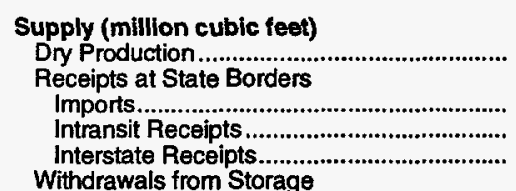 & $\begin{array}{r}0 \\
0 \\
140,431\end{array}$ & $\begin{array}{r}0 \\
0 \\
166,305\end{array}$ & $\begin{array}{r}0 \\
0 \\
205,808\end{array}$ & $\begin{array}{r}0 \\
0 \\
159,848\end{array}$ & $\begin{array}{r}0 \\
0 \\
184,516\end{array}$ \\
\hline 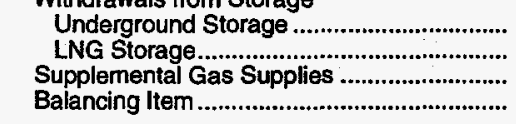 & $\begin{array}{r}0 \\
188 \\
51 \\
-1,223\end{array}$ & $\begin{array}{r}0 \\
355 \\
92 \\
-1,492\end{array}$ & $\begin{array}{r}0 \\
1,216 \\
155 \\
3,935\end{array}$ & $\begin{array}{r}0 \\
800 \\
126 \\
11,282\end{array}$ & $\begin{array}{r}0 \\
996 \\
0 \\
12,678\end{array}$ \\
\hline Total Supply & 139,447 & 165,260 & 211,114 & 172,056 & 198,189 \\
\hline
\end{tabular}

See footnotes at end of table. 


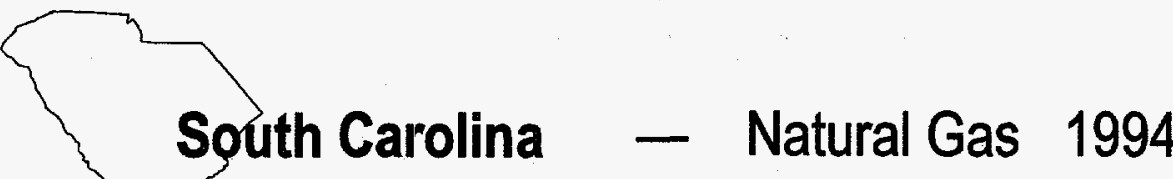

Million Percent of

Cu. Feet National Total

Million

Cu. Feet

Percent of National Total<smiles>FC1CCCCC1</smiles>

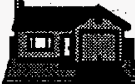

Net Interstate

Movements:

129,772

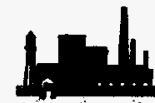

Industrial:

97,500

Marketed

Production:

0

0.00

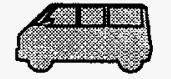

Vehicle Fuel:

2

0.12

Deliveries to Consumers:

Residential:

23,486

0.48

$\AA$

Electric

Utilities:

3,005

0.10

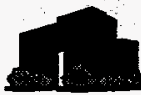

Commercial:

17,870

0.62

Total:

141,863

0.75

Table 88. Summary Statistics for Natural Gas - South Carolina, 1990-1994

\begin{tabular}{|c|c|c|c|c|c|}
\hline & 1990 & 1991 & 1992 & 1993 & 1994 \\
\hline 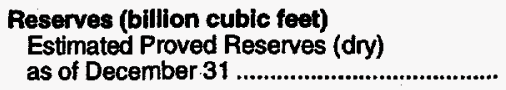 & 0 & 0 & 0 & 0 & 0 \\
\hline $\begin{array}{l}\text { Number of Gas and Gas Condensate Wells } \\
\text { Producing at End of Year.................................... }\end{array}$ & 0 & 0 & 0 & 0 & 0 \\
\hline 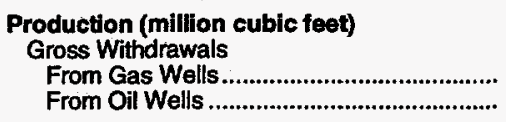 & $\begin{array}{l}0 \\
0\end{array}$ & $\begin{array}{l}0 \\
0\end{array}$ & $\begin{array}{l}0 \\
0\end{array}$ & $\begin{array}{l}0 \\
0\end{array}$ & $\begin{array}{l}0 \\
0\end{array}$ \\
\hline 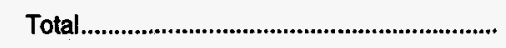 & 0 & 0 & 0 & 0 & 0 \\
\hline 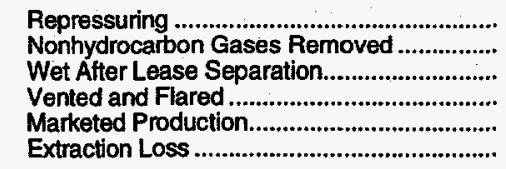 & $\begin{array}{l}0 \\
0 \\
0 \\
0 \\
0 \\
0\end{array}$ & $\begin{array}{l}0 \\
0 \\
0 \\
0 \\
0 \\
0\end{array}$ & $\begin{array}{l}0 \\
0 \\
0 \\
0 \\
0 \\
0\end{array}$ & $\begin{array}{l}0 \\
0 \\
0 \\
0 \\
0 \\
0\end{array}$ & $\begin{array}{l}0 \\
0 \\
0 \\
0 \\
0 \\
0\end{array}$ \\
\hline 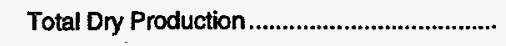 & 0 & 0 & 0 & 0 & 0 \\
\hline 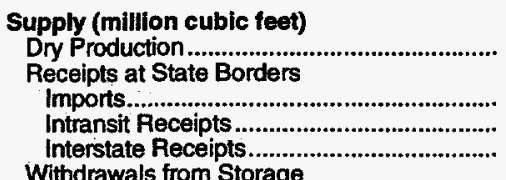 & $\begin{array}{r}0 \\
0 \\
i, 042,099\end{array}$ & $\begin{array}{r}0 \\
0 \\
1,050,481\end{array}$ & $\begin{array}{r}0 \\
0 \\
1,065,954\end{array}$ & $\begin{array}{r}0 \\
0 \\
1,024,140\end{array}$ & $\begin{array}{r}0 \\
0 \\
1,025,779\end{array}$ \\
\hline 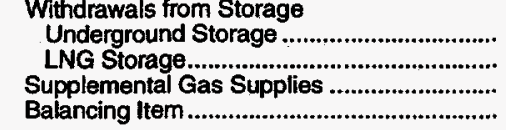 & $\begin{array}{r}0 \\
247 \\
17 \\
15,226\end{array}$ & $\begin{array}{r}0 \\
396 \\
47 \\
7,170\end{array}$ & $\begin{array}{r}0 \\
323 \\
26 \\
9,179\end{array}$ & $\begin{array}{r}0 \\
752 \\
34 \\
R_{11,296}\end{array}$ & $\begin{array}{r}0 \\
682 \\
154 \\
14,766\end{array}$ \\
\hline 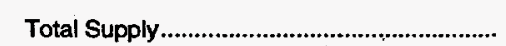 & $1,057,589$ & $1,058,094$ & $1,075,482$ & $\mathbf{R}_{1,036,222}$ & $1,041,381$ \\
\hline
\end{tabular}

See footnotes at end of table. 
Table 88. Summary Statistics for Natural Gas - South Carolina, 1990-1994 (Continued)

\begin{tabular}{|c|c|c|c|c|c|}
\hline & 1990 & 1991 & 1992 & 1993 & 1994 \\
\hline $\begin{array}{l}\text { Disposition (million cubic feet) } \\
\text { Consumption. } \\
\text { Deliveries at State Borders }\end{array}$ & 130,453 & 133,828 & 138,057 & $\mathbf{P}_{141,522}$ & 144,526 \\
\hline 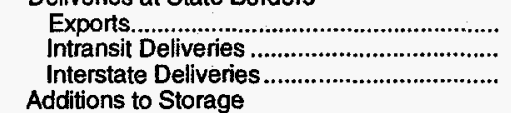 & $\begin{array}{r}0 \\
0 \\
926,742\end{array}$ & $\begin{array}{r}0 \\
0 \\
923,920\end{array}$ & $\begin{array}{r}0 \\
0 \\
937,086\end{array}$ & $\begin{array}{r}0 \\
0 \\
893,469\end{array}$ & $\begin{array}{r}0 \\
0 \\
896,007\end{array}$ \\
\hline 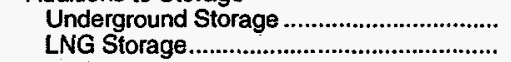 & $\begin{array}{r}0 \\
394\end{array}$ & $\begin{array}{r}0 \\
346\end{array}$ & $\begin{array}{r}0 \\
339\end{array}$ & $\begin{array}{r}0 \\
1,231\end{array}$ & $\begin{array}{r}0 \\
847\end{array}$ \\
\hline
\end{tabular}

Total Disposition

$1,057,589$

$1,058,094$

$1,075,482$

$R_{1,036,222}$

$1,041,381$

Consumption (million cubic feet)

Lease Fuel.

Pipeline Fuel......................................................

Plant Fuel.

$\begin{array}{r}0 \\ 2,858 \\ 0\end{array} \quad 2,80$

2,900

0
2,755
0

0
2,663
0

Delivered to Consumers

Residential

Industrial

Vehicle Fuel

Electric Utilities

15,394

86,831

0
6,975

19,612

15,796

85,790

9,823

127,595

131,020

Total Delivered to Consumers

130,453

133,828

22,392

16,644

94,327

1,795

Total Consumption..

135,157

138,057

24,345

$\mathrm{R}_{17,014}$
95,557

95,557
0

1,851

$\mathrm{P}_{138,767}$

$\mathrm{A}_{141,522}$

Delivered for the Account of Others

(million cubic feet)

Residential.

Commercial..........................................................

Industrial

Electric Utilities

341

20,817

0
278
25,368

0
239
31,176
0

0
$\mathrm{R}_{132}$

33,507

265

Firm Deliveries

(million cubic feet)

Residential

Commercial

Industrial.

Electric Utiities

Vehicle Fuel

NA
NA
NA
NA
NA

0

22,755

Interruptible Deliveries

(million cubic feet)

Commercial.

Industrial

Electric Utilities

Vehicle Fuel

Number of Consumers

Residential....

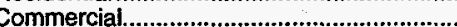

Industrial.

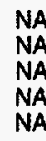

NA
NA
NA
NA

24,345
$R_{14,156}$

83,265

0
0

23,486

14,435

80,076

NA

23,486

17,870
97,500

3,005

141,863

144,526

Average Annual Consumption per Consumer

(thousand cubjc feet)

Residential.

Commercial

Industrial

386

62,739

NA

NA

NA

NA

NA

NA

339,486

39,904
1,384

344,763

39,999

1,400

NA

NA

NA

2,859

83,265

1,415

80,076

2,961

357,818

40,968
1,568

370,411
$\mathbf{R}_{42,191}$

42,191
1,625

416,773

45,487

Average Prices for Natural Gas

(dollars per thousand cubic feet)

Wellhead (Marketed Production)

Imports

Exports.

Pipeline Fuel

City Gate.

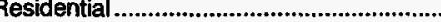

Commercial

Industrial.

Vehicle Fuel

0
0
2.06
3.14
7.17
5.90
3.35
0
1.76

$\begin{array}{r}57 \\ \quad 395 \\ \hline\end{array}$

61,279

63
406

60,158

66
$R_{403}$

58,804

56

50,571

= Revised data

$N A=$ Not Availlabie.

Notes: Deliveries to electric utilities (consumplion) are reported on the Form ElA-176, "Annual Report of Natural and Supplemental Gas Supply and Disposition." See the

discussion on electric utility data and Table A1 in Appendix A for a comparison of reporting to these two forms. Totals may not add due to independent rounding.

Sources: Energy Information Administration (EIA), Form ElA-176, "Annual Report of Natural and Supplemental Gas Supply and Disposition"; Form ElA-627, "Annual

Quantity and Value of Natural Gas Report"; Form ElA-857, "Monthly Report of Natural Gas Purchases and Deliveries to Consumers"; Form ElA-816, "Monthly Natural Gas

Liquids Report"; Form EIA-759, "Monthly Power Plant Report"; Form FERC-423, "Monthly Report of Cost and Quality of Fuels for Electric Plants"; Form EIA-191, "Underground Gas Storage Report"; Form FPC-14, "Annual Report for Importers and Exporters of Natural Gas"; U.S. Crude Oil, Natural Gas, and Natural Gas Liquids Reserves, 1994 Annual Report, DOEJEIA-0216(94); and the U.S. Minerals Management Service. 


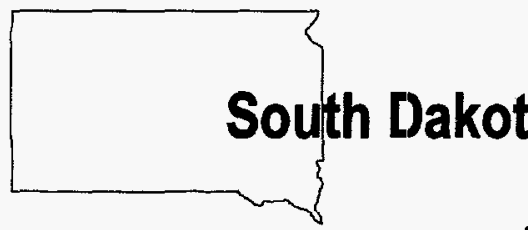

$\begin{array}{ll}\text { Million } & \text { Percent of } \\ \text { Cu. Feet } & \text { National Total }\end{array}$

Million

Cu. Feet

Percent of National Total

Net Interstate

Movements:

30,255

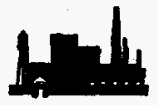

Industrial:

5,508

0.07

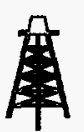

Marketed

Production:

1,437

0.01

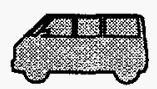

Vehicle Fuel:

Deliveries to Consumers:

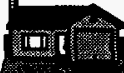

Residential:

12,056

0.25

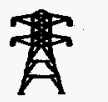

Electric

Utilities:

Commercial: $\quad 10,274 \quad 0.35$

Total:

28,002

0.15

Table 89. Summary Statistics for Natural Gas - South Dakota, 1990-1994

\begin{tabular}{|c|c|c|c|c|c|}
\hline & 1990 & 1991 & 1992 & 1993 & 1994 \\
\hline 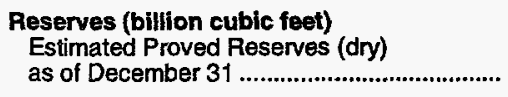 & NA & NA & NA & NA & NA \\
\hline 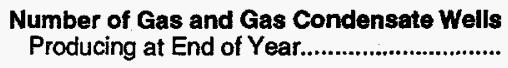 & 54 & 54 & 38 & $\mathbf{F}_{47}$ & 55 \\
\hline 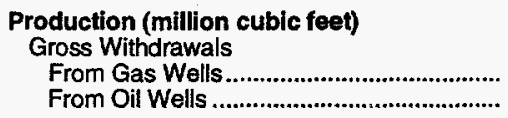 & $\begin{array}{r}549 \\
4,233\end{array}$ & $\begin{array}{r}489 \\
5,315\end{array}$ & $\begin{array}{l}1,006 \\
5,957\end{array}$ & $\begin{array}{r}R_{854} \\
R_{6,204}\end{array}$ & $\begin{array}{l}1,000 \\
6,264\end{array}$ \\
\hline Total & 4,782 & 5,804 & 6,963 & $\mathrm{P}_{7,057}$ & 7,264 \\
\hline 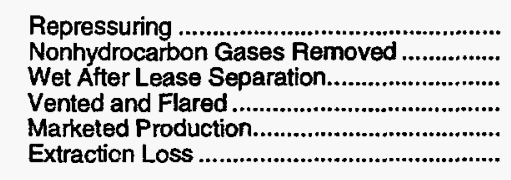 & $\begin{array}{r}253 \\
0 \\
4,529 \\
3,648 \\
881 \\
0\end{array}$ & $\begin{array}{r}77 \\
0 \\
5,726 \\
4,844 \\
882 \\
0\end{array}$ & $\begin{array}{r}30 \\
0 \\
6,932 \\
5,476 \\
1,456 \\
0\end{array}$ & $\begin{array}{r}\mathbf{R}_{19} \\
0 \\
\mathbf{R}_{7,038} \\
\mathbf{R}_{5}, 732 \\
\mathbf{R}_{1,306} \\
0\end{array}$ & $\begin{array}{r}22 \\
0 \\
7,242 \\
5,805 \\
1,437 \\
0\end{array}$ \\
\hline Total Dry Production & 881 & 882 & 1,456 & $R_{1,306}$ & 1,437 \\
\hline 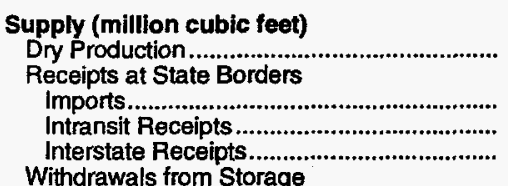 & $\begin{array}{r}0 \\
0 \\
417,567\end{array}$ & $\begin{array}{r}0 \\
0 \\
459,068\end{array}$ & $\begin{array}{r}0 \\
0 \\
532,549\end{array}$ & $\begin{array}{r}0 \\
0 \\
592,248\end{array}$ & $\begin{array}{r}0 \\
0 \\
613,908\end{array}$ \\
\hline 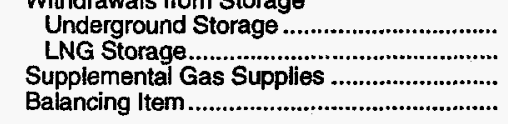 & $\begin{array}{r}0 \\
0 \\
10 \\
73\end{array}$ & $\begin{array}{r}0 \\
15 \\
3 \\
365\end{array}$ & $\begin{array}{r}0 \\
13 \\
10 \\
-1,036\end{array}$ & $\begin{array}{r}0 \\
0 \\
9 \\
R_{-650}\end{array}$ & $\begin{array}{r}0 \\
0 \\
61 \\
-773\end{array}$ \\
\hline Total Supply & 418,531 & 460,334 & 532,993 & $R_{592,913}$ & 614,633 \\
\hline
\end{tabular}

See footnotes at end of table. 
Table 89. Summary Statistics for Natural Gas - South Dakota, 1990-1994 (Continued)

\begin{tabular}{|c|c|c|c|c|c|}
\hline & 1990 & 1991 & 1992 & 1993 & 1994 \\
\hline 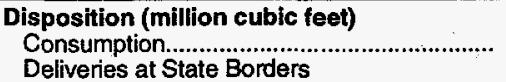 & 25,056 & 26,260 & 26,645 & $\mathrm{R}_{31,311}$ & 30,980 \\
\hline 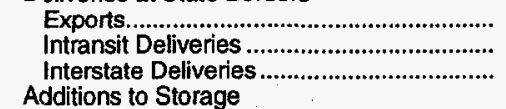 & $\begin{array}{r}0 \\
0 \\
393,476\end{array}$ & $\begin{array}{r}0 \\
0 \\
434,073\end{array}$ & $\begin{array}{r}0 \\
0 \\
506,324\end{array}$ & $\begin{array}{r}0 \\
0 \\
561,602\end{array}$ & $\begin{array}{r}0 \\
0 \\
583,653\end{array}$ \\
\hline 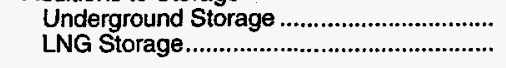 & $\begin{array}{l}0 \\
0\end{array}$ & $\begin{array}{l}0 \\
0\end{array}$ & $\begin{array}{r}0 \\
24\end{array}$ & $\begin{array}{l}0 \\
0\end{array}$ & $\begin{array}{l}0 \\
0\end{array}$ \\
\hline 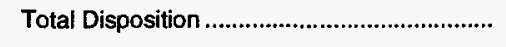 & 418,531 & 460,334 & 532,993 & $\mathrm{P}_{592,913}$ & 614,633 \\
\hline 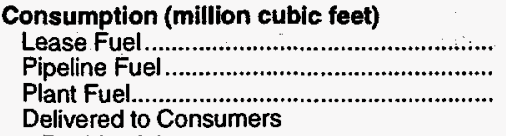 & $\begin{array}{r}158 \\
110 \\
0\end{array}$ & $\begin{array}{r}393 \\
338 \\
0\end{array}$ & $\begin{array}{r}451 \\
1,741 \\
0\end{array}$ & $\begin{array}{r}R_{452} \\
2,564 \\
0\end{array}$ & $\begin{array}{r}437 \\
2,541 \\
0\end{array}$ \\
\hline 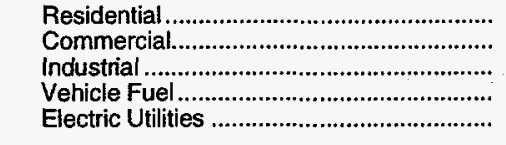 & $\begin{array}{r}10,204 \\
8,555 \\
5,793 \\
0 \\
235\end{array}$ & $\begin{array}{r}11,218 \\
9,473 \\
4,658 \\
2 \\
177\end{array}$ & $\begin{array}{r}10,791 \\
9,122 \\
4,488 \\
5 \\
48\end{array}$ & $\begin{array}{r}12,431 \\
10,696 \\
4,976 \\
7 \\
186\end{array}$ & $\begin{array}{r}12,056 \\
10,274 \\
5,508 \\
5 \\
159\end{array}$ \\
\hline Total Delivered to Consumers ............................. & 24,787 & 25,529 & 24,454 & 28,295 & 28,002 \\
\hline 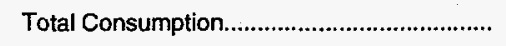 & 25,056 & 26,260 & 26,645 & $R_{31,311}$ & 30,980 \\
\hline 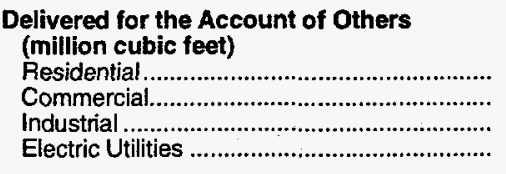 & $\begin{array}{r}0 \\
1,161 \\
3,124 \\
139\end{array}$ & $\begin{array}{r}0 \\
1,723 \\
2,090 \\
151\end{array}$ & $\begin{array}{r}0 \\
1,603 \\
2,129 \\
714\end{array}$ & $\begin{array}{r}0 \\
1,724 \\
2,428 \\
142\end{array}$ & $\begin{array}{r}0 \\
1,124 \\
3,449 \\
64\end{array}$ \\
\hline 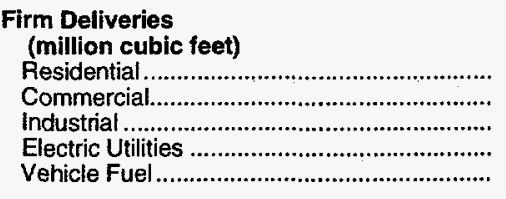 & $\begin{array}{l}\text { NA } \\
\text { NA } \\
\text { NA } \\
\text { NA } \\
\text { NA }\end{array}$ & $\begin{array}{l}\text { NA } \\
\text { NA } \\
\text { NA } \\
\text { NA } \\
\text { NA }\end{array}$ & $\begin{array}{l}\text { NA } \\
\text { NA } \\
\text { NA } \\
\text { NA } \\
\text { NA }\end{array}$ & $\begin{array}{r}12,431 \\
8,890 \\
2,935 \\
0 \\
7\end{array}$ & $\begin{array}{r}12,056 \\
9,491 \\
2,936 \\
0 \\
5\end{array}$ \\
\hline 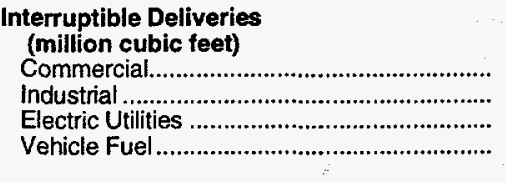 & $\begin{array}{l}\text { NA } \\
\text { NA } \\
\text { NA } \\
\text { NA }\end{array}$ & $\begin{array}{l}\text { NA } \\
\text { NA } \\
\text { NA }\end{array}$ & $\begin{array}{l}\text { NA } \\
\text { NA } \\
\text { NA } \\
\text { NA }\end{array}$ & $\begin{array}{r}1,806 \\
2,935 \\
154 \\
--\end{array}$ & $\begin{array}{r}784 \\
2,936 \\
64 \\
--\end{array}$ \\
\hline 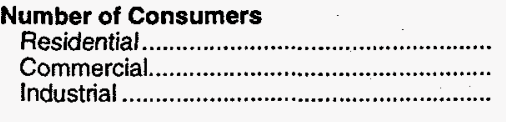 & $\begin{array}{r}105,436 \\
13,443 \\
275\end{array}$ & $\begin{array}{r}107,846 \\
13,692 \\
283\end{array}$ & $\begin{array}{r}110,291 \\
14,133 \\
319\end{array}$ & $\begin{array}{r}128,029 \\
16,523 \\
355\end{array}$ & $\begin{array}{r}119,544 \\
15,539 \\
381\end{array}$ \\
\hline 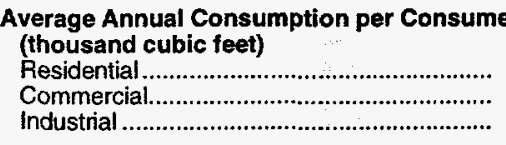 & $\begin{array}{r}97 \\
636 \\
21,067\end{array}$ & $\begin{array}{r}104 \\
692 \\
16,459\end{array}$ & $\begin{array}{r}98 \\
645 \\
14,068\end{array}$ & $\begin{array}{r}97 \\
647 \\
14,016\end{array}$ & $\begin{array}{r}101 \\
661 \\
14,456\end{array}$ \\
\hline 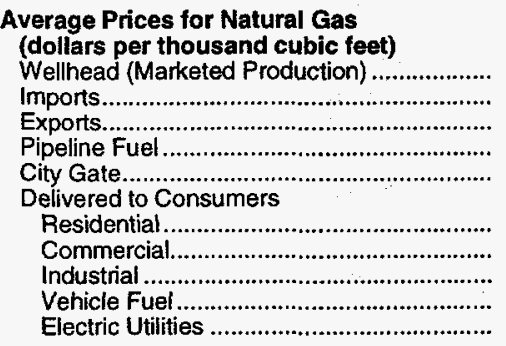 & $\begin{array}{r}1.56 \\
0 \\
0 \\
2.13 \\
3.12\end{array}$ & $\begin{array}{r}1.12 \\
0 \\
0 \\
1.42 \\
3.11\end{array}$ & $\begin{array}{r}1.79 \\
0 \\
0 \\
1.22 \\
3.10\end{array}$ & $\begin{array}{r}R_{2.13} \\
0 \\
0 \\
1.80 \\
3.35\end{array}$ & $\begin{array}{r}1.73 \\
0 \\
0 \\
1.36 \\
3.35\end{array}$ \\
\hline
\end{tabular}

$R=$ Revised data.

Notes: Deliveries to electric utilities (consumption) are reported on the Form ElA-176, "Annual Report of Natural and Supplemental Gas Supply and Disposition." See the discussion on electric utility data and Table A1 in Appendix A for a comparison of reporting to these two forms. Totals may not add due to independent rounding.

Sources: Energy Information Administration (EIA), Form EIA-176, "Annual Report of Natural and Supplemental Gas Supply and Disposition"; Form EIA-627, "Annual Quantity and Value of Natural Gas Report"; Form E|A-857, "Monthly Report of Natural Gas Purchases and Deliveries to Consumers"; Form ElA-816, "Monthly Natural Gas Liquids Report"; Form EIA-759, "Monthly Power Plant Report"; Form FERC-423, "Monthly Report of Cost and Quality of Fuels for Electric Plants"; Form EIA-191, "Underground Gas Storage Report"; Form FPC-14, "Annual Report for Importers and Exporters of Natural Gas"; U.S. Crude Oil, Natural Gas, and Natural Gas Liquids Resenves, 1994 Annual Report, DOEJEIA-0216(94); and the U.S. Minerals Management Service. 
Net Interstate

Movements:

220,082

Marketed

Production:

1,990

0.01

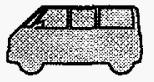

Vehicle Fuel:

5

0.29

Deliveries to Consumers:

$1]$

Residential

57,334

1.18

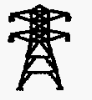

Electric

Utilities:

1,019

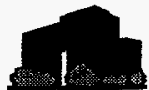

Commercial:

50,760

1.75

Table 90. Summary Statistics for Natural Gas - Tennessee, 1990-1994

\begin{tabular}{|c|c|c|c|c|c|}
\hline & 1990 & 1991 & 1992 & 1993 & 1994 \\
\hline 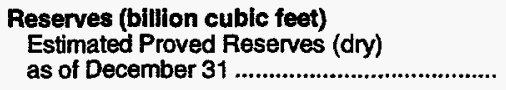 & NA & NA & NA & NA & NA \\
\hline 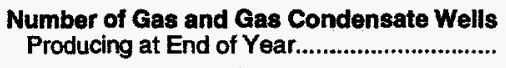 & $\mathbf{R}_{0}$ & $\mathbf{R}_{0}$ & $\mathrm{R}_{0}$ & $R_{0}$ & 0 \\
\hline 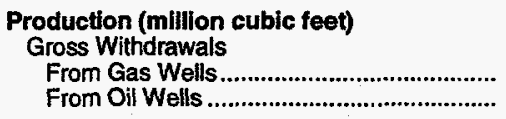 & $\begin{array}{r}0 \\
2,067\end{array}$ & $\begin{array}{r}0 \\
1,856\end{array}$ & $\begin{array}{r}0 \\
1,770\end{array}$ & $\begin{array}{r}0 \\
1,660\end{array}$ & 1,990 \\
\hline 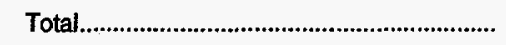 & 2,067 & 1,856 & 1,770 & 1,660 & 1,990 \\
\hline 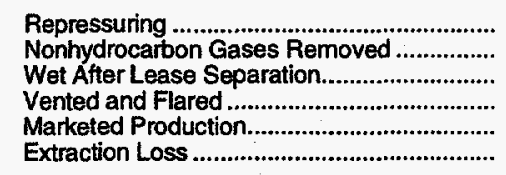 & $\begin{array}{r}\text { NA } \\
N A \\
2,067 \\
N A \\
2,067 \\
0\end{array}$ & $\begin{array}{r}\text { NA } \\
\text { NA } \\
1,856 \\
N A \\
1,856 \\
0\end{array}$ & $\begin{array}{r}\text { NA } \\
\text { NA } \\
1,770 \\
\text { NA } \\
1,770 \\
0\end{array}$ & $\begin{array}{r}\mathrm{NA} \\
\mathrm{NA} \\
1,660 \\
\mathrm{NA} \\
1,660 \\
0\end{array}$ & $\begin{array}{r}\text { NA } \\
\text { NA } \\
1,990 \\
N A \\
1,990 \\
0\end{array}$ \\
\hline Total Dry Production & 2,067 & 1,856 & 1,770 & 1,660 & 1,990 \\
\hline 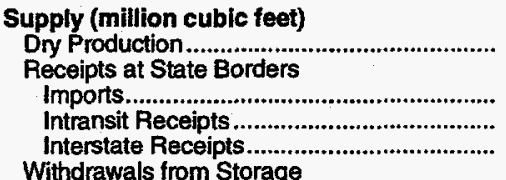 & $\begin{array}{r}2,067 \\
\\
0 \\
0 \\
3,403,840\end{array}$ & $\begin{array}{r}1,856 \\
\\
0 \\
0 \\
3,210,927\end{array}$ & $\begin{array}{r}1,770 \\
0 \\
0 \\
0 \\
3,361,934\end{array}$ & $\begin{array}{r}1,660 \\
0 \\
0 \\
0 \\
3,510,786\end{array}$ & $\begin{array}{r}1,990 \\
0 \\
0 \\
0 \\
3,878,897\end{array}$ \\
\hline 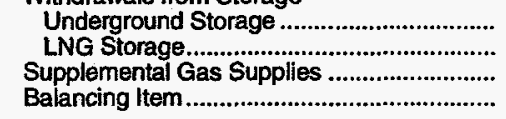 & $\begin{array}{r}0 \\
2,377 \\
3 \\
113,660\end{array}$ & $\begin{array}{r}0 \\
1,923 \\
8 \\
39,041\end{array}$ & $\begin{array}{r}0 \\
3,065 \\
12 \\
70,754\end{array}$ & $\begin{array}{r}0 \\
2,931 \\
13 \\
51,663\end{array}$ & $\begin{array}{r}0 \\
2,879 \\
84 \\
24,107\end{array}$ \\
\hline 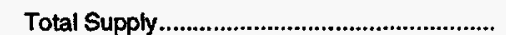 & $3,521,947$ & $3,253,755$ & $3,437,535$ & $3,567,053$ & $3,907,957$ \\
\hline
\end{tabular}


Table 90. Summary Statistics for Natural Gas - Tennessee, 1990-1994 (Continued)

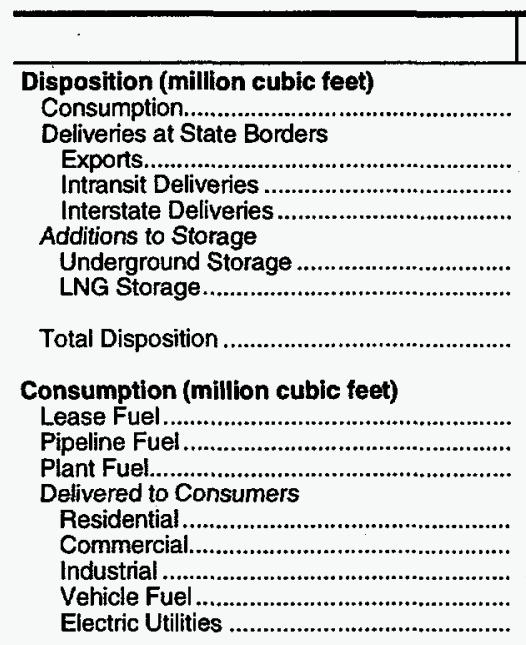

Total Delivered to Consumers.

Total Consumption

Delivered for the Account of Others

(million cubic feet)

Residential..

Commercial.....................................................

Industrial

Electric Utilities

Firm Deliveries

(million cubic feet)

Residential...

Commercial

Industrial.

Electric Utilities

Vehicle Fuel

Interruptible Deliveries

(million cubic feet)

Commercial

Industrial .

Electric Utilities

Vehicle Fuel

Number of Consumers

Residentia

Commercial.

Industrial.

Average Annual Consumption per Consumer (thousand cubic teet)

Residential

Commercial

Industrial

Average Prices for Natural Gas

(dollars per thousand cubic feet)

Wellhead (Marketed Production)

Imports.

Exports.

Pipeline Fuel

City Gate.

Delivered to Consumers

Residential.

Industrial

Vehicle Fuel

\section{0}

219,831

0
0

$3,299,107$

0
3,008

$3,521,947$

68
19,596

6

46,340

43,552

109,703

2
565

200,161

219,831

0
1,092

1,092
43,605

563

I

1991

227,108

0
0

$3,025,125$

0
1,522

$3,253,755$

41
15,756

49,357

45,953

115,786

211

211,309

227,108

0
1,961
51,462
143

NA

NA

NA

NA

NA

NA

NA

NA
NA
NA
NA

627,031

88,753

2,361

661,105

89,863

2,369

74
491

46,465

75
511
48,876
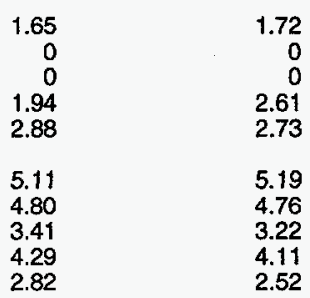

1992

241,702

0
0

$3,193,074$

0
2,759

$3,437,535$

39
16,388

52,220

46,532

126,230

2
291

225,275

241,702

0
1,680

1,680
63,666

$\begin{array}{r}219 \\ \hline\end{array}$

NA
NA
NA

NA

NA

NA

NA

NA

NA

696,140

91,999

2,425

73,363
94,860

94,860
2,512

58,919
47,002
$\mathbf{8 6 , 1 5 5}$
0
4

3,752
$\mathbf{R}_{\mathbf{8 6 , 1 5 5}}$

1,625

2,129

66,780

1,015

2,992

64,615

498

57,057

46,932

74,389

0
5

3,829

74,389

971

768,421

97,943

2,440
75

506

52,054

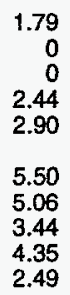

80
535

535
49,485

$\begin{array}{r}75 \\ 518 \\ \hline\end{array}$

48,725

A = Revised data.

NA $=$ Not Available.

Notes: Deliveries to electric utilities (consumption) are reported on the Form ElA-176, "Annual Report of Natural and Supplemental Gas Supply and Disposition." See the

discussion on electric utility data and Table A1 in Appendix A for a comparison of reporting to these two forms. Totals may not add due to independent rounding

Sources: Energy Information Administration (EIA), Form EIA-176, "Annual Report of Natural and Supplemental Gas Supply and Disposition"; Form EIA-627, "Annual

Quantity and Value of Natural Gas Report"; Form ElA-857, "Monthly Report of Natural Gas Purchases and Deliveries to Consumers"; Form EIA-816, "Monthly Natural Gas

Gas Storage Report"; Form FPC-14, "Annual Report for Importers and Exporters of Natural Gas"; U.S. Crude Oil, Natural Gas, and Natural Gas Liquids Reserves, 1994 Annual

Report, DOE/EIA-0216(94); and the U.S. Minerals Management Senvice. 


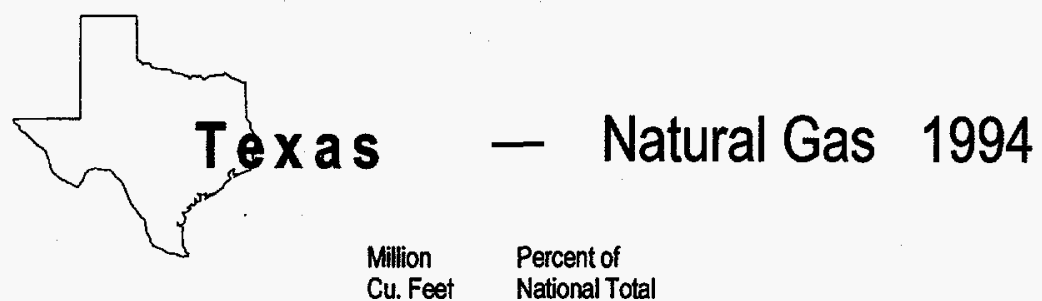

Million

Percent of

Cu. Feet National Total

Cu. Feet

National Total

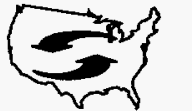

Net Interstate

Movements:

$-2,105,298$

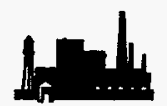

Industrial: $\quad 1,829,478$

22.37

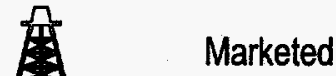

Production." $\quad 6,353,844$

32.36

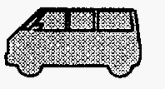

Vehicle Fuel:

45

2.60

Deliveries to Consumers:

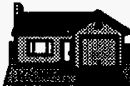

Residential:

213,433

4.40

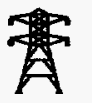

Electric

Utilities:

$1,049,205$

35.12

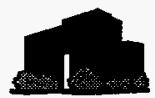

Commercial:

180,232

6.23

Total:

$3,272,393$

17.31

Table 91. Summary Statistics for Natural Gas - Texas, 1990-1994

\begin{tabular}{|c|c|c|c|c|c|}
\hline & 1990 & 1991 & 1992 & 1993 & 1994 \\
\hline $\begin{array}{l}\text { Reserves (billion cubic feet) } \\
\text { Estimated Proved Reserves (dry) } \\
\text { as of December } 31\end{array}$ & 45,492 & 42,849 & 42,089 & 41,379 & 42,357 \\
\hline 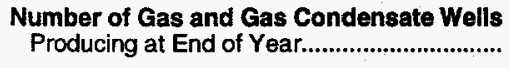 & 50,867 & 47,615 & 46,298 & 47,101 & 48,654 \\
\hline 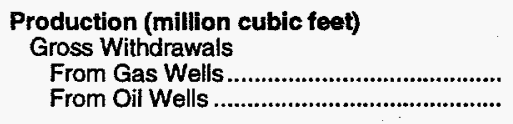 & $\begin{array}{l}5,574,740 \\
1,332,316\end{array}$ & $\begin{array}{l}5,538,692 \\
1,306,851\end{array}$ & $\begin{array}{l}5,406,256 \\
1,301,756\end{array}$ & $\begin{array}{l}\mathbf{5 , 4 7 4 , 5 5 9} \\
1,342,368\end{array}$ & $\begin{array}{l}5,643,577 \\
1,268,127\end{array}$ \\
\hline 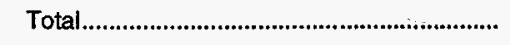 & $B, 907,056$ & $6,845,543$ & $6,708,012$ & $6,816,927$ & $6,911,705$ \\
\hline 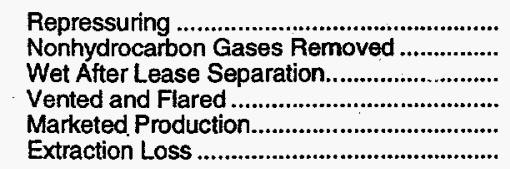 & $\begin{array}{r}380,032 \\
155,631 \\
15,371,393 \\
28,247 \\
13,343,146 \\
342,186\end{array}$ & $\begin{array}{r}360,852 \\
173,399 \\
6,311,291 \\
30,638 \\
6,280,654 \\
353,737\end{array}$ & $\begin{array}{r}362,458 \\
180,003 \\
6,165,551 \\
19,689 \\
6,145,862 \\
374,126\end{array}$ & $\begin{array}{r}348,558 \\
184,258 \\
6,284,111 \\
34,486 \\
6,249,624 \\
385,063\end{array}$ & $\begin{array}{r}319,360 \\
196,463 \\
6,395,881 \\
42,037 \\
6,353,844 \\
381,020\end{array}$ \\
\hline Total Dry Production & $13,000,960$ & $5,926,917$ & $5,771,736$ & $5,864,561$ & $5,972,824$ \\
\hline 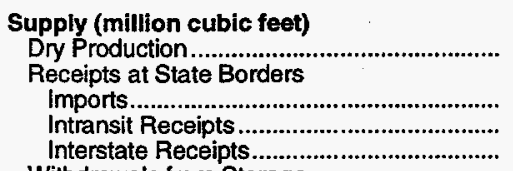 & $\begin{array}{r}15,000,960 \\
0 \\
0 \\
653,332\end{array}$ & $\begin{array}{r}0 \\
0 \\
709,907\end{array}$ & $\begin{array}{r}0 \\
0 \\
566,789\end{array}$ & $\begin{array}{r}1,678 \\
0 \\
538,183\end{array}$ & $\begin{array}{r}7,013 \\
0 \\
603,826\end{array}$ \\
\hline 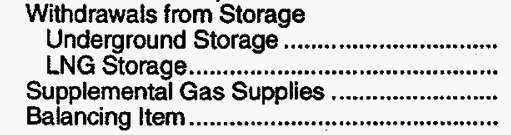 & $\begin{array}{r}150,250 \\
0 \\
1,240 \\
-131,857\end{array}$ & $\begin{array}{r}164,510 \\
0 \\
1,076 \\
-270,304\end{array}$ & $\begin{array}{r}384,042 \\
0 \\
1 \\
-64,200\end{array}$ & $\begin{array}{r}232,070 \\
0 \\
3 \\
R_{-65,481}\end{array}$ & $\begin{array}{r}257,318 \\
0 \\
1 \\
-148,241\end{array}$ \\
\hline Total Supply & $5,673,926$ & $6,532,106$ & $6,658,369$ & $R_{6,571,015}$ & $6,692,740$ \\
\hline
\end{tabular}

See footnotes at end of table. 
Table 91. Summary Statistics for Natural Gas - Texas, 1990-1994 (Continued)

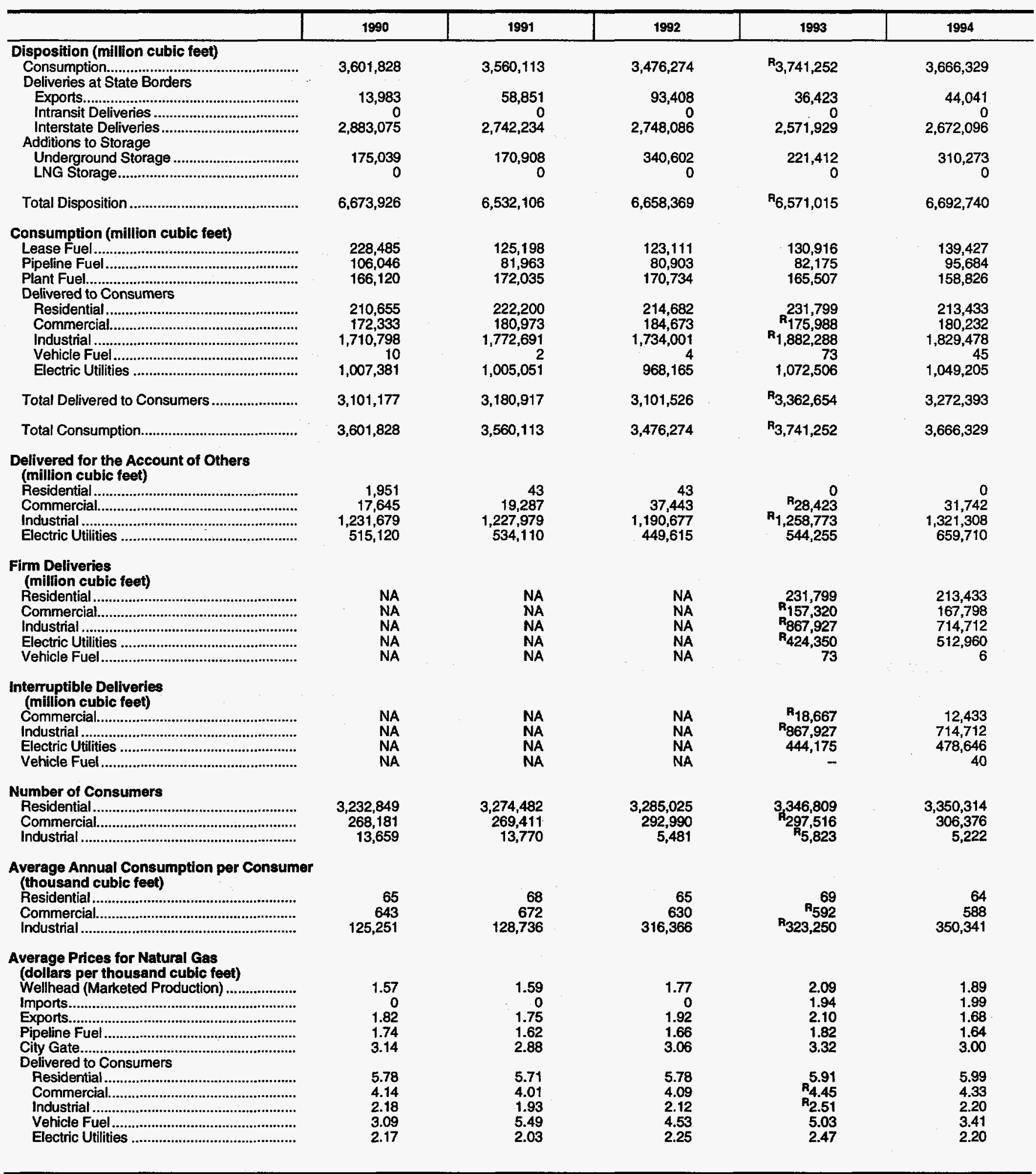

R $=$ Revised data.
NA $=$ Not Available.

Notes: Deliveries to electric utilities (consumption) are reported on the Form ElA-176, "Annual Report of Natural and Supplemental Gas Supply and Disposition." See the discussion on electric utility dala and Table A1 in Appendix A for a comparison of reporting to these two forms. Totals may not add due to independent rounding.

Sources: Energy Information Administration (EIA) Form ElA-176, "Annual Report of Natural and Supplemental Gas Supply and Disposition"; Form ElA-627, innual

Quantity and Value of Natural Gas Report"; Form ElA-857 "Monthly Report of Natural Gas Purchases and Deliveries to Consumers"; Form ElA-816, "Mionthly' Natural Gas

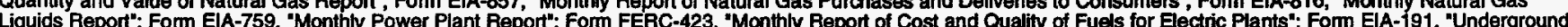

Liquids Repori, Fon ElA-759, "Monthly Power Plant Report; Form FERC-423, "Monthly Report of Cost and Quality of Fuels for Eectric Plants"; Form ElA-191, "Underground

Gas Storage Report"; Form FPC-14, "Annual Report for Importers and Exporters of Natural Gas"; U.S. Crude Oil, Natural Gas, and Natural Gas Lquids Reserves, 1994 Annual 


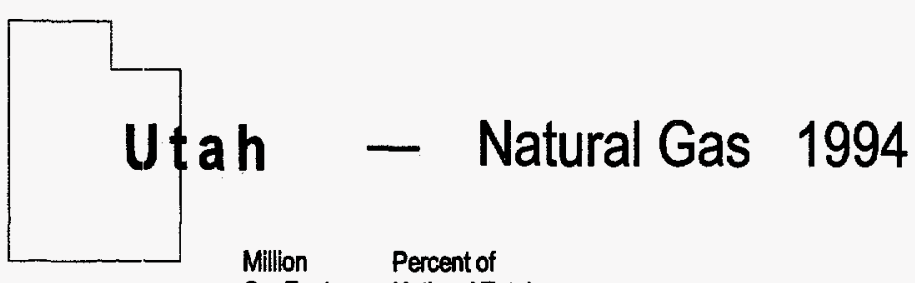

Cu. Feet National Total

Million

Percent of

Cu. Feet

National Total

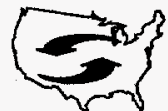

Net Interstate

Movements:

4,231

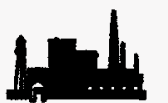

Industrial:

36,618

0.45

E Marketed

Production:

270,858

1.38

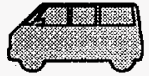

Vehicle Fuel:

$52 \quad 3.01$

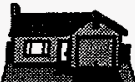

Deliveries to Consumers:

Residential:

48,922

1.01

Electric

8,900

0.30

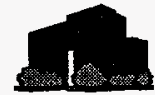

Commercial: $\quad 26,501$

0.92

Total:

120,993

0.64

Table 92. Summary Statistics for Natural Gas - Utah, 1990-1994

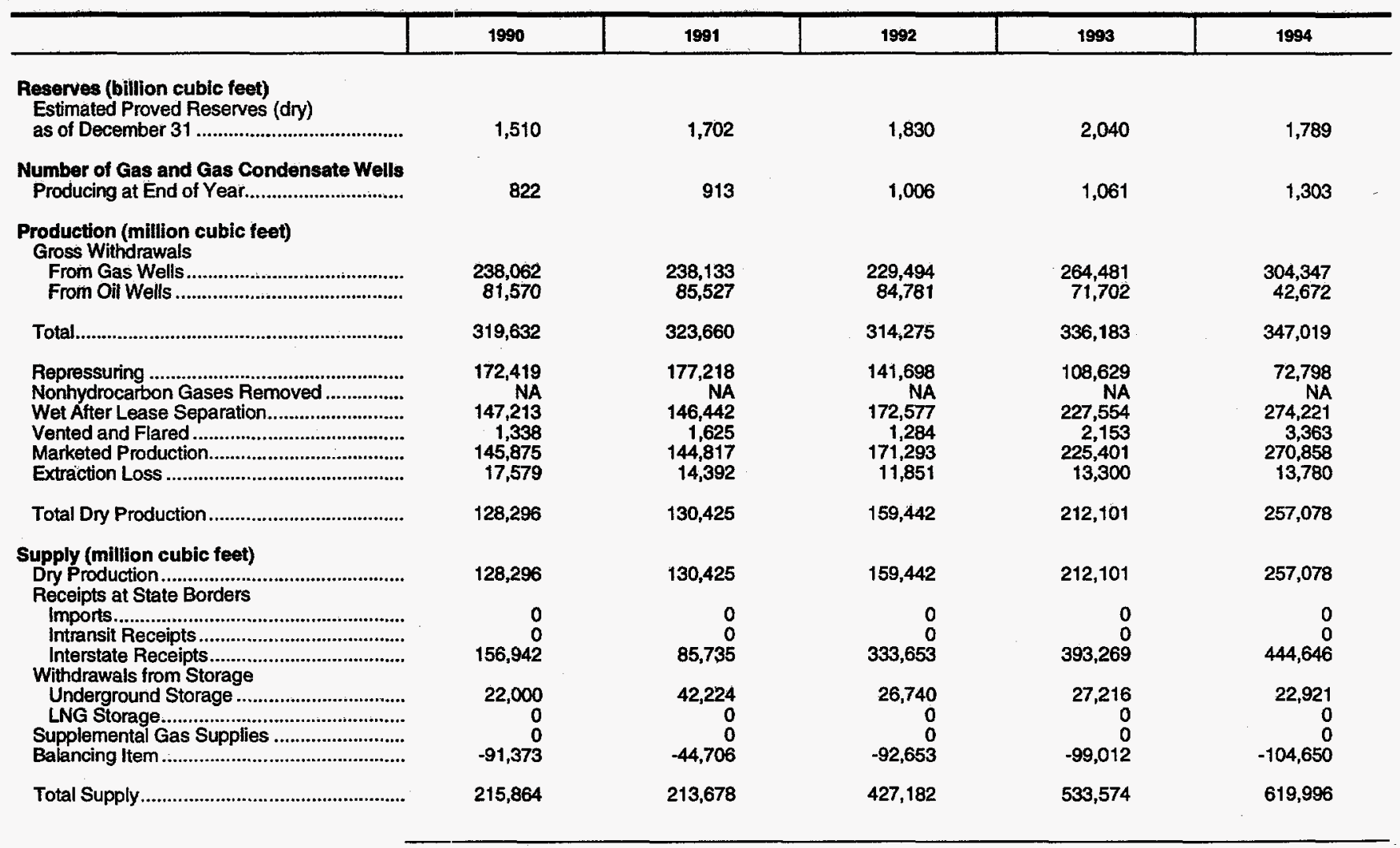

See footnotes at end of table. 
Table 92. Summary Statistics for Natural Gas - Utah, 1990-1994 (Continued)

\begin{tabular}{|c|c|c|c|c|c|}
\hline & 1990 & 1991 & 1992 & 1993 & 1994 \\
\hline $\begin{array}{l}\text { Disposition (million cubic feet) } \\
\text { Consumption............................................................. } \\
\text { Deliveries at State Borders }\end{array}$ & 116,648 & 132,766 & 122,649 & 138,044 & 137,073 \\
\hline 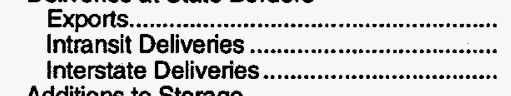 & $\begin{array}{r}0 \\
0 \\
72,020\end{array}$ & $\begin{array}{r}0 \\
0 \\
48,664\end{array}$ & $\begin{array}{r}0 \\
0 \\
273,310\end{array}$ & $\begin{array}{r}0 \\
0 \\
361,042\end{array}$ & $\begin{array}{r}0 \\
0 \\
440,415\end{array}$ \\
\hline 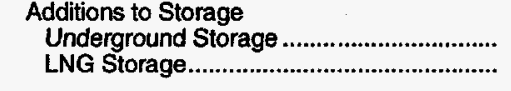 & $\begin{array}{r}27,196 \\
0\end{array}$ & $\begin{array}{r}32,248 \\
0\end{array}$ & $\begin{array}{r}31,222 \\
0\end{array}$ & $\begin{array}{r}34,488 \\
0\end{array}$ & $\begin{array}{r}42,508 \\
0\end{array}$ \\
\hline 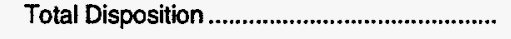 & 215,864 & 213,678 & 427,182 & 533,574 & 619,996 \\
\hline 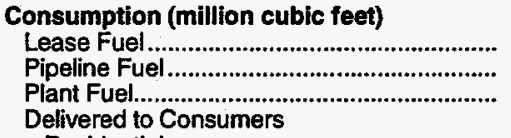 & $\begin{array}{r}10,573 \\
875 \\
9,146\end{array}$ & $\begin{array}{r}4,597 \\
864 \\
9,141\end{array}$ & $\begin{array}{l}3,866 \\
1,284 \\
\mathbf{8 , 7 4 5}\end{array}$ & $\begin{array}{l}3,241 \\
2,513 \\
9,285\end{array}$ & $\begin{array}{l}3,322 \\
2,807 \\
9,951\end{array}$ \\
\hline 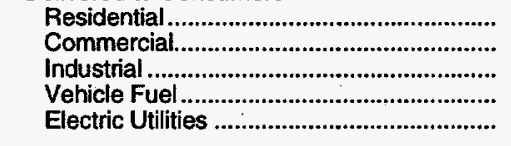 & $\begin{array}{r}43,424 \\
16,220 \\
35,502 \\
1 \\
907\end{array}$ & $\begin{array}{r}50,572 \\
19,276 \\
43,120 \\
6 \\
5,190\end{array}$ & $\begin{array}{r}44,701 \\
16,584 \\
40,878 \\
15 \\
6,576\end{array}$ & $\begin{array}{r}51,779 \\
22,588 \\
42,301 \\
33 \\
6,305\end{array}$ & $\begin{array}{r}48,922 \\
26,501 \\
36,618 \\
52 \\
8,900\end{array}$ \\
\hline Total Delivered to Consumers ............................ & 96,054 & 118,164 & 108,755 & 123,005 & 120,993 \\
\hline Total Consumption........................................... & 116,648 & 132,766 & 122,649 & 138,044 & 137,073 \\
\hline 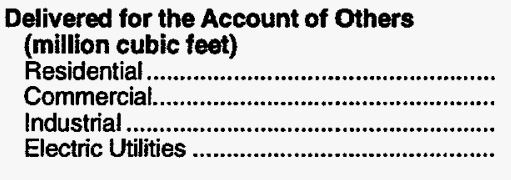 & $\begin{array}{r}0 \\
0 \\
28,108 \\
445\end{array}$ & $\begin{array}{r}0 \\
0 \\
36,534 \\
4,562\end{array}$ & $\begin{array}{r}0 \\
0 \\
36,087 \\
5,434\end{array}$ & $\begin{array}{r}0 \\
0 \\
39,387 \\
5,004\end{array}$ & $\begin{array}{r}0 \\
4,438 \\
32,238 \\
7,922\end{array}$ \\
\hline 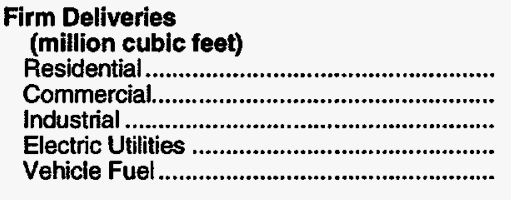 & $\begin{array}{l}\text { NA } \\
\text { NA } \\
\text { NA } \\
\text { NA } \\
\text { NA }\end{array}$ & $\begin{array}{l}\text { NA } \\
\text { NA } \\
\text { NA } \\
\text { NA } \\
\text { NA }\end{array}$ & $\begin{array}{l}\text { NA } \\
\text { NA } \\
\text { NA } \\
\text { NA } \\
\text { NA }\end{array}$ & $\begin{array}{r}51,779 \\
21,258 \\
40,410 \\
0 \\
33\end{array}$ & $\begin{array}{r}48,922 \\
20,273 \\
34,619 \\
6,200 \\
52\end{array}$ \\
\hline 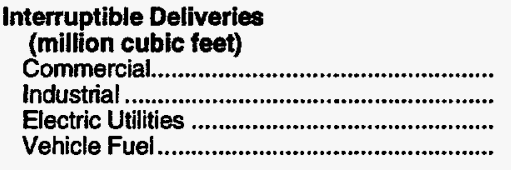 & $\begin{array}{l}\text { NA } \\
\text { NA } \\
\text { NA } \\
\text { NA }\end{array}$ & $\begin{array}{l}\text { NA } \\
\text { NA } \\
\text { NA } \\
\text { NA }\end{array}$ & $\begin{array}{l}\text { NA } \\
\text { NA } \\
\text { NA } \\
\text { NA }\end{array}$ & $\begin{array}{r}1,330 \\
40,410 \\
5,009 \\
-\end{array}$ & $\begin{array}{r}6,228 \\
34,619 \\
1,756 \\
--\end{array}$ \\
\hline 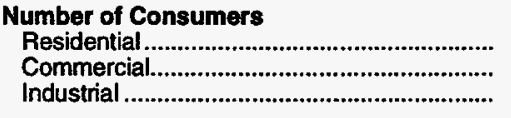 & $\begin{array}{r}453,023 \\
34,697 \\
1,508\end{array}$ & $\begin{array}{r}455,649 \\
35,627 \\
631\end{array}$ & $\begin{array}{r}467,664 \\
36,145 \\
783\end{array}$ & $\begin{array}{r}484,438 \\
37,816 \\
345\end{array}$ & $\begin{array}{r}503,583 \\
39,183 \\
252\end{array}$ \\
\hline 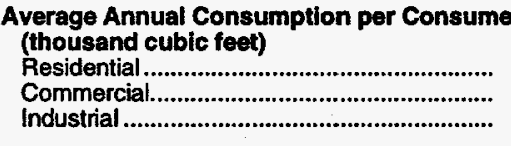 & $\begin{array}{r}96 \\
467 \\
23,542\end{array}$ & $\begin{array}{r}111 \\
541 \\
68,336\end{array}$ & $\begin{array}{r}96 \\
459 \\
52,207\end{array}$ & $\begin{array}{r}107 \\
597 \\
+22,611\end{array}$ & $\begin{array}{r}97 \\
676 \\
145,310\end{array}$ \\
\hline 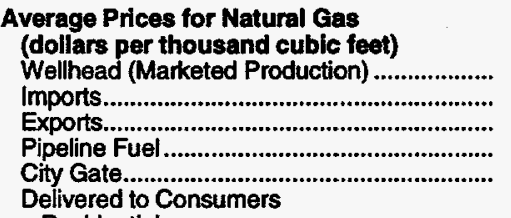 & $\begin{array}{r}1.70 \\
0 \\
0 \\
2.25 \\
3.91\end{array}$ & $\begin{array}{r}1.54 \\
0 \\
0 \\
2.51 \\
3.89\end{array}$ & $\begin{array}{r}1.63 \\
0 \\
0 \\
2.25 \\
4.09\end{array}$ & $\begin{array}{r}1.77 \\
0 \\
0 \\
1.91 \\
2.63\end{array}$ & $\begin{array}{r}1.54 \\
0 \\
0 \\
1.94 \\
3.31\end{array}$ \\
\hline 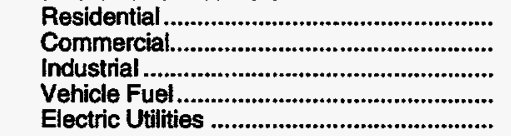 & $\begin{array}{l}5.28 \\
4.30 \\
3.62 \\
6.85 \\
5.04\end{array}$ & $\begin{array}{l}5.44 \\
4.50 \\
3.69 \\
5.52 \\
1.72\end{array}$ & $\begin{array}{l}5.44 \\
4.40 \\
3.91 \\
5.42 \\
1.87\end{array}$ & $\begin{array}{l}5.13 \\
4.06 \\
3.67 \\
5.27 \\
2.31\end{array}$ & $\begin{array}{l}4.96 \\
3.84 \\
2.74 \\
4.90 \\
2.42\end{array}$ \\
\hline
\end{tabular}

NA = Not Available.

Notes: Deliveries to electric utifities (consumption) are reported on the Form ElA-176, "Annual Report of Natural and Supplemental Gas Supply and Disposition." See the discussion on electric utility data and Table A1 in Appendix A for a comparison of reporting to these two forms. Totals may not add due to independent rounding

Sources: Energy Information Administration (EIA), Form EIA-176, "Annual Report of Natural and Supplemental Gas Supply and Disposition"; Form EIA-627, "Annual

Quantity and Value of Natural Gas Report"; Form ElA-857, "Monthly Report of Natural Gas Purchases and Deliveries to Consumers"; Form ElA-816, "Monthly Natural Gas

Liquids Report"; Form EIA-759, "Monthly Power Plant Report"; Form FERC-423, "Monthly Report of Cost and Quality of Fuels for Electric Plants"; Form EIA-191, "Underground

Gas Storage Report"; Form FPC-14, "Annual Report for Importers and Exporters of Natural Gas"; U.S. Crude Oil, Natural Gas, and Natural Gas Liquids Reserves, 1994 Annual Report, DOE/EIA-0216(94); and the U.S. Minerals Management Service. 


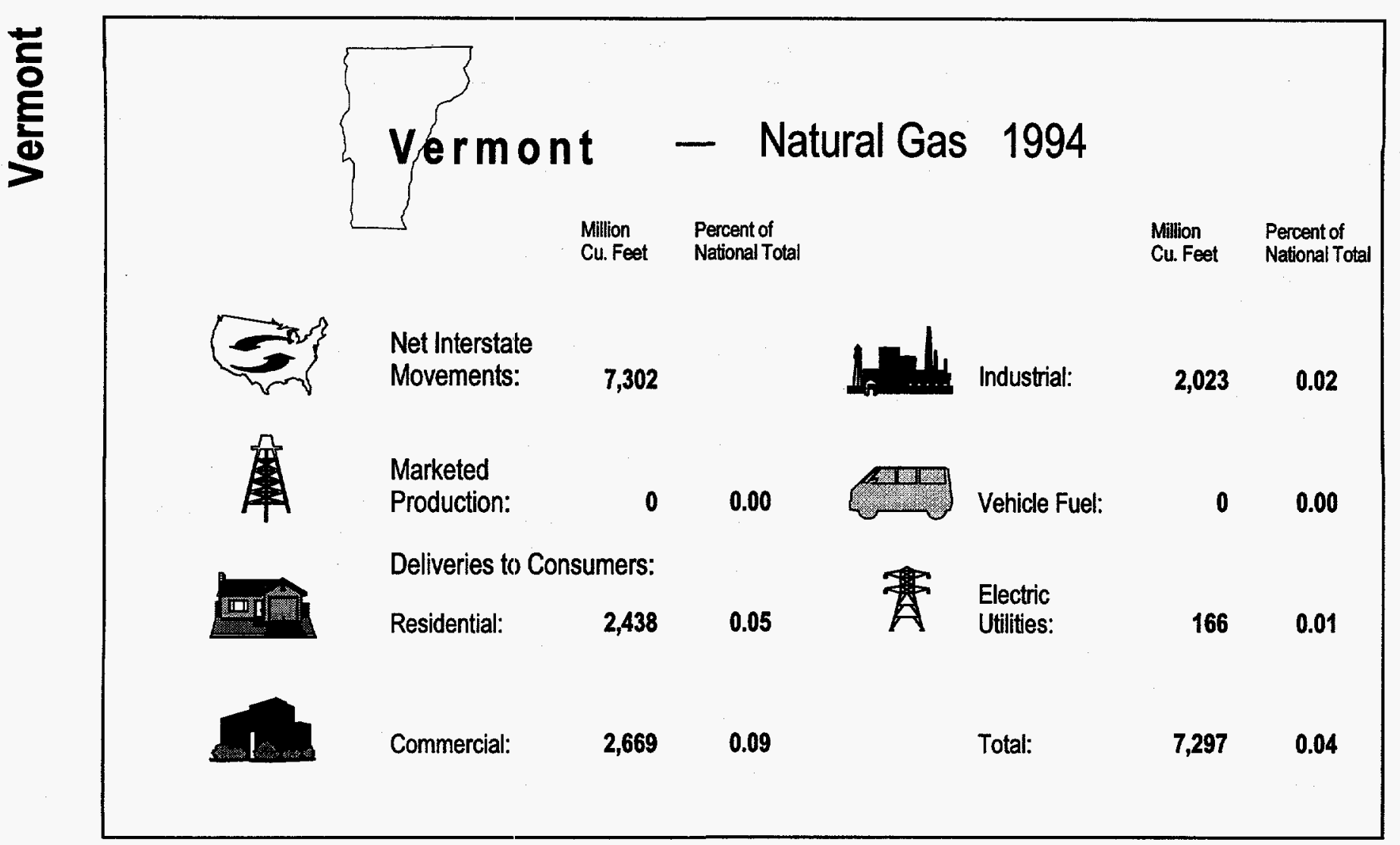

Table 93. Summary Statistics for Natural Gas - Vermont, 1990-1994

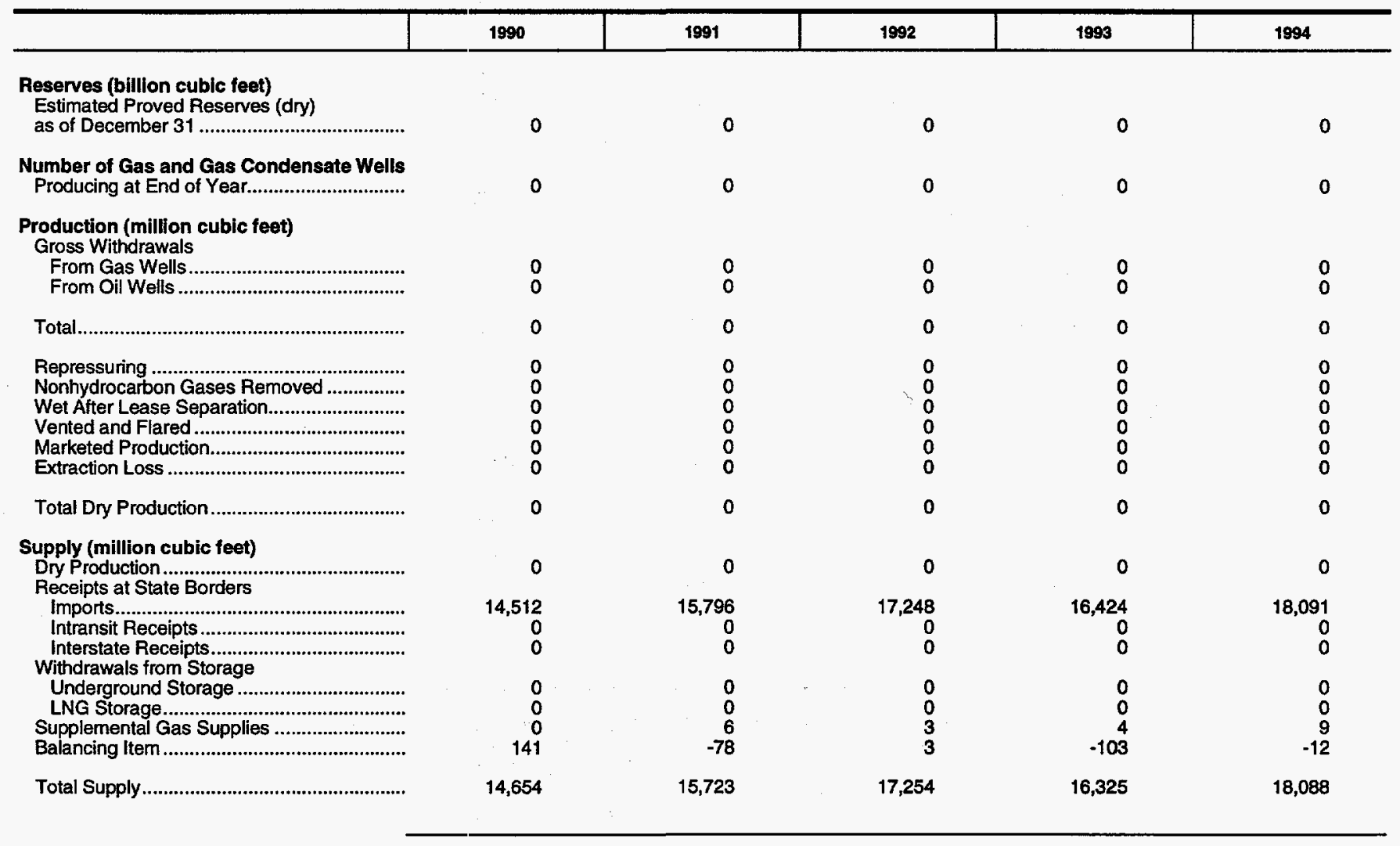


Table 93. Summary Statistics for Natural Gas - Vermont, 1990-1994 (Continued)

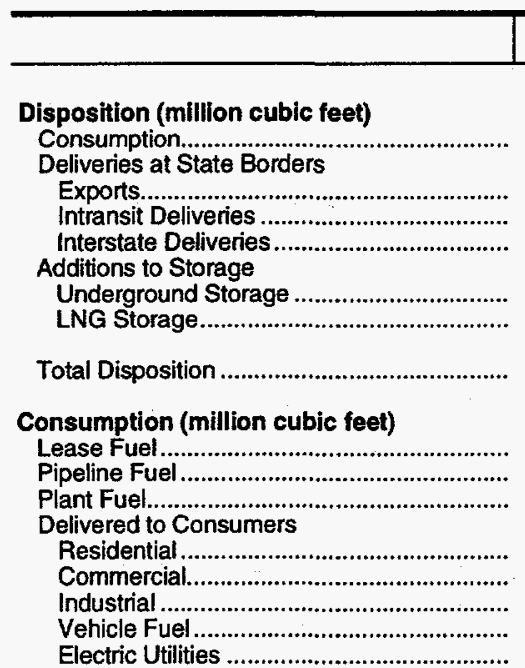

Total Consumption...

Delivered for the Account of Others
Total Delivered to Consumers.

(million cubic feet)

Residential.

Commercial.

Industrial.

Electric Utilities

Firm Deliveries

(million cubic teet)

Residential

Commercial.

Industrial ....

Electric Utilities .......................................

Vehicle

\section{Interruptible Deliveries}

(million cubic feet)

Commercial.

Industrial.

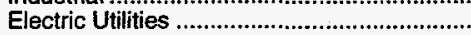

Vehicle Fuel.

Number of Consumers

Residential.

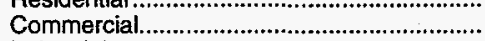

Industrial.

1990

6,766

0
0
7,887

0

14,654

0
5
0

2,150

2,049

2,049
1,878

0
685

6,761

6,766

0

0
0
0

NA

NA

NA

NA

NA

NA

NA

18,300
2,949

2,949
15

Average Annual Consumption per Consumer (thousand cubic feet)

Residential

Commercial

Industrial

117

695
125,194
Average Prices for Natural Gas

(dollars per thousand cubic feet)

Wellhead (Marketed Production)

Imports.

Pipeline Fue

City Gate.

Delivered to Consumers

Residential

Commercial

Industrial.

Vehicle Fuel.

Electric Utilities

3.04
0
2.85
2.88

5.79
5.05
3.51
0
2.42

2.80
0
2.86
2.87

6.23
5.24
2.99
0
1.72

1991

7,073

0
0
8,651

0
0

15,723

$$
\begin{aligned}
& 0 \\
& 3 \\
& 0
\end{aligned}
$$

2,203

2,058

1,717

1,091

7,069

7,073

1992

7,601

0
0
9,653

0

17,254

\section{3
0}

2,520

2,319

1,958

0
801

7,598

7,601

0
0
0
0

\section{NA}

NA

NA
NA
NA

NA
NA

NA

NA

NA

19,879

3,154
13

1993

1994

7,299

7,228

0
0

0
9,097

10,789

0
0

16,325

18,088

$\begin{array}{rr}0 & 0 \\ 3 & 3 \\ 0 & 0 \\ 2,530 & \\ 2,382 & 2,438 \\ 2,045 & 2,669 \\ 0 & 2,023 \\ 268 & 0 \\ 7,225 & 166 \\ 7,228 & 7,297 \\ & \\ & 7,299\end{array}$

7,299

NA

NA
NA

NA

20,468

3,198

18

3,314
20

3,512
24

2,438
2,669

1,908

2,382

1,945

0

0
0

1,908

165

$1,94 \overline{5}$

268

123

111

652
132,070

725
108,751

117

719
02,255

108

760
84,307

NA $=$ Not Available.

Notes: Deliveries to electric utilities (consumption) are reported on the Form ElA-176, "Annual Report of Natural and Supplemental Gas Supply and Disposition." See the discussion on electric utility data and Table A1 in Appendix A for a comparison of reporting to these two forms. Totals may not add due to independent rounding

Sources: Energy Information Administration (EIA), Form EIA-176, "Annual Report of Natural and Supplemental Gas Supply and Disposition"; Form EIA-627, "Annual

Quantity and Value of Natural Gas Report"; Form EIA-857, "Monthly Report of Natural Gas Purchases and Deliveries to Consumers"; Form ElA-816, "Monthly Natural Gas

Liquids Report"; Form EIA-759, "Monthly Power Plant Report"; Form FERC-423, "Monthly Report of Cost and Quality of Fuels for Electric Plants"; Form EIA-191, "Underground

Gas Storage Report"; Fom FPC-14, "Annual Report for Importers and Exporters of Natural Gas"; U.S. Crude Oil, Natural Gas, and Natural Gas Liquids Reserves, 1994 Annual Report, DOEE/EIA-0216(94); and the U.S. Minerals Management Service. 
Million Percent of

Cu. Feet National Total

Million Percent of

Cu. Feet National Total

Net Interstate

Movements:

140,723

Marketed

Production:

50,259

0.26

Deliveries to Consumers:

Residential:

65,176

1.34

Commercial:

52,944

1.83

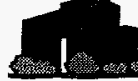

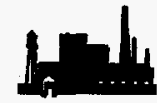

Industrial:

85,764

1.05
Vehicle Fuel:

20

1.16

Electric

Utilities:

19,219

0.64

Total:

223,122

Table 94. Summary Statistics for Natural Gas - Virginia, 1990-1994

\begin{tabular}{|c|c|c|c|c|c|}
\hline & 1990 & 1991 & 1992 & 1993 & 1994 \\
\hline 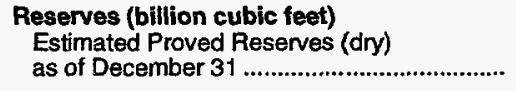 & 138 & 225 & 904 & 1,322 & 1,833 \\
\hline 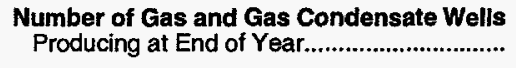 & 819 & 886 & 1,153 & 1,426 & 1,470 \\
\hline 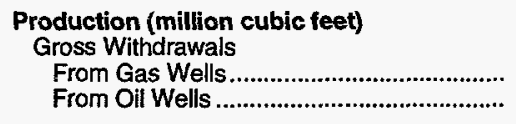 & $\begin{array}{r}14,774 \\
0\end{array}$ & $\begin{array}{r}14,906 \\
0\end{array}$ & $\begin{array}{r}24,733 \\
0\end{array}$ & $\begin{array}{r}37,840 \\
0\end{array}$ & $\begin{array}{r}50,259 \\
0\end{array}$ \\
\hline Total. & 14,774 & 14,906 & 24,733 & 37,840 & 50,259 \\
\hline 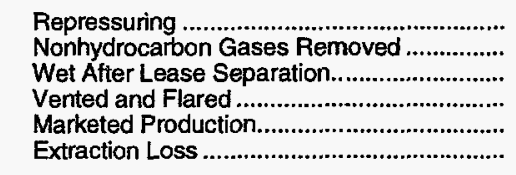 & $\begin{array}{r}0 \\
0 \\
14,774 \\
0 \\
14,774 \\
0\end{array}$ & $\begin{array}{r}0 \\
0 \\
14,906 \\
0 \\
14,906 \\
0\end{array}$ & $\begin{array}{r}0 \\
0 \\
24,733 \\
0 \\
24,733 \\
0\end{array}$ & $\begin{array}{r}0 \\
0 \\
37,840 \\
0 \\
37,840 \\
0\end{array}$ & $\begin{array}{r}0 \\
0 \\
50,259 \\
0 \\
50,259 \\
0\end{array}$ \\
\hline Total Dry Production & 14,774 & 14,906 & 24,733 & 37,840 & 50,259 \\
\hline 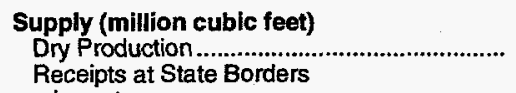 & 14,774 & 14,906 & 24,733 & 37,840 & 50,259 \\
\hline 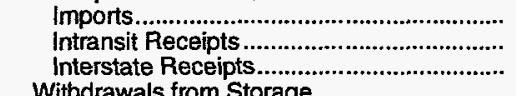 & $\begin{array}{r}0 \\
0 \\
1,059,259\end{array}$ & $\begin{array}{r}0 \\
0 \\
1,077,858\end{array}$ & $\begin{array}{r}0 \\
0 \\
1,119,418\end{array}$ & $\begin{array}{r}0 \\
0 \\
1,035,590\end{array}$ & $\begin{array}{r}0 \\
0 \\
1,025,827\end{array}$ \\
\hline 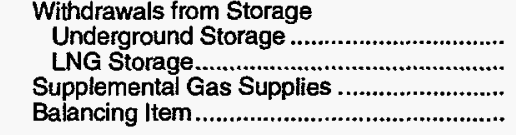 & $\begin{array}{r}0 \\
142 \\
59 \\
21,047\end{array}$ & $\begin{array}{r}0 \\
243 \\
240 \\
-1,941\end{array}$ & $\begin{array}{r}0 \\
168 \\
245 \\
-14,334\end{array}$ & $\begin{array}{r}0 \\
274 \\
538 \\
-4,287\end{array}$ & $\begin{array}{r}0 \\
236 \\
1,195 \\
38,265\end{array}$ \\
\hline 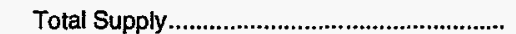 & $1,095,281$ & $1,091,307$ & $1,130,230$ & $1,069,956$ & $1,115,782$ \\
\hline
\end{tabular}

See footnotes at end of table. 
Table 94. Summary Statistics for Natural Gas - Virginia, 1990-1994 (Continued)

\begin{tabular}{|c|c|c|c|c|c|}
\hline & 1990 & 1991 & 1992 & 1993 & 1994 \\
\hline \multicolumn{6}{|l|}{ Disposition (million cubic feet) } \\
\hline \multicolumn{6}{|l|}{ Deliveries at State Borders } \\
\hline \multirow{2}{*}{\multicolumn{6}{|c|}{ 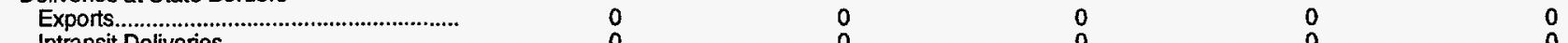 }} \\
\hline & & 0 & 0 & 0 & 0 \\
\hline 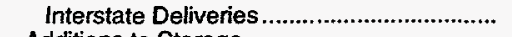 & 914,223 & 916,463 & 930,018 & 851,890 & 885,104 \\
\hline \multicolumn{6}{|l|}{ Additions to Storage } \\
\hline 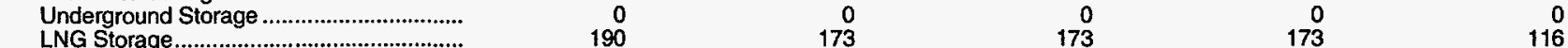 & $\begin{array}{r}0 \\
190\end{array}$ & $\begin{aligned} 0 \\
173\end{aligned}$ & $\begin{aligned} 0 \\
173\end{aligned}$ & $\begin{aligned} 0 \\
173\end{aligned}$ & $\begin{array}{r}0 \\
116\end{array}$ \\
\hline 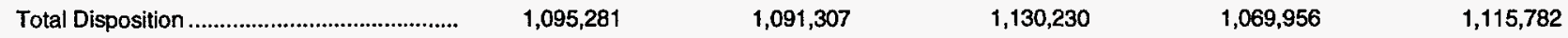 & $1,095,281$ & $1,091,307$ & $1,130,230$ & $1,069,956$ & $1,115,782$ \\
\hline \multicolumn{6}{|l|}{ Consumption (million cubic feet) } \\
\hline 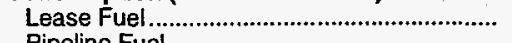 & 489 & 327 & 653 & 1,120 & 1,102 \\
\hline 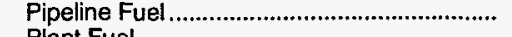 & 6,943 & 6,636 & 6,454 & 5,736 & 6,338 \\
\hline $\begin{array}{l}\text { Plant Fuel... } \\
\text { Delivered to Consumers }\end{array}$ & 0 & & & 0 & 0 \\
\hline 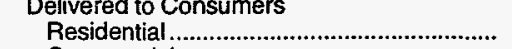 & 51,438 & 54,199 & 62,431 & 65,472 & 65,176 \\
\hline 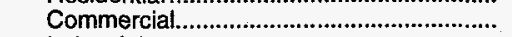 & 41,038 & 44,077 & 50,757 & 52,880 & 52,944 \\
\hline 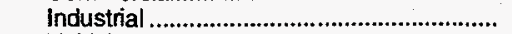 & 74,661 & 59,962 & 68,808 & 72,949 & 85,764 \\
\hline 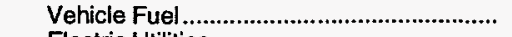 & 0 & & & 1 & 20 \\
\hline 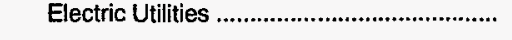 & 6,299 & 9,471 & 10,936 & 19,735 & 19,219 \\
\hline Total Delivered to Consumers ........................... & 173,437 & 167,709 & 192,932 & 211,037 & 223,122 \\
\hline Total Consumption................................................. & 180,868 & 174,671 & 200,039 & 217,893 & 230,562 \\
\hline \multicolumn{6}{|l|}{$\begin{array}{l}\text { Delivered for the Account of Others } \\
\text { (million cubic feet) }\end{array}$} \\
\hline 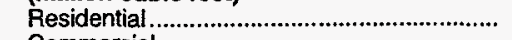 & 0 & 0 & 0 & 0 & 0 \\
\hline 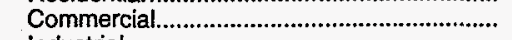 & 2,804 & 2,826 & 4,719 & 5,902 & 7,039 \\
\hline $\begin{array}{l}\text { Industrial } \\
\text { Electric Utilities }\end{array}$ & $\begin{array}{r}53,144 \\
4,945\end{array}$ & 42,709 & 51,223 & 54,791 & 67,341 \\
\hline 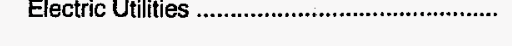 & $4,94 b$ & $\mathbf{9}, \mathbf{4 4 1}$ & 11,669 & 25,823 & \\
\hline \multicolumn{6}{|l|}{$\begin{array}{l}\text { Firm Deliveries } \\
\text { (million cubic feet) }\end{array}$} \\
\hline 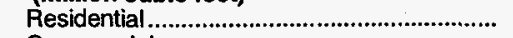 & NA & NA & NA & 65,472 & 65,176 \\
\hline 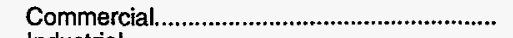 & NA & NA & NA & 38,314 & 39,093 \\
\hline Industrial & NA & NA & NA & 27,418 & 34,452 \\
\hline 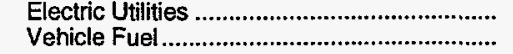 & $\begin{array}{l}\text { NA } \\
\text { NA }\end{array}$ & $\begin{array}{l}\text { NA } \\
\text { NA }\end{array}$ & $\begin{array}{l}\text { NA } \\
\text { NA }\end{array}$ & $\begin{array}{r}18,774 \\
1\end{array}$ & $\begin{array}{r}17,932 \\
18\end{array}$ \\
\hline \multirow{2}{*}{\multicolumn{6}{|c|}{$\begin{array}{l}\text { Interruptible Deliveries } \\
\text { (million cubic feet) }\end{array}$}} \\
\hline & & & & & \\
\hline 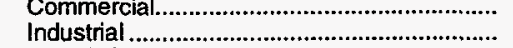 & $\begin{array}{l}\text { NA } \\
\text { NA }\end{array}$ & $\begin{array}{l}\text { NA } \\
\text { NA }\end{array}$ & $\begin{array}{l}\text { NA } \\
\text { NA }\end{array}$ & $\begin{array}{l}14,666 \\
27,418\end{array}$ & $\begin{array}{l}13,651 \\
34,452\end{array}$ \\
\hline 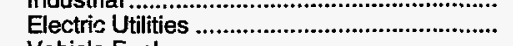 & NA & NA & NA & 7,075 & 8,865 \\
\hline 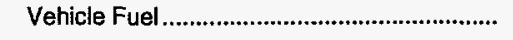 & NA & NA & NA & - & 2 \\
\hline \multicolumn{6}{|l|}{ Number of Consumers } \\
\hline 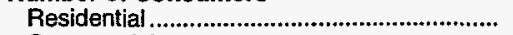 & 622,883 & 651,203 & 664,500 & 690,061 & 721,495 \\
\hline 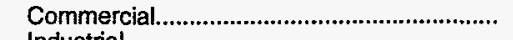 & 63,751 & 67,997 & 69,629 & 70,161 & 72,188 \\
\hline 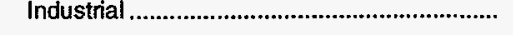 & 929 & 1,156 & 1,101 & $n_{2,706}$ & 2,740 \\
\hline \multicolumn{6}{|l|}{$\begin{array}{l}\text { Average Annual Consumption per Consumer } \\
\text { (thousand cubic feet) }\end{array}$} \\
\hline 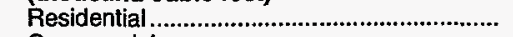 & 83 & 83 & 94 & 95 & 90 \\
\hline 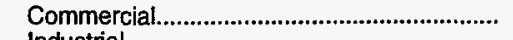 & 644 & 648 & 729 & 754 & 733 \\
\hline 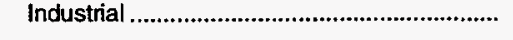 & 80,367 & 51,870 & 62,496 & ${ }^{*} 26,958$ & 31,301 \\
\hline \multicolumn{6}{|l|}{$\begin{array}{l}\text { Average Prices for Natural Gas } \\
\text { (dollars per thousand cubic feet) }\end{array}$} \\
\hline Wellhead (Marketed Production) ........................ & 2.30 & 1.88 & 1.85 & 2.29 & 2.15 \\
\hline 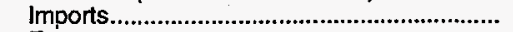 & 0 & 0 & 0 & 0 & 0 \\
\hline 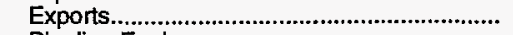 & 0 & 0 & 0 & 0 & 0 \\
\hline 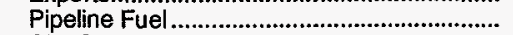 & 2.05 & 1.86 & 1.93 & 2.27 & 2.14 \\
\hline City Gate........................................................ & 3.09 & 2.76 & 2.91 & 3.33 & 3.44 \\
\hline \multicolumn{6}{|l|}{ Delivered to Consumers } \\
\hline 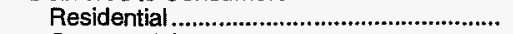 & 6.75 & 6.80 & 6.69 & 7.51 & 7.63 \\
\hline 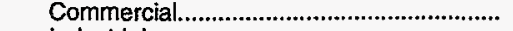 & 4.93 & 4.85 & 4.97 & 5.60 & 5.67 \\
\hline 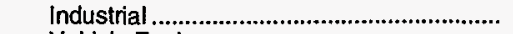 & 3.67 & 3.81 & 3.72 & 3.88 & 3.15 \\
\hline 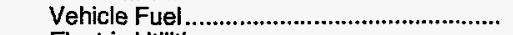 & 0 & 0 & 0 & 5.62 & 2.57 \\
\hline 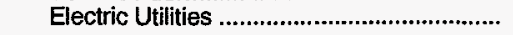 & 2.69 & 1.90 & 2.47 & 2.89 & 2.66 \\
\hline
\end{tabular}

$\mathbf{R}=$ Revised data.

NA $=$ Not Available.

Notes: Deliveries to electric utilities (consumption) are reported on the Form EIA-176, "Annual Report of Natural and Supplemental Gas Supply and Disposition." See the discussion on electric utility data and Table A1 in Appendix A for a comparison of reporting to these two forms. Totals may not add due to independent rounding.

Sources: Energy Information Administration (EIA), Form EIA-176, "Annual Report of Natural and Supplemental Gas Supply and Disposition"; Form ElA-627, "Annual Quantity and Value of Natural Gas Report"; Form EIA-857, "Monthly Report of Natural Gas Purchases and Deliveries to Consumers"; Form EIA-816, "Monthly Natural Gas Liquids Report"; Form EIA-759, "Monthly Power Plant Report"; Form FERC-423, "Monthly Report of Cost and Quality of Fuels for Electric Plants"; Form EIA-191, "Underground Gas Storage Report"; Form FPC-14, "Annual Report for Importers and Exporters of Natural Gas"; U.S. Crude Oil, Natural Gas, and Natural Gas Liquids Reserves, 1994 Annual Report, DOEJEIA-0216(94); and the U.S. Minerals Management Service. 


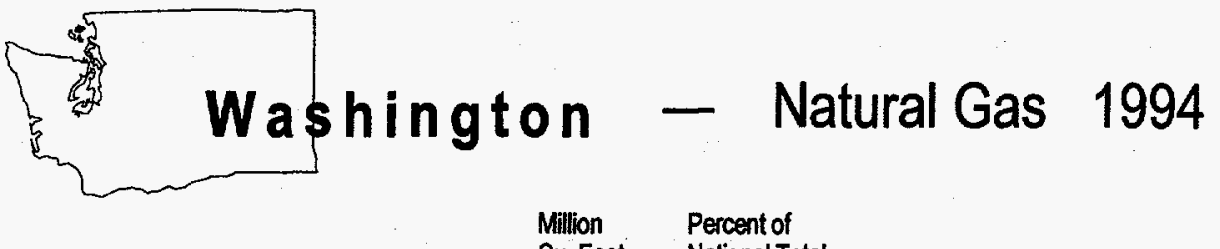

Million

Cu. Feet

Percent of National Total

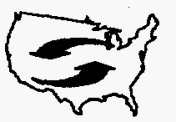

Net Interstate

Movements:

270,274

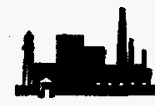

Industrial:

107,603

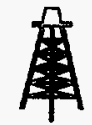

Marketed

Production:

0

0.00

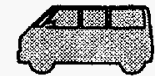

Vehicle Fuel:

155

8.96

Deliveries to Consumers:

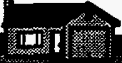

Residential:

53,144

1.10

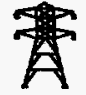

Electric

Utilities:

2,461

0.08

Commercial:

42,982

1.48

Total:

206,346

Table 95. Summary Statistics for Natural Gas — Washington, 1990-1994

\begin{tabular}{|c|c|c|c|c|c|}
\hline & 1990 & 1991 & 1992 & 1993 & 1994 \\
\hline $\begin{array}{l}\text { Reserves (billion cublc feet) } \\
\text { Estimated Proved Reserves (dry) } \\
\text { as of December } 31\end{array}$ & 0 & 0 & 0 & 0 & 0 \\
\hline 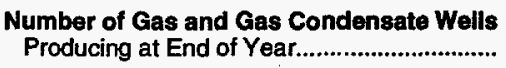 & 0 & 0 & 0 & 0 & 0 \\
\hline 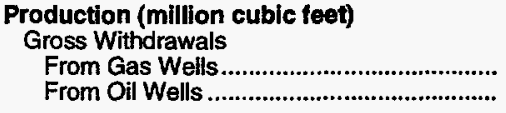 & $\begin{array}{l}0 \\
0\end{array}$ & $\begin{array}{l}0 \\
0\end{array}$ & $\begin{array}{l}0 \\
0\end{array}$ & $\begin{array}{l}0 \\
0\end{array}$ & $\begin{array}{l}0 \\
0\end{array}$ \\
\hline Total & 0 & 0 & 0 & 0 & 0 \\
\hline 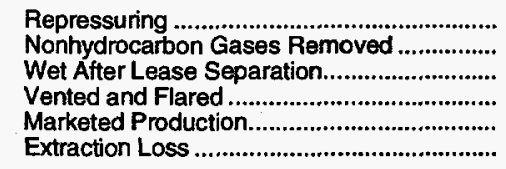 & $\begin{array}{l}0 \\
0 \\
0 \\
0 \\
0 \\
0\end{array}$ & $\begin{array}{l}0 \\
0 \\
0 \\
0 \\
0 \\
0\end{array}$ & $\begin{array}{l}0 \\
0 \\
0 \\
0 \\
0 \\
0\end{array}$ & $\begin{array}{l}0 \\
0 \\
0 \\
0 \\
0 \\
0\end{array}$ & $\begin{array}{l}0 \\
0 \\
0 \\
0 \\
0 \\
0\end{array}$ \\
\hline 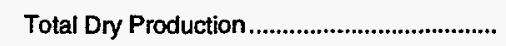 & 0 & 0 & 0 & 0 & 0 \\
\hline 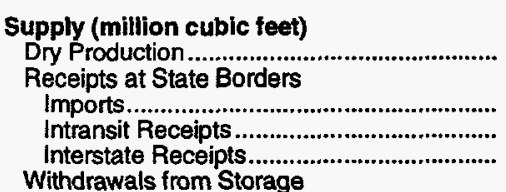 & $\begin{array}{r}168,441 \\
0 \\
508,705\end{array}$ & $\begin{array}{r}235,614 \\
0 \\
493,474\end{array}$ & $\begin{array}{r}270,477 \\
0 \\
491,200\end{array}$ & $\begin{array}{r}306,030 \\
0 \\
515,637\end{array}$ & $\begin{array}{r}323,013 \\
0 \\
713,101\end{array}$ \\
\hline 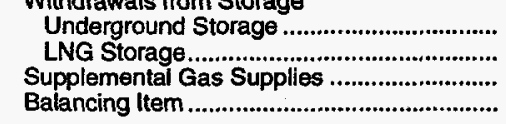 & $\begin{array}{r}15,622 \\
104 \\
181 \\
-15,658\end{array}$ & $\begin{array}{r}11,013 \\
0 \\
154 \\
-64,376\end{array}$ & $\begin{array}{r}18,960 \\
471 \\
180 \\
-55,862\end{array}$ & $\begin{array}{r}17,661 \\
7,886 \\
4 \\
-85,384\end{array}$ & $\begin{array}{r}17,133 \\
1,701 \\
0 \\
-55,281\end{array}$ \\
\hline Total Supply & 677,396 & 675,878 & 725,426 & 761,833 & 999,668 \\
\hline
\end{tabular}

See footnotes at end of table. 


\begin{tabular}{|c|c|c|c|c|c|}
\hline & 1990 & 1991 & 1992 & 1993 & 1994 \\
\hline \multicolumn{6}{|l|}{ Disposition (million cubic feet) } \\
\hline \multicolumn{5}{|l|}{ Deliveries at State Borders } & 212,782 \\
\hline \multicolumn{6}{|l|}{ 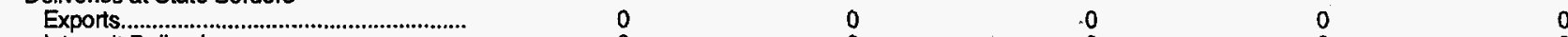 } \\
\hline Intransit Deliveries ......................................... & 0 & $\therefore 0$ & 0 & 0 & 0 \\
\hline \multirow{2}{*}{\multicolumn{6}{|c|}{ 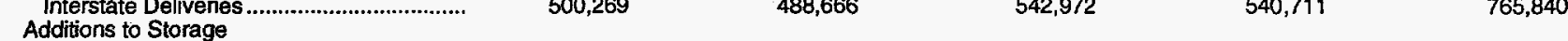 }} \\
\hline & & & & & \\
\hline \multicolumn{6}{|l|}{ 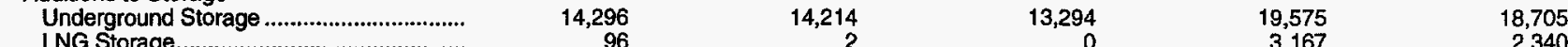 } \\
\hline 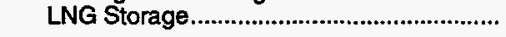 & 96 & 2 & 0 & 3,167 & 2,340 \\
\hline 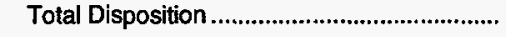 & 677,396 & 675,878 & 725,426 & 761,833 & 999,668 \\
\hline \multicolumn{6}{|l|}{ Consumption (million cubic feet) } \\
\hline 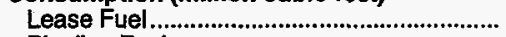 & 0 & 0 & 0 & 0 & 0 \\
\hline 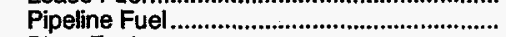 & 5,053 & 5,094 & 3,069 & 4,229 & 6,437 \\
\hline 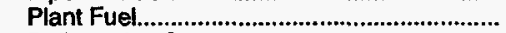 & 0 & 0 & 0 & 0 & 0 \\
\hline \multicolumn{6}{|l|}{ Delivered to Consumers } \\
\hline 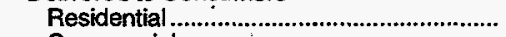 & 40,346 & 46,222 & 43,048 & 53,258 & 53,144 \\
\hline 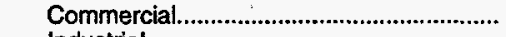 & 38,671 & 41,738 & 37,800 & 43,620 & 42,982 \\
\hline Industrial & 78,424 & 79,735 & 79,766 & 92,274 & 107,603 \\
\hline Vehicle Fuel.............................................. & 50 & 66 & 94 & 100 & 155 \\
\hline 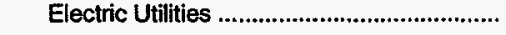 & 191 & 139 & 5,385 & 4,899 & 2,461 \\
\hline Total Delivered to Consumers ............................ & 157,682 & 167,901 & 166,092 & 194,151 & 206,346 \\
\hline 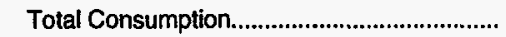 & 162,735 & 172,996 & 169,161 & 198,380 & 212,782 \\
\hline \multicolumn{6}{|l|}{$\begin{array}{l}\text { Delivered for the Account of Others } \\
\text { (million cubic feet) }\end{array}$} \\
\hline 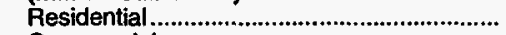 & 0 & 0 & 0 & 0 & 0 \\
\hline 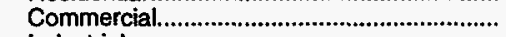 & 2,462 & 3,247 & 4,831 & 2,671 & 1,993 \\
\hline 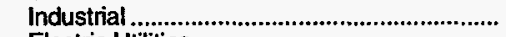 & 36,929 & 44,526 & 49,911 & 54,177 & 62,877 \\
\hline 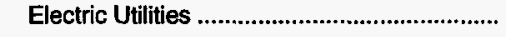 & 48 & 88 & 3,597 & 1,195 & 2,328 \\
\hline \multicolumn{6}{|l|}{$\begin{array}{l}\text { Firm Deliveries } \\
\text { (million cubic feet) }\end{array}$} \\
\hline 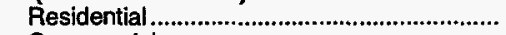 & NA & NA & NA & 53,258 & 53,144 \\
\hline 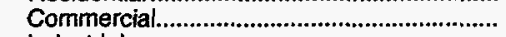 & NA & NA & NA & 33,533 & 34,631 \\
\hline 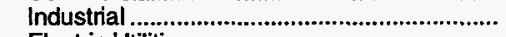 & NA & NA & NA & 45,121 & 59,059 \\
\hline 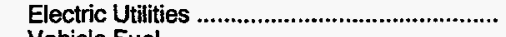 & NA & NA & NA & $\begin{array}{r}0 \\
77\end{array}$ & 2,307 \\
\hline 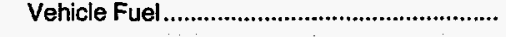 & NA & NA & NA & 77 & 60 \\
\hline \multicolumn{6}{|l|}{ Interruptible Deliveries } \\
\hline (million cubic feet) & & & & & \\
\hline 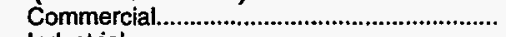 & NA & NA & NA & 10,087 & 8,352 \\
\hline 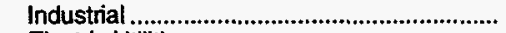 & NA & NA & NA & 45,121 & 59,059 \\
\hline 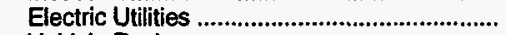 & NA & NA & NA & 1,195 & \\
\hline 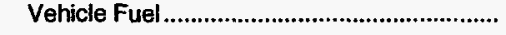 & NA & NA & NA & 23 & 95 \\
\hline \multicolumn{6}{|l|}{ Number of Consumers } \\
\hline 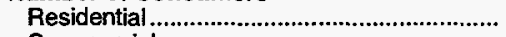 & 458,013 & 492,189 & 528,913 & 565,475 & 604,315 \\
\hline 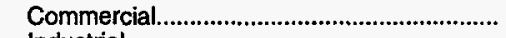 & 58,148 & 60,887 & 63,391 & 65,810 & 68,118 \\
\hline 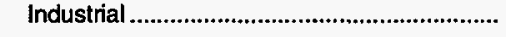 & 3,428 & 3,495 & 3,490 & 3,448 & 3,586 \\
\hline \multicolumn{6}{|l|}{$\begin{array}{l}\text { Average Annual Consumption per Consumer } \\
\text { (thousand cubic feet) }\end{array}$} \\
\hline 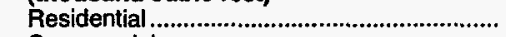 & 88 & 94 & 81 & 94 & 88 \\
\hline 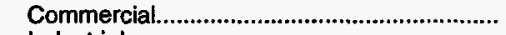 & 665 & 686 & 596 & 663 & 631 \\
\hline 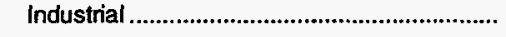 & 22,878 & 22,814 & 22,856 & 26,762 & 30,007 \\
\hline \multirow{2}{*}{\multicolumn{6}{|c|}{$\begin{array}{l}\text { Average Prices for Natural Gas } \\
\text { (dollars per thousand cubic feet) }\end{array}$}} \\
\hline & & & & & \\
\hline 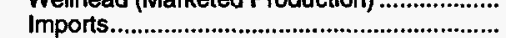 & 1.61 & 1.51 & 1.47 & 1.81 & 1.81 \\
\hline 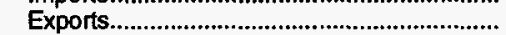 & 0 & 0 & 0 & 0 & 0 \\
\hline 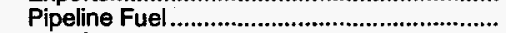 & 2.04 & 1.98 & 1.89 & 1.37 & 1.84 \\
\hline 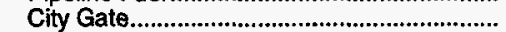 & 1.95 & 1.91 & 1.90 & 2.39 & 2.54 \\
\hline Delivered to Consumers & & & & & \\
\hline 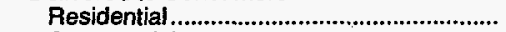 & 5.02 & 4.68 & 5.00 & 5.23 & 5.70 \\
\hline 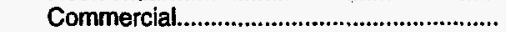 & 4.14 & 4.06 & 4.32 & 4.53 & 4.90 \\
\hline 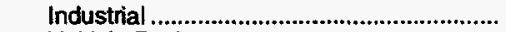 & 2.72 & 2.79 & 2.91 & 3.22 & 2.95 \\
\hline 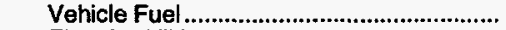 & 4.05 & 4.06 & 4.20 & 4.42 & 4.29 \\
\hline 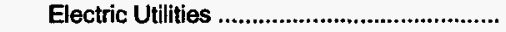 & 22.32 & 4.02 & 3.31 & 3.89 & 4.95 \\
\hline
\end{tabular}

NA $=$ Not Available

Notes: Deliveries to electric utilities (consumption) are reported on the Form ElA-176, "Annual Report of Natural and Supplemental Gas Supply and Disposition." See the discussion on electric utility dala and Table A1 in Appendix A for a comparison of reporting to these two forms. Totals may not add due to independent rounding

Sources: Energy Information Administration (EIA), Form EIA-176, "Annual Report of Nalural and Supplemental Gas Supply and Disposition"; Form EIA-627, "Annual Quantity and Value of Natural Gas Report"; Form ElA-857, "Monthly Report of Natural Gas Purchases and Deliveries to Consumers"; Form ElA-816, "Monthly Natural Gas Liquids Report"; Form EIA-759, "Monthly Power Plant Report"; Form FERC-423, "Monthly Report of Cost and Quality of Fuels for Electric Plants"; Form EIA-191, "Underground Gas Storage Report"; Form FPC-14, "Annual Report for Importers and Exporters of Natural Gas"; U.S. Crude Oil, Natural Gas, and Natural Gas Liquids Reserves, 1994 Annual Report, DOEJEIA-0216(94); and the U.S. Minerals Management Service. 


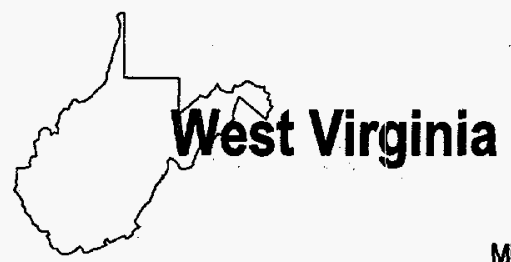

Million Percent of

Cu. Feet National Total

Net Interstate

Movements:

$-105,064$

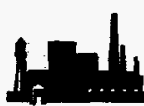

Industrial:

46,774

0.57

Marketed

Production: $\quad 182,000$

0.93

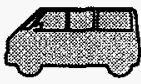

Vehicle Fuel:

0

Deliveries to Consumers:

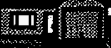

Residential:

35,201

0.73

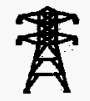

Electric

Utilities:

243

0.01

Commercial:

24,979

0.86

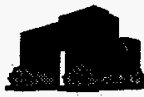

Total:

107,197

0.57
Percent of

National Total

Table 96. Summary Statistics for Natural Gas - West Virginia, 1990-1994

\begin{tabular}{|c|c|c|c|c|c|}
\hline & 1990 & 1991. & 1992 & 1993 & 1994 \\
\hline $\begin{array}{l}\text { Reserves (billion cubic feet) } \\
\text { Estimated Proved Reserves (dry) } \\
\text { as of December } 31 \text {....................................... }\end{array}$ & 2,207 & 2,528 & 2,356 & 2,439 & 2,565 \\
\hline $\begin{array}{l}\text { Number of Gas and Gas Condensate Wells } \\
\text { Producing at End of Year......................................... }\end{array}$ & 37,500 & 37,800 & 38,250 & $\mathbf{R}_{33,716}$ & 35,250 \\
\hline 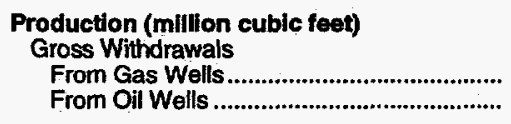 & $\begin{array}{r}178,000 \\
0\end{array}$ & $\begin{array}{r}198,605 \\
0\end{array}$ & $E_{182,000}$ & $\begin{array}{r}R_{171,024} \\
0\end{array}$ & $\begin{array}{r}182,000 \\
0\end{array}$ \\
\hline Total. & 178,000 & 198,605 & $E_{182,000}$ & $R_{171,024}$ & 182,000 \\
\hline 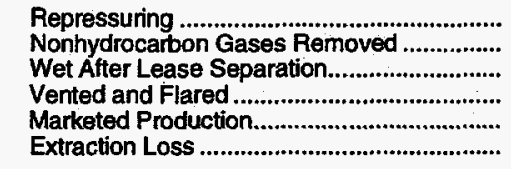 & $\begin{array}{r}\text { NA } \\
\text { NA } \\
178,000 \\
\text { NA } \\
178,000 \\
9,108\end{array}$ & $\begin{array}{r}\text { NA } \\
\text { NA } \\
198,605 \\
\text { NA } \\
198,605 \\
9,745\end{array}$ & $\begin{array}{r}\text { NA } \\
N A \\
E_{182,000} \\
N A \\
E_{182,000} \\
9,436\end{array}$ & $\begin{array}{r}\text { NA } \\
\text { NA } \\
\mathrm{R}_{171,024} \\
\text { NA } \\
\mathrm{A}_{171,024} \\
10,830\end{array}$ & $\begin{array}{r}N A \\
N A \\
182,000 \\
N A \\
182,000 \\
10,901\end{array}$ \\
\hline Total Dry Production & 168,892 & 188,860 & 172,564 & $R_{160,194}$ & 171,099 \\
\hline 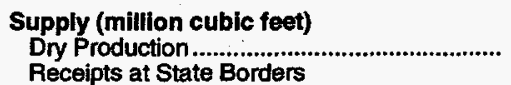 & 168,892 & 188,860 & 172,564 & $\mathrm{P}_{160,194}$ & 171,099 \\
\hline 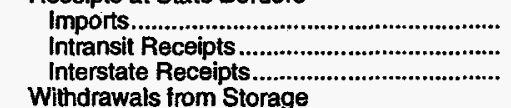 & $\begin{array}{r}0 \\
0 \\
1,463,155\end{array}$ & $\begin{array}{r}0 \\
0 \\
1,322,383\end{array}$ & $\begin{array}{r}0 \\
0 \\
0 \\
1,466,319\end{array}$ & $\begin{array}{r}0 \\
0 \\
1,517,304\end{array}$ & $\begin{array}{r}0 \\
0 \\
1,620,921\end{array}$ \\
\hline 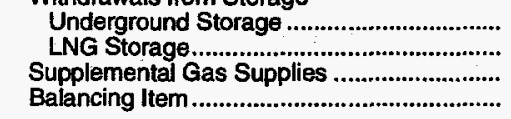 & $\begin{array}{r}82,558 \\
0 \\
0 \\
45,929\end{array}$ & $\begin{array}{r}121,139 \\
0 \\
0 \\
-62,701\end{array}$ & $\begin{array}{r}146,827 \\
0 \\
0 \\
25,017\end{array}$ & $\begin{array}{r}15 t, 836 \\
0 \\
0 \\
R_{122,550}\end{array}$ & $\begin{array}{r}156,284 \\
0 \\
0 \\
94,283\end{array}$ \\
\hline 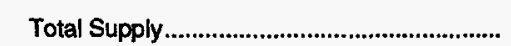 & $1,760,533$ & $1,569,682$ & $1,810,728$ & $R_{1,951,884}$ & $2,042,588$ \\
\hline
\end{tabular}

See footnotes at end of table. 
Table 96. Summary Statistics for Natural Gas - West Virginia, 1990-1994 (Continued)

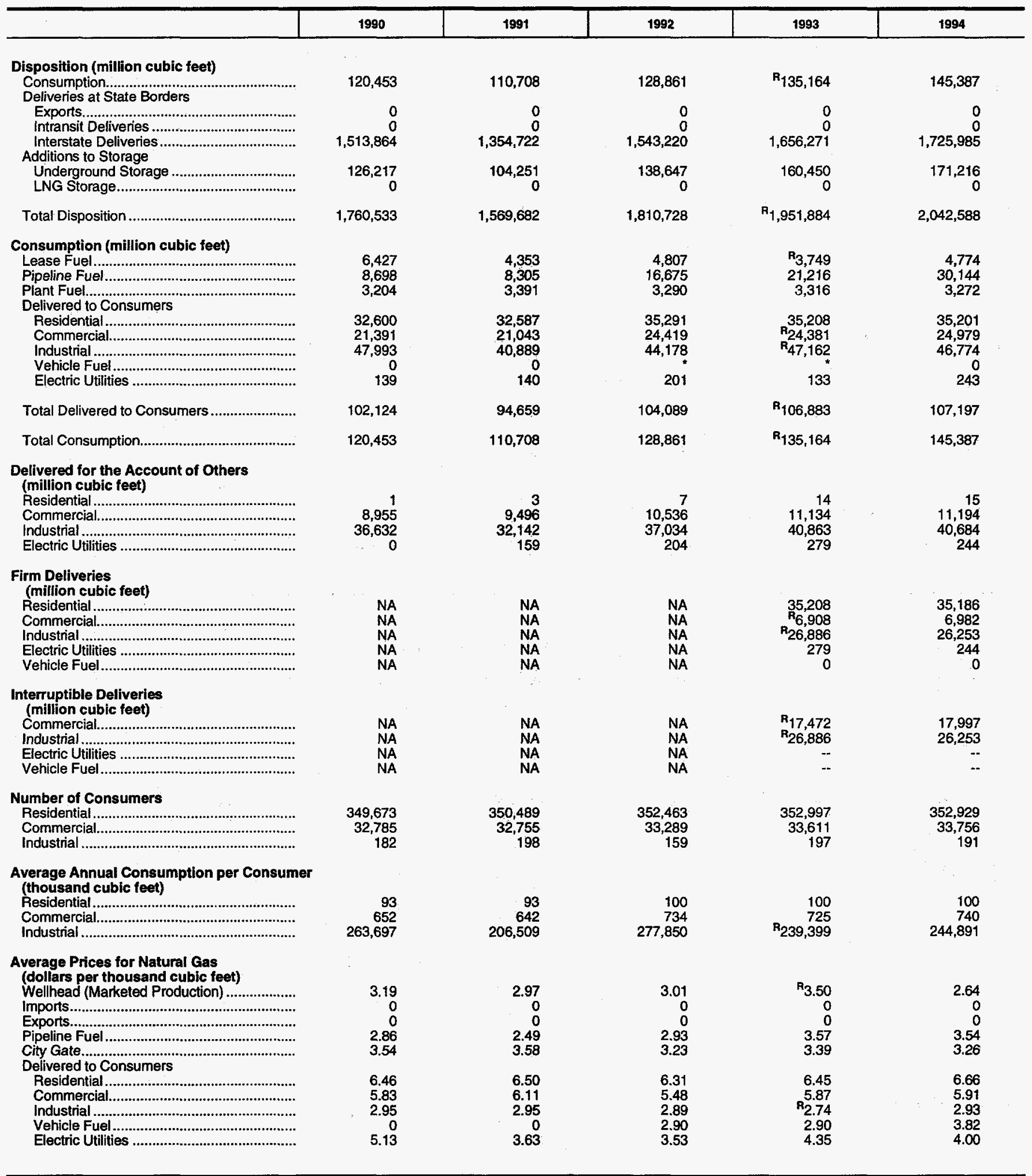

R = Revised data.

$E$ = Estimated Data

$\mathrm{NA}=$ Not Available.

- = Volume is less than 500,000 cubic feet.

Notes: Deliveries to electric utilities (consumption) are reported on the Form ElA-176, "Annual Report of Natural and Supplemental Gas Supply and Disposition." See the discussion on electric utility data and Table A1 in Appendix A for a comparison of reporting to these two forms. Totals may not add due to independent rounding.

Sources: Energy Information Administration (EIA), Form ElA-176, "Annual Report of Natural and Supplemental Gas Supply and Disposition"; Form EIA-627, "Annual Quantity and Value of Natural Gas Report"; Forn ElA-857, "Monthly Report of Natural Gas Purchases and Deliveries to Consumers"; Form ElA-816, "Monthly 'Natural Gas

Liquids Report"; Form EIA-759, "Monthly Power Plant Report"; Form FERC-423, "Monthly Report of Cost and Quality of Fuels for Electric Plants"; Form EIA-191, "Underground Gas Storage Report"; Form FPC-14, "Annual Report for Importers and Exporters of Natural Gas"; U.S. Crude Oil, Natural Gas, and Natural Gas Liquids Resenves, 1994 Annual Report, DÓE/EIA-0216(94); and the U.S. Minerals Management Service. 


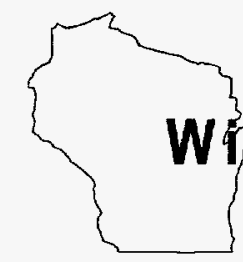

Wisconsin - Natural Gas 1994

Million Percent of

Cu. Feet National Total

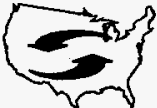

Net Interstate

Movements:

480,425

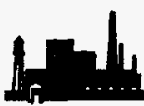

Industrial:

135,106

1.65

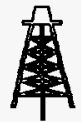

Marketed

Production:

0

0.00

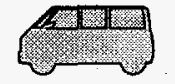

Vehicle Fuel:

36

2.08

Deliveries to Consumers:

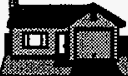

Residential:

128,175

2.64

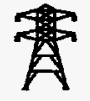

Electric

Utilities:

3,821

0.13

Commercial:

78,609

2.72

Total:

345,748

Table 97. Summary Statistics for Nlatural Gas - Wisconsin, 1990-1994

\begin{tabular}{|c|c|c|c|c|c|}
\hline & 1990 & 1991 & 1992 & 1998 & 1994 \\
\hline 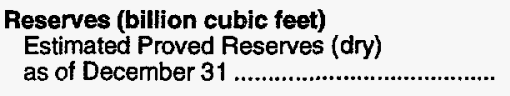 & 0 & 0 & 0 & 0 & 0 \\
\hline 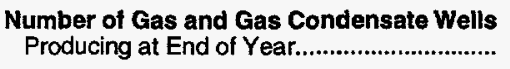 & 0 & 0 & 0 & 0 & 0 \\
\hline 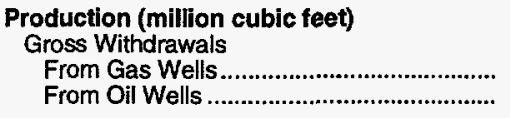 & $\begin{array}{l}0 \\
0\end{array}$ & $\begin{array}{l}0 \\
0\end{array}$ & $\begin{array}{l}0 \\
0\end{array}$ & $\begin{array}{l}0 \\
0\end{array}$ & $\begin{array}{l}0 \\
0\end{array}$ \\
\hline 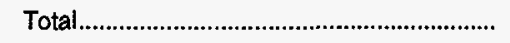 & 0 & 0 & 0 & 0 & 0 \\
\hline 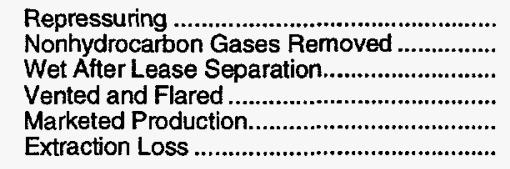 & $\begin{array}{l}0 \\
0 \\
0 \\
0 \\
0 \\
0\end{array}$ & $\begin{array}{l}0 \\
0 \\
0 \\
0 \\
0 \\
0\end{array}$ & $\begin{array}{l}0 \\
0 \\
0 \\
0 \\
0 \\
0\end{array}$ & $\begin{array}{l}0 \\
0 \\
0 \\
0 \\
0 \\
0\end{array}$ & $\begin{array}{l}0 \\
0 \\
0 \\
0 \\
0 \\
0\end{array}$ \\
\hline Total Dry Production & 0 & 0 & 0 & 0 & 0 \\
\hline 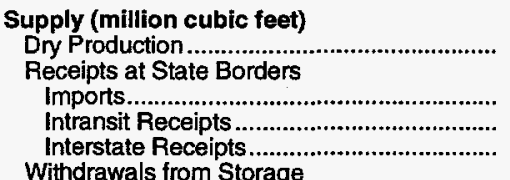 & $\begin{array}{r}0 \\
0 \\
788,801\end{array}$ & $\begin{array}{r}0 \\
0 \\
872,520\end{array}$ & $\begin{array}{r}0 \\
0 \\
1,057,942\end{array}$ & $\begin{array}{r}0 \\
0 \\
1,101,129\end{array}$ & $\begin{array}{r}0 \\
0 \\
1,179,011\end{array}$ \\
\hline 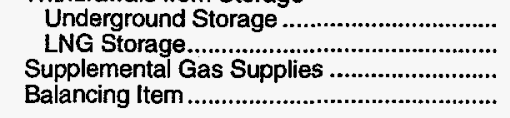 & $\begin{array}{r}0 \\
126 \\
1 \\
9,464\end{array}$ & $\begin{array}{r}0 \\
131 \\
1 \\
8,118\end{array}$ & $\begin{array}{r}0 \\
117 \\
1 \\
-20,151\end{array}$ & $\begin{array}{r}0 \\
110 \\
3 \\
-64,879\end{array}$ & $\begin{array}{r}0 \\
316 \\
5 \\
-124,878\end{array}$ \\
\hline 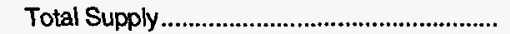 & 798,391 & 880,770 & $1,037,909$ & $1,036,363$ & $1,054,454$ \\
\hline
\end{tabular}

See footnotes at end of table. 


\begin{tabular}{|c|c|c|c|c|c|}
\hline & 1990 & 1991 & 1992 & 1993 & 1994 \\
\hline $\begin{array}{l}\text { Disposition (million cubic feet) } \\
\text { Consumption } \\
\text { Deliveries at State Borders }\end{array}$ & 309,080 & 331,524 & 331,581 & 348,014 & 355,602 \\
\hline 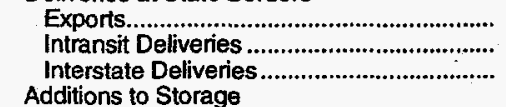 & $\begin{array}{r}0 \\
0 \\
489,196\end{array}$ & $\begin{array}{r}0 \\
0 \\
549,079\end{array}$ & $\begin{array}{r}0 \\
0 \\
706,270\end{array}$ & $\begin{array}{r}0 \\
0 \\
688,237\end{array}$ & $\begin{array}{r}0 \\
0 \\
698,586\end{array}$ \\
\hline 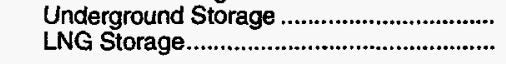 & $\begin{array}{r}0 \\
116\end{array}$ & $\begin{array}{r}0 \\
167\end{array}$ & $\begin{array}{r}0 \\
57\end{array}$ & $\begin{array}{r}0 \\
112\end{array}$ & $\begin{array}{r}0 \\
266\end{array}$ \\
\hline Total Disposition & 798,391 & 880,770 & $1,037,909$ & $1,036,363$ & $1,054,454$ \\
\hline 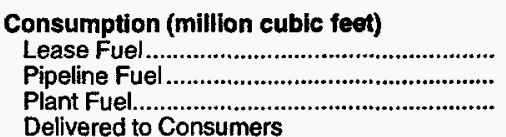 & $\begin{array}{r}0 \\
4,392 \\
0\end{array}$ & $\begin{array}{r}0 \\
4,400 \\
0\end{array}$ & $\begin{array}{r}0 \\
3,983 \\
0\end{array}$ & $\begin{array}{r}0 \\
3,630 \\
0\end{array}$ & $\begin{array}{r}0 \\
9,854 \\
0\end{array}$ \\
\hline 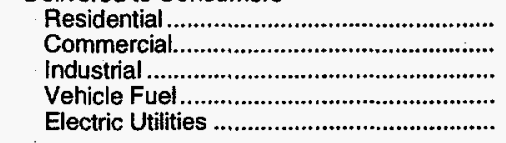 & $\begin{array}{r}114,050 \\
66,339 \\
121,896 \\
22 \\
2,381\end{array}$ & $\begin{array}{r}124,081 \\
71,516 \\
128,798 \\
23 \\
2,706\end{array}$ & $\begin{array}{r}123,405 \\
71,314 \\
130,267 \\
28 \\
2,584\end{array}$ & $\begin{array}{r}130,134 \\
77,079 \\
134,073 \\
29 \\
3,070\end{array}$ & $\begin{array}{r}128,175 \\
78,609 \\
135,106 \\
36 \\
3,821\end{array}$ \\
\hline Total Delivered to Consumers ......................... & 304,688 & 327,124 & 327,599 & 344,385 & 345,748 \\
\hline 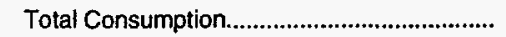 & 309,080 & 331,524 & 331,581 & 348,014 & 355,602 \\
\hline 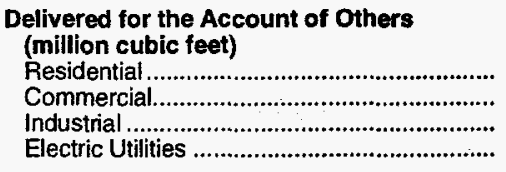 & $\begin{array}{r}0 \\
6,189 \\
67,869 \\
618\end{array}$ & $\begin{array}{r}0 \\
6,414 \\
73,777 \\
1,013\end{array}$ & $\begin{array}{r}0 \\
6,229 \\
76,161 \\
1,899\end{array}$ & $\begin{array}{r}0 \\
4,312 \\
72,890 \\
648\end{array}$ & $\begin{array}{r}0 \\
5,133 \\
69,191 \\
457\end{array}$ \\
\hline 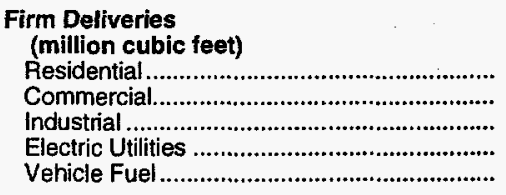 & $\begin{array}{l}\text { NA } \\
\text { NA } \\
\text { NA } \\
\text { NA }\end{array}$ & $\begin{array}{l}\text { NA } \\
\text { NA } \\
\text { NA } \\
\text { NA } \\
\text { NA }\end{array}$ & $\begin{array}{l}\text { NA } \\
\text { NA } \\
\text { NA } \\
\text { NA } \\
\text { NA }\end{array}$ & $\begin{array}{r}130,134 \\
69,316 \\
86,705 \\
1,359 \\
6\end{array}$ & $\begin{array}{r}128,175 \\
70,222 \\
106,544 \\
3,416 \\
10\end{array}$ \\
\hline 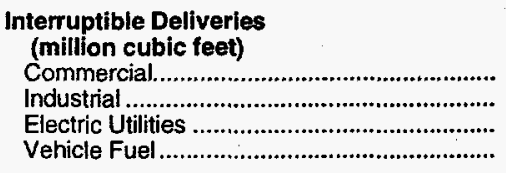 & $\begin{array}{l}\text { NA } \\
\text { NA } \\
\text { NA } \\
\text { NA }\end{array}$ & $\begin{array}{l}\text { NA } \\
\text { NA } \\
\text { NA } \\
\text { NA }\end{array}$ & $\begin{array}{l}\text { NA } \\
\text { NA } \\
\text { NA } \\
\text { NA }\end{array}$ & $\begin{array}{r}7,763 \\
86,705 \\
2,220 \\
23\end{array}$ & $\begin{array}{r}8,386 \\
106,544 \\
1,885 \\
26\end{array}$ \\
\hline 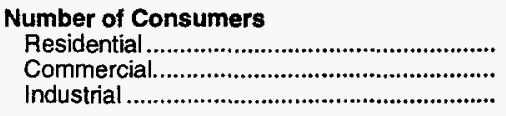 & $\begin{array}{r}1,123,557 \\
106,043 \\
7,154\end{array}$ & $\begin{array}{r}1,151,939 \\
109,616 \\
7,194\end{array}$ & $\begin{array}{r}1,182,834 \\
112,761 \\
7,396\end{array}$ & $\begin{array}{r}1,220,500 \\
115,961 \\
7,979\end{array}$ & $\begin{array}{r}1,253,333 \\
119,788 \\
7,342\end{array}$ \\
\hline 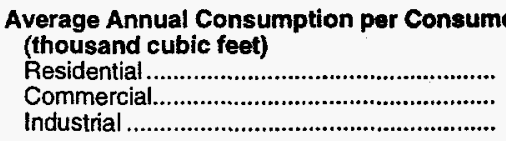 & $\begin{array}{r}102 \\
626 \\
17,039\end{array}$ & $\begin{array}{r}108 \\
652 \\
17,904\end{array}$ & $\begin{array}{r}104 \\
632 \\
17,613\end{array}$ & $\begin{array}{r}107 \\
665 \\
16,803\end{array}$ & $\begin{array}{r}102 \\
656 \\
18,402\end{array}$ \\
\hline 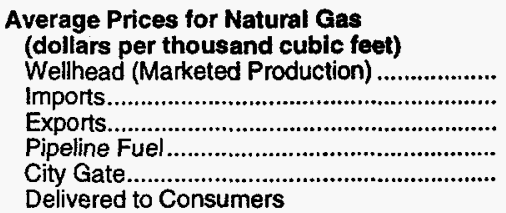 & $\begin{array}{r}0 \\
0 \\
2.14 \\
3.34\end{array}$ & $\begin{array}{r}0 \\
0 \\
1.31 \\
3.17\end{array}$ & $\begin{array}{r}0 \\
0 \\
1.26 \\
3.36\end{array}$ & $\begin{array}{r}0 \\
0 \\
0.96 \\
3.70\end{array}$ & $\begin{array}{r}0 \\
0 \\
1.36 \\
3.42\end{array}$ \\
\hline 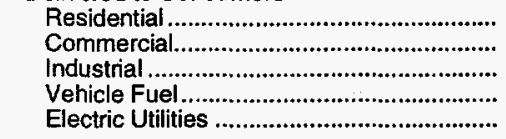 & $\begin{array}{l}5.74 \\
4.75 \\
3.39 \\
3.38 \\
2.95\end{array}$ & $\begin{array}{l}5.61 \\
4.62 \\
3.16 \\
3.44 \\
2.72\end{array}$ & $\begin{array}{l}5.87 \\
4.81 \\
3.38 \\
3.69 \\
2.42\end{array}$ & $\begin{array}{l}6.34 \\
5.16 \\
3.52 \\
3.80 \\
2.66\end{array}$ & $\begin{array}{l}6.28 \\
4.91 \\
3.36 \\
3.41 \\
2.66\end{array}$ \\
\hline
\end{tabular}

NA = Not Available.

Notes: Deliveries to electric utilities (consumption) are reported on the Form ElA-176, "Annual Report of Natural and Supplemental Gas Supply and Disposition." See the discussion on electric utility data and Table A1 in Appendix A for a comparison of reporting to these two forms. Totals may not add due to independent rounding.

Sources: Energy Information Administration (EIA), Form ElA-176, "Annual Report of Natural and Supplemental Gas Supply and Disposition"; Form EIA-627, "Annual Quantity and Value of Natural Gas Report"; Form ElA-857, "Monthly Report of Natural Gas Purchases and Deliveries to Consumers"; Form EIA-816, "Monthly Natural Gas Liquids Report"; Form EIA-759, "Monthly Power Plant Report"; Form FERC-423, "Monthly Report of Cost and Quality of Fuels for Electric Plants"; Form ElA-191, "Underground Gas Storage Report"; Form FPC-14, "Annual Report for Importers and Exporters of Natural Gas"; U.S. Crude Oil, Natural Gas, and Natural Gas Liquids Reserves, 1994 Annual Report, DOEJEIA-0216(94); and the U.S. Minerals Management Service. 


\section{Wyoming - Natural Gas 1994}

$\begin{array}{llll}\text { Million } & \text { Percent of } & \text { Million } & \text { Percent of } \\ \text { Cu. Feet } & \text { National Total } & \text { Cu. Feet } & \text { National Total }\end{array}$

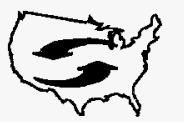

Net Interstate

Movements:

$-675,475$

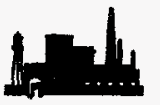

Industrial:

60,566

0.74

Marketed

Production:

696,018

3.54

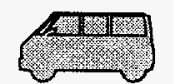

Vehicle Fuel:

18

1.04

Deliveries to Consumers:

Residential:

11,564

0.24

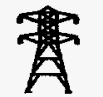

Electric

Utilities:

129

0.00

Commercial:

9,231

0.32

81,507

0.43

Table 98. Summary Statistics for Natural Gas - Wyoming, 1990-1994

\begin{tabular}{|c|c|c|c|c|c|}
\hline & 1990 & 1991 & 1992 & 1993 & 1994 \\
\hline 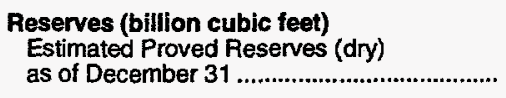 & 9,944 & 9,941 & 10,826 & 10,933 & 10,879 \\
\hline $\begin{array}{l}\text { Number of Gas and Gas Condensate Wells } \\
\text { Producing at End of Year................................... }\end{array}$ & 2,600 & 2,821 & 3,111 & 3,615 & 3,942 \\
\hline 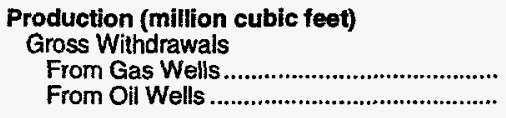 & $\begin{array}{l}570,879 \\
312,834\end{array}$ & $\begin{array}{l}652,659 \\
325,819\end{array}$ & $\begin{array}{l}751,693 \\
285,125\end{array}$ & $\begin{array}{l}880,596 \\
142,006\end{array}$ & $\begin{array}{l}949,343 \\
121,519\end{array}$ \\
\hline 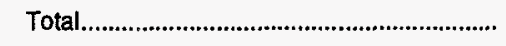 & 883,713 & 978,478 & $1,036,817$ & $1,022,602$ & $1,070,862$ \\
\hline 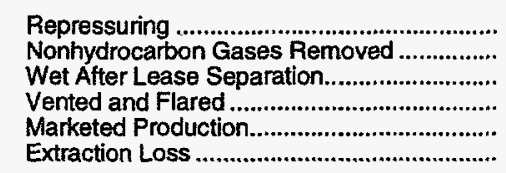 & $\begin{array}{r}56,581 \\
28,188 \\
798,944 \\
63,216 \\
735,728 \\
28,591\end{array}$ & $\begin{array}{r}90,465 \\
28,631 \\
859,382 \\
82,854 \\
776,528 \\
31,470\end{array}$ & $\begin{array}{r}81,712 \\
22,793 \\
932,312 \\
89,736 \\
842,576 \\
31,378\end{array}$ & $\begin{array}{r}R_{110,044} \\
151,239 \\
R_{761,319} \\
R_{126,362} \\
R_{634,957} \\
29,1+8\end{array}$ & $\begin{array}{r}110,064 \\
138,056 \\
822,741 \\
126,722 \\
696,018 \\
33,486\end{array}$ \\
\hline 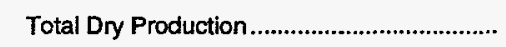 & 707,137 & 745,058 & 811,198 & $\mathrm{R}_{605,839}$ & 662,532 \\
\hline $\begin{array}{l}\text { Supply (million cubic feet) } \\
\text { Dry Production ........................................... } \\
\text { Receipts at State Borders }\end{array}$ & 707,137 & 745,058 & 811,198 & $R_{605,839}$ & 662,532 \\
\hline 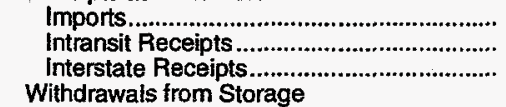 & $\begin{array}{r}0 \\
0 \\
122,436\end{array}$ & $\begin{array}{r}0 \\
0 \\
157,598\end{array}$ & $\begin{array}{r}0 \\
0 \\
193,125\end{array}$ & $\begin{array}{r}0 \\
0 \\
182,933\end{array}$ & $\begin{array}{r}0 \\
0 \\
280,941\end{array}$ \\
\hline 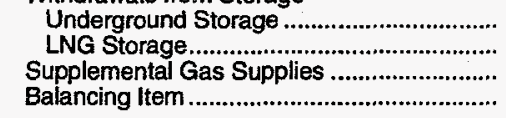 & $\begin{array}{r}8,183 \\
0 \\
0 \\
-98,936\end{array}$ & $\begin{array}{r}10,446 \\
0 \\
0 \\
-170,602\end{array}$ & $\begin{array}{r}13,876 \\
0 \\
0 \\
-110,627\end{array}$ & $\begin{array}{r}14,826 \\
0 \\
0 \\
A_{147,925}\end{array}$ & $\begin{array}{r}8,012 \\
0 \\
0 \\
121,842\end{array}$ \\
\hline 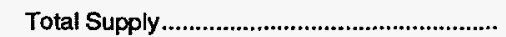 & 738,820 & 742,501 & 907,573 & 951,523 & $1,073,328$ \\
\hline
\end{tabular}

See foolnotes at end of table. 
Table 98. Summary Statistics for Natural Gas - Wyoming, 1990-1994 (Continued)

\begin{tabular}{|c|c|c|c|c|c|}
\hline & 1990 & 1991 & 1992 & 1993 & 1994 \\
\hline \multicolumn{6}{|l|}{ Disposition (million cubic feet) } \\
\hline \multicolumn{5}{|l|}{ Deliveries at State Borders } & 106,316 \\
\hline 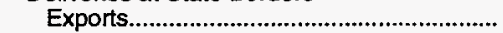 & 0 & 0 & 0 & 0 & 0 \\
\hline Intransit Deliveries ....................................... & 0 & 0 & 0 & 0 & 0 \\
\hline 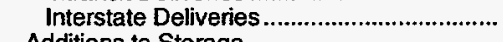 & 634,485 & 634,337 & 778,686 & 833,269 & 956,416 \\
\hline \multicolumn{6}{|l|}{ Additions to Storage } \\
\hline 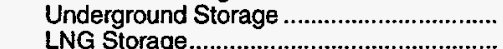 & 12,146 & 10,872 & 5,340 & 13,605 & 10,596 \\
\hline LNG Storage...................................................... & & & 0 & & 0 \\
\hline 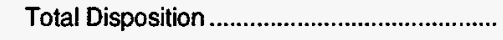 & 738,820 & 742,501 & 907,573 & 951,523 & $1,073,328$ \\
\hline \multicolumn{6}{|l|}{ Consumption (million cubic feet) } \\
\hline 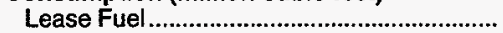 & 29,233 & 20,988 & 27,382 & 9.592 & 4,676 \\
\hline 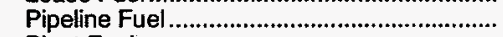 & 5,059 & 7,802 & 7,904 & 6,764 & 6,193 \\
\hline 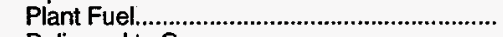 & 12,182 & 14,154 & 13,217 & 13,051 & 13,939 \\
\hline \multicolumn{6}{|l|}{ Delivered to Consumers } \\
\hline 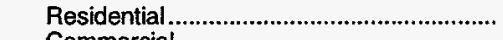 & 11,485 & 11,992 & 10,895 & 12,661 & 11,564 \\
\hline 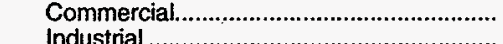 & 8,440 & 9,101 & 8,009 & 10,268 & 9,231 \\
\hline 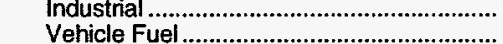 & 25,722 & 33,174 & 56,046 & 54,214 & 60,566 \\
\hline $\begin{array}{l}\text { Vehicle Fuel } \\
\text { Electric Utilities }\end{array}$ & $\begin{array}{r}0 \\
69\end{array}$ & $\begin{array}{r}5 \\
76\end{array}$ & $\begin{array}{l}10 \\
83\end{array}$ & $\begin{array}{l}12 \\
87\end{array}$ & $\begin{array}{r}18 \\
129\end{array}$ \\
\hline & & & & & \\
\hline Total Delivered to Consumers .......................... & 45,715 & 54,348 & 75,044 & 77,242 & 81,507 \\
\hline 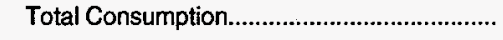 & 92,190 & 97,291 & 123,547 & 104,649 & 106,316 \\
\hline \multicolumn{6}{|l|}{$\begin{array}{l}\text { Delivered for the Account of Others } \\
\text { (million cubic feet) }\end{array}$} \\
\hline 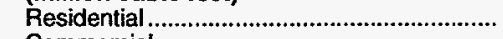 & 0 & 0 & 0 & 0 & 0 \\
\hline 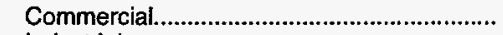 & 21 & 89 & 160 & 207 & 358 \\
\hline 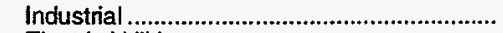 & 23,569 & 31,093 & 54,053 & 52,935 & 59,219 \\
\hline Electric Utilities ................................................ & 0 & 0 & 0 & .0 & 0 \\
\hline \multicolumn{6}{|l|}{$\begin{array}{l}\text { Firm Deliveries } \\
\text { (million cubic feet) }\end{array}$} \\
\hline 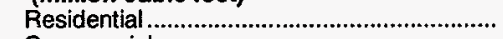 & NA & NA & NA & 12,661 & 11,564 \\
\hline 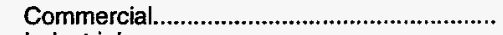 & NA & NA & NA & 5,672 & 5,025 \\
\hline 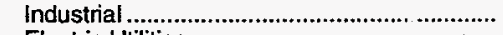 & NA & NA & NA & 43,281 & 49,896 \\
\hline 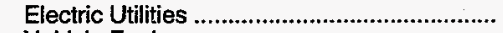 & NA & NA & NA & 88 & 79 \\
\hline 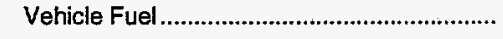 & NA & NA & NA & 12 & 18 \\
\hline \multicolumn{6}{|l|}{$\begin{array}{l}\text { Interruptible Deliveries } \\
\text { (million cubic feet) }\end{array}$} \\
\hline 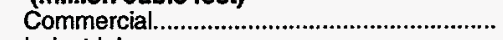 & NA & NA & NA & 4,595 & 4,205 \\
\hline 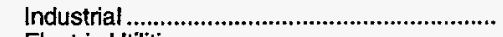 & NA & NA & NA & 43,281 & 49,896 \\
\hline 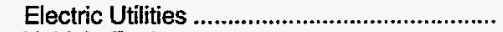 & NA & NA & NA & -- & -- \\
\hline 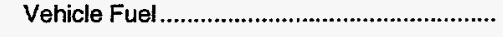 & NA & NA & NA & -- & -- \\
\hline \multicolumn{6}{|l|}{ Number of Consumers } \\
\hline 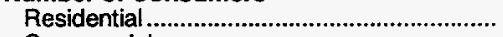 & 113,598 & 113,463 & 114,793 & 116,027 & 117,385 \\
\hline 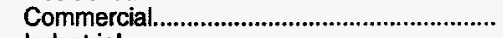 & 13,767 & 14,931 & 15,064 & 15,315 & 15,348 \\
\hline 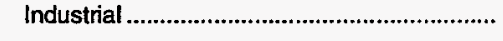 & 284 & 228 & 244 & 194 & 135 \\
\hline \multicolumn{6}{|l|}{$\begin{array}{l}\text { Average Annual Consumption per Consumer } \\
\text { (thousand cubic feet) }\end{array}$} \\
\hline 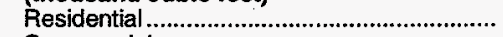 & 101 & 106 & 95 & 109 & 99 \\
\hline 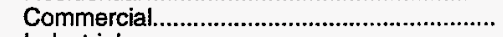 & 613 & 610 & 532 & 670 & 601 \\
\hline 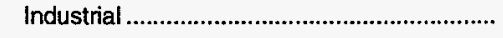 & 90,570 & 145,499 & 229,698 & 279,455 & 448,635 \\
\hline \multirow{2}{*}{\multicolumn{6}{|c|}{$\begin{array}{l}\text { Average Prices for Natural Gas } \\
\text { (dollars per thousand cubic feet) }\end{array}$}} \\
\hline & & & & & \\
\hline 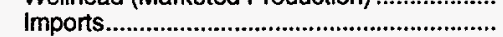 & 0 & $\begin{array}{r}1.00 \\
0\end{array}$ & $\begin{array}{r}1.10 \\
0\end{array}$ & 0 & $\begin{array}{r}0 \\
0\end{array}$ \\
\hline 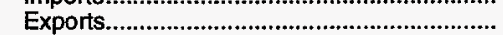 & 0 & 0 & 0 & 0 & 0 \\
\hline 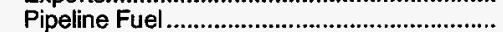 & 1.95 & 1.85 & 2.48 & 1.92 & 1.52 \\
\hline City Gate & 3.00 & 3.04 & 2.90 & 2.80 & 2.91 \\
\hline \multicolumn{6}{|l|}{ Delivered to Consumers } \\
\hline 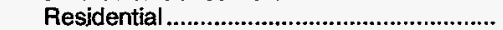 & 4.84 & 4.74 & 4.72 & 4.77 & 5.10 \\
\hline 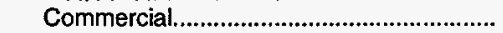 & 4.47 & 4.31 & 4.26 & 4.23 & 4.45 \\
\hline 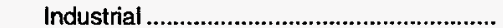 & 3.23 & 3.03 & 2.91 & 3.62 & 3.51 \\
\hline 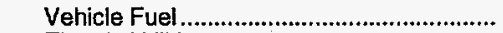 & 0 & 5.66 & 5.74 & 5.66 & 4.62 \\
\hline 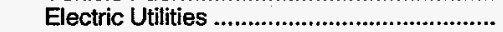 & 3.26 & 3.51 & 3.33 & 3.44 & 5.80 \\
\hline
\end{tabular}

a The state of Wyoming previously estimated lease fuel use. Starting in 1993, the data for lease fuel are actual reported data.

R $=$ Revised data.

NA $=$ Not Available.
Notes: Deliveries to electric utilities (consumption) are reported on the Form ElA-176, "Annual Report of Natural and Supplemental Gas Supply and Disposition." See the discussion on electric utility data and Table A1 in Appendix A for a comparison of reporting to these two forms. Totals may not add due to independent rounding.

Sources: Energy Information Administration (EIA), Form EIA-176, "Annual Report of Natural and Supplemental Gas Supply and Disposition"; Form EIA-627, "Annual

Quantity and Value of Natural Gas Report"; Form ElA-857, "Monthly Report of Natural Gas Purchases and Deliveries to Consumers"; Form ElA-816, "Monthly Natural Gas

Liquids Report"; Form EIA-759, "Monthly Power Plant Report"; Form FERC-423, "Monthly Report of Cost and Quality of Fuels for Electric Plants"; Form EIA-191, "Underground Gas Storage Report"; Form FPC-14, "Annual Report for Importers and Exporters of Natural Gas"; U.S. Crude Oil, Natural Gas, and Natural Gas Liquids Reserves, 1994 Annual Report, DOEJEIA-0216(94); and the U.S. Minerals Management Service. 



\section{Historical Data}

Dry production of natural gas continues to be a large contributor to the Nation's domestic energy supply. In 1973, the drop in oil imports due to the oil embargo by OPEC nations resulted in natural gas production reaching an alltime high of 21.7 trillion cubic feet. During 1994, dry production was 18.7 trillion cubic feet, 4 percent above the 1993 level (Table 99).

The early 1970's brought record levels in the total U.S. consumption of natural gas with a peak level of 22.1 trillion cubic feet in 1972. Deliveries of natural gas to the residential and electric utility sectors set record levels that same year, and deliveries to industrials peaked in 1973. Between 1982 and 1991 total U.S. consumption of natural gas remained in the range of 16 to 19 trillion cubic feet (Table 101). It rose to 20.3 trillion cubic feet in 1993 and increased slightly in 1994 (20.8 trillion cubic feet).
Industrial users are the leading consumers of natural gas. Industrial consumption reached 8.2 trillion cubic feet in 1994, up 2 percent from the 1993 level and the highest level since 1974. Much of the increase can be attributed to natural gas consumption by nonutility generators (NUG's). NUG's generate electricity for their own use and for sale to electric utilities, which in turn distribute it to consumers.

Natural gas consumption in the residential sector, the second leading consuming sector, responds largely to weather-related home-heating requirements. The winter of 1993-94 was warmer than normal. Residential consumption in 1994 was slightly lower than in 1993 and 3 percent higher than in 1992. During 1994, residential consumption was 4.8 trillion cubic feet.

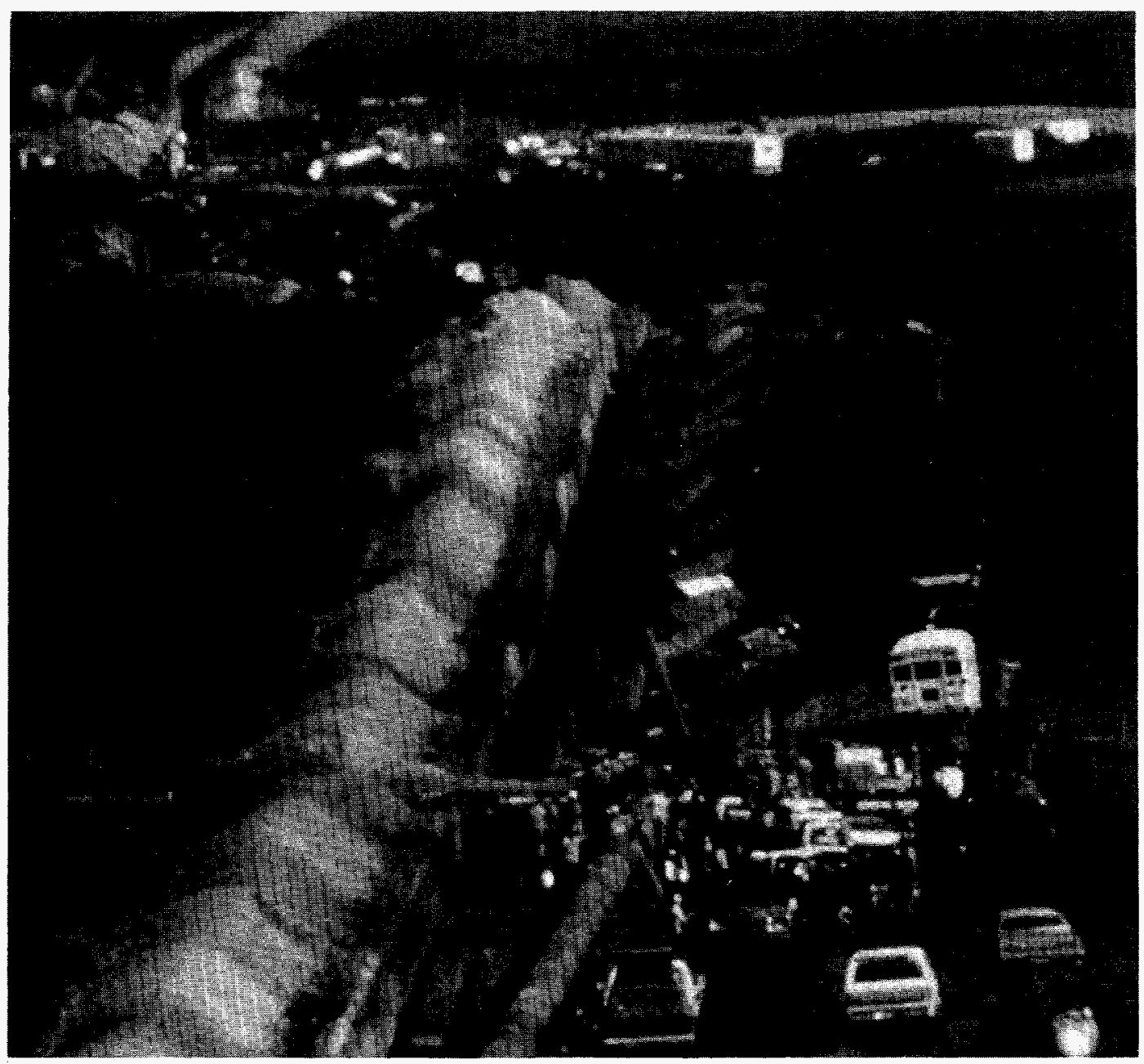

The expansion of the interstate natural gas pipeline system increases the flow to end-users. 
Commercial consumption, which also responds to variations in the weather, was at almost the same record-setting level of 2.9 trillion cubic feet set in 1993. Historically, the electric utility sector has had the third largest amount of consumption. In 1992 and 1993, it was surpassed by commercial; however in 1994 electric utility consumption was almost at 3 trillion cubic feet. Consumption increased 10 percent in the electric utility sector from 1993 to 1994.

\section{Changes to Data Collection Programs}

The Bureau of Mines, U.S. Department of the Interior, conducted surveys of the natural gas inclustry until 1977 , when this function was transferred to the newly formed U.S. Department of Energy (DOE). Since that time the Energy Information Administration (EIA) within the DOE has collected information on natural gas production, transmission, consumption, and prices. Some of the data are collected on behalf of the Federal Energy Regulatory Commission (FERC), an independent commission within the DOE, which has jurisdiction primarily in the regulation of electric utilities and the interstate natural gas industry.

The EIA made many changes to data collection forms and procedures that became effective for the collection of 1980 data. These changes provided more complete natural gas information with minimal changes in respondent burden. In some instances voluntary data collection efforts were changed to mandatory surveys. The Form EIA-176 combined and expanded two voluntary predecessor forms from the Bureau of Mines (BOM-1340-A and BOM-1341-A) into a new mandatory form.

In 1980, the Form EIA-627, "Annual Quantity and Value of Natural Gas Report," was introduced to collect data, previously collected on an informal basis, from the appropriate agencies of the producing States. In addition to volumes of gas produced from gas and oil wells, the form requested data on the quantities of nonhydrocarbon gases removed in treating or processing operations, quantities of gas used for repressuring as well as gas vented and flared, and marketed production. In 1991, the Form EIA-627 was revised to also collect quantities of fuel used on the lease.

The United States Geological Survey provided supplemental production data, including production data for the Outer Continental Shelf, until 1982, when this function was transferred to the United States Minerals Management Service.

In 1982, the Form EIA-176 was revised and expanded into the version that was used for data collection through 1986. The Form EIA-176 was reapproved in 1988 for the collection of 1987 through 1989 data. The form was changed to include collection of data on the transportation of gas to commercial consumers for the account of others and to add a short form of the EIA-176 to be filed by small companies with limited activities. In 1991, the Form EIA-176 was reapproved for the collection of 1990 through 1992 data. The major changes to the form were: deliveries of gas to be used as vehicle fuel are reported and the data reported on the form are no longer considered confidential.

In 1993, the Office of Management and Budget approved the revised Form EIA-176. All deliveries to consumers are now categorized as firm or interruptible. Commercial and industrial consumers are further categorized as nonutility power producers or as those excluding nonutility power producers. See Appendix A for further information about the history of data collection. 
Table 99. Quantity and Average Price of Natural Gas Production in the United States, 1930-1994 (Volumes in Million Cubic Feet, Prices in Dollars per Thousand Cubic Feet)

\begin{tabular}{|c|c|c|c|c|c|c|c|c|}
\hline Year & $\begin{array}{c}\text { Gross } \\
\text { Withdrawals }\end{array}$ & $\begin{array}{l}\text { Used for } \\
\text { Repressuring }\end{array}$ & $\begin{array}{c}\text { Nonhydro- } \\
\text { carbon } \\
\text { Gases } \\
\text { Removed }\end{array}$ & $\begin{array}{l}\text { Vented and } \\
\text { Flared }\end{array}$ & $\begin{array}{l}\text { Marketed } \\
\text { Production }\end{array}$ & $\begin{array}{l}\text { Extraction } \\
\text { Loss }\end{array}$ & $\begin{array}{c}\text { Dry } \\
\text { Production }\end{array}$ & $\begin{array}{c}\text { Average } \\
\text { Wellhead } \\
\text { Price of } \\
\text { Marketed } \\
\text { Praduction }\end{array}$ \\
\hline 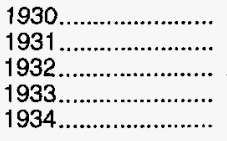 & $\begin{array}{l}\text { NA } \\
\text { NA } \\
\text { NA } \\
\text { NA } \\
\text { NA }\end{array}$ & $\begin{array}{l}\text { NA. } \\
\text { NA } \\
\text { NA } \\
\text { NA } \\
\text { NA }\end{array}$ & $\begin{array}{l}\text { NA } \\
\text { NA } \\
\text { NA } \\
\text { NA } \\
\text { NA }\end{array}$ & $\begin{array}{l}\text { NA } \\
\text { NA } \\
\text { NA } \\
\text { NA } \\
\text { NA }\end{array}$ & $\begin{array}{l}1,978,911 \\
1,721,902 \\
1,593,798 \\
1,596,673 \\
1,815,796\end{array}$ & $\begin{array}{l}75,140 \\
62,288 \\
51,816 \\
48,280 \\
52,190\end{array}$ & $\begin{array}{l}1,903,771 \\
1,659,614 \\
1,541,982 \\
1,548,393 \\
1,763,606\end{array}$ & $\begin{array}{l}0.08 \\
0.07 \\
0.06 \\
0.06 \\
0.06\end{array}$ \\
\hline 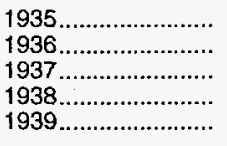 & $\begin{array}{r}\text { NA } \\
2,691,512 \\
3,084,567 \\
3,108,858 \\
3,387,095\end{array}$ & $\begin{array}{r}\text { NA } \\
73,507 \\
84,925 \\
101,551 \\
171,401\end{array}$ & $\begin{array}{l}\text { NA } \\
\text { NA } \\
\text { NA } \\
\text { NA } \\
\text { NA }\end{array}$ & $\begin{array}{r}\text { NA } \\
392,528 \\
526,159 \\
649,106 \\
677,311\end{array}$ & $\begin{array}{l}1,968,963 \\
2,225,477 \\
2,473,483 \\
2,358,201 \\
2,538,383\end{array}$ & $\begin{array}{l}55,488 \\
61,064 \\
70,210 \\
73,338 \\
73,746\end{array}$ & $\begin{array}{l}1,913,475 \\
2,164,413 \\
2,403,273 \\
2,284,863 \\
2,464,637\end{array}$ & $\begin{array}{l}0.06 \\
0.06 \\
0.05 \\
0.05 \\
0.05\end{array}$ \\
\hline $\begin{array}{l}1940 \ldots \ldots \ldots \ldots \ldots \ldots \ldots \\
1941 \ldots \ldots \ldots \ldots \ldots \ldots \ldots \ldots \ldots \\
1942 \ldots \ldots \ldots \ldots \ldots \ldots \ldots \ldots \ldots \ldots \\
1943 \ldots \ldots \ldots \ldots \ldots \ldots \ldots \ldots \ldots \\
1944 \ldots \ldots \ldots \ldots \ldots \ldots \ldots \ldots\end{array}$ & $\begin{array}{l}3,752,702 \\
4,168,116 \\
4,525,095 \\
5,024,449 \\
5,708,288\end{array}$ & $\begin{array}{l}362,916 \\
644,379 \\
752,619 \\
824,803 \\
882,979\end{array}$ & $\begin{array}{l}\text { NA } \\
\text { NA } \\
\text { NA } \\
\text { NA } \\
\text { NA }\end{array}$ & $\begin{array}{r}655,967 \\
630,212 \\
626,782 \\
684,115 \\
1,010,285\end{array}$ & $\begin{array}{l}2,733,819 \\
2,893,525 \\
3,145,694 \\
3,515,531 \\
3,815,024\end{array}$ & $\begin{array}{r}79,526 \\
115,464 \\
119,000 \\
121,788 \\
142,868\end{array}$ & $\begin{array}{l}2,654,293 \\
2,778,061 \\
3,026,694 \\
3,393,743 \\
3,672,156\end{array}$ & $\begin{array}{l}0.05 \\
0.05 \\
0.05 \\
0.05 \\
0.05\end{array}$ \\
\hline 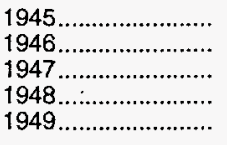 & $\begin{array}{l}6,000,161 \\
6,293,037 \\
6,733,230 \\
7,178,777 \\
7,546,825\end{array}$ & $\begin{array}{l}1,061,951 \\
1,038,242 \\
1,083,119 \\
1,220,579 \\
1,273,205\end{array}$ & $\begin{array}{l}\text { NA } \\
\text { NA } \\
\text { NA } \\
\text { NA } \\
\text { NA }\end{array}$ & $\begin{array}{r}896,208 \\
1,102,033 \\
1,067,938 \\
810,178 \\
853,884\end{array}$ & $\begin{array}{l}4,042,002 \\
4,152,762 \\
4,582,173 \\
5,148,020 \\
5,419,736\end{array}$ & $\begin{array}{l}159,936 \\
165,274 \\
188,734 \\
209,508 \\
224,332\end{array}$ & $\begin{array}{l}3,882,066 \\
3,987,488 \\
4,393,439 \\
4,938,512 \\
5,195,404\end{array}$ & $\begin{array}{l}0.05 \\
0.05 \\
0.06 \\
0.06 \\
0.06\end{array}$ \\
\hline $\begin{array}{l}1950 \ldots \ldots \ldots \ldots \ldots \ldots \\
1951 \ldots \ldots \ldots \ldots \ldots \ldots \ldots \ldots \\
1952 \ldots \ldots \ldots \ldots \ldots \ldots \ldots \ldots \\
1953 \ldots \ldots \ldots \ldots \ldots \ldots \ldots \ldots \\
1954 \ldots \ldots \ldots \ldots \ldots \ldots \ldots \ldots\end{array}$ & $\begin{array}{r}8,479,650 \\
9,689,372 \\
10,272,566 \\
10,645,798 \\
10,984,850\end{array}$ & $\begin{array}{l}1,396,546 \\
1,438,827 \\
1,410,501 \\
1,438,606 \\
1,518,737\end{array}$ & $\begin{array}{l}\text { NA } \\
\text { NA } \\
\text { NA } \\
\text { NA } \\
\text { NA }\end{array}$ & $\begin{array}{l}801,044 \\
793,186 \\
848,608 \\
810,276 \\
723,567\end{array}$ & $\begin{array}{l}6,282,060 \\
7,457,359 \\
8,013,457 \\
8,396,916 \\
8,742,546\end{array}$ & $\begin{array}{l}259,862 \\
292,400 \\
319,158 \\
340,068 \\
354,348\end{array}$ & $\begin{array}{l}6,022,198 \\
7,164,959 \\
7,694,299 \\
8,056,848 \\
8,388,198\end{array}$ & $\begin{array}{l}0.07 \\
0.07 \\
0.08 \\
0.09 \\
0.10\end{array}$ \\
\hline $\begin{array}{l}1955 \ldots \ldots \ldots \ldots \ldots \ldots . . \\
1956 \ldots \ldots \ldots \ldots \ldots \ldots \ldots \ldots \ldots \\
1957 \ldots \ldots \ldots \ldots \ldots \ldots \ldots \ldots \ldots \\
1958 \ldots \ldots \ldots \ldots \ldots \ldots \ldots . . . \\
1959 \ldots \ldots \ldots \ldots \ldots \ldots \ldots\end{array}$ & $\begin{array}{l}11,719,794 \\
12,372,905 \\
12,906,669 \\
13,146,635 \\
14,229,272\end{array}$ & $\begin{array}{l}1,540,804 \\
1,426,648 \\
1,417,263 \\
1,482,975 \\
1,612,109\end{array}$ & $\begin{array}{l}\text { NA } \\
\text { NA } \\
\text { NA } \\
\text { NA } \\
\text { NA }\end{array}$ & $\begin{array}{l}773,639 \\
864,334 \\
809,148 \\
633,412 \\
571,048\end{array}$ & $\begin{array}{r}9,405,351 \\
10,081,923 \\
10,680,258 \\
11,030,248 \\
12,046,115\end{array}$ & $\begin{array}{l}376,686 \\
418,013 \\
433,636 \\
458,040 \\
498,457\end{array}$ & $\begin{array}{r}9,028,665 \\
9,663,910 \\
10,246,622 \\
10,572,208 \\
11,547,658\end{array}$ & $\begin{array}{l}0.10 \\
0.11 \\
0.11 \\
0.12 \\
0.13\end{array}$ \\
\hline 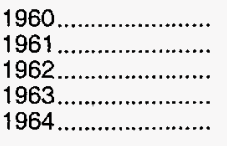 & $\begin{array}{l}15,087,911 \\
15,460,312 \\
16,038,973 \\
16,973,368 \\
17,535,553\end{array}$ & $\begin{array}{l}1,753,996 \\
1,682,754 \\
1,736,722 \\
1,843,297 \\
1,647,108\end{array}$ & $\begin{array}{l}\text { NA } \\
\text { NA } \\
\text { NA } \\
\text { NA } \\
\text { NA }\end{array}$ & $\begin{array}{l}562,877 \\
523,533 \\
425,629 \\
383,408 \\
341,853\end{array}$ & $\begin{array}{l}12,771,038 \\
13,254,025 \\
13,876,622 \\
14,746,663 \\
15,546,592\end{array}$ & $\begin{array}{l}542,890 \\
592,446 \\
623,616 \\
670,251 \\
722,565\end{array}$ & $\begin{array}{l}12,228,148 \\
12,661,579 \\
13,253,006 \\
14,076,412 \\
14,824,027\end{array}$ & $\begin{array}{l}0.14 \\
0.15 \\
0.16 \\
0.16 \\
0.15\end{array}$ \\
\hline 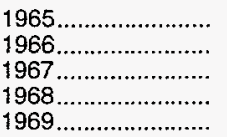 & $\begin{array}{l}17,963,100 \\
19,033,839 \\
20,251,776 \\
21,325,000 \\
22,679,195\end{array}$ & $\begin{array}{l}1,604,204 \\
1,451,516 \\
1,590,574 \\
1,486,092 \\
1,455,205\end{array}$ & $\begin{array}{l}\text { NA } \\
\text { NA } \\
\text { NA } \\
\text { NA } \\
\text { NA }\end{array}$ & $\begin{array}{l}319,143 \\
375,695 \\
489,877 \\
516,508 \\
525,750\end{array}$ & $\begin{array}{l}16,039,753 \\
17,206,628 \\
18,171,325 \\
19,322,400 \\
20,698,240\end{array}$ & $\begin{array}{l}753,473 \\
739,308 \\
784,534 \\
827,877 \\
866,560\end{array}$ & $\begin{array}{l}15,286,280 \\
16,467,320 \\
17,386,791 \\
18,494,523 \\
19,831,680\end{array}$ & $\begin{array}{l}0.16 \\
0.16 \\
0.16 \\
0.16 \\
0.17\end{array}$ \\
\hline
\end{tabular}

See footnotes at end of table. 
Table 99. Quantity and Average Price of Natural Gas Production in the United States, 1930-1994 (Continued)

\begin{tabular}{|c|c|c|c|c|c|c|c|c|}
\hline Year & $\begin{array}{c}\text { Gross } \\
\text { Withdrawals }\end{array}$ & $\begin{array}{l}\text { Used for } \\
\text { Repressuring }\end{array}$ & $\begin{array}{l}\text { Nonhydro- } \\
\text { carbon } \\
\text { Gases } \\
\text { Removed }\end{array}$ & $\begin{array}{l}\text { Vented and } \\
\text { Flared }\end{array}$ & $\begin{array}{l}\text { Marketed } \\
\text { Production }\end{array}$ & $\begin{array}{l}\text { Extraction } \\
\text { Loss }\end{array}$ & $\begin{array}{c}\text { Dry } \\
\text { Production }\end{array}$ & $\begin{array}{l}\text { Average } \\
\text { Wellhead } \\
\text { Price of } \\
\text { Marketed } \\
\text { Production }\end{array}$ \\
\hline 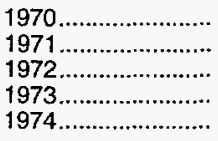 & $\begin{array}{l}23,786,453 \\
24,088,031 \\
24,016,109 \\
24,067,202 \\
22,849,793\end{array}$ & $\begin{array}{l}1,376,35 \\
1,310,45 \& \\
1,236,29 ! \\
1,171,361 \\
1,079,890\end{array}$ & $\begin{array}{l}\text { NA } \\
\text { NA } \\
\text { NA } \\
\text { NA } \\
\text { NA }\end{array}$ & $\begin{array}{l}489,460 \\
284,561 \\
248,119 \\
248,292 \\
169,381\end{array}$ & $\begin{array}{l}21,920,642 \\
22,493,012 \\
22,531,698 \\
22,647,549 \\
21,600,522\end{array}$ & $\begin{array}{l}906,413 \\
883,127 \\
907,993 \\
916,551 \\
887,490\end{array}$ & $\begin{array}{l}21,014,229 \\
21,609,885 \\
21,623,705 \\
21,730,998 \\
20,713,032\end{array}$ & $\begin{array}{l}0.17 \\
0.18 \\
0.19 \\
0.22 \\
0.30\end{array}$ \\
\hline 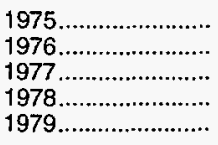 & $\begin{array}{l}21,103,530 \\
20,943,778 \\
21,097,071 \\
21,308.815 \\
21,883,353\end{array}$ & $\begin{array}{r}860,956 \\
859,410 \\
934,801 \\
1,181,432 \\
1,245,074\end{array}$ & $\begin{array}{l}\text { NA } \\
\text { NA } \\
\text { NA } \\
\text { NA } \\
\text { NA }\end{array}$ & $\begin{array}{l}133,913 \\
131,930 \\
136,807 \\
153,350 \\
167,019\end{array}$ & $\begin{array}{l}20,108,661 \\
19,952,438 \\
20,025,463 \\
19,974,033 \\
20,471,260\end{array}$ & $\begin{array}{l}872,282 \\
854,086 \\
862,563 \\
852,130 \\
807,845\end{array}$ & $\begin{array}{l}19,236,379 \\
19,098,352 \\
19,162,900 \\
19,121,903 \\
19,663,415\end{array}$ & $\begin{array}{l}0.44 \\
0.58 \\
0.79 \\
0.91 \\
1.18\end{array}$ \\
\hline 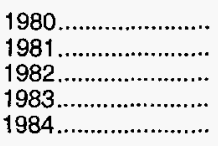 & $\begin{array}{l}21,869,692 \\
21,587,453 \\
20,272,254 \\
18,659,046 \\
20,266,522\end{array}$ & $\begin{array}{l}1,365,454 \\
1,311,735 \\
1,388,392 \\
1,458,054 \\
1,630,152\end{array}$ & $\begin{array}{l}199,063 \\
221,878 \\
208,492 \\
221,937 \\
224,118\end{array}$ & $\begin{array}{r}125,451 \\
98,017 \\
93,365 \\
94,962 \\
107,913\end{array}$ & $\begin{array}{l}20,179,724 \\
19,955,823 \\
18,582,005 \\
16,884,093 \\
18,304,339\end{array}$ & $\begin{array}{l}776,605 \\
774,562 \\
761,942 \\
789,632 \\
837,867\end{array}$ & $\begin{array}{l}19,403,119 \\
19,181,261 \\
17,820,063 \\
16,094,461 \\
17,466,472\end{array}$ & $\begin{array}{l}1.59 \\
1.98 \\
2.46 \\
2.59 \\
2.66\end{array}$ \\
\hline 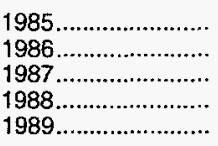 & $\begin{array}{l}19,606,699 \\
19,130,711 \\
20,140,200 \\
20,999,255 \\
21,074,425\end{array}$ & $\begin{array}{l}1,915,197 \\
1,837,552 \\
2,207,559 \\
2,478,382 \\
2,475,179\end{array}$ & $\begin{array}{l}326,497 \\
336,851 \\
376,033 \\
459,883 \\
362,457\end{array}$ & $\begin{array}{r}94,778 \\
97,633 \\
123,707 \\
142,525 \\
141,642\end{array}$ & $\begin{array}{l}17,270,227 \\
16,858,675 \\
17,432,901 \\
17,918,465 \\
18,095,147\end{array}$ & $\begin{array}{l}816,370 \\
799,645 \\
812,320 \\
815,844 \\
784,502\end{array}$ & $\begin{array}{l}16,453,857 \\
16,059,030 \\
16,620,581 \\
17,102,621 \\
17,310,645\end{array}$ & $\begin{array}{l}2.51 \\
1.94 \\
1.67 \\
1.69 \\
1.69\end{array}$ \\
\hline 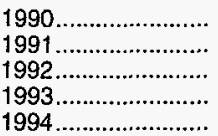 & $\begin{array}{r}21,522,622 \\
21,750,108 \\
22,132,249 \\
R_{22,725,642} \\
23,608,654\end{array}$ & $\begin{array}{r}2,489,040 \\
2,771,928 \\
2,972,552 \\
\mathbf{R}_{3,103,014} \\
3,332,644\end{array}$ & $\begin{array}{l}289,374 \\
275,831 \\
280,370 \\
413,971 \\
412,178\end{array}$ & $\begin{array}{r}150,415 \\
169,909 \\
167,519 \\
R_{226,743} \\
228,336\end{array}$ & $\begin{array}{r}18,593,792 \\
18,532,439 \\
18,711,808 \\
R_{18,981,915} \\
19,635,495\end{array}$ & $\begin{array}{l}784,118 \\
834,637 \\
871,905 \\
886,455 \\
888,500\end{array}$ & $\begin{array}{r}17,809,674 \\
17,697,802 \\
17,839,903 \\
\mathbf{R}_{18,095,460} \\
18,746,995\end{array}$ & $\begin{array}{r}1.71 \\
1.64 \\
1.74 \\
R_{2} .04 \\
1.88\end{array}$ \\
\hline
\end{tabular}

R = Revised data.

Notes: Beginning with 1965 data, all volumes are shown on a pressure base of 14.73 psia at 60 degrees Fahrenheit. For prior years, the pressure base is 14.65 psia at 60 degrees Fahrenheit

Sources: 1930-1975: Bureau of Mines, Minerals Yearbook, "Natural Gas" chapter. 1976-1978: Energy Information Administration (ElA), Energy Data Reports, Natural Gas Annual. 1979: EIA, Natural Gas Production and Consumption, 1979. 1980-1994: EIA, Form EIA-627, "Annual Quantity and Value of Natural Gas Report"; Form EIA-176,

"Annual Report of Natural and Supplemental Gas Supply and Disposition"; Form ElA-64A, "Annual Report of the Origin of Natural Gas Liquids Production"; and Form EIA-816,

"Monthly Natural Gas Liquids Report." 
Figure 21. Natural Gas Supply and Disposition in the United States, 1965-1994

Dry Production

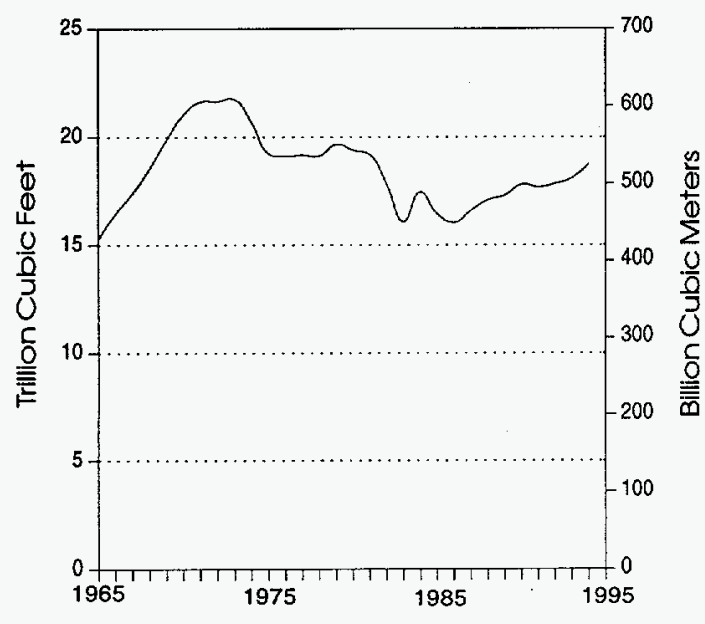

Net Additions to Storage

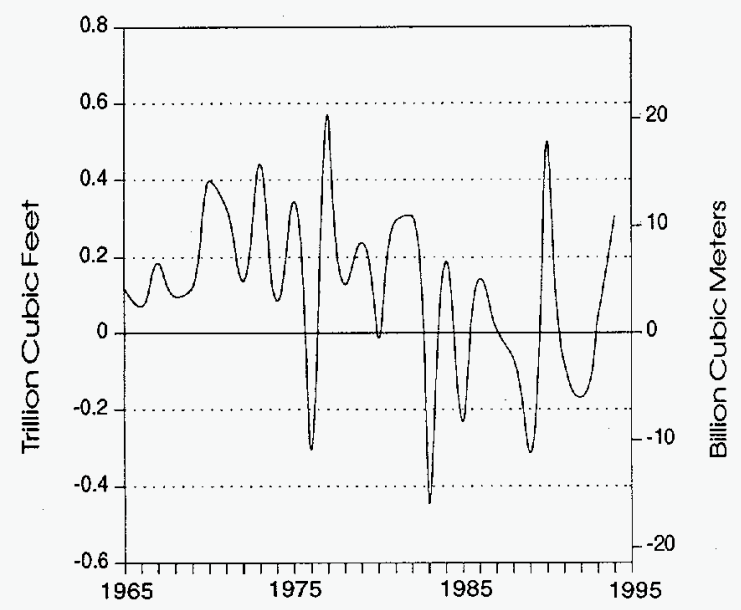

Wellhead Price

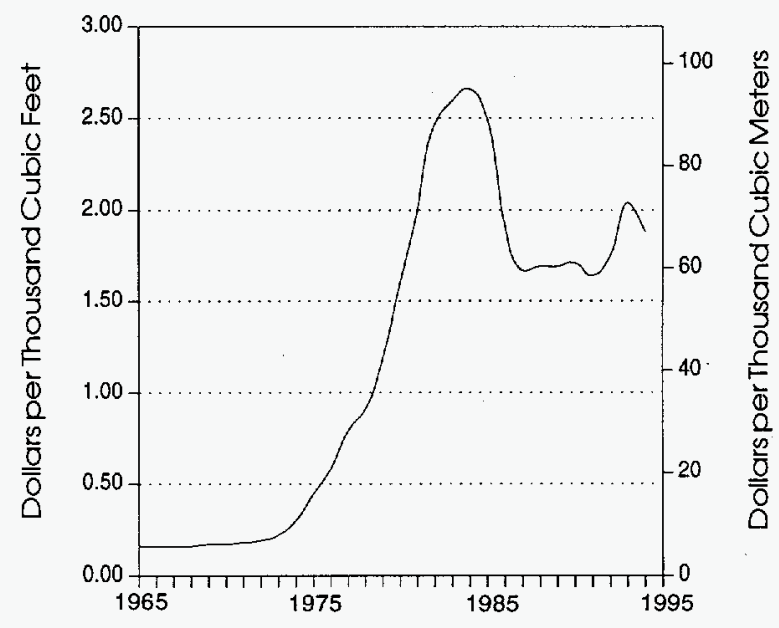

Net Imports

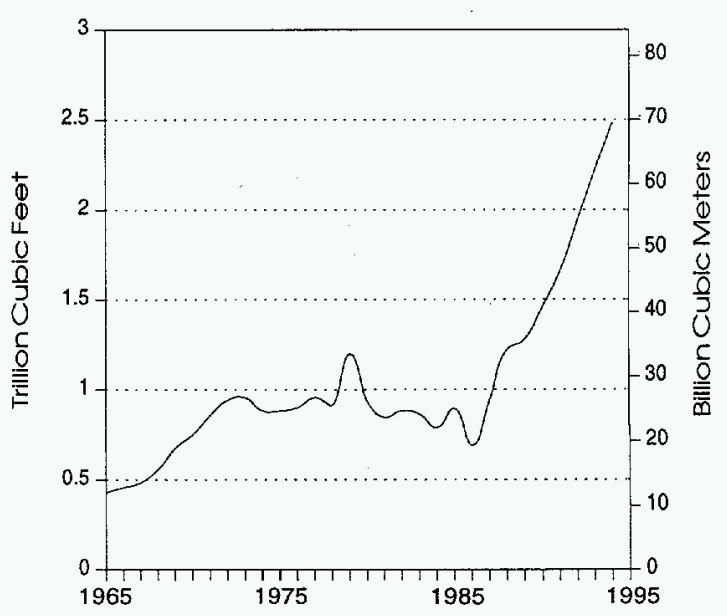

Sources: 1960-1975: Bureau of Mines, Minerals Yearbook, "Natural Gas" chapter. 1976-1978: Energy Information Administration (EIA), Energy Data Reports, Natural Gas Annual. 1979: ElA, Natural Gas Production and Consumption, 1979. 1980-1994: EIA, Form EIA-176, "Annual Report of Natural and Supplemental Gas Supply and Disposition"; Forms EIA-191/FERC-8, "Underground Gas Storage Report”; Form-64A, "Annual Report of the Origin of Natural Gas Liquids Production”; Form EIA-627, "Annual Quantity and Value of Natural Gas Report"; and Form FPC-14, "Annual Report for Imparters and Exporters of Natural Gas." 
Table 100. Supply and Disposition of Natural Gas in the United States, 1930-1994 (Million Cubic Feet)

\begin{tabular}{|c|c|c|c|c|c|c|c|c|c|}
\hline \multirow[b]{2}{*}{ Year } & \multicolumn{5}{|c|}{ Supply } & \multicolumn{4}{|c|}{ Disposition } \\
\hline & $\begin{array}{c}\text { Dry } \\
\text { Production }\end{array}$ & $\begin{array}{c}\text { Withdrawals } \\
\text { from } \\
\text { Storage }\end{array}$ & Imports & $\begin{array}{l}\text { Balancing } \\
\text { Item }\end{array}$ & Total & $\begin{array}{l}\text { Additions } \\
\text { to } \\
\text { Storage }\end{array}$ & Exports & Consumption & Total \\
\hline 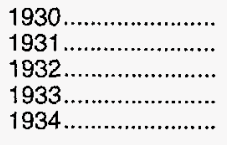 & $\begin{array}{l}1,903,771 \\
1,659,614 \\
1,541,982 \\
1,548,393 \\
1,763,606\end{array}$ & $\begin{array}{l}\text { NA } \\
\text { NA } \\
\text { NA } \\
\text { NA } \\
\text { NA }\end{array}$ & $\begin{array}{l}21 \\
44 \\
38 \\
83 \\
68\end{array}$ & $\begin{array}{l}-35,490 \\
-35,466 \\
-37,808 \\
-41,199 \\
-45,075\end{array}$ & $\begin{array}{l}1,868,302 \\
1,624,192 \\
1,504,212 \\
1,507,277 \\
1,718,599\end{array}$ & $\begin{array}{l}\text { NA } \\
\text { NA } \\
\text { NA } \\
\text { NA } \\
\text { NA }\end{array}$ & $\begin{array}{l}1,798 \\
2,231 \\
1,693 \\
2,158 \\
5,801\end{array}$ & $\begin{array}{l}1,866,504 \\
1,621,961 \\
1,502,519 \\
1,505,119 \\
1,712,798\end{array}$ & $\begin{array}{l}1,868,302 \\
1,624,192 \\
1,504,212 \\
1,507,277 \\
1,718,599\end{array}$ \\
\hline 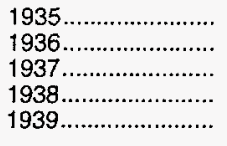 & $\begin{array}{l}1,913,475 \\
2,164,413 \\
2,403,273 \\
2,284,863 \\
2,464,637\end{array}$ & $\begin{array}{l}\text { NA } \\
\text { NA } \\
\text { NA } \\
\text { NA } \\
\text { NA }\end{array}$ & $\begin{array}{l}106 \\
152 \\
289 \\
372 \\
131\end{array}$ & $\begin{array}{l}-41,074 \\
-46,677 \\
-52,157 \\
-47,658 \\
-53,595\end{array}$ & $\begin{array}{l}1,872,507 \\
2,117,888 \\
2,351,405 \\
2,237,577 \\
2,411,173\end{array}$ & $\begin{array}{r}11,294 \\
10,998 \\
13,706 \\
14,981 \\
8,032\end{array}$ & $\begin{array}{l}6,800 \\
7,436 \\
4,868 \\
1,837 \\
3,122\end{array}$ & $\begin{array}{l}1,854,413 \\
2,099,454 \\
2,332,831 \\
2,220,759 \\
2,400,019\end{array}$ & $\begin{array}{l}1,872,507 \\
2,117,888 \\
2,351,405 \\
2,237,577 \\
2,411,173\end{array}$ \\
\hline 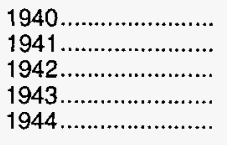 & $\begin{array}{r}2,654,293 \\
2,778,061 \\
-3,026,694 \\
3,393,743 \\
3,672,156\end{array}$ & $\begin{array}{r}\text { NA } \\
\text { NA } \\
\text { NA } \\
\text { NA } \\
33,585\end{array}$ & $\begin{array}{l}0 \\
0 \\
0 \\
0 \\
0\end{array}$ & $\begin{array}{l}-58,602 \\
-64,616 \\
-71,195 \\
-81,889 \\
-94,068\end{array}$ & $\begin{array}{l}2,595,691 \\
2,713,445 \\
2,955,499 \\
3,311,854 \\
3,611,673\end{array}$ & $\begin{array}{l}14,995 \\
16,251 \\
21,024 \\
18,953 \\
43,502\end{array}$ & $\begin{array}{r}5,563 \\
7,466 \\
8,702 \\
11,210 \\
14,576\end{array}$ & $\begin{array}{l}2,575,133 \\
2,689,728 \\
2,925,773 \\
3,281,691 \\
3,553,595\end{array}$ & $\begin{array}{l}2,595,691 \\
2,713,445 \\
2,955,499 \\
3,311,854 \\
3,611,673\end{array}$ \\
\hline $\begin{array}{l}1945 \ldots \ldots \ldots \ldots \ldots \ldots \\
1946 \ldots \ldots \ldots \ldots \ldots \ldots \ldots \ldots \\
1947 \ldots \ldots \ldots \ldots \ldots \ldots \ldots \ldots \ldots \\
1948 \ldots \ldots \ldots \ldots \ldots \ldots \ldots \ldots \ldots \\
1949 \ldots \ldots \ldots \ldots \ldots \ldots \ldots \ldots\end{array}$ & $\begin{array}{l}3,882,066 \\
3,987,488 \\
4,393,439 \\
4,938,512 \\
5,195,404\end{array}$ & $\begin{array}{r}36,167 \\
56,138 \\
86,643 \\
79,035 \\
106,368\end{array}$ & $\begin{array}{l}0 \\
0 \\
0 \\
0 \\
0\end{array}$ & $\begin{array}{r}-97,981 \\
-102,837 \\
-127,807 \\
-126,796 \\
-138,515\end{array}$ & $\begin{array}{l}3,820,252 \\
3,940,789 \\
4,352,275 \\
4,890,751 \\
5,163,257\end{array}$ & $\begin{array}{r}61,502 \\
75,458 \\
96,316 \\
136,406 \\
172,051\end{array}$ & $\begin{array}{l}18,207 \\
17,675 \\
18,149 \\
18,704 \\
20,054\end{array}$ & $\begin{array}{l}3,740,543 \\
3,847,656 \\
4,237,810 \\
4,735,641 \\
4,971,152\end{array}$ & $\begin{array}{l}3,820,252 \\
3,940,789 \\
4,352,275 \\
4,890,751 \\
5,163,257\end{array}$ \\
\hline $\begin{array}{l}1950 \ldots \ldots \ldots \ldots \ldots \ldots \ldots \\
1951 \ldots \ldots \ldots \ldots \ldots \ldots \ldots \ldots \\
1952 \ldots \ldots \ldots \ldots \ldots \ldots \ldots \ldots \\
1953 \ldots \ldots \ldots \ldots \ldots \ldots \ldots \ldots \ldots \\
1954 \ldots \ldots \ldots \ldots \ldots \ldots \ldots \ldots\end{array}$ & $\begin{array}{l}6,022,198 \\
7,164,959 \\
7,694,299 \\
8,056,848 \\
8,388,198\end{array}$ & $\begin{array}{l}175,260 \\
209,428 \\
221,909 \\
246,802 \\
330,177\end{array}$ & $\begin{array}{r}0 \\
0 \\
7,807 \\
9,225 \\
6,847\end{array}$ & $\begin{array}{l}-175,437 \\
-192,372 \\
-203,646 \\
-240,445 \\
-215,709\end{array}$ & $\begin{array}{l}6,022,021 \\
7,182,015 \\
7,720,369 \\
8,072,430 \\
8,509,513\end{array}$ & $\begin{array}{l}229,752 \\
347,690 \\
398,593 \\
404,838 \\
432,283\end{array}$ & $\begin{array}{l}25,727 \\
24,163 \\
27,456 \\
28,322 \\
28,726\end{array}$ & $\begin{array}{l}5,766,542 \\
6,810,162 \\
7,294,320 \\
7,639,270 \\
8,048,504\end{array}$ & $\begin{array}{l}6,022,021 \\
7,182,015 \\
7,720,369 \\
8,072,430 \\
8,509,513\end{array}$ \\
\hline $\begin{array}{l}1955 \ldots \ldots \ldots \ldots \ldots \ldots \ldots \\
1956 \ldots \ldots \ldots \ldots \ldots \ldots \ldots \ldots \\
1957 \ldots \ldots \ldots \ldots \ldots \ldots \ldots \ldots \ldots \\
1958 \ldots \ldots \ldots \ldots \ldots \ldots \ldots \ldots \ldots \\
1959 \ldots \ldots \ldots \ldots \ldots \ldots \ldots \ldots \ldots \ldots\end{array}$ & $\begin{array}{r}9,028,665 \\
9,663,910 \\
10,246,622 \\
10,572,208 \\
11,547,658\end{array}$ & $\begin{array}{l}437,251 \\
452,762 \\
480,981 \\
621,091 \\
668,743\end{array}$ & $\begin{array}{r}10,888 \\
10,380 \\
37,941 \\
135,797 \\
133,990\end{array}$ & $\begin{array}{l}-246,933 \\
-212,992 \\
-205,373 \\
-283,597 \\
-223,312\end{array}$ & $\begin{array}{r}9,229,871 \\
9,914,060 \\
10,560,171 \\
11,045,499 \\
12,127,079\end{array}$ & $\begin{array}{l}505,185 \\
589,232 \\
672,377 \\
704,172 \\
787,485\end{array}$ & $\begin{array}{l}31,029 \\
35,963 \\
41,655 \\
38,719 \\
18,413\end{array}$ & $\begin{array}{r}8,693,657 \\
9,288,865 \\
9,846,139 \\
10,302,608 \\
11,321,181\end{array}$ & $\begin{array}{r}9,229,871 \\
9,914,060 \\
10,560,171 \\
11,045,499 \\
12,127,079\end{array}$ \\
\hline 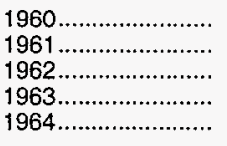 & $\begin{array}{l}12,228,148 \\
12,661,579 \\
13,253,006 \\
14,076,412 \\
14,824,027\end{array}$ & $\begin{array}{l}712,658 \\
698,050 \\
854,336 \\
916,720 \\
885,307\end{array}$ & $\begin{array}{l}155,646 \\
218,860 \\
401,534 \\
406,204 \\
443,326\end{array}$ & $\begin{array}{l}-274,231 \\
-234,808 \\
-285,726 \\
-364,658 \\
-304,435\end{array}$ & $\begin{array}{l}12,822,221 \\
13,343,681 \\
14,223,150 \\
15,034,678 \\
15,848,225\end{array}$ & $\begin{array}{r}844,352 \\
843,666 \\
940,823 \\
1,047,492 \\
1,014,814\end{array}$ & $\begin{array}{l}11,332 \\
10,747 \\
15,814 \\
16,957 \\
19,603\end{array}$ & $\begin{array}{l}11,966,537 \\
12,489,268 \\
13,266,513 \\
13,970,229 \\
14,813,808\end{array}$ & $\begin{array}{l}12,822,221 \\
13,343,681 \\
14,223,150 \\
15,034,678 \\
15,848,225\end{array}$ \\
\hline 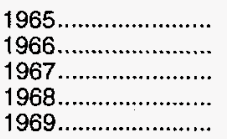 & $\begin{array}{l}15,286,280 \\
16,467,320 \\
17,386,791 \\
18,494,523 \\
19,831,680\end{array}$ & $\begin{array}{r}959,865 \\
1,141,614 \\
1,132,534 \\
1,329,536 \\
1,379,488\end{array}$ & $\begin{array}{l}456,394 \\
479,780 \\
564,226 \\
651,885 \\
726,951\end{array}$ & $\begin{array}{l}-318,711 \\
-401,203 \\
-296,214 \\
-325,062 \\
-334,168\end{array}$ & $\begin{array}{l}16,383,828 \\
17,687,511 \\
18,787,337 \\
20,150,882 \\
21,603,951\end{array}$ & $\begin{array}{l}1,077,980 \\
1,210,469 \\
1,317,363 \\
1,425,075 \\
1,498,988\end{array}$ & $\begin{array}{l}26,132 \\
24,639 \\
81,614 \\
93,745 \\
51,304\end{array}$ & $\begin{array}{l}15,279,716 \\
16,452,403 \\
17,388,360 \\
18,632,062 \\
20,056,240\end{array}$ & $\begin{array}{l}16,383,828 \\
17,687,511 \\
18,787,337 \\
20,150,882 \\
21,606,532\end{array}$ \\
\hline
\end{tabular}

See footnotes at end of table. 
Table 100. Supply and Disposition of Natural Gas in the United States, 1930-1994 (Continued) (Million Cubic Feet)

\begin{tabular}{|c|c|c|c|c|c|c|c|c|c|}
\hline \multirow[b]{2}{*}{ Year } & \multicolumn{5}{|c|}{ Supply } & \multicolumn{4}{|c|}{ Disposition } \\
\hline & $\begin{array}{c}\text { Dry } \\
\text { Production }\end{array}$ & $\begin{array}{c}\text { Withdrawals } \\
\text { from } \\
\text { Storage }\end{array}$ & Imports & $\begin{array}{l}\text { Balancing } \\
\text { Item }\end{array}$ & Total & $\begin{array}{l}\text { Additions } \\
\text { to } \\
\text { Storage }\end{array}$ & Exports & Consumption & Total \\
\hline 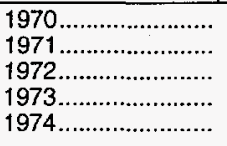 & $\begin{array}{l}21,014,229 \\
21,609,885 \\
21,623,705 \\
21,730,998 \\
20,713,032\end{array}$ & $\begin{array}{l}1,458,607 \\
1,507,630 \\
1,757,218 \\
1,532,820 \\
1,700,546\end{array}$ & $\begin{array}{r}820,780 \\
934,548 \\
1,019,496 \\
1,032,901 \\
959,284\end{array}$ & $\begin{array}{l}-227,650 \\
-338,999 \\
-328,002 \\
-195,863 \\
-288,731\end{array}$ & $\begin{array}{l}23,065,966 \\
23,713,064 \\
24,072,417 \\
24,100,856 \\
23,084,131\end{array}$ & $\begin{array}{l}1,856,767 \\
1,839,398 \\
1,892,952 \\
1,974,324 \\
1,784,209\end{array}$ & $\begin{array}{l}69,813 \\
80,212 \\
78,013 \\
77,169 \\
76,789\end{array}$ & $\begin{array}{l}21,139,386 \\
21,793,454 \\
22,101,452 \\
22,049,363 \\
21,223,133\end{array}$ & $\begin{array}{l}23,065,966 \\
23,713,064 \\
24,072,417 \\
24,100,856 \\
23,084,131\end{array}$ \\
\hline 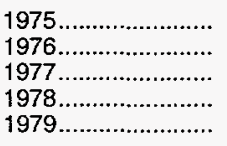 & $\begin{array}{l}19,236,379 \\
19,098,352 \\
19,162,900 \\
19,121,903 \\
19,663,415\end{array}$ & $\begin{array}{l}1,759,565 \\
2,059,898 \\
1,735,868 \\
2,150,928 \\
2,057,020\end{array}$ & $\begin{array}{r}953,008 \\
963,768 \\
1,011,002 \\
965,545 \\
1,253,383\end{array}$ & $\begin{array}{r}-235,065 \\
-216,240 \\
-41,063 \\
-287,201 \\
-372,330\end{array}$ & $\begin{array}{l}21,713,887 \\
21,905,778 \\
21,868,707 \\
21,951,175 \\
22,601,488\end{array}$ & $\begin{array}{l}2,103,619 \\
1,755,690 \\
2,306,515 \\
2,278,002 \\
2,295,034\end{array}$ & $\begin{array}{l}72,675 \\
64,711 \\
55,626 \\
52,532 \\
55,673\end{array}$ & $\begin{array}{l}19,537,593 \\
19,946,496 \\
19,520,581 \\
19,627,478 \\
20,240,761\end{array}$ & $\begin{array}{l}21,713,887 \\
21,766,897 \\
21,882,722 \\
21,958,012 \\
22,591,468\end{array}$ \\
\hline 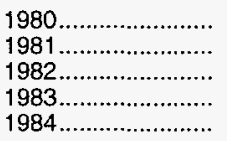 & $\begin{array}{l}19,557,709 \\
19,356,963 \\
17,964,874 \\
16,226,355 \\
17,576,449\end{array}$ & $\begin{array}{l}1,972,333 \\
1,930,092 \\
2,164,184 \\
2,269,654 \\
2,098,303\end{array}$ & $\begin{array}{l}984,767 \\
903,949 \\
933,336 \\
918,407 \\
843,060\end{array}$ & $\begin{array}{l}-639,721 \\
-500,444 \\
-537,061 \\
-703,342 \\
-217,308\end{array}$ & $\begin{array}{l}21,875,088 \\
21,690,560 \\
20,525,333 \\
18,711,074 \\
20,300,504\end{array}$ & $\begin{array}{l}1,949,064 \\
2,227,522 \\
2,472,383 \\
1,822,354 \\
2,295,138\end{array}$ & $\begin{array}{l}48,731 \\
59,372 \\
51,728 \\
54,639 \\
54,753\end{array}$ & $\begin{array}{l}19,877,293 \\
19,403,858 \\
18,001,055 \\
16,834,914 \\
17,950,524\end{array}$ & $\begin{array}{l}21,875,088 \\
21,690,752 \\
20,525,166 \\
18,711,907 \\
20,300,415\end{array}$ \\
\hline $\begin{array}{l}1985 \ldots \ldots \ldots \ldots \ldots \ldots \ldots \\
1986 \ldots \ldots \ldots \ldots \ldots \ldots \ldots \ldots \\
1987 \ldots \ldots \ldots \ldots \ldots \ldots \ldots \ldots \\
1988 \ldots \ldots \ldots \ldots \ldots \ldots \ldots \ldots \\
1989 \ldots \ldots \ldots \ldots \ldots \ldots \ldots \ldots\end{array}$ & $\begin{array}{l}16,580,220 \\
16,172,219 \\
16,721,963 \\
17,203,755 \\
17,417,390\end{array}$ & $\begin{array}{l}2,397,359 \\
1,836,693 \\
1,905,419 \\
2,270,011 \\
2,854,061\end{array}$ & $\begin{array}{r}949,715 \\
750,449 \\
992,532 \\
1,293,812 \\
1,381,520\end{array}$ & $\begin{array}{l}-428,120 \\
-494,491 \\
-443,231 \\
-452,492 \\
-217,526\end{array}$ & $\begin{array}{l}19,499,174 \\
18,264,870 \\
19,176,683 \\
20,315,086 \\
21,435,445\end{array}$ & $\begin{array}{l}2,162,603 \\
1,983,603 \\
1,911,489 \\
2,211,277 \\
2,527,750\end{array}$ & $\begin{array}{r}55,268 \\
61,271 \\
54,020 \\
73,638 \\
106,871\end{array}$ & $\begin{array}{l}17,280,943 \\
16,221,296 \\
17,210,809 \\
18,029,588 \\
18,800,826\end{array}$ & $\begin{array}{l}19,498,814 \\
18,266,170 \\
19,176,318 \\
20,314,503 \\
21,435,447\end{array}$ \\
\hline 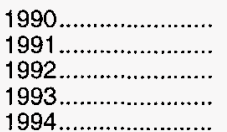 & $\begin{array}{r}17,932,480 \\
17,810,408 \\
17,957,822 \\
R_{18,214,459} \\
18,857,821\end{array}$ & $\begin{array}{l}1,986,330 \\
2,751,818 \\
2,772,308 \\
2,799,253 \\
2,562,116\end{array}$ & $\begin{array}{l}1,532,259 \\
1,773,313 \\
2,137,504 \\
2,350,115 \\
2,659,673\end{array}$ & $\begin{array}{r}-151,863 \\
-499,779 \\
-507,565 \\
\mathbf{R}_{-109,593} \\
-296,577\end{array}$ & $\begin{array}{r}21,299,206 \\
21,835,760 \\
22,360,069 \\
\mathbf{R}_{23,254,234} \\
23,783,033\end{array}$ & $\begin{array}{l}2,499,264 \\
2,671,632 \\
2,599,426 \\
2,834,955 \\
2,864,757\end{array}$ & $\begin{array}{r}85,565 \\
129,244 \\
216,282 \\
140,183 \\
178,066\end{array}$ & $\begin{array}{r}18,715,090 \\
19,035,156 \\
19,544,364 \\
\mathrm{R}_{20,279,095} \\
20,755,471\end{array}$ & $\begin{array}{r}21,299,919 \\
21,836,032 \\
22,360,072 \\
\mathrm{R}_{23,254,233} \\
23,798,294\end{array}$ \\
\hline
\end{tabular}

$\mathrm{A}=$ Revised data

NA $=$ Not Available

Notes: Beginning with 1980 data, dry production and consumption volumes did include supplemental gas supplies, and storage volumes did include liquefied natural gas (LNG). Beginning with 1976 data, total supply and disposition do not balance on equivalent data in Table 1 due to the exclusion of intransit receipts and deliveries. Beginning with 1965 data, all volumes are shown on a pressure base of 14.73 psia at degrees Fahrenheit. For prior years, the pressure base is 14.65 psia at 60 degrees Fahrenheit.

Sources: 1930-1975: Bureau of Mines, Minerals Yearbook, "Natural Gas" chapter. 1976-1978: Energy Information Administration (ElA), Energy Data Report, Natural Gas Annual. 1979: ElA, Natural Gas Production and Consumption, 1979. 1980-1994: ElA, Form ElA-176, "Annual Report of Natural and Supplemental Gas Supply and

Disposition"; Form E]A-191, "Underground Gas Storage Report"; Form ElA-64A, "Annual Report of the Origin of Natural Gas Liquids Production"; Form EIA-627, "Annual Quantity and Value of Natural Gas Report"; Form ElA-191, "Underground Gas Storage Report"; and Form FPC-14, "Annual Report for Importers and Exporters of Natural Gas." 
Figure 22. Natural Gas Delivered to Consumers in the United States, 1930 - 1994

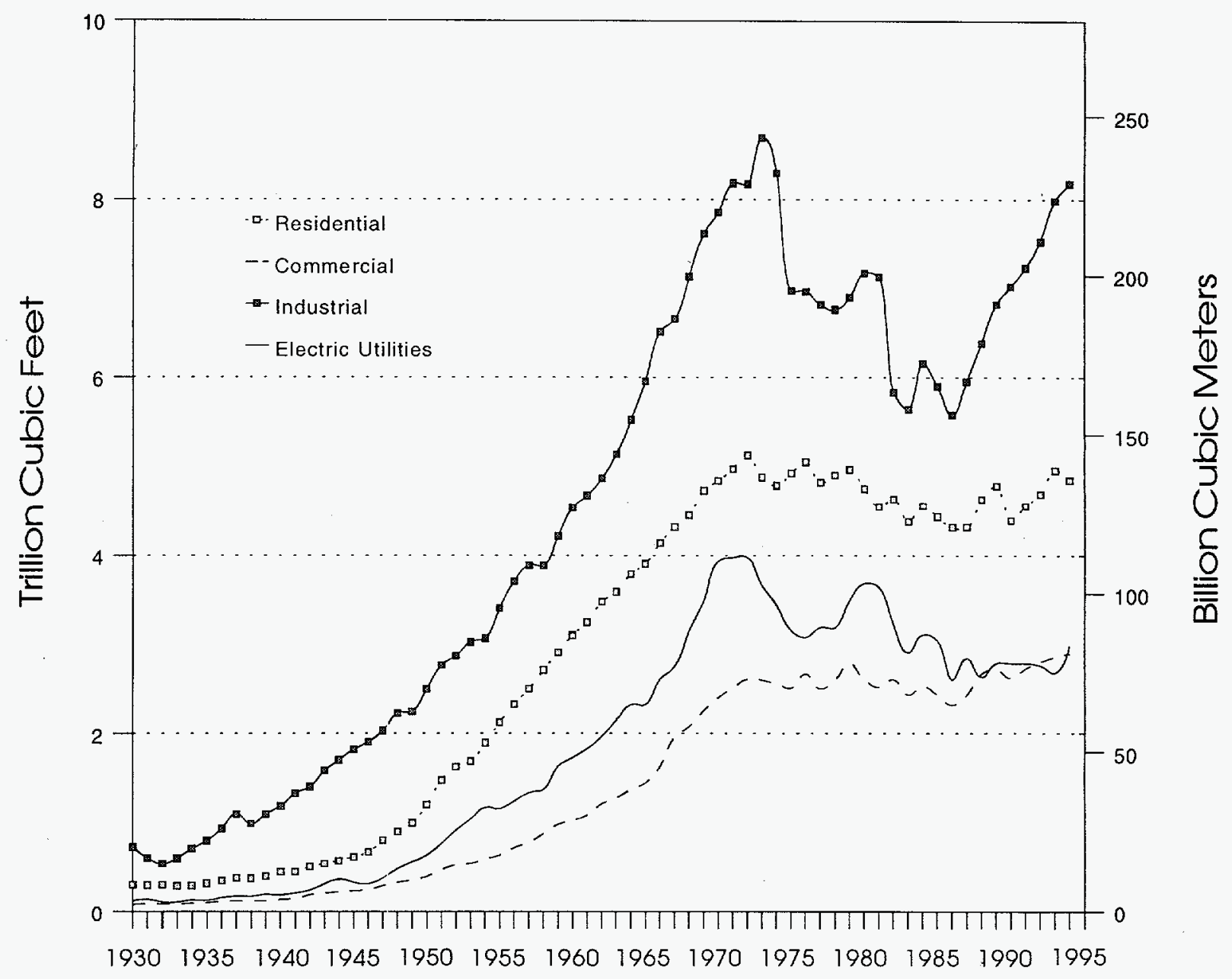

Sources: 1930-1975: Bureau of Mines, Minerals Yearbook, "Natural Gas" chapter. 1976-1978: Energy Information Administration (ElA), Energy Data Reports, Natural Gas Annual. 1979: EIA, Natural Gas Production and Consumption, 1979. 1980-1994: Form EIA-176, "Annual Report of Natural and Supplemental Gas Supply and Disposition" and Form ElA-759 "Monthly Power Plant Reprort." 
Table 101. Natural Gas Consumption in the United States, 1930-1994

(Million Cubic Feet)

\begin{tabular}{|c|c|c|c|c|c|c|c|c|c|}
\hline \multirow{2}{*}{ Year } & \multirow{2}{*}{$\begin{array}{c}\text { Lease } \\
\text { and } \\
\text { Plant Fuel }\end{array}$} & \multirow{2}{*}{$\begin{array}{l}\text { Pipeline } \\
\text { Fuel }\end{array}$} & \multicolumn{6}{|c|}{ Delivered to Consumers } & \multirow{2}{*}{$\begin{array}{c}\text { Total } \\
\text { Consumption }\end{array}$} \\
\hline & & & Residential & Commercial & Industrial & Vehicle Fuel & $\begin{array}{l}\text { Electric } \\
\text { Utilities }\end{array}$ & Total & \\
\hline 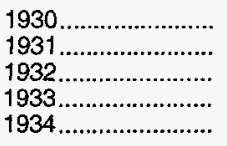 & $\begin{array}{l}648,025 \\
509,077 \\
477,562 \\
442,879 \\
502,352\end{array}$ & $\begin{array}{l}\text { NA } \\
\text { NA } \\
\text { NA } \\
\text { NA } \\
\text { NA }\end{array}$ & $\begin{array}{l}295,700 \\
294,406 \\
298,520 \\
283,197 \\
288,236\end{array}$ & $\begin{array}{l}80,707 \\
86,491 \\
87,367 \\
85,577 \\
91,261\end{array}$ & $\begin{array}{l}721,782 \\
593,644 \\
531,831 \\
590,865 \\
703,053\end{array}$ & $\begin{array}{l}\text { NA } \\
\text { NA } \\
\text { NA } \\
\text { NA } \\
\text { NA }\end{array}$ & $\begin{array}{l}120,290 \\
138,343 \\
107,239 \\
102,601 \\
127,896\end{array}$ & $\begin{array}{l}1,218,479 \\
1,112,884 \\
1,024,957 \\
1,062,240 \\
1,210,446\end{array}$ & $\begin{array}{l}1,866,504 \\
1,621,961 \\
1,502,519 \\
1,505,119 \\
1,712,798\end{array}$ \\
\hline 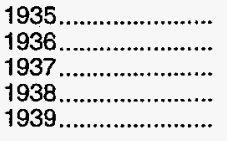 & $\begin{array}{l}524,926 \\
557,404 \\
581,110 \\
585,865 \\
607,138\end{array}$ & $\begin{array}{l}\text { NA } \\
\text { NA } \\
\text { NA } \\
\text { NA } \\
\text { NA }\end{array}$ & $\begin{array}{l}313,498 \\
343,346 \\
371,844 \\
367,772 \\
391,153\end{array}$ & $\begin{array}{l}100,187 \\
111,623 \\
117,390 \\
114,296 \\
118,334\end{array}$ & $\begin{array}{r}790,563 \\
931,001 \\
1,091,920 \\
982,838 \\
1,092,263\end{array}$ & $\begin{array}{l}\text { NA } \\
\text { NA } \\
\text { NA } \\
\text { NA } \\
\text { NA }\end{array}$ & $\begin{array}{l}125,239 \\
156,080 \\
170,567 \\
169,988 \\
191,131\end{array}$ & $\begin{array}{l}1,329,487 \\
1,542,050 \\
1,751,721 \\
1,634,894 \\
1,792,881\end{array}$ & $\begin{array}{l}1,854,413 \\
2,099,454 \\
2,332,831 \\
2,220,759 \\
2,400,019\end{array}$ \\
\hline 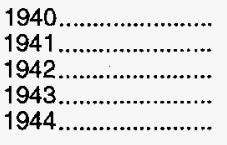 & $\begin{array}{l}632,335 \\
570,694 \\
602,063 \\
659,198 \\
712,312\end{array}$ & $\begin{array}{l}\text { NA } \\
\text { NA } \\
\text { NA } \\
\text { NA } \\
\text { NA }\end{array}$ & $\begin{array}{l}443,646 \\
442,067 \\
498,537 \\
529,444 \\
562,183\end{array}$ & $\begin{array}{l}134,644 \\
144,844 \\
183,603 \\
204,793 \\
220,747\end{array}$ & $\begin{array}{l}1,181,352 \\
1,326,967 \\
1,402,834 \\
1,582,680 \\
1,698,608\end{array}$ & $\begin{array}{l}\text { NA } \\
\text { NA } \\
\text { NA } \\
\text { NA } \\
\text { NA }\end{array}$ & $\begin{array}{l}183,156 \\
205,156 \\
238,736 \\
305,576 \\
359,745\end{array}$ & $\begin{array}{l}1,942,798 \\
2,119,034 \\
2,323,710 \\
2,622,493 \\
2,841,283\end{array}$ & $\begin{array}{l}2,575,133 \\
2,689,728 \\
2,925,773 \\
3,281,691 \\
3,553,595\end{array}$ \\
\hline 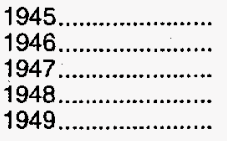 & $\begin{array}{l}757,016 \\
732,535 \\
745,027 \\
812,005 \\
835,297\end{array}$ & $\begin{array}{l}\text { NA } \\
\text { NA } \\
\text { NA } \\
\text { NA } \\
\text { NA }\end{array}$ & $\begin{array}{l}607,400 \\
660,820 \\
802,150 \\
896,348 \\
992,544\end{array}$ & $\begin{array}{l}230,099 \\
241,802 \\
285,213 \\
323,054 \\
347,818\end{array}$ & $\begin{array}{l}1,819,838 \\
1,905,575 \\
2,032,383 \\
2,226,137 \\
2,245,372\end{array}$ & $\begin{array}{l}\text { NA } \\
\text { NA } \\
\text { NA } \\
\text { NA } \\
\text { NA }\end{array}$ & $\begin{array}{l}326,190 \\
306,924 \\
373,037 \\
478,097 \\
550,121\end{array}$ & $\begin{array}{l}2,983,527 \\
3,115,121 \\
3,492,783 \\
3,923,636 \\
4,135,855\end{array}$ & $\begin{array}{l}3,740,543 \\
3,847,656 \\
4,237,810 \\
4,735,641 \\
4,971,152\end{array}$ \\
\hline 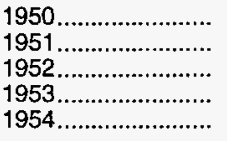 & $\begin{array}{r}927,611 \\
1,149,470 \\
1,164,596 \\
1,131,017 \\
1,102,535\end{array}$ & $\begin{array}{l}125,546 \\
192,496 \\
207,207 \\
230,314 \\
230,615\end{array}$ & $\begin{array}{l}1,198,369 \\
1,474,725 \\
1,621,966 \\
1,685,503 \\
1,894,248\end{array}$ & $\begin{array}{l}387,838 \\
464,309 \\
515,669 \\
530,650 \\
584,957\end{array}$ & $\begin{array}{l}2,498,259 \\
2,765,264 \\
2,874,765 \\
3,027,514 \\
3,070,651\end{array}$ & $\begin{array}{l}\text { NA } \\
\text { NA } \\
\text { NA } \\
\text { NA } \\
\text { NA }\end{array}$ & $\begin{array}{r}628,919 \\
763,898 \\
910,117 \\
1,034,272 \\
1,165,498\end{array}$ & $\begin{array}{l}4,713,385 \\
5,468,196 \\
5,922,517 \\
6,277,939 \\
6,715,354\end{array}$ & $\begin{array}{l}5,766,542 \\
6,810,162 \\
7,294,320 \\
7,639,270 \\
8,048,504\end{array}$ \\
\hline 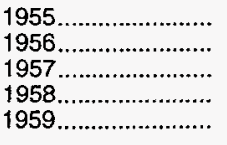 & $\begin{array}{l}1,130,985 \\
1,002,537 \\
1,046,084 \\
1,146,064 \\
1,238,945\end{array}$ & $\begin{array}{l}245,246 \\
295,972 \\
299,235 \\
312,221 \\
349,348\end{array}$ & $\begin{array}{l}2,123,952 \\
2,327,564 \\
2,500,269 \\
2,714,251 \\
2,912,601\end{array}$ & $\begin{array}{l}629,219 \\
716,871 \\
775,916 \\
871,774 \\
975,107\end{array}$ & $\begin{array}{l}3,410,975 \\
3,706,610 \\
3,888,494 \\
3,885,445 \\
4,216,671\end{array}$ & $\begin{array}{l}\text { NA } \\
\text { NA } \\
\text { NA } \\
\text { NA } \\
\text { NA }\end{array}$ & $\begin{array}{l}1,153,280 \\
1,239,311 \\
1,336,141 \\
1,372,853 \\
1,628,509\end{array}$ & $\begin{array}{l}7,317,426 \\
7,990,356 \\
8,500,820 \\
8,844,323 \\
9,732,888\end{array}$ & $\begin{array}{r}8,693,657 \\
9,288,865 \\
9,846,139 \\
10,302,608 \\
11,321,181\end{array}$ \\
\hline 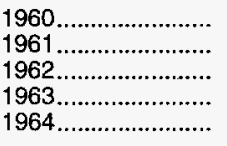 & $\begin{array}{l}1,236,781 \\
1,288,762 \\
1,369,512 \\
1,411,088 \\
1,370,835\end{array}$ & $\begin{array}{l}347,075 \\
377,607 \\
382,496 \\
423,783 \\
435,570\end{array}$ & $\begin{array}{l}3,103,167 \\
3,248,578 \\
3,478,563 \\
3,589,021 \\
3,787,292\end{array}$ & $\begin{array}{l}1,020,222 \\
1,076,849 \\
1,206,668 \\
1,267,783 \\
1,374,717\end{array}$ & $\begin{array}{l}4,534,530 \\
4,672,355 \\
4,863,300 \\
5,134,081 \\
5,522,498\end{array}$ & $\begin{array}{l}\text { NA } \\
\text { NA } \\
\text { NA } \\
\text { NA } \\
\text { NA }\end{array}$ & $\begin{array}{l}1,724,762 \\
1,825,117 \\
1,965,974 \\
2,144,473 \\
2,322,896\end{array}$ & $\begin{array}{l}10,382,681 \\
10,822,899 \\
11,514,505 \\
12,135,358 \\
13,007,403\end{array}$ & $\begin{array}{l}11,966,537 \\
12,489,268 \\
13,266,513 \\
13,970,229 \\
14,813,808\end{array}$ \\
\hline 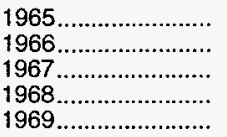 & $\begin{array}{l}1,156,224 \\
1,033,400 \\
1,140,966 \\
1,237,131 \\
1,345,648\end{array}$ & $\begin{array}{l}500,524 \\
535,353 \\
575,752 \\
590,965 \\
630,962\end{array}$ & $\begin{array}{l}3,902,802 \\
4,138,259 \\
4,313,304 \\
4,450,354 \\
4,728,281\end{array}$ & $\begin{array}{l}1,443,648 \\
1,622,740 \\
1,958,970 \\
2,075,736 \\
2,253,206\end{array}$ & $\begin{array}{l}5,955,417 \\
6,512,702 \\
6,653,016 \\
7,129,967 \\
7,610,501\end{array}$ & $\begin{array}{l}\text { NA } \\
\text { NA } \\
\text { NA } \\
\text { NA } \\
\text { NA }\end{array}$ & $\begin{array}{l}2,321,101 \\
2,609,949 \\
2,746,352 \\
3,147,909 \\
3,487,642\end{array}$ & $\begin{array}{l}13,622,968 \\
14,883,650 \\
15,671,642 \\
16,803,966 \\
18,079,630\end{array}$ & $\begin{array}{l}15,279,716 \\
16,452,403 \\
17,388,360 \\
18,632,062 \\
20,056,240\end{array}$ \\
\hline
\end{tabular}

See footnotes at end of table. 
Table 101. Natural Gas Consumption In the United States, 1930-1994 (Continued) (Million Cubic Feet)

\begin{tabular}{|c|c|c|c|c|c|c|c|c|c|}
\hline \multirow{2}{*}{ Year } & \multirow{2}{*}{$\begin{array}{c}\text { Lease } \\
\text { and } \\
\text { Plant Fuel }\end{array}$} & \multirow{2}{*}{$\begin{array}{l}\text { Pipeline } \\
\text { Fuel }\end{array}$} & \multicolumn{6}{|c|}{ Delivered to Consumers } & \multirow{2}{*}{$\begin{array}{c}\text { Total } \\
\text { Consumption }\end{array}$} \\
\hline & & & Residential & Commercial & Industrial & Vehicle Fuel & $\begin{array}{l}\text { Electric } \\
\text { Utilities }\end{array}$ & Total & \\
\hline 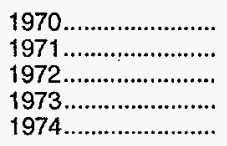 & $\begin{array}{l}1,398,758 \\
1,413,650 \\
1,455,563 \\
1,495,915 \\
1,477,386\end{array}$ & $\begin{array}{l}722,166 \\
742,592 \\
766,156 \\
728,177 \\
668,792\end{array}$ & $\begin{array}{l}4,837,432 \\
4,971,690 \\
5,125,982 \\
4,879,387 \\
4,786,128\end{array}$ & $\begin{array}{l}2,398,510 \\
2,508,977 \\
2,607,982 \\
2,597,037 \\
2,555,617\end{array}$ & $\begin{array}{l}7,850,660 \\
8,180,527 \\
8,168,855 \\
8,688,675 \\
8,291,782\end{array}$ & $\begin{array}{l}\text { NA } \\
\text { NA } \\
\text { NA } \\
\text { NA } \\
\text { NA }\end{array}$ & $\begin{array}{l}3,931,860 \\
3,976,018 \\
3,976,914 \\
3,660,172 \\
3,443,428\end{array}$ & $\begin{array}{l}19,018,462 \\
19,637,212 \\
19,879,733 \\
19,825,271 \\
19,076,955\end{array}$ & $\begin{array}{l}21,139,386 \\
21,793,454 \\
22,101,452 \\
22,049,363 \\
21,223,133\end{array}$ \\
\hline 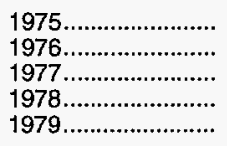 & $\begin{array}{l}1,396,277 \\
1,634,355 \\
1,659,145 \\
1,647,911 \\
1,498,530\end{array}$ & $\begin{array}{l}582,963 \\
548,323 \\
532,669 \\
530,451 \\
600,964\end{array}$ & $\begin{array}{l}4,924,124 \\
5,051,360 \\
4,821,485 \\
4,903,006 \\
4,965,365\end{array}$ & $\begin{array}{l}2,508,293 \\
2,667,740 \\
2,500,793 \\
2,601,106 \\
2,785,961\end{array}$ & $\begin{array}{l}6,968,267 \\
6,963,850 \\
6,815,289 \\
6,756,641 \\
6,899,418\end{array}$ & $\begin{array}{l}\text { NA } \\
\text { NA } \\
\text { NA } \\
\text { NA } \\
\text { NA }\end{array}$ & $\begin{array}{l}3,157,669 \\
3,080,868 \\
3,191,200 \\
3,188,363 \\
3,490,523\end{array}$ & $\begin{array}{l}17,558,353 \\
17,763,818 \\
17,328,767 \\
17,449,116 \\
18,141,267\end{array}$ & $\begin{array}{l}19,537,593 \\
19,946,496 \\
19,520,581 \\
19,627,478 \\
20,240,761\end{array}$ \\
\hline 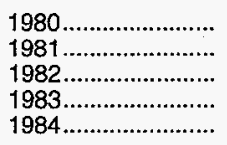 & $\begin{array}{r}1,026,438 \\
927,591 \\
1,109,398 \\
978,249 \\
1,076,881\end{array}$ & $\begin{array}{l}634,622 \\
642,325 \\
596,411 \\
490,042 \\
528,754\end{array}$ & $\begin{array}{l}4,752,082 \\
4,546,450 \\
4,633,035 \\
4,380,599 \\
4,555,465\end{array}$ & $\begin{array}{l}2,610,895 \\
2,519,791 \\
2,605,523 \\
2,432,547 \\
2,524,244\end{array}$ & $\begin{array}{l}7,171,661 \\
7,127,547 \\
5,831,170 \\
5,642,708 \\
6,153,841\end{array}$ & $\begin{array}{l}\text { NA } \\
\text { NA } \\
\text { NA } \\
\text { NA } \\
\text { NA }\end{array}$ & $\begin{array}{l}3,681,595 \\
3,640,154 \\
3,225,518 \\
2,910,767 \\
3,111,342\end{array}$ & $\begin{array}{l}18,216,233 \\
17,833,942 \\
16,295,245 \\
15,366,621 \\
16,344,893\end{array}$ & $\begin{array}{l}19,877,293 \\
19,403,858 \\
18,001,055 \\
16,834,914 \\
17,950,524\end{array}$ \\
\hline 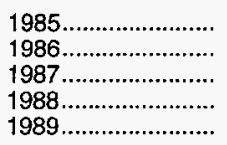 & $\begin{array}{r}966,047 \\
922,524 \\
1,149,383 \\
1,095,883 \\
1,069,902\end{array}$ & $\begin{array}{l}503,766 \\
485,041 \\
519,170 \\
613,912 \\
629,308\end{array}$ & $\begin{array}{l}4,433,377 \\
4,313,969 \\
4,314,833 \\
4,630,330 \\
4,780,638\end{array}$ & $\begin{array}{l}2,432,382 \\
2,318,335 \\
2,430,064 \\
2,670,465 \\
2,717,722\end{array}$ & $\begin{array}{l}5,901,288 \\
5,579,057 \\
5,953,308 \\
6,383,382 \\
6,816,244\end{array}$ & $\begin{array}{l}\text { NA } \\
\text { NA } \\
\text { NA } \\
\text { NA } \\
\text { NA }\end{array}$ & $\begin{array}{l}3,044,083 \\
2,602,370 \\
2,844,051 \\
2,635,616 \\
2,787,012\end{array}$ & $\begin{array}{l}15,811,130 \\
14,813,731 \\
15,542,256 \\
16,319,793 \\
17,101,615\end{array}$ & $\begin{array}{l}17,280,943 \\
16,221,296 \\
17,210,809 \\
18,029,588 \\
18,800,826\end{array}$ \\
\hline 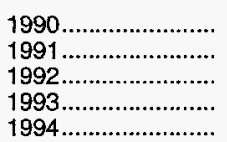 & $\begin{array}{r}1,236,392 \\
1,129,268 \\
1,170,821 \\
R_{1}, 171,940 \\
1,160,556\end{array}$ & $\begin{array}{l}659,816 \\
601,305 \\
587,710 \\
624,308 \\
685,327\end{array}$ & $\begin{array}{r}4,391,324 \\
4,555,659 \\
4,690,065 \\
R_{4,956,445} \\
4,847,702\end{array}$ & $\begin{array}{r}2,622,721 \\
2,728,581 \\
2,802,751 \\
\mathrm{R}_{2,861,569} \\
2,895,034\end{array}$ & $\begin{array}{r}7,018,414 \\
7,230,962 \\
7,526,898 \\
\mathbf{R}_{7,981,433} \\
8,177,975\end{array}$ & $\begin{array}{r}270 \\
367 \\
511 \\
960 \\
1,730\end{array}$ & $\begin{array}{l}2,786,153 \\
2,789,014 \\
2,765,608 \\
2,682,440 \\
2,987,146\end{array}$ & $\begin{array}{r}16,818,882 \\
17,304,582 \\
17,785,833 \\
\mathbf{R}_{18,482,847} \\
18,909,587\end{array}$ & $\begin{array}{r}18,715,090 \\
19,035,156 \\
19,544,364 \\
R_{20,279,095} \\
20,755,471\end{array}$ \\
\hline
\end{tabular}

$\mathrm{R}=$ Revised data.

NA = Not Available. Notes: Beginning with 1965 data, all volumes are show'n on a pressure base of 14.73 psia at 60 degrees
degrees Fahrenheit. Number of vehicle fuel consumers cjenerally refers to the number of fueling stations.

degrees Fahrenheit. Number of vehicle fuel consumers generally refers to the number of fueling stations.
Sources: 1930-1975: Bureau of Mines, Minerals Yearbook, "Natural Gas" chapter. 1976-1978: Energy Information Administration (ElA), Energy Data Reports, Natural Gas Sources: 1930-1975: Bureau of Mines, Minerals Yearbook, "Natural Gas" chapter. 1976-1978: Energy Information Administration (ElA), Energy Data Reports, Natura
Annual. 1979: EIA, Natural Gas Production and Consumption, 1979. 1980-1989: ElA, Form EIA-176, "Annual Report of Natural and Supplemental Gas Supply and

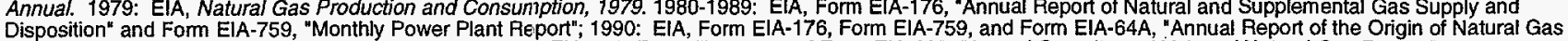
Liquids Production"; 1991-1994: EIA, Form EIA-176, Forn EIA-759, Form ElA-64A, and Form EIA-627, "Annual Quantity and Value of Natural Gas Report." 
Figure 23. Average Price of Natural Gas Delivered to U.S. Consumers, 1967 - 1994

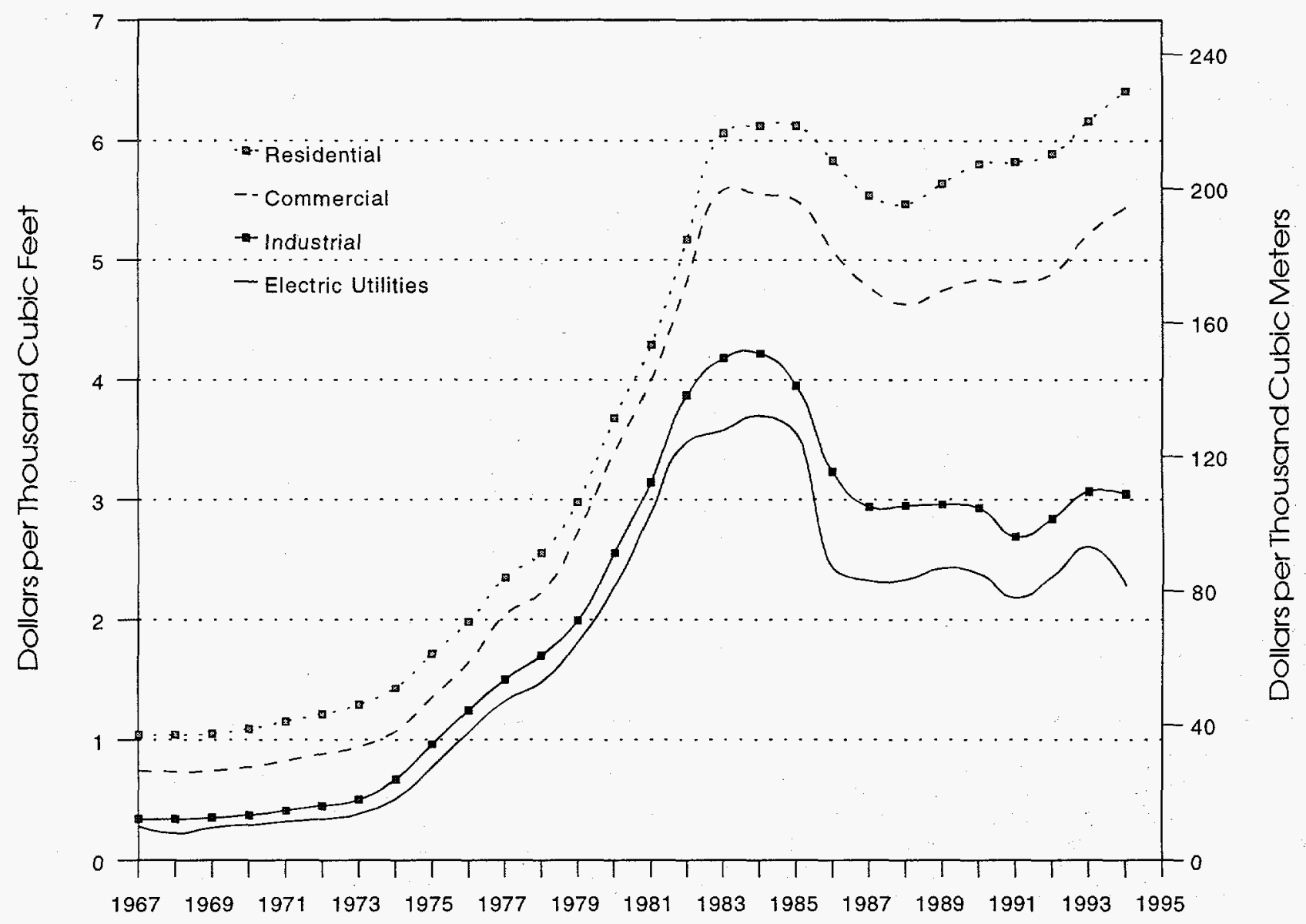

Sources: Electric Utilities: 1967-1977: Federal Power Commission (FPC). 1978-1993: Energy information Administration (ElA), Form ElA-759, "Monthly Power Plant Report;" Federal Energy Regulatory Commission (FERC), Form FERC-423, "Cost and Quality of Fuels for Electric Utility Plants" and Form ElA-176, "Annual Report of Natural and Supplemental Gas Supply and Disposition." All other data: 1967-1975: Bureau of Mines, Minerals Yearbook, "Natural Gas" chapter. 1976-1978: Energy Information Administration, Energy Data Report, Natural Gas Annual. 1979: Energy Information Administration, Natural Gas Production and Consumption 1979, 1980-1994: Energy Information Administration (EIA), Form ElA-176, "Annual Report of Natural and Supplemental Gas Supply and Disposition" and Federal Energy Regulatory Commission (FERC), Form FERC-423, "Monthly Report of Cost and Quality of Fueis for Electric Plants." 
Table 102. Average Price of Natural Gas Delivered to U. S. Consumers, 1967-1994 (Dollars per Thousand Cubic Feet)

\begin{tabular}{|c|c|c|c|c|c|}
\hline \multirow{2}{*}{ Year } & \multicolumn{5}{|c|}{ Delivered to Consumers } \\
\hline & Residential & Commercial & Industrial & Vehicle & $\begin{array}{l}\text { Electric } \\
\text { Utilities }\end{array}$ \\
\hline 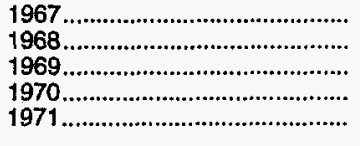 & $\begin{array}{l}1.04 \\
1.04 \\
1.05 \\
1.09 \\
1.15\end{array}$ & $\begin{array}{l}0.74 \\
0.73 \\
0.74 \\
0.77 \\
0.82\end{array}$ & $\begin{array}{l}0.34 \\
0.34 \\
0.35 \\
0.37 \\
0.41\end{array}$ & $\begin{array}{l}\text { NA } \\
\text { NA } \\
\text { NA } \\
\text { NA } \\
\text { NA }\end{array}$ & $\begin{array}{l}0.28 \\
0.22 \\
0.27 \\
0.29 \\
0.32\end{array}$ \\
\hline 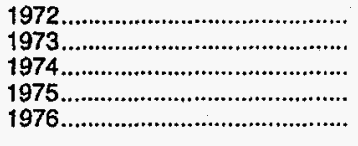 & $\begin{array}{l}1.21 \\
1.29 \\
1.43 \\
1.71 \\
1.98\end{array}$ & $\begin{array}{l}0.88 \\
0.94 \\
1.07 \\
1.35 \\
1.64\end{array}$ & $\begin{array}{l}0.45 \\
0.50 \\
0.67 \\
0.96 \\
1.24\end{array}$ & $\begin{array}{l}\text { NA } \\
\text { NA } \\
\text { NA } \\
\text { NA } \\
\text { NA }\end{array}$ & $\begin{array}{l}0.34 \\
0.38 \\
0.51 \\
0.77 \\
1.06\end{array}$ \\
\hline 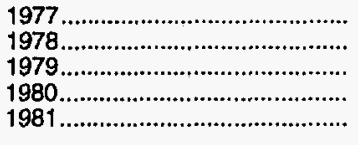 & $\begin{array}{l}2.35 \\
2.56 \\
2.98 \\
3.68 \\
4.29\end{array}$ & $\begin{array}{l}2.04 \\
2.23 \\
2.73 \\
3.39 \\
4.00\end{array}$ & $\begin{array}{l}1.50 \\
1.70 \\
1.99 \\
2.56 \\
3.14\end{array}$ & $\begin{array}{l}\text { NA } \\
\text { NA } \\
\text { NA } \\
\text { NA } \\
\text { NA }\end{array}$ & $\begin{array}{l}1.32 \\
1.48 \\
1.81 \\
2.27 \\
2.89\end{array}$ \\
\hline 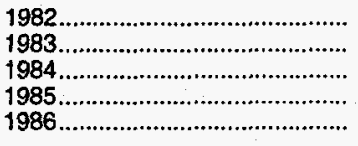 & $\begin{array}{l}5.17 \\
6.06 \\
6.12 \\
6.12 \\
5.83\end{array}$ & $\begin{array}{l}4.82 \\
5.59 \\
5.55 \\
5.50 \\
5.08\end{array}$ & $\begin{array}{l}3.87 \\
4.18 \\
4.22 \\
3.95 \\
3.23\end{array}$ & $\begin{array}{l}\text { NA } \\
\text { NA } \\
\text { NA } \\
\text { NA } \\
\text { NA }\end{array}$ & $\begin{array}{l}3.48 \\
3.58 \\
3.70 \\
3.55 \\
2.43\end{array}$ \\
\hline 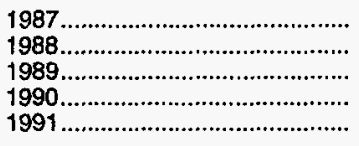 & $\begin{array}{l}5.54 \\
5.47 \\
5.64 \\
5.80 \\
5.82\end{array}$ & $\begin{array}{l}4.77 \\
4.63 \\
4.74 \\
4.83 \\
4.81\end{array}$ & $\begin{array}{l}2.94 \\
2.95 \\
2.96 \\
2.93 \\
2.69\end{array}$ & $\begin{array}{r}\text { NA } \\
\text { NA } \\
\text { NA } \\
3.39 \\
3.96\end{array}$ & $\begin{array}{l}2.32 \\
2.33 \\
2.43 \\
2.38 \\
2.18\end{array}$ \\
\hline 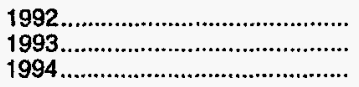 & $\begin{array}{l}5.89 \\
6.16 \\
6.41\end{array}$ & $\begin{array}{r}4.88 \\
\mathrm{R}_{5.22} \\
5.44\end{array}$ & $\begin{array}{r}2.84 \\
R_{3.07} \\
3.05\end{array}$ & $\begin{array}{l}4.05 \\
4.27 \\
4.13\end{array}$ & $\begin{array}{l}2.36 \\
2.61 \\
2.28\end{array}$ \\
\hline
\end{tabular}

$R$ = Revised data.

NA $=$ Not Available.

Notes: Beginning in 1987, prices for deliveries to consumers are calculated using only on-system sales data. Due to large amounts of missing data for values of lease and plant fuel since 1987, prices are not estimated. In previous years, imputations were made for missing data and a price was calculated using reported and imputed data to derive a total value for gas consumed. Since total consumption value estimates were not made since 1987, no lease and plant fuel value estimate was made.

Sources: Electric Utilities: 1967-1977: Federal Power Commission (FPC). 1978-1994: Energy Information Administration (EIA), Form EIA-759, "Monthly Power Plant

Report"; Federal Energy Regulatory Commission (FERC), Form FERC-423, "Cost and Quality of Fuels for Electric Utility Plants"; and Form EIA-176, "Annual Report of Natural

and Supplemental Gas Supply and Disposition." All other data: 1967-1975: Bureau of Mines, Minerals Yearbook, "Natural Gas" chapter. 1976-1978: Energy information Administration. Energy Data Repor, Natural Gas Annual. 1979: Energy Information Administration, Natural Gas Production and Consumption, 1979. 1980-1994: Energy Information Administration (EIA), Form EIA-176, "Annual Report of Natural and Supplemental Gas Supply and Disposition." 
Appendix A

Summary of Data

Collection

Operations and

Report

Methodology 



\section{Summary of Data Collection Operations and Report Methodology}

The 1994 data for the Natural Gas Annual are taken primarily from Form EIA-176, "Annual Report of Natural and Supplemental Gas Supply and Disposition" and Form EIA627, "Annual Quantity and Value of Natural Gas Report." Each of these surveys and all other sources of data for this report are discussed separately in the following sections.

\section{Form EIA-176}

\section{Survey Design}

The original version of Form EIA-176 was approved in 1980 with a mandatory response requirement. Prior to 1980 , published data were based on voluntary responses to Bureau of Mines, U.S. Department of the Interior predecessor Forms BOM-6-1340-A and BOM-6-1341-A of the same title.

In 1982, the scope of the revised EIA-176 survey was expanded to collect the number of electric utility consumers in each State, volumes of gas transported to industrial and electric utility consumers, detailed information on volumes transported across State borders by the respondent for others and for the responding company, and detailed information on other disposition. These changes were incorporated to provide more complete survey information with a minimal change in respondent burden. The 1982 revision of the Form EIA- 176 continues to be the basis for the current version of this form.

In 1988, the Form EIA-176 was revised to include data collection for deliveries of natural gas to commercial consumers for the account of others. The revised form was approved for use during report years 1987 through 1989. A short version of Form EIA-176 was also approved in 1988. Companies engaged in purchase and delivery activities but not in transportation and storage activities may file the short form. Usually, these companies are municipals handling small volumes of gas.

In 1990, the Form EIA-176 was revised to include more detailed information for gas withdrawn from storage facilities, gas added to storage facilities, deliveries of companyowned natural gas and natural gas transported for the account of others. The revised form was approved for use beginning with report year 1990 .
Upon the Office of Management and Budget's approval in 1993, the Form EIA-176 was revised. All deliveries to consumers are now categorized as firm or interruptible. Commercial and industrial consumers are further categorized as nonutility power producers or as those excluding nonutility power producers.

Data reported on this form are no longer considered proprietary. Response to the form continues to be mandatory.

In February 1995, forms for report year 1994 were mailed to all identified interstate natural gas pipeline companies; intrastate natural gas pipeline companies; investor and municipally owned natural gas distributors; underground natural gas storage operators; synthetic natural gas plant operators; field, well, or processing plant operators that deliver natural gas directly to consumers (including their own industrial facilities) other than for lease or plant use or processing; and field, well, or processing-plant operators that transport gas to, across, or from a State border through field or gathering facilities. Detailed instructions for completing the form were included in each survey package. Completed forms were returned to the Data Operations Branch of the Reserves and Natural Gas Division, where each was checked for errors, corrected as necessary, and processed into computer-generated State and national data summaries.

\section{Response Statistics}

Each company and its parent company or subsidiaries were required to file if they met the survey specifications. The original mailing totaled 2,050 questionnaire packages. To this original mailing, 23 names were added and 97 were deleted as a result of the survey processing. Additions were the result of comparisons of the mailing list to other survey mailing lists. Deletions resulted from post office returns and determinations that companies were out of business, sold, or not within the scope of the survey. After all updates, the survey universe was 1,976 responses from approximately 1,800 companies.

Following the original mailing, second request mailing, and nonrespondents follow-up, 1,962 responses were entered into the data base. There were fourteen nonrespondents. 


\section{Summary of Form EIA-176 Data Reporting Re- quirements}

The Form EIA-176 is a five-page form consisting of seven parts. Part I of the form contains identifying information including the company identification number, the company name and address, the State for which the report is filed, and address correction information. Part II contains certification information. The body of the form (Parts III-VII) is a multiline schedule for reporting all supplies of natural gas and supplemental gaseous fuels and their disposition within the State indicated. Respondents filed completed forms with the EIA in Washington, D.C. Data for the year 1994 were due April 3, 1995.

Computer edit programs verified the report year, State code, and arithmetic totals. Further tests were made to ensure that all necessary data elements were present and that the data were reasonable and internally consistent. The computerized edit system produced error listingsi with messages for each failed edit test. To resolve problems, respondents were contacted by telephone and were required to file amended forms with corrected data.

All natural gas and supplemental gaseous fuels volumes were reported on a physical custody basis in thousand cubic feet, and dollar values were reported to the nearest whole dollar. All volumes were reported at 14.73 pounds per square inch absolute pressure and 60 degrees Fahrenheit. Other minor report standards were specified in the instructions booklet to assure that the filed data were consistent and could be readily processed.

\section{Comparison of the Form EIA-176 with Other Data Sources}

Comparison of the EIA-176 data with data from similar series is another method of ensuring the validity of the data published in this report. This comparison on a companyby-company basis showed significant differences that respondents were required to reconcile.

Data on imports and exports of natural gas, as collected by the EIA-176 survey, were checked by comparing individual responses with the mandatory submissions of Form FPC14, "Annual Report for Importers and Exporters of Natural Gas." Where discrepancies were noted, respondents were required to file corrected reports.

Similarly, data on the underground storage of natural gas were compared with submissions of Form EIA-191, "Underground Gas Storage Report." If significant differences were noted, companies were contacted to reconcile the discrepancies. During 1994, the 103 companies filing the Form EIA-191 reported total injections of 2,726 billion cubic feet and total withdrawals of 2,408 billion cubic feet. This compares to 2,796 billion cubic feet of injections and 2,491 billion cubic feet of withdrawals, as reported on the Form EIA-176.
Data on deliveries to residential, commercial, and industrial consumers were compared with data submitted on Form EIA-857, "Monthly Report of Natural Gas Purchases and Deliveries to Consumers." Where discrepancies were noted, respondents were required to file corrected reports for either and sometimes both surveys. Numerous telephone calls were made to clarify any misunderstandings concerning the correct filing of both forms. Typical errors included electric utility volumes combined with industrial volumes, sale for resale volumes reported as industrial consumption, cogeneration volumes not reported on Form EIA-857, and misinterpretation of general instructions.

A discussion of the comparison of the data on deliveries to electric utilities filed on Form EIA-176 and that reported in the EIA publication, Electric Power Annual, is included in this Appendix under "Electric Utility Data."

\section{Routine Form EIA-176 Edit Checks}

A series of manual and computerized edit checks were used to screen the Form EIA-176. The edits performed included validity, arithmetic, and analytical checks. A computerized check was also made for consistency with previous filings.

The incoming forms for the survey were reviewed prior to keying. This prescan determined if the respondent identification (ID) number and the company name and address were correct, if the data on the form appeared complete and reasonable, and if the certifying information was complete.

Manual checks on the data were also made. Each form was prescanned to determine that data were reported on the correct lines. The flow of gas through interstate pipelines was checked at the company level to assure that each delivery from a State was matched with a corresponding receipt in an adjoining State.

\section{Form EIA-627}

\section{Survey Design}

Beginning with 1980 data, natural gas production data previously obtained on an informal basis from appropriate State agencies were collected on Form EIA-627 (Figure A3). This form was designed by the EIA to collect annual natural gas production data from the appropriate State agencies under a standard data reporting system within the limits imposed by the diversity of data collection systems of the various producing States. It was also designed to avoid duplication of the efforts involved in the collection of production and value data by producing States and to avoid an unnecessary respondent burden on gas and oil well operators. 
Figure A1. Form EIA-176

EIA-176 (Revised 1993)

U.S. DEPARTMENT OF ENERGY

Energy Information Administration Washington DC 20585
Form Approved OMB No. 19050175 Expires 12/31/96

\section{ANNUAL REPORT OF NATURAL AND SUPPLEMENTAL GAS SUPPLY AND DISPOSITION, 19}

This repon is mandatory under the Federal Energy Adm inistration Act of 1974 (Public Law 93-275). For the provisions concerning the confidentiality of information and sanctions statement, see Sections VI and VI of the instructions. Public reporting burden for this collection of inform ation is estimated to average 20.8 hours per response, including the time of reviewing instructions, searching existing data sources, gathering and maintaining the data needed, and completing and reviewing the collection of information. Send comments regarding this burden estimate or any other aspect of this collection of information including, suggestions for reducing this burden, to the Energy information Administration, Otfice of Statistical

Standards, 1000 Independence Ave. SW, Washington, DC 20585; and to the Office of information and Regulatory Aftairs, Office of Managem ent and Budget, Washington, DC 20503.

\section{EIA USE}

RESPONDENT COPY.

Retain for your files.

\section{Affix mailing label or enter mail address}

Control (ID) No.

Name:

Operations in (State):

Street or Post Office Box:

City, State, Zip Code:

Attention: .

\section{CATION}

2.0 Company Name

3.0 Report State

\begin{tabular}{c|c|c} 
EIA & 4.0 Resubmitta \\
\hline & & Date
\end{tabular}

\section{Date}

Name and address on mailing label are correct.

Change name, attention line, and/or mail address as indicated below.

Company was sold to, or merged with, company entered below.

Company went out of business. Customer accounts taken over by company entered below.

Other changes, corrections, or cormments:

5.1 Change company name and/or address to:

a. Company Name: . . . . . . . . . . . . . . . . . . . .

b. Operations in (State): . . . . . . . . . . . . . . .

c. Street or Post Office Box:

d. City, State, Zip Code:

e. Attention:
6.0 Contact person:

Name:

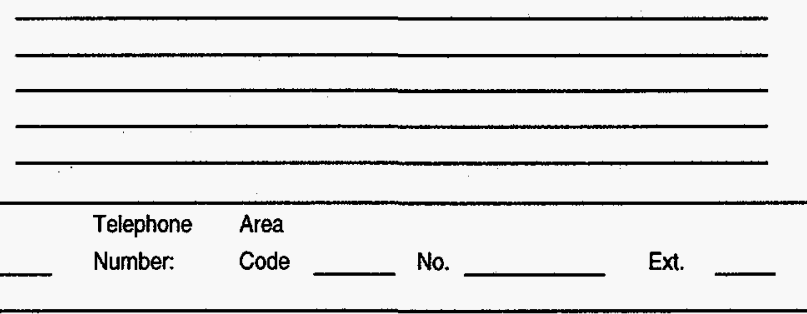

PART Il: CERTIFICATION AND DISCLOSURE STATEMENT

1.0 I certify that (Check appropriate box):

a. The information proviced herein and appended hereto is true and accurate or, where indicated on the form, are reasonable estimates to the best of my knowledge.

b. My company does not meet any of the criteria set forth in Section II, "Who must report," of the instructions and is therefore not required to complete and submit a Form EIA-176 for the report State.

\begin{tabular}{|l|l|}
\hline 2.0 Name & 3.0 Title \\
\hline 4.0 Signature & 5.0 Date \\
\hline
\end{tabular}

Title 18, USC 1001, makes it a crime for any person knowingly and willingly to make to any agency or department of the United States any false, fictitious or fraudulent statements as to any matter within its jurisdiction. 
EIA-176, ANNUAL REPORT OF NATURAL AND SUPPLEMENTAL GAS SUPPLY AND DISPOSITION, 19

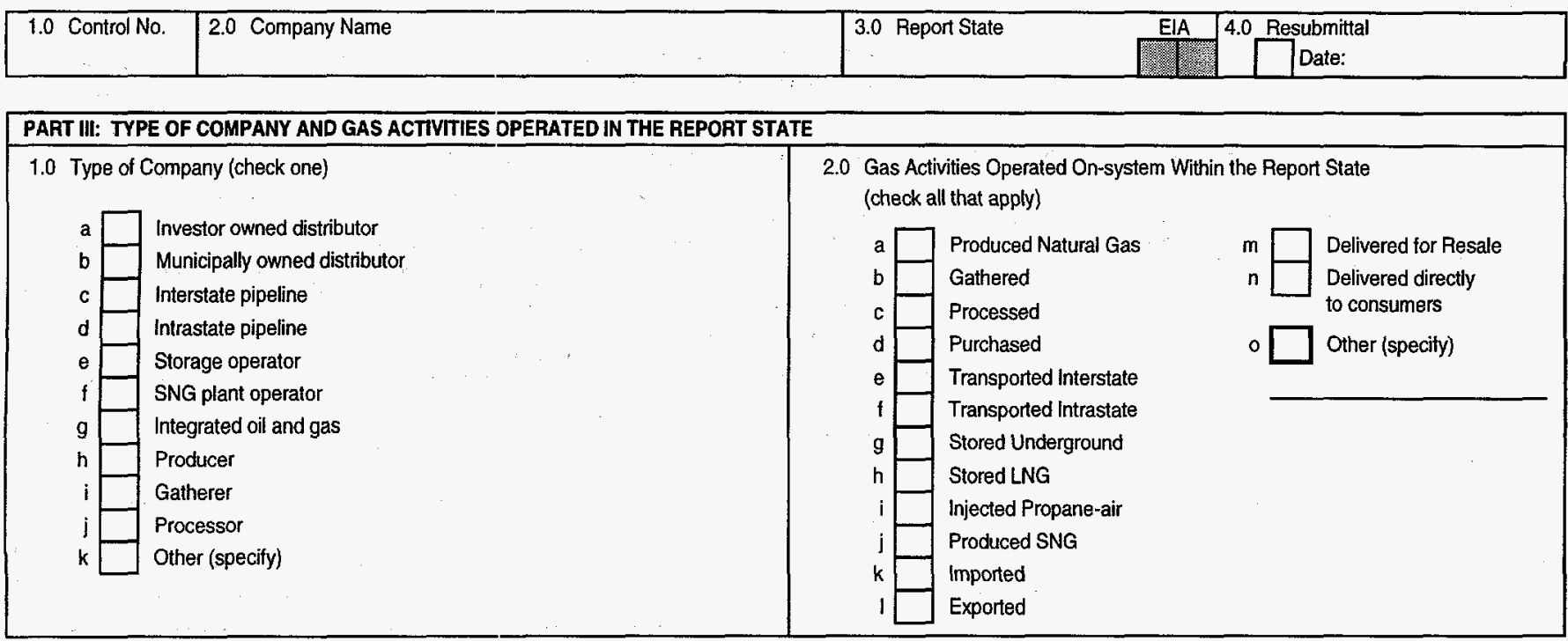

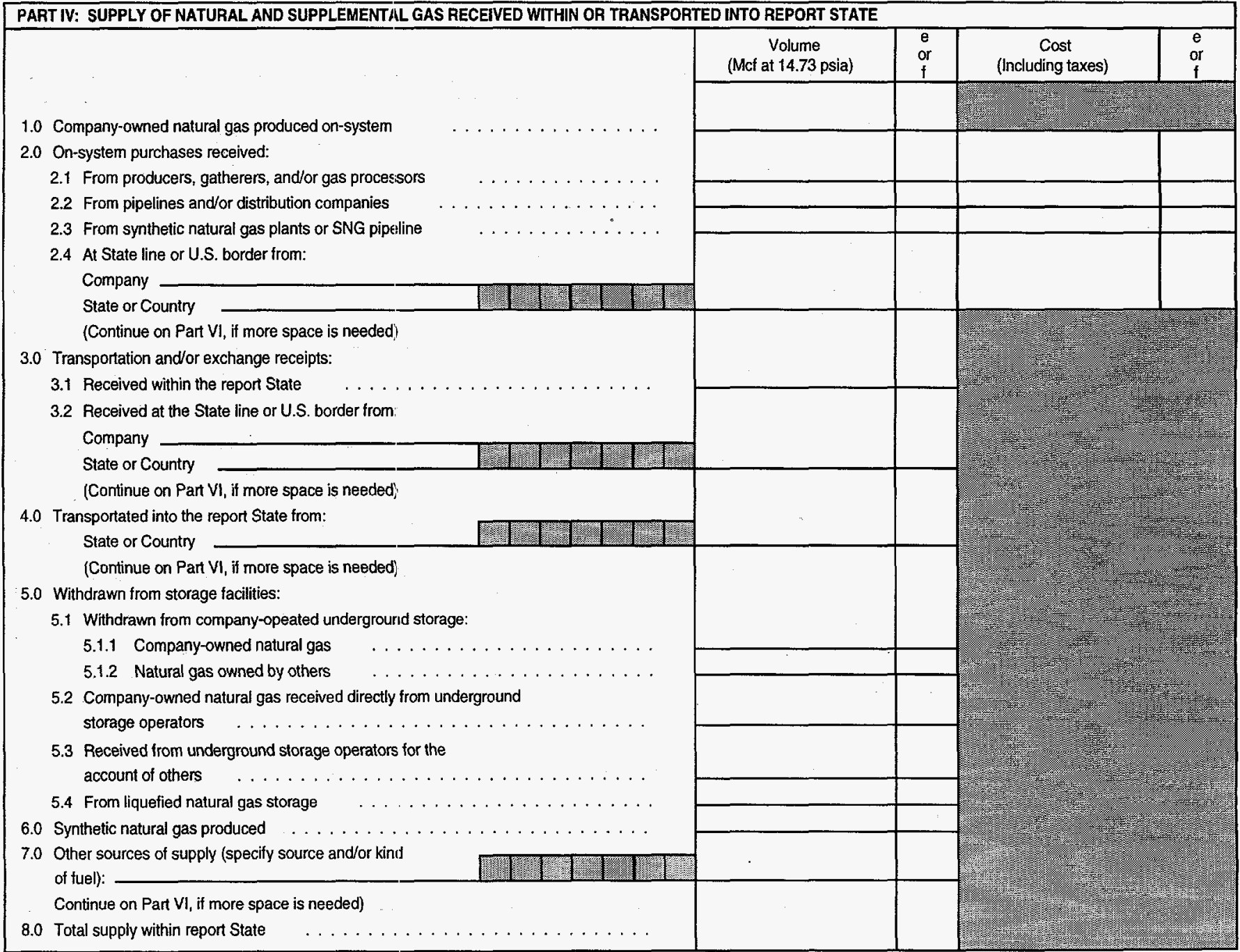


EIA-176, ANNUAL REPORT OF NATURAL AND SUPPLEMENTAL GAS SUPPLY AND DISPOSITION, 19

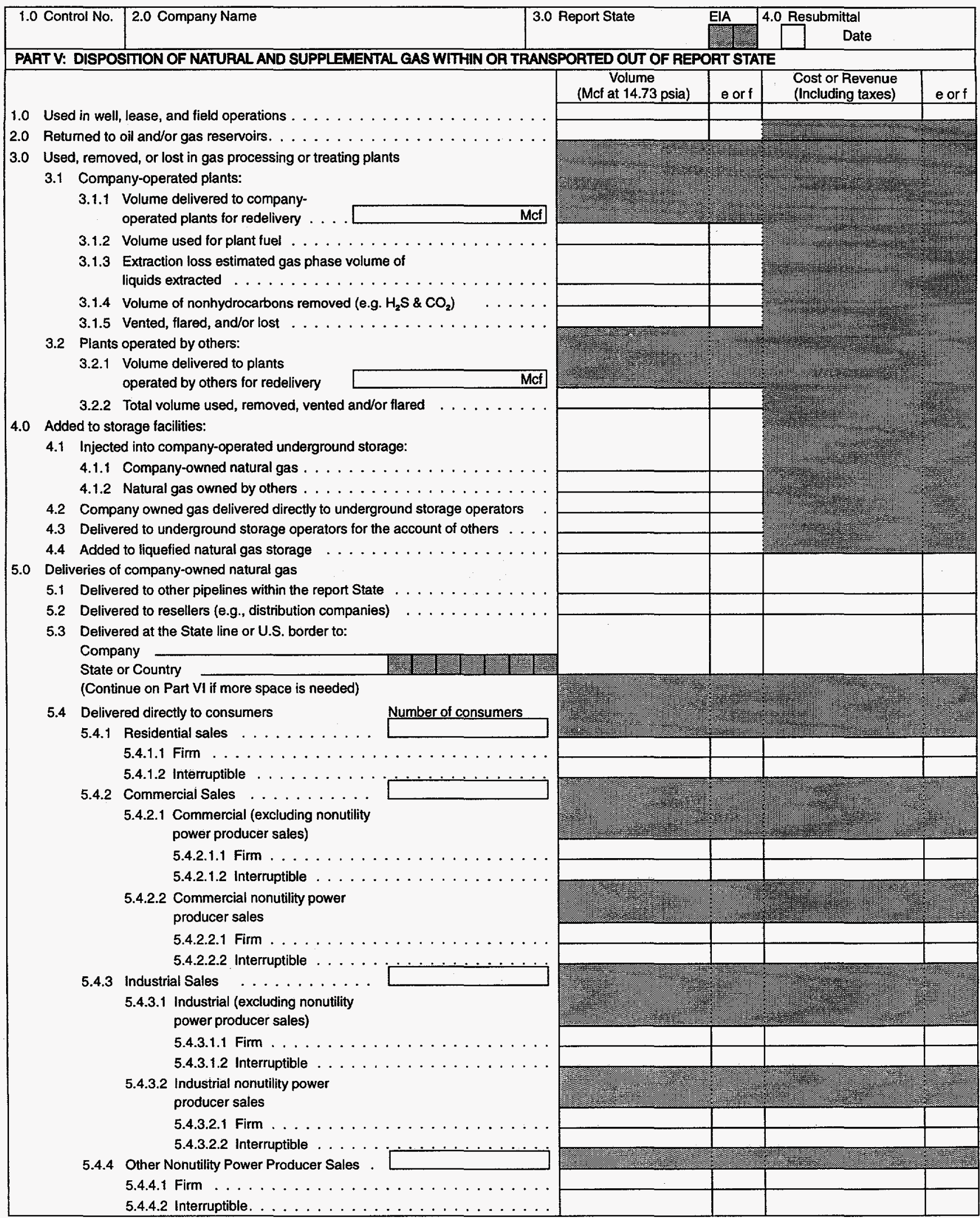


EIA-176, ANNUAL REPORT OF NATURAL AND SUPPLEMENTAL GAS SUPPLY AND DISPOSITION, 19

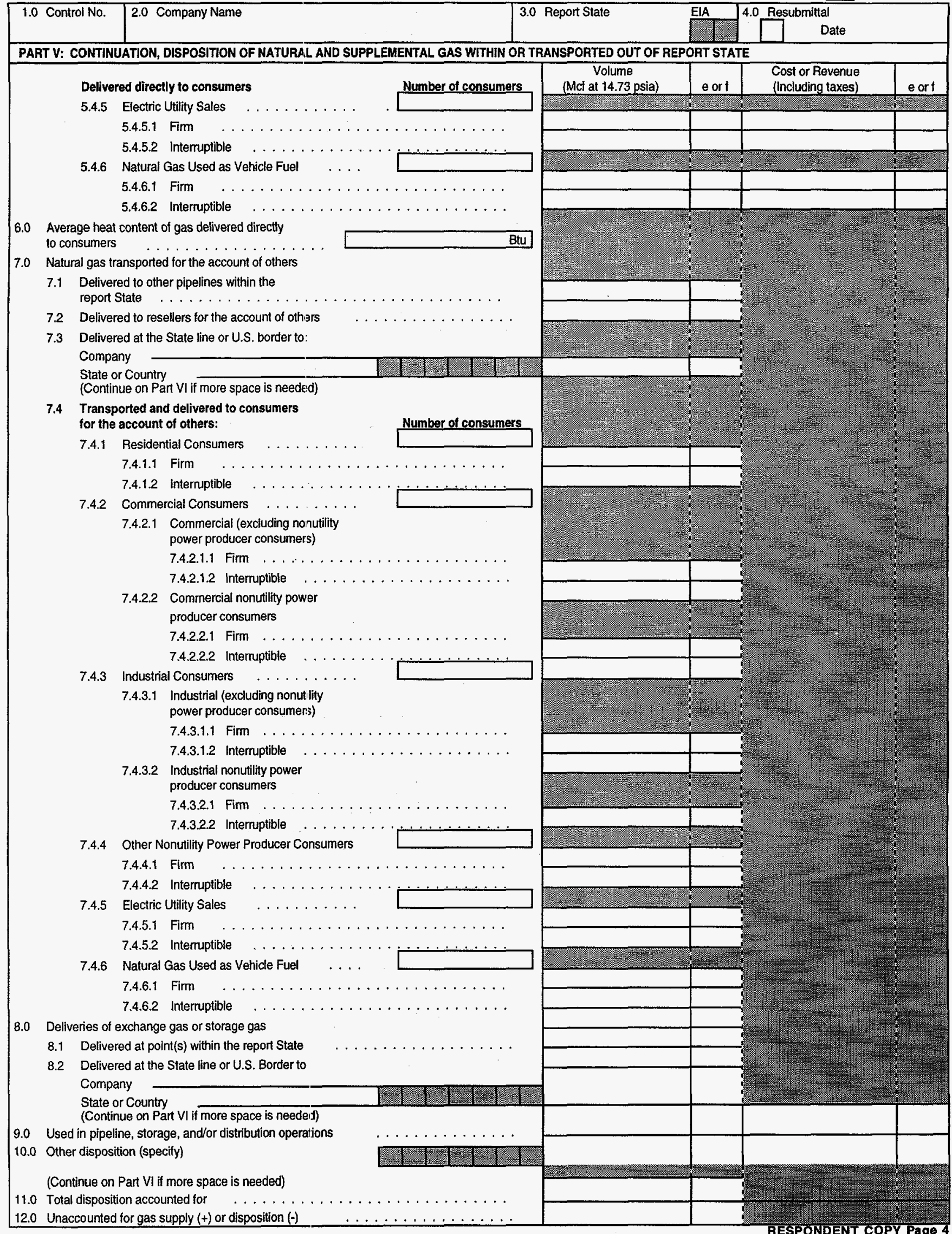


EIA-176, ANNUAL REPORT OF NATURAL AND SUPPLEMENTAL GAS SUPPLY AND DISPOSITION, 19

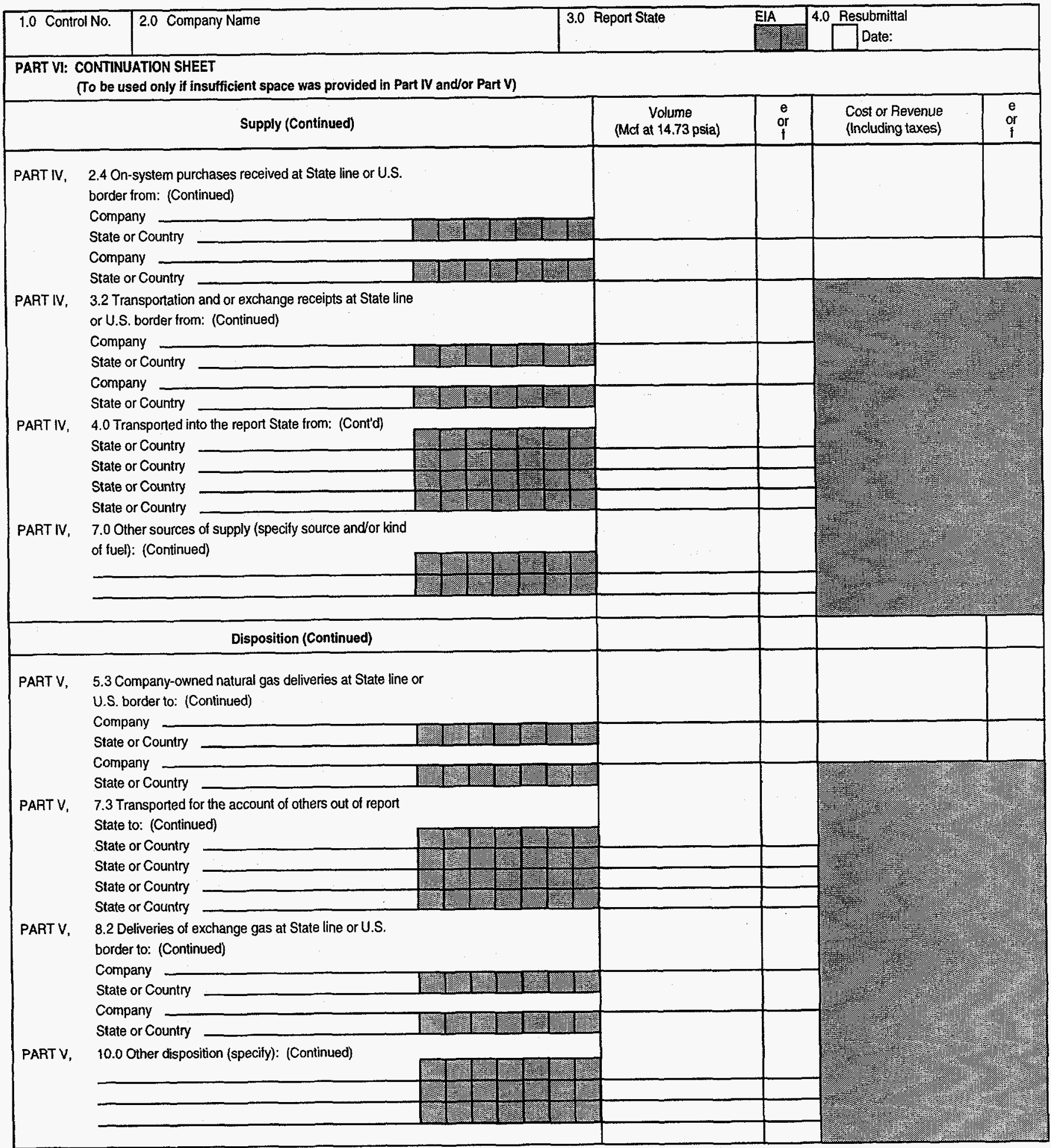


EIA-176, ANNUAL REPORT OF NATURAL AND SUPPLEMENTAL GAS SUPPLY AND DISPOSITION, 19

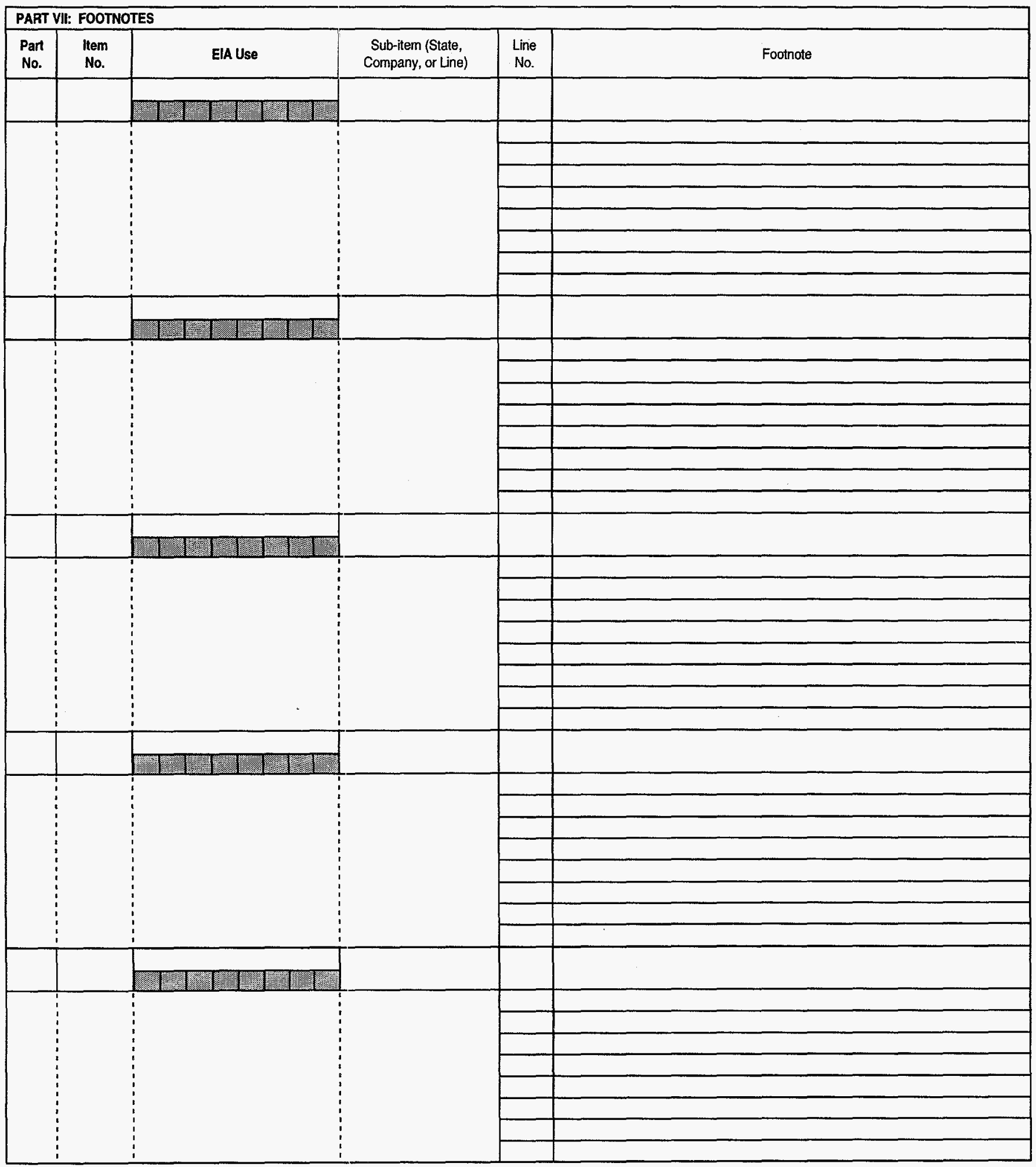


Figure A2. Form EIA-176, Short Form

EIA-176 (Revised 1993)

U.S. DEPARTMENT OF ENERGY

Energy Information Administration

Washington DC 20585

Form Approved

OMB No. 19050175

Expires: 12/31/96

SHORT

FORM

\section{ANNUAL REPORT OF NATURAL AND SUPPLEMENTAL GAS SUPPLY AND DISPOSITION, 19}

This report is mandatory under the Federal Energy Adm in istration Act of 1974 (Public Law 93-275). For the provisions concerning the confidentiality of information and sanctions statement, see Sections VI and VII of the instructions. Public reporting burden for this collection of information is estimated to average 20.8 hours per response, including the time of reviewing instructions, searching existing data sources, gathering and maintaining the data needed, and completing and reviewing the collection of information. Send comm ents regarding this burden estimate or any other aspect of this collection of information including, suggestions for reducing this burden, to the Energy Information Administration, Office of Statistical

Standards, 1000 independence Ave. SW, Washington, DC 20585; and to the Office of information and Regulatory Affairs, Otfice of Management and Budget, Washington, DC 20503.

\section{EIA USE}

RESPONDENT COPY.

Retain for your files.

Control (ID) No.

Name:

Operations in (State):

Street or Post Office Box:

City, State, Zip Code:

Attention:

PART I: IDENTIFICATION

\begin{tabular}{|c|c|c|c|c|}
\hline 1.0 Control No. & 2.0 Company Name & 3.0 Report State & EIA & 4.0 Resubmitta \\
\hline
\end{tabular}

5.0 Company status, name, and/or address change or correction. (Check appropriate box)

a. Name and address on mailing label are correct.

b. Change name, attention line, and/or mail address as indicated below.

c. Company was sold to, or merged with, company entered below.

d. Company went out of business. Customer accounts taken over by company entered below.

e. Other changes, corrections, or comments:

5.1 Change company name and/or address to:
a. Company Name: . . . . . . . . . . . . . . . . . . . .
b. Operations in (State): . . . . . . . . . . . . .
c. Street or Post Office Box: ................
d. City, State, Zip Code: . . . . . . . . . . . . . . . . . .
e. Attention: . . . . . . . . . . . . . . . . .

6.0 Contact person:

Name:

Telephone Area

Number: Code

No.

Ext.

PART II: CERTIFICATION AND DISCLOSURE STATEMENT

1.0 I certify that (Check appropriate box):

a. The information proviced herein and appended hereto is true and accurate or, where indicated on the form, are reasonable estimates to the best of my knowledge.

b. My company does not meet any of the criteria set forth in Section II, "Who must report, " of the instructions and is therefore not required to complete and submit a Form EIA-176 tor the report State.

\begin{tabular}{|l|l|}
\hline 2.0 Name & 3.0 Title \\
\hline 4.0 Signafure & 5.0 Date \\
\hline
\end{tabular}

Title 18, USC 1001, makes it a crime for any person knowingly and willingly to make to any agency or department of the United States any false, fictitious or fraudulent statements as to any matter within its juris diction. 
EIA-176, ANNUAL REPORT OF NATURAL AND SUPPLEMENTAL GAS SUPPLY AND DISPOSITION, 19

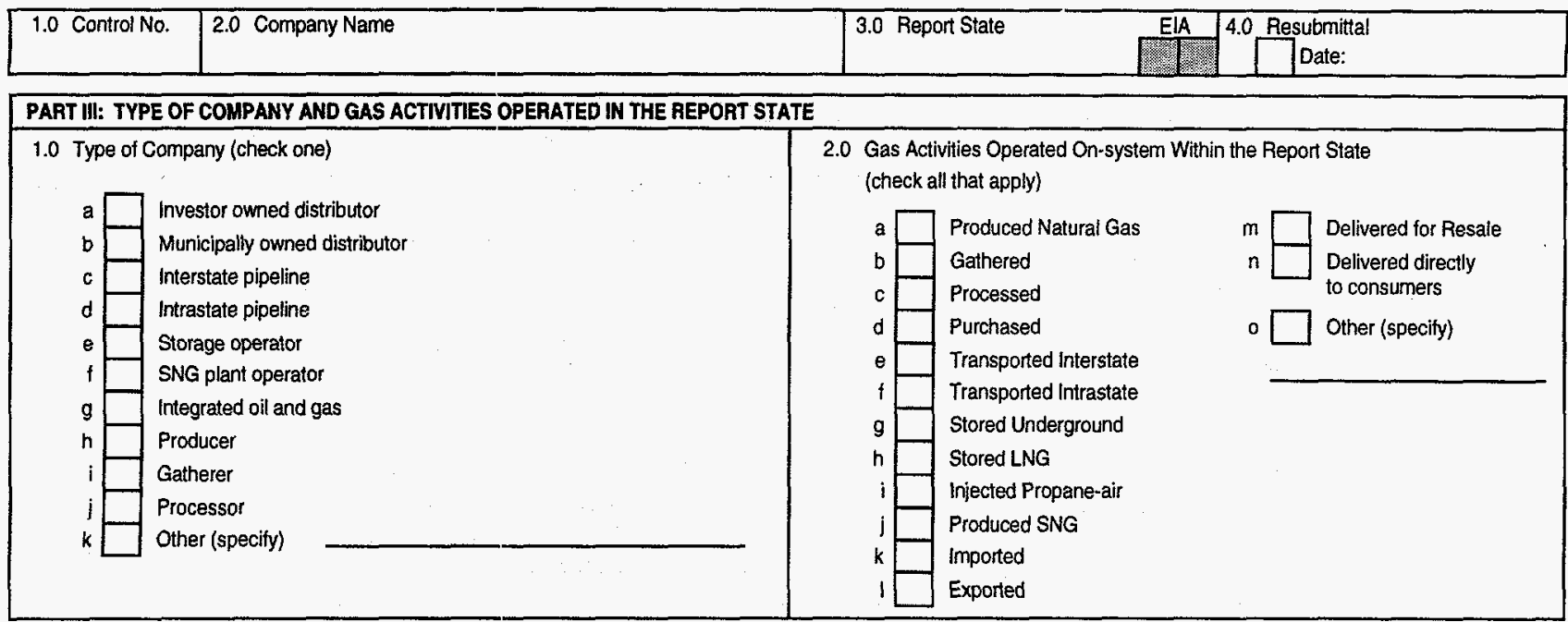

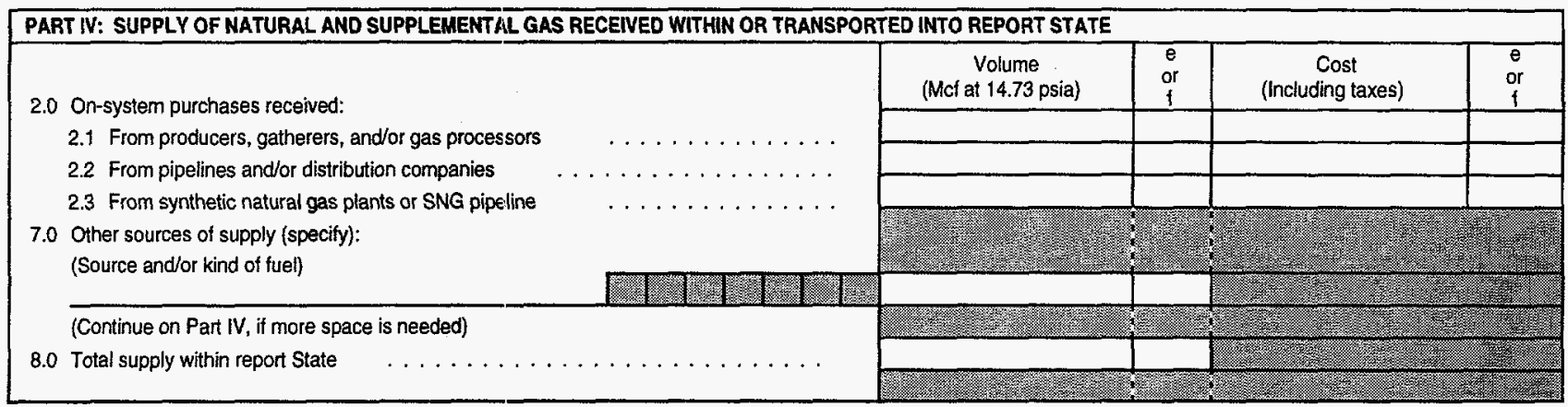

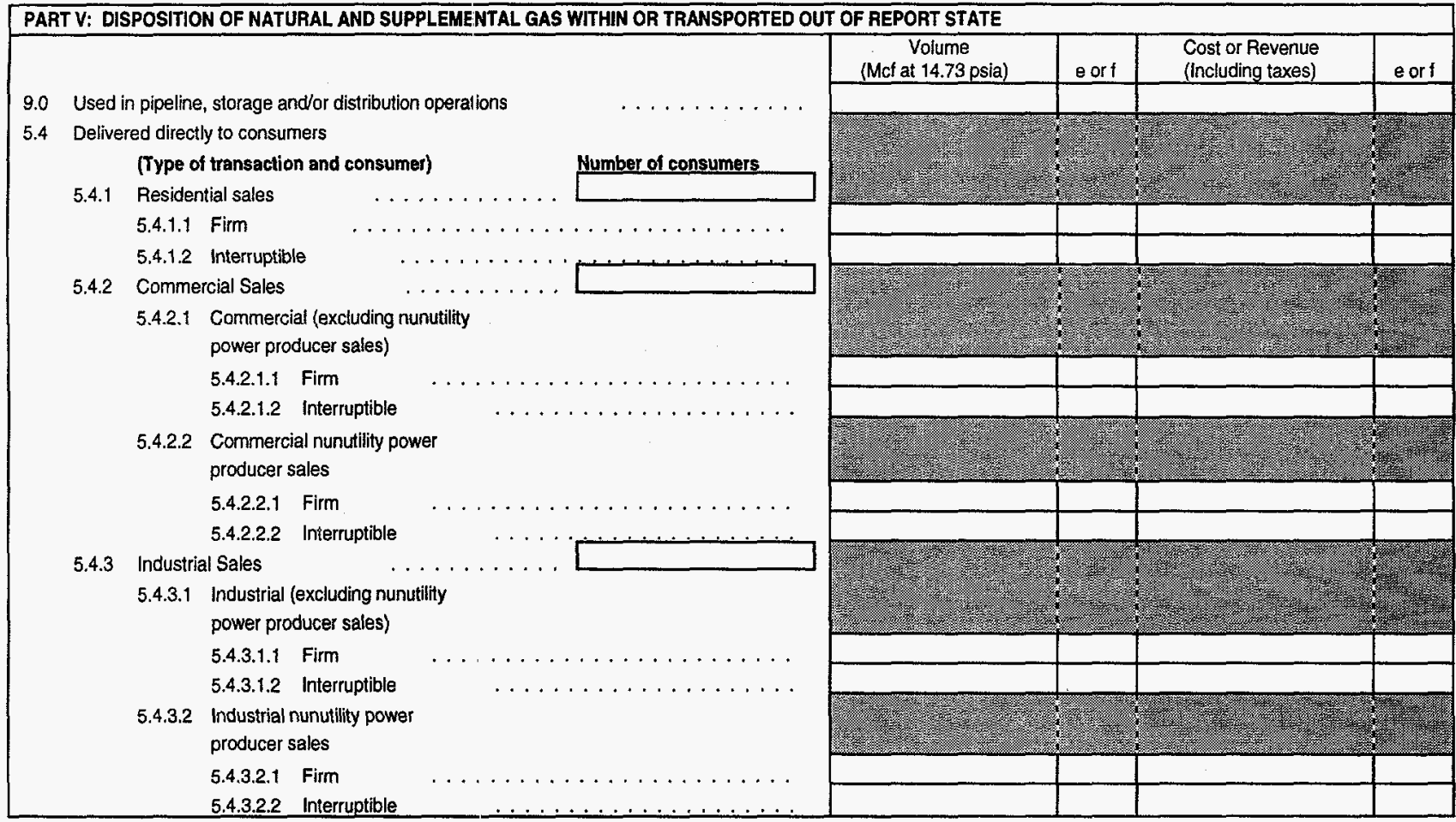


EIA-176, ANNUAL REPORT OF NATURAL AND SUPPLEMENTAL GAS SUPPLY AND DISPOSITION, 19

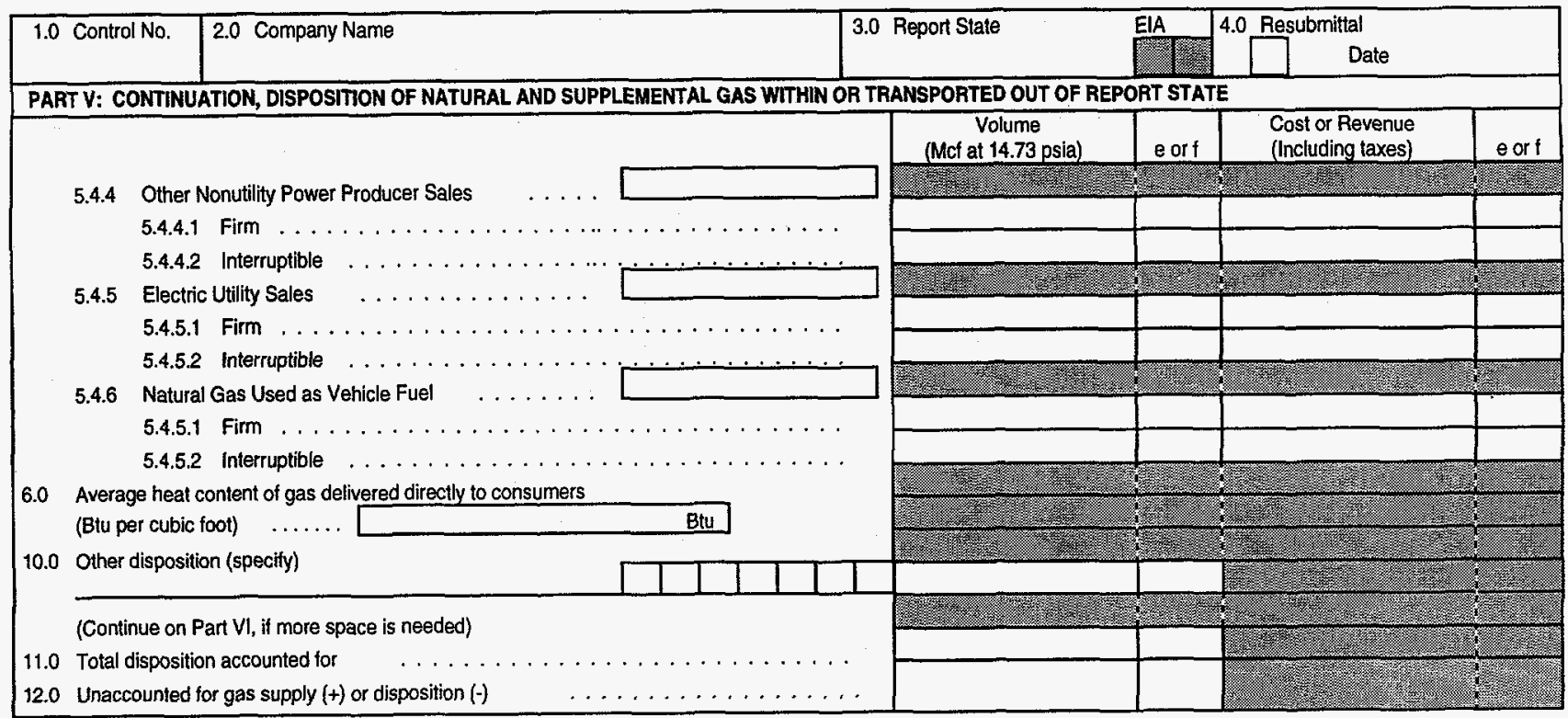


EIA-176, ANNUAL REPORT OF NATURAL AND SUPPLEMENTAL GAS SUPPLY AND DISPOSITION, 19

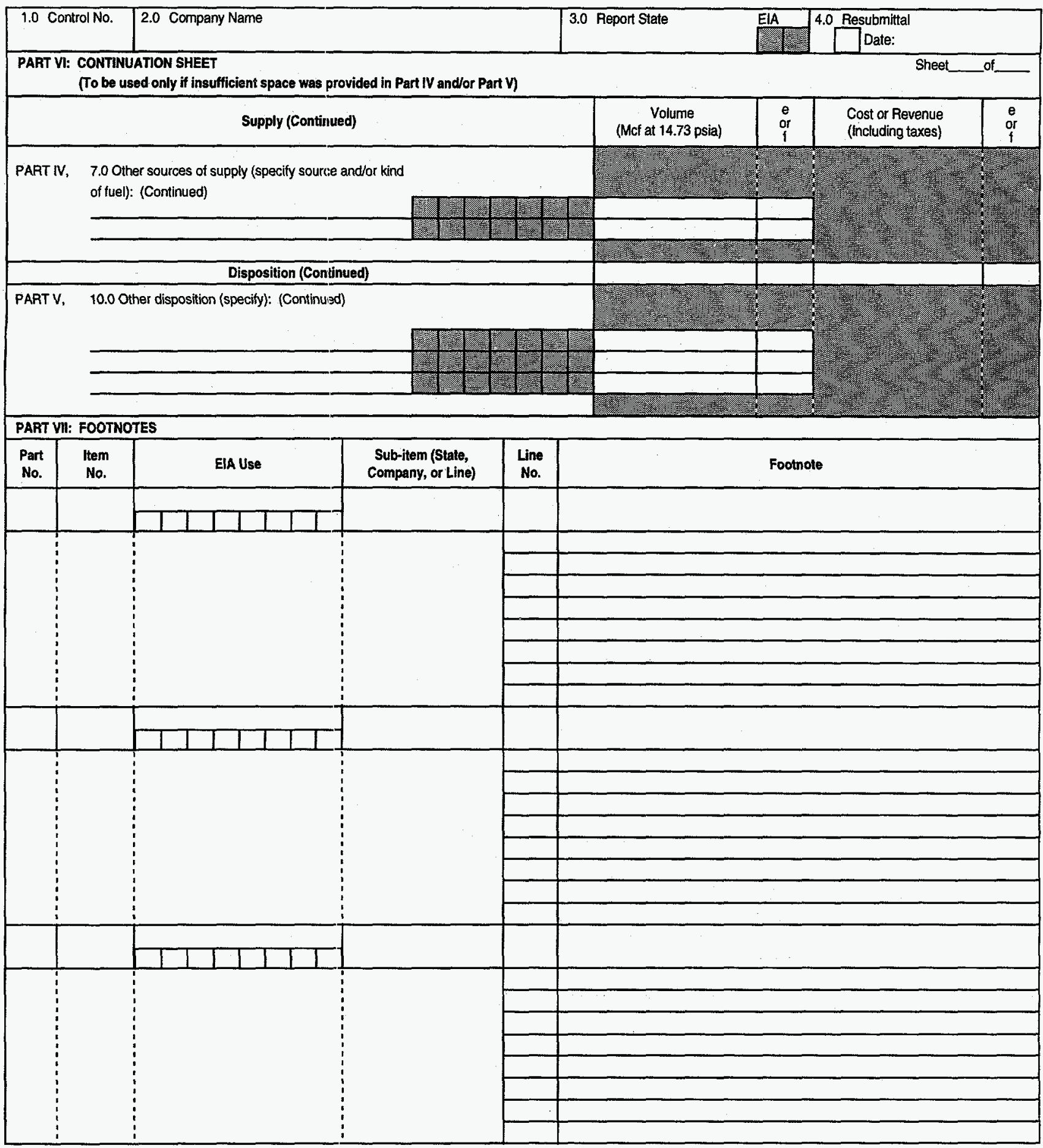


In 1993, the Office of Management and Budget approved the Form EIA-627 for use in report years 1994 through 1996. In June 1995, forms for report year 1994 were mailed to the appropriate agencies in 33 States. Completed forms were returned to the Data Operations Branch of the Reserves and Natural Gas Division for review, processing, and compilation.

\section{Response Statistics}

Of the 33 natural gas producing States, 30 participated in the voluntary EIA- 627 survey by filing the completed form or by responding to telephone contacts. Data for the three nonresponding States (Illinois, Pennsylvania, and West Virginia) were estimated. Data on the quantities of nonhydrocarbon gases removed in 1994 were reported by the appropriate agencies of 22 of the 33 producing States. The 22 States accounted for 57 percent of total 1994 gross withdrawals. In addition, the gross withdrawal data from Kansas, Louisiana, Montana, and Oklahoma, which together accounted for 40 percent of total production, excluded all or most of the nonhydrocarbon gases removed on leases. Gross withdrawals from Louisiana excludes most quantities of nonhydrocarbon gases removed on leases. Nonhydrocarbon gases removed have been excluded from gross withdrawals in Missouri.

The commercial recovery of methane from coal beds contribute a significant amount to the production totals in a number of States. Coal bed methane seams production quantities (in million cubic feet) are included in gross withdrawals totals for the following States: Alabama $(110,100)$, Colorado $(178,608)$, and New Mexico $(529,705)$.

\section{Summary of EIA-627 Data Reporting Require- ments}

The Form EIA-627 is a two-page form divided into four parts. Part I requests identifying information including the name and location of the responding State agency and the name and telephone number of a contact person within the agency. Part II collects data on the number of producing gas wells, the production of natural gas including gross withdrawals from both gas and oil wells; volumes returned to formation for repressuring, pressure maintenance, and cycling; quantities vented and flared; quantities of nonhydrocarbon gases removed; quantities of fuel used on lease; and marketed production. Part III of the form is for reporting the value of marketed production. Part IV is space to be used by the respondent to explain data elements reported that may be based on definitions differing from those applied to data in previous years.

\section{Routine Form EIA-627 Edit Checks}

Each filing of the Form EIA-627 is manually checked for reasonableness and mathematical accuracy. Volumes are converted, as necessary, to a standard 14.73 psia pressure base. Value data are compared to the previous year's data for reasonableness. When data on nonhydrocarbon gases removed, gas vented and flared, and gas used for repressuring are not reported for a State that historically reported one or more of these items, a volume is imputed. The imputation is based on the average ratio of gas volumes in the missing category to total gross withdrawals in States with values reporting gas in that category. This average ratio is applied to the volume of total gross withdrawals reported by the State to calculate the volume for the missing items. State agencies are contacted by telephone in order to correct errors. Amended filings or resubmissions are not a requirement, since participation in the survey is voluntary.

\section{Comparison of the Form EIA-627 with Other Data Sources}

Annual production data, as reported on the Form EIA-627, are compared to the sum of monthly data previously reported to the Interstate Oil and Gas Compact Commission (IOGCC). Beginning in January 1995, the data were collected on the Form EIA-895, "Monthly Quantity of Natural Gas Report." The Form EIA-895 was designed in 1994 to replace the discontinued IOGCC form. The comparison is made in order to assure the reasonableness of the data reported on the Form EIA-627. Any significant differences are resolved by contacting the reporting State.

For discussion of the comparison of production data collected on Form EIA-627 and that collected on Form EIA-23, "Annual Survey of Domestic Oil and Gas Reserves," see the EIA report, U.S. Crude Oil, Natural Gas, and Natural Gas Liquids Reserves, 1994 Annual Report.

\section{Electric Utility Data}

The electric utility data published in this report are taken from the Forms EIA-759, "Monthly Power Plant Report" and FERC-423, "Monthly Report of Cost and Quality of Fuels for Electric Plants." These data were used in order to maintain consistency among EIA publications. Electric data are necessary on the Form EIA-176 to provide a supply/disposition balance on the form. Differences in the two surveys are apparent in the results published in Table 15, "Natural Gas Deliveries to Consumers by State," and Table 18, "Natural Gas Delivered to Electric Utilities for the 


\section{ANNUAL QUANTITY AND VALUE OF NATURAL GAS REPORT}

This report is voluntary under Public Law 93-275. For the provisions concerning the confidentiality of intormation and sanctions, see Sections $V$ and VI of the instructions. Public reporting burden for this collection of inform ation is est mated to average 4.7 hours per response, including the time of reviewing instruction, searching existing data sources, gathering and maintaining the data needed, and completing and reviewing the collection of information. Send Independence Avenue, S. W W ashington, DC 20585; and to the Office of Information and Regulatory Aftairs, Office of Management and Budget, Washington, DC 20503.

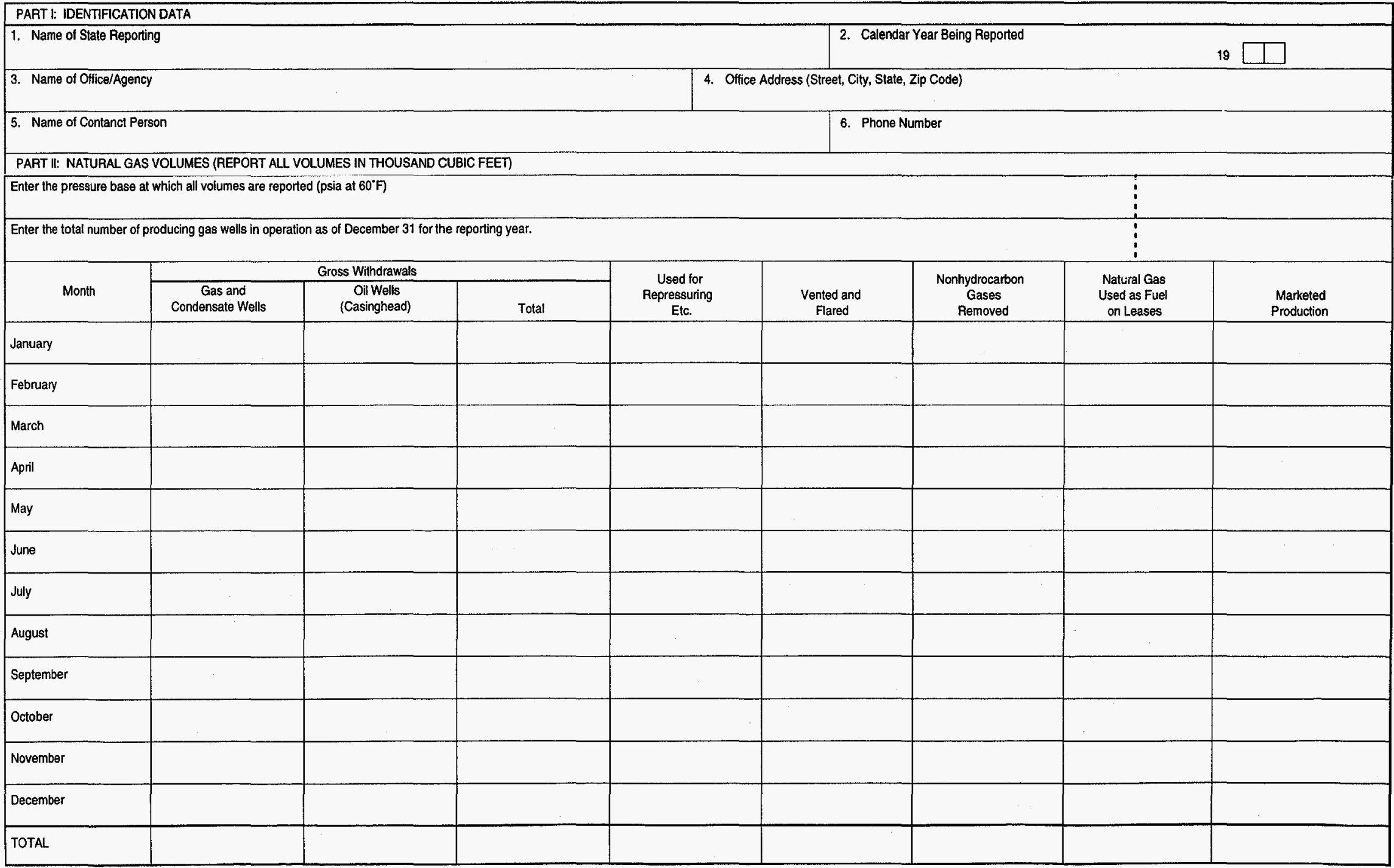

PART II: NATURAL GAS VOLUMES (AEPORT ALL VOLUMES IN THOUSAND CUBBC FEET)

Enter the pressure base at which all volumes are reported (psia at 60. $\mathrm{F}$ )

Enter the total number of producing gas wells in operation as of December 31 for the reporting year. 
EIA-627, ANNUAL QUANTITY AND VALUE OF NATURAL GAS REPORT

PART III: VALUE OF MARKETEO PRODUCTION

The value reported should represent wellhead sales prices, including charges for natural gas plant liquids subsequently removed from the gas and for gathering and compression in addition to State production, severance, and/or similar taxes.

Does your computation of wellhead value agree with the above conditions?

Yes

Complete the table below only if your method of reporting is inconsistent with the above note. For each item, enter an " $X$ " in the appropriate column to indicate whether the item is included or excluded from the amount reported.

\begin{tabular}{|l|l|l|}
\hline \multicolumn{1}{|c|}{ Item } & \multicolumn{1}{|c|}{ Included } & Excluded \\
\hline Natural gas plant liquids & & \\
\hline Lease condensate & & \\
\hline Gathering and compression charges & & \\
\hline State production, severance, and/or similar taxes & & \\
\hline
\end{tabular}

\begin{tabular}{|c|c|c|}
\hline \multirow[b]{2}{*}{ Month } & \multicolumn{2}{|c|}{ Quantity and Volume of Marketed Production by Month } \\
\hline & Volume (thousand cubic feet) & Value (thousand dollars) \\
\hline \multicolumn{3}{|l|}{ January } \\
\hline \multicolumn{3}{|l|}{ February } \\
\hline \multicolumn{3}{|l|}{ March } \\
\hline \multicolumn{3}{|l|}{ April } \\
\hline \multicolumn{3}{|l|}{ May } \\
\hline \multicolumn{3}{|l|}{ June } \\
\hline \multicolumn{3}{|l|}{ July } \\
\hline \multicolumn{3}{|l|}{ August } \\
\hline \multicolumn{3}{|l|}{ September } \\
\hline \multicolumn{3}{|l|}{ October } \\
\hline \multicolumn{3}{|l|}{ November } \\
\hline \multicolumn{3}{|l|}{ December } \\
\hline TOTAL. & & \\
\hline
\end{tabular}

PART IV: COMMENTS

Enter on separate sheet(s) any additional comments including identification and explanation of any data elements submitted based on definitions differing from those provided for the previous year.

Retum completed forms to: $\quad$ Energy Information Administration: El-441

Mail Station: BG-094, Forrestal Building

U.S. Department of Energy

Washington, D.C. 20585
Attn: Form ElA-627 
Account of Others by State," where volumes in Table 18 sometimes exceed volumes in Table 15. A State-by-State comparison of the reported volumes of natural gas, as collected on the Forms EIA-176 and EIA-759 is shown in Table A1. The national totals differ by 196 billion cubic feet or 7 percent in relative terms.

While processing the data reported on the Form EIA-176, the EIA made special efforts to determine the reasons for the differences in reporting of electric utility data on the Forms EIA-176 and EIA-759. Typical instances of misreporting occurred in the reporting of gas clelivered to electric utilities for the account of others. Some companies reported these deliveries under sales for resale. Others reported them under transportation, exchange and/or storage deliveries. A few others reported them under transported to industrials. Companies making mistakes were asked to refile, and new companies were asked to file when they were found making deliveries of gas. Most companies were cooperative, and their refilings and new filings improved the accuracy of the data.

\section{Other Data Sources}

The U.S. Minerals Management Service (USMMS) supplied data on the quantity and value of natural gas production and the number of producing wells in the Gulf of Mexico Outer Continental Shelf. Monthly reports to the IOGCC were the source of information for gross withdrawals from gas and oil wells in Wyoming. Volumes of extraction losses were reported on Form EIA-64A, "Annual Report of the Origin of Natural Gas Liquids Production." Heat (Btu) content extraction loss was estimated from data reported on Form EIA-64A and Form EIA-816, "Monthly Natural Gas Liquids Report." Volumes and prices of natural gas imports and exports were reported on the Form FPC-14, "Annual Report for Importers and Exporters of Natural Gas." These data are nonproprietary and are filed annually by each individual or organization having authorization to import and export natural gas.

\section{Report Methodology}

\section{Natural Gas Consumed as a Vehicle Fuel}

Data on deliveries of natural gas delivered for use as a vehicle fuel were collected for the first time in 1990. In 1990 and 1991 deliveries of natural gas for vehicle fuel use were included with volumes delivered to commercial consumers. Beginning with the Natural Gas Annual 1992, vehicle fuel volumes are no longer included with cornmercial volumes.

\section{Natural Gas Balancing Item}

The natural gas balancing item represents the difference between the sum of the components of natural gas supply and the sum of the components of natural gas disposition. It is calculated for each State as the result of a comparison between total reported supply and total reported disposition (Table 2). In the formula used, total reported supply is the sum of marketed production, net interstate movements, net movements across U.S. borders, and supplemental gaseous fuels supply. Total reported disposition is the sum of extraction loss, net storage changes (net additions to storage), and consumption. When this calculation results in a negative quantity for the balancing item it represents an excess of reported supply in relation to reported disposition, and positive quantities indicate the opposite situation.

The differences between supply and demand represent quantities lost, the net result of gas company conversions of flow data metered at varying temperature and pressure conditions to a standard temperature and pressure base, metering inaccuracies, the effect of variations in company accounting and billing practices, differences between billing cycle and calendar-period time frames, and imbalances resulting from EIA's merger of data reporting systems, which vary in scope, format, definitions, and type of respondents. The balancing items in individual States may also reflect the underreporting on Form EIA-176 of gas transported across State borders for the account of others by some interstate pipelines.

\section{Natural Gas Processed and Extraction Loss}

Extraction loss is the reduction in the volume of natural gas available for disposition resulting from the removal of natural gas liquid constituents at natural gas processing plants. It represents that portion of the "raw" gas stream that is transferred from the natural gas supply chain to the petroleum and natural gas liquids supply chain. Extraction loss does not include the reduction in volume resulting from the removal of nonhydrocarbon constituents or gas used as fuel, vented, flared, or otherwise disposed of within natural gas processing plants. Extraction loss also results in a reduction in the total heat (Btu) content of the natural gas stream equal to the heat content of the liquids extracted.

The Form EIA-64A, "Annual Report of the Origin of Natural Gas Liquids Production," collects data on the volume of natural gas received for processing, the total quantity of natural gas liquids produced, and the resulting shrinkage (defined as extraction loss in this report) from all natural gas processing- and cycling-plant operators. The quantity of natural gas received and liquids produced are reported by State of origin of the natural gas. Shrinkage volumes are calculated and reported by plant operators based upon the 
chemical composition of the liquids extracted using standard conversion factors specified in the form instructions. A description of the Form EIA-64A survey is presented in the EIA publication, U.S. Crude Oil, Natural Gas, and Natural Gas Liquids Reserves, 1994 Annual Report.

The heat (Btu) content of liquids extracted is not reported on the Form EIA-64A. Therefore, in order to estimate the extraction loss heat content, data reported on the Form EIA-816, "Monthly Natural Gas Liquids Report," were used to determine the individual products contained in the total liquids reported on Form EIA-64A. A description of the Form EIA-816 survey is presented in the EIA publication, Petroleum Supply Annual 1994, Volume II.

To estimate the quantities of individual products extracted in each State, data from the Form EIA-64A survey were used to determine the total liquids production, and data from the Form EIA-816 survey were used to estimate the quantities of the individual products contained in those total liquids.

The Form EIA-816 captures information on the quantity of individual components (i.e., ethane, propane, normal butane, isobutane, and pentanes plus) produced or contained in mixes of plant liquids as determined by chemical analysis. The volumetric ratios of the individual components to the total liquids, as calculated from the 12 monthly Form EIA816 reports for each State, were applied to the annual total liquids production, as reported on the Form EIA-64A, to estimate the quantities of individual components removed at gas-processing plants (Table A4).

The heat (Btu) content of extracted liquids was estimated by applying conversion factors to the estimated quantities of products extracted in each State. These conversion factors, in million Btu per barrel of liquid produced, were ethane, 3.082; propane, 3.836; normal butane, 4.326; isobutane, 3.974; and pentane plus, 4.620. It should be noted that, at the State level, extraction losses are not necessarily related to State production. All gas processed in 11 States originated, or was produced in those States; but part of the gas processed in the other 12 States originated outside of the State in which the gas was processed. Gas produced from 9 States (Arizona, Indiana, Maryland, Missouri, New York, Oregon, South Dakota, Tennessee, and Virginia) was not processed.

For comparative purposes, the quantities of natural gas delivered to processing plants, total liquids extracted, and estimated volumetric and heat content extraction losses by State or origin of the gas (i.e., the State in which the gas was produced) are shown in Table A5.

\section{Lease and Plant Fuel}

Lease and plant fuel represent those quantities of natural gas used in well, field, and/or lease operations (such as gas used in drilling operations, heaters, dehydrators, and field compressors) and as fuel in natural gas processing plants.

Lease fuel data were collected for report year 1994, on the Form EIA-627, "Annual Quantity and Value of Natural Gas Report." Of the 30 States reporting on the Form EIA-627, 19 States reported quantities of natural gas used as lease fuel. Most of Nevada's marketed production is consumed as lease fuel, 16 million cubic feet. In the absence of reporting quantities on the Form EIA-627, the Form EIA-176 was used to estimate lease fuel quantities. Although EIA recognizes that lease data collected on the Form EIA-176 do not constitute a census or result from a statistically selected sample, the data collected in the survey provide the best information available to the EIA for estimating such usage. To estimate lease use during 1994 (Table 14), several simplifying assumptions were made:

- The quantity of gas used for lease fuel was assumed to be a function of gross withdrawals of natural gas from gas and oil wells.

- The average proportion of company-owned on-system production reported as used in lease operations by respondents to the Form EIA-176 was assumed to be typical of the average use by all operators as a proportion of gross withdrawals.

- Average usage was calculated separately for Alaska and for the lower 48 States to reflect the distinctive field operations in Alaska, particularly on the North Slope.

Form EIA-176 respondents reported volumes of companyowned onsystem production amounting to 12 percent of 1994 gross withdrawals ( 33 percent of withdrawals in Alaska and 8 percent of withdrawals in the lower 48 States). Lease use reported by respondents averaged 0.02891 thousand cubic feet per thousand cubic feet of reported production in Alaska and 0.02602 thousand cubic feet per thousand cubic feet of reported production in the lower 48 States. The fuel-use estimates shown in Table 14 were calculated by applying the above ratios to the gross withdrawals from the various States (Table 3), not reporting lease use on the EIA-627.

Beginning with the 1990 survey, data on natural gas used as processing plant fuel were collected from the Form EIA64A, "Annual Report of the Origin of Natural Gas Liquids Production." For 1989 and prior years, the quantity of gas used as plant fuel was assumed to be a function of the quantity of natural gas delivered to natural gas processing plants. The average proportion of gas delivered to company-operated onsystem plants used as fuel by respondents to the Form EIA-176 was assumed to be typical of the average use by all plant operators as a proportion of total gas delivered to all plants within each State. 


\section{Marketed Production}

Marketed production of natural gas is taken from responses to Part II of the Form EIA-627. It is the quantity of natural gas produced that is available for marketing and is reported in Tables 3 and 7. It refers to quantities of gas available after processes related to production are complete. These processes are repressuring, pressure maintenance, cycling, venting and flaring, removing nonhydrocarbon gases, using fuel on the lease.

Average wellhead prices are calculated from volumes and values reported in Part III of the Form EIA-627. These data are shown as "Reported Wellhead Value" in Table 7. The volumes in this section refer to the actual amounts of natural gas reported to the States as sold.

In many States, the marketed production volumes are larger than the reported wellhead value volumes. Differences in these volumes generally result from differences in definition and reporting requirements for separate data systems in the State. For example, while production quantities of federal, tribal, and State royalty gas are included in marketed production, some State reporting rules exclude these quantities from reported wellhead value volumes.

\section{Census Divisions}

The Bureau of the Census, U.S. Department of Commerce, has grouped the 50 States and the District of Columbia into
Census divisions. Some of the tables and graphs in this report show data by Census division. These groupings are:

New England: Connecticut, Maine, Massachusetts, New Hampshire, Rhode Island, and Vermont.

Middle Atlantic: New Jersey, New York, and Pennsylvania.

East North Central: Illinois, Indiana, Michigan, Ohio, and Wisconsin.

West North Central: Iowa, Kansas, Minnesota, Missouri, Nebraska, North Dakota, and South Dakota.

South Atlantic: Delaware, District of Columbia, Florida, Georgia, Maryland, North Carolina, South Carolina, Virginia, and West Virginia.

East South Central: Alabama, Kentucky, Mississippi, and Tennessee.

West South Central: Arkansas, Louisiana, Oklahoma, and Texas.

Mountain: Arizona, Colorado, Idaho, Montana, Nevada, New Mexico, Utah, and Wyoming.

Pacific Contiguous: California, Oregon, and Washington.

Pacific Noncontiguous: Alaska and Hawaii. 
Table A1. Comparison of Electric Utility Natural Gas Consumption Data by State, 1994 (Million Cubic Feet)

\begin{tabular}{|c|c|c|c|c|}
\hline State & $\begin{array}{c}\text { Form } \\
\text { EIA-176 }\end{array}$ & $\begin{array}{c}\text { Form } \\
\text { ElA-759 }\end{array}$ & Difference & MDP \\
\hline 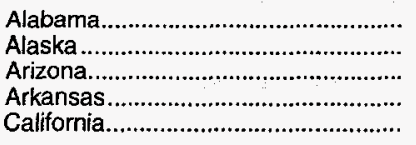 & $\begin{array}{r}3,652 \\
28,246 \\
33,230 \\
19,419 \\
596,774\end{array}$ & $\begin{array}{r}3,834 \\
29,048 \\
23,716 \\
24,977 \\
601,290\end{array}$ & $\begin{array}{r}182 \\
801 \\
-9,513 \\
5,558 \\
4,515\end{array}$ & $\begin{array}{r}5.0 \\
2.8 \\
40.1 \\
28.6 \\
0.8\end{array}$ \\
\hline 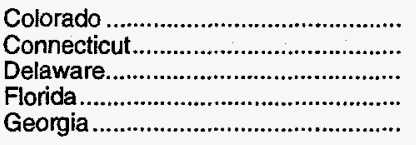 & $\begin{array}{r}4,219 \\
7,854 \\
17,468 \\
184,345 \\
1,137\end{array}$ & $\begin{array}{r}4,881 \\
8,002 \\
17,399 \\
180,697 \\
1,028\end{array}$ & $\begin{array}{r}662 \\
149 \\
-69 \\
-3,648 \\
-109\end{array}$ & $\begin{array}{r}15.7 \\
1.9 \\
0.4 \\
2.0 \\
10.6\end{array}$ \\
\hline 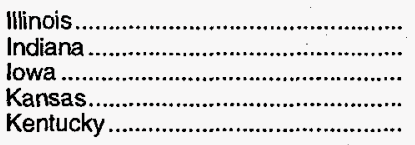 & $\begin{array}{r}33,599 \\
9,411 \\
2,474 \\
20,456 \\
269\end{array}$ & $\begin{array}{r}34,505 \\
9,009 \\
2,696 \\
27,279 \\
350\end{array}$ & $\begin{array}{r}906 \\
-402 \\
222 \\
6,824 \\
81\end{array}$ & $\begin{array}{r}2.7 \\
4.5 \\
9.0 \\
33.4 \\
30.0\end{array}$ \\
\hline 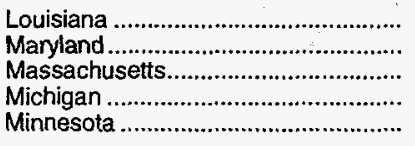 & $\begin{array}{r}208,096 \\
12,887 \\
41,343 \\
18,218 \\
3,996\end{array}$ & $\begin{array}{r}277,116 \\
12,718 \\
38,567 \\
18,218 \\
5,826\end{array}$ & $\begin{array}{r}69,020 \\
-168 \\
-2,776 \\
0 \\
1,830\end{array}$ & $\begin{array}{r}33.2 \\
1.3 \\
7.2 \\
0 \\
45.8\end{array}$ \\
\hline 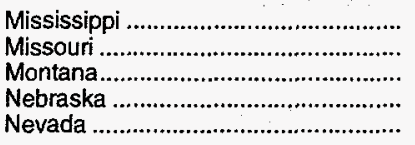 & $\begin{array}{r}33,643 \\
3,154 \\
663 \\
618 \\
32,377\end{array}$ & $\begin{array}{r}82,541 \\
4,351 \\
632 \\
3,061 \\
32,246\end{array}$ & $\begin{array}{r}48,898 \\
1,197 \\
-30 \\
2,443 \\
-130\end{array}$ & $\begin{array}{r}145.3 \\
37.9 \\
4.8 \\
395.3 \\
0.4\end{array}$ \\
\hline 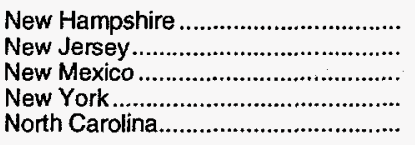 & $\begin{array}{r}1,271 \\
35,412 \\
26,573 \\
169,798 \\
852\end{array}$ & $\begin{array}{r}1,277 \\
42,625 \\
32,214 \\
182,521 \\
871\end{array}$ & $\begin{array}{r}5 \\
7,214 \\
5,641 \\
12,723 \\
19\end{array}$ & $\begin{array}{r}0.4 \\
20.4 \\
21.2 \\
7.5 \\
2.2\end{array}$ \\
\hline 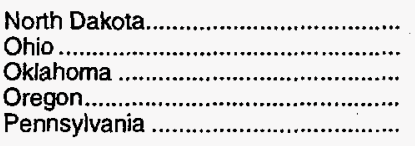 & $\begin{array}{r}2 \\
2,688 \\
158,059 \\
26,310 \\
14,153\end{array}$ & $\begin{array}{r}3 \\
2,818 \\
153,109 \\
26,132 \\
12,716\end{array}$ & $\begin{array}{r}0 \\
130 \\
-4,950 \\
-177 \\
-1,437\end{array}$ & $\begin{array}{r}7.8 \\
4.8 \\
3.2 \\
0.7 \\
11.3\end{array}$ \\
\hline 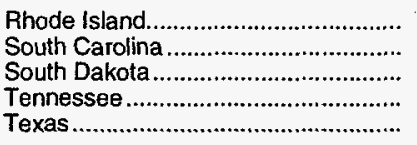 & $\begin{array}{r}0 \\
3,021 \\
64 \\
971 \\
991,606\end{array}$ & $\begin{array}{r}546 \\
3,005 \\
159 \\
1,019 \\
1,049,205\end{array}$ & $\begin{array}{r}546 \\
-15 \\
95 \\
47 \\
57,599\end{array}$ & $\begin{array}{r}0 \\
0.5 \\
149.3 \\
4.9 \\
5.8\end{array}$ \\
\hline 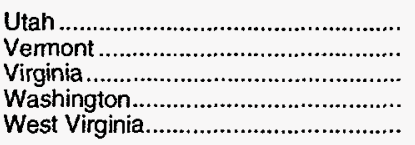 & $\begin{array}{r}7,956 \\
165 \\
26,797 \\
2,328 \\
244\end{array}$ & $\begin{array}{r}8,900 \\
166 \\
19,219 \\
2,461 \\
243\end{array}$ & $\begin{array}{r}944 \\
2 \\
-7,578 \\
133 \\
-1\end{array}$ & $\begin{array}{r}11.9 \\
1.0 \\
39.4 \\
5.7 \\
0.5\end{array}$ \\
\hline 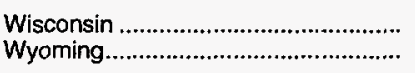 & $\begin{array}{r}5,301 \\
79\end{array}$ & $\begin{array}{r}3,821 \\
129\end{array}$ & $\begin{array}{r}-1,480 \\
51\end{array}$ & $\begin{array}{l}38.7 \\
64.2\end{array}$ \\
\hline 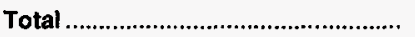 & $2,791,198$ & $2,987,146$ & 195,948 & 7.0 \\
\hline
\end{tabular}

a Relative comparisons are expressed as the maximum difference percentage (MOP), or the absolute value of the difference between two volumes divided by the smaller of the twa volumes, multiplied by 100 .

Note: Totals may not equal sum of components due to independent rounding. 
Table A2. Natural Gas Unaccounted for by State, 1990-1994 (Million Cubic Feet)

\begin{tabular}{|c|c|c|c|c|c|}
\hline State & 1990 & 1991 & 1992 & 1993 & 1994 \\
\hline 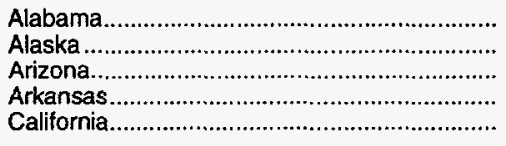 & $\begin{array}{r}67 \\
2,397 \\
17,347 \\
4,177 \\
4,341\end{array}$ & $\begin{array}{r}1,908 \\
114 \\
23,029 \\
13,145 \\
80,522\end{array}$ & $\begin{array}{r}3,062 \\
-14 \\
53,366 \\
4,424 \\
11,493\end{array}$ & $\begin{array}{r}3,937 \\
1,206 \\
168,056 \\
4,199 \\
181,570\end{array}$ & $\begin{array}{r}5,295 \\
2,669 \\
-1,782 \\
5,650 \\
217,809\end{array}$ \\
\hline 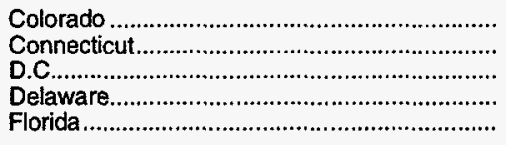 & $\begin{array}{r}9,499 \\
-441 \\
457 \\
-132 \\
-1,006\end{array}$ & $\begin{array}{r}10,580 \\
2,284 \\
771 \\
1,527 \\
402\end{array}$ & $\begin{array}{r}9,372 \\
946 \\
620 \\
170 \\
-275\end{array}$ & $\begin{array}{r}18,893 \\
2,682 \\
610 \\
689 \\
2,255\end{array}$ & $\begin{array}{r}9,023 \\
1,491 \\
528 \\
479 \\
6,063\end{array}$ \\
\hline 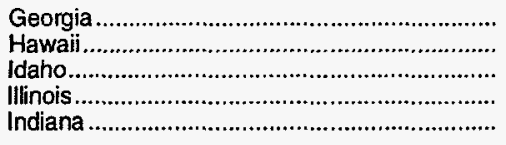 & $\begin{array}{r}-2,313 \\
29 \\
3,379 \\
-2,391 \\
-2,394\end{array}$ & $\begin{array}{r}7,039 \\
31 \\
-415 \\
-16,276 \\
2,261\end{array}$ & $\begin{array}{r}6,624 \\
16 \\
5,755 \\
17,107 \\
8,676\end{array}$ & $\begin{array}{r}5,346 \\
24 \\
-6,264 \\
10,576 \\
11,840\end{array}$ & $\begin{array}{r}2,104 \\
53 \\
5,572 \\
5,205 \\
979\end{array}$ \\
\hline 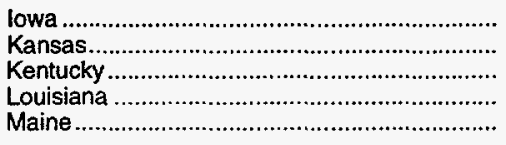 & $\begin{array}{r}18,357 \\
-473 \\
2,769 \\
-3,929 \\
120\end{array}$ & $\begin{array}{r}9,222 \\
11,288 \\
-836 \\
-6,069 \\
45\end{array}$ & $\begin{array}{r}9,307 \\
17,699 \\
6,660 \\
15,242 \\
26\end{array}$ & $\begin{array}{r}5,668 \\
12,330 \\
8,238 \\
16,283 \\
24\end{array}$ & $\begin{array}{r}4,963 \\
4,823 \\
4,534 \\
58,677 \\
-131\end{array}$ \\
\hline 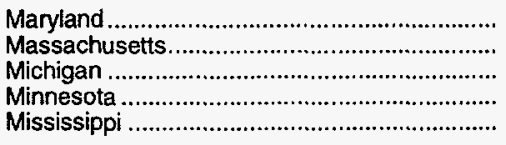 & $\begin{array}{r}-3,484 \\
-447 \\
1,249 \\
-14,294 \\
-6,959\end{array}$ & $\begin{array}{r}13,400 \\
-3,225 \\
16,865 \\
-17,472 \\
-5,013\end{array}$ & $\begin{array}{r}6,019 \\
331 \\
8,913 \\
-62,450 \\
8,000\end{array}$ & $\begin{array}{r}-5,891 \\
-2,960 \\
91,266 \\
-183,638 \\
9,503\end{array}$ & $\begin{array}{r}3,620 \\
12,201 \\
-14,398 \\
-15,824 \\
35,615\end{array}$ \\
\hline 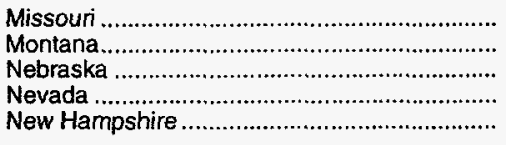 & $\begin{array}{r}1,271 \\
-26,971 \\
6,150 \\
2,095 \\
-219\end{array}$ & $\begin{array}{r}4,142 \\
1,872 \\
4,546 \\
22 \\
6\end{array}$ & $\begin{array}{r}13,773 \\
1,491 \\
2,292 \\
2,111 \\
678\end{array}$ & $\begin{array}{r}6,784 \\
-1,713 \\
2,227 \\
1,393 \\
4,008\end{array}$ & $\begin{array}{r}2,253 \\
79 \\
22,179 \\
-1 \\
327\end{array}$ \\
\hline 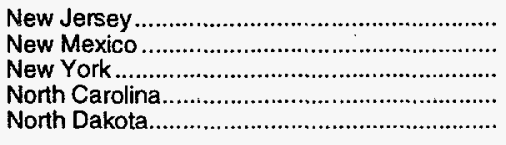 & $\begin{array}{r}723 \\
10 \\
-3,003 \\
-246 \\
-53\end{array}$ & $\begin{array}{r}6,120 \\
2,095 \\
11,958 \\
2,906 \\
-976\end{array}$ & $\begin{array}{r}39,471 \\
813 \\
28,140 \\
2,414 \\
-233\end{array}$ & $\begin{array}{r}5,811 \\
6,205 \\
99,726 \\
5,208 \\
-260\end{array}$ & $\begin{array}{r}-31,893 \\
7,827 \\
84,743 \\
2,906 \\
728\end{array}$ \\
\hline 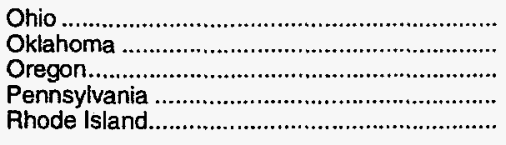 & $\begin{array}{r}12,381 \\
13,234 \\
3,062 \\
-2,571 \\
6,164\end{array}$ & $\begin{array}{r}9,636 \\
7,913 \\
-2,395 \\
13,505 \\
2,092\end{array}$ & $\begin{array}{r}8,537 \\
16,062 \\
-764 \\
11,380 \\
-1,100\end{array}$ & $\begin{array}{r}17,285 \\
11,498 \\
1,298 \\
6,164 \\
-960\end{array}$ & $\begin{array}{r}10,641 \\
8,976 \\
57 \\
-1,317 \\
-1,603\end{array}$ \\
\hline 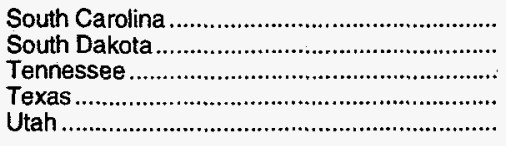 & $\begin{array}{r}-1,878 \\
-62 \\
-2,376 \\
-1,640 \\
4,507\end{array}$ & $\begin{array}{r}746 \\
-518 \\
38,691 \\
12,288 \\
-2,949\end{array}$ & $\begin{array}{r}1,110 \\
413 \\
6,920 \\
17,549 \\
-6,193\end{array}$ & $\begin{array}{r}1,064 \\
445 \\
8,280 \\
-22,703 \\
5,267\end{array}$ & $\begin{array}{r}298 \\
999 \\
2,884 \\
-28,914 \\
6,461\end{array}$ \\
\hline 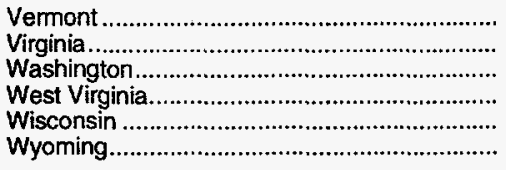 & $\begin{array}{r}-151 \\
-613 \\
5,778 \\
-6,101 \\
-671 \\
4,903\end{array}$ & $\begin{array}{r}76 \\
1,383 \\
-1,735 \\
10,297 \\
926 \\
3,050\end{array}$ & $\begin{array}{r}-3 \\
963 \\
247 \\
10,233 \\
3,614 \\
3,234\end{array}$ & $\begin{array}{r}103 \\
6,869 \\
51,049 \\
3,420 \\
-345 \\
1,368\end{array}$ & $\begin{array}{r}14 \\
-831 \\
70,679 \\
7,790 \\
1,218 \\
1,681\end{array}$ \\
\hline Total & 39,650 & 270,826 & 294,236 & 575,933 & 523,402 \\
\hline
\end{tabular}

Source: Energy Information Administration (EIA), Form ElA-176, "Annual Report of Natural and Supplemental Gas Supply and Disposition." 
Table A3. Natural Gas Processed and Liquids Extracted at Natural Gas Processing Plants by State, 1994

\begin{tabular}{|c|c|c|c|c|c|}
\hline \multirow{2}{*}{$\begin{array}{l}\text { Plant } \\
\text { Location }\end{array}$} & \multicolumn{3}{|c|}{$\begin{array}{l}\text { Volume of Natural Gas } \\
\text { Delivered to Processing Plants" } \\
\text { (million cubic feet) }\end{array}$} & \multirow{2}{*}{$\begin{array}{l}\text { Total } \\
\text { Liquids } \\
\text { Extracted } \\
\text { (thousand } \\
\text { barreis) }\end{array}$} & \multirow{2}{*}{$\begin{array}{l}\text { Extraction } \\
\text { Loss } \\
\text { (million } \\
\text { cubic } \\
\text { foet) }\end{array}$} \\
\hline & $\begin{array}{c}\text { State } \\
\text { Production }\end{array}$ & $\begin{array}{l}\text { Out of State } \\
\text { Production }\end{array}$ & $\begin{array}{l}\text { Natural } \\
\text { Gas } \\
\text { Processed }\end{array}$ & & \\
\hline 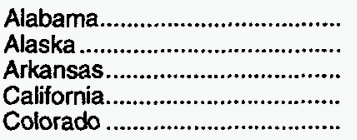 & $\begin{array}{r}134,635 \\
2,667,254 \\
216,024 \\
228,346 \\
353,547\end{array}$ & $\begin{array}{r}1,560 \\
0 \\
2,686 \\
0 \\
308\end{array}$ & $\begin{array}{r}136,195 \\
2,667,254 \\
218,710 \\
228,346 \\
353,855\end{array}$ & $\begin{array}{r}4,167 \\
26,071 \\
458 \\
9,425 \\
14,546\end{array}$ & $\begin{array}{r}5,647 \\
30,945 \\
553 \\
11,250 \\
19,612\end{array}$ \\
\hline 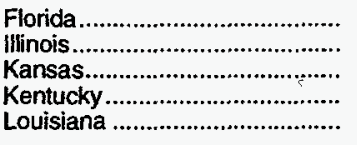 & $\begin{array}{r}7,311 \\
685 \\
819,544 \\
41,637 \\
4,071,043\end{array}$ & $\begin{array}{r}2,954 \\
0 \\
146,130 \\
1,240 \\
129,083\end{array}$ & $\begin{array}{r}10,265 \\
685 \\
965,674 \\
42,877 \\
4,200,126\end{array}$ & $\begin{array}{r}1,768 \\
69 \\
33,762 \\
1,453 \\
91,569\end{array}$ & $\begin{array}{r}1,789 \\
80 \\
46,936 \\
2,024 \\
128,583\end{array}$ \\
\hline 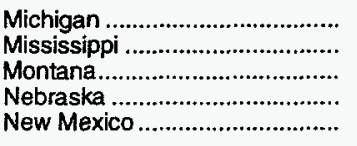 & $\begin{array}{r}196,000 \\
5,052 \\
10,388 \\
586 \\
799,285\end{array}$ & $\begin{array}{r}0 \\
0 \\
30 \\
0 \\
1,551\end{array}$ & $\begin{array}{r}196,000 \\
5,052 \\
10,418 \\
586 \\
800,836\end{array}$ & $\begin{array}{r}4,900 \\
337 \\
474 \\
6 \\
60,740\end{array}$ & $\begin{array}{r}6,371 \\
425 \\
631 \\
8 \\
86,607\end{array}$ \\
\hline 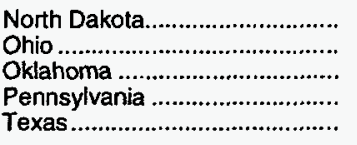 & $\begin{array}{r}55,150 \\
2,930 \\
1,072,307 \\
4,307 \\
4,123,600\end{array}$ & $\begin{array}{r}0 \\
0 \\
20,427 \\
2,826 \\
36,951\end{array}$ & $\begin{array}{r}55,150 \\
2,930 \\
1,092,734 \\
7,133 \\
4,160,551\end{array}$ & $\begin{array}{r}4,278 \\
78 \\
71,936 \\
394 \\
267,602\end{array}$ & $\begin{array}{r}5,671 \\
104 \\
101,564 \\
513 \\
381,020\end{array}$ \\
\hline 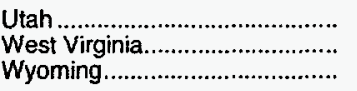 & $\begin{array}{r}349,631 \\
84,801 \\
820,504\end{array}$ & $\begin{array}{r}18,954 \\
29,064 \\
1,185\end{array}$ & $\begin{array}{l}368,585 \\
113,865 \\
821,689\end{array}$ & $\begin{array}{r}9,763 \\
7,369 \\
24,818\end{array}$ & $\begin{array}{l}13,780 \\
10,901 \\
33,486\end{array}$ \\
\hline 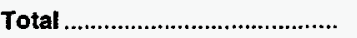 & $16,064,567$ & 394,949 & $16,459,516$ & 635,983 & 888,500 \\
\hline
\end{tabular}

a "State Production" refers to gas delivered to processing plants located in the same State in which the gas was produced. "Out of State Production" relers to gas produced in other States and delivered to plants located in the State listed for processing.

6 "Totals Liquids Extracted" represents the fotal quantity of liquids extracted from natural gas at natural gas processing plants located in the State.

Note: Totals may not equal sum of components due to independent rounding.

Source: Energy Information Administration (EIA), Form EIA-64A, "Annual Report of the Origin of Natural Gas Liquids Production." 
Table A4. Estimated Composition of Liquids Extracted at Natural Gas Processing Plants and the Resulting Heat Content Extraction Loss by State, 1994

\begin{tabular}{|c|c|c|c|c|c|c|}
\hline \multirow{2}{*}{ State } & \multicolumn{5}{|c|}{ Estimated Components and Products in Liquids Extracted } & \multirow{2}{*}{$\frac{\begin{array}{c}\text { Estimated Heat } \\
\text { Content } \\
\text { Extraction Loss' }\end{array}}{\text { Heat Content }}$} \\
\hline & Ethane & Propane & Isobutane & N-Butane & Pentanes Plus & \\
\hline $\begin{array}{l}\text { Alabama.................... } \\
\text { Alaska ....................... } \\
\text { Arkansas..................... } \\
\text { California..................... } \\
\text { Colorado ...................... }\end{array}$ & $\begin{array}{r}0 \\
0 \\
58 \\
16 \\
3,939\end{array}$ & $\begin{array}{r}1,568 \\
543 \\
87 \\
2,330 \\
4,686\end{array}$ & $\begin{array}{r}189 \\
2,546 \\
52 \\
2,057 \\
839\end{array}$ & $\begin{array}{r}1,167 \\
8,172 \\
69 \\
1,504 \\
2,158\end{array}$ & $\begin{array}{r}1,243 \\
14,810 \\
192 \\
3,518 \\
2,924\end{array}$ & $\begin{array}{r}17,557 \\
115,975 \\
1,904 \\
39,921 \\
56,294\end{array}$ \\
\hline 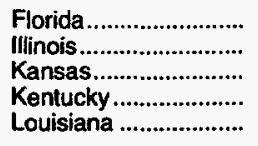 & $\begin{array}{r}703 \\
0 \\
4,791 \\
101 \\
31,024\end{array}$ & $\begin{array}{r}576 \\
25 \\
14,589 \\
803 \\
25,658\end{array}$ & $\begin{array}{r}0 \\
0 \\
3,015 \\
77 \\
9,077\end{array}$ & $\begin{array}{r}351 \\
0 \\
6,018 \\
275 \\
9,123\end{array}$ & $\begin{array}{r}138 \\
44 \\
5,348 \\
196 \\
16,685\end{array}$ & $\begin{array}{r}6,532 \\
299 \\
133,457 \\
5,796 \\
346,671\end{array}$ \\
\hline $\begin{array}{l}\text { Michigan ................. } \\
\text { Mississippi .................. } \\
\text { Montana...................... } \\
\text { Nebraska ................... } \\
\text { New Mexico ................ }\end{array}$ & $\begin{array}{r}673 \\
0 \\
0 \\
0 \\
25,030\end{array}$ & $\begin{array}{r}1,798 \\
106 \\
186 \\
0 \\
17,831\end{array}$ & $\begin{array}{r}783 \\
0 \\
25 \\
0 \\
3,747\end{array}$ & $\begin{array}{r}682 \\
100 \\
122 \\
0 \\
6,346\end{array}$ & $\begin{array}{r}964 \\
131 \\
141 \\
0 \\
7,786\end{array}$ & $\begin{array}{r}19,487 \\
1,445 \\
1,992 \\
0 \\
223,857\end{array}$ \\
\hline $\begin{array}{l}\text { North Dakota............... } \\
\text { Ohio ........................... } \\
\text { Oklahoma ................... } \\
\text { Pennsylvania .............. } \\
\text { Texas ........................... }\end{array}$ & $\begin{array}{r}8 \\
0 \\
29,273 \\
0 \\
89,464\end{array}$ & $\begin{array}{r}1,933 \\
30 \\
22,074 \\
163 \\
70,177\end{array}$ & $\begin{array}{r}0 \\
5 \\
3,486 \\
29 \\
38,143\end{array}$ & $\begin{array}{r}1,210 \\
18 \\
7,794 \\
97 \\
28,384\end{array}$ & $\begin{array}{r}1,128 \\
24 \\
9,309 \\
104 \\
42,434\end{array}$ & $\begin{array}{r}17,881 \\
328 \\
265,473 \\
1,645 \\
1,012,259\end{array}$ \\
\hline $\begin{array}{l}\text { Utah ....................... } \\
\text { West Virginia............ } \\
\text { Wyoming..................... }\end{array}$ & $\begin{array}{l}2,714 \\
2,388 \\
6,748\end{array}$ & $\begin{array}{l}2,738 \\
2,933 \\
7,840\end{array}$ & $\begin{array}{r}260 \\
379 \\
2,118\end{array}$ & $\begin{array}{r}1,181 \\
871 \\
3,505\end{array}$ & $\begin{array}{r}2,871 \\
799 \\
4,607\end{array}$ & $\begin{array}{l}38,270 \\
27,572 \\
95,735\end{array}$ \\
\hline Total .......................... & 195,930 & 178,674 & 66,827 & 79,147 & 115,396 & $2,430,350$ \\
\hline \multicolumn{7}{|c|}{ 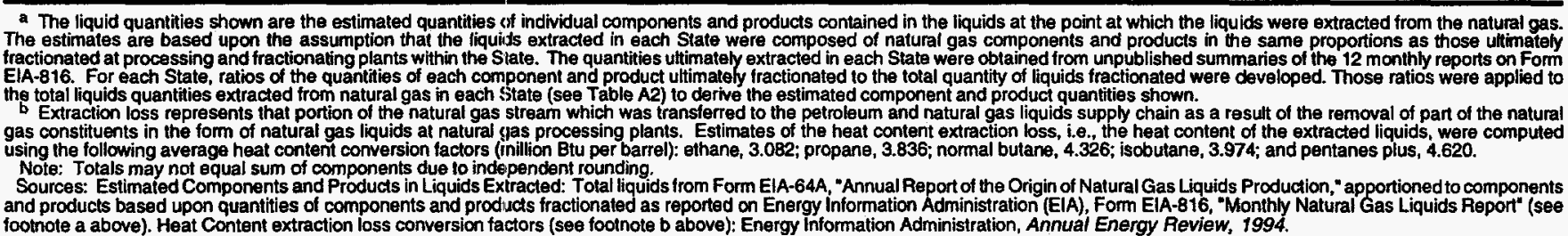 } \\
\hline
\end{tabular}


Table A5. Natural Gas Processed, Liquids Extracted, and Estimated Extraction Loss by State of Origin (Production) of Natural Gas, 1994

\begin{tabular}{|c|c|c|c|c|c|c|}
\hline \multirow{2}{*}{ State } & \multicolumn{3}{|c|}{$\begin{array}{l}\text { Volume of Natural Gas Delivered } \\
\text { to Processing Plants } \\
\text { (million cubic feet) }\end{array}$} & \multirow{2}{*}{$\begin{array}{l}\text { Total } \\
\text { Liquids } \\
\text { Extracted } \\
\text { (thousand } \\
\text { barrels) }\end{array}$} & \multicolumn{2}{|c|}{ Extraction Loss } \\
\hline & $\begin{array}{l}\text { Located Within } \\
\text { the State }\end{array}$ & $\begin{array}{l}\text { Located Outside } \\
\text { of the State }\end{array}$ & $\begin{array}{l}\text { Total } \\
\text { Processed }\end{array}$ & & $\begin{array}{l}\text { Volume } \\
\text { (million cubic } \\
\text { feet }\end{array}$ & $\begin{array}{c}\text { Estimated } \\
\text { Heat } \\
\text { Content } \\
\text { (billion Btu) }\end{array}$ \\
\hline $\begin{array}{l}\text { Alabama.................... } \\
\text { Alaska ........................ } \\
\text { Arkansas.................... } \\
\text { California................... } \\
\text { Colorado ...................... }\end{array}$ & $\begin{array}{r}134,635 \\
2,667,254 \\
216,024 \\
228,346 \\
353,547\end{array}$ & $\begin{array}{r}2,954 \\
0 \\
11 \\
0 \\
4,673\end{array}$ & $\begin{array}{r}137,589 \\
2,667,254 \\
216,035 \\
228,346 \\
358,220\end{array}$ & $\begin{array}{r}4,633 \\
26,071 \\
332 \\
9,425 \\
14,665\end{array}$ & $\begin{array}{r}6,097 \\
30,945 \\
547 \\
11,250 \\
19,776\end{array}$ & $\begin{array}{r}19,254 \\
115,975 \\
1,380 \\
39,921 \\
56,755\end{array}$ \\
\hline 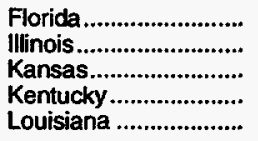 & $\begin{array}{r}7,311 \\
685 \\
819,544 \\
41,637 \\
4,071,043\end{array}$ & $\begin{array}{r}0 \\
0 \\
2,435 \\
28,881 \\
912\end{array}$ & $\begin{array}{r}7,311 \\
685 \\
821,979 \\
70,518 \\
4,071,955\end{array}$ & $\begin{array}{r}1,255 \\
69 \\
28,982 \\
2,568 \\
88,491\end{array}$ & $\begin{array}{r}1,274 \\
80 \\
40,059 \\
4,730 \\
124,715\end{array}$ & $\begin{array}{r}4,637 \\
299 \\
114,543 \\
9,962 \\
335,018\end{array}$ \\
\hline $\begin{array}{l}\text { Michigan ................... } \\
\text { Mississippi ................. } \\
\text { Montana.................... } \\
\text { Nebraska .................. } \\
\text { New Mexico ............... }\end{array}$ & $\begin{array}{r}196,000 \\
5,052 \\
10,388 \\
586 \\
799,285\end{array}$ & $\begin{array}{r}0 \\
1,560 \\
412 \\
0 \\
844\end{array}$ & $\begin{array}{r}196,000 \\
6,612 \\
10,800 \\
586 \\
800,129\end{array}$ & $\begin{array}{r}4,900 \\
384 \\
515 \\
6 \\
60,599\end{array}$ & $\begin{array}{r}6,371 \\
490 \\
646 \\
8 \\
86,517\end{array}$ & $\begin{array}{r}19,487 \\
1,643 \\
2,149 \\
0 \\
223,339\end{array}$ \\
\hline $\begin{array}{l}\text { North Dakota.............. } \\
\text { Ohio ......................... } \\
\text { Oklahoma .................. } \\
\text { Pennsylvania .............. } \\
\text { Tennessee .................. }\end{array}$ & $\begin{array}{r}55,150 \\
2,930 \\
1,072,307 \\
4,307 \\
0\end{array}$ & $\begin{array}{r}30 \\
0 \\
104,566 \\
183 \\
1,240\end{array}$ & $\begin{array}{r}55,180 \\
2,930 \\
1,176,873 \\
4,490 \\
1,240\end{array}$ & $\begin{array}{r}4,283 \\
78 \\
75,538 \\
362 \\
26\end{array}$ & $\begin{array}{r}5,673 \\
104 \\
106,242 \\
327 \\
59\end{array}$ & $\begin{array}{r}17,902 \\
328 \\
279,671 \\
1,508 \\
104\end{array}$ \\
\hline 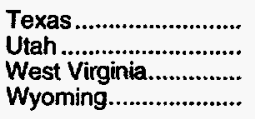 & $\begin{array}{r}4,123,600 \\
349,631 \\
84,801 \\
820,504\end{array}$ & $\begin{array}{r}227,701 \\
261 \\
2,826 \\
15,460\end{array}$ & $\begin{array}{r}4,351,301 \\
349,892 \\
87,627 \\
835,964\end{array}$ & $\begin{array}{r}272,109 \\
9,422 \\
6,260 \\
25,010\end{array}$ & $\begin{array}{r}387,166 \\
13,086 \\
8,322 \\
34,017\end{array}$ & $\begin{array}{r}1,029,605 \\
36,933 \\
23,440 \\
96,498\end{array}$ \\
\hline 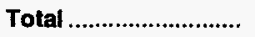 & $16,064,567$ & 394,949 & $16,459,516$ & 635,983 & 888,500 & $2,430,350$ \\
\hline
\end{tabular}

Notes: This table shows the volume of natural gas delivered to processing plants, the quantity of natural gas liquids extracted, and the estimated volumetric and heat content extraction losses traced back to the State of origin of the gas, i.e., to the State from which the gas was produced whether processed within or outside of the producing State. Totals may not equal sum of components

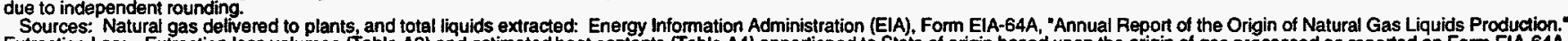
Extraction Loss: Extraction loss volumes (Table A3) and estimated heat contents (Table A4) apportioned to State of origin based upon the origin of gas processed as reported on Form EIA-64A. 


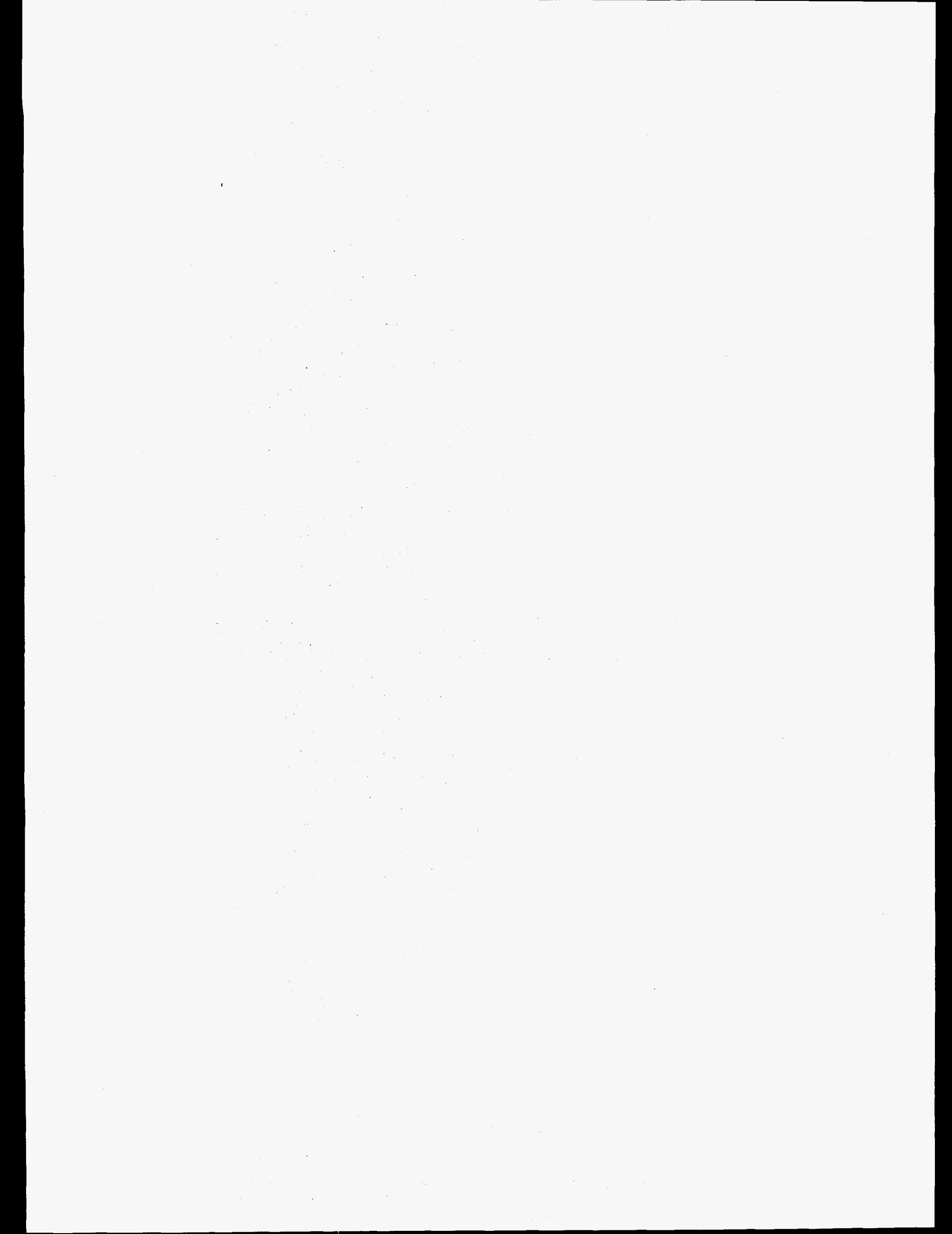


Appendix B

Metric and

Thermal

Conversion Tables 


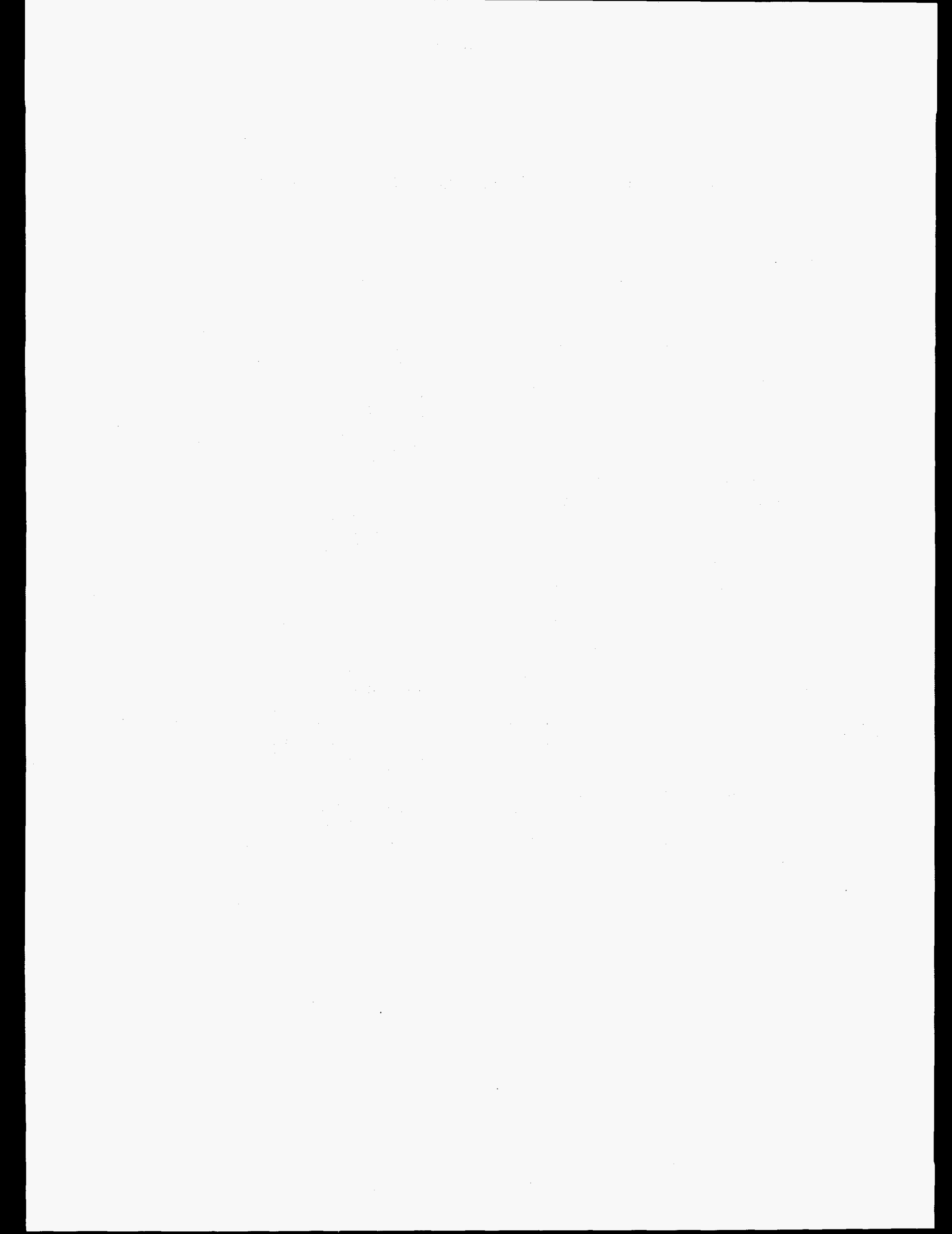




\section{Metric and Thermal Conversion Tables}

\section{Metric Conversions}

Table B1 presents Summary Statistics for Natural Gas in the United States for 1990 through 1994 in metric units of measure. Volumes are shown in cubic meters instead of cubic feet. Prices are shown in dollars per thousand cubic meters instead of dollars per thousand cubic feet. The data in this table have been converted from the data that appear in Table 1 of this report.

\section{Thermal Conversions}

Table B2 presents the thermal (Btu) conversion factors and the converted data for natural gas supply and disposition from 1990 through 1994. A brief documentation for the thermal conversion factors follows:

- Marketed Production. The conversion factor is calculated by adding the total heat content of dry production to the total heat content of extraction loss and dividing the resulting sum by the total quantity of dry production and extraction loss (see below).

- Extraction Loss. The conversion factor is obtained from Appendix $A$ of this publication.

- Dry Production. The conversion factor is assumed to be the same as the thermal conversion factors for consumption (see below).

- Receipts at U.S. Borders. The conversion factors for imports are obtained from Form FPC-14, "Annual Report for Importers and Exporters of Natural Gas." Intransit receipts are assumed to have the same average heat content as imports.
- Withdrawals from Storage. Both underground and LNG storage withdrawals are assumed to have the same heat content as consumption (see below).

- Supplemental Gas Supplies. This conversion factor is assumed to be the same as that for consumption (see below).

- Balancing ltem. This conversion factor is calculated by subtracting the total heat content of all other items of supply from the heat content of total disposition (from Table B2) and dividing the difference by the balancing item quantity.

- Consumption. The thermal conversion factor for total consumption (lease fuel, plant fuel, pipeline fuel, and deliveries to consumers) is the average heat content for deliveries to end users as reported on Form EIA-176, "Annual Report of Natural and Supplemental Gas Supply and Disposition." Average heat content of electric utility consumption is obtained from EIA's Electric Power Annual. The factor for nonutility consumption is calculated by subtracting the total heat content of electric utility consumption from the heat content of total consumption and dividing the difference by the quantity of nonutility consumption (total consumption less electric utility consumption).

- Deliveries at U.S. Borders. The conversion factor for exports is obtained from Form FPC-14. Intransit deliveries are assumed to have the same average heat content as exports.

- Additions to Storage. Additions to both underground and LNG storage are assumed to have the same heat content as consumption (see above). 
Table B1. Summary Statistics for Natural Gas in the United States, Metric Equivalents, 1990-1994

\begin{tabular}{|c|c|c|c|c|c|}
\hline & 1990 & 1991 & 1992 & 1993 & 1994 \\
\hline 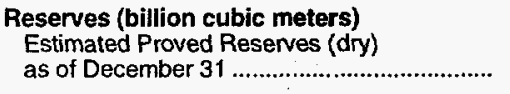 & 4,795 & 4,731 & 4,673 & 4,599 & 4,639 \\
\hline 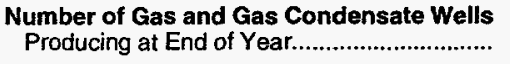 & $R_{269,100}$ & $R_{276,337}$ & $\mathrm{~A}_{275,414}$ & $R_{282,152}$ & 287,206 \\
\hline $\begin{array}{l}\text { Production (million cubic meters) } \\
\text { Gross Withdrawals } \\
\text { From Gas Wells } \ldots \ldots \ldots \ldots \ldots \ldots \ldots \ldots \ldots \ldots \ldots \ldots \ldots \ldots \ldots \ldots \ldots \ldots \ldots \ldots \ldots \ldots \ldots \ldots \ldots \ldots \ldots \ldots \ldots \ldots \ldots\end{array}$ & $\begin{array}{l}454,586 \\
154,866\end{array}$ & $\begin{array}{l}453,568 \\
162,326\end{array}$ & $\begin{array}{l}457,738 \\
168,977\end{array}$ & $\begin{array}{l}\mathbf{R}_{472,640} \\
\mathbf{R}_{170,878}\end{array}$ & $\begin{array}{l}492,076 \\
176,445\end{array}$ \\
\hline 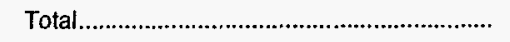 & 609,452 & 615,893 & 626,714 & ${ }^{A} 643,517$ & 668,522 \\
\hline 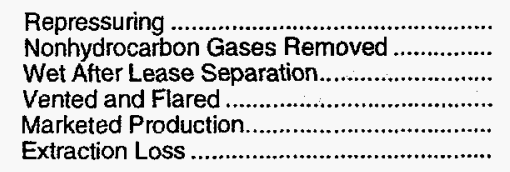 & $\begin{array}{r}-70,482 \\
-8,194 \\
530,776 \\
-4,259 \\
526,517 \\
-22,204\end{array}$ & $\begin{array}{r}-78,492 \\
-7,811 \\
529,591 \\
-4,811 \\
524,779 \\
-23,634\end{array}$ & $\begin{array}{r}-84,173 \\
-7,939 \\
534,602 \\
-4,744 \\
529,859 \\
-24,690\end{array}$ & $\begin{array}{r}A_{-87,867} \\
-11,722 \\
R_{543,928} \\
R_{-6,421} \\
R_{537,507} \\
-25,102\end{array}$ & $\begin{array}{r}-94,370 \\
-11,672 \\
562,480 \\
-6,466 \\
556,014 \\
-25,159\end{array}$ \\
\hline 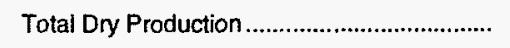 & 504,313 & 501,145 & 505,169 & $R_{512,406}$ & 530,855 \\
\hline $\begin{array}{l}\text { Supply (million cubic meters) } \\
\text { Dry Production } \\
\text { Receipts at U.S. Borders }\end{array}$ & 504,313 & 501,145 & 505,169 & $\mathbf{R}_{512,406}$ & 530,855 \\
\hline 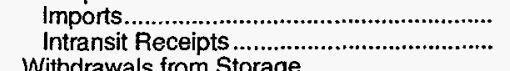 & $\begin{array}{l}43,389 \\
10,092\end{array}$ & $\begin{array}{l}50,215 \\
10,275\end{array}$ & $\begin{array}{l}60,527 \\
13,767\end{array}$ & $\begin{array}{r}66,548 \\
9,177\end{array}$ & $\begin{array}{l}75,313 \\
13,812\end{array}$ \\
\hline 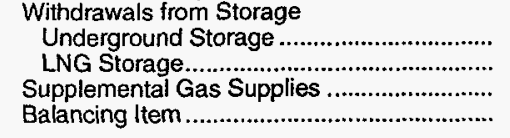 & $\begin{array}{r}54,759 \\
1,488 \\
3,477 \\
-4,300\end{array}$ & $\begin{array}{r}76,136 \\
1,787 \\
3,189 \\
-14,152\end{array}$ & $\begin{array}{r}77,129 \\
1,374 \\
3,339 \\
-14,373\end{array}$ & $\begin{array}{r}76,939 \\
2,327 \\
3,370 \\
\mathrm{R}_{-3,103}\end{array}$ & $\begin{array}{r}70,549 \\
2,002 \\
3,138 \\
-8,398\end{array}$ \\
\hline 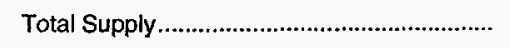 & 613,217 & 628,594 & 646,932 & $\mathbf{A}_{667,663}$ & 687,271 \\
\hline 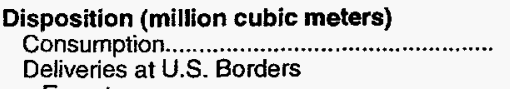 & 529,951 & 539,015 & 553,434 & $R_{574,239}$ & 587,729 \\
\hline 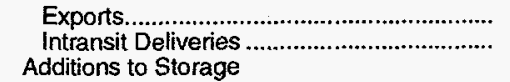 & $\begin{array}{r}2,423 \\
10,072\end{array}$ & $\begin{array}{r}3,660 \\
10,267\end{array}$ & $\begin{array}{r}6,124 \\
13,767\end{array}$ & $\begin{array}{l}3,970 \\
9,177\end{array}$ & $\begin{array}{r}5,042 \\
13,380\end{array}$ \\
\hline 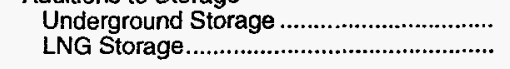 & $\begin{array}{r}68,908 \\
1,864\end{array}$ & $\begin{array}{r}73,861 \\
1,791\end{array}$ & $\begin{array}{r}72,361 \\
1,247\end{array}$ & $\begin{array}{r}78,147 \\
2,130\end{array}$ & $\begin{array}{r}79,182 \\
1,939\end{array}$ \\
\hline 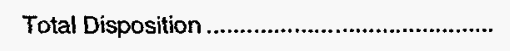 & 613,217 & 628,594 & 646,932 & $\mathrm{R}_{667,663}$ & 687,271 \\
\hline 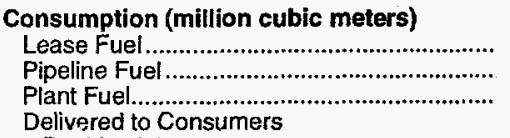 & $\begin{array}{l}22,872 \\
18,684 \\
12,138\end{array}$ & $\begin{array}{l}19,038 \\
17,027 \\
12,939\end{array}$ & $\begin{array}{l}20,112 \\
16,642 \\
13,042\end{array}$ & $\begin{array}{r}R_{20,476} \\
17,678 \\
12,709\end{array}$ & $\begin{array}{l}20,860 \\
19,406 \\
12,003\end{array}$ \\
\hline 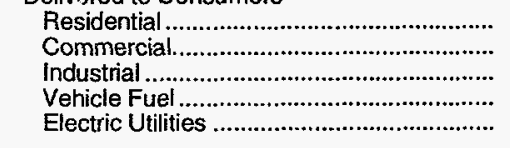 & $\begin{array}{r}124,348 \\
74,267 \\
198,739 \\
8 \\
78,895\end{array}$ & $\begin{array}{r}129,002 \\
77,265 \\
204,758 \\
10 \\
78,976\end{array}$ & $\begin{array}{r}132,808 \\
79,365 \\
213,138 \\
14 \\
78,313\end{array}$ & $\begin{array}{r}\mathbf{R}_{140,351} \\
\mathbf{R}_{81,030} \\
\mathbf{R}_{226,009} \\
27 \\
75,958\end{array}$ & $\begin{array}{r}137,271 \\
81,978 \\
231,574 \\
49 \\
84,586\end{array}$ \\
\hline Total Delivered to Consumers ............................ & 476,257 & 490,010 & 503,638 & $\mathbf{R}_{523,375}$ & 535,459 \\
\hline 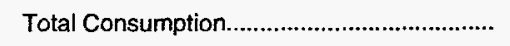 & 529,951 & 539,015 & 553,434 & $R_{574,239}$ & 587,729 \\
\hline 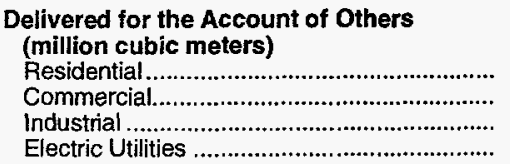 & $\begin{array}{r}886 \\
9,982 \\
128,687 \\
39,370\end{array}$ & $\begin{array}{r}1,032 \\
11,494 \\
137,731 \\
44,743\end{array}$ & $\begin{array}{r}1,173 \\
13,337 \\
148,624 \\
48,064\end{array}$ & $\begin{array}{r}1,255 \\
R_{13,028} \\
R_{159,845} \\
46,962\end{array}$ & $\begin{array}{r}1,199 \\
16,963 \\
173,406 \\
59,242\end{array}$ \\
\hline
\end{tabular}

See footnotes at end of table. 
Table B1. Summary Statistics for Natural Gas in the United States, Metric Equivalents, 1990-1994 (Continued)

\begin{tabular}{|c|c|c|c|c|c|}
\hline & 1990 & 1991 & 1992 & 1993 & 1994 \\
\hline 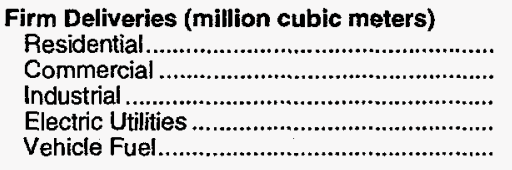 & $\begin{array}{l}\text { NA } \\
\text { NA } \\
\text { NA } \\
\text { NA } \\
\text { NA }\end{array}$ & $\begin{array}{l}\text { NA } \\
\text { NA } \\
\text { NA } \\
\text { NA } \\
\text { NA }\end{array}$ & $\begin{array}{l}\text { NA } \\
\text { NA } \\
\text { NA } \\
\text { NA } \\
\text { NA }\end{array}$ & $\begin{array}{r}140,372 \\
\mathbf{R}_{72,312} \\
\mathbf{R}_{130,961} \\
\mathbf{R}_{43,100} \\
22\end{array}$ & $\begin{array}{r}137,166 \\
72,477 \\
134,644 \\
44,762 \\
38\end{array}$ \\
\hline 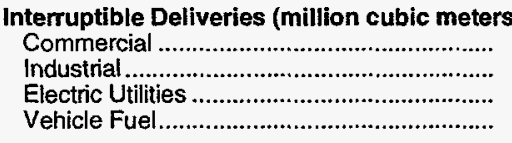 & $\begin{array}{l}\text { NA } \\
\text { NA } \\
\text { NA } \\
\text { NA }\end{array}$ & $\begin{array}{l}\text { NA } \\
\text { NA } \\
\text { NA } \\
\text { NA }\end{array}$ & $\begin{array}{l}\text { NA } \\
\text { NA } \\
\text { NA } \\
\text { NA }\end{array}$ & $\begin{array}{r}R_{8,718} \\
R_{95,048} \\
26,135 \\
5\end{array}$ & $\begin{array}{r}9,501 \\
96,930 \\
34,276 \\
11\end{array}$ \\
\hline 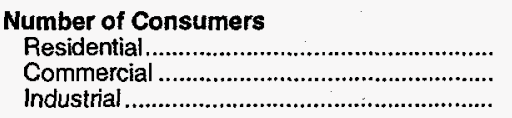 & $\begin{array}{r}50,187,178 \\
4,236,280 \\
218,341\end{array}$ & $\begin{array}{r}51,593,206 \\
4,357,252 \\
216,529\end{array}$ & $\begin{array}{r}52,331,397 \\
4,409,699 \\
209,616\end{array}$ & $\begin{array}{r}52,535,411 \\
\mathbf{R}_{4,464,906} \\
\mathbf{R}_{209,666}\end{array}$ & $\begin{array}{r}53,393,142 \\
4,537,500 \\
202,953\end{array}$ \\
\hline 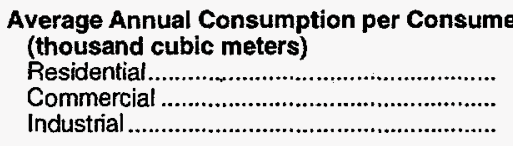 & $\begin{array}{r}2 \\
18 \\
910\end{array}$ & $\begin{array}{r}2 \\
18 \\
946\end{array}$ & $\begin{array}{r}3 \\
18 \\
1,017\end{array}$ & $\begin{array}{r}3 \\
R_{18} \\
R_{1,078}\end{array}$ & $\begin{array}{r}3 \\
18 \\
1,141\end{array}$ \\
\hline 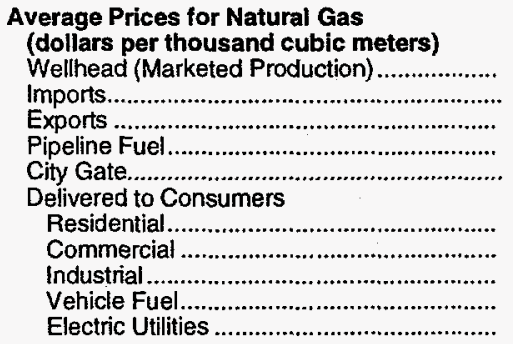 & $\begin{array}{r}60.39 \\
68.51 \\
109.48 \\
68.86 \\
107.00\end{array}$ & $\begin{array}{r}205.53 \\
169.86 \\
95.00 \\
139.85 \\
76.99\end{array}$ & $\begin{array}{r}61.45 \\
65.33 \\
79.46 \\
73.10 \\
106.30\end{array}$ & $\begin{array}{r}217.54 \\
R_{184.34} \\
R_{108.42} \\
150.79 \\
92.17\end{array}$ & $\begin{array}{r}226.37 \\
192.11 \\
107.71 \\
145.85 \\
80.52\end{array}$ \\
\hline
\end{tabular}

$R=$ Revised data.

NA = Not Available. Notes: Beginning in 1987, prices for gas delivered to consumers are calculated using only on-system sales data. No imputations are made for prices of gas delivered for the account of others.
in previous years, prices were calculated using reported values and values imputed for gas delivereo for the account of others. The United States includes the 50 States and the District of Columbia. In previous years, prices were calculated using reported values and values imputed for gas delivered for the account of others. The United States includes the 50 States and the District of Columbia. Sources: Energy Information Administration (EIA), Form EIA-176, "Annual Report of Natural and Supplemental Gas Supply and Disposition"; Form EIA-627, "Annual Quantity and Value of Natural Gas Report"; Form EIA-857, "Monthly Report of Natural Gas Purchases and Deliveries to Consumers"; Form ElA-816, "Monthiy Natural Gas Liquids Report"; Form ElA-759, "Monthly Power Plant Report; Form FERC-423, "Monthly Report of Cost and Quality of Fuels tor Electric Importers and Exporters of Natural Gas"; U.S. Crude Oil, Natural Gas, and Natural Gas Liquids Reserves, 1994 Annual Report, DOE/ElA-0216(94); and the U.S. Minerals Management Service. 
Table B2. Thermal Conversion Factors and Data, 1990-1994

\begin{tabular}{|c|c|c|c|c|c|}
\hline & 1990 & 1991 & 1992 & 1993 & 1994 \\
\hline \multicolumn{6}{|l|}{$\begin{array}{l}\text { Conversion Factor } \\
\text { (Btu per cubic foot) }\end{array}$} \\
\hline 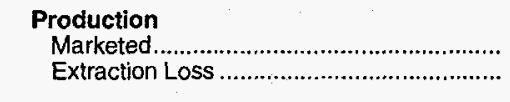 & $\begin{array}{l}1,105 \\
2,797\end{array}$ & $\begin{array}{l}1,108 \\
2,753\end{array}$ & $\begin{array}{l}1,110 \\
2,753\end{array}$ & $\begin{array}{l}1,106 \\
2,740\end{array}$ & $\begin{array}{l}1,105 \\
2,735\end{array}$ \\
\hline Total Dry Production & 1,031 & 1,030 & 1,030 & 1,027 & 1,028 \\
\hline $\begin{array}{l}\text { Supply } \\
\text { Dry Production ................................................... } \\
\text { Receipts at U.S. Borders }\end{array}$ & 1,031 & 1,030 & 1,030 & 1,027 & 1,028 \\
\hline 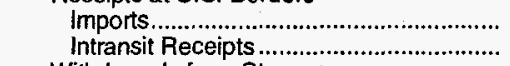 & $\begin{array}{l}1,012 \\
1,012\end{array}$ & $\begin{array}{l}1,014 \\
1,014\end{array}$ & $\begin{array}{l}1,011 \\
1,011\end{array}$ & $\begin{array}{l}1,020 \\
1,020\end{array}$ & $\begin{array}{l}1,022 \\
1,022\end{array}$ \\
\hline 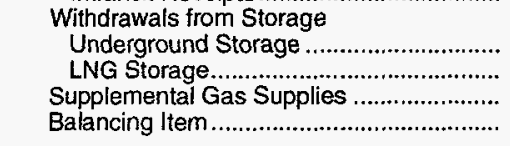 & $\begin{array}{r}1,031 \\
1,031 \\
1,031 \\
835\end{array}$ & $\begin{array}{r}1,030 \\
1,030 \\
1,030 \\
971\end{array}$ & $\begin{array}{r}1,030 \\
1,030 \\
1,030 \\
949\end{array}$ & $\begin{array}{r}1,027 \\
1,027 \\
1,027 \\
997\end{array}$ & $\begin{array}{r}1,028 \\
1,028 \\
1,028 \\
937\end{array}$ \\
\hline 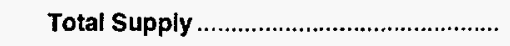 & NA & NA & NA & NA & NA \\
\hline 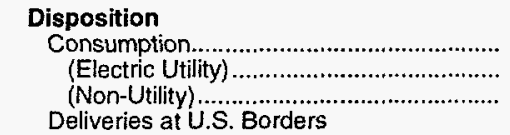 & $\begin{array}{r}1,031 \\
(1,034) \\
(1,030)\end{array}$ & $\begin{array}{r}1,030 \\
(1,024) \\
(1,031)\end{array}$ & $\begin{array}{r}1,030 \\
(1,022) \\
(1,031)\end{array}$ & $\begin{array}{r}1,027 \\
(1,022) \\
(1,028)\end{array}$ & $\begin{array}{r}1,028 \\
(1,022) \\
(1,029)\end{array}$ \\
\hline Exports.t. & $\begin{array}{l}1,018 \\
1,018\end{array}$ & $\begin{array}{l}1,022 \\
1,022\end{array}$ & $\begin{array}{l}1,018 \\
1,018\end{array}$ & $\begin{array}{l}1,016 \\
1,016\end{array}$ & $\begin{array}{l}1,011 \\
1,011\end{array}$ \\
\hline 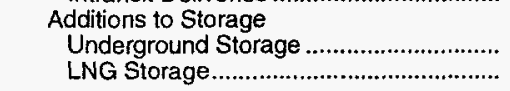 & $\begin{array}{l}1,031 \\
1,031\end{array}$ & $\begin{array}{l}1,030 \\
1,030\end{array}$ & $\begin{array}{l}1,030 \\
1,030\end{array}$ & $\begin{array}{l}1,027 \\
1,027\end{array}$ & $\begin{array}{l}1,028 \\
1,028\end{array}$ \\
\hline 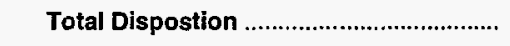 & NA & NA & NA & NA & NA \\
\hline \multicolumn{6}{|l|}{$\begin{array}{l}\text { Summary Data } \\
\text { (billion Btu) }\end{array}$} \\
\hline 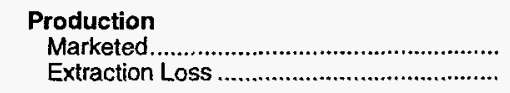 & $\begin{array}{l}20,554,878 \\
-2,193,104\end{array}$ & $\begin{array}{r}20,526,272 \\
-2,297,536\end{array}$ & $\begin{array}{r}20,775,302 \\
-2,400,203\end{array}$ & $\begin{array}{r}21,344,603 \\
-2,428,668\end{array}$ & $\begin{array}{r}21,702,261 \\
-2,430,350\end{array}$ \\
\hline 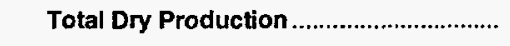 & $18,361,774$ & $18,228,736$ & $18,375,100$ & $18,915,935$ & $19,271,911$ \\
\hline $\begin{array}{l}\text { Supply } \\
\text { Dry Production... } \\
\text { Receipts at U.S. Borders }\end{array}$ & $18,361,774$ & $18,228,736$ & $18,375,100$ & $18,915,935$ & $19,271,911$ \\
\hline 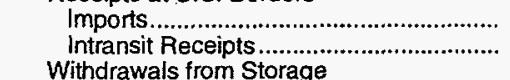 & $\begin{array}{r}1,550,996 \\
360,678\end{array}$ & $\begin{array}{r}1,798,967 \\
367,941\end{array}$ & $\begin{array}{r}2,161,448 \\
491,511\end{array}$ & $\begin{array}{r}2,398,159 \\
330,718\end{array}$ & $\begin{array}{r}2,682,436 \\
498,653\end{array}$ \\
\hline 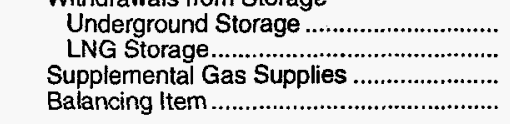 & $\begin{array}{r}1,993,734 \\
54,172 \\
126,613 \\
-126,769\end{array}$ & $\begin{array}{r}2,769,390 \\
64,983 \\
115,984 \\
-485,314\end{array}$ & $\begin{array}{r}2,805,487 \\
49,990 \\
121,457 \\
-481,767\end{array}$ & $\begin{array}{r}2,790,425 \\
84,408 \\
122,212 \\
-412,557\end{array}$ & $\begin{array}{r}2,561,187 \\
72,668 \\
113,929 \\
-277,959\end{array}$ \\
\hline 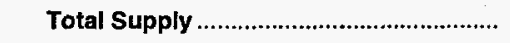 & $22,321,197$ & $22,860,687$ & $23,523,226$ & $24,229,301$ & $24,922,825$ \\
\hline 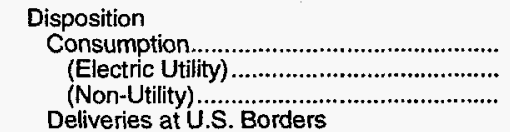 & $\begin{array}{r}19,295,258 \\
(2,880,882) \\
(16,414,376)\end{array}$ & $\begin{array}{r}19,606,211 \\
(2,855,950) \\
(16,747,888)\end{array}$ & $\begin{array}{r}20,130,695 \\
(2,826,451) \\
(17,304,244)\end{array}$ & $\begin{array}{r}20,846,168 \\
(2,741,453) \\
(18,104,715)\end{array}$ & $\begin{array}{r}21,336,624 \\
(3,052,864) \\
(18,283,760)\end{array}$ \\
\hline $\begin{array}{c}\text { Exports. } \\
\text { Intransit Deliveries . } \\
\text { Additions to Storage }\end{array}$ & $\begin{array}{r}87,108 \\
362,090\end{array}$ & $\begin{array}{l}132,130 \\
370,565\end{array}$ & $\begin{array}{l}220,211 \\
494,912\end{array}$ & $\begin{array}{l}142,405 \\
329,229\end{array}$ & $\begin{array}{l}163,522 \\
477,710\end{array}$ \\
\hline 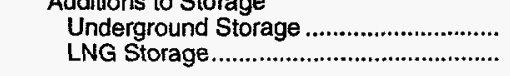 & $\begin{array}{r}2,508,886 \\
67,855\end{array}$ & $\begin{array}{r}2,686,624 \\
65,157\end{array}$ & $\begin{array}{r}2,632,055 \\
45,354\end{array}$ & $\begin{array}{r}2,834,251 \\
77,248\end{array}$ & $\begin{array}{r}2,874,574 \\
70,395\end{array}$ \\
\hline Total Disposition & $2: 2,321,197$ & $22,860,687$ & $23,523,226$ & $24,229,301$ & $24,922,825$ \\
\hline
\end{tabular}

NA $=$ Not Avaitable

Notes: See accompanying text for conversion factor documentation. Items appearing in parentheses are subsets of other items for which data are shown in this table and are not involved in the summing of supply and disposition. Totals may not equal stim of components due to independent rounding.

Sources: Eneroy Information Administration (EIA). Form EIA-1527, "Annual Quantity and Value of Natural Gas Report"; Form E|A-176, "Annual Report of Natural and Supplemental Gas Supply and Disposition"; Form ElA-64A. "Annual Repont of the Origin of Natural Gas Liquids Production"; Form FPC-14. "Annual Report for Importers and Exporters of Natural Gas"; Forms EIA-191/FERC-8, "Underground Gas Storage Report"; and Forma EIA-767, "Steam-Electric Plant Operating and Design Report." 
Appendix C

Selected

Natural Gas

and Related

Reports 



\section{Selected Natural Gas and Related Reports}

\section{Recurring Natural Gas Reports}

- Natural Gas Monthly, DOE/EIA-0130. Published monthly.

Other Reports Covering Natural Gas, Natural Gas Liquids, and Other Energy Sources

- Monthly Energy Review, DOE/EIA-0035. Published monthly. Provides national aggregate data for natural gas, natural gas liquids, and other energy sources.

- Short-Term Energy Outlook, DOE/EIA-0202. Published quarterly. Provides forecasts for next six quarters for natural gas and other energy sources.

- Natural Gas 1995: Issues and Trends, DOE/EIA0560(20), November 1995.

- U.S. Crude Oil, Natural Gas, and Natural Gas Liquids Reserves -1994 Annual Report, DOE/EIA-0216(94), September 1995.

- Annual Energy Review 1994, DOE/ EIA-0384(93), July 1995. Published annually.

- State Energy Data Report, Consumption Estimates, 1960-1992, DOE/EIA-0214(93), July 1995.

- Annual Report to Congress 1994, DOE/ EIA0173(94), April 1995. Published annually.

- Annual Energy Outlook 1995, DOE/ EIA-0383(95), January 1995. Published annually.
- State Energy Price and Expenditure Report 1992, DOE/EIA-0376(92), December 1994.

\section{One-Time Reports}

- The Value of Underground Storage in Today's Natural Gas Industry, DOE/EIA-0591, March 1995.

- U.S. Production of Natural Gas from Tight Reservoirs, DOE/EIA-TR-0574, October 1993.

- Natural Gas Productive Capacity for the Lower 48 States 1980 Through 1995, DOE/EIA-0542(95), July 1994.

- Largest U.S. Oil and Gas Fields, DOE/EIA-TR-0567, August 1993.

- Geologic Distributions of U.S. Oil and Gas, DOE/EIA0557, July 1992 .

- Capacity and Service on the Interstate Natural Gas Pipeline System 1990, DOE/EIA-0556, June 1992.

\section{Reference Reports}

- Directory of Energy Data Collection Forms, DOE/ EIA-0249(93), December 1993.

- Oil and Gas Field Code Master List, 1993, EIA0370(93), December 1993. 


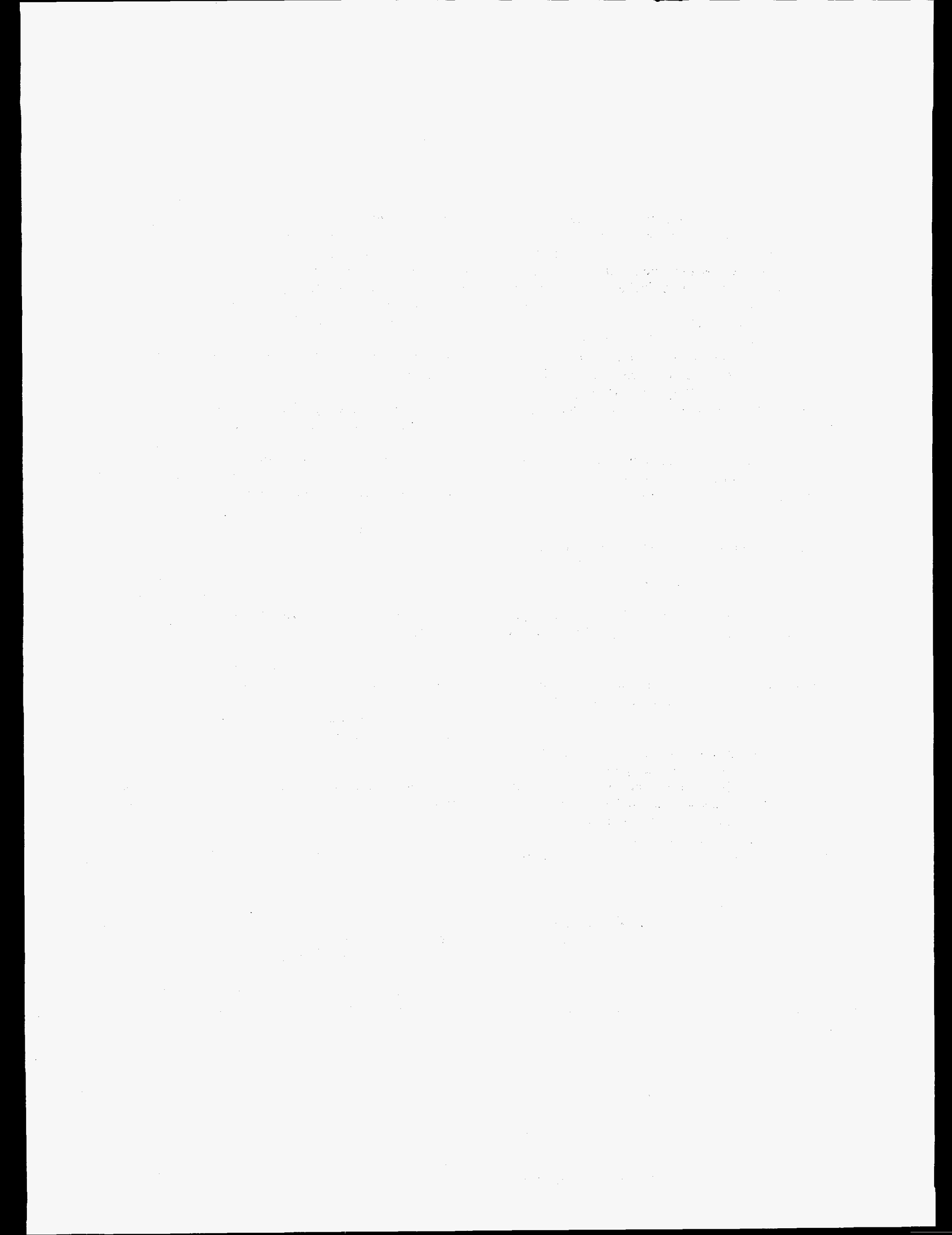




\section{Glossary}

Balancing Item: Represents differences between the sum of the components of natural gas supply and the sum of the components of natural gas disposition. These differences may be due to quantities lost or to the effects of data-reporting problems. Reporting problems include differences due to the net result of conversions of flow data metered at varying temperature and pressure bases and converted to a standard temperature and pressure base; the effect of variations in company accounting and billing practices; differences between billing cycle and calendar period time frames; and imbalances resulting from the merger of datareporting systems that vary in scope, format, definitions, and type of respondents.

Biomass Gas: A medium Btu gas containing methane and carbon dioxide, resulting from the action of microorganisms on organic materials such as a landfill.

British Thermal Unit (Btu): The quantity of heat required to raise the temperature of 1 pound of water by 1 degree Fahrenheit at or near 39.2 degrees Fahrenheit.

City Gate: A point or measuring station at which a gas distribution company receives gas from a pipeline company or transmission system.

Coke Oven Gas: The gaseous portion of volatile substances driven off in the coking process after other coal chemicals are removed.

Commercial Consumption: Gas used by nonmanufacturing establishments or agencies primarily engaged in the sale of goods or services. Included are such establishments as hotels, restaurants, wholesale and retail stores and other service enterprises; gas used by establishments engaged in agriculture, forestry, and fisheries; and gas used by local, State, and Federal agencies engaged in nonmanufacturing activities.

Delivered: The physical transfer of natural, synthetic, and/or supplemental gas from facilities operated by the responding company to facilities operated by others or to consumers.

Dry Natural Gas Production: Marketed production less extraction loss.
Electric Utility: An enterprise that is engaged in the generation, transmission, or distribution of electric energy primarily for use by the public and that is the major power supplier within a designated service area. Electric utilities include investor-owned, publicly-owned, cooperativelyowned, and government-owned (municipals, Federal agencies, State projects, and public power districts) systems.

Electric Utility Consumption: Gas used as fuel in electric utility plants.

Exports: Natural gas deliveries out of the continental United States and Alaska to foreign countries.

Extraction Loss: The reduction in volume of natural gas due to the removal of natural gas liquid constituents such as ethane, propane, and butane at natural gas processing plants.

Flared: Natural gas burned in flares at the base site or at gas-processing plants.

Gas Condensate Well: A gas well that produces from a gas reservoir containing considerable quantities of liquid hydrocarbons in the pentane and heavier range generally described as "condensate."

Gas Well: A well completed for the production of natural gas from one or more gas zones or reservoirs.

Gross Withdrawals: Full well-stream volume, including all natural gas plant liquids and all nonhydrocarbon gases, but excluding lease condensate.

Heating Value: The average number of British thermal units per cubic foot of natural gas as determined from tests of fuel samples.

Imports: Gas receipts into the United States from a foreign country.

Industrial Consumers: Establishments engaged in a process which creates or changes raw or unfinished materials into another form or product. Generation of electricity, other than by electric utilities, is included.

Industrial Consumption: Natural gas used by manufacturing and mining establishments for heat, power, and chemical feedstock. 
Intransit Deliveries: Redeliveries to a foreign country of foreign gas received for transportation across U.S. territory and deliveries of U.S. gas to a foreign country for transportation across its territory and redelivery to the United States.

Intransit Receipts: Receipts of foreign gas for transportation across U.S. territory and redelivery to a foreign country and redeliveries to the United States of U.S. gas transported across foreign territory.

Lease Fuel: Natural gas used in well, field, and lease operations (such as gas used in drilling cperations, heaters, dehydrators, and field compressors).

Liquefied Natural Gas (LNG): Natural gas (primarily methane) that has been liquefied by reducing its temperature to minus 260 degrees Fahrenheit at atmospheric pressure.

Manufactured Gas: A gas obtained by clestructive distillation of coal, or by the thermal decomposition of oil, or by the reaction of steam passing through a bed of heated coal or coke. Examples are coal gases, coke oven gases, producer gas, blast furnace gas, blue (water) gas, carbureted water gas. Btu content varies widely.

Marketed Production: Gross withdrawals less gas used for repressuring, quantities vented and flared, and nonhydrocarbon gases removed in treating or processing operations.

Natural Gas: A mixture of hydrocarbon compounds and small quantities of various nonhydrocarbons existing in the gaseous phase or in solution with crude oil in natural underground reservoirs at reservoir conditions.

Natural Gas, Wet After Lease Separation: The volume of natural gas remaining after removal of lease condensate in lease and/or field separation facilities, if any, and after exclusion of nonhydrocarbon gases where they occur in sufficient quantity to render the gas unmarketable. Natural gas liquids may be recovered from volumes of natural gas, wet after lease separation, at natural gas processing plants.

Nonhydrocarbon Gases: Typical nonhydrocarbon gases which may be present in reservoir natural gras, such as carbon dioxide, helium, hydrogen sulfide, and nitrogen.

Nonutility Power Producers: A comprany which owns electric generating capacity and is not an electric utility. Nonutility power producers include qualifying cogenerators, qualifying small power producers, and other nonutility generators (including independent power producers).

Offshore Reserves and Production: Unless otherwise indicated, reserves and production that are in either State or Federal domains, located seaward of the coastline.
Oil Well (Casinghead) Gas: Associated and dissolved gas produced along with crude oil from oil completions.

Outer Continental Shelf: Offshore Federal domain.

Pipeline: A continuous pipe conduit, complete with such equipment as valves, compressor stations, communications systems, and meters, for transporting natural and/or supplemental gas from one point to another, usually from a point in or beyond the producing field or processing plant to another pipeline or to points of use. Also refers to a company operating such facilities.

Pipeline Fuel: Gas consumed in the operation of pipelines, primarily in compressors.

Plant Fuel: Natural gas used as fuel in natural gas processing plants.

Production, Wet After Lease Separation: Gross withdrawals less gas used for repressuring and nonhydrocarbon gases removed in treating or processing operations.

Propane-air: A mixture of propane and air resulting in a gaseous fuel suitable for pipeline distribution.

Proved Reserves: The estimated quantities that analysis of geological and engineering data demonstrate with reasonable certainty to be recoverable in future years from known oil and gas reservoirs under existing economic and operating conditions.

Receipts: Gas physically transferred into the responding company's transportation, storage, and/or distribution facilities.

Refinery Gas: Noncondensate gas collected in petroleum refineries.

Repressuring: The injection of gas into oil or gas reservoir formations to effect greater ultimate recovery.

Residential Consumption: Gas used in private dwellings, including apartments, for heating, air-conditioning, cooking, water heating, and other household uses.

Storage Additions: Volumes of gas injected or otherwise added to underground natural gas reservoirs or liquefied natural gas storage.

Storage Withdrawals: Volumes of gas withdrawn from underground storage or liquefied natural gas storage. 
Supplemental Gaseous Fuels Supplies: Synthetic natural gas, propane-air, coke oven gas, refinery gas, biomass gas, air injected for Btu stabilization, and manufactured gas commingled and distributed with natural gas.

Synthetic Natural Gas (SNG): A manufactured product chemically similar in most respects to natural gas, resulting from the conversion or reforming of petroleum hydrocarbons or from coal gasification. It may easily be substituted for or interchanged with pipeline quality natural gas.

Therm: One hundred thousand British thermal units.

Underground Storage: The storage of natural gas in underground reservoirs at a different location from which it was produced.
Underground Storage Injections: Gas from extraneous sources put into underground storage reservoirs.

Underground Storage Withdrawals: Gas removed from underground storage reservoirs.

Unit Value, Consumption: Total price per specified unit, including all taxes, at the point of consumption.

Unit Value, Wellhead: The wellhead sales price, including charges for natural gas plant liquids subsequently removed from the gas, gathering and compression charges, and State production, severance, and/or similar charges.

Vented: Gas released into the air on the base site or at processing plants. 


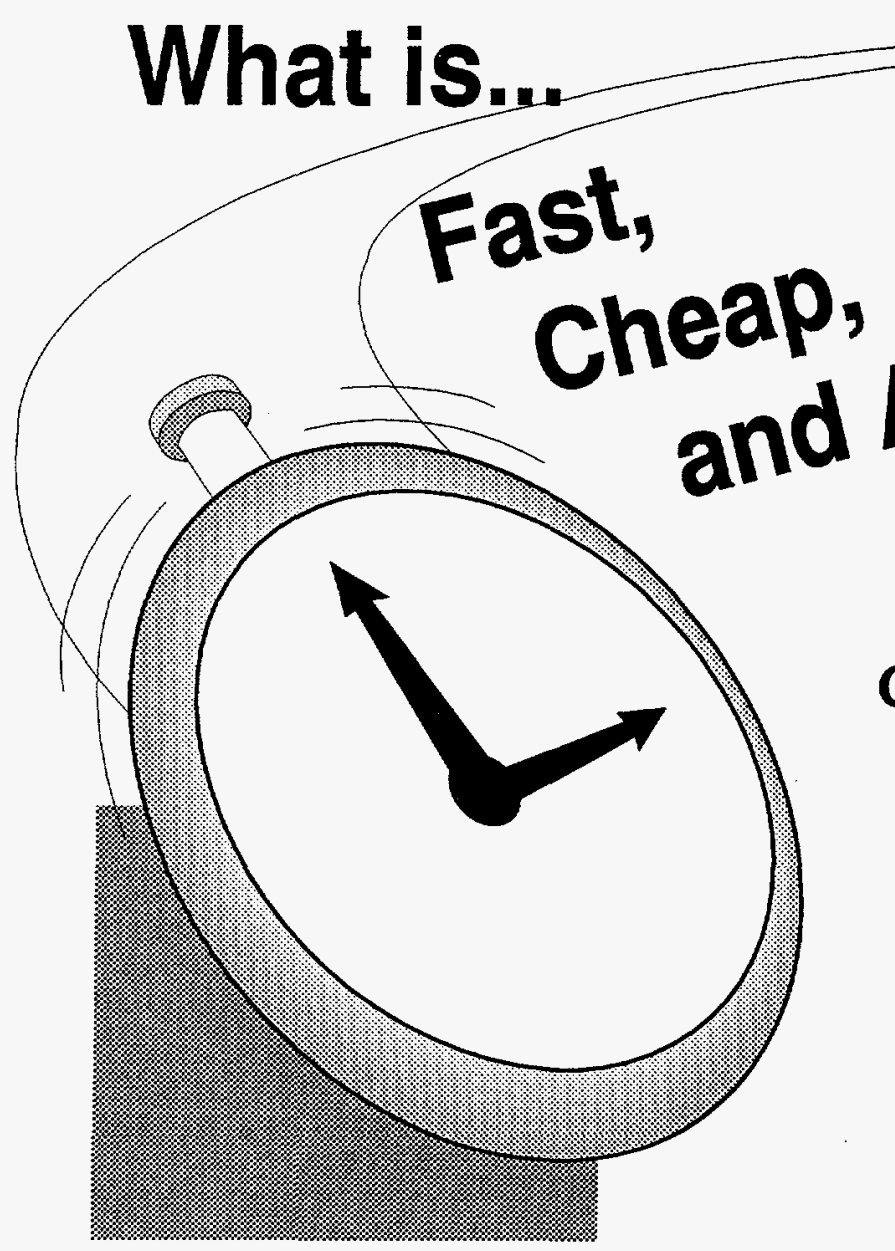

\section{Available NOW?!

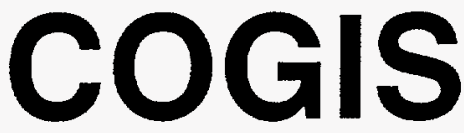

COGIS (Comprehensive Oil and Gas Information Source) offers the latest oil and gas data published by the Energy Information Administration (EIA) through the Economic Bulletin Board (EBB). Selected data series from the Petroleum Supply Monthly, the Petroleum Marketing Monthly, the Natural Gas Monthly, the Weekly Petroleum Status Report, and the Winter Fuels Report are now available.

Why wait days or weeks, when current data are available now? Anyone with a workstation connected to an Internet node, or with a personal computer and modem, can have immediate access to oil and gas industry information.

For information, call EIA's National Energy Information Center, 202-586-8800. To open an account, call the U.S. Department of Commerce, Office of Business Analysis, 202-482-1986.

Current fee schedule is listed below.
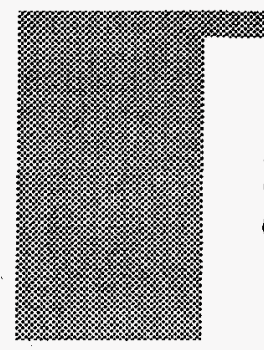

\section{Charge}

Annual Fee

Connect Charge Credit

Connect Charges (per minute based on

eastern time)

Weekdays: 8:00 a.m. - noon

Noon - 6:00 p.m.

6:00 p.m. - 8:00 a.m.

(Also weekends and holidays)

\section{Means Used to Access the EBB}

\begin{tabular}{ccc}
\hline Up to 2400 Baud & 9600 Baud & Internet (telnet only) \\
$\$ 45.00$ & $\$ 45.00$ & $\$ 45.00$ \\
$\$ 20.00$ & $\$ 20.00$ & $\$ 20.00$
\end{tabular}

$\$ 0.20$

$\$ 0.15$

$\$ 0.40$

$\$ 0.25$

$\$ 0.40$

$\$ 0.05$

$\$ 0.10$

$\$ 0.25$

$\$ 0.10$

Annual Flat Fee Option (cannot use

account between 8:00 a.m. and noon)

Maximum 1 hour per day

Maximum 4 hours per day 